
Technology Administration

National Institute of Standards and Technology

\author{
NISTIR 5093
}

Experimental Thermal Conductivity Values for Mixtures of R32, R125, R134a, and Propane
R.A. Perkins
E. Schwarzberg
X. Gao

OC

100

.456

N0.5093

1999 



\title{
Experimental Thermal Conductivity Values for Mixtures of R32, R125, R134a, and Propane
}

\author{
R.A. Perkins \\ E. Schwarzberg \\ X. Gao
}

Physical and Chemical Properties Division Chemical Science and Technology Laboratory National Institute of Standards and Technology Boulder, Colorado 80303-3328

Final Report

December 1999

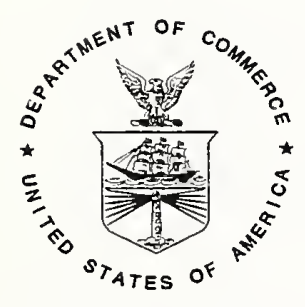

U.S. DEPARTMENT OF COMMERCE, William M. Daley, Secretary TECHNOLOGY ADMINISTRATION, Dr. Cheryl L. Shavers, Under Secretary of Commerce for Technology NATIONAL INSTITUTE OF STANDARDS AND TECHNOLOGY, Raymond G. Kammer, Director 



\section{CONTENTS}

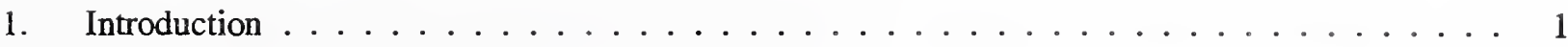

2. Transient Results Using Anodized Tantalum Wires . . . . . . . . . . . . . . 3

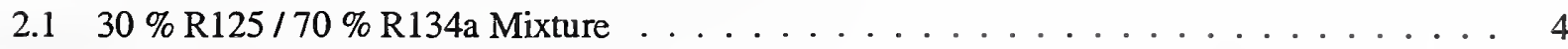

$2.270 \% \mathrm{R} 125 / 30 \% \mathrm{R} 134 \mathrm{a}$ Mixture $\ldots \ldots \ldots \ldots \ldots \ldots \ldots \ldots$

$2.330 \% \mathrm{R} 32 / 70 \%$ Propane Mixture . . . . . . . . . . . . . . . . 6

$2.470 \% \mathrm{R} 32 / 30 \%$ Propane Mixture . . . . . . . . . . . . . . . . . . 7

$2.530 \% \mathrm{R} 32 / 70 \% \mathrm{R} 134 \mathrm{a}$ Mixture $\ldots \ldots \ldots \ldots \ldots \ldots \ldots$

$2.670 \% \mathrm{R} 32 / 30 \% \mathrm{R} 134 \mathrm{a}$ Mixture $\ldots \ldots \ldots \ldots \ldots \ldots \ldots \ldots$

$2.730 \% \mathrm{R} 134 \mathrm{a} / 70 \%$ Propane Mixture . . . . . . . . . . . . . . . 10

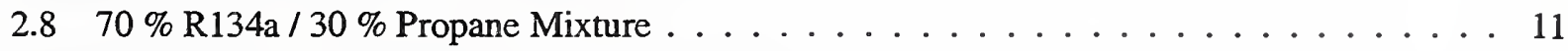

$2.933 \% \mathrm{R} 32 / 33 \% \mathrm{R} 125 / 33 \% \mathrm{R} 134 \mathrm{a}$ Mixture $\ldots \ldots \ldots \ldots \ldots \ldots \ldots \ldots$

$2.1030 \% \mathrm{R} 32 / 10 \% \mathrm{R} 125 / 60 \% \mathrm{R} 134 \mathrm{a}$ Mixture . . . . . . . . . . . . . . . . . . . . . . . . .

3. Transient Results Using Bare Tungsten Wires . . . . . . . . . . . . . . . 14

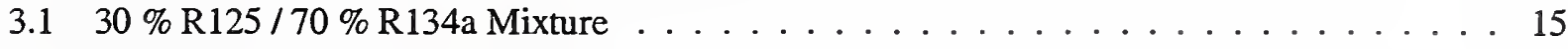

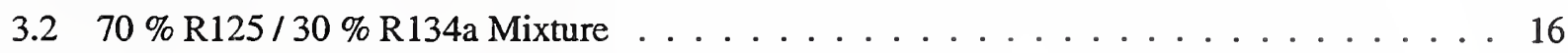

$3.330 \% \mathrm{R} 32 / 70 \%$ Propane Mixture . . . . . . . . . . . . . . . . . 17

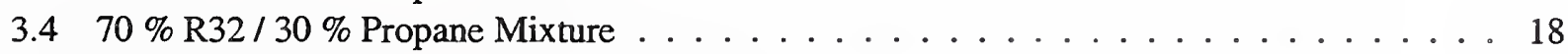

$3.530 \% \mathrm{R} 32 / 70 \% \mathrm{R} 134 \mathrm{a}$ Mixture $\ldots \ldots \ldots \ldots \ldots \ldots \ldots \ldots$

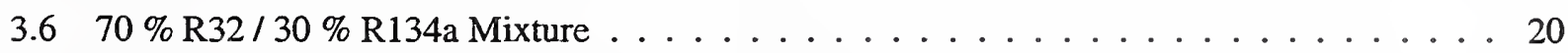

$3.730 \% \mathrm{R} 134 \mathrm{a} / 70 \%$ Propane Mixture . . . . . . . . . . . . . . . 21

$3.870 \% \mathrm{R} 134 \mathrm{a} / 30 \%$ Propane Mixture . . . . . . . . . . . . . . . 22

$3.933 \% \mathrm{R} 32 / 33 \% \mathrm{R} 125 / 33 \% \mathrm{R} 134 \mathrm{a}$ Mixture $\ldots \ldots \ldots \ldots \ldots \ldots$

$3.1030 \% \mathrm{R} 32 / 10 \% \mathrm{R} 125 / 60 \% \mathrm{R} 134 \mathrm{a}$ Mixture . . . . . . . . . . . . . . . 24

4. Steady-State Results Using Bare Tungsten Wires . . . . . . . . . . . . . . . . . . 25

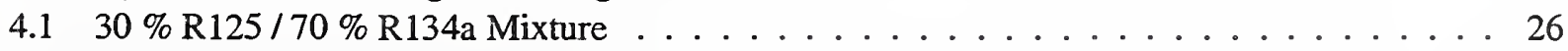

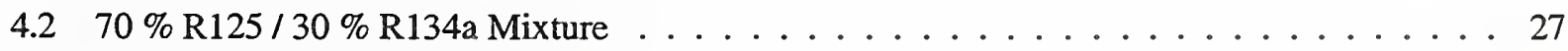

$4.330 \% \mathrm{R} 32 / 70 \%$ Propane Mixture . . . . . . . . . . . . . . . . 28

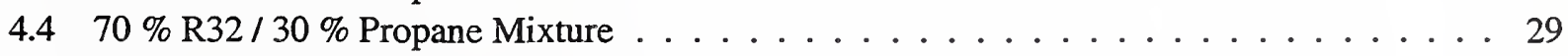

$4.530 \% \mathrm{R} 32 / 70 \% \mathrm{R} 134 \mathrm{a}$ Mixture . . . . . . . . . . . . . . . . 30

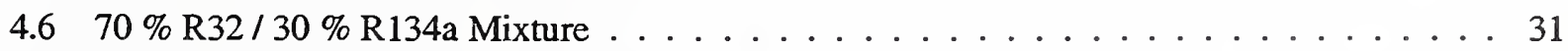

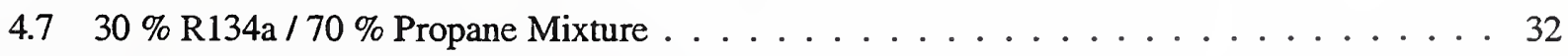

$4.870 \% \mathrm{R} 134 \mathrm{a} / 30 \%$ Propane Mixture . . . . . . . . . . . . . . 33

$4.933 \% \mathrm{R} 32 / 33 \% \mathrm{R} 125 / 33 \% \mathrm{R} 134 \mathrm{a}$ Mixture . . . . . . . . . . . . . . . . 34

$4.1030 \% \mathrm{R} 32 / 10 \% \mathrm{R} 125 / 60 \% \mathrm{R} 134 \mathrm{a}$ Mixture . . . . . . . . . . . . . . . . . . . . . . .

5. Summary of Deviations Relative to REFPROP $6.01 \ldots \ldots \ldots \ldots$

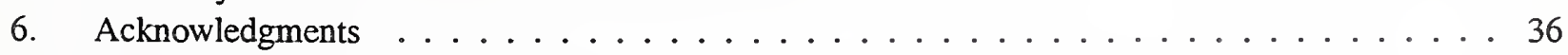

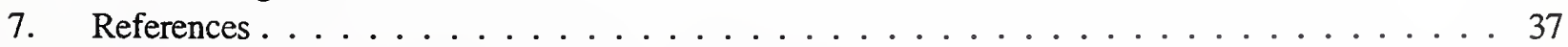

8. Tables of transient results for anodized tantalum wires . . . . . . . . . . . . . . . 39

9. Tables of transient results for bare tungsten wires . . . . . . . . . . . . . . . . . . 84

10. Tables of steady-state results for bare tungsten wires . . . . . . . . . . . . 137 


\section{List of Tables}

Table 1. Summary of deviations between the thermal conductivity data for each mixture and REFPROP 6.01.

Table 2. Thermal conductivity of the binary $30 \% \mathrm{R} 125 / 70 \% \mathrm{R} 134 \mathrm{a}$ mixture in the compressed liquid phase. . . . . . . . . . . . . . . . . . . 39

Table 3. Thermal conductivity of the binary $70 \% \mathrm{R} 125 / 30 \% \mathrm{R} 134 \mathrm{a}$ mixture in the compressed liquid phase. . . . . . . . . . . . . . . . . . 43

Table 4. Thermal conductivity of the binary $30 \% \mathrm{R} 32 / 70 \%$ propane mixture in the compressed

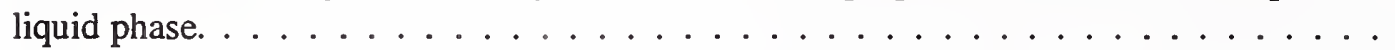

Table 5. Thermal conductivity of the binary $70 \% \mathrm{R} 32 / 30 \%$ propane mixture in the compressed liquid phase. . . . . . . . . . . . . . . . . . . 51

Table 6. Thermal conductivity of the binary $30 \%$ R $32 / 70 \%$ R134a mixture in the compressed liquid phase. . . . . . . . . . . . . . . . . . 55

Table 7. Thermal conductivity of the binary $70 \% \mathrm{R} 32 / 30 \% \mathrm{R} 134 \mathrm{a}$ mixture in the compressed liquid phase. . . . . . . . . . . . . . . . . . . . 60

Table 8 . Thermal conductivity of the binary $30 \% \mathrm{R} 134 \mathrm{a} / 70 \%$ propane mixture in the compressed liquid phase. . . . . . . . . . . . . . . . . . . 63

Table 9. Thermal conductivity of the binary $70 \% \mathrm{R} 134 \mathrm{a} / 30 \%$ propane mixture in the compressed liquid phase obtained with wire assembly $1 . \ldots \ldots \ldots 7$

Table 10. Thermal conductivity of the binary $70 \% \mathrm{R} 134 \mathrm{a} / 30 \%$ propane mixture in the compressed liquid phase obtained with wire assembly $2 \ldots \ldots \ldots . \ldots \ldots$

Table 11. Thermal conductivity of the ternary $33 \% \mathrm{R} 32 / 33 \% \mathrm{R} 125 / 33 \% \mathrm{R} 134 \mathrm{a}$ mixture in the compressed liquid phase. . . . . . . . . . . . . . . . . 74

Table 12. Thermal conductivity of the ternary $30 \% \mathrm{R} 32 / 10 \% \mathrm{R} 125$ / $60 \% \mathrm{R} 134 \mathrm{a}$ mixture in the compressed liquid phase. . . . . . . . . . . . . . . . . 79

Table 13. Thermal conductivity of the binary $30 \% \mathrm{R} 125 / 70 \% \mathrm{R} 134 \mathrm{a}$ mixture in the vapor phase. . 84

Table 14. Thermal conductivity of the binary $70 \% \mathrm{R} 125 / 30 \% \mathrm{R} 134 \mathrm{a}$ mixture in the vapor phase. . 90

Table 15. Thermal conductivity of the binary $30 \%$ R $32 / 70 \%$ propane mixture in the vapor phase. . 95

Table 16. Thermal conductivity of the binary $70 \%$ R $32 / 30 \%$ propane mixture in the vapor phase. . 99

Table 17. Thermal conductivity of the binary $30 \% \mathrm{R} 32 / 70 \% \mathrm{R} 134$ a mixture in the vapor phase. . . 109

Table 18. Thermal conductivity of the binary $70 \% \mathrm{R} 32 / 30 \% \mathrm{R} 134 \mathrm{a}$ mixture in the vapor phase. . 115

Table 19. Thermal conductivity of the binary $30 \% \mathrm{R} 134 \mathrm{a} / 70 \%$ propane mixture in the vapor phase. 121

Table 20. Thermal conductivity of the binary $70 \% \mathrm{R} 134 \mathrm{a} / 30 \%$ propane mixture in the vapor phase. 123

Table 21. Thermal conductivity of the ternary $33 \%$ R32 / $33 \%$ R125/33\% R134a mixture in the vapor phase. . . . . . . . . . . . . . . . . . . . . . . 129

Table 22. Thermal conductivity of the ternary $30 \% \mathrm{R} 32 / 10 \% \mathrm{R} 125$ / $60 \% \mathrm{R} 134 \mathrm{a}$ mixture in the vapor phase. . . . . . . . . . . . . . . . . . . . 131

Table 23. Thermal conductivity of the binary $30 \% \mathrm{R} 125 / 70 \% \mathrm{R} 134 \mathrm{a}$ mixture in the dilute gas measured with the steady-state technique. . . . . . . . . . . . 137

Table 24. Thermal conductivity of the binary $70 \% \mathrm{R} 125 / 30 \% \mathrm{R} 134 \mathrm{a}$ mixture in the dilute gas measured with the steady-state technique. . . . . . . . . . . . . 141

Table 25. Thermal conductivity of the binary $30 \% \mathrm{R} 32 / 70 \%$ propane mixture in the dilute gas measured with the steady-state technique. . . . . . . . . . . . . 146

Table 26. Thermal conductivity of the binary $70 \%$ R32/30\% propane mixture in the dilute gas measured with the steady-state technique. . . . . . . . . . . . . 152 
Table 27. Thermal conductivity of the binary $30 \% \mathrm{R} 32 / 70 \% \mathrm{R} 134 \mathrm{a}$ mixture in the dilute gas measured with the steady-state technique with bare tungsten hot wires. . . . . . . . . 157

Table 28. Thermal conductivity of the binary $70 \% \mathrm{R} 32$ / $30 \% \mathrm{R} 134 \mathrm{a}$ mixture in the dilute gas measured with the steady-state technique. . . . . . . . . . . . 164

Table 29. Thermal conductivity of the binary $30 \% \mathrm{R} 134 \mathrm{a} / 70 \%$ propane mixture in the dilute gas measured with the steady-state technique. . . . . . . . . . . . 171

Table 30. Thermal conductivity of the binary $70 \% \mathrm{R} 134 \mathrm{a} / 30 \%$ propane mixture in the dilute gas measured using the steady-state technique. . . . . . . . . . . 173

Table 31. Thermal conductivity of the ternary $33 \% \mathrm{R} 32$ / $33 \% \mathrm{R} 125$ / $33 \%$ R134a mixture in the dilute gas measured using the steady-state technique. . . . . . . . . . . . . . 179

Table 32. Thermal conductivity of the ternary $30 \%$ R 32 / $10 \%$ R 125 / $60 \%$ R 134 a mixture in the dilute gas measured using the steady-state technique. . . . . . . . . . . . 182 


\section{List of Figures}

Figure 1. Relative deviations of the thermal conductivity data for the binary $30 \%$ R125/70\% R134a mixture in the compressed liquid phase from the predictions of REFPROP 6.01. . .

Figure 2. Relative deviations of the thermal conductivity data for the binary $70 \%$ R125/30\% R134a mixture in the compressed liquid phase from the predictions of REFPROP 6.01. . .

Figure 3. Relative deviations of the thermal conductivity data for the binary $30 \%$ R32/70\% propane mixture in the compressed liquid phase from the predictions of REFPROP 6.01 .

Figure 4. Relative deviations of the thermal conductivity data for the binary $70 \%$ R32/30\% propane mixture in the compressed liquid phase from the predictions of REFPROP 6.01. .

Figure 5. Relative deviations of the thermal conductivity data for the binary $30 \%$ R 32 / $70 \%$ R $134 a$ mixture in the compressed liquid phase from the predictions of REFPROP 6.01. . . . . . 8

Figure 6. Relative deviations of the thermal conductivity data for the binary $70 \%$ R 32 / $30 \%$ R $134 a$ mixture in the compressed liquid phase from the predictions of REFPROP 6.01. . . . . .

Figure 7. Relative deviations of the thermal conductivity data for the binary $30 \% \mathrm{R} 134 \mathrm{a} / 70 \%$ propane mixture in the compressed liquid phase from the predictions of REFPROP 6.01. . 10

Figure 8. Relative deviations of the thermal conductivity data for the binary $70 \%$ R $134 \mathrm{a} / 30 \%$ propane mixture in the compressed liquid phase from the predictions of REFPROP 6.01. .

Figure 9. Relative deviations of the thermal conductivity data for the ternary $33 \% \mathrm{R} 32 / 33 \% \mathrm{R} 125$ $133 \% \mathrm{R} 134 \mathrm{a}$ mixture in the compressed liquid phase from the predictions of REFPROP

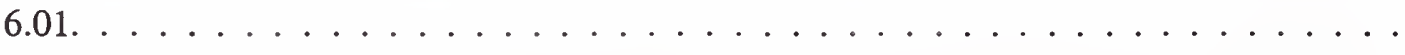

Figure 10. Relative deviations of the thermal conductivity data for the ternary $30 \%$ R32/ $10 \%$ R 125 $160 \%$ R134a mixture in the compressed liquid phase from the predictions of REFPROP

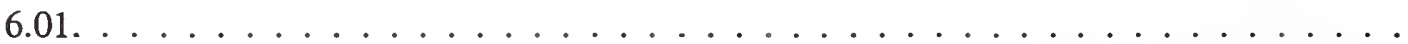

Figure 11. Relative deviations of the transient thermal conductivity data for the binary $30 \% \mathrm{R} 125$ / $70 \%$ R134a mixture in the vapor phase from the predictions of REFPROP 6.01 . . . . . 15

Figure 12. Relative deviations of the transient thermal conductivity data for the binary $70 \% \mathrm{R} 125$ / $30 \%$ R134a mixture in the vapor phase from the predictions of REFPROP 6.01. . . . . 16

Figure 13. Relative deviations of the transient thermal conductivity data for the binary $30 \%$ R32/ 70 $\%$ propane mixture in the vapor phase from the predictions of REFPROP $6.01 \ldots \ldots$

Figure 14. Relative deviations of the transient thermal conductivity data for the binary $70 \% \mathrm{R} 32$ / 30 $\%$ propane mixture in the vapor phase from the predictions of REFPROP 6.01 . . . . . . .

Figure 15. Relative deviations of the transient thermal conductivity data for the binary $30 \% \mathrm{R} 32 / 70$ $\%$ R134a mixture in the vapor phase from the predictions of REFPROP 6.01. . . . . . .

Figure 16. Relative deviations of the transient thermal conductivity data for the binary $70 \% \mathrm{R} 32$ / 30 $\%$ R134a mixture in the vapor phase from the predictions of REFPROP 6.01. . . . . . . 20

Figure 17. Relative deviations of the transient thermal conductivity data for the binary $30 \%$ R134a / $70 \%$ propane mixture in the vapor phase from the predictions of REFPROP 6.01. . . . . . 21

Figure 18. Relative deviations of the transient thermal conductivity data for the binary $70 \% \mathrm{R} 134 \mathrm{a} /$ $30 \%$ propane mixture in the vapor phase from the predictions of REFPROP 6.01. . . . . .

Figure 19. Relative deviations of the transient thermal conductivity data for the termary $33 \%$ R32 / $33 \% \mathrm{R} 125 / 33 \% \mathrm{R} 134 \mathrm{a}$ mixture in the vapor phase from the predictions of REFPROP

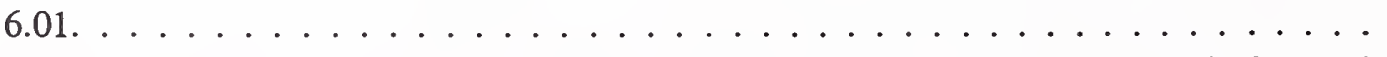

Figure 20. Relative deviations of transient thermal conductivity data for the ternary $30 \% \mathrm{R} 32 / 10 \%$ R125 / $60 \%$ R134a mixture in the vapor phase from the predictions of REFPROP 6.01. . . 
Figure 21. Relative deviations of the steady-state thermal conductivity data for the binary $30 \% \mathrm{R} 125$ $170 \%$ R134a mixture in the vapor phase from the predictions of REFPROP 6.01. . . . 26

Figure 22. Relative deviations of the steady-state thermal conductivity data for the binary $70 \% \mathrm{R} 125$ / $30 \%$ R134a mixture in the vapor phase from the predictions of REFPROP 6.01. . . . . . 27

Figure 23. Relative deviations of the steady-state thermal conductivity data for the binary $30 \% \mathrm{R} 32$ $170 \%$ propane mixture in the vapor phase from the predictions of REFPROP 6.01. . . . 28

Figure 24. Relative deviations of the steady-state thermal conductivity data for the binary $70 \%$ R32 $130 \%$ mixture in the vapor phase from the predictions of REFPROP 6.01 . . . . . . . . 29

Figure 25. Relative deviations of the steady-state thermal conductivity data for the binary $30 \%$ R32 $170 \%$ R134a mixture in the vapor phase from the predictions of REFPROP 6.01. . . . . . 30

Figure 26. Relative deviations of the steady-state thermal conductivity data for the binary $70 \%$ R32 / $30 \%$ R134a mixture in the vapor phase from the predictions of REFPROP 6.01. . . . . 31

Figure 27. Relative deviations of the steady-state thermal conductivity data for the binary $30 \% \mathrm{R} 134 \mathrm{a}$ $170 \%$ propane mixture in the vapor phase from the predictions of REFPROP 6.01. . . . . 32

Figure 28. Relative deviations of the steady-state thermal conductivity data for the binary $70 \% \mathrm{R} 134 \mathrm{a}$ / $30 \%$ propane mixture in the vapor phase from the predictions of REFPROP 6.01. . . . . 33

Figure 29. Relative deviations of the steady-state thermal conductivity data for the ternary $33 \%$ R 32 / $33 \% \mathrm{R} 125 / 33 \% \mathrm{R} 134 \mathrm{a}$ mixture in the vapor phase from the predictions of REFPROP

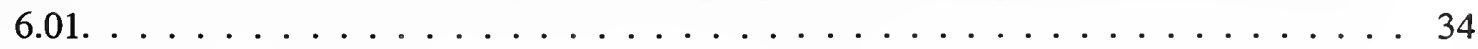

Figure 30. Relative deviations of the steady-state thermal conductivity data for the ternary $30 \% \mathrm{R} 32$ / $10 \%$ R125 / $60 \%$ R134a mixture in the vapor phase from the predictions of REFPROP

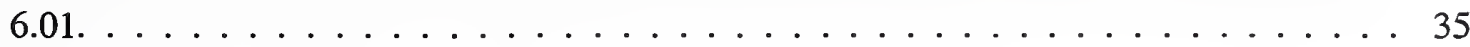





\title{
Experimental thermal conductivity values for mixtures of $\mathrm{R} 32, \mathrm{R} 125, \mathrm{R} 134 \mathrm{a}$, and propane
}

\begin{abstract}
R.A. Perkins, E. Schwarzberg, X. Gao
Experimental measurements are reported for the thermal conductivity of eight binary and two ternary mixtures containing difluoromethane (R32), pentafluoroethane (R125), 1,1,1,2tetrafluoroethane (R134a), and propane (R290). The mixtures were prepared gravimetrically from the pure components for accurate knowledge of the composition. Both the vapor and liquid phases of each mixture were studied over a temperature range from $245 \mathrm{~K}$ to $345 \mathrm{~K}$ with maximum pressures of $10 \mathrm{MPa}-20 \mathrm{MPa}$. The thermal conductivity data for the vapor phase of each mixture was obtained using transient and steady-state hot wires. Bare tungsten hot wires ( $4 \mu \mathrm{m}$ diameter) were used for the vapor-phase measurements with both the transient and steady-state modes of operation. The extremely small wire diameter minimized corrections to the transient results that account for the finite wire diameter. Steady-state measurements allowed measurements at lower pressures where the transient thermal wave penetrates to the outer cell wall in less than $1 \mathrm{~s}$. Comparison between the transient and steady-state vapor results provides a cross-check of the reliability of the vapor results. Anodized tantalum hot wires (25 $\mu \mathrm{m}$ diameter) were used for the liquid-phase measurements with the transient mode of operation. The anodized layer of tantalum pentoxide provides the electrical insulation required for accurate measurements in these highly polar and moderately electrically conducting liquids. The measurements cover the temperature region from $229 \mathrm{~K}$ to $347 \mathrm{~K}$ in the subcritical-vapor and compressed-liquid phases. A total of 5683 measurements are reported with an estimated uncertainty of $\pm 3 \%$. The data are compared with REFPROP version 6.01 , a wide range extended corresponding states correlation for the thermophysical properties of alternative refrigerants and their mixtures.
\end{abstract}

Key Words: hot wire; mixtures; propane; R32; R125; R134a; steady state; thermal conductivity; transient.

\section{Introduction}

This report is the archival record for the results of steady-state and transient hot-wire measurements on eight binary and two temary mixtures containing difluoromethane (R32), pentafluoroethane (R125), 1,1,1,2tetrafluoroethane (R134a), and propane (R290). The 10 mixtures were prepared gravimetrically from the pure gas samples. Each of the pure gas samples was verified to have a molar purity greater than 99.9 mol $\%$ using gas chromatography. All mixture compositions reported in this work are on a molar basis. The quantity of data obtained is so large that an electronically accessible version is necessary to facilitate use of the data. All of the hot-wire results described in this Interagency Report are available in an ASCII form at the NIST anonymous ftp site:

$$
\text { ftp://ftp.boulder.nist.gov/pub/fluids/NIST_Data/Hot-Wire/ }
$$

Each of the two hot-wire instruments used in this study have been described elsewhere [1,2]. These hot-wire instruments have two hot wires of different lengths, normally bare $12.7 \mu \mathrm{m}$ diameter hot wires made from platinum, which are operated in a differential mode using a Wheatstone bridge circuit to eliminate effects due to axial conduction near the ends of the wires. These transient hot-wire instruments are highly automated and cover a wide range of fluid conditions with a combined temperature range of $30 \mathrm{~K}$ to 
$750 \mathrm{~K}$ and pressures from $0.05 \mathrm{MPa}$ to $70 \mathrm{MPa}$. The low-temperature system [1] has been used previously to study the thermal conductivity of oxygen [3], hydrogen $[4,5]$, methane $[4,6]$, ethane $[4,7]$, methaneethane mixtures [8,9], propane [4,10], argon [11-15], nitrogen [11, 16], nitrogen-oxygen-argon mixtures [17], neon-nitrogen mixtures [18], R134a [19, 20], and R123 [21]. The high-temperature instrument [2] has been used to study nitrogen, argon, toluene, R134a [19, 20], and R123 [21].

The present measurements did not rely on the polarization technique [19-21] that we have used previously with bare hot wires to minimize errors due to the electrical conductivity of the liquid phase of these refrigerants. Instead, the present liquid-phase measurements used an anodized tantalum pentoxide layer to provide direct electrical insulation between the tantalum hot wires ( $25 \mu \mathrm{m}$ diameter) and the fluid [22]. The vapor phase of these refrigerant mixtures was studied tungsten hot wires of with extremely small diameter $(4 \mu \mathrm{m})$ operating in both transient and steady-state modes. The small wire diameter minimizes corrections to the transient results, while the use of two different measurement modes allows an assessment of the reliability of the measurements through intercomparison of the results. Finally, replicated measurements are made at each fixed cell temperature and pressure, with about five different applied powers, to provide a check of the reproducibility of the measurements. It should be noted that each different power level yields a thermal conductivity result at a slightly different experimental temperature, thus each individual thermal conductivity datum is reported separately rather than averaged.

Measurements of the thermal conductivity of polar mixtures are more difficult and less reliable than the measurements on nonpolar gas mixtures, such as methane and ethane, that were studied previously. Since the present mixtures are significantly below their critical point at room temperature, they must be prepared and stored as a single-phase gas, below the mixture dew point, at a relatively low pressure. The equation of state model used to predict the properties of these mixtures [23] is not nearly as accurate as those used for other pure fluids, yet is required for corrections to the transient results. Finally, the uncertainty increases due to the polar nature of the refrigerant mixtures. These polar fluids are very good solvents, with a moderate electrical conductivity. Combined, these factors lead to a degradation in the uncertainty estimated for the thermal conductivity, about $\pm 3 \%$, and thermal diffusivity is not reported since the uncertainty is estimated to be about $\pm 30 \%$. The uncertainty of the thermal diffusivity increases further in the dilute vapor phase where large corrections are required for the finite wire diameter that are based on the mixture predictions of REFPROP [23] for both density and heat capacity.

Recorded in the tables are the run-point numbers; the pressure $P_{\text {exp }}$, temperature (ITS 90) $T_{\text {exp }}$, and the calculated density $\rho_{\text {calc }}[23]$ of the fluid to which the thermal conductivity is assigned; the applied power per unit length of the wire $Q$; the experimental thermal conductivity $\lambda_{\exp }$ and its $2 \sigma$ uncertainty value (STAT); the cell temperature $T_{\text {cell }}$, which is provided to characterize the experiment temperature rise. STAT is the uncertainty of the slope, at the $2 \sigma$ level, as determined in the data reduction program $[1,15]$. STAT is a direct measure of the precision of the thermal conductivity. A STAT of 0.001 , for example, corresponds to a precision of $0.1 \%$ in thermal conductivity. The tables of steady-state hot-wire results also include the start time $t_{\text {start }}$, and end time $t_{\text {end }}$, of the steady-state temperature rise measurment that was used to determine the thermal conductivity. Also included are the average wire temperature rise $\Delta T_{\text {avg }}$, and the Rayleigh number $N_{R a}$, which characterizes the sensitivity of the fluid to the onset of convection. Larger Rayleigh numbers indicate that convection will occur at shorter experiment times and will be more significant. Finally, the precision of the steady-state experiment is characterized by TBAND which is the relative uncertainty in percent of the measured temperature rise, and hence the experimental thermal conductivity, at $3 \sigma$ confidence over the time interval from $t_{\text {start }}$ to $t_{\text {end }}$. Deviations of the thermal conductivity data from the extended corresponding states predictions in REFPROP 6.01 [23] are plotted as a function of the fluid density for the liquid measurements and as a function of temperature for the vapor measurements. 


\section{Transient Results Using Anodized Tantalum Wires}

The ten mixture supply cylinders were maintained in the single-phase gas state during storage and use. The cylinders were heated during use to increase the supply pressure and to minimize adsorption. The supply pressure was not adequate to operate the air-driven diaphragm compressors that are normally used to increase the pressure in the hot-wire cells. Thus, liquid-phase measurements required condensation of the sample into the cell at the lowest temperature studied. The cell was then pressurized solely by increasing the temperature to the next isotherm. This allowed uniform characterization of the fluid in terms of density, but restricted study to the region in the vicinity of the saturated liquid since the measurements are at relatively high reduced temperatures and the fluid becomes increasingly compressible near the critical point.

The measurements in the liquid phase were made using relatively large ( $25 \mu \mathrm{m}$ diameter) tantalum hot wires that were anodized insitu to obtain an electrically insulating layer of tantalum pentoxide for good isolation from the electrically conducting liquid phases of these mixtures. The relatively large diameter requires excessively large corrections for the finite wire diameter for the gas phase, but the magnitude of the corrections remains acceptable for liquid-phase measurements, even considering the relatively large uncertainty of the thermophysical properties used for the corrections. The densities reported in the tables have been calculated using the mixture model REFPROP 6.01 [23]. The relatively large wire diameter has the added advantage of reducing the electrical field strength in the vicinity of the wire during hot-wire measurements.

The temperature of the tantalum hot-wire cell was maintained with a refrigerated liquid circulator that allowed measurements from about $250 \mathrm{~K}$ to $350 \mathrm{~K}$. This circulator provided convenient control of the temperature over the range needed for the present measurements. Furthermore, the minimum temperature of $250 \mathrm{~K}$ allowed all of the mixtures to be condensed into the measurement cell at pressures above the bubble point of the mixtures. However, temperature gradient was observed in the measurement cell at the highest temperatures that was larger than is normally present when the measurement cells are used in a furnace. This temperature gradient made the liquids more susceptible to convection at the highest temperatures. 


\section{$2.130 \%$ R125 / $70 \%$ R134a Mixture}

The precise molar composition of the mixture was $0.30015 \mathrm{R} 125+0.69985 \mathrm{R} 134 \mathrm{a}$. Results for 142 measurements, at temperatures from $244 \mathrm{~K}$ to $347 \mathrm{~K}$, are reported in Table 1. Deviations between the measured thermal conductivity and the predictions of the mixture model REFPROP 6.01 [23] are shown in Figure 1. The lower densities correspond to the higher temperatures where the thermal conductivity critical enhancement becomes significant.

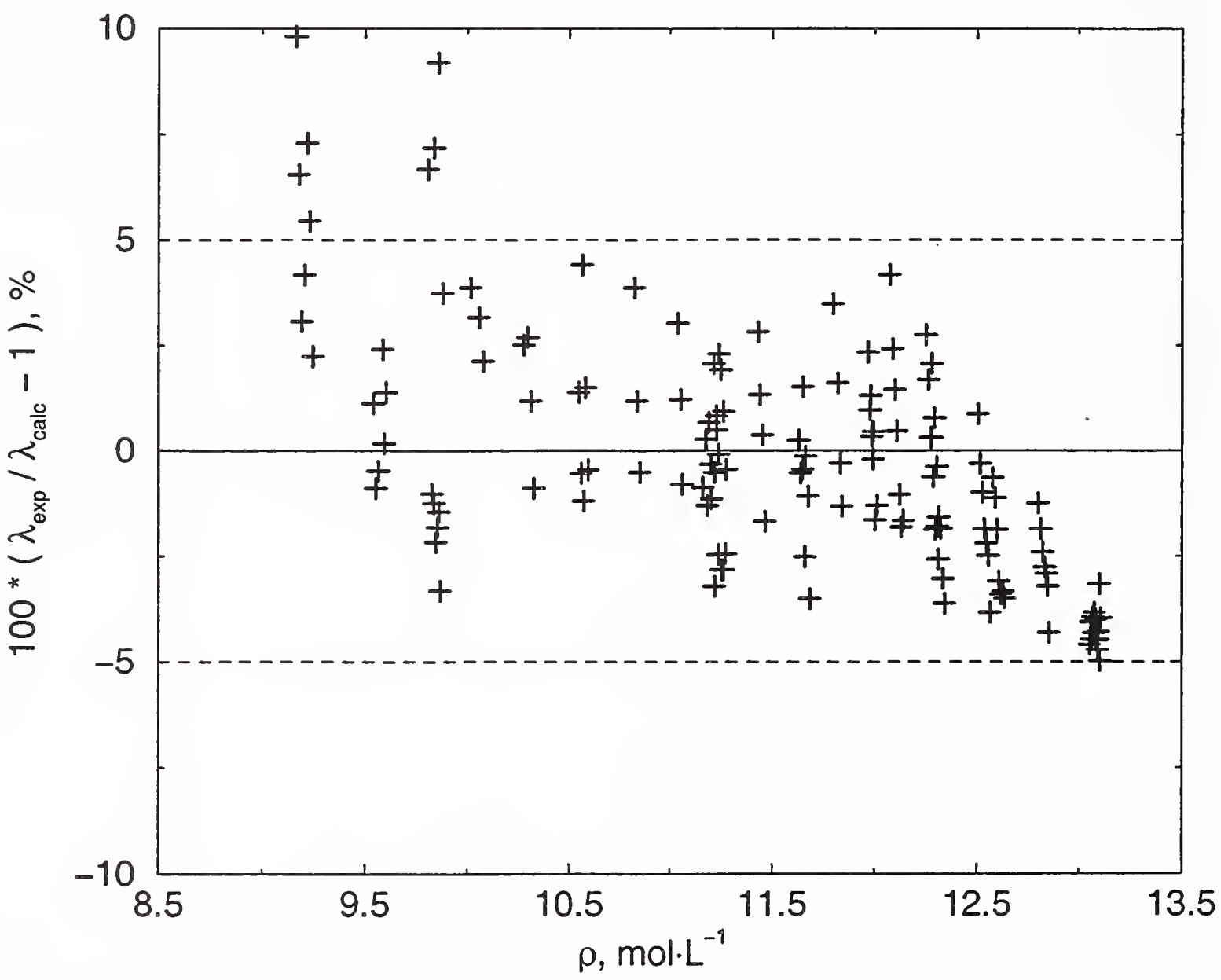

Figure 1. Relative deviations of the thermal conductivity data for the binary $30 \%$ R $125 / 70 \%$ R $134 \mathrm{a}$ mixture in the compressed liquid phase from the predictions of REFPROP 6.01. 


\section{$2.270 \%$ R125 / $30 \%$ R134a Mixture}

The precise molar composition of the mixture was $0.70000 \mathrm{R} 125+0.30000 \mathrm{R} 134 \mathrm{a}$. Results for 136 measurements, at temperatures from $247 \mathrm{~K}$ to $330 \mathrm{~K}$, are reported in Table 2 . Relative deviations between the measured thermal conductivity and the predictions of the mixture model REFPROP 6.01 [23] are shown in Figure 2. The lower densities correspond to the higher temperatures where the thermal conductivity critical enhancement becomes significant.

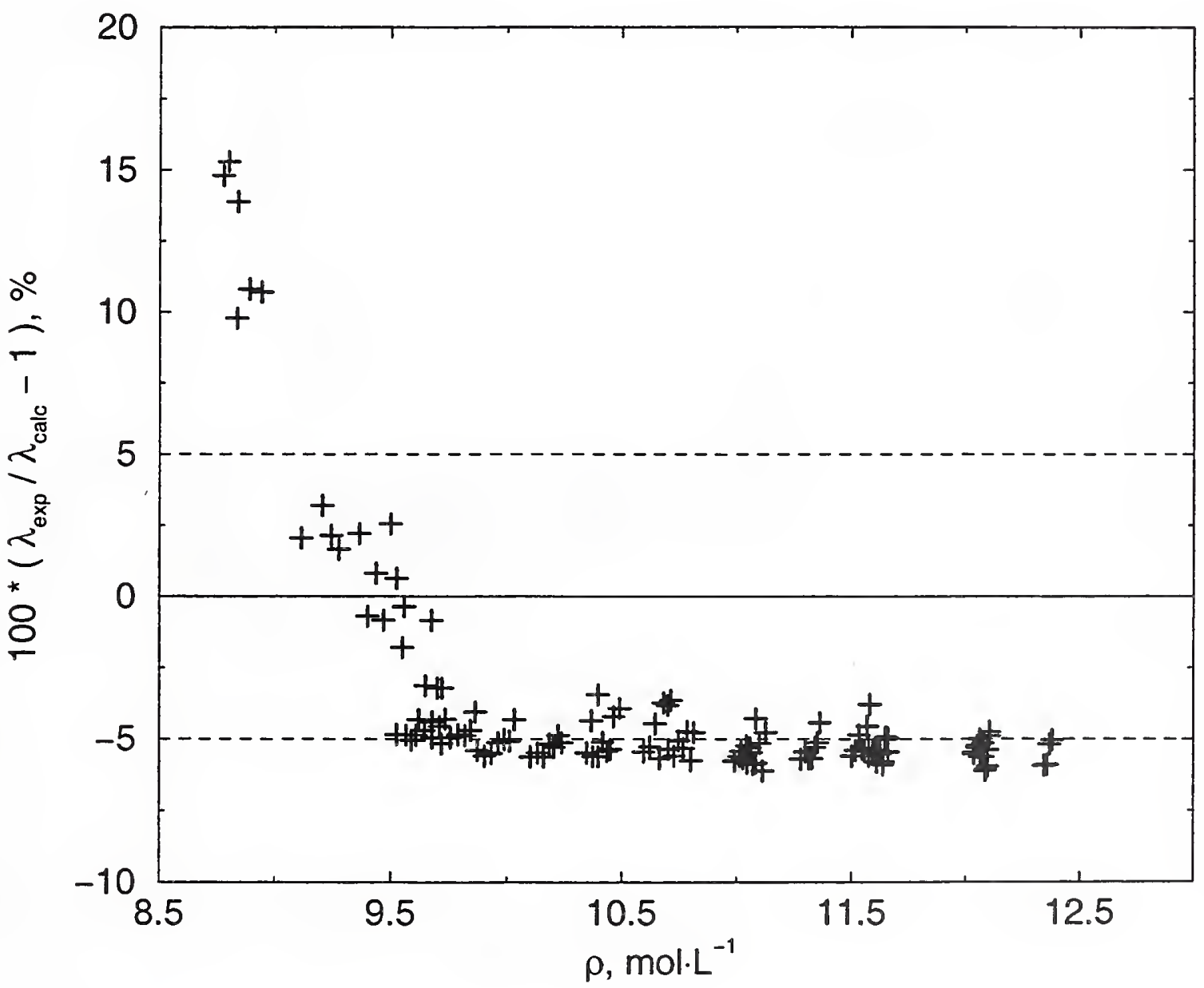

Figure 2. Relative deviations of the thermal conductivity data for the binary $70 \% \mathrm{R} 125 / 30 \% \mathrm{R} 134 \mathrm{a}$ mixture in the compressed liquid phase from the predictions of REFPROP 6.01. 


\section{$2.330 \%$ R32 / $70 \%$ Propane Mixture}

The precise molar composition of the mixture was $0.29992 \mathrm{R} 32+0.70008$ propane. Results for 159 measurements, at temperatures from $245 \mathrm{~K}$ to $347 \mathrm{~K}$, are reported in Table 3. Relative deviations between the measured thermal conductivity and the predictions of the mixture model REFPROP 6.01 [23] are shown in Figure 3. The lower densities correspond to the higher temperatures where the thermal conductivity critical enhancement becomes significant.

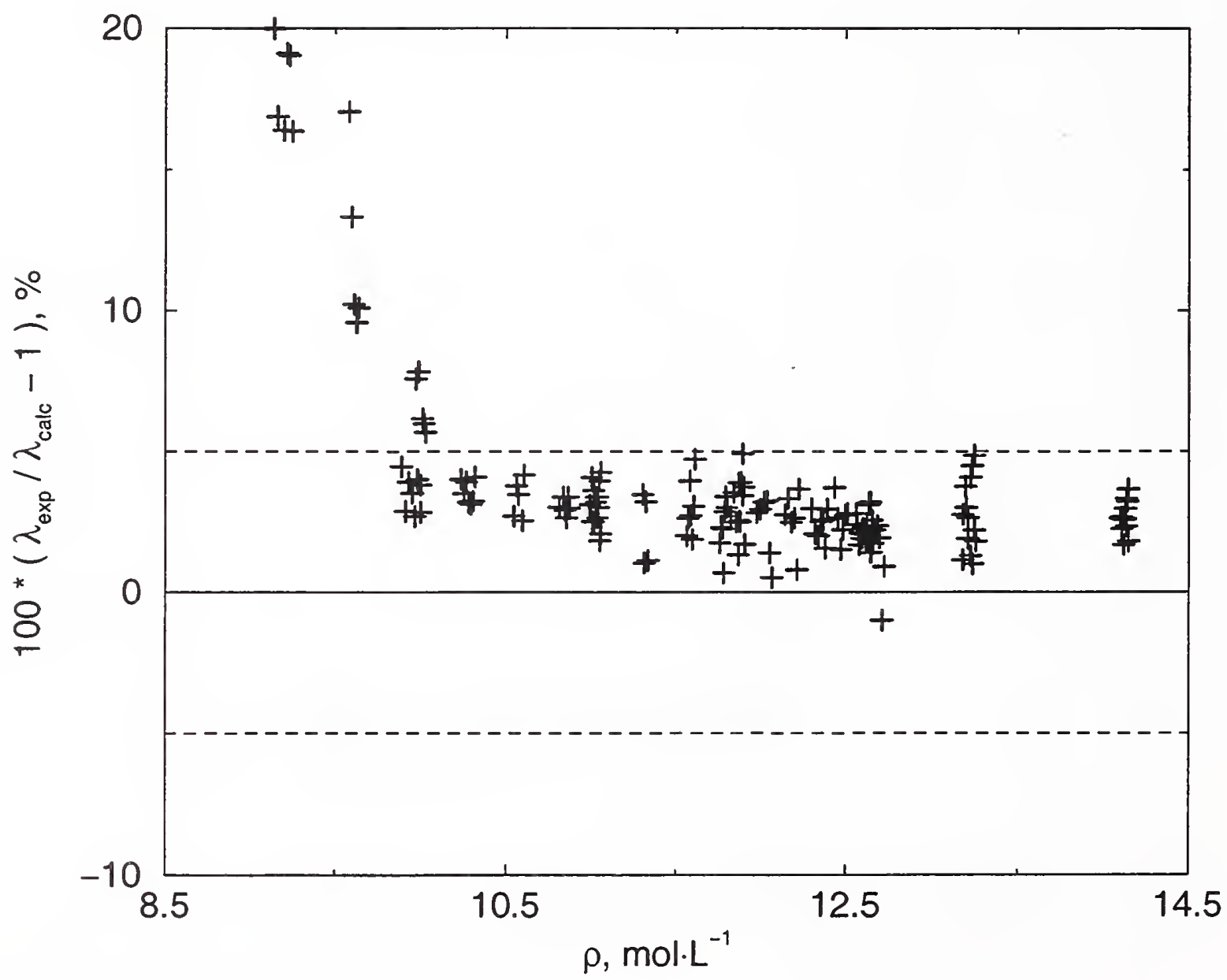

Figure 3. Relative deviations of the thermal conductivity data for the binary $30 \% \mathrm{R} 32 / 70 \%$ propane mixture in the compressed liquid phase from the predictions of REFPROP 6.01. 


\section{$2.470 \%$ R32 / $30 \%$ Propane Mixture}

The precise molar composition of the mixture was $0.70005 \mathrm{R} 32+0.29995$ propane. Results for 148 measurements, at temperatures from $254 \mathrm{~K}$ to $330 \mathrm{~K}$, are reported in Table 4 . Relative deviations between the measured thermal conductivity and the predictions of the mixture model REFPROP 6.01 [23] are shown in Figure 4. The lower densities correspond to the higher temperatures where the thermal conductivity critical enhancement becomes significant.

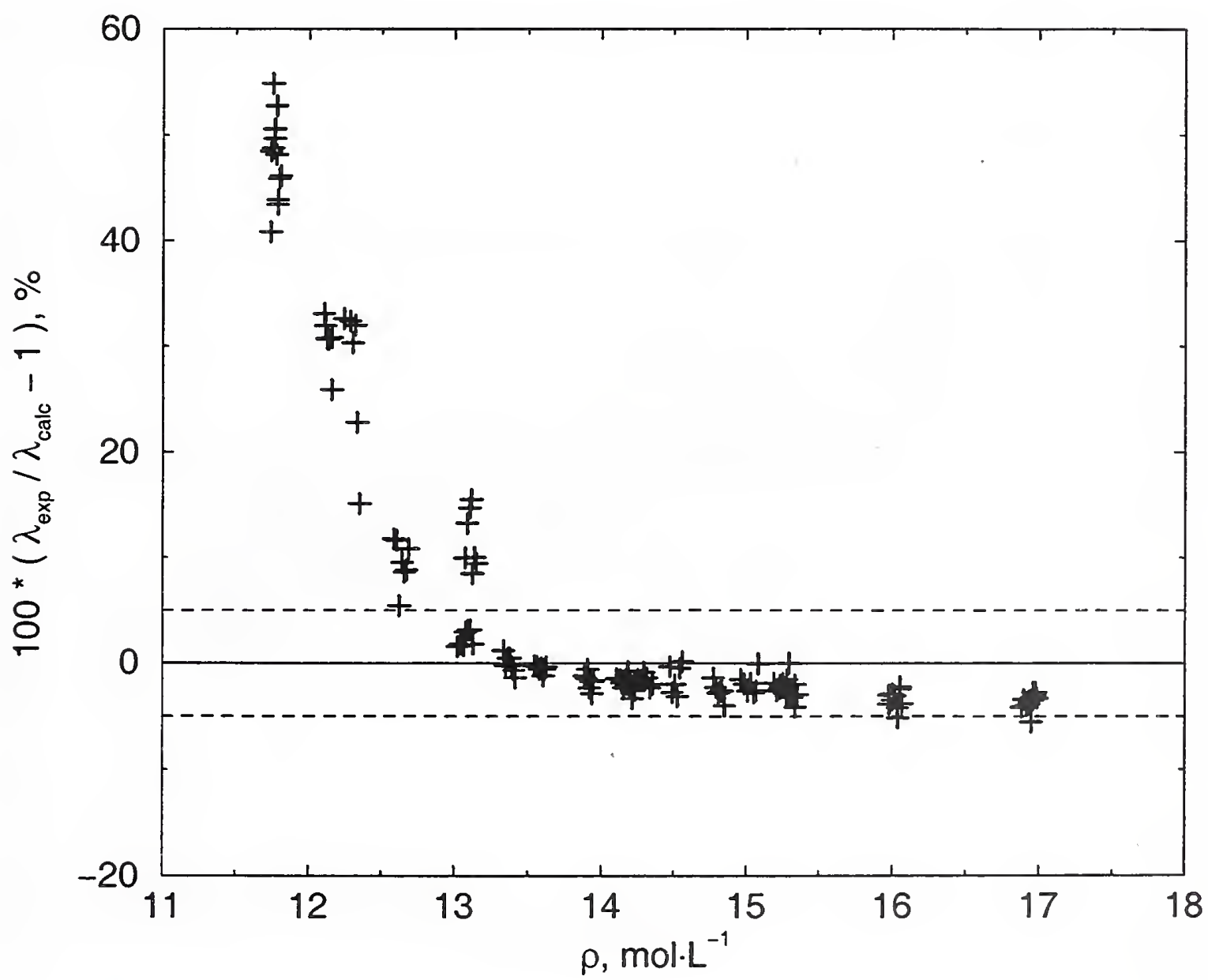

Figure 4. Relative deviations of the thermal conductivity data for the binary $70 \% \mathrm{R} 32$ / $30 \%$ propane mixture in the compressed liquid phase from the predictions of REFPROP 6.01 . 


\section{$2.530 \%$ R32 / $70 \%$ R134a Mixture}

The precise molar composition of the mixture was $0.29955 \mathrm{R} 32+0.70044 \mathrm{R} 134 \mathrm{a}$. Results for 179 measurements, at temperatures from $255 \mathrm{~K}$ to $360 \mathrm{~K}$, are reported in Table 5. Relative deviations between the measured thermal conductivity and the predictions of the mixture model REFPROP 6.01 [23] are shown in Figure 5. The lower densities correspond to the higher temperatures where the thermal conductivity critical enhancement becomes significant.

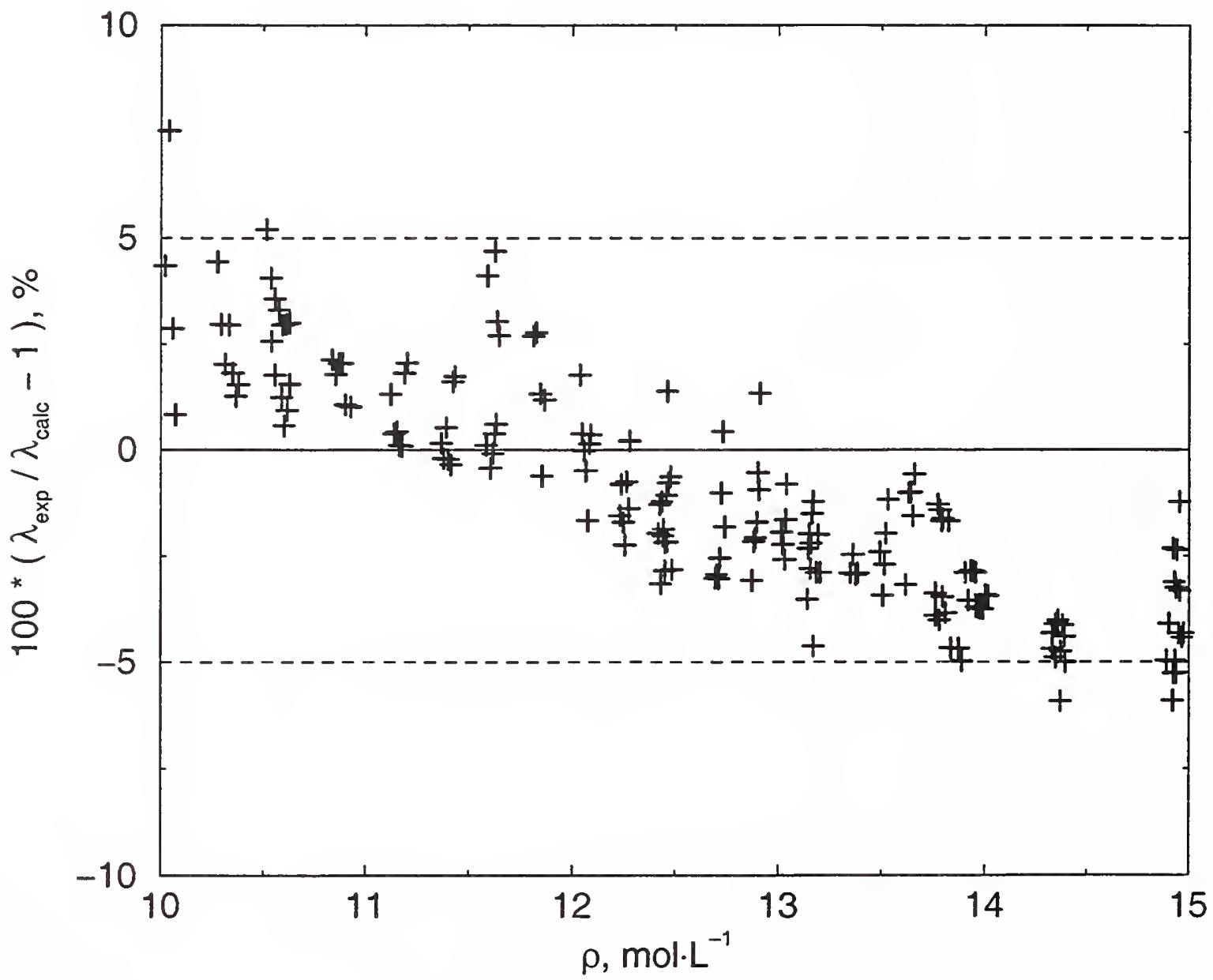

Figure 5. Relative deviations of the thermal conductivity data for the binary $30 \%$ R32/ $70 \%$ R $134 a$ mixture in the compressed liquid phase from the predictions of REFPROP 6.01. 


\section{$2.670 \%$ R32 / $30 \%$ R134a Mixture}

The precise molar composition of the mixture was $0.70031 \mathrm{R} 32+0.29969 \mathrm{R} 134 \mathrm{a}$. Results for 130 measurements, at temperatures from $254 \mathrm{~K}$ to $346 \mathrm{~K}$, are reported in Table 6 . Relative deviations between the measured thermal conductivity and the predictions of the mixture model REFPROP 6.01 [23] are shown in Figure 6 . The lower densities correspond to the higher temperatures where the thermal conductivity critical enhancement becomes significant.

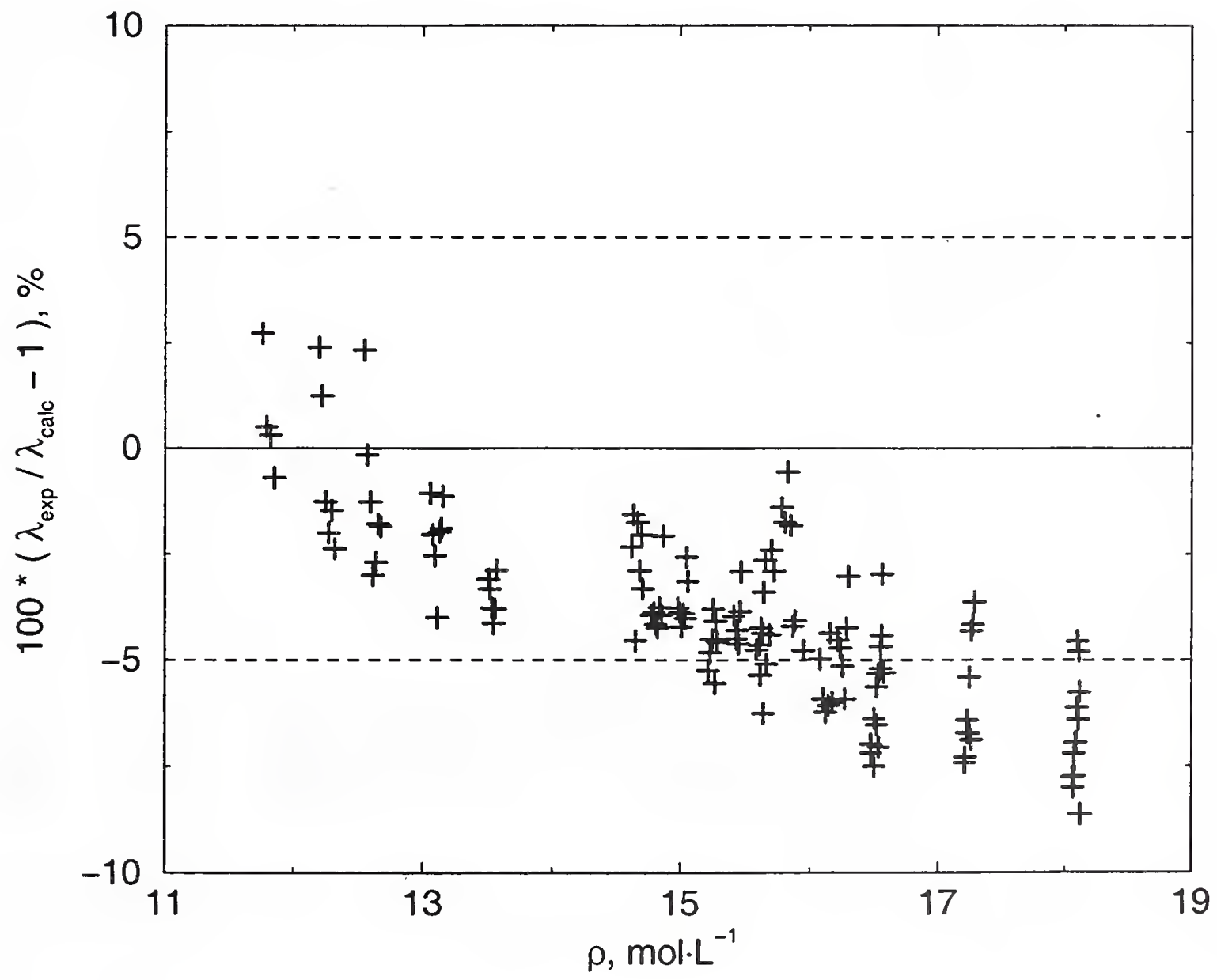

Figure 6. Relative deviations of the thermal conductivity data for the binary $70 \% \mathrm{R} 32$ / $30 \%$ R $134 \mathrm{a}$ mixture in the compressed liquid phase from the predictions of REFPROP 6.01. 


\section{$2.730 \%$ R134a / 70 \% Propane Mixture}

The precise molar composition of the mixture was $0.30033 \mathrm{R} 134 \mathrm{a}+0.69966$ propane. Results for 165 measurements, at temperatures from $254 \mathrm{~K}$ to $347 \mathrm{~K}$, are reported in Table 7. Relative deviations between the measured thermal conductivity and the predictions of the mixture model REFPROP 6.01 [23] are shown in Figure 7. The lower densities correspond to the higher temperatures where the thermal conductivity critical enhancement becomes significant.

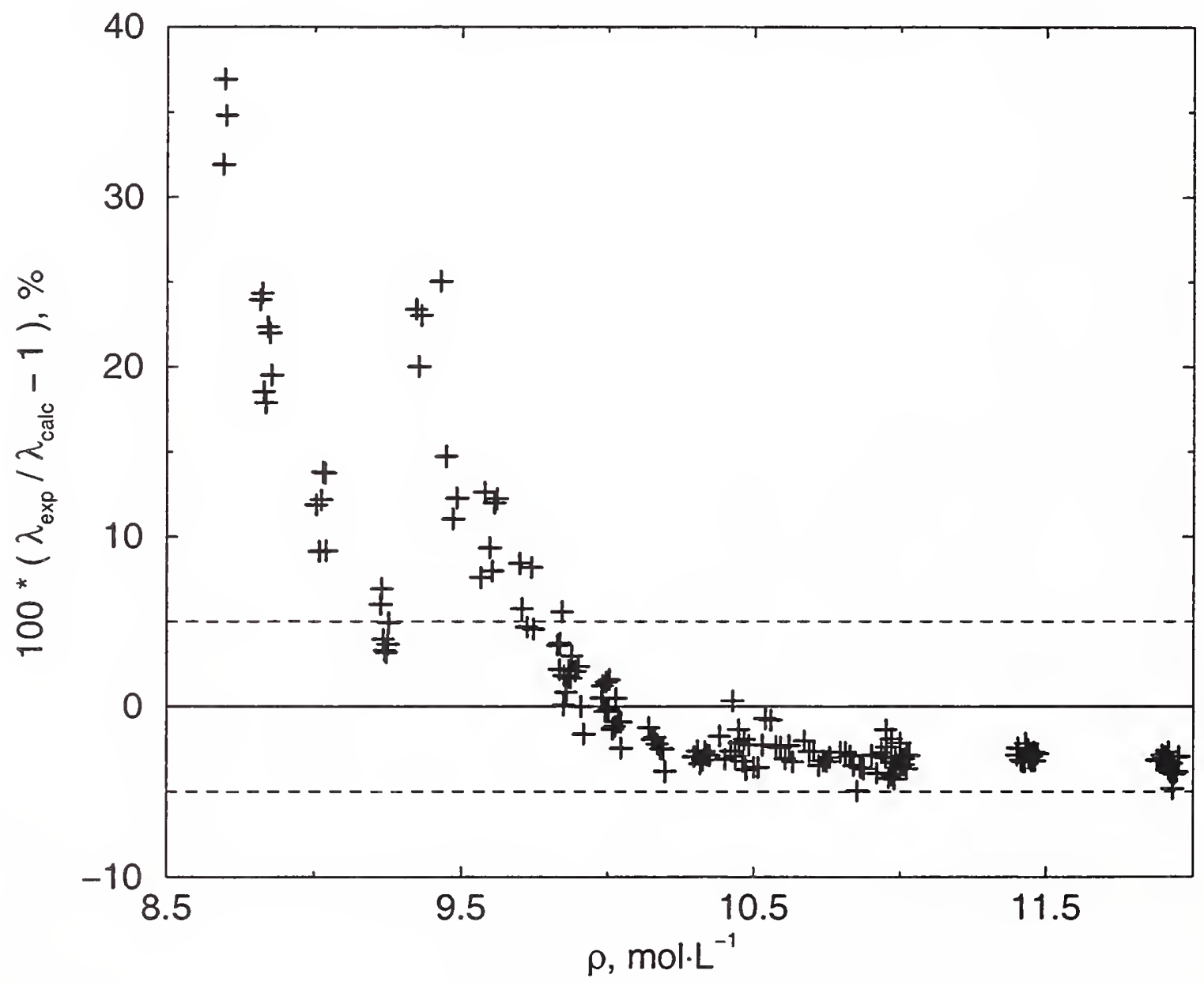

Figure 7. Relative deviations of the thermal conductivity data for the binary $30 \%$ R134a / $70 \%$ propane mixture in the compressed liquid phase from the predictions of REFPROP 6.01. 


\section{$2.870 \%$ R134a / 30 \% Propane Mixture}

The precise molar composition of the mixture was $0.70138 \mathrm{R} 134 \mathrm{a}+0.29862$ propane. The hot-wire cell failed during this measurement series with assembly 1 measurements obtained prior to this failure and assembly 2 measurements obtained with the rebuilt hot-wire cell. Results for 138 measurements, using assembly 1 at temperatures from $250 \mathrm{~K}$ to $319 \mathrm{~K}$, are reported in Table 8 . Results for 108 measurements, using assembly 2 at temperatures from 316 to $347 \mathrm{~K}$, are reported in Table 9. Relative deviations between the measured thermal conductivity and the predictions of the mixture model REFPROP 6.01 [23] are shown in Figure 8. The lower densities correspond to the higher temperatures where the thermal conductivity critical enhancement becomes significant. Good agreement is found between 2 separate hot-wire cells for this mixture.

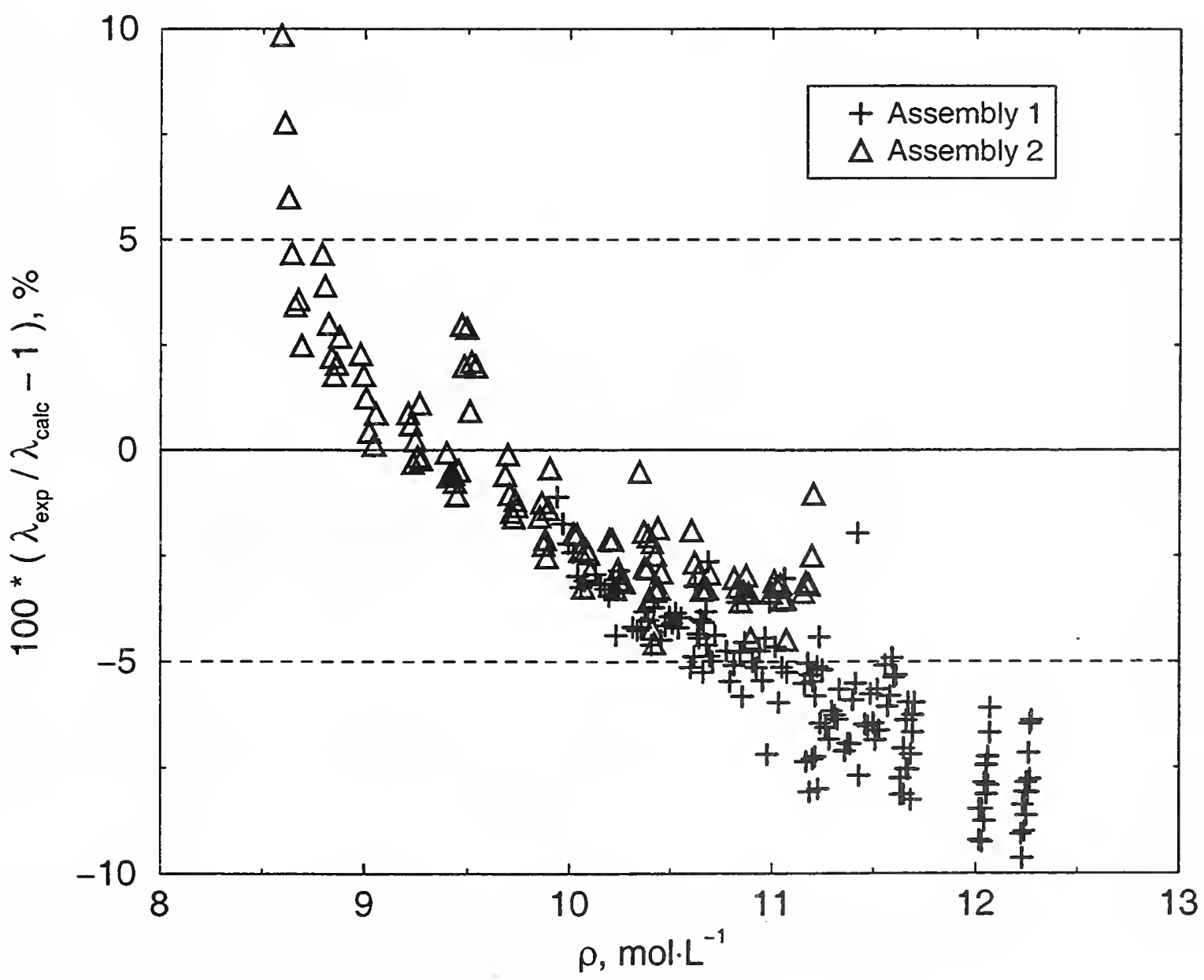

Figure 8. Relative deviations of the thermal conductivity data for the binary $70 \% \mathrm{R} 134 \mathrm{a} / 30 \%$ propane mixture in the compressed liquid phase from the predictions of REFPROP 6.01. 


\section{$2.933 \%$ R32 / 33 \% R125 / 33 \% R134a Mixture}

The precise molar composition of the mixture was $0.33298 \mathrm{R} 32+0.33342 \mathrm{R} 125+0.33359 \mathrm{R} 134 \mathrm{a}$. Results for 175 measurements, at temperatures from $250 \mathrm{~K}$ to $344 \mathrm{~K}$, are reported in Table 10 . Relative deviations between the measured thermal conductivity and the predictions of the mixture model REFPROP 6.01 [23] are shown in Figure 9. The lower densities correspond to the higher temperatures where the thermal conductivity critical enhancement becomes significant.

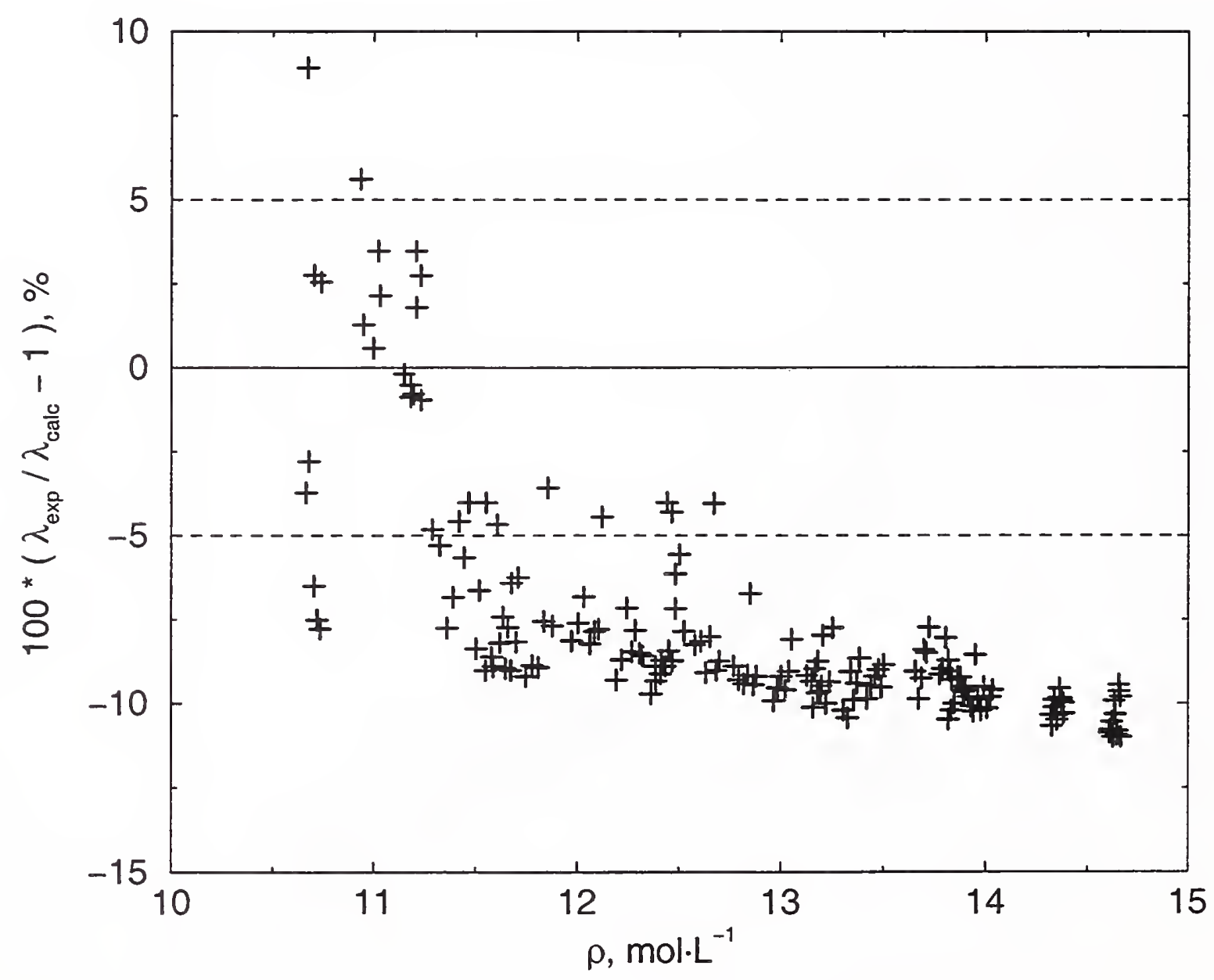

Figure 9. Relative deviations of the thermal conductivity data for the ternary $33 \% \mathrm{R} 32$ / $33 \% \mathrm{R} 125 / 33 \%$ $\mathrm{R} 134 \mathrm{a}$ mixture in the compressed liquid phase from the predictions of REFPROP 6.01. 


\section{$2.1030 \% \mathrm{R} 32$ / $10 \% \mathrm{R} 125$ / $60 \%$ R134a Mixture}

The precise molar composition of the mixture was $0.30027 \mathrm{R} 32+0.09995 \mathrm{R} 125+0.59977 \mathrm{R} 134 \mathrm{a}$. Results for 182 measurements, at temperatures from $250 \mathrm{~K}$ to $345 \mathrm{~K}$, are reported in Table 11 . Relative deviations between the measured thermal conductivity and the predictions of the mixture model REFPROP 6.01 [23] are shown in Figure 10. The lower densities correspond to the higher temperatures where the thermal conductivity critical enhancement becomes significant.

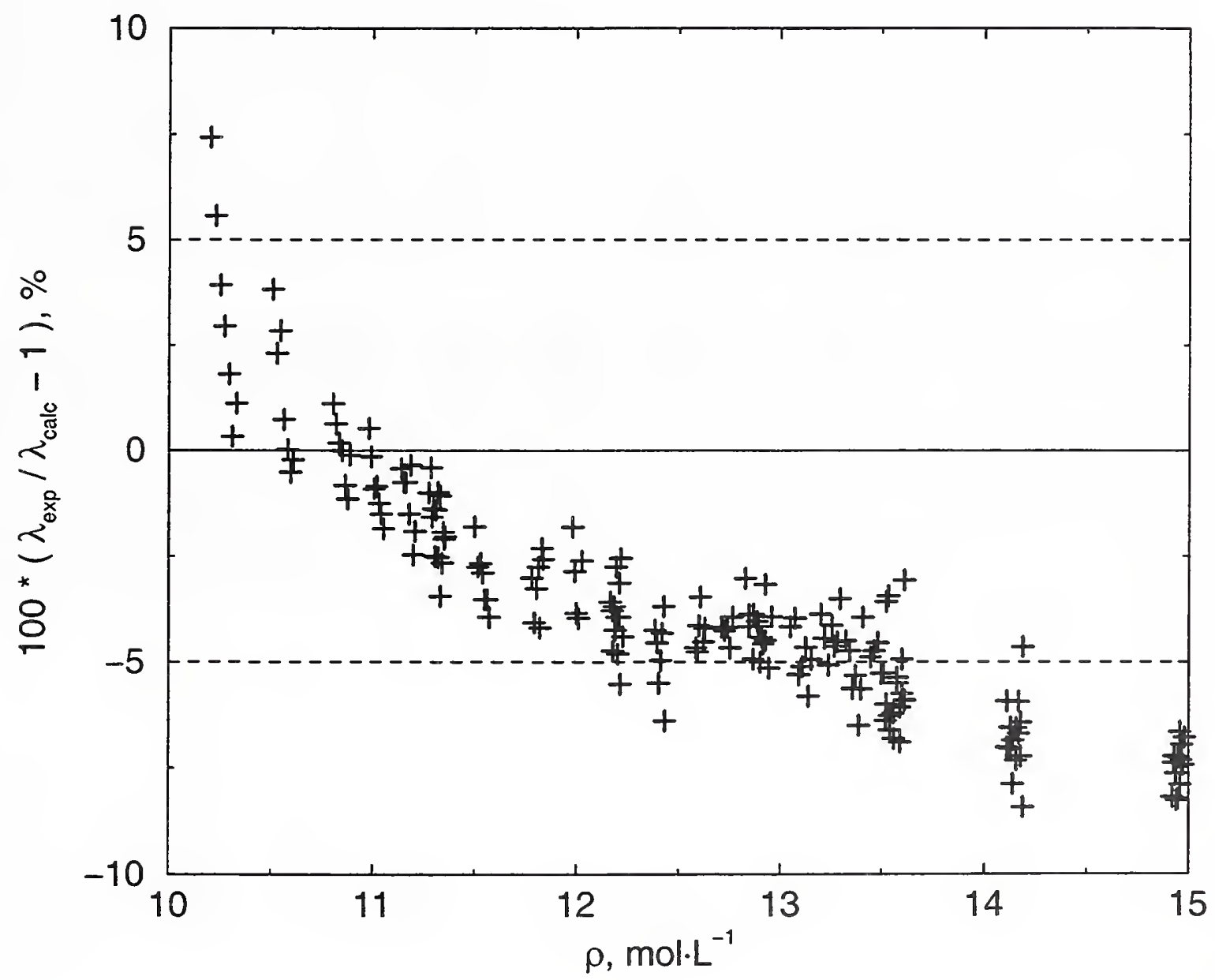

Figure 10. Relative deviations of the thermal conductivity data for the ternary $30 \% \mathrm{R} 32 / 10 \% \mathrm{R} 125 / 60$ $\% \mathrm{R} 134 \mathrm{a}$ mixture in the compressed liquid phase from the predictions of REFPROP 6.01. 


\section{Transient Results Using Bare Tungsten Wires}

The ten mixture supply cylinders were maintained in the single-phase gas state during storage and use. The cylinders were heated during use to increase the supply pressure and minimize effects due to adsorption. The supply pressure was not adequate to operate the air-driven diaphragm compressors that are normally used to increase the pressure in the hot-wire cells. The vapor measurements were made at pressures up to the heated sample bottle pressure and do not extend to the saturated vapor at the highest temperatures.

The measurements in the vapor phase were made using extremely small $4 \mu \mathrm{m}$ diameter tungsten hot wires. These bare tungsten hot wires are much smaller than the bare $12 \mu \mathrm{m}$ diameter platinum hot wires that have been used for the measurements on nonpolar fluids. Corrections for the finite wire diameter are much larger for vapors than they are for liquids due to the greater difference between the volumetric specific heat of the fluid relative to that of the wire. The relatively small diameter wire requires much smaller corrections for the finite wire diameter, which becomes very important considering the magnitude of the correction in the vapor phase and the relatively large uncertainty in the thermophysical properties of these mixtures that are required for the corrections. However, the extremely small wire diameter dramatically increases the electrical field strength in the vicinity of the wire during hot-wire measurements. This did not impact the vapor measurements, but did make the bare tungsten wires unusable for liquid phase measurements in these refrigerant mixtures. The fluid polarization effect with the $4 \mu \mathrm{m}$ diameter tungsten wires was much more pronounced than that observed for $12 \mu \mathrm{m}$ diameter platinum hot wires in a pure R134a liquid sample.

The temperature of the tantalum hot-wire cell was maintained with a refrigerated liquid circulator that allowed measurements from about $250 \mathrm{~K}$ to $350 \mathrm{~K}$. This circulator provided convenient control of the temperature over the range needed for the present measurements. The pressure and density of the gas phase mixtures were quite low at the lowest temperatures. This makes vapor measurements very difficult since the thermal diffusivity, and hence the rate at which the transient thermal wave penetrates to the outer cell wall, increase dramatically at these low pressures. This penetration time was less than 1 second for vapor measurements at the lowest temperatures. At these very low densities, the steady-state hot-wire measurements reported in section 4 are considered more reliable. The densities reported in the tables have been calculated using the mixture model REFPROP 6.01 [23]. 


\section{$3.130 \%$ R125 / 70 \% R134a Mixture}

The precise molar composition of the mixture was $0.30015 \mathrm{R} 125+0.69985 \mathrm{R} 134 \mathrm{a}$. Results for 248 measurements, at temperatures from $263 \mathrm{~K}$ to $345 \mathrm{~K}$ (0.14 MPa to $0.55 \mathrm{MPa})$, are reported in Table 13. Relative deviations between the measured thermal conductivity and the predictions of the mixture model REFPROP 6.01 [23] are shown in Figure 11. The thermal conductivity critical enhancement becomes more significant as the temperature increases and is likely responsible for the increasing deviations in Figure 11.

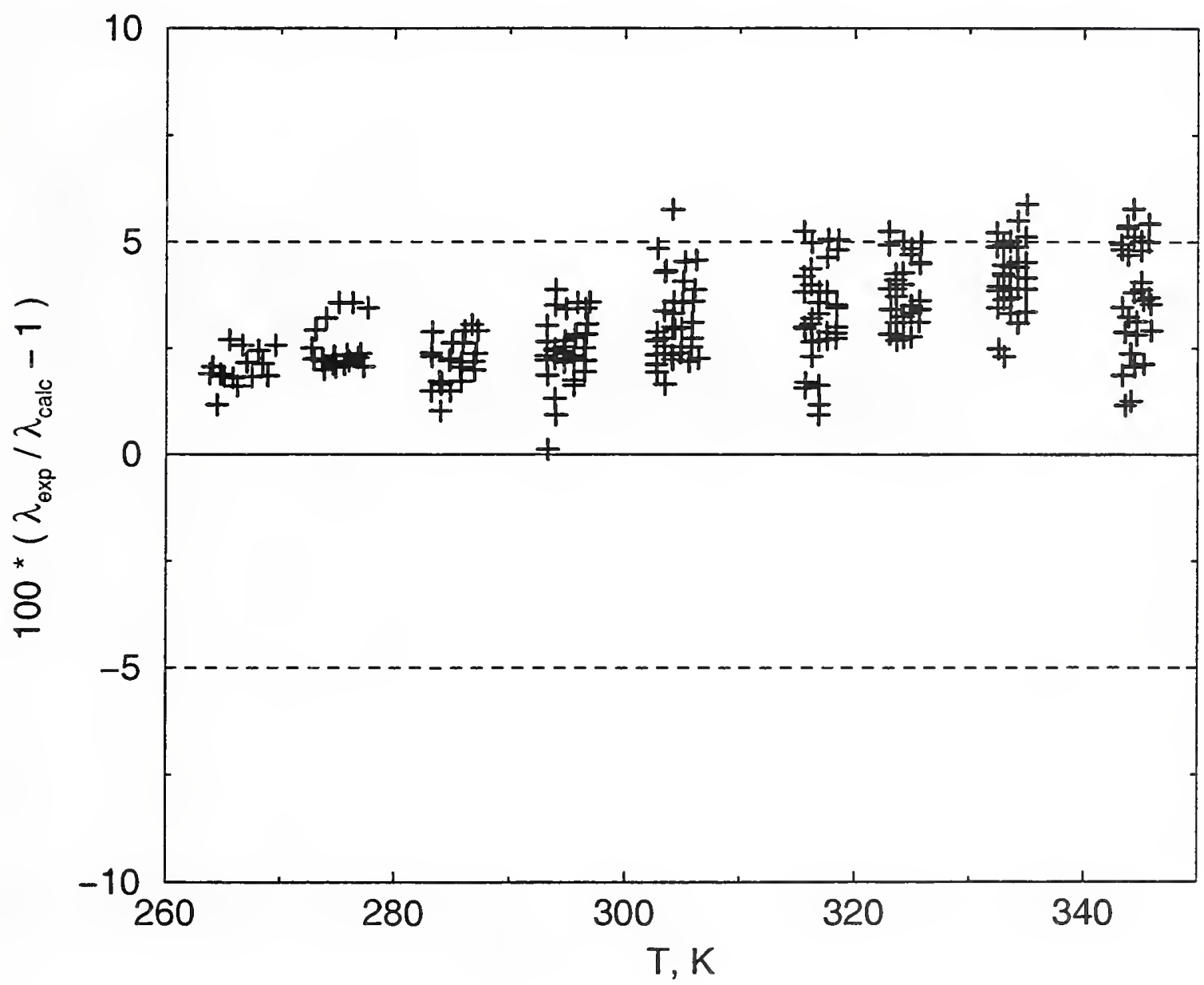

Figure 11. Relative deviations of the transient thermal conductivity data for the binary $30 \%$ R $125 / 70 \%$ R134a mixture in the vapor phase from the predictions of REFPROP 6.01. 


\section{$3.270 \% \mathrm{R} 125$ / $30 \%$ R134a Mixture}

The precise molar composition of the mixture was $0.70000 \mathrm{R} 125+0.30000 \mathrm{R} 134 \mathrm{a}$. Results for 202 measurements, at temperatures from $260 \mathrm{~K}$ to $344 \mathrm{~K}(0.09 \mathrm{MPa}$ to $0.62 \mathrm{MPa})$, are reported in Table 14. Relative deviations between the measured thermal conductivity and the predictions of the mixture model REFPROP 6.01 [23] are shown in Figure 12. The thermal conductivity critical enhancement becomes more significant as the temperature increases and is likely responsible for the increasing deviations in Figure 12.

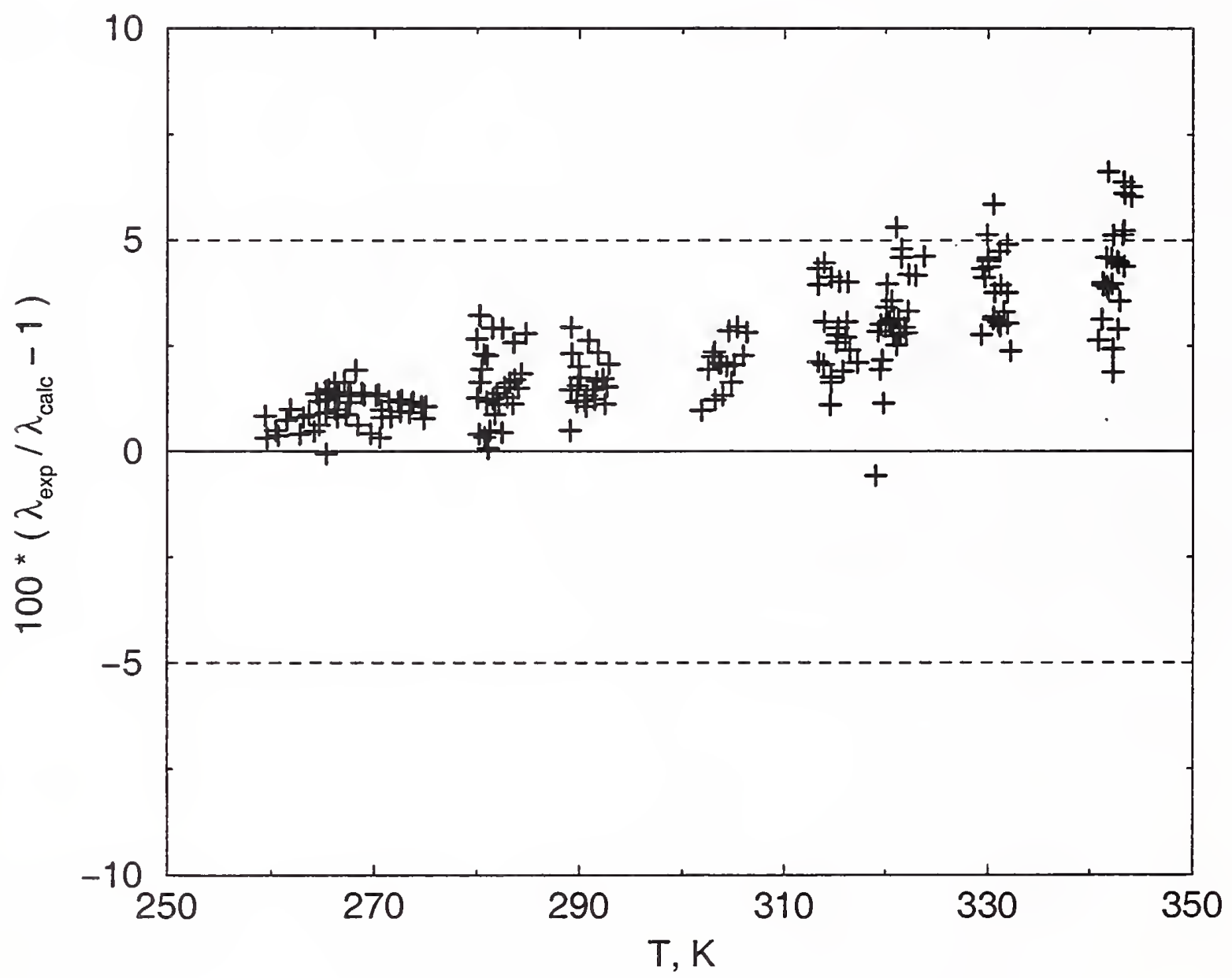

Figure 12. Relative deviations of the transient thermal conductivity data for the binary $70 \%$ R125/30\% R134a mixture in the vapor phase from the predictions of REFPROP 6.01 . 


\section{$3.330 \%$ R32 / $70 \%$ Propane Mixture}

The precise molar composition of the mixture was $0.29992 \mathrm{R} 32+0.70008$ propane. Results for 139 measurements, at temperatures from $229 \mathrm{~K}$ to $341 \mathrm{~K}(0.03 \mathrm{MPa}$ to $1.1 \mathrm{MPa}$ ), are reported in Table 15 . Relative deviations between the measured thermal conductivity and the predictions of the mixture model REFPROP 6.01 [23] are shown in Figure 13. The thermal conductivity critical enhancement becomes more significant as the temperature increases and is likely responsible for the increasing deviations in Figure 13.

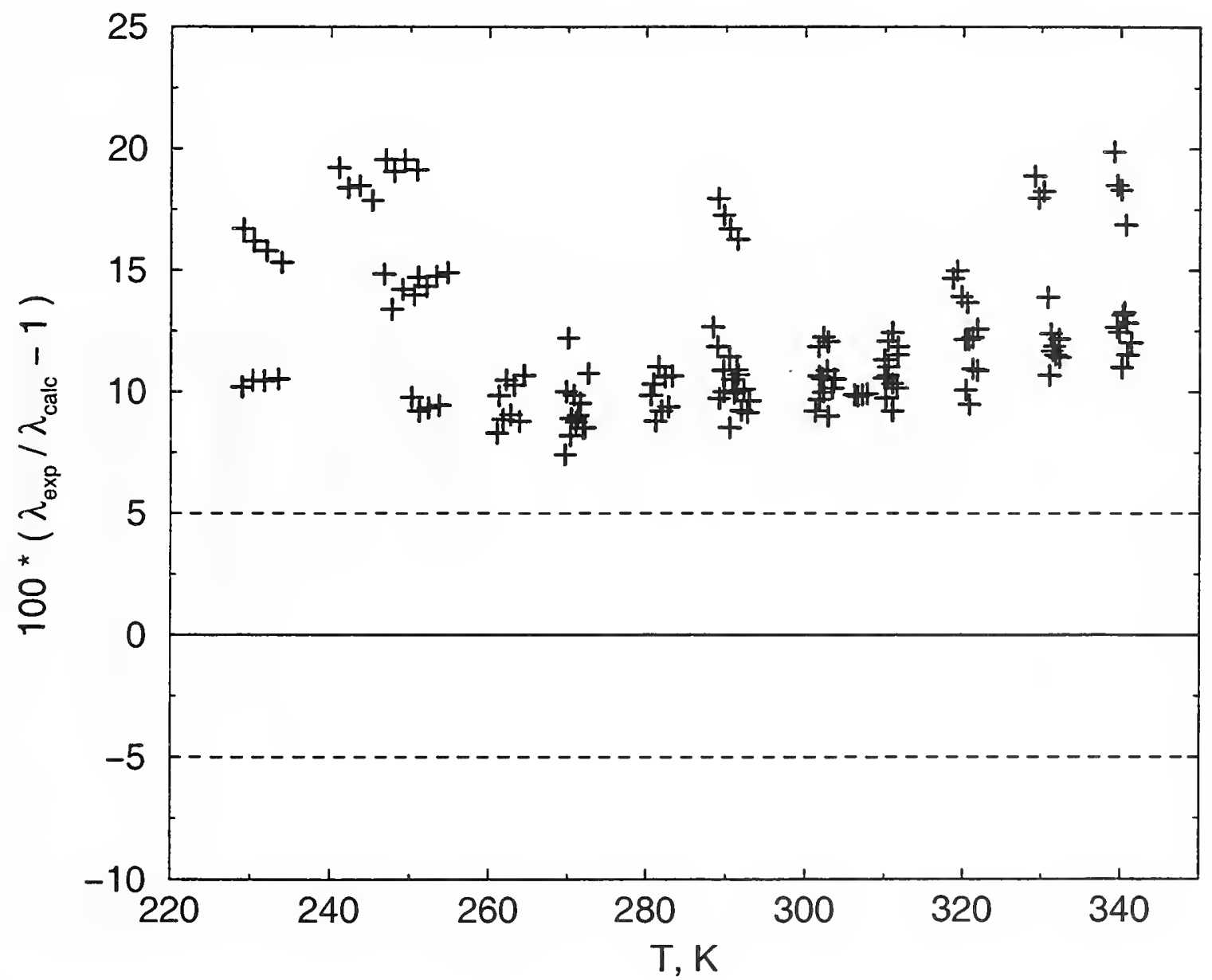

Figure 13. Relative deviations of the transient thermal conductivity data for the binary $30 \%$ R32/70\% propane mixture in the vapor phase from the predictions of REFPROP 6.01. 


\section{$3.470 \%$ R32 / $30 \%$ Propane Mixture}

The precise molar composition of the mixture was $0.70005 \mathrm{R} 32+0.29995$ propane. Results for 395 measurements, at temperatures from $258 \mathrm{~K}$ to $336 \mathrm{~K}(0.09 \mathrm{MPa}$ to $0.32 \mathrm{MPa})$, are reported in Table 16. Relative deviations between the measured thermal conductivity and the predictions of the mixture model REFPROP 6.01 [23] are shown in Figure 14. The thermal conductivity critical enhancement becomes more significant as the temperature increases and is likely responsible for the increasing deviations in Figure 14.

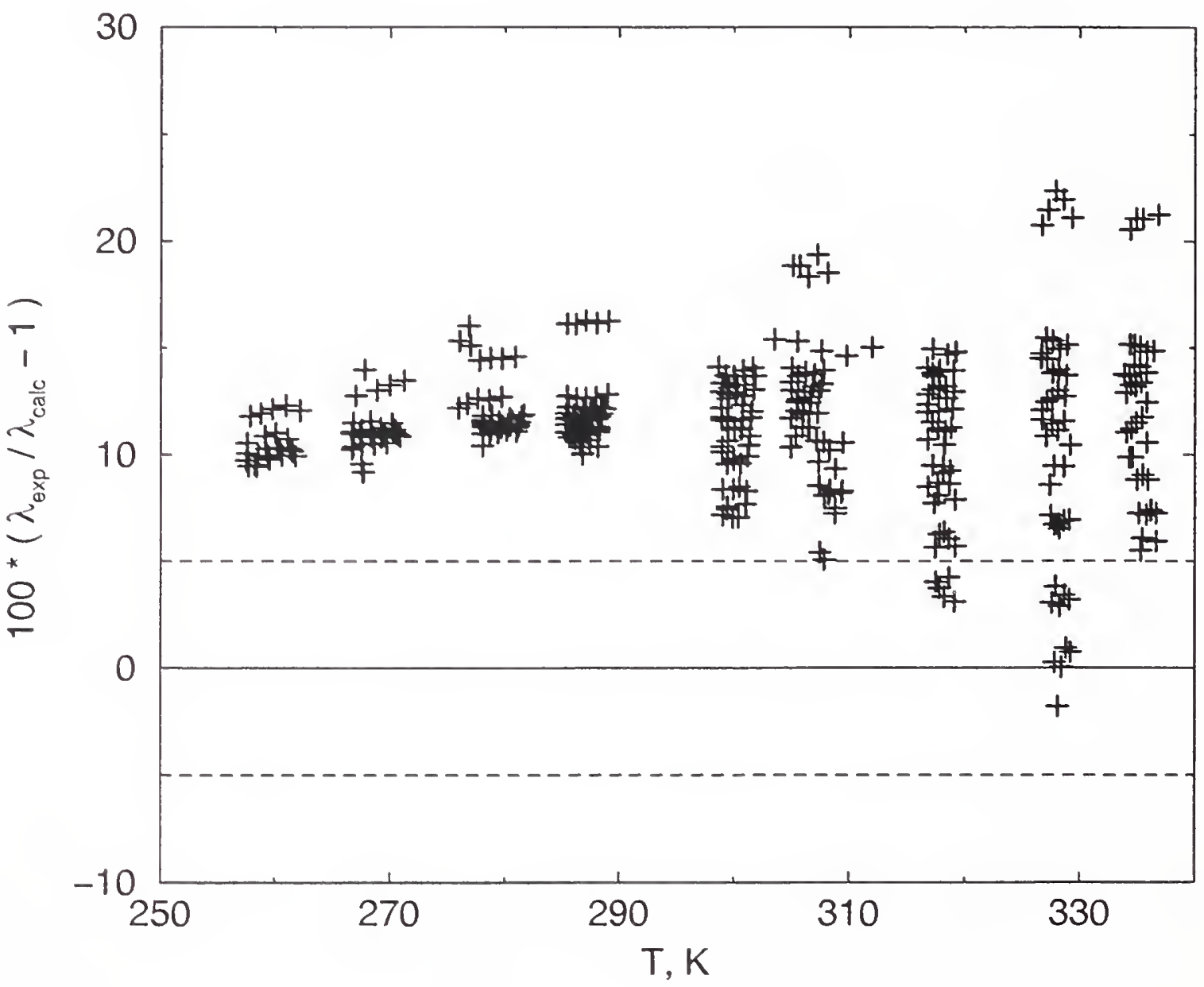

Figure 14. Relative deviations of the transient thermal conductivity data for the binary $70 \%$ R32/30\% propane mixture in the vapor phase from the predictions of REFPROP 6.01. 


\section{$3.530 \% \mathrm{R} 32 / 70 \% \mathrm{R} 134 \mathrm{a}$ Mixture}

The precise molar composition of the mixture was $0.29955 \mathrm{R} 32+0.70044 \mathrm{R} 134 \mathrm{a}$. Results for 235 measurements, at temperatures from $258 \mathrm{~K}$ to $343 \mathrm{~K}(0.09 \mathrm{MPa}$ to $0.45 \mathrm{MPa})$, are reported in Table 17 . Relative deviations between the measured thermal conductivity and the predictions of the mixture model REFPROP 6.01 [23] are shown in Figure 15. The thermal conductivity critical enhancement becomes more significant as the temperature increases and is likely responsible for the increasing deviations in Figure 15.

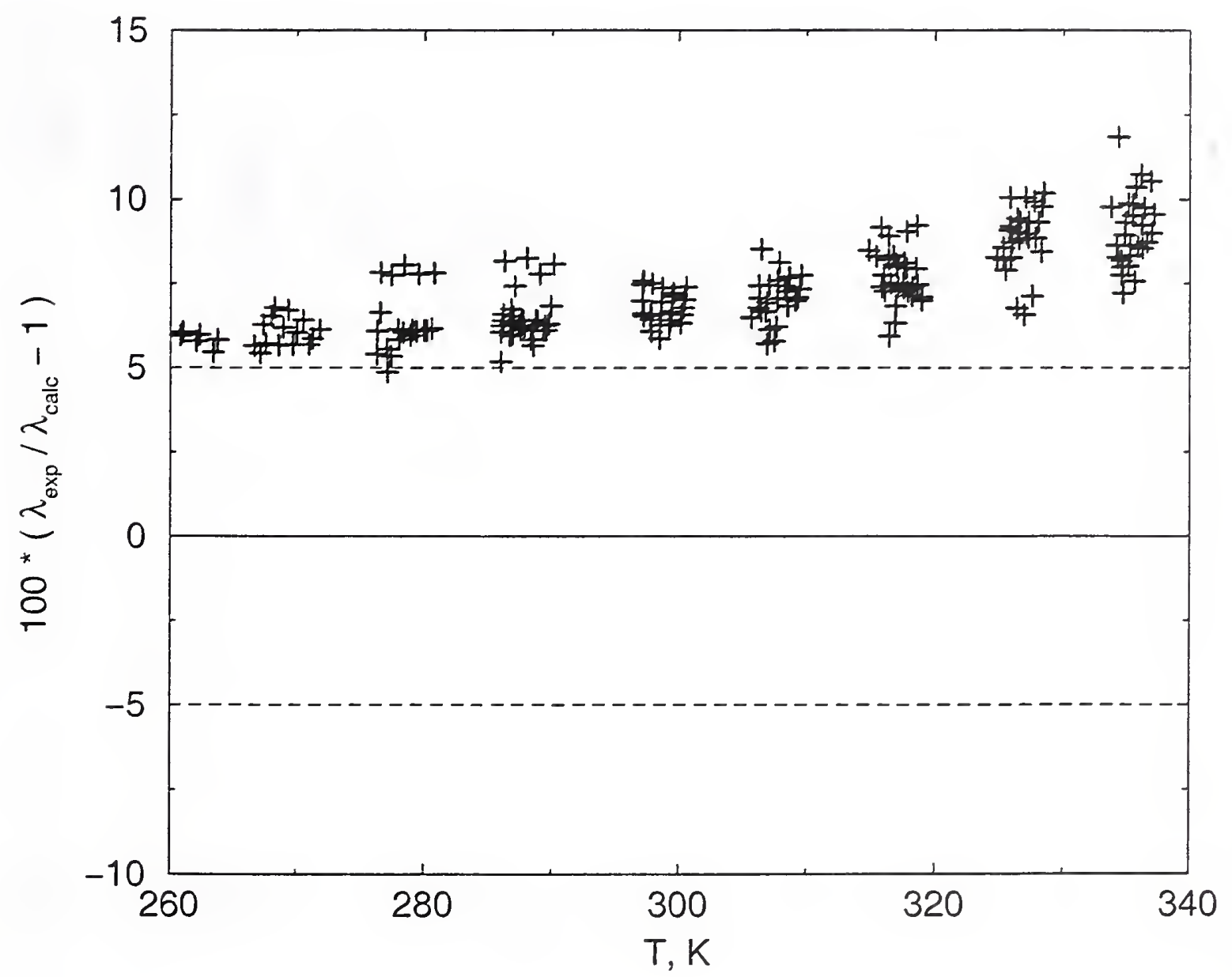

Figure 15. Relative deviations of the transient thermal conductivity data for the binary $30 \% \mathrm{R} 32 / 70 \%$ R134a mixture in the vapor phase from the predictions of REFPROP 6.01. 


\subsection{0 \% R32 / 30 \% R134a Mixture}

The precise molar composition of the mixture was $0.70031 \mathrm{R} 32+0.29969 \mathrm{R} 134 \mathrm{a}$. Results for 231 measurements, at temperatures from $259 \mathrm{~K}$ to $348 \mathrm{~K}(0.09 \mathrm{MPa}$ to $0.56 \mathrm{MPa})$, are reported in Table 18 . Relative deviations between the measured thermal conductivity and the predictions of the mixture model REFPROP 6.01 [23] are shown in Figure 16. The thermal conductivity critical enhancement becomes more significant as the temperature increases and is likely responsible for the increasing deviations in Figure 16.

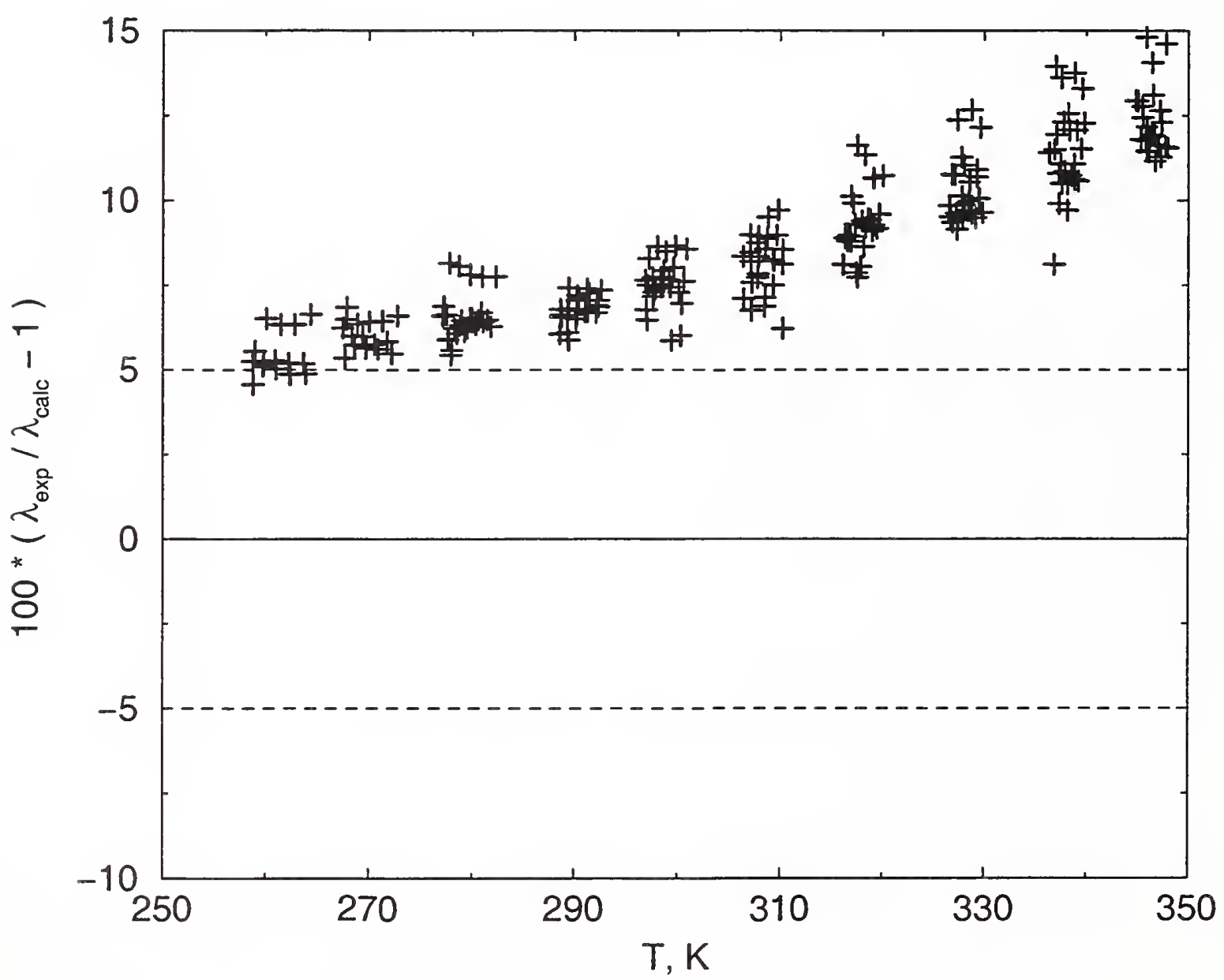

Figure 16. Relative deviations of the transient thermal conductivity data for the binary $70 \% \mathrm{R} 32 / 30 \%$ R134a mixture in the vapor phase from the predictions of REFPROP 6.01. 


\section{$3.730 \%$ R134a / 70 \% Propane Mixture}

The precise molar composition of the mixture was $0.30033 \mathrm{R} 134 \mathrm{a}+0.69966$ propane. Results for 51 measurements, at temperatures from $243 \mathrm{~K}$ to $302 \mathrm{~K}(0.12 \mathrm{MPa}$ to $0.72 \mathrm{MPa})$, are reported in Table 19 . Relative deviations between the measured thermal conductivity and the predictions of the mixture model REFPROP 6.01 [23] are shown in Figure 17. The thermal conductivity critical enhancement becomes more significant as the temperature increases and is likely responsible for the increasing deviations in Figure 17.

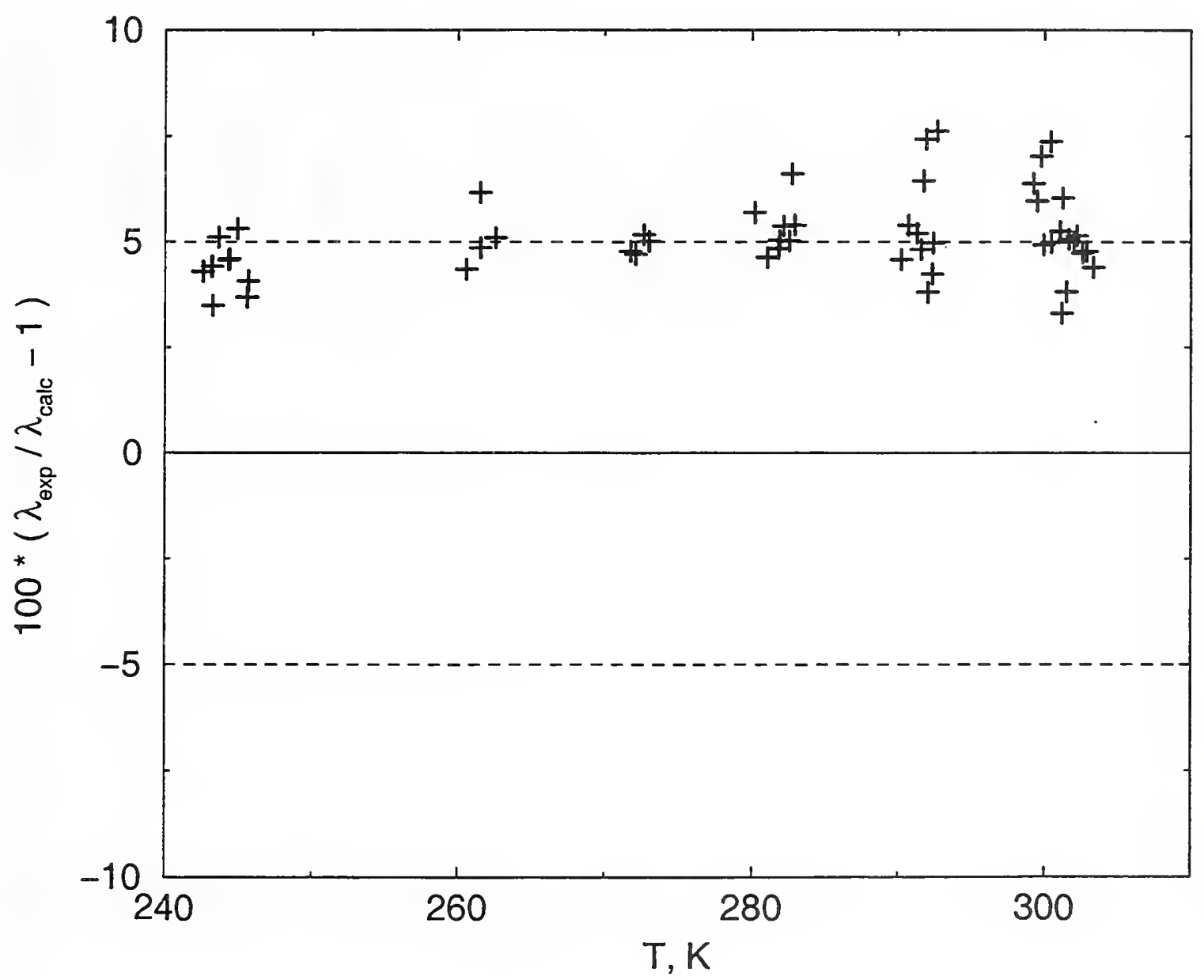

Figure 17. Relative deviations of the transient thermal conductivity data for the binary $30 \%$ R134a / $70 \%$ propane mixture in the vapor phase from the predictions of REFPROP 6.01 . 


\section{$3.870 \%$ R134a / $30 \%$ Propane Mixture}

The precise molar composition of the mixture was $0.69948 \mathrm{R} 134 \mathrm{a}+0.30052$ propane. Results for 233 measurements, at temperatures from $258 \mathrm{~K}$ to $348 \mathrm{~K}(0.09 \mathrm{MPa}$ to $0.56 \mathrm{MPa})$, are reported in Table 20 . Relative deviations between the measured thermal conductivity and the predictions of the mixture model REFPROP 6.01 [23] are shown in Figure 18. The thermal conductivity critical enhancement becomes more significant as the temperature increases and is likely responsible for the increasing deviations in Figure 18.

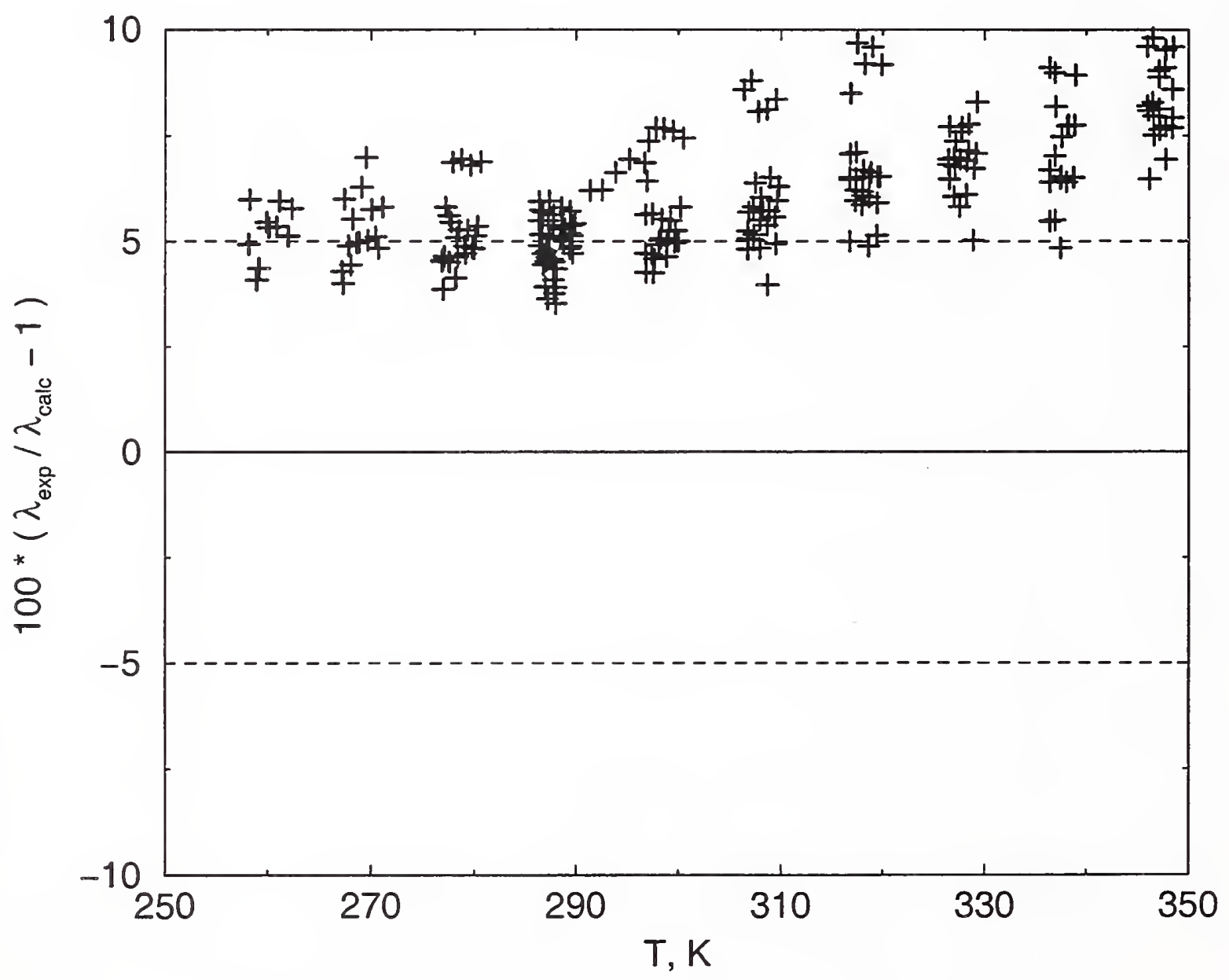

Figure 18. Relative deviations of the transient thermal conductivity data for the binary $70 \% \mathrm{R} 134 \mathrm{a} / 30 \%$ propane mixture in the vapor phase from the predictions of REFPROP 6.01. 


\section{$3.933 \% \mathbf{R} 32$ / 33 \% R125 / 33 \% R134a Mixture}

The precise molar composition of the mixture was $0.33298 \mathrm{R} 32+0.33342 \mathrm{R} 125+0.33359 \mathrm{R} 134 \mathrm{a}$. Results for 81 measurements, at temperatures from $263 \mathrm{~K}$ to $341 \mathrm{~K}(0.10 \mathrm{MPa}$ to $0.30 \mathrm{MPa})$, are reported in Table 21. Relative deviations between the measured thermal conductivity and the predictions of the mixture model REFPROP 6.01 [23] are shown in Figure 19. The thermal conductivity critical enhancement becomes more significant as the temperature increases and is likely responsible for the increasing deviations in Figure 19.

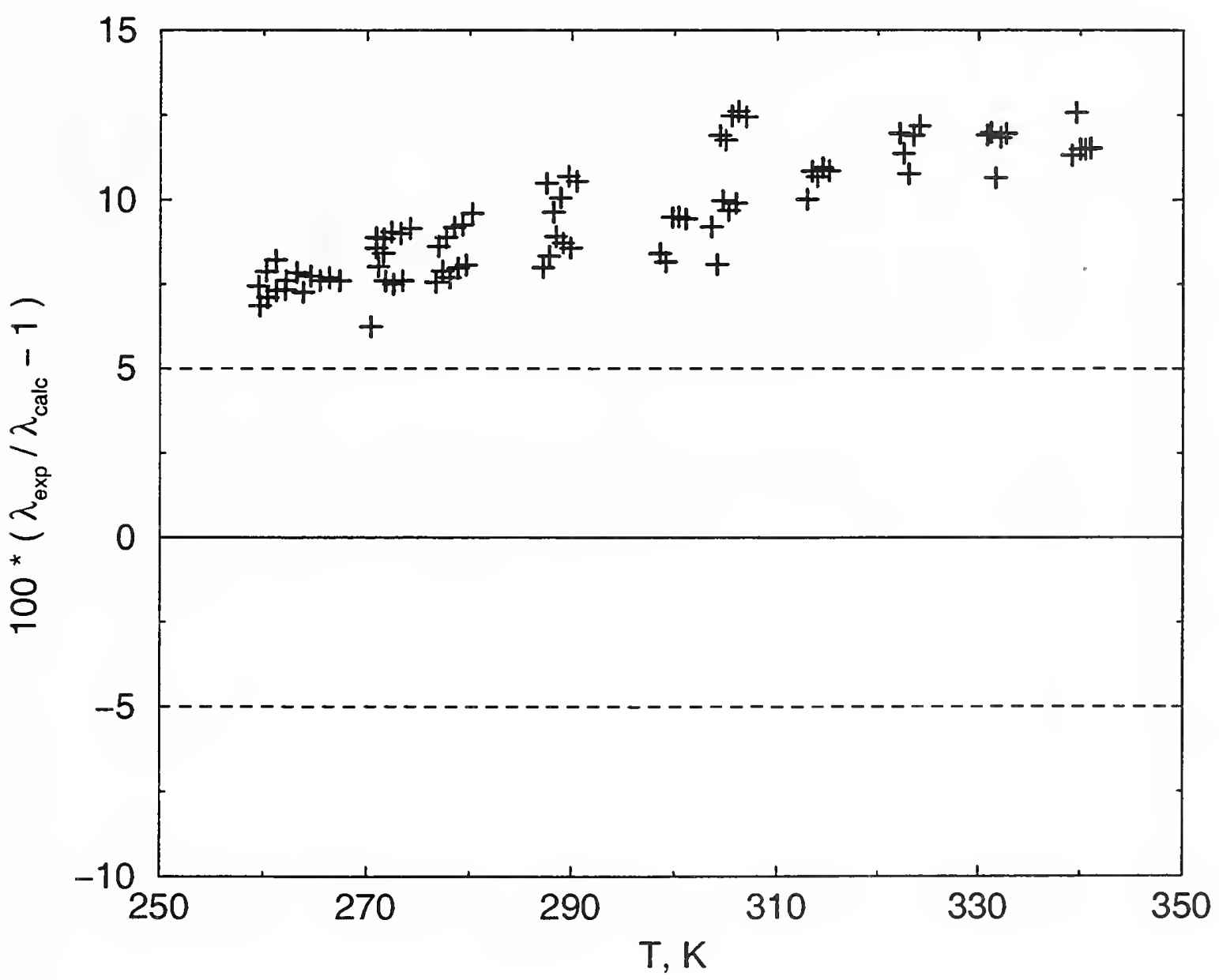

Figure 19. Relative deviations of the transient thermal conductivity data for the termary $33 \% \mathrm{R} 32 / 33 \%$ R125/33\% R134a mixture in the vapor phase from the predictions of REFPROP 6.01. 


\section{$3.1030 \% \mathrm{R} 32$ / $10 \% \mathrm{R} 125$ / 60 \% R134a Mixture}

The precise molar composition of the mixture was $0.30027 \mathrm{R} 32+0.09995 \mathrm{R} 125+0.59977 \mathrm{R} 134 \mathrm{a}$. Results for 230 measurements, at temperatures from $258 \mathrm{~K}$ to $345 \mathrm{~K}(0.09 \mathrm{MPa}$ to $0.53 \mathrm{MPa})$, are reported in Table 22. Relative deviations between the measured thermal conductivity and the predictions of the mixture model REFPROP 6.01 [23] are shown in Figure 20. The thermal conductivity critical enhancement becomes more significant as the temperature increases and is likely responsible for the increasing deviations in Figure 20.

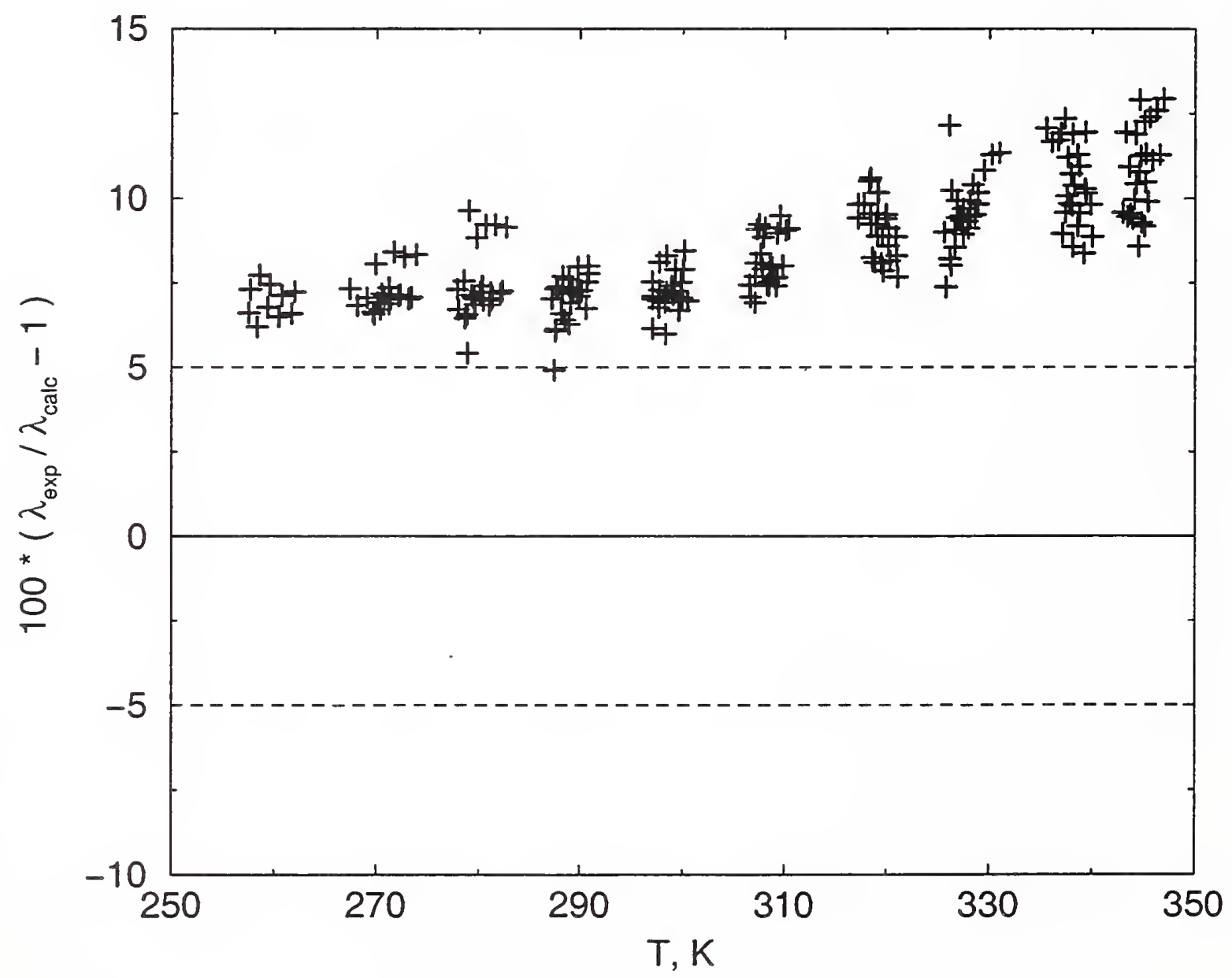

Figure 20. Relative deviations of transient thermal conductivity data for the ternary $30 \%$ R32/ $10 \%$ R 125 $160 \%$ R134a mixture in the vapor phase from the predictions of REFPROP 6.01 . 


\section{Steady-State Results Using Bare Tungsten Wires}

The ten mixture supply cylinders were maintained in the single-phase gas state during storage and use. The cylinders were heated during use to increase the supply pressure and to minimize effects due to adsorption. The supply pressure was not adequate to operate the air-driven diaphragm compressors that are normally used to increase the pressure in the hot-wire cells. The vapor measurements were made at pressures up to the heated sample bottle pressure and do not extend to the saturated vapor at the highest temperatures. The temperature of the tantalum hot-wire cell was maintained with a refrigerated liquid circulator that allowed measurements from about $250 \mathrm{~K}$ to $350 \mathrm{~K}$. This circulator provided convenient control of the temperature over the range needed for the present measurements.

The tables of steady-state results include several new parameters that were not included in the transient tables. First, TBAND is a direct measure of the precision of the temperature rise measurement, and hence the thermal conductivity, at a $3 \sigma$ uncertainty level over the steady-state fit limits from $t_{\text {start }}$ to $t_{\text {end }}$ that are also provided in the tables for reference. Fluid convection is the key concern with steady-state measurements of thermal conductivity. The hot-wire cells are designed for transient measurements, so the fluid gap between the inner wire and the outer concentric cell wall where the fluid resides is larger than is optimum for steady-state measurements. The hot-wire cells can be used reliably only for steady-state measurements of low-pressure gases where convection is not likely. The measure of the convective stability of the gas is the Rayleigh number $N_{R a}$ and is included in the tables for each point for reference. Experience has shown that $N_{R a}$ should be less than 70000 for our measurement geometry. The absence of fluid convection can further be verified by examination of the consistency of measurements at different applied power levels. At least four different applied power levels were measured at each initial fluid state point.

The agreement between the transient results of the previous section and the steady-state results presented here is quite good. At very low densities, the steady-state hot-wire measurements are considered more reliable than the transient measurements, since no corrections are required for either the finite wire diameter or the outer boundary. The densities reported in the tables have been calculated using the mixture model REFPROP 6.01 [23]. 


\section{$4.130 \%$ R125 / 70 \% R134a Mixture}

The precise molar composition of the mixture was $0.30015 \mathrm{R} 125+0.69985 \mathrm{R} 134 \mathrm{a}$. Results for 160 measurements, at temperatures from $263 \mathrm{~K}$ to $345 \mathrm{~K}(0.07 \mathrm{MPa}$ to $0.54 \mathrm{MPa})$, are reported in Table 23 . Relative deviations between the measured thermal conductivity and the predictions of the mixture model REFPROP 6.01 [23] are shown in Figure 21. The thermal conductivity critical enhancement becomes more significant as the temperature increases and is likely responsible for the increasing deviations in Figure 21.

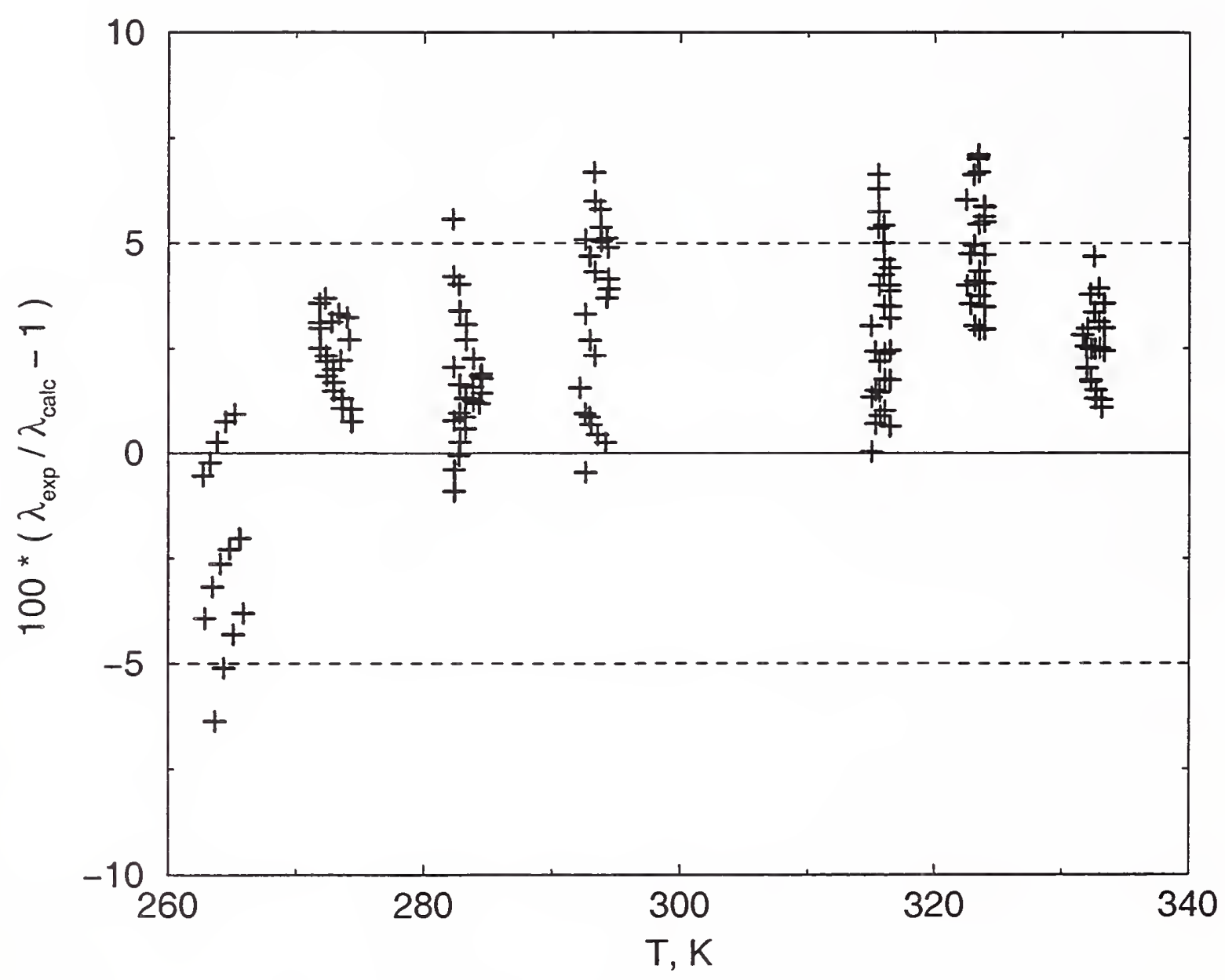

Figure 21. Relative deviations of the steady-state thermal conductivity data for the binary $30 \% \mathrm{R} 125 / 70$ \% R134a mixture in the vapor phase from the predictions of REFPROP 6.01. 


\section{$4.270 \%$ R125/30 \% R134a Mixture}

The precise molar composition of the mixture was $0.70000 \mathrm{R} 125+0.30000 \mathrm{R} 134 \mathrm{a}$. Results for 205 measurements, at temperatures from $260 \mathrm{~K}$ to $344 \mathrm{~K}(0.09 \mathrm{MPa}$ to $0.62 \mathrm{MPa})$, are reported in Table 24 . Relative deviations between the measured thermal conductivity and the predictions of the mixture model REFPROP 6.01 [23] are shown in Figure 22. The thermal conductivity critical enhancement becomes more significant as the temperature increases and is likely responsible for the increasing deviations in Figure 22.

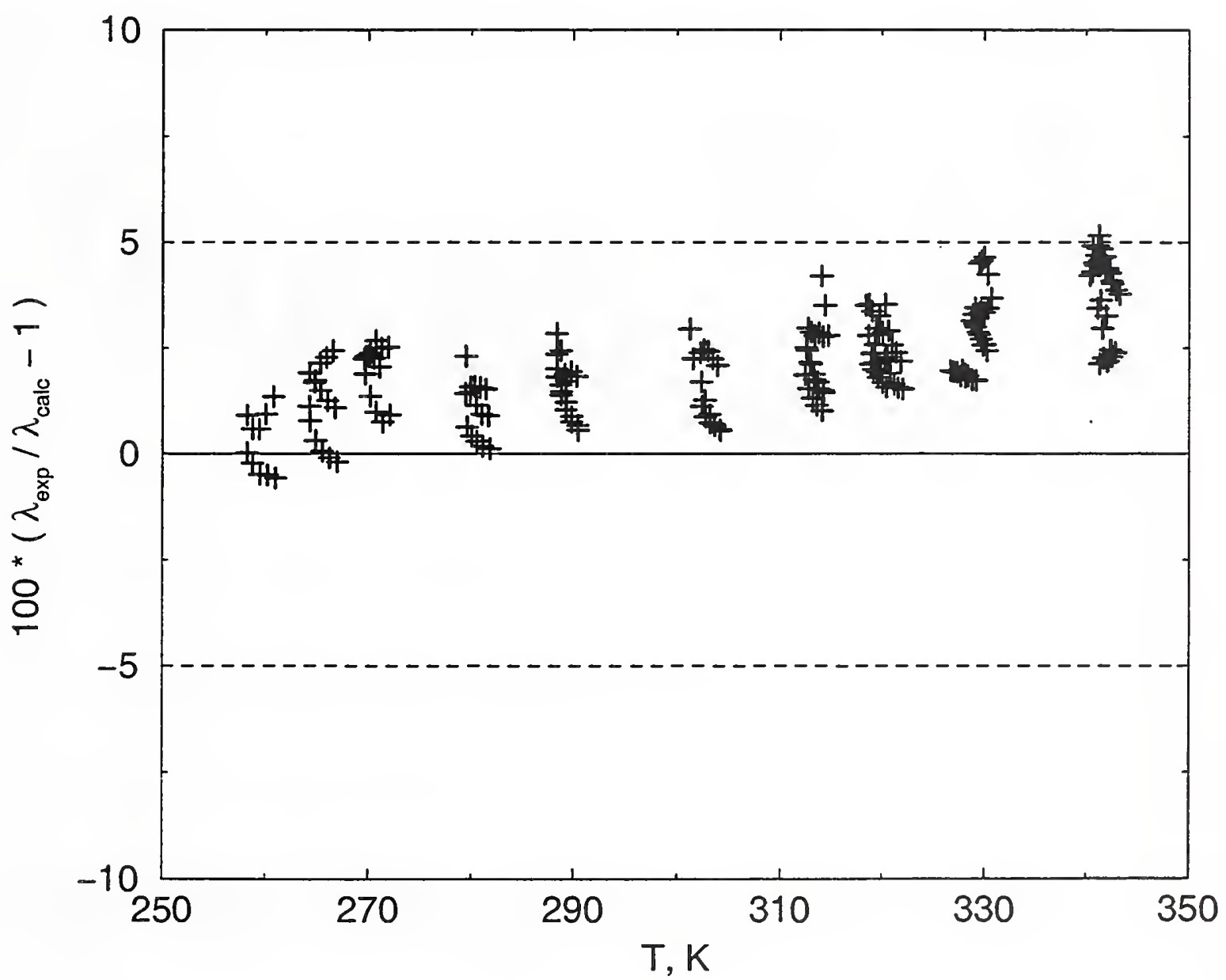

Figure 22. Relative deviations of the steady-state thermal conductivity data for the binary $70 \% \mathrm{R} 125 / 30$ $\%$ R134a mixture in the vapor phase from the predictions of REFPROP 6.01 . 


\section{$4.330 \%$ R32 / 70 \% Propane Mixture}

The precise molar composition of the mixture was $0.29992 \mathrm{R} 32+0.70008$ propane. Results for 225 measurements, at temperatures from $229 \mathrm{~K}$ to $341 \mathrm{~K}(0.03 \mathrm{MPa}$ to $1.12 \mathrm{MPa})$, are reported in Table 25 . Relative deviations between the measured thermal conductivity and the predictions of the mixture model REFPROP 6.01 [23] are shown in Figure 23. The thermal conductivity critical enhancement becomes more significant as the temperature increases and is likely responsible for the increasing deviations in Figure 23.

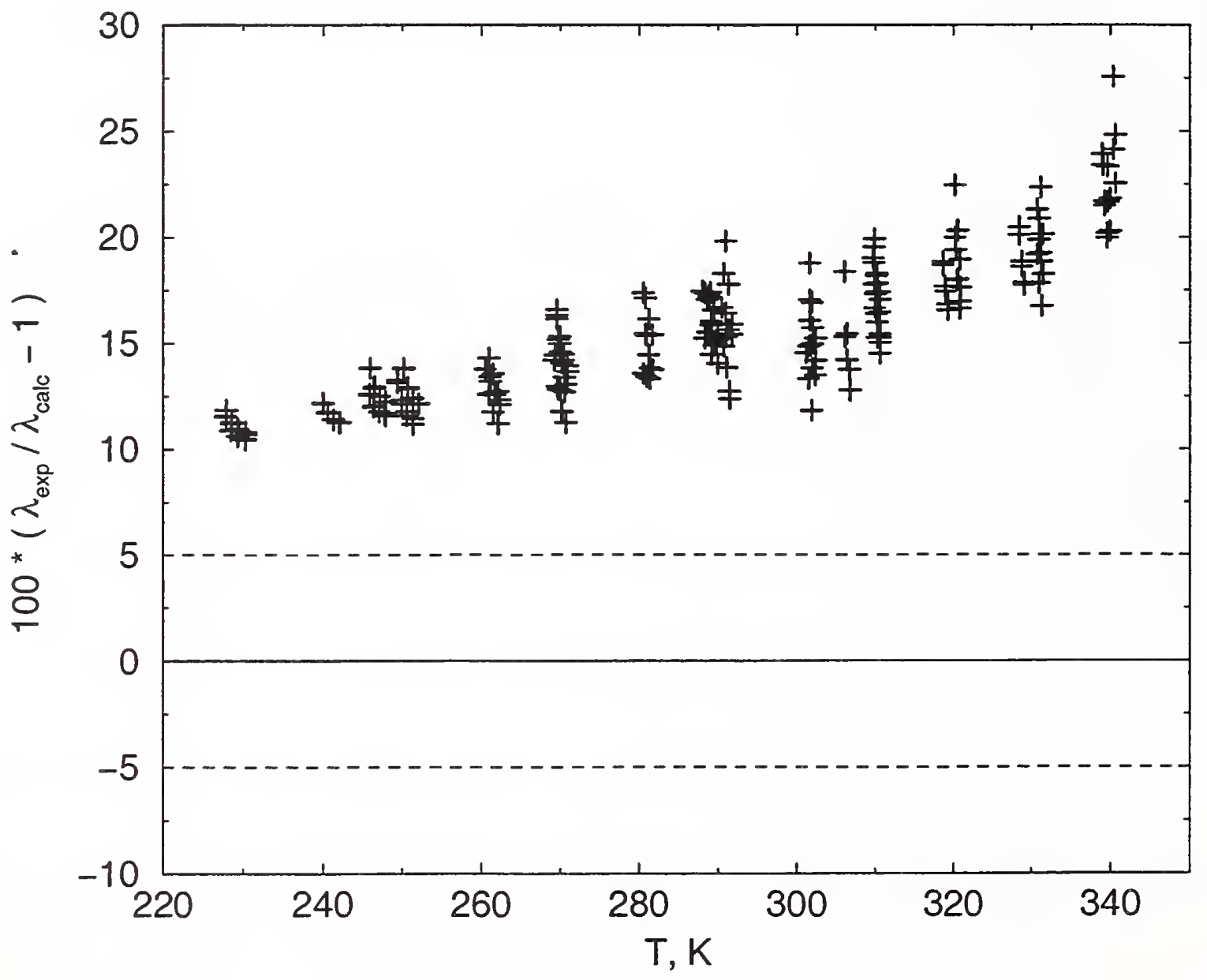

Figure 23. Relative deviations of the steady-state thermal conductivity data for the binary $30 \% \mathrm{R} 32 / 70 \%$ propane mixture in the vapor phase from the predictions of REFPROP 6.01. 


\section{$4.470 \%$ R32 / 30 \% Propane Mixture}

The precise molar composition of the mixture was $0.70005 \mathrm{R} 32+0.29995$ propane. Results for 193 measurements, at temperatures from $258 \mathrm{~K}$ to $328 \mathrm{~K}(0.10 \mathrm{MPa}$ to $1.33 \mathrm{MPa})$, are reported in Table 26 . Relative deviations between the measured thermal conductivity and the predictions of the mixture model REFPROP 6.01 [23] are shown in Figure 24. The thermal conductivity critical enhancement becomes more significant as the temperature increases and is likely responsible for the increasing deviations in Figure 24.

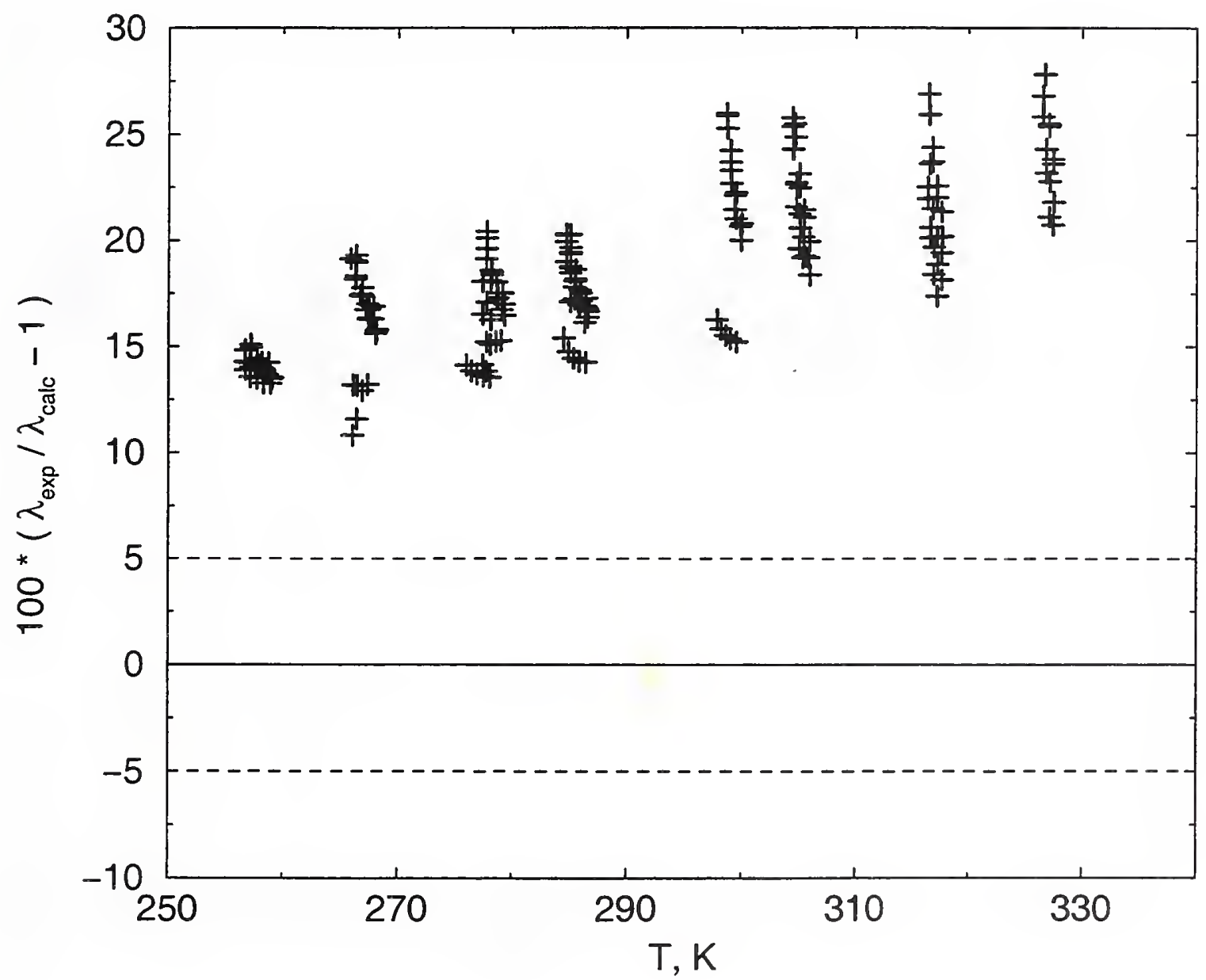

Figure 24. Relative deviations of the steady-state thermal conductivity data for the binary $70 \% \mathrm{R} 32 / 30 \%$ mixture in the vapor phase from the predictions of REFPROP 6.01. 


\section{$4.530 \%$ R32 / 70 \% R134a Mixture}

The precise molar composition of the mixture was $0.29955 \mathrm{R} 32+0.70044 \mathrm{R} 134 \mathrm{a}$. Results for 266 measurements, at temperatures from $258 \mathrm{~K}$ to $343 \mathrm{~K}(0.09 \mathrm{MPa}$ to $0.45 \mathrm{MPa})$, are reported in Table 27 . Relative deviations between the measured thermal conductivity and the predictions of the mixture model REFPROP 6.01 [23] are shown in Figure 25. The thermal conductivity critical enhancement becomes more significant as the temperature increases and is likely responsible for the increasing deviations in Figure 25.

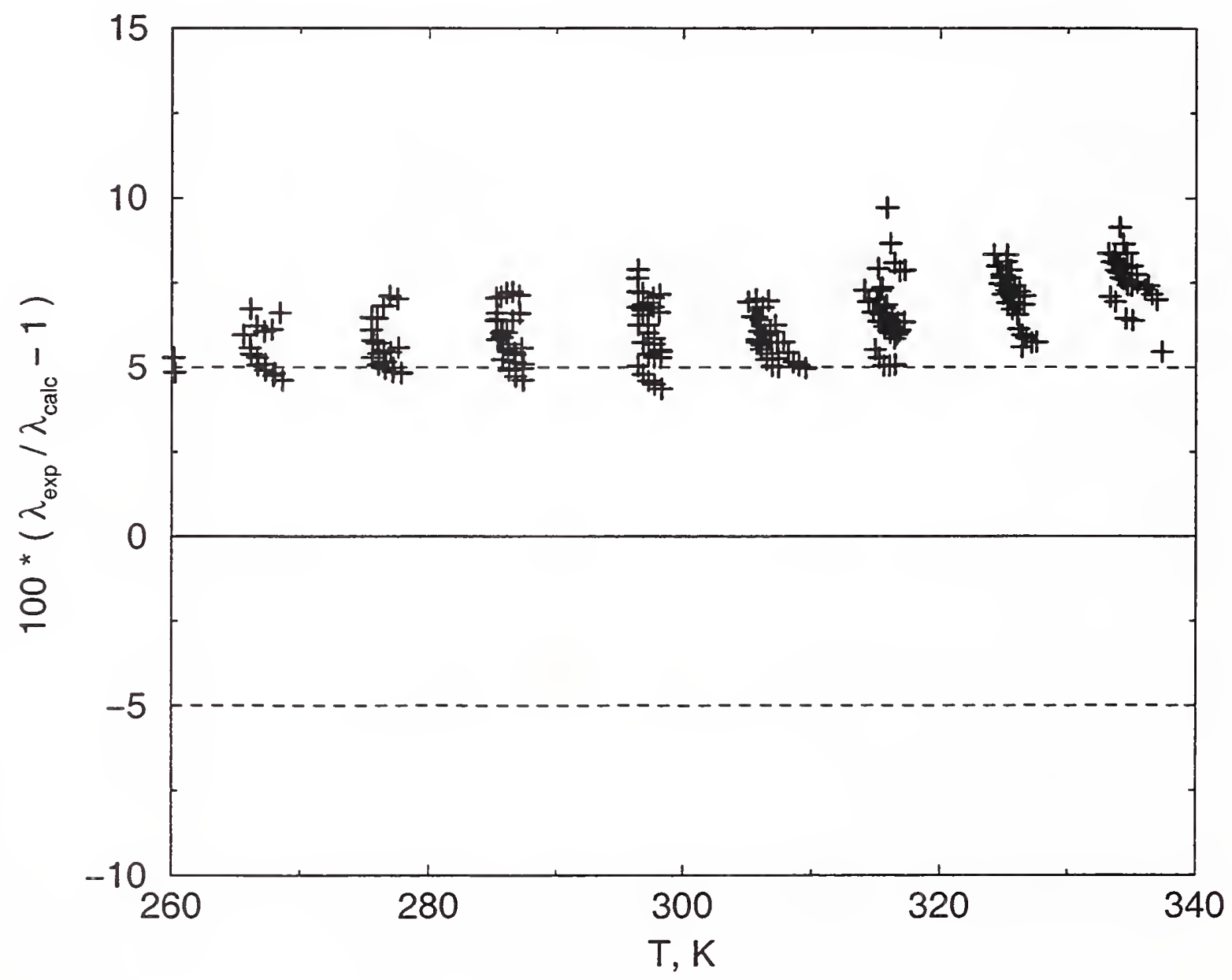

Figure 25. Relative deviations of the steady-state thermal conductivity data for the binary $30 \% \mathrm{R} 32 / 70 \%$ $\mathrm{R} 134 \mathrm{a}$ mixture in the vapor phase from the predictions of REFPROP 6.01. 


\subsection{0 \% R32 / 30 \% R134a Mixture}

The precise molar composition of the mixture was $0.70031 \mathrm{R} 32+0.29969 \mathrm{R} 134 \mathrm{a}$. Results for 267 measurements, at temperatures from $259 \mathrm{~K}$ to $348 \mathrm{~K}(0.09 \mathrm{MPa}$ to $0.56 \mathrm{MPa})$, are reported in Table 28 . Relative deviations between the measured thermal conductivity and the predictions of the mixture model REFPROP 6.01 [23] are shown in Figure 26. The thermal conductivity critical enhancement becomes more significant as the temperature increases and is likely responsible for the increasing deviations in Figure 26.

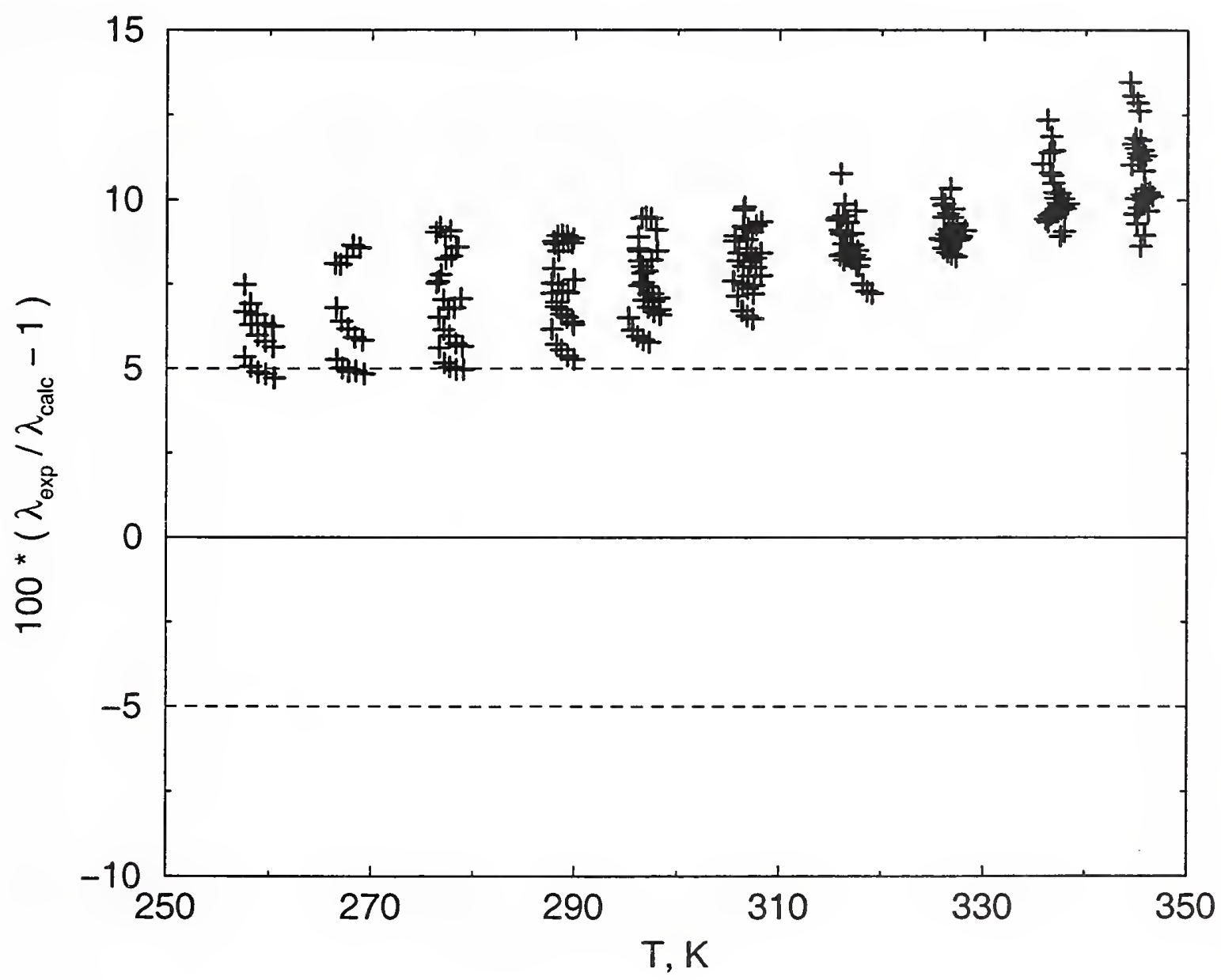

Figure 26. Relative deviations of the steady-state thermal conductivity data for the binary $70 \% \mathrm{R} 32 / 30 \%$ R134a mixture in the vapor phase from the predictions of REFPROP 6.01. 


\section{7 $30 \%$ R134a / $70 \%$ Propane Mixture}

The precise molar composition of the mixture was $0.30033 \mathrm{R} 134 \mathrm{a}+0.69966$ propane. Results for 55 measurements, at temperatures from $243 \mathrm{~K}$ to $302 \mathrm{~K}(0.11 \mathrm{MPa}$ to $0.30 \mathrm{MPa})$, are reported in Table 29 . Relative deviations between the measured thermal conductivity and the predictions of the mixture model REFPROP 6.01 [23] are shown in Figure 27. The thermal conductivity critical enhancement becomes more significant as the temperature increases and is likely responsible for the increasing deviations in Figure 27.

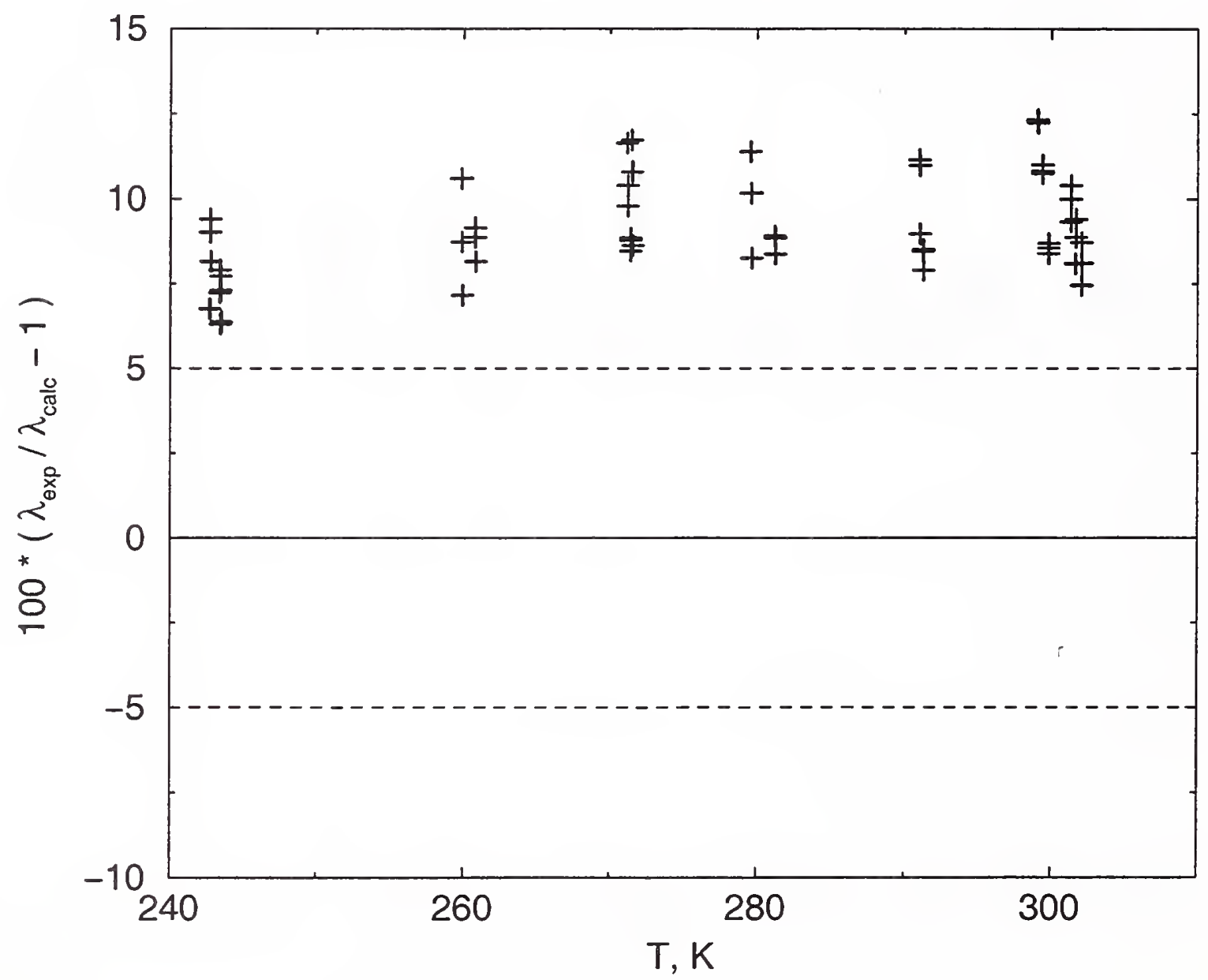

Figure 27. Relative deviations of the steady-state thermal conductivity data for the binary $30 \% \mathrm{R} 134 \mathrm{a} / 70$ $\%$ propane mixture in the vapor phase from the predictions of REFPROP 6.01 . 


\subsection{0 \% R134a / 30 \% Propane Mixture}

The precise molar composition of the mixture was $0.69948 \mathrm{R} 134 \mathrm{a}+0.30051$ propane. Results for 235 measurements, at temperatures from $258 \mathrm{~K}$ to $348 \mathrm{~K}(0.09 \mathrm{MPa}$ to $0.56 \mathrm{MPa})$, are reported in Table 30 . Relative deviations between the measured thermal conductivity and the predictions of the mixture model REFPROP 6.01 [23] are shown in Figure 28. The thermal conductivity critical enhancement becomes more significant as the temperature increases and is likely responsible for the increasing deviations in Figure 28.

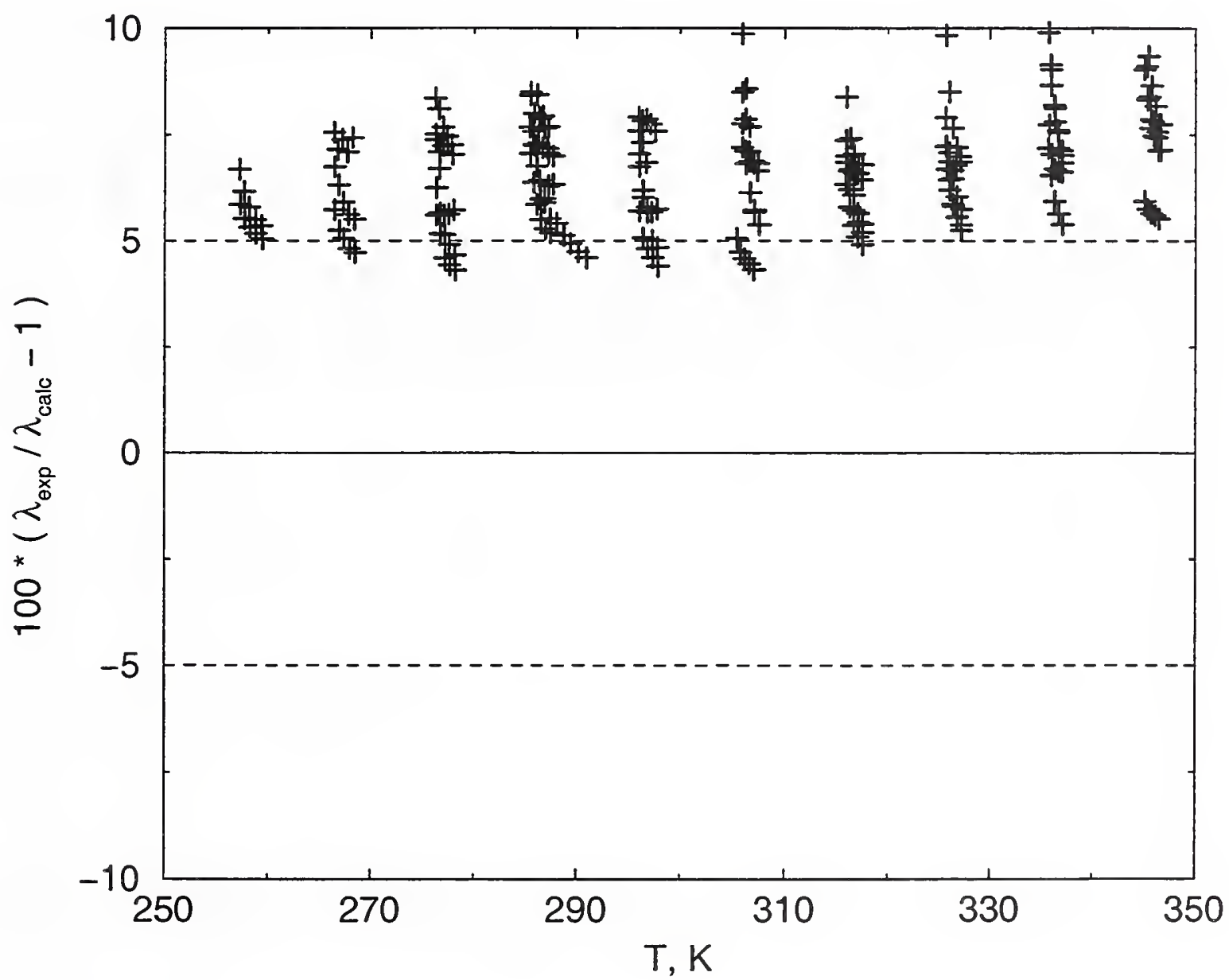

Figure 28. Relative deviations of the steady-state thermal conductivity data for the binary $70 \% \mathrm{R} 134 \mathrm{a} / 30$ $\%$ propane mixture in the vapor phase from the predictions of REFPROP 6.01 . 


\section{$4.933 \%$ R32 / $33 \%$ R125 / 33 \% R134a Mixture}

The precise molar composition of the mixture was $0.33298 \mathrm{R} 32+0.33342 \mathrm{R} 125+0.33359 \mathrm{R} 134 \mathrm{a}$. Results for 107 measurements, at temperatures from $263 \mathrm{~K}$ to $341 \mathrm{~K}(0.10 \mathrm{MPa}$ to $0.30 \mathrm{MPa})$, are reported in Table 31. Relative deviations between the measured thermal conductivity and the predictions of the mixture model REFPROP 6.01 [23] are shown in Figure 29. The thermal conductivity critical enhancement becomes more significant as the temperature increases and is likely responsible for the increasing deviations in Figure 29.

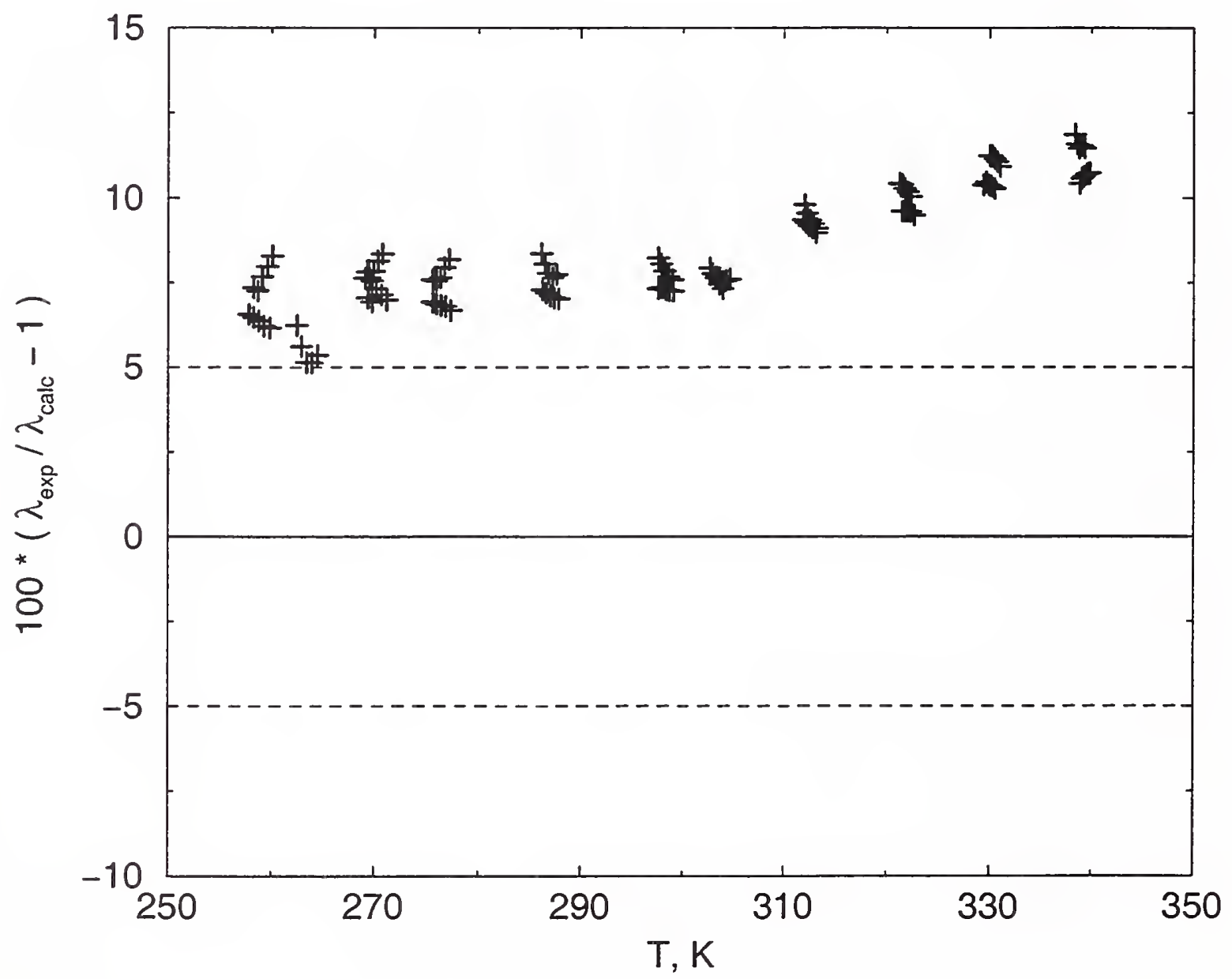

Figure 29. Relative deviations of the steady-state thermal conductivity data for the ternary $33 \%$ R $32 / 33 \%$ R125/33\% R134a mixture in the vapor phase from the predictions of REFPROP 6.01. 


\section{$4.1030 \% \mathbf{R} 32$ / 10 \% R125 / 60 \% R134a Mixture}

The precise molar composition of the mixture was $0.30027 \mathrm{R} 32+0.09995 \mathrm{R} 125+0.59977 \mathrm{R} 134 \mathrm{a}$. Results for 263 measurements, at temperatures from $258 \mathrm{~K}$ to $345 \mathrm{~K}(0.09 \mathrm{MPa}$ to $0.53 \mathrm{MPa})$, are reported in Table 32. Relative deviations between the measured thermal conductivity and the predictions of the mixture model REFPROP 6.01 [23] are shown in Figure 30. The thermal conductivity critical enhancement becomes more significant as the temperature increases and is likely responsible for the increasing deviations in Figure 30.

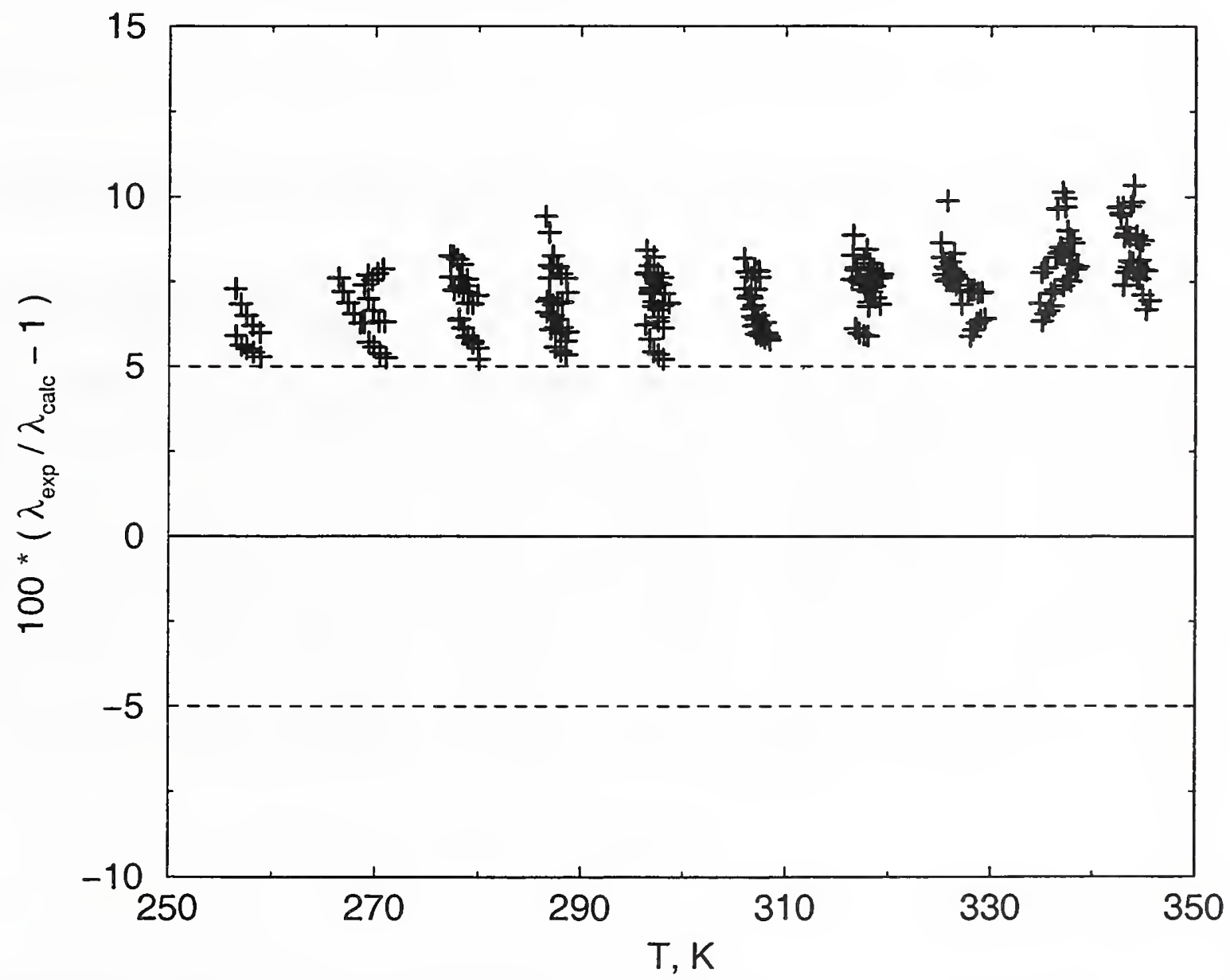

Figure 30. Relative deviations of the steady-state thermal conductivity data for the ternary $30 \% \mathrm{R} 32 / 10 \%$ $\mathrm{R} 125$ / $60 \% \mathrm{R} 134 \mathrm{a}$ mixture in the vapor phase from the predictions of REFPROP 6.01 . 


\section{Summary of Deviations Relative to REFPROP 6.01}

The mixture data have been compared to the NIST REFPROP 6.01 database [23], with deviations plots provided for each mixture. A summary of the statistics of these deviations is provided in Table 1. In the vapor phase, REFPROP 6.01 predicts the thermal conductivity much better for mixtures of polar refrigerants that it does for polar refrigerants mixtures with nonpolar propane. REFPROP 6.01 does better at predicting the thermal conductivity of the liquid phase than the vapor phase. The data is generally higher than the REFPROP predictions in both the liquid and vapor phases as the critical point is approached. This is likely due to an increasing contribution from the critical enhancement of the thermal conductivity. The deviations are characterized in terms of the average absolute deviations (AAD), where

$$
A A D=\frac{1}{n_{\text {points }}} \sum_{k=1}^{n_{\text {points }}} 100\left|\frac{\lambda_{\text {exp }}-\lambda_{\text {calc }}}{\lambda_{\text {calc }}}\right| .
$$

Table 1. Summary of deviations between the thermal conductivity data for each mixture and REFPROP 6.01 .

\begin{tabular}{|c|c|c|c|c|c|c|c|c|c|}
\hline \multirow[t]{2}{*}{ Mixture } & \multirow{2}{*}{$\begin{array}{l}\text { Composition } \\
\text { (mole fraction) }\end{array}$} & \multicolumn{2}{|c|}{ Data range } & \multicolumn{6}{|c|}{ Number of points, AAD ( \%) } \\
\hline & & \multirow{2}{*}{$\frac{T(K)}{244-347}$} & \multirow{2}{*}{$\frac{\rho\left(\mathrm{mol} \cdot \mathrm{L}^{-1}\right)}{0.027-13.1}$} & \multicolumn{2}{|c|}{$\begin{array}{c}\text { liquid } \\
\text { (transient) }\end{array}$} & \multicolumn{2}{|c|}{$\begin{array}{c}\text { vapor } \\
\text { (steady-state) }\end{array}$} & \multicolumn{2}{|c|}{$\begin{array}{c}\text { vapor } \\
\text { (transient) }\end{array}$} \\
\hline \multirow[t]{2}{*}{ R125/R134a } & $0.3002 / 0.6998$ & & & 142 & 3.01 & 160 & 3.14 & 248 & 2.28 \\
\hline & $0.7000 / 0.3000$ & $247-344$ & $0.034-12.4$ & 136 & 5.10 & 205 & 2.23 & 202 & 2.37 \\
\hline \multirow[t]{2}{*}{ R32/propane } & $0.2999 / 0.7001$ & $229-347$ & $0.012-14.2$ & 159 & 5.10 & 225 & 15.4 & 139 & 12.1 \\
\hline & $0.7001 / 0.2999$ & $254-336$ & $0.040-17.0$ & 136 & 8.83 & 193 & 18.5 & 395 & 11.4 \\
\hline \multirow[t]{2}{*}{$\mathrm{R} 32 / 134 \mathrm{a}$} & $0.2996 / 0.7004$ & $255-360$ & $0.032-14.9$ & 179 & 2.39 & 266 & 6.61 & 235 & 7.65 \\
\hline & $0.7003 / 0.2997$ & $254-348$ & $0.033-18.1$ & 130 & 4.11 & 267 & 8.38 & 231 & 8.65 \\
\hline \multirow[t]{2}{*}{ R134a/propane } & $0.3003 / 0.6997$ & $243-347$ & $0.037-12.3$ & 165 & 5.64 & 55 & 6.67 & 51 & 6.06 \\
\hline & $0.7012 / 0.2986$ & $250-348$ & $0.057-11.9$ & 246 & 5.46 & 235 & 9.18 & 233 & 5.10 \\
\hline \multirow[t]{2}{*}{$\mathrm{R} 32 / 125 / 134 \mathrm{a}$} & $0.333 / 0.333 / 0.334$ & $250-344$ & $0.033-14.7$ & 175 & 8.04 & 107 & 8.40 & 81 & 9.43 \\
\hline & $0.300 / 0.100 / 0.600$ & $250-345$ & $0.033-15.0$ & 182 & 4.02 & 263 & 7.26 & 230 & 8.52 \\
\hline
\end{tabular}

\section{Acknowledgments}

The support of the U.S. Department of Energy, Office of Building Technologies for this work on the thermophysical properties of alternative refrigerant mixtures is gratefully acknowledged. The data presented here will be used for further development of the NIST thermophysical-property database for alternative refrigerant mixtures - REFPROP. 


\section{References}

[1] Roder, H.M. A transient hot wire thermal conductivity apparatus for fluids, J. Res. Nat. Bur. Stand. (U.S.) 86, 457 (1981).

[2] Perkins, R.A.; Roder, H.M.; Nieto de Castro, C.A. A high temperature transient hot-wire thermal conductivity apparatus for fluids, J. Res. Natl. Inst. Stand. Technol. (U.S.) 96, 247 (1991).

[3] Roder, H.M., The thermal conductivity values of oxygen, J. Res. Nat. Bur. Stand. (U.S.) 86, 279 (1982).

[4] Roder, H.M. Experimental thermal conductivity values for hydrogen, methane, ethane, and propane, Natl. Bur. Stand. (U.S.) NBSIR 84-3006 (1984).

[5] Roder, H.M. The thermal conductivity of hydrogen for temperatures between 78 and $310 \mathrm{~K}$ with pressures to $70 \mathrm{MPa}$, Int. J. Thermophys. 5, 323 (1984).

[6] Roder, H.M. The thermal conductivity of methane for temperatures between 110 and $310 \mathrm{~K}$ with pressures to $70 \mathrm{MPa}$, Int. J. Thermophys. 6, 119 (1985).

[7] Roder, H.M.; Nieto de Castro, C.A. The thermal conductivity of ethane for temperatures between 110 and $325 \mathrm{~K}$ at pressures to $70 \mathrm{MPa}$, High Temp. High Press. 17, 453 (1985).

[8] Roder, H.M.; Friend, D.G. Experimental thermal conductivity values for mixtures of methane and ethane, Natl. Bur. Stand. (U.S.) NBSIR 85-3024 (1985).

[9] Roder, H.M.; Friend, D.G. The thermal conductivity of methane-ethane mixtures at temperatures between 140 and $330 \mathrm{~K}$ and at pressures to $70 \mathrm{MPa}$, Int. J. Thermophys. 6, 607 (1985).

[10] Roder, H.M.; Nieto de Castro, C.A. The thermal conductivity of liquid propane, J. Chem. Eng. Data 17, 12 (1982).

[11] Roder, H.M.; Perkins, R.A.; Nieto de Castro, C.A. Experimental thermal conductivity, thermal diffusivity, and specific heat values of argon and nitrogen, Natl. Inst. Stand. Technol. NISTIR 88-3902 (1988).

[12] Roder, H.M.; Nieto de Castro, C.A.; Mardolcar, U.V. The thermal conductivity of liquid argon for temperatures between 110 and $140 \mathrm{~K}$ with pressures to $70 \mathrm{MPa}$, Int. J. Thermophys. 8, 521 (1987).

[13] Roder, H.M.; Perkins, R.A.; Nieto de Castro, C.A. The thermal conductivity and heat capacity of gaseous argon, Int. J. Thermophys. 10, 1141 (1989).

[14] Perkins, R.A.; Friend, D.G.; Roder, H.M.; Nieto de Castro, C.A. Thermal conductivity surface of argon: A fresh analysis, Int. J. Thermophys. 12, 965 (1991).

[15] Nieto de Castro, C.A.; Taxis, B.; Roder, H.M.; Wakeham, W.A. Thermal diffusivity measurement by the transient hot-wire technique: A reappraisal, Int. J. Thermophys. 9, 293 (1988).

[16] Perkins, R.A.; Roder, H.M.; Friend, D.G.; Nieto de Castro, C.A. The thermal conductivity and heat capacity of fluid nitrogen, Physica A173, 332 (1991).

[17] Perkins, R.A.; Cieszkiewicz, M.T. Experimental thermal conductivity, thermal diffusivity, and specific heat values for mixtures of nitrogen, oxygen, and argon, Natl. Inst. Stand. Technol. NISTIR 3961 (1991) 
[18] Perkins, R.A.; Roder, H.M. Experimental thermal conductivity and thermal diffusivity values for neon and mixtures of neon and nitrogen, Natl. Inst. Stand. Technol. NISTIR 5088 (1999).

[19] Laesecke, A.; Perkins, R.A.; Nieto de Castro, C.A. Thermal conductivity of R134a, Fluid Phase Equilib. 80, 263 (1992).

[20] Perkins, R.A.; Laesecke, A.; Nieto de Castro, C.A. Polarized transient hot-wire thermal conductivity measurements, Fluid Phase Equilib. 80, 275 (1992).

[21] Laesecke, A.; Perkins, R.A.; Howley, J.B. An improved correlation for the thermal conductivity of HCFC123 (2,2-dichloro-1,1,1-trifluoroethane), Int. J. Refrig. 19, 231 (1996).

[22] Perkins, R.A. Measurement of the thermal properties of electrically conducting fluids using coated transient hot wires, Proc. 12th Symp. on Energy Eng. Sci., U.S. Department of Energy, Argonne, Illinois, p. 90-97, (1994).

[23] McLinden, M.O.; Klein, S.A.; Lemmon, E.W.; Peskin, A.P. NIST thermodynamic and transport properties of refrigerants and refrigerant mixtures-REFPROP, Natl. Inst. Stand. Technol. Standard Reference Database 23, 6.01 (1998). 


\section{Tables of transient results for anodized tantalum wires}

Table 2. Thermal conductivity of the binary $30 \% \mathrm{R} 125 / 70 \% \mathrm{R} 134 \mathrm{a}$ mixture in the compressed liquid phase.

\begin{tabular}{|c|c|c|c|c|c|c|c|}
\hline $\begin{array}{l}\text { Run } \\
\text { point }\end{array}$ & $\begin{array}{l}P_{\text {exp }} \\
\mathrm{MPa}\end{array}$ & $\begin{array}{c}Q \\
\mathrm{~W} \cdot \mathrm{m}^{-1}\end{array}$ & $\begin{array}{c}T_{\text {exp }} \\
\mathrm{K}\end{array}$ & $\begin{array}{c}\rho_{\text {calc }} \\
\mathrm{mol}^{\prime} \mathrm{L}^{-1}\end{array}$ & $\begin{array}{c}\lambda_{\exp } \\
\mathrm{W} \cdot \mathrm{m}^{-1} \cdot \mathrm{K}^{-1}\end{array}$ & STAT & $\begin{array}{c}T_{\text {cell }} \\
\mathrm{K}\end{array}$ \\
\hline 1001 & 0.353 & 0.17781 & 244.420 & 13.1040 & 0.09385 & 0.005 & 243.632 \\
\hline 1003 & 0.345 & 0.22535 & 244.640 & 13.0971 & 0.09437 & 0.004 & 243.633 \\
\hline 1005 & 0.346 & 0.27846 & 244.871 & 13.0902 & 0.09388 & 0.003 & 243.629 \\
\hline 1007 & 0.338 & 0.33721 & 245.125 & 13.0824 & 0.09440 & 0.003 & 243.625 \\
\hline 1009 & 0.336 & 0.40161 & 245.412 & 13.0737 & 0.09399 & 0.002 & 243.621 \\
\hline 1011 & 0.336 & 0.47168 & 245.709 & 13.0647 & 0.09372 & 0.003 & 243.611 \\
\hline 1013 & 0.333 & 0.54749 & 246.051 & 13.0543 & 0.09345 & 0.003 & 243.608 \\
\hline 1015 & 0.355 & 0.17778 & 244.302 & 13.1076 & 0.09481 & 0.005 & 243.522 \\
\hline 1017 & 0.353 & 0.22524 & 244.516 & 13.1011 & 0.09546 & 0.003 & 243.522 \\
\hline 1019 & 0.351 & 0.27829 & 244.765 & 13.0935 & 0.09414 & 0.004 & 243.525 \\
\hline 1021 & 0.349 & 0.33698 & 245.019 & 13.0858 & 0.09401 & 0.003 & 243.516 \\
\hline 1023 & 0.347 & 0.40134 & 245.315 & 13.0769 & 0.09447 & .0 .003 & 243.520 \\
\hline 1025 & 0.345 & 0.47129 & 245.617 & 13.0678 & 0.09424 & 0.003 & 243.514 \\
\hline 1027 & 0.344 & 0.54714 & 245.956 & 13.0575 & 0.09398 & 0.003 & 243.512 \\
\hline 1029 & 0.850 & 0.18357 & 253.054 & 12.8562 & 0.09087 & 0.005 & 252.225 \\
\hline 1031 & 0.859 & 0.23251 & 253.299 & 12.8489 & 0.09173 & 0.003 & 252.234 \\
\hline 1033 & 0.865 & 0.28727 & 253.553 & 12.8413 & 0.09189 & 0.002 & 252.237 \\
\hline 1035 & 0.854 & 0.34794 & 253.837 & 12.8322 & 0.09189 & 0.003 & 252.241 \\
\hline 1037 & 0.843 & 0.41439 & 254.144 & 12.8223 & 0.09207 & 0.002 & 252.245 \\
\hline 1039 & 0.839 & 0.48675 & 254.476 & 12.8119 & 0.09242 & 0.002 & 252.246 \\
\hline 1041 & 0.851 & 0.56483 & 254.818 & 12.8017 & 0.09283 & 0.002 & 252.239 \\
\hline 1043 & 0.908 & 0.18840 & 260.209 & 12.6343 & 0.08846 & 0.005 & 259.332 \\
\hline 1045 & 0.909 & 0.23870 & 260.456 & 12.6265 & 0.08850 & 0.003 & 259.338 \\
\hline 1047 & 0.907 & 0.29493 & 260.728 & 12.6177 & 0.08832 & 0.003 & 259.340 \\
\hline 1049 & 0.902 & 0.35712 & 261.031 & 12.6079 & 0.08845 & 0.003 & 259.353 \\
\hline 1051 & 0.899 & 0.42528 & 261.355 & 12.5974 & 0.08938 & 0.002 & 259.350 \\
\hline 1053 & 0.899 & 0.49940 & 261.698 & 12.5865 & 0.08988 & 0.002 & 259.356 \\
\hline 1055 & 0.905 & 0.57955 & 262.083 & 12.5744 & 0.09014 & 0.003 & 259.360 \\
\hline 1057 & 7.458 & 0.19493 & 269.851 & 12.5627 & 0.08781 & 0.006 & 268.941 \\
\hline 1059 & 7.459 & 0.24685 & 270.106 & 12.5551 & 0.08887 & 0.004 & 268.950 \\
\hline 1061 & 7.440 & 0.30483 & 270.400 & 12.5457 & 0.08901 & 0.003 & 268.964 \\
\hline 1063 & 7.419 & 0.36904 & 270.696 & 12.5362 & 0.08916 & 0.003 & 268.967 \\
\hline 1065 & 7.410 & 0.43949 & 271.034 & 12.5258 & 0.08979 & 0.004 & 268.968 \\
\hline 1067 & 7.435 & 0.51609 & 271.389 & 12.5160 & 0.09027 & 0.004 & 268.969 \\
\hline 1069 & 7.462 & 0.59898 & 271.773 & 12.5054 & 0.09121 & 0.005 & 268.973 \\
\hline 1071 & 1.539 & 0.19504 & 269.945 & 12.3429 & 0.08455 & 0.005 & 268.996 \\
\hline 1073 & 1.530 & 0.24687 & 270.200 & 12.3341 & 0.08491 & 0.004 & 269.000 \\
\hline 1075 & 1.523 & 0.30486 & 270.495 & 12.3241 & 0.08577 & 0.003 & 269.009 \\
\hline 1077 & 1.522 & 0.36926 & 270.813 & 12.3136 & 0.08586 & 0.004 & 269.010 \\
\hline 1079 & 1.545 & 0.43987 & 271.146 & 12.3034 & 0.08675 & 0.004 & 269.009 \\
\hline 1081 & 1.562 & 0.51661 & 271.513 & 12.2920 & 0.08760 & 0.005 & 269.015 \\
\hline
\end{tabular}


Table 2. Thermal conductivity of the binary $30 \% \mathrm{R} 125 / 70 \% \mathrm{R} 134 \mathrm{a}$ mixture in the compressed liquid phase (continued).

\begin{tabular}{|c|c|c|c|c|c|c|c|}
\hline $\begin{array}{l}\text { Run } \\
\text { point }\end{array}$ & $\begin{array}{l}P_{\text {exp }} \\
\mathrm{MPa}\end{array}$ & $\begin{array}{c}Q \\
\mathrm{~W} \cdot \mathrm{m}^{-1}\end{array}$ & $\begin{array}{c}T_{\exp } \\
\mathrm{K}\end{array}$ & $\begin{array}{c}\rho_{\text {calc }} \\
\mathrm{mol} \cdot \mathrm{L}^{-1}\end{array}$ & $\begin{array}{c}\lambda_{\exp } \\
\mathrm{W} \cdot \mathrm{m}^{-1} \cdot \mathrm{K}^{-1}\end{array}$ & STAT & $\begin{array}{c}T_{\text {cell }} \\
\mathrm{K}\end{array}$ \\
\hline 1083 & 1.576 & 0.59963 & 271.910 & 12.2793 & 0.08859 & 0.006 & 269.018 \\
\hline 1085 & 8.787 & 0.20145 & 279.768 & 12.3123 & 0.08576 & 0.005 & 278.784 \\
\hline 1087 & 8.827 & 0.25495 & 280.017 & 12.3062 & 0.08633 & 0.004 & 278.789 \\
\hline 1089 & 8.846 & 0.31489 & 280.313 & 12.2980 & 0.08617 & 0.004 & 278.789 \\
\hline 1091 & 8.818 & 0.38133 & 280.630 & 12.2873 & 0.08710 & 0.004 & 278.791 \\
\hline 1093 & 8.804 & 0.45422 & 280.977 & 12.2763 & 0.08775 & 0.005 & 278.787 \\
\hline 1095 & 8.787 & 0.53360 & 281.359 & 12.2641 & 0.08881 & 0.006 & 278.795 \\
\hline 1097 & 8.779 & 0.61929 & 281.750 & 12.2519 & 0.08962 & 0.008 & 278.786 \\
\hline 1099 & 4.423 & 0.20153 & 279.749 & 12.1398 & 0.08387 & 0.006 & 278.747 \\
\hline 1101 & 4.397 & 0.25515 & 280.033 & 12.1295 & 0.08361 & 0.004 & 278.763 \\
\hline 1103 & 4.385 & 0.31502 & 280.316 & 12.1196 & 0.08413 & 0.004 & 278.763 \\
\hline 1105 & 4.378 & 0.38148 & 280.647 & 12.1085 & 0.08525 & 0.005 & 278.763 \\
\hline 1107 & 4.392 & 0.45427 & 281.008 & 12.0972 & 0.08596 & 0.006 & 278.766 \\
\hline 1109 & 4.426 & 0.53348 & 281.382 & 12.0863 & 0.08667 & 0.008 & 278.767 \\
\hline 1111 & 4.443 & 0.61919 & 281.791 & 12.0735 & 0.08810 & 0.009 & 278.775 \\
\hline 1113 & 1.552 & 0.20157 & 279.746 & 12.0113 & 0.08225 & 0.004 & 278.730 \\
\hline 1115 & 1.550 & 0.25511 & 280.033 & 12.0012 & 0.08184 & 0.004 & 278.746 \\
\hline 1117 & 1.545 & 0.31494 & 280.332 & 11.9905 & 0.08288 & 0.004 & 278.751 \\
\hline 1119 & 1.525 & 0.38136 & 280.661 & 11.9779 & 0.08398 & 0.004 & 278.754 \\
\hline 1127 & 8.014 & 0.15907 & 289.124 & 11.9915 & 0.08419 & 0.006 & 288.342 \\
\hline 1129 & 8.021 & 0.20774 & 289.398 & 11.9831 & 0.08400 & 0.004 & 288.355 \\
\hline 1131 & 8.067 & 0.26288 & 289.666 & 11.9765 & 0.08444 & 0.003 & 288.355 \\
\hline 1133 & 8.086 & 0.32464 & 289.998 & 11.9668 & 0.08551 & 0.004 & 288.374 \\
\hline 1141 & 4.769 & 0.15909 & 289.196 & 11.8398 & 0.08054 & 0.006 & 288.373 \\
\hline 1143 & 4.774 & 0.20771 & 289.437 & 11.8318 & 0.08125 & 0.004 & 288.381 \\
\hline 1145 & 4.739 & 0.26294 & 289.746 & 11.8194 & 0.08267 & 0.003 & 288.395 \\
\hline 1149 & 4.719 & 0.39338 & 290.403 & 11.7958 & 0.08399 & 0.005 & 288.405 \\
\hline 1155 & 1.896 & 0.15915 & 289.366 & 11.6835 & 0.07668 & 0.005 & 288.516 \\
\hline 1157 & 1.886 & 0.20792 & 289.603 & 11.6742 & 0.07843 & 0.004 & 288.517 \\
\hline 1159 & 1.872 & 0.26318 & 289.905 & 11.6622 & 0.07902 & 0.004 & 288.522 \\
\hline 1161 & 1.861 & 0.32508 & 290.235 & 11.6493 & 0.08019 & 0.004 & 288.535 \\
\hline 1169 & 7.886 & 0.16400 & 299.246 & 11.6553 & 0.07786 & 0.005 & 298.384 \\
\hline 1171 & 7.878 & 0.21435 & 299.506 & 11.6462 & 0.07936 & 0.004 & 298.389 \\
\hline 1173 & 7.902 & 0.27143 & 299.808 & 11.6373 & 0.07919 & 0.004 & 298.391 \\
\hline 1175 & 7.922 & 0.33523 & 300.137 & 11.6273 & 0.07970 & 0.006 & 298.391 \\
\hline 1183 & 4.391 & 0.16435 & 299.229 & 11.4636 & 0.07593 & 0.005 & 298.350 \\
\hline 1185 & 4.381 & 0.21461 & 299.509 & 11.4527 & 0.07737 & 0.004 & 298.363 \\
\hline 1187 & 4.368 & 0.27156 & 299.825 & 11.4402 & 0.07796 & 0.003 & 298.371 \\
\hline 1189 & 4.404 & 0.33528 & 300.156 & 11.4301 & 0.07906 & 0.004 & 298.366 \\
\hline 1197 & 1.476 & 0.16435 & 299.233 & 11.2740 & 0.07438 & 0.005 & 298.330 \\
\hline 1199 & 1.469 & 0.21455 & 299.523 & 11.2616 & 0.07528 & 0.003 & 298.346 \\
\hline 1201 & 1.469 & 0.27136 & 299.837 & 11.2487 & 0.07589 & 0.003 & 298.349 \\
\hline 1211 & 1.484 & 0.16431 & 299.276 & 11.2728 & 0.07292 & 0.005 & 298.369 \\
\hline
\end{tabular}


Table 2. Thermal conductivity of the binary $30 \% \mathrm{R} 125 / 70 \% \mathrm{R} 134 \mathrm{a}$ mixture in the compressed liquid phase (continued).

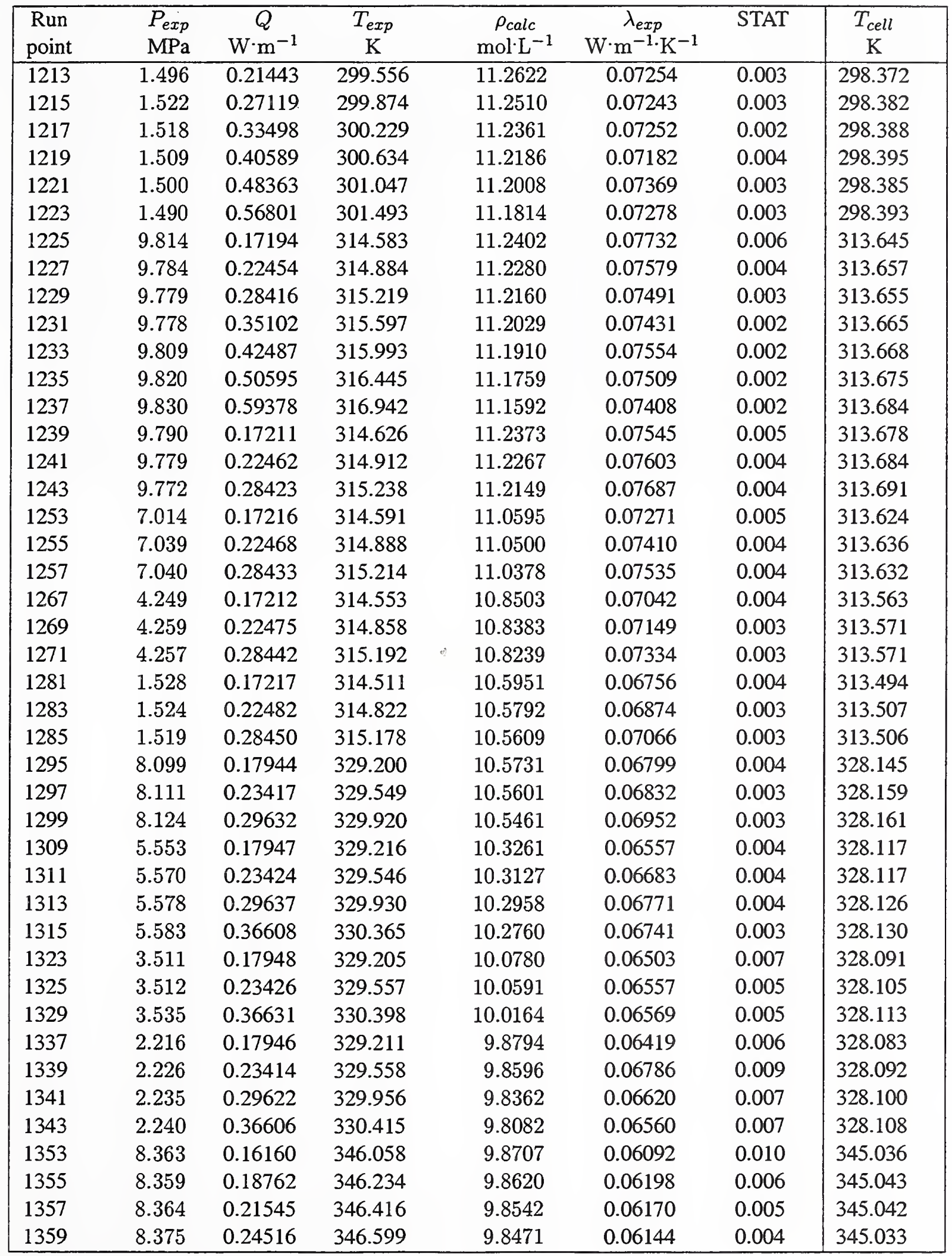


Table 2. Thermal conductivity of the binary $30 \% \mathrm{R} 125 / 70 \% \mathrm{R} 134 \mathrm{a}$ mixture in the compressed liquid phase (continued).

\begin{tabular}{|ccccccc|c|}
\hline $\begin{array}{c}\text { Run } \\
\text { point }\end{array}$ & $\begin{array}{c}P_{\text {exp }} \\
\mathrm{MPa}\end{array}$ & $\begin{array}{c}Q \\
\mathrm{~W} \cdot \mathrm{m}^{-1}\end{array}$ & $\begin{array}{c}T_{\text {exp }} \\
\mathrm{K}\end{array}$ & $\begin{array}{c}\rho_{\text {calc }} \\
\mathrm{mol} \cdot \mathrm{L}^{-1}\end{array}$ & $\begin{array}{c}\lambda_{\text {exp }} \\
\mathrm{W} \cdot \mathrm{m}^{-1} \cdot \mathrm{K}^{-1}\end{array}$ & STAT & $\begin{array}{c}T_{\text {cell }} \\
\mathrm{K}\end{array}$ \\
\hline 1361 & 8.380 & 0.27677 & 346.816 & 9.8375 & 0.06192 & 0.003 & 345.042 \\
1363 & 8.382 & 0.31039 & 347.041 & 9.8272 & 0.06198 & 0.004 & 345.043 \\
1365 & 6.384 & 0.13789 & 345.871 & 9.6022 & 0.06135 & 0.009 & 344.988 \\
1367 & 6.383 & 0.16162 & 346.050 & 9.5922 & 0.06053 & 0.007 & 345.006 \\
1369 & 6.394 & 0.18734 & 346.207 & 9.5854 & 0.06187 & 0.007 & 344.999 \\
1373 & 6.402 & 0.24502 & 346.620 & 9.5642 & 0.05994 & 0.004 & 345.014 \\
1375 & 6.402 & 0.27681 & 346.826 & 9.5529 & 0.05962 & 0.004 & 345.012 \\
1377 & 6.397 & 0.31054 & 347.043 & 9.5400 & 0.06073 & 0.004 & 345.019 \\
1379 & 4.505 & 0.13780 & 345.870 & 9.2461 & 0.05890 & 0.009 & 344.961 \\
1381 & 4.496 & 0.16148 & 346.043 & 9.2321 & 0.06081 & 0.010 & 344.975 \\
1383 & 4.493 & 0.18728 & 346.196 & 9.2209 & 0.06193 & 0.010 & 344.969 \\
1385 & 4.491 & 0.21523 & 346.407 & 9.2058 & 0.05981 & 0.007 & 344.979 \\
1387 & 4.495 & 0.24502 & 346.625 & 9.1916 & 0.05903 & 0.006 & 344.987 \\
1389 & 4.503 & 0.27671 & 346.819 & 9.1798 & 0.06115 & 0.007 & 344.988 \\
1391 & 4.506 & 0.31031 & 347.044 & 9.1647 & 0.06324 & 0.010 & 344.995 \\
\hline
\end{tabular}


Table 3. Thermal conductivity of the binary $70 \% \mathrm{R} 125$ / $30 \% \mathrm{R} 134 \mathrm{a}$ mixture in the compressed liquid phase.

\begin{tabular}{|c|c|c|c|c|c|c|c|}
\hline $\begin{array}{l}\text { Run } \\
\text { point }\end{array}$ & $\begin{array}{l}P_{\text {exp }} \\
\mathrm{MPa}\end{array}$ & $\begin{array}{c}Q \\
\mathrm{~W} \cdot \mathrm{m}^{-1}\end{array}$ & $\begin{array}{c}T_{\exp } \\
\mathrm{K}\end{array}$ & $\begin{array}{c}\rho_{\text {calc }} \\
\mathrm{mol} \cdot \mathrm{L}^{-1}\end{array}$ & $\begin{array}{c}\lambda_{\exp } \\
\mathrm{W} \cdot \mathrm{m}^{-1} \cdot \mathrm{K}^{-1}\end{array}$ & STAT & $\begin{array}{c}T_{\text {cell }} \\
\mathrm{K}\end{array}$ \\
\hline 1007 & 0.326 & 0.33687 & 247.603 & 12.3804 & 0.08405 & 0.003 & 245.753 \\
\hline 1009 & 0.319 & 0.40147 & 247.950 & 12.3691 & 0.08378 & 0.002 & 245.751 \\
\hline 1011 & 0.313 & 0.47180 & 248.341 & 12.3563 & 0.08304 & 0.002 & 245.747 \\
\hline 1013 & 0.308 & 0.54789 & 248.756 & 12.3428 & 0.08284 & 0.002 & 245.742 \\
\hline 1015 & 0.500 & 0.14141 & 256.286 & 12.1037 & 0.08048 & 0.011 & 255.502 \\
\hline 1017 & 0.501 & 0.18457 & 256.533 & 12.0955 & 0.07957 & 0.006 & 255.508 \\
\hline 1019 & 0.502 & 0.23355 & 256.828 & 12.0857 & 0.07933 & 0.005 & 255.514 \\
\hline 1021 & 0.503 & 0.28844 & 257.136 & 12.0754 & 0.07992 & 0.004 & 255.511 \\
\hline 1023 & 0.503 & 0.34924 & 257.499 & 12.0632 & 0.07988 & 0.003 & 255.515 \\
\hline 1025 & 0.502 & 0.41603 & 257.873 & 12.0507 & 0.07954 & 0.002 & 255.510 \\
\hline 1027 & 0.501 & 0.48845 & 258.302 & 12.0362 & 0.07914 & 0.002 & 255.511 \\
\hline 1029 & 0.488 & 0.14146 & 256.268 & 12.1038 & 0.08060 & 0.010 & 255.482 \\
\hline 1031 & 0.486 & 0.18452 & 256.510 & 12.0957 & 0.08001 & 0.007 & 255.483 \\
\hline 1033 & 0.485 & 0.23319 & 256.798 & 12.0860 & 0.07937 & 0.005 & 255.489 \\
\hline 1035 & 0.485 & 0.28804 & 257.121 & 12.0752 & 0.07956 & 0.003 & 255.497 \\
\hline 1037 & 0.484 & 0.34920 & 257.476 & 12.0633 & 0.07940 & 0.003 & 255.492 \\
\hline 1039 & 0.482 & 0.41618 & 257.853 & 12.0505 & 0.07949 & 0.002 & 255.492 \\
\hline 1041 & 0.480 & 0.48902 & 258.269 & 12.0364 & 0.07918 & 0.002 & 255.487 \\
\hline 1043 & 1.207 & 0.14845 & 270.116 & 11.6579 & 0.07464 & 0.009 & 269.245 \\
\hline 1045 & 1.212 & 0.19352 & 270.400 & 11.6481 & 0.07489 & 0.006 & 269.254 \\
\hline 1047 & 1.219 & 0.24480 & 270.721 & 11.6369 & 0.07415 & 0.004 & 269.260 \\
\hline 1049 & 1.215 & 0.30248 & 271.081 & 11.6238 & 0.07420 & 0.003 & 269.265 \\
\hline 1051 & 1.212 & 0.36650 & 271.478 & 11.6093 & 0.07383 & 0.002 & 269.265 \\
\hline 1053 & 1.204 & 0.43683 & 271.914 & 11.5931 & 0.07388 & 0.002 & 269.270 \\
\hline 1055 & 1.199 & 0.51350 & 272.377 & 11.5760 & 0.07379 & 0.002 & 269.260 \\
\hline 1057 & 1.198 & 0.14845 & 270.123 & 11.6573 & 0.07498 & 0.009 & 269.250 \\
\hline 1059 & 1.206 & 0.19355 & 270.412 & 11.6473 & 0.07484 & 0.006 & 269.262 \\
\hline 1061 & 1.206 & 0.24497 & 270.727 & 11.6360 & 0.07407 & 0.004 & 269.261 \\
\hline 1063 & 1.203 & 0.30289 & 271.087 & 11.6230 & 0.07424 & 0.003 & 269.266 \\
\hline 1065 & 1.200 & 0.36700 & 271.481 & 11.6086 & 0.07410 & 0.002 & 269.266 \\
\hline 1067 & 1.194 & 0.43735 & 271.910 & 11.5927 & 0.07393 & 0.002 & 269.265 \\
\hline 1069 & 1.188 & 0.51373 & 272.381 & 11.5753 & 0.07358 & 0.002 & 269.259 \\
\hline 1071 & 9.949 & 0.15553 & 284.622 & 11.5797 & 0.07603 & 0.009 & 283.707 \\
\hline 1073 & 9.941 & 0.20296 & 284.932 & 11.5695 & 0.07536 & 0.005 & 283.722 \\
\hline 1075 & 9.908 & 0.25716 & 285.259 & 11.5578 & 0.07459 & 0.004 & 283.723 \\
\hline 1077 & 9.899 & 0.31790 & 285.631 & 11.5456 & 0.07452 & 0.003 & 283.723 \\
\hline 1079 & 9.889 & 0.38529 & 286.047 & 11.5320 & 0.07471 & 0.002 & 283.721 \\
\hline 1081 & 9.885 & 0.45899 & 286.495 & 11.5175 & 0.07416 & 0.002 & 283.719 \\
\hline 1083 & 9.893 & 0.53909 & 286.995 & 11.5020 & 0.07386 & 0.001 & 283.724 \\
\hline 1085 & 5.461 & 0.15607 & 284.629 & 11.3616 & 0.07254 & 0.008 & 283.674 \\
\hline 1087 & 5.451 & 0.20353 & 284.924 & 11.3507 & 0.07195 & 0.005 & 283.683 \\
\hline 1089 & 5.453 & 0.25734 & 285.282 & 11.3382 & 0.07170 & 0.004 & 283.697 \\
\hline 1091 & 5.478 & 0.31797 & 285.673 & 11.3258 & 0.07130 & 0.003 & 283.693 \\
\hline
\end{tabular}


Table 3. Thermal conductivity of the binary $70 \% \mathrm{R} 125$ / $30 \% \mathrm{R} 134 \mathrm{a}$ mixture in the compressed liquid phase (continued).

\begin{tabular}{|c|c|c|c|c|c|c|c|}
\hline $\begin{array}{l}\text { Run } \\
\text { point }\end{array}$ & $\begin{array}{l}P_{\text {exp }} \\
\mathrm{MPa}\end{array}$ & $\begin{array}{c}Q \\
W \cdot m^{-1}\end{array}$ & $\begin{array}{c}T_{e x p} \\
\mathrm{~K}\end{array}$ & $\begin{array}{c}\rho_{\text {calc }} \\
\mathrm{mol}^{-1} \mathrm{~L}^{-1}\end{array}$ & $\begin{array}{c}\lambda_{\exp } \\
\mathrm{W} \cdot \mathrm{m}^{-1} \cdot \mathrm{K}^{-1}\end{array}$ & STAT & $\begin{array}{c}T_{\text {cell }} \\
\mathrm{K}\end{array}$ \\
\hline 1093 & 5.494 & 0.38530 & 286.092 & 11.3119 & 0.07115 & 0.002 & 283.699 \\
\hline 1095 & 5.503 & 0.45927 & 286.561 & 11.2958 & 0.07114 & 0.002 & 283.693 \\
\hline 1097 & 5.503 & 0.53960 & 287.069 & 11.2777 & 0.07078 & 0.001 & 283.699 \\
\hline 1099 & 1.463 & 0.15595 & 284.621 & 11.1256 & 0.06921 & 0.008 & 283.638 \\
\hline 1101 & 1.472 & 0.20346 & 284.944 & 11.1133 & 0.06820 & 0.005 & 283.659 \\
\hline 1103 & 1.479 & 0.25714 & 285.308 & 11.0991 & 0.06868 & 0.004 & 283.661 \\
\hline 1105 & 1.480 & 0.31787 & 285.704 & 11.0832 & 0.06807 & 0.003 & 283.664 \\
\hline 1107 & 1.473 & 0.38532 & 286.155 & 11.0645 & 0.06811 & 0.002 & 283.673 \\
\hline 1109 & 1.456 & 0.45926 & 286.643 & 11.0435 & 0.06805 & 0.002 & 283.676 \\
\hline 1111 & 1.444 & 0.53975 & 287.168 & 11.0212 & 0.06768 & 0.002 & 283.675 \\
\hline 1113 & 9.709 & 0.16297 & 299.399 & 11.0850 & 0.07032 & 0.008 & 298.377 \\
\hline 1115 & 9.689 & 0.21267 & 299.727 & 11.0727 & 0.06913 & 0.005 & 298.383 \\
\hline 1117 & 9.681 & 0.26945 & 300.104 & 11.0594 & 0.06938 & 0.004 & 298.391 \\
\hline 1119 & 9.674 & 0.33327 & 300.517 & 11.0449 & 0.06889 & 0.003 & 298.391 \\
\hline 1121 & 9.669 & 0.40357 & 300.969 & 11.0291 & 0.06883 & 0.002 & 298.394 \\
\hline 1123 & 9.668 & 0.48069 & 301.473 & 11.0118 & 0.06871 & 0.002 & 298.389 \\
\hline 1125 & 9.670 & 0.56486 & 302.029 & 10.9927 & 0.06842 & 0.001 & 298.394 \\
\hline 1127 & 5.448 & 0.16351 & 299.405 & 10.8154 & 0.06668 & 0.010 & 298.336 \\
\hline 1129 & 5.450 & 0.21337 & 299.739 & 10.8025 & 0.06593 & 0.005 & 298.343 \\
\hline 1131 & 5.448 & 0.26980 & 300.150 & 10.7862 & 0.06642 & 0.004 & 298.365 \\
\hline 1133 & 5.447 & 0.33340 & 300.570 & 10.7696 & 0.06589 & 0.003 & 298.356 \\
\hline 1135 & 5.435 & 0.40394 & 301.048 & 10.7498 & 0.06586 & 0.002 & 298.359 \\
\hline 1137 & 5.425 & 0.48147 & 301.575 & 10.7282 & 0.06533 & 0.002 & 298.360 \\
\hline 1139 & 5.420 & 0.56590 & 302.149 & 10.7049 & 0.06525 & 0.001 & 298.355 \\
\hline 1141 & 4.129 & 0.16351 & 299.425 & 10.7161 & 0.06621 & 0.007 & 298.339 \\
\hline 1143 & 4.125 & 0.21332 & 299.759 & 10.7020 & 0.06596 & 0.005 & 298.346 \\
\hline 1145 & 4.116 & 0.26971 & 300.162 & 10.6845 & 0.06586 & 0.004 & 298.356 \\
\hline 1147 & 4.116 & 0.33355 & 300.602 & 10.6662 & 0.06447 & 0.002 & 298.355 \\
\hline 1149 & 4.109 & 0.40415 & 301.086 & 10.6454 & 0.06501 & 0.002 & 298.355 \\
\hline 1151 & 4.110 & 0.48175 & 301.612 & 10.6233 & 0.06432 & 0.002 & 298.351 \\
\hline 1153 & 4.107 & 0.56604 & 302.202 & 10.5980 & 0.06398 & 0.001 & 298.362 \\
\hline 1157 & 1.728 & 0.21327 & 299.794 & 10.4913 & 0.06350 & 0.005 & 298.329 \\
\hline 1165 & 1.705 & 0.48179 & 301.683 & 10.3988 & 0.06293 & 0.002 & 298.338 \\
\hline 1167 & 1.702 & 0.56618 & 302.290 & 10.3689 & 0.06211 & 0.001 & 298.342 \\
\hline 1169 & 8.826 & 0.17100 & 314.955 & 10.4658 & 0.06434 & 0.007 & 313.803 \\
\hline 1171 & 8.830 & 0.22294 & 315.325 & 10.4518 & 0.06352 & 0.005 & 313.809 \\
\hline 1173 & 8.837 & 0.28249 & 315.736 & 10.4365 & 0.06336 & 0.003 & 313.804 \\
\hline 1175 & 8.844 & 0.34931 & 316.219 & 10.4185 & 0.06339 & 0.003 & 313.810 \\
\hline 1177 & 8.847 & 0.42324 & 316.730 & 10.3989 & 0.06292 & 0.002 & 313.800 \\
\hline 1179 & 8.846 & 0.50426 & 317.298 & 10.3766 & 0.06273 & 0.001 & 313.795 \\
\hline 1181 & 8.821 & 0.59278 & 317.924 & 10.3501 & 0.06257 & 0.001 & 313.794 \\
\hline 1183 & 6.194 & 0.17121 & 314.905 & 10.2397 & 0.06136 & 0.007 & 313.702 \\
\hline 1185 & 6.203 & 0.22329 & 315.280 & 10.2242 & 0.06138 & 0.005 & 313.710 \\
\hline
\end{tabular}


Table 3. Thermal conductivity of the binary $70 \% \mathrm{R} 125 / 30 \% \mathrm{R} 134 \mathrm{a}$ mixture in the compressed liquid phase (continued).

\begin{tabular}{|c|c|c|c|c|c|c|c|}
\hline $\begin{array}{c}\text { Run } \\
\text { point }\end{array}$ & $\begin{array}{l}P_{\text {exp }} \\
\mathrm{MPa}\end{array}$ & $\begin{array}{c}Q \\
\mathrm{~W} \cdot \mathrm{m}^{-1}\end{array}$ & $\begin{array}{c}T_{\exp } \\
\mathrm{K}\end{array}$ & $\begin{array}{c}\rho_{\text {calc }} \\
\mathrm{mol} \cdot \mathrm{L}^{-1}\end{array}$ & $\begin{array}{c}\lambda_{\exp } \\
\mathrm{W} \cdot \mathrm{m}^{-1} \cdot \mathrm{K}^{-1}\end{array}$ & STAT & $\begin{array}{c}T_{\text {cell }} \\
\mathrm{K}\end{array}$ \\
\hline 1187 & 6.208 & 0.28252 & 315.704 & 10.2063 & 0.06098 & 0.003 & 313.710 \\
\hline 1189 & 6.210 & 0.34935 & 316.189 & 10.1851 & 0.06087 & 0.002 & 313.700 \\
\hline 1191 & 6.207 & 0.42342 & 316.736 & 10.1607 & 0.06041 & 0.002 & 313.713 \\
\hline 1193 & 6.191 & 0.50453 & 317.305 & 10.1339 & 0.06027 & 0.001 & 313.698 \\
\hline 1195 & 6.176 & 0.59296 & 317.942 & 10.1038 & 0.05994 & 0.001 & 313.688 \\
\hline 1197 & 4.192 & 0.17103 & 314.773 & 10.0332 & 0.05972 & 0.007 & 313.554 \\
\hline 1199 & 4.170 & 0.22305 & 315.158 & 10.0115 & 0.05911 & 0.004 & 313.551 \\
\hline 1201 & 4.162 & 0.28229 & 315.602 & 9.9885 & 0.05896 & 0.003 & 313.549 \\
\hline 1203 & 4.156 & 0.34917 & 316.093 & 9.9631 & 0.05868 & 0.002 & 313.548 \\
\hline 1205 & 4.151 & 0.42340 & 316.645 & 9.9346 & 0.05824 & 0.002 & 313.541 \\
\hline 1207 & 4.163 & 0.50452 & 317.246 & 9.9054 & 0.05797 & 0.001 & 313.539 \\
\hline 1209 & 4.172 & 0.59278 & 317.897 & 9.8731 & 0.05782 & 0.001 & 313.531 \\
\hline 1211 & 2.793 & 0.17096 & 314.562 & 9.8641 & 0.05821 & 0.007 & 313.318 \\
\hline 1213 & 2.807 & 0.22292 & 314.948 & 9.8447 & 0.05770 & 0.004 & 313.321 \\
\hline 1215 & 2.812 & 0.28206 & 315.400 & 9.8201 & 0.05741 & 0.003 & 313.320 \\
\hline 1217 & 2.808 & 0.34868 & 315.902 & 9.7912 & 0.05721 & 0.002 & 313.320 \\
\hline 1219 & 2.790 & 0.42269 & 316.459 & 9.7567 & 0.05688 & 0.002 & 313.320 \\
\hline 1221 & 2.784 & 0.50402 & 317.089 & 9.7193 & 0.05647 & 0.001 & 313.318 \\
\hline 1223 & 2.780 & 0.59257 & 317.754 & 9.6796 & 0.05629 & 0.001 & 313.312 \\
\hline 1225 & 1.923 & 0.17095 & 314.514 & 9.7348 & 0.05687 & 0.006 & 313.251 \\
\hline 12.27 & 1.926 & 0.22314 & 314.925 & 9.7102 & 0.05655 & 0.004 & 313.264 \\
\hline 1229 & 1.912 & 0.28247 & 315.374 & 9.6800 & 0.05640 & 0.003 & 313.269 \\
\hline 1231 & 1.905 & 0.34919 & 315.898 & 9.6459 & 0.05598 & 0.002 & 313.265 \\
\hline 1233 & 1.901 & 0.42320 & 316.457 & 9.6095 & 0.05563 & 0.002 & 313.260 \\
\hline 1235 & 1.897 & 0.50438 & 317.094 & 9.5676 & 0.05532 & 0.001 & 313.265 \\
\hline 1237 & 1.912 & 0.59260 & 317.766 & 9.5261 & 0.05502 & 0.002 & 313.260 \\
\hline 1239 & 7.435 & 0.17824 & 329.392 & 9.7204 & 0.05854 & 0.006 & 328.100 \\
\hline 1241 & 7.437 & 0.23259 & 329.821 & 9.7005 & 0.05839 & 0.004 & 328.104 \\
\hline 1243 & 7.428 & 0.29455 & 330.288 & 9.6772 & 0.05958 & 0.003 & 328.108 \\
\hline 1245 & 7.414 & 0.36423 & 330.843 & 9.6491 & 0.05805 & 0.002 & 328.113 \\
\hline 1247 & 7.408 & 0.44142 & 331.433 & 9.6200 & 0.05717 & 0.002 & 328.109 \\
\hline 1249 & 7.408 & 0.52606 & 332.078 & 9.5888 & 0.05655 & 0.001 & 328.104 \\
\hline 1251 & 7.423 & 0.61799 & 332.758 & 9.5577 & 0.05896 & 0.001 & 328.108 \\
\hline 1253 & 6.155 & 0.17839 & 329.384 & 9.5506 & 0.05778 & 0.006 & 328.064 \\
\hline 1255 & 6.150 & 0.23271 & 329.813 & 9.5276 & 0.05902 & 0.005 & 328.083 \\
\hline 1257 & 6.137 & 0.29458 & 330.296 & 9.5003 & 0.05997 & 0.004 & 328.083 \\
\hline 1259 & 6.132 & 0.36443 & 330.856 & 9.4700 & 0.05773 & 0.006 & 328.090 \\
\hline 1261 & 6.130 & 0.44179 & 331.447 & 9.4382 & 0.05845 & 0.002 & 328.087 \\
\hline 1263 & 6.137 & 0.52647 & 332.120 & 9.4029 & 0.05732 & 0.002 & 328.090 \\
\hline 1265 & 6.147 & 0.61858 & 332.816 & 9.3668 & 0.05875 & 0.002 & 328.095 \\
\hline 1271 & 4.815 & 0.29493 & 330.340 & 9.2779 & 0.05747 & 0.003 & 328.070 \\
\hline 1273 & 4.817 & 0.36464 & 330.881 & 9.2452 & 0.05752 & 0.003 & 328.072 \\
\hline 1275 & 4.813 & 0.44183 & 331.497 & 9.2064 & 0.05786 & 0.003 & 328.082 \\
\hline
\end{tabular}


Table 3. Thermal conductivity of the binary $70 \% \mathrm{R} 125 / 30 \% \mathrm{R} 134 \mathrm{a}$ mixture in the compressed liquid phase (continued).

\begin{tabular}{|ccccccc|c|}
\hline $\begin{array}{c}\text { Run } \\
\text { point }\end{array}$ & $\begin{array}{c}P_{\text {exp }} \\
\mathrm{MPa}\end{array}$ & $\begin{array}{c}Q \\
\mathrm{~W} \cdot \mathrm{m}^{-1}\end{array}$ & $\begin{array}{c}T_{\text {exp }} \\
\mathrm{K}\end{array}$ & $\begin{array}{c}\rho_{\text {calc }} \\
\mathrm{mol} \cdot \mathrm{L}^{-1}\end{array}$ & $\begin{array}{c}\lambda_{\text {exp }} \\
\mathrm{W} \cdot \mathrm{m}^{-1} \cdot \mathrm{K}^{-1}\end{array}$ & STAT & $\begin{array}{c}T_{\text {cell }} \\
\mathrm{K}\end{array}$ \\
\hline 1279 & 4.799 & 0.61891 & 332.907 & 9.1145 & 0.05653 & 0.003 & 328.070 \\
1287 & 3.483 & 0.36465 & 330.915 & 8.9431 & 0.06040 & 0.006 & 328.072 \\
1289 & 3.476 & 0.44198 & 331.530 & 8.8934 & 0.06013 & 0.007 & 328.071 \\
1291 & 3.475 & 0.52667 & 332.234 & 8.8368 & 0.05904 & 0.007 & 328.076 \\
1293 & 3.472 & 0.61890 & 332.936 & 8.7786 & 0.06212 & 0.010 & 328.068 \\
1295 & 2.718 & 0.17839 & 329.410 & 8.8422 & 0.06173 & 0.009 & 328.046 \\
1297 & 2.710 & 0.23264 & 329.852 & 8.8002 & 0.06247 & 0.010 & 328.059 \\
\hline
\end{tabular}


Table 4. Thermal conductivity of the binary $30 \% \mathrm{R} 32 / 70 \%$ propane mixture in the compressed liquid phase.

\begin{tabular}{|c|c|c|c|c|c|c|c|}
\hline $\begin{array}{l}\text { Run } \\
\text { point }\end{array}$ & $\begin{array}{l}P_{\text {exp }} \\
\mathrm{MPa}\end{array}$ & $\begin{array}{c}Q \\
\mathrm{~W} \cdot \mathrm{m}^{-1}\end{array}$ & $\begin{array}{c}T_{\exp } \\
\mathrm{K}\end{array}$ & $\begin{array}{c}\rho_{\text {calc }} \\
\mathrm{mol} \cdot \mathrm{L}^{-1}\end{array}$ & $\begin{array}{c}\lambda_{\exp } \\
\mathrm{W} \cdot \mathrm{m}^{-1} \cdot \mathrm{K}^{-1}\end{array}$ & STAT & $\begin{array}{c}T_{\text {cell }} \\
\mathrm{K}\end{array}$ \\
\hline 1001 & 0.444 & 0.13799 & 245.516 & 14.1535 & 0.11761 & 0.010 & 244.857 \\
\hline 1003 & 0.444 & 0.13794 & 245.519 & 14.1534 & 0.11537 & 0.010 & 244.852 \\
\hline 1005 & 0.445 & 0.18018 & 245.675 & 14.1476 & 0.11697 & 0.007 & 244.851 \\
\hline 1007 & 0.445 & 0.18009 & 245.658 & 14.1482 & 0.11748 & 0.007 & 244.836 \\
\hline 1009 & 0.445 & 0.22805 & $245: 853$ & 14.1410 & 0.11580 & 0.005 & 244.843 \\
\hline 1011 & 0.445 & 0.22809 & 245.847 & 14.1412 & 0.11698 & 0.005 & 244.830 \\
\hline 1013 & 0.445 & 0.28176 & 246.061 & 14.1332 & 0.11597 & 0.004 & 244.829 \\
\hline 1015 & 0.445 & 0.28180 & 246.060 & 14.1332 & 0.11642 & 0.010 & 244.823 \\
\hline 1017 & 0.445 & 0.34121 & 246.295 & 14.1245 & 0.11581 & 0.003 & 244.814 \\
\hline 1019 & 0.444 & 0.34126 & 246.297 & 14.1244 & 0.11475 & 0.003 & 244.817 \\
\hline 1021 & 0.444 & 0.40643 & 246.556 & 14.1147 & 0.11527 & 0.002 & 244.809 \\
\hline 1023 & 0.444 & 0.40645 & 246.550 & 14.1149 & 0.11574 & 0.002 & 244.806 \\
\hline 1025 & 0.444 & 0.47741 & 246.848 & 14.1037 & 0.11553 & 0.002 & 244.796 \\
\hline 1027 & 0.444 & 0.47739 & 246.824 & 14.1046 & 0.11552 & 0.001 & 244.791 \\
\hline 1031 & 1.606 & 0.15070 & 269.819 & 13.2566 & 0.10169 & 0.008 & 269.024 \\
\hline 1033 & 1.604 & 0.19690 & 270.023 & 13.2478 & 0.10198 & 0.006 & 269.030 \\
\hline 1035 & 1.592 & 0.19686 & 270.013 & 13.2474 & 0.10482 & 0.006 & 269.029 \\
\hline 1037 & 1.586 & 0.24920 & 270.247 & 13.2371 & 0.10060 & 0.004 & 269.031 \\
\hline 1039 & 1.586 & 0.24927 & 270.266 & 13.2363 & 0.10425 & 0.004 & 269.035 \\
\hline 1041 & 1.576 & 0.30790 & 270.518 & 13.2250 & 0.10070 & 0.003 & 269.026 \\
\hline 1043 & 1.583 & 0.30789 & 270.535 & 13.2247 & 0.10363 & 0.003 & 269.037 \\
\hline 1045 & 1.581 & 0.37284 & 270.827 & 13.2121 & 0.10194 & 0.002 & 269.033 \\
\hline 1047 & 1.579 & 0.37287 & 270.819 & 13.2123 & 0.10116 & 0.002 & 269.029 \\
\hline 1049 & 1.574 & 0.44403 & 271.149 & 13.1979 & 0.10212 & 0.002 & 269.030 \\
\hline 1051 & 1.561 & 0.44406 & 271.156 & 13.1968 & 0.10289 & 0.002 & 269.034 \\
\hline 1053 & 1.561 & 0.52158 & 271.529 & 13.1808 & 0.09996 & 0.003 & 269.037 \\
\hline 1055 & 1.561 & 0.52163 & 271.533 & 13.1806 & 0.10160 & 0.002 & 269.036 \\
\hline 1057 & 3.064 & 0.15837 & 284.469 & 12.7211 & 0.09385 & 0.009 & 283.612 \\
\hline 1059 & 3.064 & 0.20681 & 284.712 & 12.7100 & 0.09193 & 0.005 & 283.611 \\
\hline 1061 & 3.037 & 0.26181 & 284.971 & 12.6960 & 0.09449 & 0.003 & 283.614 \\
\hline 1063 & 3.057 & 0.32338 & 285.259 & 12.6844 & 0.09475 & 0.003 & 283.614 \\
\hline 1065 & 3.065 & 0.39162 & 285.600 & 12.6695 & 0.09444 & 0.002 & 283.615 \\
\hline 1067 & 3.066 & 0.46645 & 285.968 & 12.6526 & 0.09455 & 0.002 & 283.615 \\
\hline 1069 & 3.071 & 0.54775 & 286.386 & 12.6338 & 0.09490 & 0.001 & 283.620 \\
\hline 1071 & 2.289 & 0.15837 & 284.456 & 12.6574 & 0.09373 & 0.007 & 283.599 \\
\hline 1073 & 2.278 & 0.20696 & 284.687 & 12.6456 & 0.09328 & 0.005 & 283.603 \\
\hline 1075 & 2.278 & 0.26182 & 284.963 & 12.6326 & 0.09475 & 0.003 & 283.608 \\
\hline 1077 & 2.281 & 0.32349 & 285.272 & 12.6183 & 0.09338 & 0.002 & 283.611 \\
\hline 1079 & 2.279 & 0.39172 & 285.617 & 12.6018 & 0.09356 & 0.002 & 283.613 \\
\hline 1081 & 2.271 & 0.46655 & 286.000 & 12.5829 & 0.09314 & 0.002 & 283.615 \\
\hline 1083 & 2.272 & 0.54790 & 286.406 & 12.5636 & 0.09318 & 0.001 & 283.618 \\
\hline 1085 & 10.307 & 0.16600 & 299.300 & 12.6427 & 0.09600 & 0.007 & 298.381 \\
\hline 1087 & 10.311 & 0.21515 & 299.538 & 12.6335 & 0.09452 & 0.005 & 298.386 \\
\hline
\end{tabular}


Table 4. Thermal conductivity of the binary $30 \%$ R32 / $70 \%$ propane mixture in the compressed liquid phase (continued).

\begin{tabular}{|c|c|c|c|c|c|c|c|}
\hline $\begin{array}{l}\text { Run } \\
\text { point }\end{array}$ & $\begin{array}{l}P_{\text {exp }} \\
\mathrm{MPa}\end{array}$ & $\begin{array}{c}Q \\
W \cdot m^{-1}\end{array}$ & $\begin{array}{c}T_{\exp } \\
\mathrm{K}\end{array}$ & $\begin{array}{c}\rho_{\text {calc }} \\
\mathrm{mol} \cdot \mathrm{L}^{-1}\end{array}$ & $\begin{array}{c}\lambda_{\exp } \\
\mathrm{W} \cdot \mathrm{m}^{-1} \cdot \mathrm{K}^{-1}\end{array}$ & STAT & $\begin{array}{c}T_{\text {cell }} \\
\mathrm{K}\end{array}$ \\
\hline 1089 & 10.316 & 0.27416 & 299.838 & 12.6219 & 0.09490 & 0.004 & 298.391 \\
\hline 1091 & 10.313 & 0.33926 & 300.141 & 12.6096 & 0.09449 & 0.003 & 298.393 \\
\hline 1093 & 10.318 & 0.41083 & 300.502 & 12.5955 & 0.09418 & 0.002 & 298.391 \\
\hline 1095 & 10.320 & 0.48923 & 300.918 & 12.5789 & 0.09376 & 0.002 & 298.389 \\
\hline 1097 & 10.318 & 0.57446 & 301.331 & 12.5622 & 0.09462 & 0.001 & 298.394 \\
\hline 1101 & 8.684 & 0.21697 & 299.538 & 12.5111 & 0.09378 & 0.005 & 298.367 \\
\hline 1103 & 8.686 & 0.27461 & 299.828 & 12.4991 & 0.09359 & 0.004 & 298.374 \\
\hline 1105 & 8.692 & 0.33932 & 300.167 & 12.4854 & 0.09294 & 0.002 & 298.380 \\
\hline 1107 & 8.697 & 0.41094 & 300.507 & 12.4714 & 0.09211 & 0.002 & 298.377 \\
\hline 1109 & 8.701 & 0.48941 & 300.921 & 12.4542 & 0.09297 & 0.002 & 298.380 \\
\hline 1111 & 8.706 & 0.57472 & 301.363 & 12.4359 & 0.09381 & 0.002 & 298.382 \\
\hline 1113 & 7.145 & 0.16605 & 299.297 & 12.3956 & 0.09235 & 0.007 & 298.353 \\
\hline 1115 & 7.144 & 0.21688 & 299.559 & 12.3840 & 0.09090 & 0.005 & 298.359 \\
\hline 1117 & 7.147 & 0.27466 & 299.848 & 12.3714 & 0.09170 & 0.003 & 298.364 \\
\hline 1119 & 7.147 & 0.33938 & 300.179 & 12.3567 & 0.09148 & 0.002 & 298.371 \\
\hline 1121 & 7.149 & 0.41107 & 300.549 & 12.3404 & 0.09081 & 0.002 & 298.375 \\
\hline 1123 & 7.151 & 0.48960 & 300.953 & 12.3225 & 0.09068 & 0.002 & 298.377 \\
\hline 1125 & 7.153 & 0.57502 & 301.405 & 12.3025 & 0.09128 & 0.001 & 298.377 \\
\hline 1129 & 5.305 & 0.16592 & 299.299 & 12.2289 & 0.09079 & 0.007 & 298.351 \\
\hline 1131 & 5.304 & 0.21681 & 299.573 & 12.2158 & 0.08803 & 0.005 & 298.359 \\
\hline 1133 & 5.301 & 0.27465 & 299.883 & 12.2007 & 0.08951 & 0.003 & 298.362 \\
\hline 1135 & 5.298 & 0.33941 & 300.213 & 12.1845 & 0.08919 & 0.002 & 298.369 \\
\hline 1137 & 5.297 & 0.41091 & 300.578 & 12.1669 & 0.08976 & 0.002 & 298.363 \\
\hline 1139 & 5.295 & 0.48951 & 300.989 & 12.1469 & 0.08901 & 0.002 & 298.368 \\
\hline 1143 & 3.729 & 0.16592 & 299.274 & 12.0689 & 0.08589 & 0.006 & 298.344 \\
\hline 1145 & 3.731 & 0.21683 & 299.561 & 12.0544 & 0.08650 & 0.004 & 298.359 \\
\hline 1147 & 3.735 & 0.27463 & 299.851 & 12.0399 & 0.08799 & 0.003 & 298.357 \\
\hline 1149 & 3.737 & 0.33931 & 300.211 & 12.0214 & 0.08774 & 0.002 & 298.363 \\
\hline 1151 & 3.742 & 0.41086 & 300.586 & 12.0024 & 0.08730 & 0.002 & 298.360 \\
\hline 1153 & 3.745 & 0.48928 & 301.010 & 11.9805 & 0.08695 & 0.001 & 298.363 \\
\hline 1157 & 2.264 & 0.15659 & 299.266 & 11.8987 & 0.08769 & 0.007 & 298.353 \\
\hline 1159 & 2.262 & 0.19562 & 299.455 & 11.8878 & 0.08664 & 0.005 & 298.349 \\
\hline 1161 & 2.262 & 0.23914 & 299.707 & 11.8736 & 0.08646 & 0.004 & 298.360 \\
\hline 1163 & 2.265 & 0.28711 & 299.958 & 11.8597 & 0.08507 & 0.003 & 298.359 \\
\hline 1165 & 2.271 & 0.33940 & 300.237 & 11.8445 & 0.08583 & 0.002 & 298.362 \\
\hline 1167 & 2.276 & 0.39607 & 300.548 & 11.8275 & 0.08490 & 0.002 & 298.360 \\
\hline 1169 & 9.287 & 0.12768 & 314.564 & 11.9081 & 0.08615 & 0.009 & 313.813 \\
\hline 1171 & 9.289 & 0.14981 & 314.681 & 11.9030 & 0.08793 & 0.008 & 313.807 \\
\hline 1173 & 9.289 & 0.17378 & 314.828 & 11.8963 & 0.08757 & 0.006 & 313.822 \\
\hline 1177 & 9.290 & 0.22702 & 315.102 & 11.8838 & 0.08661 & 0.005 & 313.824 \\
\hline 1179 & 9.292 & 0.25632 & 315.269 & 11.8764 & 0.08656 & 0.004 & 313.829 \\
\hline 1181 & 9.293 & 0.28742 & 315.415 & 11.8699 & 0.08543 & 0.003 & 313.823 \\
\hline 1183 & 8.281 & 0.12776 & 314.577 & 11.8033 & 0.08603 & 0.009 & 313.807 \\
\hline
\end{tabular}


Table 4. Thermal conductivity of the binary $30 \% \mathrm{R} 32 / 70 \%$ propane mixture in the compressed liquid phase (continued).

\begin{tabular}{|c|c|c|c|c|c|c|c|}
\hline $\begin{array}{l}\text { Run } \\
\text { point }\end{array}$ & $\begin{array}{l}P_{e x p} \\
M P a\end{array}$ & $\begin{array}{c}Q \\
\mathrm{~W} \cdot \mathrm{m}^{-1}\end{array}$ & $\begin{array}{c}T_{\exp } \\
\mathrm{K}\end{array}$ & $\begin{array}{c}\rho_{\text {calc }} \\
\mathrm{mol} \cdot \mathrm{L}^{-1}\end{array}$ & $\begin{array}{c}\lambda_{\exp } \\
\mathrm{W} \cdot \mathrm{m}^{-1} \cdot \mathrm{K}^{-1}\end{array}$ & STAT & $\begin{array}{c}T_{\text {cell }} \\
\mathrm{K}\end{array}$ \\
\hline 1185 & 8.280 & 0.14994 & 314.705 & 11.7971 & 0.08631 & 0.008 & 313.818 \\
\hline 1187 & 8.283 & 0.17376 & 314.828 & 11.7916 & 0.08578 & 0.006 & 313.824 \\
\hline 1189 & 8.283 & 0.19950 & 314.976 & 11.7845 & 0.08382 & 0.006 & 313.830 \\
\hline 1191 & 8.283 & 0.22720 & 315.131 & 11.7771 & 0.08510 & 0.004 & 313.834 \\
\hline 1193 & 8.284 & 0.25654 & 315.287 & 11.7697 & 0.08505 & 0.004 & 313.830 \\
\hline 1195 & 8.277 & 0.28767 & 315.468 & 11.7602 & 0.08449 & 0.003 & 313.841 \\
\hline 1197 & 6.612 & 0.12773 & 314.555 & 11.6123 & 0.08524 & 0.009 & 313.809 \\
\hline 1199 & 6.613 & 0.14988 & 314.707 & 11.6045 & 0.08370 & 0.006 & 313.828 \\
\hline 1201 & 6.614 & 0.17377 & 314.862 & 11.5964 & 0.08260 & 0.008 & 313.827 \\
\hline 1203 & 6.615 & 0.19956 & 315.010 & 11.5888 & 0.08325 & 0.007 & 313.840 \\
\hline 1205 & 6.616 & 0.22706 & 315.149 & 11.5817 & 0.08423 & 0.004 & 313.828 \\
\hline 1207 & 6.615 & 0.25641 & 315.310 & 11.5731 & 0.08301 & 0.003 & 313.837 \\
\hline 1209 & 6.615 & 0.28756 & 315.490 & 11.5636 & 0.08240 & 0.003 & 313.841 \\
\hline 1217 & 4.804 & 0.19949 & 315.011 & 11.3396 & 0.07911 & 0.007 & 313.822 \\
\hline 1219 & 4.807 & 0.22708 & 315.180 & 11.3299 & 0.08071 & 0.006 & 313.827 \\
\hline 1221 & 4.806 & 0.25644 & 315.357 & 11.3193 & 0.07885 & 0.005 & 313.835 \\
\hline 1223 & 4.808 & 0.28756 & 315.522 & 11.3098 & 0.08073 & 0.003 & 313.828 \\
\hline 1225 & 3.031 & 0.12773 & 314.624 & 11.0603 & 0.07860 & 0.008 & 313.805 \\
\hline 1227 & 3.032 & 0.14988 & 314.750 & 11.0517 & 0.07827 & 0.007 & 313.810 \\
\hline 1229 & 3.033 & 0.17368 & 314.895 & 11.0417 & 0.07771 & 0.006 & 313.819 \\
\hline 1231 & 3.035 & 0.19943 & 315.055 & 11.0307 & 0.07747 & 0.005 & 313.832 \\
\hline 1233 & 3.036 & 0.22706 & 315.214 & 11.0196 & 0.07769 & 0.004 & 313.831 \\
\hline 1235 & 3.036 & 0.25645 & 315.383 & 11.0077 & 0.07797 & 0.003 & 313.828 \\
\hline 1237 & 3.039 & 0.28760 & 315.559 & 10.9956 & 0.07707 & 0.003 & 313.837 \\
\hline 1239 & 8.834 & 0.13361 & 330.592 & 11.0619 & 0.07829 & 0.009 & 329.730 \\
\hline 1241 & 8.835 & 0.15709 & 330.715 & 11.0554 & 0.07803 & 0.008 & 329.727 \\
\hline 1243 & 8.833 & 0.18244 & 330.873 & 11.0466 & 0.07890 & 0.006 & 329.744 \\
\hline 1245 & 8.832 & 0.20953 & 331.022 & 11.0385 & 0.07854 & 0.005 & 329.741 \\
\hline 1247 & 8.836 & 0.23846 & 331.187 & 11.0301 & 0.07905 & 0.004 & 329.739 \\
\hline 1249 & 8.837 & 0.26924 & 331.375 & 11.0200 & 0.07827 & 0.003 & 329.747 \\
\hline 1251 & 8.838 & 0.30191 & 331.553 & 11.0106 & 0.07819 & 0.003 & 329.740 \\
\hline 1253 & 7.630 & 0.13373 & 330.570 & 10.8732 & 0.07709 & 0.009 & 329.704 \\
\hline 1255 & 7.630 & 0.15723 & 330.720 & 10.8642 & 0.07732 & 0.007 & 329.723 \\
\hline 1257 & 7.630 & 0.18245 & 330.862 & 10.8558 & 0.07667 & 0.007 & 329.710 \\
\hline 1259 & 7.629 & 0.20946 & 331.029 & 10.8459 & 0.07681 & 0.004 & 329.731 \\
\hline 1261 & 7.631 & 0.23839 & 331.192 & 10.8365 & 0.07709 & 0.004 & 329.729 \\
\hline 1265 & 7.633 & 0.30183 & 331.578 & 10.8139 & 0.07663 & 0.004 & 329.736 \\
\hline 1269 & 6.270 & 0.15719 & 330.720 & 10.6084 & 0.07550 & 0.007 & 329.699 \\
\hline 1271 & 6.270 & 0.18236 & 330.876 & 10.5979 & 0.07416 & 0.006 & 329.708 \\
\hline 1275 & 6.270 & 0.23832 & 331.188 & 10.5767 & 0.07471 & 0.004 & 329.694 \\
\hline 1277 & 6.271 & 0.26914 & 331.395 & 10.5628 & 0.07483 & 0.003 & 329.711 \\
\hline 1279 & 6.272 & 0.30177 & 331.597 & 10.5493 & 0.07390 & 0.003 & 329.720 \\
\hline 1281 & 5.005 & 0.13374 & 330.585 & 10.3196 & 0.07297 & 0.009 & 329.685 \\
\hline
\end{tabular}


Table 4. Thermal conductivity of the binary $30 \% \mathrm{R} 32$ / $70 \%$ propane mixture in the compressed liquid phase (continued).

\begin{tabular}{|c|c|c|c|c|c|c|c|}
\hline $\begin{array}{l}\text { Run } \\
\text { point }\end{array}$ & $\begin{array}{l}P_{e x p} \\
\mathrm{MPa}\end{array}$ & $\begin{array}{c}Q \\
\mathrm{~W} \cdot \mathrm{m}^{-1}\end{array}$ & $\begin{array}{c}T_{\exp } \\
\mathrm{K}\end{array}$ & $\begin{array}{c}\rho_{\text {calc }} \\
\mathrm{mol} \cdot \mathrm{L}^{-1}\end{array}$ & $\begin{array}{c}\lambda_{\exp } \\
\mathrm{W} \cdot \mathrm{m}^{-1} \cdot \mathrm{K}^{-1}\end{array}$ & STAT & $\begin{array}{c}T_{\text {cell }} \\
\mathrm{K}\end{array}$ \\
\hline 1283 & 5.005 & 0.15716 & 330.737 & 10.3075 & 0.07224 & 0.007 & 329.703 \\
\hline 1285 & 5.006 & 0.18230 & 330.888 & 10.2956 & 0.07209 & 0.005 & 329.703 \\
\hline 1287 & 5.007 & 0.20935 & 331.054 & 10.2825 & 0.07205 & 0.004 & 329.709 \\
\hline 1289 & 5.007 & 0.23825 & 331.229 & 10.2684 & 0.07249 & 0.003 & 329.709 \\
\hline 1291 & 5.009 & 0.26911 & $331: 425$ & 10.2529 & 0.07208 & 0.003 & 329.711 \\
\hline 1293 & 5.009 & 0.30176 & 331.626 & 10.2365 & 0.07236 & 0.003 & 329.714 \\
\hline 1295 & 3.978 & 0.13370 & 330.584 & 9.9990 & 0.06998 & 0.008 & 329.672 \\
\hline 1297 & 3.978 & 0.15714 & 330.738 & 9.9836 & 0.07075 & 0.007 & 329.690 \\
\hline 1299 & 3.979 & 0.18229 & 330.893 & 9.9685 & 0.06974 & 0.005 & 329.695 \\
\hline 1301 & 3.979 & 0.20931 & 331.055 & 9.9520 & 0.07027 & 0.004 & 329.696 \\
\hline 1303 & 3.979 & 0.23825 & 331.248 & 9.9326 & 0.07047 & 0.003 & 329.699 \\
\hline 1305 & 3.980 & 0.26904 & 331.428 & 9.9142 & 0.06965 & 0.003 & 329.702 \\
\hline 1307 & 3.980 & 0.30169 & 331.647 & 9.8916 & 0.07073 & 0.003 & 329.710 \\
\hline 1311 & 8.348 & 0.11724 & 346.097 & 10.0312 & 0.07338 & 0.011 & 345.305 \\
\hline 1313 & 8.349 & 0.13957 & 346.246 & 10.0211 & 0.07355 & 0.008 & 345.308 \\
\hline 1315 & 8.349 & 0.16381 & 346.388 & 10.0112 & 0.07364 & 0.007 & 345.305 \\
\hline 1317 & 8.349 & 0.19003 & 346.554 & 9.9997 & 0.07177 & 0.006 & 345.304 \\
\hline 1319 & 8.348 & 0.21817 & 346.726 & 9.9876 & 0.07483 & 0.006 & 345.311 \\
\hline 1321. & 8.348 & 0.24828 & 346.925 & 9.9736 & 0.07455 & 0.005 & 345.315 \\
\hline $1327^{\circ}$ & 7.024 & 0.13952 & 346.225 & 9.6405 & 0.07443 & 0.009 & 345.278 \\
\hline 1329 & 7.024 & 0.16372 & 346.383 & 9.6272 & 0.07395 & 0.007 & 345.281 \\
\hline 1331 & 7.025 & 0.18996 & 346.538 & 9.6142 & 0.07442 & 0.007 & 345.279 \\
\hline 1333 & 7.025 & 0.21814 & 346.711 & 9.5995 & 0.07702 & 0.007 & 345.277 \\
\hline 1335 & 7.026 & 0.24822 & 346.887 & 9.5844 & 0.08043 & 0.008 & 345.282 \\
\hline 1337 & 5.972 & 0.09677 & 345.908 & 9.2455 & 0.07837 & 0.016 & 345.241 \\
\hline 1339 & 5.972 & 0.11721 & 346.062 & 9.2285 & 0.08092 & 0.013 & 345.256 \\
\hline 1341 & 5.972 & 0.13951 & 346.194 & 9.2141 & 0.08097 & 0.014 & 345.258 \\
\hline 1343 & 5.973 & 0.16376 & 346.361 & 9.1959 & 0.07831 & 0.011 & 345.261 \\
\hline 1347 & 5.974 & 0.21812 & 346.702 & 9.1582 & 0.07867 & 0.009 & 345.265 \\
\hline 1349 & 5.975 & 0.24823 & 346.888 & 9.1375 & 0.08170 & 0.011 & 345.265 \\
\hline
\end{tabular}


Table 5. Thermal conductivity of the binary $70 \% \mathrm{R} 32$ / $30 \%$ propane mixture in the compressed liquid phase.

\begin{tabular}{|c|c|c|c|c|c|c|c|}
\hline $\begin{array}{l}\text { Run } \\
\text { point }\end{array}$ & $\begin{array}{l}P_{\text {exp }} \\
\mathrm{MPa}\end{array}$ & $\begin{array}{c}Q \\
\mathrm{~W} \cdot \mathrm{m}^{-1}\end{array}$ & $\begin{array}{c}T_{e x p} \\
\mathrm{~K}\end{array}$ & $\begin{array}{c}\rho_{\text {calc }} \\
\mathrm{mol} \cdot \mathrm{L}^{-1}\end{array}$ & $\begin{array}{c}\lambda_{\exp } \\
\mathrm{W} \cdot \mathrm{m}^{-1} \cdot \mathrm{K}^{-1}\end{array}$ & STAT & $\begin{array}{c}T_{\text {cell }} \\
\mathrm{K}\end{array}$ \\
\hline 1001 & 0.703 & 0.23593 & 254.064 & 16.9738 & 0.12192 & 0.007 & 253.113 \\
\hline 1003 & 0.704 & 0.29088 & 254.280 & 16.9624 & 0.12206 & 0.004 & 253.108 \\
\hline 1005 & 0.704 & 0.35201 & 254.543 & 16.9485 & 0.12104 & 0.003 & 253.111 \\
\hline 1007 & 0.705 & 0.41939 & 254.820 & 16.9339 & 0.12078 & 0.003 & 253.109 \\
\hline 1009 & 0.705 & 0.49254 & 255.130 & 16.9174 & 0.12013 & 0.002 & 253.116 \\
\hline 1011 & 0.704 & 0.57149 & 255.453 & 16.9003 & 0.12052 & 0.001 & 253.113 \\
\hline 1013 & 0.704 & 0.65686 & 255.811 & 16.8811 & 0.11946 & 0.001 & 253.114 \\
\hline 1017 & 0.703 & 0.18614 & 253.854 & 16.9848 & 0.12176 & 0.008 & 253.118 \\
\hline 1019 & 0.703 & 0.23563 & 254.051 & 16.9744 & 0.12221 & 0.005 & 253.111 \\
\hline 1021 & 0.704 & 0.29107 & 254.287 & 16.9620 & 0.12126 & 0.004 & 253.117 \\
\hline 1023 & 0.704 & 0.35257 & 254.542 & 16.9485 & 0.11873 & 0.005 & 253.103 \\
\hline 1025 & 0.704 & 0.42014 & 254.825 & 16.9336 & 0.12044 & 0.003 & 253.105 \\
\hline 1027 & 0.705 & 0.49352 & 255.133 & 16.9173 & 0.12007 & 0.002 & 253.102 \\
\hline 1033 & 1.201 & 0.25005 & 271.203 & 16.0527 & 0.11153 & 0.005 & 270.114 \\
\hline 1035 & 1.199 & 0.30880 & 271.469 & 16.0366 & 0.10850 & 0.004 & 270.120 \\
\hline 1037 & 1.196 & 0.37397 & 271.777 & 16.0179 & 0.11043 & 0.003 & 270.119 \\
\hline 1039 & 1.193 & 0.44560 & 272.075 & 15.9998 & 0.11043 & 0.002 & 270.119 \\
\hline 1041 & 1.191 & 0.52331 & 272.436 & 15.9778 & 0.11008 & 0.002 & 270.113 \\
\hline 1045 & 1.182 & 0.19762 & 270.957 & 16.0657 & 0.11020 & 0.006 & 270.104 \\
\hline 1047 & 1.180 & 0.25015 & 271.196 & 16.0512 & 0.11175 & 0.005 & 270.109 \\
\hline 1049 & 1.177 & 0.30929 & 271.454 & 16.0356 & 0.10944 & 0.009 & 270.099 \\
\hline 1051 & 1.175 & 0.37475 & 271.758 & 16.0172 & 0.11047 & 0.003 & 270.101 \\
\hline 1053 & 1.173 & 0.44627 & 272.077 & 15.9979 & 0.10981 & 0.005 & 270.094 \\
\hline 1055 & 1.171 & 0.52387 & 272.421 & 15.9770 & 0.10916 & 0.002 & 270.101 \\
\hline 1057 & 2.578 & 0.15846 & 284.587 & 15.3532 & 0.10328 & 0.008 & 283.875 \\
\hline 1059 & 2.576 & 0.20693 & 284.833 & 15.3370 & 0.10196 & 0.007 & 283.882 \\
\hline 1061 & 2.575 & 0.26218 & 285.109 & 15.3188 & 0.10269 & 0.005 & 283.880 \\
\hline 1065 & 2.587 & 0.39238 & 285.716 & 15.2802 & 0.10269 & 0.003 & 283.879 \\
\hline 1067 & 2.592 & 0.46724 & 286.101 & 15.2552 & 0.10299 & 0.002 & 283.897 \\
\hline 1069 & 2.593 & 0.54878 & 286.472 & 15.2307 & 0.10289 & 0.002 & 283.885 \\
\hline 1073 & 2.569 & 0.20716 & 284.840 & 15.3357 & 0.10406 & 0.006 & 283.885 \\
\hline 1075 & 2.567 & 0.26209 & 285.108 & 15.3179 & 0.10279 & 0.004 & 283.887 \\
\hline 1077 & 2.562 & 0.32398 & 285.398 & 15.2983 & 0.10246 & 0.003 & 283.884 \\
\hline 1079 & 2.563 & 0.39251 & 285.746 & 15.2756 & 0.10228 & 0.002 & 283.889 \\
\hline 1081 & 2.558 & 0.46754 & 286.086 & 15.2524 & 0.10334 & 0.002 & 283.889 \\
\hline 1083 & 2.558 & 0.54909 & 286.488 & 15.2257 & 0.10252 & 0.002 & 283.900 \\
\hline 1085 & 10.720 & 0.16635 & 299.161 & 15.2954 & 0.10593 & 0.007 & 298.388 \\
\hline 1087 & 10.712 & 0.21699 & 299.421 & 15.2802 & 0.10419 & 0.005 & 298.405 \\
\hline 1089 & 10.716 & 0.27440 & 299.702 & 15.2650 & 0.10327 & 0.003 & 298.405 \\
\hline 1091 & 10.738 & 0.33896 & 300.008 & 15.2500 & 0.10331 & 0.002 & 298.403 \\
\hline 1093 & 10.747 & 0.41075 & 300.355 & 15.2314 & 0.10288 & 0.002 & 298.398 \\
\hline 1095 & 10.752 & 0.48970 & 300.725 & 15.2112 & 0.10242 & 0.001 & 298.404 \\
\hline 1097 & 10.758 & 0.57510 & 301.140 & 15.1884 & 0.10292 & 0.002 & 298.406 \\
\hline
\end{tabular}


Table 5. Thermal conductivity of the binary $70 \% \mathrm{R} 32$ / $30 \%$ propane mixture in the compressed liquid phase (continued).

\begin{tabular}{|c|c|c|c|c|c|c|c|}
\hline $\begin{array}{l}\text { Run } \\
\text { point }\end{array}$ & $\begin{array}{l}P_{\text {exp }} \\
\mathrm{MPa}\end{array}$ & $\begin{array}{c}Q \\
\mathrm{~W} \cdot \mathrm{m}^{-1}\end{array}$ & $\begin{array}{c}T_{\exp } \\
\mathrm{K}\end{array}$ & $\begin{array}{c}\rho_{\text {calc }} \\
\mathrm{mol} \cdot \mathrm{L}^{-1}\end{array}$ & $\begin{array}{c}\lambda_{\exp } \\
\mathrm{W} \cdot \mathrm{m}^{-1} \cdot \mathrm{K}^{-1}\end{array}$ & STAT & $\begin{array}{c}T_{\text {cell }} \\
\mathrm{K}\end{array}$ \\
\hline 1099 & 8.545 & 0.16619 & 299.127 & 15.0836 & 0.10360 & 0.008 & 298.361 \\
\hline 1101 & 8.551 & 0.21703 & 299.404 & 15.0676 & 0.10089 & 0.005 & 298.378 \\
\hline 1103 & 8.557 & 0.27468 & 299.694 & 15.0510 & 0.10049 & 0.003 & 298.383 \\
\hline 1105 & 8.561 & 0.33931 & 300.033 & 15.0310 & 0.10119 & 0.002 & 298.396 \\
\hline 1107 & 8.556 & 0.41075 & 300.378 & 15.0098 & 0.10023 & 0.002 & 298.390 \\
\hline 1109 & 8.551 & 0.48937 & 300.759 & 14.9863 & 0.10066 & 0.002 & 298.388 \\
\hline 1111 & 8.533 & 0.57539 & 301.178 & 14.9590 & 0.10079 & 0.001 & 298.391 \\
\hline 1115 & 6.421 & 0.15675 & 299.114 & 14.8477 & 0.09722 & 0.007 & 298.364 \\
\hline 1117 & 6.413 & 0.19576 & 299.325 & 14.8330 & 0.09838 & 0.007 & 298.375 \\
\hline 1119 & 6.419 & 0.23920 & 299.526 & 14.8206 & 0.09823 & 0.004 & 298.366 \\
\hline 1121 & 6.445 & 0.28716 & 299.777 & 14.8074 & 0.09796 & 0.003 & 298.376 \\
\hline 1123 & 6.453 & 0.33970 & 300.037 & 14.7914 & 0.09828 & 0.002 & 298.371 \\
\hline 1125 & 6.461 & 0.39640 & 300.325 & 14.7735 & 0.09897 & 0.002 & 298.375 \\
\hline 1129 & 4.215 & 0.15658 & 299.098 & 14.5613 & 0.09828 & 0.007 & 298.348 \\
\hline 1131 & 4.202 & 0.19568 & 299.329 & 14.5427 & 0.09747 & 0.005 & 298.360 \\
\hline 1133 & 4.195 & 0.23913 & 299.553 & 14.5253 & 0.09478 & 0.004 & 298.361 \\
\hline 1135 & 4.197 & 0.28710 & 299.786 & 14.5085 & 0.09568 & 0.003 & 298.356 \\
\hline 1137 & 4.228 & 0.33952 & 300.074 & 14.4919 & 0.09481 & 0.003 & 298.361 \\
\hline 1139 & 4.238 & 0.39648 & 300.351 & 14.4730 & 0.09689 & 0.002 & 298.359 \\
\hline 1143 & 2.869 & 0.15670 & 299.116 & 14.3542 & 0.09410 & 0.007 & 298.347 \\
\hline 1145 & 2.865 & 0.19563 & 299.327 & 14.3368 & 0.09367 & 0.007 & 298.350 \\
\hline 1147 & 2.857 & 0.23925 & 299.564 & 14.3166 & 0.09432 & 0.004 & 298.350 \\
\hline 1149 & 2.853 & 0.28714 & 299.811 & 14.2962 & 0.09462 & 0.003 & 298.363 \\
\hline 1151 & 2.878 & 0.33927 & 300.085 & 14.2784 & 0.09379 & 0.002 & 298.363 \\
\hline 1153 & 2.889 & 0.39604 & 300.382 & 14.2562 & 0.09367 & 0.002 & 298.356 \\
\hline 1155 & 2.094 & 0.12221 & 298.955 & 14.2343 & 0.09245 & 0.010 & 298.360 \\
\hline 1157 & 2.093 & 0.15674 & 299.149 & 14.2180 & 0.09158 & 0.007 & 298.375 \\
\hline 1159 & 2.093 & 0.19571 & 299.373 & 14.1990 & 0.09248 & 0.005 & 298.379 \\
\hline 1161 & 2.093 & 0.23922 & 299.585 & 14.1810 & 0.09246 & 0.005 & 298.371 \\
\hline 1163 & 2.093 & 0.28738 & 299.843 & 14.1590 & 0.09248 & 0.003 & 298.373 \\
\hline 1165 & 2.093 & 0.33974 & 300.122 & 14.1352 & 0.09246 & 0.003 & 298.377 \\
\hline 1167 & 2.094 & 0.39630 & 300.416 & 14.1099 & 0.09230 & 0.002 & 298.385 \\
\hline 1169 & 8.929 & 0.12765 & 313.797 & 14.2030 & 0.09317 & 0.010 & 313.158 \\
\hline 1171 & 8.943 & 0.14942 & 313.916 & 14.1969 & 0.09333 & 0.008 & 313.165 \\
\hline 1173 & 8.952 & 0.17323 & 314.045 & 14.1895 & 0.09406 & 0.007 & 313.163 \\
\hline 1175 & 8.955 & 0.19923 & 314.182 & 14.1807 & 0.09355 & 0.005 & 313.166 \\
\hline 1177 & 8.956 & 0.22706 & 314.328 & 14.1710 & 0.09317 & 0.004 & 313.162 \\
\hline 1179 & 8.945 & 0.25672 & 314.484 & 14.1589 & 0.09238 & 0.003 & 313.169 \\
\hline 1181 & 8.936 & 0.28777 & 314.662 & 14.1455 & 0.09262 & 0.003 & 313.172 \\
\hline 1183 & 7.250 & 0.12771 & 313.787 & 13.9491 & 0.09089 & 0.009 & 313.133 \\
\hline 1185 & 7.246 & 0.14974 & 313.896 & 13.9404 & 0.08976 & 0.008 & 313.138 \\
\hline 1187 & 7.244 & 0.17372 & 314.041 & 13.9293 & 0.09065 & 0.008 & 313.140 \\
\hline 1189 & 7.255 & 0.19942 & 314.182 & 13.9205 & 0.09003 & 0.005 & 313.141 \\
\hline
\end{tabular}


Table 5. Thermal conductivity of the binary $70 \% \mathrm{R} 32$ / $30 \%$ propane mixture in the compressed liquid phase (continued).

\begin{tabular}{|c|c|c|c|c|c|c|c|}
\hline $\begin{array}{c}\text { Run } \\
\text { point }\end{array}$ & $\begin{array}{l}P_{\text {exp }} \\
\mathrm{MPa} \\
\end{array}$ & $\begin{array}{c}Q \\
\mathrm{~W} \cdot \mathrm{m}^{-1}\end{array}$ & $\begin{array}{c}T_{\exp } \\
\mathrm{K} \\
\end{array}$ & $\begin{array}{c}\rho_{\text {calc }} \\
\mathrm{mol} \cdot \mathrm{L}^{-1}\end{array}$ & $\begin{array}{c}\lambda_{\exp } \\
\mathrm{W} \cdot \mathrm{m}^{-1} \cdot \mathrm{K}^{-1}\end{array}$ & STAT & $\begin{array}{c}T_{\text {cell }} \\
\mathrm{K}\end{array}$ \\
\hline 1191 & 7.259 & 0.22686 & 314.338 & 13.9097 & 0.09149 & 0.005 & 313.153 \\
\hline 1193 & 7.263 & 0.25619 & 314.489 & 13.8991 & 0.09063 & 0.003 & 313.147 \\
\hline 1195 & 7.266 & 0.28727 & 314.655 & 13.8871 & 0.09076 & 0.003 & 313.140 \\
\hline 1197 & 5.461 & 0.12765 & 313.750 & 13.6277 & 0.08906 & 0.009 & 313.078 \\
\hline 1199 & 5.462 & 0.14970 & 313.891 & 13.6160 & 0.08872 & 0.007 & 313.110 \\
\hline 1201 & 5.464 & 0.17362 & 314.037 & 13.6039 & 0.08807 & 0.006 & 313.120 \\
\hline 1203 & 5.467 & 0.19939 & 314.167 & 13.5935 & 0.08868 & 0.005 & 313.112 \\
\hline 1205 & 5.467 & 0.22704 & 314.335 & 13.5793 & 0.08840 & 0.004 & 313.123 \\
\hline 1207 & 5.459 & 0.25652 & 314.498 & 13.5636 & 0.08855 & 0.003 & 313.123 \\
\hline 1209 & 5.453 & 0.28778 & 314.675 & 13.5471 & 0.08842 & 0.003 & 313.131 \\
\hline 1211 & 4.507 & 0.12773 & 313.772 & 13.4190 & 0.08625 & 0.009 & 313.101 \\
\hline 1213 & 4.504 & 0.14985 & 313.902 & 13.4063 & 0.08675 & 0.007 & 313.112 \\
\hline 1215 & 4.505 & 0.17373 & 314.043 & 13.3933 & 0.08698 & 0.006 & 313.110 \\
\hline 1217 & 4.513 & 0.19949 & 314.188 & 13.3815 & 0.08754 & 0.005 & 313.117 \\
\hline 1219 & 4.517 & 0.22701 & 314.344 & 13.3679 & 0.08744 & 0.004 & 313.121 \\
\hline 1221 & 4.520 & 0.25628 & 314.513 & 13.3528 & 0.08671 & 0.003 & 313.129 \\
\hline 1223 & 4.523 & 0.28732 & 314.674 & 13.3382 & 0.08781 & 0.003 & 313.121 \\
\hline 1225 & 3.380 & 0.12764 & 313.754 & 13.1283 & 0.08645 & 0.011 & 313.080 \\
\hline 1227 & 3.382 & 0.14968 & 313.897 & 13.1134 & 0.08755 & 0.008 & 313.098 \\
\hline 1229 & 3.384 & 0.17355 & 314.042 & 13.0982 & 0.08739 & 0.006 & 313.110 \\
\hline 1231 & 3.385 & 0.19931 & 314.192 & 13.0822 & 0.08709 & 0.005 & 313.117 \\
\hline 1233 & 3.385 & 0.22689 & 314.343 & 13.0657 & 0.08584 & 0.004 & 313.107 \\
\hline 1235 & 3.384 & 0.25641 & 314.512 & 13.0469 & 0.08578 & 0.003 & 313.106 \\
\hline 1237 & 3.378 & 0.28777 & 314.705 & 13.0234 & 0.08538 & 0.003 & 313.127 \\
\hline 1239 & 9.151 & 0.13337 & 328.697 & 13.1526 & 0.09448 & 0.010 & 327.994 \\
\hline 1241 & 9.140 & 0.15649 & 328.853 & 13.1380 & 0.09495 & 0.008 & 328.011 \\
\hline 1243 & 9.138 & 0.18139 & 328.978 & 13.1274 & 0.09331 & 0.006 & 328.000 \\
\hline 1245 & 9.149 & 0.20821 & 329.133 & 13.1174 & 0.10094 & 0.007 & 328.009 \\
\hline 1247 & 9.156 & 0.23693 & 329.271 & 13.1078 & 0.09988 & 0.007 & 327.998 \\
\hline 1249 & 9.158 & 0.26750 & 329.464 & 13.0927 & 0.09811 & 0.005 & 328.005 \\
\hline 1251 & 9.160 & 0.29985 & 329.655 & 13.0776 & 0.09436 & 0.005 & 328.012 \\
\hline 1253 & 7.191 & 0.13333 & 328.688 & 12.6865 & 0.09159 & 0.011 & 327.963 \\
\hline 1255 & 7.193 & 0.15635 & 328.821 & 12.6742 & 0.08945 & 0.008 & 327.968 \\
\hline 1257 & 7.193 & 0.18114 & 328.978 & 12.6590 & 0.08915 & 0.006 & 327.973 \\
\hline 1259 & 7.188 & 0.20811 & 329.136 & 12.6422 & 0.08992 & 0.006 & 327.980 \\
\hline 1261 & 7.184 & 0.23693 & 329.307 & 12.6241 & 0.08589 & 0.004 & 327.977 \\
\hline 1263 & 7.182 & 0.26755 & 329.473 & 12.6073 & 0.09175 & 0.005 & 327.971 \\
\hline 1265 & 7.181 & 0.30024 & 329.669 & 12.5877 & 0.09174 & 0.005 & 327.978 \\
\hline 1267 & 6.110 & 0.13336 & 328.677 & 12.3509 & 0.09317 & 0.011 & 327.960 \\
\hline 1269 & 6.110 & 0.15635 & 328.817 & 12.3349 & 0.10234 & 0.012 & 327.966 \\
\hline 1271 & 6.108 & 0.18121 & 328.946 & 12.3196 & 0.11602 & 0.012 & 327.974 \\
\hline 1273 & 6.109 & 0.20817 & 329.104 & 12.3017 & 0.11305 & 0.013 & 327.974 \\
\hline 1275 & 6.114 & 0.23691 & 329.274 & 12.2840 & 0.11631 & 0.013 & 327.986 \\
\hline
\end{tabular}


Table 5. Thermal conductivity of the binary $70 \% \mathrm{R} 32$ / $30 \%$ propane mixture in the compressed liquid phase (continued).

\begin{tabular}{|ccccccc|c|}
\hline $\begin{array}{c}\text { Run } \\
\text { point }\end{array}$ & $\begin{array}{c}P_{\text {exp }} \\
\mathrm{MPa}\end{array}$ & $\begin{array}{c}Q \\
\mathrm{~W} \cdot \mathrm{m}^{-1}\end{array}$ & $\begin{array}{c}T_{\text {exp }} \\
\mathrm{K}\end{array}$ & $\begin{array}{c}\rho_{\text {calc }} \\
\mathrm{mol} \cdot \mathrm{L}^{-1}\end{array}$ & $\begin{array}{c}\lambda_{\text {exp }} \\
\mathrm{W} \cdot \mathrm{m}^{-1} \cdot \mathrm{K}^{-1}\end{array}$ & STAT & $\begin{array}{c}T_{\text {cell }} \\
\mathrm{K}\end{array}$ \\
\hline 1279 & 6.119 & 0.29997 & 329.630 & 12.2443 & 0.11628 & 0.014 & 327.979 \\
1281 & 5.540 & 0.09255 & 328.455 & 12.1611 & 0.11238 & 0.023 & 327.964 \\
1283 & 5.539 & 0.10388 & 328.495 & 12.1555 & 0.10479 & 0.017 & 327.971 \\
1287 & 5.541 & 0.12862 & 328.665 & 12.1351 & 0.11194 & 0.017 & 327.985 \\
1289 & 5.544 & 0.14213 & 328.749 & 12.1258 & 0.11193 & 0.015 & 327.982 \\
1291 & 5.545 & 0.15629 & 328.836 & 12.1149 & 0.11382 & 0.015 & 327.989 \\
1293 & 5.546 & 0.17107 & 328.918 & 12.1047 & 0.11564 & 0.016 & 327.994 \\
1299 & 4.778 & 0.11581 & 328.555 & 11.7856 & 0.13342 & 0.027 & 327.971 \\
1301 & 4.780 & 0.12859 & 328.625 & 11.7755 & 0.14553 & 0.026 & 327.976 \\
1303 & 4.782 & 0.14205 & 328.712 & 11.7631 & 0.15245 & 0.029 & 327.980 \\
1305 & 4.784 & 0.15626 & 328.789 & 11.7523 & 0.14704 & 0.030 & 327.976 \\
1307 & 4.785 & 0.17114 & 328.899 & 11.7350 & 0.12707 & 0.018 & 327.974 \\
1309 & 4.780 & 0.09256 & 328.427 & 11.8066 & 0.14041 & 0.032 & 327.978 \\
1311 & 4.782 & 0.10389 & 328.500 & 11.7962 & 0.13967 & 0.026 & 327.982 \\
1313 & 4.783 & 0.11588 & 328.586 & 11.7838 & 0.13443 & 0.022 & 327.988 \\
1315 & 4.785 & 0.12869 & 328.628 & 11.7782 & 0.15978 & 0.025 & 327.981 \\
1317 & 4.786 & 0.14220 & 328.729 & 11.7627 & 0.14972 & 0.027 & 327.997 \\
1319 & 4.787 & 0.15627 & 328.789 & 11.7537 & 0.16691 & 0.030 & 327.992 \\
1321 & 4.788 & 0.17101 & 328.890 & 11.7383 & 0.14610 & 0.026 & 327.989 \\
\hline
\end{tabular}


Table 6. Thermal conductivity of the binary $30 \% \mathrm{R} 32$ / $70 \% \mathrm{R} 134$ a mixture in the compressed liquid phase.

\begin{tabular}{|c|c|c|c|c|c|c|c|}
\hline $\begin{array}{l}\text { Run } \\
\text { point }\end{array}$ & $\begin{array}{l}P_{\text {exp }} \\
\mathrm{MPa}\end{array}$ & $\begin{array}{c}Q \\
\mathrm{~W} \cdot \mathrm{m}^{-1}\end{array}$ & $\begin{array}{c}T_{\exp } \\
\mathrm{K}\end{array}$ & $\begin{array}{c}\rho_{\text {calc }} \\
\mathrm{mol} \cdot \mathrm{L}^{-1}\end{array}$ & $\frac{\lambda_{\exp }}{\mathrm{W} \cdot \mathrm{m}^{-1} \cdot \mathrm{K}^{-1}}$ & STAT & $\begin{array}{c}T_{\text {cell }} \\
\mathrm{K}\end{array}$ \\
\hline 1003 & 0.324 & 0.18777 & 255.431 & 14.9369 & 0.10774 & 0.007 & 254.483 \\
\hline 1005 & 0.324 & 0.23774 & 255.633 & 14.9295 & 0.10778 & 0.006 & 254.476 \\
\hline 1007 & 0.325 & 0.29368 & 255.879 & 14.9205 & 0.10851 & 0.004 & 254.471 \\
\hline 1011 & 0.326 & 0.42342 & 256.439 & 14.9001 & 0.10638 & 0.002 & 254.448 \\
\hline 1013 & 0.325 & 0.49716 & 256.746 & 14.8888 & 0.10534 & 0.002 & 254.435 \\
\hline 1021 & 0.323 & 0.29244 & 254.983 & 14.9532 & 0.11014 & 0.004 & 253.598 \\
\hline 1023 & 0.324 & 0.35405 & 255.290 & 14.9420 & 0.10876 & 0.003 & 253.621 \\
\hline 1025 & 0.324 & 0.42150 & 255.598 & 14.9308 & 0.10590 & 0.002 & 253.615 \\
\hline 1027 & 0.324 & 0.49479 & 255.917 & 14.9191 & 0.10480 & 0.002 & 253.612 \\
\hline 1031 & 0.325 & 0.18665 & 254.405 & 14.9743 & 0.10716 & 0.007 & 253.485 \\
\hline 1033 & 0.325 & 0.23647 & 254.616 & 14.9666 & 0.10695 & 0.005 & 253.478 \\
\hline 1035 & 0.325 & 0.29239 & 254.888 & 14.9567 & 0.10795 & 0.004 & 253.504 \\
\hline 1037 & 0.325 & 0.35398 & 255.137 & 14.9477 & 0.10679 & 0.003 & 253.476 \\
\hline 1039 & 0.325 & 0.42158 & 255.438 & 14.9367 & 0.10570 & 0.002 & 253.478 \\
\hline 1041 & 0.325 & 0.49514 & 255.772 & 14.9245 & 0.10552 & 0.002 & 253.471 \\
\hline 1045 & 0.980 & 0.19804 & 270.641 & 14.3914 & 0.09890 & 0.006 & 269.605 \\
\hline 1047 & 0.969 & 0.25081 & 270.897 & 14.3809 & 0.09960 & 0.005 & 269.607 \\
\hline 1049 & 0.957 & 0.30986 & 271.178 & 14.3695 & 0.09886 & 0.004 & 269.610 \\
\hline 1051 & 0.969 & 0.37506 & 271.485 & 14.3581 & 0.09942 & 0.002 & 269.607 \\
\hline 1053 & 0.989 & 0.44649 & 271.829 & 14.3455 & 0.09918 & 0.004 & 269.607 \\
\hline 1055 & 1.001 & 0.52418 & 272.214 & 14.3310 & 0.09880 & 0.002 & 269.614 \\
\hline 1059 & 0.987 & 0.19795 & 270.655 & 14.3911 & 0.09947 & 0.006 & 269.612 \\
\hline 1063 & 0.969 & 0.30976 & 271.174 & 14.3702 & 0.09778 & 0.004 & 269.614 \\
\hline 1065 & 0.963 & 0.37509 & 271.491 & 14.3576 & 0.09933 & 0.007 & 269.615 \\
\hline 1067 & 0.960 & 0.44656 & 271.821 & 14.3445 & 0.09842 & 0.006 & 269.609 \\
\hline 1069 & 0.982 & 0.52430 & 272.217 & 14.3301 & 0.09844 & 0.001 & 269.615 \\
\hline 1075 & 5.037 & 0.26309 & 285.159 & 14.0159 & 0.09601 & 0.004 & 283.766 \\
\hline 1077 & 5.039 & 0.32490 & 285.486 & 14.0033 & 0.09589 & 0.003 & 283.779 \\
\hline 1079 & 4.984 & 0.39345 & 285.777 & 13.9893 & 0.09541 & 0.003 & 283.744 \\
\hline 1081 & 4.961 & 0.46862 & 286.172 & 13.9728 & 0.09523 & 0.003 & 283.768 \\
\hline 1083 & 4.987 & 0.54999 & 286.576 & 13.9583 & 0.09508 & 0.001 & 283.761 \\
\hline 1085 & 3.387 & 0.15869 & 284.594 & 13.9561 & 0.09570 & 0.008 & 283.704 \\
\hline 1087 & 3.346 & 0.20766 & 284.892 & 13.9421 & 0.09557 & 0.006 & 283.764 \\
\hline 1089 & 3.325 & 0.26299 & 285.155 & 13.9305 & 0.09544 & 0.004 & 283.756 \\
\hline 1091 & 3.313 & 0.32487 & 285.467 & 13.9173 & 0.09464 & 0.004 & 283.758 \\
\hline 1093 & 3.302 & 0.39346 & 285.802 & 13.9032 & 0.09507 & 0.003 & 283.753 \\
\hline 1095 & 3.295 & 0.46851 & 286.195 & 13.8870 & 0.09300 & 0.002 & 283.756 \\
\hline 1097 & 3.297 & 0.55012 & 286.604 & 13.8706 & 0.09308 & 0.001 & 283.757 \\
\hline 1101 & 1.328 & 0.20763 & 284.879 & 13.8352 & 0.09256 & 0.006 & 283.743 \\
\hline 1103 & 1.322 & 0.26287 & 285.163 & 13.8230 & 0.09513 & 0.004 & 283.742 \\
\hline 1105 & 1.314 & 0.32472 & 285.483 & 13.8091 & 0.09298 & 0.003 & 283.748 \\
\hline 1107 & 1.315 & 0.39327 & 285.833 & 13.7943 & 0.09315 & 0.004 & 283.750 \\
\hline 1109 & 1.320 & 0.46837 & 286.189 & 13.7796 & 0.09248 & 0.002 & 283.726 \\
\hline 1111 & 1.345 & 0.55010 & 286.631 & 13.7623 & 0.09239 & 0.002 & 283.745 \\
\hline
\end{tabular}


Table 6. Thermal conductivity of the binary $30 \% \mathrm{R} 32$ / $70 \% \mathrm{R} 134 \mathrm{a}$ mixture in the compressed liquid phase (continued).

\begin{tabular}{|c|c|c|c|c|c|c|c|}
\hline $\begin{array}{l}\text { Run } \\
\text { point }\end{array}$ & $\begin{array}{l}P_{e x p} \\
\mathrm{MPa}\end{array}$ & $\begin{array}{c}Q \\
\mathrm{~W} \cdot \mathrm{m}^{-1}\end{array}$ & $\begin{array}{c}T_{\exp } \\
\mathrm{K}\end{array}$ & $\begin{array}{c}\rho_{\text {calc }} \\
\mathrm{mol} \cdot \mathrm{L}^{-1}\end{array}$ & $\begin{array}{c}\lambda_{\exp } \\
\mathrm{W} \cdot \mathrm{m}^{-1} \cdot \mathrm{K}^{-1}\end{array}$ & STAT & $\begin{array}{c}T_{\text {cell }} \\
\mathrm{K}\end{array}$ \\
\hline 1117 & 11.673 & 0.19615 & 299.505 & 13.7980 & 0.09559 & 0.007 & 298.422 \\
\hline 1119 & 11.681 & 0.23963 & 299.711 & 13.7909 & 0.09545 & 0.004 & 298.417 \\
\hline 1121 & 11.683 & 0.28753 & 299.952 & 13.7821 & 0.09559 & 0.003 & 298.418 \\
\hline 1123 & 11.694 & 0.33988 & 300.240 & 13.7722 & 0.09560 & 0.002 & 298.424 \\
\hline 1125 & 11.691 & 0.39659 & 300.508 & 13.7622 & 0.09354 & 0.002 & 298.424 \\
\hline 1131 & 8.937 & 0.19598 & 299.496 & 13.6600 & 0.09478 & 0.006 & 298.402 \\
\hline 1133 & 8.959 & 0.23949 & 299.726 & 13.6523 & 0.09377 & 0.004 & 298.410 \\
\hline 1135 & 8.963 & 0.28751 & 299.949 & 13.6439 & 0.09420 & 0.003 & 298.403 \\
\hline 1137 & 8.975 & 0.33999 & 300.226 & 13.6339 & 0.09407 & 0.002 & 298.405 \\
\hline 1139 & 8.975 & 0.39669 & 300.540 & 13.6218 & 0.09194 & 0.002 & 298.417 \\
\hline 1145 & 6.595 & 0.19614 & 299.501 & 13.5303 & 0.09256 & 0.006 & 298.389 \\
\hline 1147 & 6.618 & 0.23967 & 299.730 & 13.5224 & 0.09175 & 0.004 & 298.399 \\
\hline 1149 & 6.632 & 0.28769 & 299.975 & 13.5132 & 0.09100 & 0.003 & 298.395 \\
\hline 1151 & 6.650 & 0.34001 & 300.256 & 13.5029 & 0.09025 & 0.003 & 298.400 \\
\hline 1153 & 6.658 & 0.39666 & 300.542 & 13.4918 & 0.09103 & 0.002 & 298.394 \\
\hline 1161 & 4.310 & 0.23956 & 299.730 & 13.3818 & 0.08920 & 0.005 & 298.382 \\
\hline 1163 & 4.315 & 0.28759 & 299.969 & 13.3718 & 0.08907 & 0.005 & 298.367 \\
\hline 1165 & 4.330 & 0.34002 & 300.268 & 13.3599 & 0.08934 & 0.003 & 298.382 \\
\hline 1167 & 4.343 & 0.39676 & 300.569 & 13.3478 & 0.08883 & 0.002 & 298.383 \\
\hline 1171 & 1.446 & 0.15695 & 299.308 & 13.2044 & 0.08707 & 0.008 & 298.360 \\
\hline 1173 & 1.440 & 0.19597 & 299.506 & 13.1947 & 0.08772 & 0.006 & 298.356 \\
\hline 1175 & 1.436 & 0.23960 & 299.741 & 13.1833 & 0.08684 & 0.005 & 298.357 \\
\hline 1177 & 1.430 & 0.28770 & 299.998 & 13.1707 & 0.08528 & 0.003 & 298.354 \\
\hline 1179 & 1.428 & 0.34020 & 300.276 & 13.1574 & 0.08665 & 0.002 & 298.355 \\
\hline 1181 & 1.433 & 0.39704 & 300.602 & 13.1424 & 0.08588 & 0.002 & 298.366 \\
\hline 1187 & 10.745 & 0.17408 & 314.669 & 13.1700 & 0.08890 & 0.006 & 313.675 \\
\hline 1189 & 10.735 & 0.19980 & 314.785 & 13.1647 & 0.08860 & 0.005 & 313.661 \\
\hline 1191 & 10.729 & 0.22730 & 314.927 & 13.1587 & 0.08792 & 0.004 & 313.647 \\
\hline 1193 & 10.714 & 0.25667 & 315.072 & 13.1519 & 0.08805 & 0.004 & 313.643 \\
\hline 1195 & 10.696 & 0.28790 & 315.229 & 13.1446 & 0.08768 & 0.003 & 313.628 \\
\hline 1201 & 8.607 & 0.17383 & 314.450 & 13.0395 & 0.08770 & 0.006 & 313.451 \\
\hline 1203 & 8.617 & 0.19961 & 314.576 & 13.0348 & 0.08693 & 0.005 & 313.444 \\
\hline 1205 & 8.621 & 0.22731 & 314.730 & 13.0287 & 0.08607 & 0.004 & 313.440 \\
\hline 1207 & 8.625 & 0.25672 & 314.894 & 13.0220 & 0.08629 & 0.003 & 313.447 \\
\hline 1209 & 8.630 & 0.28794 & 315.045 & 13.0159 & 0.08647 & 0.003 & 313.429 \\
\hline 1211 & 6.618 & 0.12785 & 314.117 & 12.9115 & 0.08805 & 0.009 & 313.355 \\
\hline 1213 & 6.621 & 0.14994 & 314.262 & 12.9052 & 0.08600 & 0.008 & 313.366 \\
\hline 1215 & 6.626 & 0.17385 & 314.397 & 12.8996 & 0.08629 & 0.006 & 313.373 \\
\hline 1217 & 6.633 & 0.19964 & 314.519 & 12.8947 & 0.08525 & 0.005 & 313.363 \\
\hline 1219 & 6.637 & 0.22728 & 314.690 & 12.8873 & 0.08487 & 0.004 & 313.369 \\
\hline 1221 & 6.645 & 0.25664 & 314.842 & 12.8811 & 0.08474 & 0.003 & 313.364 \\
\hline 1223 & 6.647 & 0.28781 & 315.019 & 12.8733 & 0.08390 & 0.003 & 313.360 \\
\hline 1225 & 4.441 & 0.12775 & 314.082 & 12.7388 & 0.08338 & 0.009 & 313.301 \\
\hline
\end{tabular}


Table 6. Thermal conductivity of the binary $30 \% \mathrm{R} 32$ / $70 \% \mathrm{R} 134 \mathrm{a}$ mixture in the compressed liquid phase (continued).

\begin{tabular}{|c|c|c|c|c|c|c|c|}
\hline $\begin{array}{l}\text { Run } \\
\text { point }\end{array}$ & $\begin{array}{l}P_{e x p} \\
\mathrm{MPa}\end{array}$ & $\begin{array}{c}Q \\
W \cdot \mathrm{m}^{-1}\end{array}$ & $\begin{array}{c}T_{\exp } \\
\mathrm{K}\end{array}$ & $\begin{array}{c}\rho_{\text {calc }} \\
\mathrm{mol} \cdot \mathrm{L}^{-1}\end{array}$ & $\begin{array}{c}\lambda_{\exp } \\
\mathrm{W} \cdot \mathrm{m}^{-1} \cdot \mathrm{K}^{-1}\end{array}$ & STAT & $\begin{array}{c}T_{\text {cell }} \\
\mathrm{K}\end{array}$ \\
\hline 1227 & 4.437 & 0.14998 & 314.211 & 12.7323 & 0.08520 & 0.008 & 313.311 \\
\hline 1229 & 4.433 & 0.17394 & 314.348 & 12.7253 & 0.08390 & 0.006 & 313.313 \\
\hline 1231 & 4.432 & 0.19971 & 314.506 & 12.7176 & 0.08257 & 0.005 & 313.322 \\
\hline 1233 & 4.438 & 0.22734 & 314.648 & 12.7112 & 0.08211 & 0.004 & 313.306 \\
\hline 1235 & 4.443 & 0.25670 & 314.826 & 12.7031 & 0.08211 & 0.004 & 313.315 \\
\hline 1237 & 4.449 & 0.28790 & 315.000 & 12.6950 & 0.08196 & 0.003 & 313.316 \\
\hline 1239 & 1.729 & 0.12771 & 314.050 & 12.4848 & 0.07982 & 0.009 & 313.253 \\
\hline 1241 & 1.716 & 0.14996 & 314.190 & 12.4757 & 0.08146 & 0.007 & 313.266 \\
\hline 1243 & 1.712 & 0.17385 & 314.337 & 12.4672 & 0.08127 & 0.006 & 313.276 \\
\hline 1245 & 1.709 & 0.19964 & 314.474 & 12.4593 & 0.08008 & 0.004 & 313.268 \\
\hline 1247 & 1.706 & 0.22734 & 314.650 & 12.4494 & 0.07998 & 0.004 & 313.275 \\
\hline 1249 & 1.704 & 0.25678 & 314.814 & 12.4400 & 0.08000 & 0.003 & 313.268 \\
\hline 1251 & 1.701 & 0.28800 & 315.004 & 12.4292 & 0.07903 & 0.003 & 313.275 \\
\hline 1253 & 9.385 & 0.13322 & 329.033 & 12.4625 & 0.08378 & 0.009 & 328.191 \\
\hline 1255 & 9.391 & 0.15620 & 329.175 & 12.4567 & 0.08168 & 0.009 & 328.196 \\
\hline 1257 & 9.388 & 0.18125 & 329.329 & 12.4494 & 0.08021 & 0.006 & 328.213 \\
\hline 1259 & 9.377 & 0.20827 & 329.481 & 12.4416 & 0.08091 & 0.005 & 328.206 \\
\hline 1261 & 9.371 & 0.23718 & 329.641 & 12.4338 & 0.08135 & 0.004 & 328.203 \\
\hline 1263 & 9.366 & 0.26797 & 329.825 & 12.4250 & 0.08121 & 0.003 & 328.208 \\
\hline 1265 & 9.365 & 0.30059 & 330.024 & 12.4158 & 0.08058 & 0.003 & 328.216 \\
\hline 1267 & 7.370 & 0.13329 & 329.027 & 12.2788 & 0.08082 & 0.009 & 328.169 \\
\hline 1269 & 7.368 & 0.15641 & 329.168 & 12.2717 & 0.07947 & 0.007 & 328.185 \\
\hline 1271 & 7.365 & 0.18133 & 329.325 & 12.2637 & 0.07989 & 0.006 & 328.192 \\
\hline 1273 & 7.364 & 0.20837 & 329.485 & 12.2557 & 0.07866 & 0.004 & 328.193 \\
\hline 1275 & 7.365 & 0.23734 & 329.652 & 12.2475 & 0.07900 & 0.004 & 328.192 \\
\hline 1277 & 7.368 & 0.26816 & 329.841 & 12.2385 & 0.07962 & 0.003 & 328.192 \\
\hline 1279 & 7.372 & 0.30074 & 330.028 & 12.2296 & 0.07895 & 0.003 & 328.195 \\
\hline 1281 & 5.579 & 0.13328 & 329.039 & 12.0904 & 0.07896 & 0.009 & 328.164 \\
\hline 1283 & 5.578 & 0.15640 & 329.177 & 12.0828 & 0.07873 & 0.008 & 328.173 \\
\hline 1285 & 5.576 & 0.18145 & 329.338 & 12.0740 & 0.07724 & 0.006 & 328.174 \\
\hline 1287 & 5.575 & 0.20841 & 329.500 & 12.0652 & 0.07807 & 0.009 & 328.182 \\
\hline 1289 & 5.578 & 0.23733 & 329.671 & 12.0562 & 0.07834 & 0.004 & 328.177 \\
\hline 1291 & 5.583 & 0.26807 & 329.860 & 12.0467 & 0.07857 & 0.003 & 328.189 \\
\hline 1293 & 5.591 & 0.30061 & 330.043 & 12.0376 & 0.07959 & 0.003 & 328.185 \\
\hline 1297 & 3.826 & 0.15640 & 329.191 & 11.8660 & 0.07735 & 0.009 & 328.160 \\
\hline 1299 & 3.825 & 0.18146 & 329.346 & 11.8566 & 0.07589 & 0.006 & 328.168 \\
\hline 1301 & 3.827 & 0.20842 & 329.513 & 11.8468 & 0.07729 & 0.005 & 328.171 \\
\hline 1305 & 3.837 & 0.26798 & 329.878 & 11.8261 & 0.07824 & 0.004 & 328.185 \\
\hline 1307 & 3.842 & 0.30053 & 330.075 & 11.8148 & 0.07808 & 0.003 & 328.184 \\
\hline 1309 & 2.290 & 0.13325 & 329.030 & 11.6484 & 0.07639 & 0.008 & 328.143 \\
\hline 1311 & 2.288 & 0.15635 & 329.173 & 11.6384 & 0.07656 & 0.007 & 328.151 \\
\hline 1313 & 2.287 & 0.18146 & 329.338 & 11.6269 & 0.07779 & 0.006 & 328.159 \\
\hline 1319 & 2.288 & 0.26809 & 329.882 & 11.5896 & 0.07698 & 0.004 & 328.177 \\
\hline
\end{tabular}


Table 6. Thermal conductivity of the binary $30 \% \mathrm{R} 32$ / $70 \% \mathrm{R} 134 \mathrm{a}$ mixture in the compressed liquid phase (continued).

\begin{tabular}{|c|c|c|c|c|c|c|c|}
\hline $\begin{array}{l}\text { Run } \\
\text { point }\end{array}$ & $\begin{array}{l}P_{\text {exp }} \\
\mathrm{MPa}\end{array}$ & $\begin{array}{c}Q \\
\mathrm{~W} \cdot \mathrm{m}^{-1}\end{array}$ & $\begin{array}{c}T_{\exp } \\
\mathrm{K}\end{array}$ & $\begin{array}{c}\rho_{\text {calc }} \\
\mathrm{mol} \cdot \mathrm{L}^{-1}\end{array}$ & $\frac{\lambda_{\exp }}{\mathrm{W} \cdot \mathrm{m}^{-1} \cdot \mathrm{K}^{-1}}$ & STAT & $\begin{array}{c}T_{\text {cell }} \\
\mathrm{K}\end{array}$ \\
\hline 1325 & 8.655 & 0.16301 & 344.399 & 11.6335 & 0.07543 & 0.007 & 343.297 \\
\hline 1327 & 8.656 & 0.18917 & 344.562 & 11.6249 & 0.07518 & 0.006 & 343.300 \\
\hline 1329 & 8.657 & 0.21725 & 344.721 & 11.6166 & 0.07476 & 0.005 & 343.290 \\
\hline 1331 & 8.662 & 0.24732 & 344.919 & 11.6065 & 0.07443 & 0.004 & 343.293 \\
\hline 1335 & 8.668 & 0.31324 & 345.320 & 11.5856 & 0.07464 & 0.003 & 343.290 \\
\hline 1337 & 7.111 & 0.13882 & 344.233 & 11.4333 & 0.07437 & 0.010 & 343.246 \\
\hline 1339 & 7.109 & 0.16298 & 344.389 & 11.4237 & 0.07419 & 0.007 & 343.252 \\
\hline 1341 & 7.109 & 0.18914 & 344.554 & 11.4139 & 0.07267 & 0.006 & 343.261 \\
\hline 1343 & 7.107 & 0.21736 & 344.739 & 11.4027 & 0.07266 & 0.006 & 343.263 \\
\hline 1345 & 7.109 & 0.24745 & 344.931 & 11.3915 & 0.07312 & 0.004 & 343.266 \\
\hline 1347 & 7.113 & 0.27943 & 345.127 & 11.3804 & 0.07250 & 0.004 & 343.264 \\
\hline 1349 & 7.114 & 0.31331 & 345.342 & 11.3677 & 0.07265 & 0.003 & 343.260 \\
\hline 1351 & 5.657 & 0.13878 & 344.219 & 11.1998 & 0.07246 & 0.009 & 343.219 \\
\hline 1353 & 5.657 & 0.16294 & 344.384 & 11.1887 & 0.07219 & 0.007 & 343.227 \\
\hline 1355 & 5.652 & 0.18916 & 344.556 & 11.1765 & 0.07085 & 0.006 & 343.235 \\
\hline 1357 & 5.651 & 0.21733 & 344.742 & 11.1637 & 0.07076 & 0.005 & 343.242 \\
\hline 1359 & 5.651 & 0.24739 & 344.938 & 11.1505 & 0.07089 & 0.004 & 343.249 \\
\hline 1361 & 5.649 & 0.27945 & 345.146 & 11.1361 & 0.07074 & 0.004 & 343.248 \\
\hline 1363 & 5.648 & 0.31347 & 345.373 & 11.1205 & 0.07128 & 0.003 & 343.257 \\
\hline 1365 & 4.294 & 0.13881 & 344.223 & 10.9280 & 0.06937 & 0.009 & 343.205 \\
\hline 1369 & 4.294 & 0.18921 & 344.555 & 10.9021 & 0.06921 & 0.006 & 343.219 \\
\hline 1371 & 4.295 & 0.21736 & 344.751 & 10.8869 & 0.06978 & 0.005 & 343.228 \\
\hline 1373 & 4.296 & 0.24745 & 344.956 & 10.8708 & 0.06965 & 0.004 & 343.233 \\
\hline 1375 & 4.297 & 0.27945 & 345.160 & 10.8549 & 0.06934 & 0.004 & 343.225 \\
\hline 1377 & 4.299 & 0.31338 & 345.370 & 10.8386 & 0.06946 & 0.004 & 343.220 \\
\hline 1379 & 3.141 & 0.13878 & 344.214 & 10.6296 & 0.06836 & 0.010 & 343.187 \\
\hline 1381 & 3.141 & 0.16302 & 344.383 & 10.6134 & 0.06823 & 0.006 & 343.203 \\
\hline 1383 & 3.141 & 0.18923 & 344.565 & 10.5959 & 0.06808 & 0.006 & 343.214 \\
\hline 1385 & 3.140 & 0.21734 & 344.754 & 10.5776 & 0.06820 & 0.005 & 343.216 \\
\hline 1387 & 3.141 & 0.24744 & 344.948 & 10.5587 & 0.06825 & 0.004 & 343.214 \\
\hline 1389 & 3.143 & 0.27940 & 345.173 & 10.5371 & 0.06844 & 0.005 & 343.218 \\
\hline 1391 & 3.145 & 0.31335 & 345.385 & 10.5168 & 0.06911 & 0.005 & 343.215 \\
\hline 1393 & 7.998 & 0.14446 & 359.354 & 10.6293 & 0.06798 & 0.008 & 358.255 \\
\hline 1395 & 7.991 & 0.16971 & 359.519 & 10.6167 & 0.06747 & 0.007 & 358.261 \\
\hline 1397 & 7.992 & 0.19693 & 359.719 & 10.6032 & 0.06713 & 0.006 & 358.268 \\
\hline 1399 & 7.992 & 0.22614 & 359.906 & 10.5902 & 0.06749 & 0.005 & 358.272 \\
\hline 1403 & 7.985 & 0.29078 & 360.344 & 10.5583 & 0.06762 & 0.004 & 358.276 \\
\hline 1405 & 7.984 & 0.32613 & 360.582 & 10.5415 & 0.06805 & 0.003 & 358.272 \\
\hline 1407 & 6.895 & 0.14444 & 359.313 & 10.3773 & 0.06600 & 0.008 & 358.196 \\
\hline 1409 & 6.896 & 0.16955 & 359.490 & 10.3638 & 0.06572 & 0.007 & 358.212 \\
\hline 1411 & 6.896 & 0.19672 & 359.687 & 10.3483 & 0.06597 & 0.005 & 358.224 \\
\hline 1413 & 6.896 & 0.22602 & 359.900 & 10.3314 & 0.06663 & 0.005 & 358.239 \\
\hline 1415 & 6.895 & 0.25742 & 360.140 & 10.3123 & 0.06587 & 0.004 & 358.262 \\
\hline
\end{tabular}


Table 6. Thermal conductivity of the binary $30 \% \mathrm{R} 32$ / $70 \% \mathrm{R} 134 \mathrm{a}$ mixture in the compressed liquid phase (continued).

\begin{tabular}{|ccccccc|c|}
\hline $\begin{array}{c}\text { Run } \\
\text { point }\end{array}$ & $\begin{array}{c}P_{\text {exp }} \\
\mathrm{MPa}\end{array}$ & $\begin{array}{c}Q \\
\mathrm{~W} \cdot \mathrm{m}^{-1}\end{array}$ & $\begin{array}{c}T_{\text {exp }} \\
\mathrm{K}\end{array}$ & $\begin{array}{c}\rho_{\text {calc }} \\
\mathrm{mol} \cdot \mathrm{L}^{-1}\end{array}$ & $\begin{array}{c}\lambda_{\text {exp }} \\
\mathrm{W} \cdot \mathrm{m}^{-1} \cdot \mathrm{K}^{-1}\end{array}$ & STAT & $\begin{array}{c}T_{\text {cell }} \\
\mathrm{K}\end{array}$ \\
\hline 1417 & 6.898 & 0.29080 & 360.404 & 10.2920 & 0.06636 & 0.004 & 358.296 \\
1419 & 6.903 & 0.32621 & 360.675 & 10.2716 & 0.06723 & 0.004 & 358.324 \\
1421 & 5.979 & 0.14449 & 359.771 & 10.0726 & 0.06334 & 0.008 & 358.630 \\
1423 & 5.992 & 0.16965 & 360.000 & 10.0560 & 0.06455 & 0.006 & 358.689 \\
1425 & 6.005 & 0.19687 & 360.235 & 10.0384 & 0.06768 & 0.005 & 358.744 \\
1427 & 6.017 & 0.22615 & 360.493 & 10.0186 & 0.06530 & 0.005 & 358.793 \\
\hline
\end{tabular}


Table 7. Thermal conductivity of the binary $70 \% \mathrm{R} 32 / 30 \% \mathrm{R} 134 \mathrm{a}$ mixture in the compressed liquid phase.

\begin{tabular}{|c|c|c|c|c|c|c|c|}
\hline $\begin{array}{l}\text { Run } \\
\text { point }\end{array}$ & $\begin{array}{l}P_{\text {exp }} \\
\mathrm{MPa}\end{array}$ & $\begin{array}{c}Q \\
W \cdot \mathrm{m}^{-1}\end{array}$ & $\begin{array}{c}T_{\exp } \\
\mathrm{K} \\
\end{array}$ & $\begin{array}{c}\rho_{\text {calc }} \\
\mathrm{mol} \cdot \mathrm{L}^{-1}\end{array}$ & $\begin{array}{c}\lambda_{\exp } \\
\mathrm{W} \cdot \mathrm{m}^{-1} \cdot \mathrm{K}^{-1}\end{array}$ & STAT & $\begin{array}{c}T_{\text {cell }} \\
\mathrm{K}\end{array}$ \\
\hline 1003 & 0.401 & 0.18622 & 253.784 & 18.1163 & 0.12946 & 0.007 & 253.093 \\
\hline 1005 & 0.402 & 0.23602 & 253.978 & 18.1070 & 0.12857 & 0.005 & 253.081 \\
\hline 1007 & 0.403 & 0.29134 & 254.183 & 18.0972 & 0.12881 & 0.004 & 253.078 \\
\hline 1009 & 0.402 & 0.35269 & 254.430 & 18.0854 & 0.12766 & 0.003 & 253.067 \\
\hline 1011 & 0.401 & 0.41998 & 254.685 & 18.0731 & 0.12721 & 0.002 & 253.057 \\
\hline 1013 & 0.400 & 0.49358 & 254.948 & 18.0604 & 0.12641 & 0.002 & 253.040 \\
\hline 1017 & 0.400 & 0.18615 & 253.710 & 18.1197 & 0.12609 & 0.007 & 253.023 \\
\hline 1019 & 0.400 & 0.23574 & 253.890 & 18.1111 & 0.13059 & 0.005 & 253.005 \\
\hline 1021 & 0.401 & 0.29130 & 254.109 & 18.1007 & 0.13077 & 0.004 & 253.001 \\
\hline 1023 & 0.401 & 0.35273 & 254.364 & 18.0885 & 0.12772 & 0.003 & 252.997 \\
\hline 1025 & 0.401 & 0.41977 & 254.602 & 18.0771 & 0.12665 & 0.002 & 252.991 \\
\hline 1027 & 0.401 & 0.49287 & 254.880 & 18.0638 & 0.12617 & 0.002 & 252.981 \\
\hline 1035 & 0.597 & 0.30897 & 271.096 & 17.2623 & 0.11830 & 0.011 & 269.820 \\
\hline 1037 & 0.597 & 0.37411 & 271.399 & 17.2464 & 0.11830 & 0.007 & 269.838 \\
\hline 1039 & 0.597 & 0.44562 & 271.688 & 17.2312 & 0.11847 & 0.002 & 269.832 \\
\hline 1041 & 0.598 & 0.52341 & 272.013 & 17.2142 & 0.11733 & 0.001 & 269.838 \\
\hline 1045 & 0.598 & 0.19810 & 270.646 & 17.2859 & 0.12229 & 0.006 & 269.851 \\
\hline 1047 & 0.598 & 0.25040 & 270.889 & 17.2732 & 0.12151 & 0.005 & 269.856 \\
\hline 1049 & 0.596 & 0.30944 & 271.146 & 17.2597 & 0.12119 & 0.003 & 269.863 \\
\hline 1051 & 0.596 & 0.37472 & 271.415 & 17.2455 & 0.11978 & 0.002 & 269.864 \\
\hline 1053 & 0.598 & 0.44593 & 271.732 & 17.2289 & 0.11812 & 0.002 & 269.879 \\
\hline 1055 & 0.597 & 0.52361 & 272.057 & 17.2118 & 0.11716 & 0.001 & 269.874 \\
\hline 1057 & 1.335 & 0.15915 & 284.590 & 16.5750 & 0.11260 & 0.008 & 283.909 \\
\hline 1059 & 1.319 & 0.20758 & 284.822 & 16.5606 & 0.11340 & 0.005 & 283.921 \\
\hline 1061 & 1.346 & 0.26218 & 285.057 & 16.5491 & 0.11242 & 0.004 & 283.913 \\
\hline 1063 & 1.369 & 0.32394 & 285.335 & 16.5350 & 0.11035 & 0.003 & 283.914 \\
\hline 1065 & 1.384 & 0.39241 & 285.642 & 16.5185 & 0.11071 & 0.002 & 283.915 \\
\hline 1067 & 1.366 & 0.46747 & 285.983 & 16.4976 & 0.10949 & 0.002 & 283.924 \\
\hline 1069 & 1.338 & 0.54880 & 286.360 & 16.4738 & 0.10957 & 0.001 & 283.934 \\
\hline 1073 & 1.345 & 0.20749 & 284.806 & 16.5633 & 0.11502 & 0.005 & 283.914 \\
\hline 1075 & 1.355 & 0.26226 & 285.066 & 16.5493 & 0.11299 & 0.004 & 283.920 \\
\hline 1077 & 1.366 & 0.32393 & 285.337 & 16.5346 & 0.11214 & 0.003 & 283.916 \\
\hline 1079 & 1.371 & 0.39252 & 285.647 & 16.5173 & 0.11163 & 0.002 & 283.922 \\
\hline 1081 & 1.374 & 0.46744 & 286.001 & 16.4971 & 0.11065 & 0.002 & 283.929 \\
\hline 1083 & 1.372 & 0.54889 & 286.363 & 16.4761 & 0.10981 & 0.001 & 283.926 \\
\hline 1087 & 8.723 & 0.21686 & 299.355 & 16.3004 & 0.11225 & 0.005 & 298.406 \\
\hline 1089 & 8.717 & 0.27434 & 299.627 & 16.2857 & 0.11080 & 0.004 & 298.404 \\
\hline 1091 & 8.724 & 0.33868 & 299.925 & 16.2705 & 0.10888 & 0.003 & 298.412 \\
\hline 1093 & 8.752 & 0.40974 & 300.258 & 16.2548 & 0.10952 & 0.002 & 298.412 \\
\hline 1095 & 8.762 & 0.48803 & 300.617 & 16.2366 & 0.10978 & 0.002 & 298.413 \\
\hline 1097 & 8.769 & 0.57367 & 300.993 & 16.2171 & 0.10977 & 0.002 & 298.403 \\
\hline 1101 & 6.974 & 0.21717 & 299.349 & 16.1716 & 0.10776 & 0.005 & 298.387 \\
\hline 1103 & 6.978 & 0.27443 & 299.621 & 16.1569 & 0.10930 & 0.004 & 298.381 \\
\hline 1105 & 6.983 & 0.33904 & 299.933 & 16.1401 & 0.10737 & 0.003 & 298.381 \\
\hline
\end{tabular}


Table 7. Thermal conductivity of the binary $70 \% \mathrm{R} 32 / 30 \% \mathrm{R} 134 \mathrm{a}$ mixture in the compressed liquid phase (continued).

\begin{tabular}{|c|c|c|c|c|c|c|c|}
\hline $\begin{array}{l}\text { Run } \\
\text { point }\end{array}$ & $\begin{array}{l}P_{\text {exp }} \\
\mathrm{MPa}\end{array}$ & $\begin{array}{c}Q \\
\mathrm{~W} \cdot \mathrm{m}^{-1}\end{array}$ & $\begin{array}{c}T_{\text {exp }} \\
\mathrm{K}\end{array}$ & $\begin{array}{c}\rho_{\text {calc }} \\
\mathrm{mol} \cdot \mathrm{L}^{-1}\end{array}$ & $\begin{array}{c}\lambda_{\exp } \\
\mathrm{W} \cdot \mathrm{m}^{-1} \cdot \mathrm{K}^{-1} \\
\end{array}$ & STAT & $\begin{array}{c}T_{\text {cell }} \\
\mathrm{K}\end{array}$ \\
\hline 1107 & 6.990 & 0.41076 & 300.265 & 16.1224 & 0.10704 & 0.002 & 298.397 \\
\hline 1109 & 6.975 & 0.48894 & 300.617 & 16.1016 & 0.10715 & 0.002 & 298.398 \\
\hline 1111 & 6.945 & 0.57431 & 301.028 & 16.0764 & 0.10785 & 0.001 & 298.405 \\
\hline 1123 & 5.086 & 0.48930 & 300.628 & 15.9463 & 0.10672 & 0.002 & 298.368 \\
\hline 1129 & 3.555 & 0.21720 & 299.397 & 15.8851 & 0.10680 & 0.006 & 298.393 \\
\hline 1131 & 3.543 & 0.27476 & 299.676 & 15.8671 & 0.10649 & 0.009 & 298.379 \\
\hline 1133 & 3.559 & 0.33989 & 299.968 & 15.8507 & 0.10882 & 0.010 & 298.371 \\
\hline 1135 & 3.570 & 0.41167 & 300.321 & 15.8301 & 0.10997 & 0.008 & 298.400 \\
\hline 1137 & 3.580 & 0.48967 & 300.686 & 15.8086 & 0.10846 & 0.005 & 298.383 \\
\hline 1139 & 3.591 & 0.57462 & 301.077 & 15.7855 & 0.10860 & 0.005 & 298.374 \\
\hline 1141 & 1.689 & 0.16650 & 299.125 & 15.7249 & 0.10637 & 0.007 & 298.366 \\
\hline 1143 & 1.688 & 0.21691 & 299.406 & 15.7065 & 0.10671 & 0.009 & 298.381 \\
\hline 1145 & 1.670 & 0.27451 & 299.670 & 15.6875 & 0.10448 & 0.003 & 298.383 \\
\hline 1147 & 1.658 & 0.33938 & 299.985 & 15.6657 & 0.10358 & 0.002 & 298.386 \\
\hline 1149 & 1.657 & 0.41083 & 300.333 & 15.6427 & 0.10222 & 0.002 & 298.387 \\
\hline 1151 & 1.649 & 0.48953 & 300.704 & 15.6172 & 0.10286 & 0.002 & 298.378 \\
\hline 1153 & 1.657 & 0.57590 & 301.121 & 15.5904 & 0.10317 & 0.002 & 298.382 \\
\hline 1159 & 11.236 & 0.23865 & 314.994 & 15.6572 & 0.10615 & 0.004 & 313.876 \\
\hline 1161 & 11.227 & 0.27483 & 315.183 & 15.6462 & 0.10528 & 0.003 & 313.883 \\
\hline 1163 & 11.222 & 0.31404 & 315.363 & 15.6358 & 0.10420 & 0.003 & 313.873 \\
\hline 1165 & 11.232 & 0.35593 & 315.565 & 15.6257 & 0.10423 & 0.002 & 313.869 \\
\hline 1167 & 11.236 & 0.40005 & 315.778 & 15.6144 & 0.10370 & 0.002 & 313.879 \\
\hline 1171 & 9.027 & 0.20517 & 314.841 & 15.4699 & 0.10399 & 0.007 & 313.857 \\
\hline 1173 & 9.035 & 0.23871 & 314.997 & 15.4616 & 0.10296 & 0.005 & 313.858 \\
\hline 1175 & 9.049 & 0.27498 & 315.184 & 15.4520 & 0.10213 & 0.006 & 313.864 \\
\hline 1177 & 9.054 & 0.31399 & 315.378 & 15.4412 & 0.10234 & 0.003 & 313.868 \\
\hline 1179 & 9.062 & 0.35590 & 315.570 & 15.4307 & 0.10203 & 0.002 & 313.855 \\
\hline 1181 & 9.056 & 0.40049 & 315.805 & 15.4165 & 0.10242 & 0.003 & 313.868 \\
\hline 1183 & 7.063 & 0.17092 & 314.623 & 15.2895 & 0.10058 & 0.007 & 313.798 \\
\hline 1185 & 7.055 & 0.20197 & 314.797 & 15.2778 & 0.10094 & 0.005 & 313.822 \\
\hline 1187 & 7.061 & 0.23622 & 314.980 & 15.2669 & 0.09944 & 0.005 & 313.827 \\
\hline 1189 & 7.080 & 0.27326 & 315.158 & 15.2579 & 0.10103 & 0.003 & 313.824 \\
\hline 1191 & 7.087 & 0.31272 & 315.356 & 15.2463 & 0.10023 & 0.003 & 313.824 \\
\hline 1193 & 7.079 & 0.35495 & 315.562 & 15.2326 & 0.09981 & 0.002 & 313.828 \\
\hline 1195 & 7.062 & 0.39958 & 315.793 & 15.2163 & 0.09925 & 0.002 & 313.828 \\
\hline 1197 & 5.033 & 0.17359 & 314.631 & 15.0631 & 0.09979 & 0.007 & 313.784 \\
\hline 1199 & 5.034 & 0.20443 & 314.794 & 15.0521 & 0.10024 & 0.005 & 313.796 \\
\hline 1201 & 5.023 & 0.23837 & 314.973 & 15.0387 & 0.09872 & 0.004 & 313.797 \\
\hline 1203 & 5.013 & 0.27503 & 315.160 & 15.0247 & 0.09870 & 0.003 & 313.798 \\
\hline 1205 & 5.011 & 0.31421 & 315.357 & 15.0110 & 0.09828 & 0.003 & 313.804 \\
\hline 1207 & 5.012 & 0.35571 & 315.581 & 14.9959 & 0.09846 & 0.002 & 313.804 \\
\hline 1209 & 5.032 & 0.39955 & 315.795 & 14.9837 & 0.09844 & 0.002 & 313.805 \\
\hline 1211 & 3.493 & 0.17422 & 314.622 & 14.8683 & 0.09898 & 0.007 & 313.758 \\
\hline
\end{tabular}


Table 7. Thermal conductivity of the binary $70 \% \mathrm{R} 32$ / $30 \% \mathrm{R} 134 \mathrm{a}$ mixture in the compressed liquid phase (continued).

\begin{tabular}{|c|c|c|c|c|c|c|c|}
\hline $\begin{array}{l}\text { Run } \\
\text { point }\end{array}$ & $\begin{array}{l}P_{\text {exp }} \\
\mathrm{MPa}\end{array}$ & $\begin{array}{c}Q \\
\mathrm{~W} \cdot \mathrm{m}^{-1}\end{array}$ & $\begin{array}{c}T_{\exp } \\
\mathrm{K}\end{array}$ & $\begin{array}{c}\rho_{\text {calc }} \\
\mathrm{mol} \cdot \mathrm{L}^{-1}\end{array}$ & $\begin{array}{c}\lambda_{\exp } \\
\mathrm{W} \cdot \mathrm{m}^{-1} \cdot \mathrm{K}^{-1}\end{array}$ & STAT & $\begin{array}{c}T_{\text {cell }} \\
\mathrm{K}\end{array}$ \\
\hline 1213 & 3.498 & 0.20514 & 314.789 & 14.8566 & 0.09709 & 0.005 & 313.772 \\
\hline 1215 & 3.484 & 0.23893 & 314.980 & 14.8406 & 0.09711 & 0.004 & 313.784 \\
\hline 1217 & 3.475 & 0.27542 & 315.176 & 14.8249 & 0.09653 & 0.003 & 313.784 \\
\hline 1219 & 3.474 & 0.31450 & 315.370 & 14.8105 & 0.09647 & 0.003 & 313.782 \\
\hline 1221 & 3.471 & 0.35626 & 315.575 & 14.7948 & 0.09659 & 0.003 & 313.779 \\
\hline 1223 & 3.469 & 0.40052 & 315.809 & 14.7771 & 0.09637 & 0.003 & 313.788 \\
\hline 1225 & 2.372 & 0.17432 & 314.600 & 14.7098 & 0.09633 & 0.006 & 313.727 \\
\hline 1227 & 2.380 & 0.20515 & 314.759 & 14.6984 & 0.09743 & 0.005 & 313.731 \\
\hline 1229 & 2.384 & 0.23883 & 314.938 & 14.6849 & 0.09650 & 0.004 & 313.741 \\
\hline 1231 & 2.388 & 0.27514 & 315.132 & 14.6702 & 0.09745 & 0.003 & 313.745 \\
\hline 1233 & 2.390 & 0.31382 & 315.345 & 14.6536 & 0.09470 & 0.004 & 313.749 \\
\hline 1235 & 2.396 & 0.35534 & 315.549 & 14.6382 & 0.09733 & 0.003 & 313.748 \\
\hline 1237 & 2.395 & 0.39967 & 315.782 & 14.6195 & 0.09644 & 0.003 & 313.746 \\
\hline 1311 & 9.969 & 0.16344 & 345.504 & 13.5736 & 0.08721 & 0.007 & 344.614 \\
\hline 1313 & 9.964 & 0.18974 & 345.670 & 13.5602 & 0.08634 & 0.006 & 344.619 \\
\hline 1315 & 9.963 & 0.21803 & 345.833 & 13.5475 & 0.08596 & 0.005 & 344.624 \\
\hline 1317 & 9.967 & 0.24808 & 345.999 & 13.5356 & 0.08616 & 0.004 & 344.622 \\
\hline 1319 & 9.972 & 0.28010 & 346.185 & 13.5223 & 0.08644 & 0.003 & 344.626 \\
\hline 1321 & 9.974 & 0.31425 & 346.373 & 13.5085 & 0.08651 & 0.003 & 344.621 \\
\hline 1323 & 7.862 & 0.13951 & 345.350 & 13.1563 & 0.08530 & 0.010 & 344.572 \\
\hline 1325 & 7.869 & 0.16360 & 345.507 & 13.1437 & 0.08457 & 0.008 & 344.588 \\
\hline 1327 & 7.872 & 0.18970 & 345.653 & 13.1314 & 0.08441 & 0.008 & 344.582 \\
\hline 1329 & 7.864 & 0.21799 & 345.825 & 13.1139 & 0.08263 & 0.005 & 344.587 \\
\hline 1331 & 7.859 & 0.24812 & 346.017 & 13.0956 & 0.08367 & 0.004 & 344.603 \\
\hline 1333 & 7.859 & 0.28015 & 346.201 & 13.0787 & 0.08395 & 0.003 & 344.607 \\
\hline 1335 & 7.858 & 0.31407 & 346.388 & 13.0614 & 0.08463 & 0.004 & 344.600 \\
\hline 1337 & 6.101 & 0.13944 & 345.325 & 12.6756 & 0.08107 & 0.009 & 344.530 \\
\hline 1339 & 6.103 & 0.16363 & 345.521 & 12.6545 & 0.08097 & 0.007 & 344.579 \\
\hline 1341 & 6.103 & 0.18967 & 345.669 & 12.6378 & 0.08014 & 0.005 & 344.571 \\
\hline 1343 & 6.099 & 0.21784 & 345.840 & 12.6171 & 0.07974 & 0.005 & 344.579 \\
\hline 1345 & 6.098 & 0.24806 & 346.019 & 12.5962 & 0.08097 & 0.005 & 344.579 \\
\hline 1347 & 6.097 & 0.28021 & 346.218 & 12.5732 & 0.08169 & 0.004 & 344.588 \\
\hline 1349 & 6.102 & 0.31412 & 346.414 & 12.5524 & 0.08361 & 0.005 & 344.594 \\
\hline 1351 & 5.164 & 0.13942 & 345.354 & 12.3223 & 0.07823 & 0.008 & 344.548 \\
\hline 1353 & 5.161 & 0.16356 & 345.515 & 12.2992 & 0.07877 & 0.007 & 344.569 \\
\hline 1355 & 5.159 & 0.18958 & 345.682 & 12.2754 & 0.07818 & 0.006 & 344.574 \\
\hline 1357 & 5.158 & 0.21763 & 345.857 & 12.2509 & 0.07859 & 0.007 & 344.584 \\
\hline 1359 & 5.158 & 0.24772 & 346.041 & 12.2252 & 0.08041 & 0.005 & 344.588 \\
\hline 1361 & 5.163 & 0.27968 & 346.223 & 12.2020 & 0.08119 & 0.005 & 344.585 \\
\hline 1365 & 4.286 & 0.13947 & 345.393 & 11.8537 & 0.07661 & 0.009 & 344.588 \\
\hline 1367 & 4.284 & 0.16360 & 345.536 & 11.8261 & 0.07720 & 0.008 & 344.599 \\
\hline 1369 & 4.283 & 0.18949 & 345.709 & 11.7933 & 0.07717 & 0.006 & 344.608 \\
\hline 1371 & 4.283 & 0.21760 & 345.876 & 11.7615 & 0.07872 & 0.007 & 344.607 \\
\hline
\end{tabular}


Table 8 . Thermal conductivity of the binary $30 \% \mathrm{R} 134 \mathrm{a} / 70 \%$ propane mixture in the compressed liquid phase.

\begin{tabular}{|c|c|c|c|c|c|c|c|}
\hline $\begin{array}{l}\text { Run } \\
\text { point }\end{array}$ & $\begin{array}{l}P_{\text {exp }} \\
\mathrm{MPa} \\
\end{array}$ & $\begin{array}{c}Q \\
\mathrm{~W} \cdot \mathrm{m}^{-1}\end{array}$ & $\begin{array}{c}T_{\exp } \\
\mathrm{K}\end{array}$ & $\begin{array}{c}\rho_{\text {calc }} \\
\mathrm{mol}^{-1} \mathrm{~L}^{-1}\end{array}$ & $\begin{array}{c}\lambda_{\exp } \\
\mathrm{W} \cdot \mathrm{m}^{-1} \cdot \mathrm{K}^{-1}\end{array}$ & STAT & $\begin{array}{c}T_{\text {cell }} \\
\mathrm{K}\end{array}$ \\
\hline 1001 & 0.425 & 0.14348 & 254.557 & 11.9309 & 0.09463 & 0.007 & 253.856 \\
\hline 1003 & 0.424 & 0.18757 & 254.793 & 11.9240 & 0.09540 & 0.005 & 253.853 \\
\hline 1005 & 0.424 & 0.23755 & 255.055 & 11.9163 & 0.09540 & 0.004 & 253.843 \\
\hline 1007 & 0.424 & 0.29339 & 255.320 & 11.9086 & 0.09597 & 0.002 & 253.825 \\
\hline 1009 & 0.424 & 0.35523 & 255.633 & 11.8994 & 0.09570 & 0.004 & 253.816 \\
\hline 1011 & 0.425 & 0.42313 & 255.977 & 11.8893 & 0.09541 & 0.002 & 253.797 \\
\hline 1013 & 0.425 & 0.49702 & 256.349 & 11.8784 & 0.09526 & 0.001 & 253.787 \\
\hline 1015 & 0.427 & 0.14324 & 253.848 & 11.9517 & 0.09671 & 0.008 & 253.145 \\
\hline 1017 & 0.426 & 0.18719 & 254.098 & 11.9444 & 0.09577 & 0.005 & 253.160 \\
\hline 1019 & 0.426 & 0.23694 & 254.365 & 11.9366 & 0.09553 & 0.008 & 253.167 \\
\hline 1021 & 0.425 & 0.29263 & 254.673 & 11.9275 & 0.09594 & 0.003 & 253.178 \\
\hline 1023 & 0.424 & 0.35418 & 255.005 & 11.9178 & 0.09638 & 0.002 & 253.184 \\
\hline 1025 & 0.425 & 0.42160 & 255.360 & 11.9074 & 0.09573 & 0.002 & 253.182 \\
\hline 1027 & 0.425 & 0.49502 & 255.739 & 11.8963 & 0.09583 & 0.001 & 253.187 \\
\hline 1029 & 1.186 & 0.15209 & 270.935 & 11.4701 & 0.08892 & 0.009 & 270.122 \\
\hline 1031 & 1.184 & 0.19859 & 271.191 & 11.4621 & 0.08846 & 0.005 & 270.119 \\
\hline 1033 & 1.144 & 0.25153 & 271.491 & 11.4509 & 0.08846 & 0.004 & 270.131 \\
\hline 1035 & 1.118 & 0.31087 & 271.844 & 11.4386 & 0.08863 & 0.002 & 270.131 \\
\hline 1037 & 1.113 & 0.37655 & 272.212 & 11.4268 & 0.08783 & 0.002 & 270.130 \\
\hline 1039 & 1.117 & 0.44842 & 272.611 & 11.4145 & 0.08773 & 0.001 & 270.125 \\
\hline 1041 & 1.124 & 0.52688 & 273.074 & 11.4003 & 0.08775 & 0.002 & 270.138 \\
\hline 1045 & 1.097 & 0.19867 & 271.193 & 11.4579 & 0.08880 & 0.005 & 270.113 \\
\hline 1047 & 1.130 & 0.25124 & 271.485 & 11.4504 & 0.08824 & 0.003 & 270.117 \\
\hline 1049 & 1.160 & 0.31052 & 271.828 & 11.4411 & 0.08823 & 0.003 & 270.123 \\
\hline 1051 & 1.174 & 0.37646 & 272.200 & 11.4301 & 0.08881 & 0.002 & 270.119 \\
\hline 1053 & 1.184 & 0.44836 & 272.610 & 11.4178 & 0.08771 & 0.002 & 270.127 \\
\hline 1055 & 1.186 & 0.52690 & 273.060 & 11.4037 & 0.08817 & 0.002 & 270.126 \\
\hline 1057 & 1.357 & 0.15947 & 284.846 & 11.0304 & 0.08221 & 0.006 & 283.936 \\
\hline 1059 & 1.393 & 0.20828 & 285.146 & 11.0224 & 0.08214 & 0.004 & 283.946 \\
\hline 1061 & 1.404 & 0.26356 & 285.475 & 11.0119 & 0.08182 & 0.003 & 283.943 \\
\hline 1063 & 1.408 & 0.32528 & 285.849 & 10.9996 & 0.08132 & 0.002 & 283.948 \\
\hline 1065 & 1.410 & 0.39358 & 286.264 & 10.9857 & 0.08125 & 0.002 & 283.947 \\
\hline 1067 & 1.412 & 0.46876 & 286.718 & 10.9704 & 0.08109 & 0.001 & 283.954 \\
\hline 1069 & 1.412 & 0.55067 & 287.216 & 10.9534 & 0.08152 & 0.001 & 283.949 \\
\hline 1073 & 1.397 & 0.20834 & 285.150 & 11.0225 & 0.08148 & 0.004 & 283.946 \\
\hline 1075 & 1.399 & 0.26364 & 285.486 & 11.0113 & 0.08151 & 0.003 & 283.945 \\
\hline 1077 & 1.403 & 0.32542 & 285.849 & 10.9992 & 0.08237 & 0.002 & 283.943 \\
\hline 1079 & 1.396 & 0.39410 & 286.271 & 10.9846 & 0.08152 & 0.002 & 283.947 \\
\hline 1081 & 1.402 & 0.46912 & 286.724 & 10.9695 & 0.08213 & 0.002 & 283.950 \\
\hline 1083 & 1.396 & 0.55100 & 287.234 & 10.9518 & 0.08232 & 0.003 & 283.956 \\
\hline 1085 & 8.767 & 0.16693 & 299.362 & 10.9783 & 0.08220 & 0.008 & 298.405 \\
\hline 1087 & 8.787 & 0.21778 & 299.675 & 10.9700 & 0.08232 & 0.004 & 298.412 \\
\hline 1089 & 8.795 & 0.27562 & 300.022 & 10.9601 & 0.08201 & 0.003 & 298.417 \\
\hline
\end{tabular}


Table 8 . Thermal conductivity of the binary $30 \% \mathrm{R} 134 \mathrm{a} / 70 \%$ propane mixture in the compressed liquid phase (continued).

\begin{tabular}{|c|c|c|c|c|c|c|c|}
\hline $\begin{array}{l}\text { Run } \\
\text { point }\end{array}$ & $\begin{array}{l}P_{\text {exp }} \\
\mathrm{MPa}\end{array}$ & $\begin{array}{c}Q Q \\
\mathrm{~W} \cdot \mathrm{m}^{-1}\end{array}$ & $\begin{array}{c}T_{\exp } \\
\mathrm{K}\end{array}$ & $\begin{array}{c}\rho_{\text {calc }} \\
\mathrm{mol} \cdot \mathrm{L}^{-1}\end{array}$ & $\begin{array}{c}\lambda_{\exp } \\
\mathrm{W} \cdot \mathrm{m}^{-1} \cdot \mathrm{K}^{-1}\end{array}$ & STAT & $\begin{array}{l}T_{\text {cell }} \\
\mathrm{K}\end{array}$ \\
\hline 1091 & 8.801 & 0.34049 & 300.407 & 10.9489 & 0.08283 & 0.002 & 298.415 \\
\hline 1093 & 8.798 & 0.41223 & 300.854 & 10.9353 & 0.08289 & 0.002 & 298.420 \\
\hline 1095 & 8.763 & 0.49101 & 301.314 & 10.9196 & 0.08171 & 0.001 & 298.420 \\
\hline 1097 & 8.749 & 0.57685 & 301.846 & 10.9029 & 0.08232 & 0.001 & 298.431 \\
\hline 1099 & 6.874 & 0.16699 & 299.371 & 10.8750 & 0.08084 & 0.006 & 298.397 \\
\hline 1101 & 6.861 & 0.21819 & 299.689 & 10.8643 & 0.08076 & 0.004 & 298.400 \\
\hline 1103 & 6.855 & 0.27578 & 300.037 & 10.8531 & 0.07956 & 0.003 & 298.399 \\
\hline 1105 & 6.884 & 0.34039 & 300.434 & 10.8422 & 0.08056 & 0.002 & 298.409 \\
\hline 1107 & 6.891 & 0.41179 & 300.869 & 10.8289 & 0.08084 & 0.002 & 298.412 \\
\hline 1109 & 6.894 & 0.49027 & 301.360 & 10.8136 & 0.08081 & 0.002 & 298.414 \\
\hline 1111 & 6.898 & 0.57631 & 301.883 & 10.7972 & 0.08058 & 0.001 & 298.406 \\
\hline 1113 & 4.939 & 0.16691 & 299.376 & 10.7601 & 0.07934 & 0.006 & 298.381 \\
\hline 1115 & 4.936 & 0.21798 & 299.709 & 10.7490 & 0.07901 & 0.004 & 298.395 \\
\hline 1117 & 4.936 & 0.27539 & 300.044 & 10.7378 & 0.07889 & 0.003 & 298.386 \\
\hline 1119 & 4.918 & 0.34041 & 300.460 & 10.7228 & 0.07848 & 0.002 & 298.396 \\
\hline 1121 & 4.904 & 0.41229 & 300.921 & 10.7065 & 0.07891 & 0.002 & 298.398 \\
\hline 1123 & 4.903 & 0.49102 & 301.404 & 10.6903 & 0.07868 & 0.001 & 298.397 \\
\hline 1125 & 4.927 & 0.57673 & 301.944 & 10.6737 & 0.07893 & 0.002 & 298.401 \\
\hline 1127 & 3.007 & 0.16688 & 299.383 & 10.6337 & 0.07693 & 0.005 & 298.370 \\
\hline 1129 & 3.007 & 0.21791 & 299.721 & 10.6217 & 0.07749 & 0.004 & 298.388 \\
\hline 1131 & 2.980 & 0.27538 & 300.087 & 10.6069 & 0.07671 & 0.003 & 298.390 \\
\hline 1133 & 2.970 & 0.34040 & 300.507 & 10.5912 & 0.07700 & 0.002 & 298.393 \\
\hline 1135 & 2.974 & 0.41200 & 300.963 & 10.5752 & 0.07684 & 0.002 & 298.397 \\
\hline 1137 & 2.996 & 0.49064 & 301.470 & 10.5585 & 0.07780 & 0.003 & 298.395 \\
\hline 1139 & 3.005 & 0.57710 & 302.027 & 10.5391 & 0.07762 & 0.002 & 298.392 \\
\hline 1141 & 1.558 & 0.16689 & 299.403 & 10.5286 & 0.07585 & 0.005 & 298.367 \\
\hline 1143 & 1.539 & 0.21773 & 299.717 & 10.5154 & 0.07473 & 0.003 & 298.374 \\
\hline 1145 & 1.525 & 0.27529 & 300.111 & 10.4995 & 0.07451 & 0.003 & 298.379 \\
\hline 1147 & 1.520 & 0.34026 & 300.540 & 10.4829 & 0.07525 & 0.002 & 298.389 \\
\hline 1149 & 1.545 & 0.41220 & 300.998 & 10.4674 & 0.07530 & 0.002 & 298.384 \\
\hline 1151 & 1.555 & 0.49154 & 301.506 & 10.4488 & 0.07549 & 0.002 & 298.389 \\
\hline 1153 & 1.564 & 0.57772 & 302.066 & 10.4281 & 0.07649 & 0.003 & 298.387 \\
\hline 1155 & 8.614 & 0.17504 & 315.325 & 10.4738 & 0.07630 & 0.005 & 314.252 \\
\hline 1157 & 8.621 & 0.22806 & 315.664 & 10.4634 & 0.07652 & 0.003 & 314.253 \\
\hline 1159 & 8.626 & 0.28856 & 316.060 & 10.4508 & 0.07717 & 0.004 & 314.261 \\
\hline 1161 & 8.631 & 0.35667 & 316.512 & 10.4365 & 0.07643 & 0.002 & 314.267 \\
\hline 1163 & 8.628 & 0.43224 & 316.975 & 10.4212 & 0.07648 & 0.002 & 314.252 \\
\hline 1165 & 8.611 & 0.51555 & 317.523 & 10.4021 & 0.07588 & 0.001 & 314.260 \\
\hline 1167 & 8.605 & 0.60558 & 318.106 & 10.3826 & 0.07665 & 0.002 & 314.259 \\
\hline 1169 & 6.795 & 0.17518 & 315.309 & 10.3473 & 0.07483 & 0.005 & 314.213 \\
\hline 1171 & 6.789 & 0.20589 & 315.513 & 10.3398 & 0.07475 & 0.004 & 314.220 \\
\hline 1173 & 6.803 & 0.23916 & 315.738 & 10.3331 & 0.07482 & 0.003 & 314.222 \\
\hline 1175 & 6.811 & 0.27564 & 315.986 & 10.3251 & 0.07437 & 0.003 & 314.231 \\
\hline
\end{tabular}


Table 8 . Thermal conductivity of the binary $30 \% \mathrm{R} 134 \mathrm{a} / 70 \%$ propane mixture in the compressed liquid phase (continued).

\begin{tabular}{|c|c|c|c|c|c|c|c|}
\hline $\begin{array}{l}\text { Run } \\
\text { point }\end{array}$ & $\begin{array}{l}P_{\text {exp }} \\
\mathrm{MPa}\end{array}$ & $\begin{array}{c}Q \\
\mathrm{~W} \cdot \mathrm{m}^{-1}\end{array}$ & $\begin{array}{c}T_{\exp } \\
\mathrm{K}\end{array}$ & $\begin{array}{c}\rho_{\text {calc }} \\
\mathrm{mol} \cdot \mathrm{L}^{-1}\end{array}$ & $\begin{array}{c}\lambda_{\exp } \\
\mathrm{W} \cdot \mathrm{m}^{-1} \cdot \mathrm{K}^{-1}\end{array}$ & STAT & $\begin{array}{c}T_{\text {cell }} \\
\mathrm{K}\end{array}$ \\
\hline 1177 & 6.815 & 0.31465 & 316.226 & 10.3171 & 0.07412 & 0.003 & 314.221 \\
\hline 1179 & 6.815 & 0.35666 & 316.497 & 10.3077 & 0.07455 & 0.002 & 314.216 \\
\hline 1181 & 6.802 & 0.40142 & 316.791 & 10.2965 & 0.07420 & 0.002 & 314.218 \\
\hline 1183 & 4.865 & 0.17518 & 315.292 & 10.1969 & 0.07176 & 0.005 & 314.127 \\
\hline 1185 & 4.867 & 0.20607 & 315.497 & 10.1894 & 0.07260 & 0.004 & 314.156 \\
\hline 1187 & 4.877 & 0.23925 & 315.729 & 10.1816 & 0.07253 & 0.003 & 314.175 \\
\hline 1189 & 4.879 & 0.27565 & 316.013 & 10.1711 & 0.07260 & 0.004 & 314.187 \\
\hline 1191 & 4.881 & 0.31493 & 316.232 & 10.1630 & 0.07277 & 0.002 & 314.176 \\
\hline 1193 & 4.881 & 0.35697 & 316.504 & 10.1528 & 0.07257 & 0.002 & 314.168 \\
\hline 1195 & 4.882 & 0.40159 & 316.804 & 10.1416 & 0.07294 & 0.002 & 314.171 \\
\hline 1197 & 3.162 & 0.17508 & 315.262 & 10.0465 & 0.07034 & 0.005 & 314.123 \\
\hline 1199 & 3.165 & 0.20720 & 320.299 & 9.8363 & 0.07118 & 0.004 & 314.135 \\
\hline 1201 & 3.170 & 0.23962 & 315.734 & 10.0280 & 0.07219 & 0.006 & 314.141 \\
\hline 1203 & 3.169 & 0.27615 & 315.992 & 10.0174 & 0.07077 & 0.005 & 314.135 \\
\hline 1205 & 3.155 & 0.31502 & 316.262 & 10.0050 & 0.07144 & 0.007 & 314.147 \\
\hline 1207 & 3.149 & 0.35679 & 316.551 & 9.9925 & 0.07120 & 0.003 & 314.139 \\
\hline 1209 & 3.150 & 0.40122 & 316.850 & 9.9802 & 0.07163 & 0.004 & 314.140 \\
\hline 1211 & 3.155 & 0.17452 & 315.278 & 10.0452 & 0.07137 & 0.005 & 314.132 \\
\hline 1213 & 3.151 & 0.20588 & 315.500 & 10.0357 & 0.07127 & 0.005 & 314.138 \\
\hline 1215 & 3.149 & 0.23990 & 315.742 & 10.0257 & 0.07099 & 0.004 & 314.144 \\
\hline 1217 & 3.157 & 0.27632 & 315.966 & 10.0173 & 0.07081 & 0.003 & 314.135 \\
\hline 1219 & 3.163 & 0.31539 & 316.240 & 10.0067 & 0.07274 & 0.003 & 314.144 \\
\hline 1221 & 3.167 & 0.35711 & 316.527 & 9.9952 & 0.07250 & 0.003 & 314.140 \\
\hline 1223 & 3.171 & 0.40139 & 316.827 & 9.9833 & 0.07221 & 0.003 & 314.137 \\
\hline 1225 & 1.899 & 0.17503 & 315.266 & 9.9187 & 0.06894 & 0.005 & 314.101 \\
\hline 1227 & 1.898 & 0.20616 & 315.496 & 9.9086 & 0.06993 & 0.004 & 314.114 \\
\hline 1229 & 1.907 & 0.23932 & 315.709 & 9.9002 & 0.07151 & 0.003 & 314.117 \\
\hline 1231 & 1.913 & 0.27553 & 315.976 & 9.8890 & 0.07119 & 0.004 & 314.118 \\
\hline 1233 & 1.916 & 0.31491 & 316.243 & 9.8775 & 0.07171 & 0.003 & 314.114 \\
\hline 1235 & 1.918 & 0.35697 & 316.535 & 9.8647 & 0.07065 & 0.003 & 314.116 \\
\hline 1237 & 1.907 & 0.40150 & 316.850 & 9.8494 & 0.06930 & 0.003 & 314.114 \\
\hline 1239 & 6.962 & 0.13370 & 328.834 & 9.8731 & 0.07285 & 0.008 & 327.960 \\
\hline 1241 & 6.961 & 0.15659 & 328.995 & 9.8669 & 0.07313 & 0.006 & 327.971 \\
\hline 1243 & 6.958 & 0.18142 & 329.197 & 9.8590 & 0.07208 & 0.005 & 327.986 \\
\hline 1245 & 6.958 & 0.20827 & 329.386 & 9.8518 & 0.07272 & 0.005 & 327.991 \\
\hline 1247 & 6.961 & 0.23699 & 329.569 & 9.8451 & 0.07553 & 0.005 & 327.995 \\
\hline 1249 & 6.961 & 0.26800 & 329.793 & 9.8366 & 0.07400 & 0.004 & 327.996 \\
\hline 1251 & 6.963 & 0.30091 & 330.022 & 9.8280 & 0.07381 & 0.004 & 327.992 \\
\hline 1253 & 5.684 & 0.13370 & 328.861 & 9.7458 & 0.07305 & 0.009 & 327.976 \\
\hline 1255 & 5.684 & 0.15686 & 329.026 & 9.7390 & 0.07584 & 0.007 & 327.982 \\
\hline 1259 & 5.683 & 0.20853 & 329.411 & 9.7232 & 0.07290 & 0.010 & 328.002 \\
\hline 1263 & 5.685 & 0.26780 & 329.833 & 9.7062 & 0.07353 & 0.004 & 328.008 \\
\hline 1265 & 5.684 & 0.30033 & 330.042 & 9.6976 & 0.07556 & 0.006 & 327.998 \\
\hline
\end{tabular}


Table 8 . Thermal conductivity of the binary $30 \% \mathrm{R} 134 \mathrm{a} / 70 \%$ propane mixture in the compressed liquid phase (continued).

\begin{tabular}{|c|c|c|c|c|c|c|c|}
\hline $\begin{array}{l}\text { Run } \\
\text { point }\end{array}$ & $\begin{array}{l}P_{\text {exp }} \\
\mathrm{MPa}\end{array}$ & $\begin{array}{c}Q \\
\mathrm{~W} \cdot \mathrm{m}^{-1}\end{array}$ & $\begin{array}{c}T_{\exp } \\
\mathrm{K}\end{array}$ & $\begin{array}{c}\rho_{\text {calc }} \\
\mathrm{mol} \cdot \mathrm{L}^{-1}\end{array}$ & $\begin{array}{c}\lambda_{\exp } \\
\mathrm{W} \cdot \mathrm{m}^{-1} \cdot \mathrm{K}^{-1}\end{array}$ & STAT & $\begin{array}{c}T_{\text {cell }} \\
\mathrm{K}\end{array}$ \\
\hline 1267 & 4.551 & 0.13371 & 328.886 & 9.6195 & 0.07731 & 0.011 & 327.995 \\
\hline 1269 & 4.551 & 0.15682 & 329.051 & 9.6123 & 0.07702 & 0.009 & 327.997 \\
\hline 1271 & 4.551 & 0.18157 & 329.233 & 9.6043 & 0.07358 & 0.007 & 327.997 \\
\hline 1273 & 4.551 & 0.20851 & 329.424 & 9.5959 & 0.07459 & 0.007 & 327.997 \\
\hline 1277 & 4.552 & 0.26791 & 329.839 & 9.5779 & 0.07717 & 0.007 & 328.014 \\
\hline 1279 & 4.555 & 0.30038 & 330.096 & 9.5668 & 0.07283 & 0.005 & 328.013 \\
\hline 1281 & 3.477 & 0.13373 & 328.895 & 9.4853 & 0.07519 & 0.011 & 327.995 \\
\hline 1285 & 3.481 & 0.18152 & 329.255 & 9.4687 & 0.07395 & 0.007 & 328.006 \\
\hline 1289 & 3.470 & 0.23739 & 329.645 & 9.4486 & 0.07690 & 0.007 & 328.011 \\
\hline 1293 & 3.476 & 0.30089 & 330.078 & 9.4285 & 0.08723 & 0.012 & 328.014 \\
\hline 1295 & 2.614 & 0.13368 & 328.890 & 9.3638 & 0.08355 & 0.013 & 327.991 \\
\hline 1297 & 2.615 & 0.15666 & 329.068 & 9.3547 & 0.08030 & 0.011 & 328.000 \\
\hline 1299 & 2.616 & 0.18146 & 329.241 & 9.3458 & 0.08372 & 0.011 & 328.000 \\
\hline 1309 & 7.574 & 0.11723 & 345.889 & 9.2543 & 0.06927 & 0.010 & 345.083 \\
\hline 1311 & 7.575 & 0.13017 & 346.005 & 9.2495 & 0.06831 & 0.009 & 345.100 \\
\hline 1313 & 7.570 & 0.14411 & 346.105 & 9.2446 & 0.06793 & 0.008 & 345.102 \\
\hline 1315 & 7.568 & 0.15870 & 346.223 & 9.2392 & 0.06796 & 0.006 & 345.107 \\
\hline 1317 & 7.564 & 0.17404 & 346.327 & 9.2342 & 0.06836 & 0.006 & 345.101 \\
\hline 1319 & 7.566 & 0.19014 & 346.433 & 9.2300 & 0.07052 & 0.006 & 345.090 \\
\hline 1321 & 7.572 & 0.20670 & 346.564 & 9.2251 & 0.06980 & 0.005 & 345.096 \\
\hline 1323 & 6.060 & 0.11746 & 345.883 & 9.0408 & 0.06954 & 0.010 & 345.058 \\
\hline 1325 & 6.061 & 0.13044 & 345.985 & 9.0360 & 0.07322 & 0.010 & 345.072 \\
\hline 1327 & 6.055 & 0.14418 & 346.104 & 9.0294 & 0.07319 & 0.009 & 345.083 \\
\hline 1329 & 6.052 & 0.15870 & 346.207 & 9.0238 & 0.07177 & 0.008 & 345.080 \\
\hline 1331 & 6.052 & 0.17405 & 346.329 & 9.0179 & 0.06932 & 0.007 & 345.080 \\
\hline 1335 & 6.059 & 0.20676 & 346.570 & 9.0072 & 0.07138 & 0.007 & 345.077 \\
\hline 1337 & 4.969 & 0.11741 & 345.864 & 8.8561 & 0.07583 & 0.013 & 345.045 \\
\hline 1339 & 4.971 & 0.13029 & 345.969 & 8.8506 & 0.07817 & 0.013 & 345.059 \\
\hline 1341 & 4.971 & 0.14399 & 346.084 & 8.8444 & 0.07847 & 0.012 & 345.064 \\
\hline 1343 & 4.971 & 0.15852 & 346.213 & 8.8372 & 0.07413 & 0.010 & 345.075 \\
\hline 1345 & 4.967 & 0.17391 & 346.326 & 8.8302 & 0.07467 & 0.011 & 345.071 \\
\hline 1347 & 4.966 & 0.19001 & 346.433 & 8.8241 & 0.08033 & 0.013 & 345.065 \\
\hline 1349 & 4.965 & 0.20683 & 346.550 & 8.8174 & 0.07985 & 0.012 & 345.069 \\
\hline 1351 & 4.203 & 0.11741 & 345.863 & 8.7010 & 0.09111 & 0.020 & 345.056 \\
\hline 1353 & 4.203 & 0.13035 & 345.957 & 8.6954 & 0.09414 & 0.019 & 345.058 \\
\hline 1355 & 4.204 & 0.14398 & 346.074 & 8.6883 & 0.08713 & 0.017 & 345.062 \\
\hline
\end{tabular}


Table 9. Thermal conductivity of the binary $70 \% \mathrm{R} 134 \mathrm{a} / 30 \%$ propane mixture in the compressed liquid phase obtained with wire assembly 1 .

\begin{tabular}{|c|c|c|c|c|c|c|c|}
\hline $\begin{array}{l}\text { Run } \\
\text { point }\end{array}$ & $\begin{array}{l}P_{\text {exp }} \\
\mathrm{MPa}\end{array}$ & $\begin{array}{c}Q \\
\mathrm{~W} \cdot \mathrm{m}^{-1}\end{array}$ & $\begin{array}{c}T_{e x p} \\
\mathrm{~K}\end{array}$ & $\begin{array}{c}\rho_{\text {calc }} \\
\mathrm{mol} \cdot \mathrm{L}^{-1}\end{array}$ & $\begin{array}{c}\lambda_{\exp } \\
\mathrm{W} \cdot \mathrm{m}^{-1} \cdot \mathrm{K}^{-1}\end{array}$ & STAT & $\begin{array}{c}T_{\text {cell }} \\
\mathrm{K}\end{array}$ \\
\hline 1003 & 0.456 & 0.18030 & 250.965 & 12.2673 & 0.09048 & 0.007 & 250.046 \\
\hline 1005 & 0.455 & 0.22845 & 251.216 & 12.2599 & 0.08977 & 0.005 & 250.048 \\
\hline 1007 & 0.456 & 0.28226 & 251.480 & 12.2520 & 0.08909 & 0.004 & 250.030 \\
\hline 1009 & 0.456 & 0.34181 & 251.781 & 12.2430 & 0.08877 & 0.005 & 250.012 \\
\hline 1011 & 0.457 & 0.40718 & 252.096 & 12.2337 & 0.08837 & 0.002 & 250.000 \\
\hline 1013 & 0.458 & 0.47805 & 252.452 & 12.2231 & 0.08764 & 0.002 & 249.986 \\
\hline 1017 & 0.457 & 0.18004 & 250.710 & 12.2749 & 0.09067 & 0.007 & 249.796 \\
\hline 1019 & 0.457 & 0.22803 & 250.960 & 12.2675 & 0.08939 & 0.005 & 249.795 \\
\hline 1021 & 0.457 & 0.28174 & 251.247 & 12.2590 & 0.08900 & 0.004 & 249.785 \\
\hline 1023 & 0.457 & 0.34109 & 251.535 & 12.2504 & 0.08842 & 0.003 & 249.780 \\
\hline 1025 & 0.457 & 0.40634 & 251.878 & 12.2402 & 0.08796 & 0.002 & 249.770 \\
\hline 1027 & 0.457 & 0.47739 & 252.235 & 12.2296 & 0.08728 & 0.003 & 249.761 \\
\hline 1031 & 0.783 & 0.18535 & 257.992 & 12.0686 & 0.08730 & 0.007 & 257.021 \\
\hline 1033 & 0.786 & 0.23464 & 258.255 & 12.0608 & 0.08672 & 0.005 & 257.023 \\
\hline 1035 & 0.787 & 0.28979 & 258.548 & 12.0520 & 0.08643 & 0.004 & 257.016 \\
\hline 1037 & 0.789 & 0.35086 & 258.888 & 12.0417 & 0.08594 & 0.003 & 257.024 \\
\hline 1039 & 0.786 & 0.41775 & 259.243 & 12.0308 & 0.08529 & 0.002 & 257.026 \\
\hline 1041 . & 0.783 & 0.49061 & 259.637 & 12.0187 & 0.08511 & 0.002 & 257.024 \\
\hline 1045 & 0.784 & 0.18533 & 257.985 & 12.0689 & 0.08778 & 0.007 & 257.018 \\
\hline 1047 & 0.781 & 0.23448 & 258.253 & 12.0607 & 0.08618 & 0.005 & 257.016 \\
\hline 1049 & 0.784 & 0.28958 & 258.545 & 12.0519 & 0.08588 & 0.004 & 257.020 \\
\hline 1051 & 0.781 & 0.35059 & 258.883 & 12.0416 & 0.08522 & 0.003 & 257.023 \\
\hline 1053 & 0.781 & 0.41749 & 259.238 & 12.0308 & 0.08469 & 0.002 & 257.018 \\
\hline 1055 & 0.779 & 0.49025 & 259.631 & 12.0188 & 0.08454 & 0.002 & 257.024 \\
\hline 1059 & 1.075 & 0.19386 & 270.640 & 11.6889 & 0.08214 & 0.006 & 269.594 \\
\hline 1061 & 1.075 & 0.24534 & 270.936 & 11.6795 & 0.08130 & 0.004 & 269.595 \\
\hline 1063 & 1.075 & 0.30294 & 271.275 & 11.6688 & 0.08208 & 0.003 & 269.596 \\
\hline 1065 & 1.077 & 0.36658 & 271.625 & 11.6577 & 0.08162 & 0.002 & 269.599 \\
\hline 1067 & 1.077 & 0.43645 & 272.032 & 11.6447 & 0.08092 & 0.002 & 269.607 \\
\hline 1069 & 1.077 & 0.51256 & 272.444 & 11.6315 & 0.08022 & 0.002 & 269.598 \\
\hline 1071 & 1.079 & 0.14854 & 270.372 & 11.6977 & 0.08250 & 0.009 & 269.583 \\
\hline 1073 & 1.080 & 0.19392 & 270.643 & 11.6891 & 0.08183 & 0.006 & 269.600 \\
\hline 1075 & 1.081 & 0.24534 & 270.948 & 11.6794 & 0.08049 & 0.004 & 269.596 \\
\hline 1077 & 1.080 & 0.30294 & 271.277 & 11.6689 & 0.08089 & 0.003 & 269.607 \\
\hline 1079 & 1.080 & 0.36684 & 271.639 & 11.6573 & 0.08073 & 0.002 & 269.606 \\
\hline 1081 & 1.082 & 0.43693 & 272.039 & 11.6447 & 0.08011 & 0.002 & 269.607 \\
\hline 1083 & 1.080 & 0.51312 & 272.469 & 11.6308 & 0.07991 & 0.002 & 269.611 \\
\hline 1087 & 9.163 & 0.20341 & 284.931 & 11.6082 & 0.08298 & 0.006 & 283.818 \\
\hline 1089 & 9.165 & 0.25738 & 285.253 & 11.5991 & 0.08282 & 0.004 & 283.832 \\
\hline 1091 & 9.159 & 0.31784 & 285.595 & 11.5890 & 0.08304 & 0.003 & 283.827 \\
\hline 1093 & 9.161 & 0.38483 & 285.973 & 11.5782 & 0.08220 & 0.002 & 283.831 \\
\hline 1095 & 9.162 & 0.45817 & 286.398 & 11.5660 & 0.08185 & 0.002 & 283.826 \\
\hline 1097 & 9.160 & 0.53809 & 286.850 & 11.5528 & 0.08242 & 0.002 & 283.827 \\
\hline
\end{tabular}


Table 9. Thermal conductivity of the binary $70 \% \mathrm{R} 134 \mathrm{a} / 30 \%$ propane mixture in the compressed liquid phase obtained with wire assembly 1 (continued).

\begin{tabular}{|c|c|c|c|c|c|c|c|}
\hline $\begin{array}{l}\text { Run } \\
\text { point }\end{array}$ & $\begin{array}{l}P_{\text {exp }} \\
\mathrm{MPa}\end{array}$ & $\begin{array}{c}Q \\
\mathrm{~W} \cdot \mathrm{m}^{-1}\end{array}$ & $\begin{array}{c}T_{\exp } \\
\mathrm{K}\end{array}$ & $\begin{array}{c}\rho_{\text {calc }} \\
\mathrm{mol} \cdot \mathrm{L}^{-1}\end{array}$ & $\begin{array}{c}\lambda_{\exp } \\
\mathrm{W} \cdot \mathrm{m}^{-1} \cdot \mathrm{K}^{-1}\end{array}$ & STAT & $\begin{array}{c}T_{\text {cell }} \\
\mathrm{K}\end{array}$ \\
\hline 1099 & 7.020 & 0.15584 & 284.659 & 11.5238 & 0.08052 & 0.008 & 283.800 \\
\hline 1101 & 7.020 & 0.20340 & 284.938 & 11.5154 & 0.08114 & 0.006 & 283.801 \\
\hline 1103 & 7.023 & 0.25725 & 285.251 & 11.5062 & 0.08012 & 0.004 & 283.809 \\
\hline 1105 & 7.022 & 0.31756 & 285.607 & 11.4955 & 0.08027 & 0.003 & 283.809 \\
\hline 1107 & 7.023 & 0.38457 & 285.993 & 11.4840 & 0.08065 & 0.002 & 283.816 \\
\hline 1109 & 7.026 & 0.45820 & 286.417 & 11.4714 & 0.07990 & 0.002 & 283.818 \\
\hline 1111 & 7.024 & 0.53815 & 286.892 & 11.4570 & 0.07975 & 0.002 & 283.821 \\
\hline 1113 & 4.949 & 0.15580 & 284.659 & 11.4280 & 0.07815 & 0.008 & 283.781 \\
\hline 1115 & 4.947 & 0.20337 & 284.943 & 11.4190 & 0.08241 & 0.007 & 283.797 \\
\hline 1117 & 4.947 & 0.25712 & 285.279 & 11.4085 & 0.07950 & 0.005 & 283.804 \\
\hline 1119 & 4.947 & 0.31734 & 285.612 & 11.3981 & 0.07906 & 0.003 & 283.798 \\
\hline 1121 & 4.946 & 0.38441 & 286.013 & 11.3855 & 0.07814 & 0.002 & 283.807 \\
\hline 1123 & 4.948 & 0.45789 & 286.435 & 11.3723 & 0.07797 & 0.002 & 283.804 \\
\hline 1125 & 4.945 & 0.53783 & 286.906 & 11.3574 & 0.07765 & 0.001 & 283.808 \\
\hline 1129 & 3.196 & 0.20333 & 284.956 & 11.3313 & 0.07808 & 0.006 & 283.792 \\
\hline 1131 & 3.196 & 0.25715 & 285.281 & 11.3207 & 0.07742 & 0.005 & 283.802 \\
\hline 1133 & 3.188 & 0.31750 & 285.639 & 11.3086 & 0.07733 & 0.003 & 283.804 \\
\hline 1135 & 3.169 & 0.38463 & 286.050 & 11.2942 & 0.07721 & 0.002 & 283.813 \\
\hline 1137 & 3.157 & 0.45775 & 286.482 & 11.2794 & 0.07654 & 0.002 & 283.809 \\
\hline 1139 & 3.144 & 0.53720 & 286.937 & 11.2637 & 0.07654 & 0.001 & 283.812 \\
\hline 1141 & 1.432 & 0.15604 & 284.677 & 11.2461 & 0.07699 & 0.008 & 283.783 \\
\hline 1143 & 1.429 & 0.20387 & 284.975 & 11.2358 & 0.07594 & 0.006 & 283.788 \\
\hline 1145 & 1.424 & 0.25804 & 285.289 & 11.2248 & 0.07471 & 0.004 & 283.791 \\
\hline 1147 & 1.415 & 0.31861 & 285.658 & 11.2117 & 0.07507 & 0.003 & 283.790 \\
\hline 1149 & 1.414 & 0.38559 & 286.056 & 11.1980 & 0.07486 & 0.002 & 283.795 \\
\hline 1151 & 1.411 & 0.45904 & 286.493 & 11.1828 & 0.07413 & 0.002 & 283.792 \\
\hline 1153 & 1.418 & 0.53869 & 286.962 & 11.1670 & 0.07442 & 0.002 & 283.797 \\
\hline 1155 & 10.084 & 0.16294 & 299.329 & 11.2298 & 0.07892 & 0.009 & 298.388 \\
\hline 1157 & 10.084 & 0.21292 & 299.626 & 11.2210 & 0.07829 & 0.005 & 298.395 \\
\hline 1159 & 10.087 & 0.26950 & 299.981 & 11.2106 & 0.07765 & 0.004 & 298.404 \\
\hline 1161 & 10.082 & 0.33259 & 300.359 & 11.1991 & 0.07776 & 0.003 & 298.402 \\
\hline 1163 & 10.083 & 0.40245 & 300.770 & 11.1869 & 0.07773 & 0.002 & 298.400 \\
\hline 1165 & 10.082 & 0.47897 & 301.243 & 11.1727 & 0.07775 & 0.002 & 298.407 \\
\hline 1167 & 10.084 & 0.56259 & 301.725 & 11.1585 & 0.07722 & 0.001 & 298.400 \\
\hline 1173 & 7.399 & 0.26925 & 300.001 & 11.0716 & 0.07586 & 0.009 & 298.376 \\
\hline 1175 & 7.402 & 0.33237 & 300.416 & 11.0586 & 0.07732 & 0.008 & 298.386 \\
\hline 1177 & 7.402 & 0.40252 & 300.811 & 11.0461 & 0.07563 & 0.002 & 298.386 \\
\hline 1179 & 7.402 & 0.47977 & 301.287 & 11.0310 & 0.07486 & 0.002 & 298.388 \\
\hline 1181 & 7.407 & 0.56348 & 301.789 & 11.0153 & 0.07560 & 0.002 & 298.387 \\
\hline 1183 & 5.536 & 0.16316 & 299.334 & 10.9883 & 0.07566 & 0.008 & 298.365 \\
\hline 1185 & 5.534 & 0.21278 & 299.644 & 10.9780 & 0.07300 & 0.005 & 298.367 \\
\hline 1187 & 5.536 & 0.26890 & 300.002 & 10.9662 & 0.07479 & 0.004 & 298.368 \\
\hline 1189 & 5.535 & 0.33219 & 300.391 & 10.9532 & 0.07391 & 0.003 & 298.376 \\
\hline
\end{tabular}


Table 9. Thermal conductivity of the binary $70 \% \mathrm{R} 134 \mathrm{a} / 30 \%$ propane mixture in the compressed liquid phase obtained with wire assembly 1 (continued).

\begin{tabular}{|c|c|c|c|c|c|c|c|}
\hline $\begin{array}{l}\text { Run } \\
\text { point }\end{array}$ & $\begin{array}{l}P_{e x p} \\
\mathrm{MPa} \\
\end{array}$ & $\begin{array}{c}Q \\
\mathrm{~W} \cdot \mathrm{m}^{-1}\end{array}$ & $\begin{array}{c}T_{\exp } \\
\mathrm{K} \\
\end{array}$ & $\begin{array}{c}\rho_{\text {calc }} \\
\mathrm{mol} \cdot \mathrm{L}^{-1}\end{array}$ & $\begin{array}{c}\lambda_{\exp } \\
\mathrm{W} \cdot \mathrm{m}^{-1} \cdot \mathrm{K}^{-1}\end{array}$ & STAT & $\begin{array}{c}T_{\text {cell }} \\
\mathrm{K}\end{array}$ \\
\hline 1191 & 5.534 & 0.40241 & 300.833 & 10.9384 & 0.07422 & 0.002 & 298.379 \\
\hline 1193 & 5.533 & 0.47952 & 301.300 & 10.9227 & 0.07376 & 0.002 & 298.376 \\
\hline 1195 & 5.535 & 0.56345 & 301.831 & 10.9051 & 0.07365 & 0.001 & 298.384 \\
\hline 1197 & 3.548 & 0.16315 & 299.340 & 10.8663 & 0.07308 & 0.008 & 298.353 \\
\hline 1199 & 3.551 & 0.21278 & 299.655 & 10.8554 & 0.07206 & 0.005 & 298.361 \\
\hline 1201 & 3.552 & 0.26902 & 300.017 & 10.8427 & 0.07263 & 0.004 & 298.365 \\
\hline 1203 & 3.556 & 0.33228 & 300.418 & 10.8288 & 0.07329 & 0.005 & 298.368 \\
\hline 1205 & 3.555 & 0.40243 & 300.864 & 10.8129 & 0.07206 & 0.002 & 298.373 \\
\hline 1207 & 3.551 & 0.47952 & 301.352 & 10.7953 & 0.07159 & 0.001 & 298.372 \\
\hline 1209 & 3.550 & 0.56363 & 301.867 & 10.7769 & 0.07186 & 0.001 & 298.373 \\
\hline 1211 & 1.411 & 0.16315 & 299.359 & 10.7190 & 0.07093 & 0.008 & 298.344 \\
\hline 1213 & 1.411 & 0.21288 & 299.695 & 10.7062 & 0.07037 & 0.006 & 298.349 \\
\hline 1215 & 1.410 & 0.26907 & 300.086 & 10.6913 & 0.07027 & 0.007 & 298.364 \\
\hline 1217 & 1.410 & 0.33227 & 300.461 & 10.6770 & 0.07028 & 0.005 & 298.365 \\
\hline 1219 & 1.414 & 0.40264 & 300.907 & 10.6601 & 0.06965 & 0.002 & 298.365 \\
\hline 1221 & 1.410 & 0.48002 & 301.395 & 10.6411 & 0.06997 & 0.002 & 298.364 \\
\hline 1223 & 1.411 & 0.56389 & 301.939 & 10.6200 & 0.07053 & 0.003 & 298.368 \\
\hline 1225 & 9.298 & 0.17116 & 315.547 & 10.6854 & 0.07358 & 0.007 & 314.484 \\
\hline 1227 & 9.300 & 0.22336 & 315.891 & 10.6744 & 0.07262 & 0.005 & 314.495 \\
\hline 1229 & 9.301 & 0.28259 & 316.266 & 10.6623 & 0.07232 & 0.003 & 314.498 \\
\hline 1231 & 9.303 & 0.34886 & 316.706 & 10.6481 & 0.07220 & 0.003 & 314.510 \\
\hline 1233 & 9.305 & 0.42240 & 317.170 & 10.6331 & 0.07180 & 0.002 & 314.509 \\
\hline 1235 & 9.306 & 0.50296 & 317.699 & 10.6159 & 0.07124 & 0.001 & 314.516 \\
\hline 1237 & 9.307 & 0.59054 & 318.260 & 10.5976 & 0.07088 & 0.001 & 314.512 \\
\hline 1239 & 7.263 & 0.17140 & 315.569 & 10.5504 & 0.07065 & 0.007 & 314.495 \\
\hline 1241 & 7.265 & 0.22363 & 315.930 & 10.5381 & 0.07036 & 0.005 & 314.508 \\
\hline 1243 & 7.267 & 0.28280 & 316.326 & 10.5246 & 0.07045 & 0.004 & 314.522 \\
\hline 1245 & 7.268 & 0.34912 & 316.762 & 10.5095 & 0.07012 & 0.002 & 314.525 \\
\hline 1247 & 7.270 & 0.42278 & 317.254 & 10.4926 & 0.07003 & 0.002 & 314.529 \\
\hline 1249 & 7.271 & 0.50365 & 317.777 & 10.4745 & 0.06969 & 0.001 & 314.525 \\
\hline 1251 & 7.273 & 0.59164 & 318.369 & 10.4538 & 0.06925 & 0.001 & 314.537 \\
\hline 1253 & 5.506 & 0.17140 & 315.685 & 10.4174 & 0.06890 & 0.007 & 314.579 \\
\hline 1255 & 5.506 & 0.22352 & 316.043 & 10.4043 & 0.06818 & 0.005 & 314.596 \\
\hline 1257 & 5.506 & 0.28251 & 316.445 & 10.3895 & 0.06840 & 0.003 & 314.601 \\
\hline 1259 & 5.507 & 0.34908 & 316.889 & 10.3730 & 0.06834 & 0.002 & 314.606 \\
\hline 1261 & 5.508 & 0.42309 & 317.379 & 10.3549 & 0.06791 & 0.002 & 314.605 \\
\hline 1263 & 5.509 & 0.50415 & 317.928 & 10.3345 & 0.06764 & 0.001 & 314.611 \\
\hline 1265 & 5.510 & 0.59224 & 318.514 & 10.3126 & 0.06745 & 0.001 & 314.608 \\
\hline 1267 & 3.431 & 0.17135 & 315.698 & 10.2440 & 0.06699 & 0.007 & 314.573 \\
\hline 1269 & 3.431 & 0.22335 & 316.063 & 10.2293 & 0.06586 & 0.004 & 314.582 \\
\hline 1271 & 3.431 & 0.28235 & 316.478 & 10.2125 & 0.06645 & 0.003 & 314.593 \\
\hline 1273 & 3.430 & 0.34906 & 316.929 & 10.1940 & 0.06606 & 0.002 & 314.588 \\
\hline 1275 & 3.431 & 0.42300 & 317.436 & 10.1733 & 0.06594 & 0.002 & 314.597 \\
\hline
\end{tabular}


Table 9. Thermal conductivity of the binary $70 \% \mathrm{R} 134 \mathrm{a} / 30 \%$ propane mixture in the compressed liquid phase obtained with wire assembly 1 (continued).

\begin{tabular}{|ccccccc|c|}
\hline $\begin{array}{c}\text { Run } \\
\text { point }\end{array}$ & $\begin{array}{c}P_{\text {exp }} \\
\mathrm{MPa}\end{array}$ & $\begin{array}{c}Q \\
\mathrm{~W} \cdot \mathrm{m}^{-1}\end{array}$ & $\begin{array}{c}T_{\text {exp }} \\
\mathrm{K}\end{array}$ & $\begin{array}{c}\rho_{\text {calc }} \\
\mathrm{mol} \cdot \mathrm{L}^{-1}\end{array}$ & $\begin{array}{c}\lambda_{\text {exp }} \\
\mathrm{W} \cdot \mathrm{m}^{-1} \cdot \mathrm{K}^{-1}\end{array}$ & STAT & $\begin{array}{c}T_{\text {cell }} \\
\mathrm{K}\end{array}$ \\
\hline 1277 & 3.430 & 0.50404 & 317.992 & 10.1504 & 0.06577 & 0.002 & 314.601 \\
1279 & 3.430 & 0.59214 & 318.596 & 10.1254 & 0.06565 & 0.002 & 314.604 \\
1281 & 1.677 & 0.17127 & 315.702 & 10.0736 & 0.06448 & 0.006 & 314.549 \\
1283 & 1.677 & 0.22319 & 316.090 & 10.0562 & 0.06419 & 0.004 & 314.571 \\
1285 & 1.677 & 0.28225 & 316.484 & 10.0385 & 0.06417 & 0.004 & 314.565 \\
1287 & 1.677 & 0.34914 & 316.959 & 10.0171 & 0.06429 & 0.002 & 314.570 \\
1289 & 1.677 & 0.42336 & 317.467 & 9.9940 & 0.06417 & 0.002 & 314.565 \\
1291 & 1.677 & 0.50443 & 318.031 & 9.9682 & 0.06419 & 0.002 & 314.563 \\
1293 & 1.677 & 0.59256 & 318.636 & 9.9402 & 0.06430 & 0.002 & 314.572 \\
\hline
\end{tabular}


Table 10. Thermal conductivity of the binary $70 \% \mathrm{R} 134 \mathrm{a} / 30 \%$ propane mixture in the compressed liquid phase obtained with wire assembly 2 .

\begin{tabular}{|c|c|c|c|c|c|c|c|}
\hline $\begin{array}{l}\text { Run } \\
\text { point }\end{array}$ & $\begin{array}{l}P_{\text {exp }} \\
\mathrm{MPa}\end{array}$ & $\begin{array}{c}Q \\
\mathrm{~W} \cdot \mathrm{m}^{-1}\end{array}$ & $\begin{array}{c}T_{\exp } \\
\mathrm{K}\end{array}$ & $\begin{array}{c}\rho_{\text {calc }} \\
\mathrm{mol} \cdot \mathrm{L}^{-1}\end{array}$ & $\begin{array}{c}\lambda_{\exp } \\
\mathrm{W} \cdot \mathrm{m}^{-1} \cdot \mathrm{K}^{-1}\end{array}$ & STAT & $\begin{array}{c}T_{\text {cell }} \\
\mathrm{K}\end{array}$ \\
\hline 2019 & 19.667 & 0.29355 & 316.257 & 11.2013 & 0.08294 & 0.003 & 314.544 \\
\hline 2021 & 19.674 & 0.36257 & 316.693 & 11.1902 & 0.08162 & 0.002 & 314.558 \\
\hline 2023 & 19.677 & 0.43884 & 317.146 & 11.1786 & 0.08094 & 0.001 & 314.554 \\
\hline 2025 & 19.682 & 0.52226 & 317.671 & 11.1651 & 0.08083 & 0.001 & 314.568 \\
\hline 2027 & 19.687 & 0.61302 & 318.210 & 11.1513 & 0.08049 & 0.001 & 314.574 \\
\hline 2029 & 16.412 & 0.17771 & 315.667 & 11.0702 & 0.07808 & 0.005 & 314.616 \\
\hline 2031 & 16.415 & 0.23195 & 316.004 & 11.0611 & 0.07871 & 0.003 & 314.624 \\
\hline 2033 & 16.418 & 0.29329 & 316.386 & 11.0508 & 0.07856 & 0.002 & 314.629 \\
\hline 2035 & 16.419 & 0.36261 & 316.822 & 11.0388 & 0.07871 & 0.002 & 314.639 \\
\hline 2037 & 16.421 & 0.43946 & 317.306 & 11.0256 & 0.07849 & 0.001 & 314.647 \\
\hline 2039 & 16.421 & 0.52333 & 317.829 & 11.0112 & 0.07844 & 0.001 & 314.652 \\
\hline 2041 & 16.422 & 0.61475 & 318.396 & 10.9955 & 0.07805 & 0.001 & 314.661 \\
\hline 2043 & 12.975 & 0.17777 & 315.727 & 10.8958 & 0.07542 & 0.005 & 314.645 \\
\hline 2045 & 12.980 & 0.23218 & 316.076 & 10.8858 & 0.07610 & 0.003 & 314.651 \\
\hline 2047 & 12.986 & 0.29361 & 316.478 & 10.8742 & 0.07627 & 0.002 & 314.668 \\
\hline 2049 & 12.987 & 0.36293 & 316.909 & 10.8616 & 0.07585 & 0.002 & 314.663 \\
\hline 2051 & 12.991 & 0.43966 & 317.410 & 10.8469 & 0.07551 & 0.001 & 314.674 \\
\hline 2053 & 12.992 & 0.52342 & 317.936 & 10.8314 & 0.07557 & 0.001 & 314.677 \\
\hline 2055 & 12.993 & 0.61461 & 318.520 & 10.8140 & 0.07554 & 0.001 & 314.682 \\
\hline 2057 & 9.463 & 0.17776 & 315.769 & 10.6921 & 0.07346 & 0.008 & 314.659 \\
\hline 2059 & 9.465 & 0.23214 & 316.133 & 10.6804 & 0.07308 & 0.003 & 314.664 \\
\hline 2061 & 9.466 & 0.29349 & 316.552 & 10.6670 & 0.07293 & 0.002 & 314.683 \\
\hline 2063 & 9.471 & 0.36258 & 317.007 & 10.6526 & 0.07275 & 0.002 & 314.684 \\
\hline 2065 & 9.474 & 0.43910 & 317.509 & 10.6365 & 0.07283 & 0.001 & 314.687 \\
\hline 2067 & 9.477 & 0.52314 & 318.051 & 10.6190 & 0.07283 & 0.001 & 314.691 \\
\hline 2069 & 9.480 & 0.61449 & 318.659 & 10.5994 & 0.07316 & 0.001 & 314.694 \\
\hline 2071 & 6.007 & 0.17769 & 315.838 & 10.4538 & 0.06996 & 0.005 & 314.677 \\
\hline 2073 & 6.010 & 0.23200 & 316.199 & 10.4410 & 0.06956 & 0.003 & 314.688 \\
\hline 2075 & 6.012 & 0.29332 & 316.639 & 10.4252 & 0.06993 & 0.002 & 314.706 \\
\hline 2077 & 6.014 & 0.36245 & 317.107 & 10.4083 & 0.06995 & 0.002 & 314.699 \\
\hline 2079 & 6.016 & 0.43899 & 317.633 & 10.3892 & 0.06983 & 0.001 & 314.714 \\
\hline 2081 & 6.017 & 0.52284 & 318.204 & 10.3684 & 0.06967 & 0.001 & 314.715 \\
\hline 2083 & 6.017 & 0.61414 & 318.821 & 10.3457 & 0.07040 & 0.002 & 314.724 \\
\hline 2085 & 12.052 & 0.13553 & 328.952 & 10.4365 & 0.07204 & 0.007 & 328.100 \\
\hline 2086 & 12.051 & 0.17390 & 329.219 & 10.4279 & 0.07091 & 0.005 & 328.095 \\
\hline 2087 & 12.050 & 0.21704 & 329.509 & 10.4186 & 0.06998 & 0.005 & 328.095 \\
\hline 2088 & 12.051 & 0.26519 & 329.840 & 10.4082 & 0.07012 & 0.003 & 328.104 \\
\hline 2089 & 12.052 & 0.31838 & 330.176 & 10.3975 & 0.07050 & 0.002 & 328.095 \\
\hline 2090 & 12.050 & 0.37648 & 330.591 & 10.3841 & 0.07083 & 0.002 & 328.111 \\
\hline 2091 & 12.049 & 0.43956 & 331.007 & 10.3707 & 0.07071 & 0.001 & 328.104 \\
\hline 2092 & 9.679 & 0.13559 & 328.962 & 10.2684 & 0.06880 & 0.007 & 328.069 \\
\hline 2094 & 9.680 & 0.17395 & 329.224 & 10.2595 & 0.06874 & 0.005 & 328.079 \\
\hline 2096 & 9.679 & 0.21698 & 329.521 & 10.2492 & 0.06859 & 0.004 & 328.085 \\
\hline
\end{tabular}


Table 10 . Thermal conductivity of the binary $70 \% \mathrm{R} 134 \mathrm{a} / 30 \%$ propane mixture in the compressed liquid phase obtained with wire assembly 2 (continued).

\begin{tabular}{|c|c|c|c|c|c|c|c|}
\hline $\begin{array}{l}\text { Run } \\
\text { point }\end{array}$ & $\begin{array}{l}P_{\text {exp }} \\
\mathrm{MPa}\end{array}$ & $\begin{array}{c}Q \\
\mathrm{~W} \cdot \mathrm{m}^{-1}\end{array}$ & $\begin{array}{c}T_{\exp } \\
\mathrm{K}\end{array}$ & $\begin{array}{c}\rho_{\text {calc }} \\
\mathrm{mol} \cdot \mathrm{L}^{-1}\end{array}$ & $\frac{\lambda_{\exp }}{\mathrm{W} \cdot \mathrm{m}^{-1} \cdot \mathrm{K}^{-1}}$ & STAT & $\begin{array}{c}T_{\text {cell }} \\
\mathrm{K}\end{array}$ \\
\hline 2098 & 9.680 & 0.26539 & 329.857 & 10.2377 & 0.06869 & 0.003 & 328.084 \\
\hline 2100 & 9.680 & 0.31869 & 330.234 & 10.2247 & 0.06824 & 0.002 & 328.087 \\
\hline 2102 & 9.680 & 0.37683 & 330.632 & 10.2109 & 0.06887 & 0.002 & 328.090 \\
\hline 2104 & 9.680 & 0.43971 & 331.077 & 10.1955 & 0.06873 & 0.001 & 328.095 \\
\hline 2106 & 7.605 & 0.13556 & 328.955 & 10.1016 & 0.06666 & 0.006 & 328.056 \\
\hline 2108 & 7.604 & 0.17402 & 329.231 & 10.0912 & 0.06683 & 0.005 & 328.066 \\
\hline 2110 & 7.606 & 0.21723 & 329.558 & 10.0792 & 0.06677 & 0.003 & 328.073 \\
\hline 2112 & 7.607 & 0.26552 & 329.887 & 10.0669 & 0.06607 & 0.002 & 328.076 \\
\hline 2114 & 7.607 & 0.31874 & 330.266 & 10.0527 & 0.06648 & 0.002 & 328.082 \\
\hline 2116 & 7.607 & 0.37692 & 330.692 & 10.0367 & 0.06658 & 0.001 & 328.087 \\
\hline 2118 & 7.607 & 0.43966 & 331.125 & 10.0204 & 0.06642 & 0.002 & 328.083 \\
\hline 2120 & 5.497 & 0.13557 & 328.974 & 9.9036 & 0.06555 & 0.006 & 328.052 \\
\hline 2122 & 5.498 & 0.15888 & 329.151 & 9.8965 & 0.06486 & 0.005 & 328.058 \\
\hline 2124 & 5.500 & 0.18402 & 329.341 & 9.8888 & 0.06407 & 0.004 & 328.061 \\
\hline 2126 & 5.501 & 0.21143 & 329.550 & 9.8803 & 0.06425 & 0.003 & 328.065 \\
\hline 2128 & 5.502 & 0.24062 & 329.748 & 9.8721 & 0.06409 & 0.003 & 328.066 \\
\hline 2130 & 5.503 & 0.27177 & 329.985 & 9.8623 & 0.06461 & 0.002 & 328.072 \\
\hline 2132 & 5.504 & 0.30482 & 330.217 & 9.8527 & 0.06430 & 0.002 & 328.068 \\
\hline 2134 & 4.009 & 0.13558 & 328.981 & 9.7404 & 0.06282 & 0.006 & 328.037 \\
\hline 2136 & 4.010 & 0.15890 & 329.171 & 9.7319 & 0.06282 & 0.005 & 328.050 \\
\hline 2138 & 4.010 & 0.18397 & 329.349 & 9.7239 & 0.06250 & 0.004 & 328.047 \\
\hline 2140 & 4.013 & 0.21125 & 329.571 & 9.7141 & 0.06247 & 0.003 & 328.064 \\
\hline 2142 & 4.014 & 0.24066 & 329.789 & 9.7043 & 0.06264 & 0.003 & 328.065 \\
\hline 2144 & 4.016 & 0.27183 & 330.016 & 9.6940 & 0.06313 & 0.002 & 328.065 \\
\hline 2146 & 4.017 & 0.30497 & 330.258 & 9.6830 & 0.06271 & 0.002 & 328.067 \\
\hline 2148 & 2.458 & 0.13556 & 328.994 & 9.5384 & 0.06237 & 0.006 & 328.034 \\
\hline 2152 & 2.459 & 0.18404 & 329.380 & 9.5184 & 0.06224 & 0.005 & 328.054 \\
\hline 2154 & 2.459 & 0.21132 & 329.590 & 9.5076 & 0.06141 & 0.003 & 328.052 \\
\hline 2156 & 2.461 & 0.24079 & 329.812 & 9.4963 & 0.06254 & 0.005 & 328.056 \\
\hline 2158 & 2.462 & 0.27196 & 330.052 & 9.4840 & 0.06186 & 0.003 & 328.061 \\
\hline 2160 & 2.464 & 0.30498 & 330.301 & 9.4711 & 0.06235 & 0.003 & 328.063 \\
\hline 2162 & 7.836 & 0.14146 & 345.821 & 9.4567 & 0.06206 & 0.006 & 344.816 \\
\hline 2164 & 7.837 & 0.16569 & 346.017 & 9.4484 & 0.06164 & 0.005 & 344.827 \\
\hline 2166 & 7.837 & 0.19218 & 346.228 & 9.4394 & 0.06174 & 0.004 & 344.836 \\
\hline 2168 & 7.837 & 0.22075 & 346.443 & 9.4304 & 0.06177 & 0.003 & 344.835 \\
\hline 2170 & 7.837 & 0.25130 & 346.672 & 9.4206 & 0.06166 & 0.003 & 344.833 \\
\hline 2172 & 7.839 & 0.28376 & 346.930 & 9.4097 & 0.06156 & 0.002 & 344.845 \\
\hline 2174 & 7.841 & 0.31815 & 347.185 & 9.3990 & 0.06181 & 0.002 & 344.845 \\
\hline 2176 & 6.450 & 0.14179 & 345.858 & 9.2761 & 0.06006 & 0.006 & 344.824 \\
\hline 2178 & 6.450 & 0.16618 & 346.047 & 9.2672 & 0.06078 & 0.005 & 344.836 \\
\hline 2180 & 6.451 & 0.19249 & 346.272 & 9.2567 & 0.05994 & 0.004 & 344.850 \\
\hline 2182 & 6.451 & 0.22081 & 346.504 & 9.2459 & 0.06007 & 0.004 & 344.853 \\
\hline 2184 & 6.452 & 0.25148 & 346.728 & 9.2353 & 0.05966 & 0.003 & 344.846 \\
\hline
\end{tabular}


Table 10. Thermal conductivity of the binary $70 \% \mathrm{R} 134 \mathrm{a} / 30 \%$ propane mixture in the compressed liquid phase obtained with wire assembly 2 (continued).

\begin{tabular}{|ccccccc|c|}
\hline $\begin{array}{c}\text { Run } \\
\text { point }\end{array}$ & $\begin{array}{c}P_{\text {exp }} \\
\mathrm{MPa}\end{array}$ & $\begin{array}{c}Q \\
\mathrm{~W} \cdot \mathrm{m}^{-1}\end{array}$ & $\begin{array}{c}T_{\text {exp }} \\
\mathrm{K}\end{array}$ & $\begin{array}{c}\rho_{\text {calc }} \\
\mathrm{mol} \cdot \mathrm{L}^{-1}\end{array}$ & $\begin{array}{c}\lambda_{\text {exp }} \\
\mathrm{W} \cdot \mathrm{m}^{-1} \cdot \mathrm{K}^{-1}\end{array}$ & STAT & $\begin{array}{c}T_{\text {cell }} \\
\mathrm{K}\end{array}$ \\
\hline 2186 & 6.453 & 0.28412 & 346.983 & 9.2234 & 0.06010 & 0.002 & 344.854 \\
2188 & 6.453 & 0.31858 & 347.261 & 9.2102 & 0.06015 & 0.002 & 344.856 \\
2190 & 5.044 & 0.14180 & 345.923 & 9.0554 & 0.05821 & 0.006 & 344.860 \\
2192 & 5.045 & 0.16604 & 346.129 & 9.0446 & 0.05769 & 0.006 & 344.872 \\
2196 & 5.047 & 0.22096 & 346.576 & 9.0208 & 0.05768 & 0.003 & 344.889 \\
2198 & 5.048 & 0.25165 & 346.813 & 9.0082 & 0.05805 & 0.003 & 344.886 \\
2200 & 5.049 & 0.28423 & 347.074 & 8.9941 & 0.05824 & 0.003 & 344.890 \\
2202 & 5.050 & 0.31895 & 347.348 & 8.9792 & 0.05842 & 0.003 & 344.892 \\
2204 & 4.100 & 0.14179 & 345.929 & 8.8776 & 0.05748 & 0.006 & 344.855 \\
2206 & 4.100 & 0.16611 & 346.155 & 8.8640 & 0.05699 & 0.005 & 344.878 \\
2208 & 4.102 & 0.19254 & 346.360 & 8.8519 & 0.05675 & 0.004 & 344.880 \\
2210 & 4.103 & 0.22114 & 346.593 & 8.8378 & 0.05689 & 0.003 & 344.887 \\
2212 & 4.104 & 0.25177 & 346.839 & 8.8228 & 0.05724 & 0.004 & 344.888 \\
2214 & 4.104 & 0.28431 & 347.096 & 8.8071 & 0.05766 & 0.003 & 344.888 \\
2216 & 4.105 & 0.31879 & 347.371 & 8.7901 & 0.05799 & 0.004 & 344.886 \\
2218 & 3.275 & 0.14174 & 345.934 & 8.6887 & 0.05608 & 0.006 & 344.858 \\
2220 & 3.276 & 0.16604 & 346.133 & 8.6749 & 0.05664 & 0.005 & 344.873 \\
2222 & 3.276 & 0.19248 & 346.351 & 8.6596 & 0.05649 & 0.004 & 344.880 \\
2224 & 3.276 & 0.22099 & 346.583 & 8.6432 & 0.05715 & 0.005 & 344.884 \\
2226 & 3.277 & 0.25171 & 346.827 & 8.6259 & 0.05788 & 0.005 & 344.891 \\
2228 & 3.278 & 0.28418 & 347.079 & 8.6078 & 0.05892 & 0.006 & 344.889 \\
2230 & 3.279 & 0.31859 & 347.340 & 8.5890 & 0.06021 & 0.008 & 344.886 \\
\hline
\end{tabular}


Table 11. Thermal conductivity of the ternary $33 \% \mathrm{R} 32$ / $33 \% \mathrm{R} 125$ / $33 \% \mathrm{R} 134 \mathrm{a}$ mixture in the compressed liquid phase.

\begin{tabular}{|c|c|c|c|c|c|c|c|}
\hline $\begin{array}{l}\text { Run } \\
\text { point }\end{array}$ & $\begin{array}{l}P_{\text {exp }} \\
\mathrm{MPa} \\
\end{array}$ & $\begin{array}{c}Q \\
\mathrm{~W} \cdot \mathrm{m}^{-1}\end{array}$ & $\begin{array}{c}T_{\exp } \\
\mathrm{K} \\
\end{array}$ & $\begin{array}{c}\rho_{\text {calc }} \\
\mathrm{mol} \cdot \mathrm{L}^{-1} \\
\end{array}$ & $\begin{array}{c}\lambda_{\exp } \\
\mathrm{W} \cdot \mathrm{m}^{-1} \cdot \mathrm{K}^{-1} \\
\end{array}$ & STAT & $\begin{array}{c}T_{\text {cell }} \\
\mathrm{K} \\
\end{array}$ \\
\hline 1003 & 0.449 & 0.17952 & 249.522 & 14.6630 & 0.09745 & 0.008 & 248.667 \\
\hline 1005 & 0.448 & 0.22696 & 249.751 & 14.6543 & 0.09766 & 0.006 & 248.667 \\
\hline 1007 & 0.447 & 0.28023 & 250.010 & 14.6444 & 0.09622 & 0.004 & 248.663 \\
\hline 1009 & 0.446 & 0.33957 & 250.295 & 14.6336 & 0.09697 & 0.003 & 248.657 \\
\hline 1011 & 0.445 & 0.40445 & 250.610 & 14.6216 & 0.09627 & 0.002 & 248.644 \\
\hline 1013 & 0.446 & 0.47533 & 250.949 & 14.6088 & 0.09582 & 0.002 & 248.646 \\
\hline 1017 & 0.449 & 0.17955 & 249.425 & 14.6666 & 0.09646 & 0.008 & 248.574 \\
\hline 1019 & 0.449 & 0.22708 & 249.654 & 14.6580 & 0.09754 & 0.005 & 248.573 \\
\hline 1021 & 0.448 & 0.28047 & 249.923 & 14.6478 & 0.09637 & 0.004 & 248.571 \\
\hline 1023 & 0.446 & 0.33979 & 250.205 & 14.6370 & 0.09666 & 0.003 & 248.569 \\
\hline 1025 & 0.445 & 0.40473 & 250.524 & 14.6249 & 0.09593 & 0.002 & 248.567 \\
\hline 1027 & 0.445 & 0.47545 & 250.868 & 14.6118 & 0.09594 & 0.002 & 248.561 \\
\hline 1031 & 1.163 & 0.18516 & 257.569 & 14.3830 & 0.09384 & 0.007 & 256.667 \\
\hline 1033 & 1.149 & 0.23429 & 257.821 & 14.3726 & 0.09382 & 0.005 & 256.667 \\
\hline 1035 & 1.171 & 0.28932 & 258.121 & 14.3619 & 0.09394 & 0.004 & 256.676 \\
\hline 1037 & 1.182 & 0.35024 & 258.411 & 14.3510 & 0.09313 & 0.003 & 256.672 \\
\hline 1039 & 1.193 & 0.41708 & 258.751 & 14.3382 & 0.09336 & 0.003 & 256.673 \\
\hline 1041 & 1.202 & 0.48992 & 259.127 & 14.3239 & 0.09282 & 0.002 & 256.673 \\
\hline 1045 & 1.156 & 0.18485 & 257.573 & 14.3826 & 0.09355 & 0.008 & 256.667 \\
\hline 1047 & 1.156 & 0.23407 & 257.835 & 14.3724 & 0.09376 & 0.005 & 256.676 \\
\hline 1049 & 1.177 & 0.28910 & 258.108 & 14.3626 & 0.09370 & 0.004 & 256.676 \\
\hline 1051 & 1.189 & 0.35013 & 258.415 & 14.3512 & 0.09301 & 0.003 & 256.678 \\
\hline 1053 & 1.198 & 0.41712 & 258.757 & 14.3382 & 0.09317 & 0.002 & 256.670 \\
\hline 1055 & 1.203 & 0.49007 & 259.119 & 14.3243 & 0.09254 & 0.002 & 256.675 \\
\hline 1059 & 4.656 & 0.19364 & 270.472 & 14.0342 & 0.09018 & 0.007 & 269.488 \\
\hline 1061 & 4.664 & 0.24505 & 270.753 & 14.0236 & 0.08990 & 0.005 & 269.497 \\
\hline 1063 & 4.590 & 0.30270 & 271.061 & 14.0082 & 0.08945 & 0.004 & 269.497 \\
\hline 1065 & 4.561 & 0.36656 & 271.389 & 13.9939 & 0.08980 & 0.003 & 269.501 \\
\hline 1067 & 4.539 & 0.43665 & 271.763 & 13.9782 & 0.08904 & 0.003 & 269.497 \\
\hline 1069 & 4.529 & 0.51308 & 272.162 & 13.9620 & 0.08912 & 0.002 & 269.493 \\
\hline 1073 & 2.911 & 0.19386 & 270.478 & 13.9512 & 0.08998 & 0.007 & 269.481 \\
\hline 1075 & 2.947 & 0.24515 & 270.752 & 13.9419 & 0.08851 & 0.005 & 269.476 \\
\hline 1077 & 2.991 & 0.30268 & 271.060 & 13.9316 & 0.08852 & 0.003 & 269.483 \\
\hline 1079 & 3.009 & 0.36664 & 271.400 & 13.9188 & 0.08861 & 0.003 & 269.477 \\
\hline 1081 & 3.024 & 0.43675 & 271.766 & 13.9047 & 0.08860 & 0.002 & 269.481 \\
\hline 1083 & 2.984 & 0.51307 & 272.172 & 13.8862 & 0.08848 & 0.002 & 269.485 \\
\hline 1087 & 1.754 & 0.19372 & 270.469 & 13.8939 & 0.08836 & 0.007 & 269.473 \\
\hline 1089 & 1.681 & 0.24519 & 270.751 & 13.8785 & 0.08853 & 0.005 & 269.472 \\
\hline 1091 & 1.656 & 0.30292 & 271.053 & 13.8646 & 0.08839 & 0.004 & 269.471 \\
\hline 1093 & 1.636 & 0.36687 & 271.404 & 13.8490 & 0.08758 & 0.002 & 269.472 \\
\hline 1095 & 1.639 & 0.43704 & 271.777 & 13.8336 & 0.08724 & 0.002 & 269.472 \\
\hline 1097 & 1.704 & 0.51325 & 272.199 & 13.8193 & 0.08686 & 0.001 & 269.477 \\
\hline 1101 & 12.172 & 0.20325 & 284.840 & 13.8377 & 0.08866 & 0.006 & 283.787 \\
\hline
\end{tabular}


Table 11. Thermal conductivity of the ternary $33 \% \mathrm{R} 32$ / $33 \% \mathrm{R} 125$ / 33 \% R134a mixture in the compressed liquid phase (continued).

\begin{tabular}{|c|c|c|c|c|c|c|c|}
\hline $\begin{array}{l}\text { Run } \\
\text { point }\end{array}$ & $\begin{array}{l}P_{\text {exp }} \\
\mathrm{MPa}\end{array}$ & $\begin{array}{c}Q \\
\mathrm{~W} \cdot \mathrm{m}^{-1}\end{array}$ & $\begin{array}{c}T_{\exp } \\
\mathrm{K}\end{array}$ & $\begin{array}{c}\rho_{\text {calc }} \\
\mathrm{mol} \cdot \mathrm{L}^{-1}\end{array}$ & $\begin{array}{c}\lambda_{\exp } \\
\mathrm{W} \cdot \mathrm{m}^{-1} \cdot \mathrm{K}^{-1}\end{array}$ & STAT & $\begin{array}{c}T_{\text {cell }} \\
\mathrm{K}\end{array}$ \\
\hline 1103 & 12.196 & 0.25741 & 285.123 & 13.8285 & 0.08876 & 0.005 & 283.784 \\
\hline 1105 & 12.241 & 0.31793 & 285.451 & 13.8186 & 0.08892 & 0.003 & 283.790 \\
\hline 1107 & 12.252 & 0.38492 & 285.799 & 13.8065 & 0.08934 & 0.002 & 283.784 \\
\hline 1109 & 12.265 & 0.45832 & 286.209 & 13.7922 & 0.08842 & 0.002 & 283.792 \\
\hline 1111 & 12.263 & 0.53840 & 286.622 & 13.7770 & 0.08812 & 0.002 & 283.791 \\
\hline 1115 & 9.753 & 0.20347 & 284.831 & 13.7243 & 0.08852 & 0.007 & 283.767 \\
\hline 1117 & 9.742 & 0.25724 & 285.142 & 13.7119 & 0.08776 & 0.005 & 283.776 \\
\hline 1119 & 9.739 & 0.31798 & 285.457 & 13.6998 & 0.08769 & 0.003 & 283.775 \\
\hline 1121 & 9.770 & 0.38500 & 285.822 & 13.6875 & 0.08688 & 0.002 & 283.765 \\
\hline 1123 & 9.800 & 0.45863 & 286.222 & 13.6738 & 0.08625 & 0.002 & 283.774 \\
\hline 1125 & 9.818 & 0.53873 & 286.651 & 13.6583 & 0.08673 & 0.001 & 283.769 \\
\hline 1129 & 5.499 & 0.20322 & 284.835 & 13.5005 & 0.08489 & 0.006 & 283.738 \\
\hline 1131 & 5.522 & 0.25711 & 285.146 & 13.4888 & 0.08425 & 0.004 & 283.743 \\
\hline 1133 & 5.535 & 0.31789 & 285.477 & 13.4759 & 0.08451 & 0.003 & 283.749 \\
\hline 1135 & 5.520 & 0.38506 & 285.840 & 13.4598 & 0.08419 & 0.002 & 283.748 \\
\hline 1137 & 5.468 & 0.45896 & 286.275 & 13.4387 & 0.08374 & 0.002 & 283.751 \\
\hline 1139 & 5.456 & 0.53922 & 286.724 & 13.4191 & 0.08323 & 0.002 & 283.755 \\
\hline 1143 & 3.564 & 0.20341 & 284.840 & 13.3857 & 0.08367 & 0.006 & 283.730 \\
\hline 1145 & 3.552 & 0.25745 & 285.151 & 13.3713 & 0.08296 & 0.004 & 283.737 \\
\hline 1147 & 3.574 & 0.31811 & 285.508 & 13.3571 & 0.08243 & 0.003 & 283.742 \\
\hline 1149 & 3.616 & 0.38525 & 285.872 & 13.3437 & 0.08293 & 0.002 & 283.743 \\
\hline 1151 & 3.635 & 0.45867 & 286.293 & 13.3265 & 0.08172 & 0.002 & 283.742 \\
\hline 1153 & 3.646 & 0.53874 & 286.749 & 13.3071 & 0.08169 & 0.001 & 283.747 \\
\hline 1157 & 1.563 & 0.20343 & 284.848 & 13.2555 & 0.08285 & 0.006 & 283.730 \\
\hline 1159 & 1.574 & 0.25734 & 285.170 & 13.2414 & 0.08148 & 0.004 & 283.739 \\
\hline 1161 & 1.546 & 0.31809 & 285.511 & 13.2236 & 0.08083 & 0.003 & 283.738 \\
\hline 1163 & 1.506 & 0.38553 & 285.912 & 13.2021 & 0.08099 & 0.002 & 283.742 \\
\hline 1165 & 1.490 & 0.45940 & 286.349 & 13.1805 & 0.08061 & 0.002 & 283.748 \\
\hline 1167 & 1.486 & 0.53977 & 286.807 & 13.1586 & 0.08007 & 0.002 & 283.745 \\
\hline 1171 & 10.775 & 0.21322 & 299.568 & 13.2076 & 0.08288 & 0.006 & 298.384 \\
\hline 1173 & 10.786 & 0.26973 & 299.887 & 13.1955 & 0.08150 & 0.004 & 298.381 \\
\hline 1175 & 10.825 & 0.33312 & 300.249 & 13.1834 & 0.08206 & 0.003 & 298.389 \\
\hline 1177 & 10.839 & 0.40341 & 300.659 & 13.1679 & 0.08173 & 0.002 & 298.398 \\
\hline 1179 & 10.848 & 0.48041 & 301.096 & 13.1510 & 0.08127 & 0.002 & 298.393 \\
\hline 1181 & 10.815 & 0.56436 & 301.573 & 13.1299 & 0.08121 & 0.001 & 298.390 \\
\hline 1185 & 8.318 & 0.21313 & 299.564 & 13.0555 & 0.08105 & 0.006 & 298.363 \\
\hline 1187 & 8.292 & 0.26973 & 299.887 & 13.0402 & 0.08020 & 0.004 & 298.365 \\
\hline 1189 & 8.259 & 0.33334 & 300.273 & 13.0216 & 0.07961 & 0.003 & 298.374 \\
\hline 1191 & 8.247 & 0.40382 & 300.681 & 13.0034 & 0.07971 & 0.002 & 298.376 \\
\hline 1193 & 8.260 & 0.48103 & 301.130 & 12.9851 & 0.07929 & 0.002 & 298.370 \\
\hline 1195 & 8.296 & 0.56479 & 301.624 & 12.9664 & 0.07883 & 0.001 & 298.372 \\
\hline 1197 & 5.610 & 0.16336 & 299.267 & 12.8801 & 0.07829 & 0.009 & 298.336 \\
\hline 1199 & 5.611 & 0.21327 & 299.578 & 12.8659 & 0.07797 & 0.005 & 298.346 \\
\hline
\end{tabular}


Table 11. Thermal conductivity of the ternary $33 \% \mathrm{R} 32$ / $33 \% \mathrm{R} 125$ / $33 \% \mathrm{R} 134 \mathrm{a}$ mixture in the compressed liquid phase (continued).

\begin{tabular}{|c|c|c|c|c|c|c|c|}
\hline $\begin{array}{l}\text { Run } \\
\text { point }\end{array}$ & $\begin{array}{l}P_{e x p} \\
\mathrm{MPa}\end{array}$ & $\begin{array}{c}Q \\
\mathrm{~W} \cdot \mathrm{m}^{-1}\end{array}$ & $\begin{array}{c}T_{\exp } \\
\mathrm{K}\end{array}$ & $\begin{array}{c}\rho_{\text {calc }} \\
\mathrm{mol}^{\prime} \mathrm{L}^{-1}\end{array}$ & $\begin{array}{c}\lambda_{\exp } \\
\mathrm{W} \cdot \mathrm{m}^{-1} \cdot \mathrm{K}^{-1}\end{array}$ & STAT & $\begin{array}{c}T_{\text {cell }} \\
\mathrm{K}\end{array}$ \\
\hline 1201 & 5.654 & 0.26975 & 299.905 & 12.8542 & 0.07986 & 0.004 & 298.354 \\
\hline 1203 & 5.673 & 0.33313 & 300.289 & 12.8379 & 0.07791 & 0.003 & 298.350 \\
\hline 1205 & 5.683 & 0.40355 & 300.720 & 12.8187 & 0.07755 & 0.002 & 298.359 \\
\hline 1207 & 5.638 & 0.48100 & 301.172 & 12.7943 & 0.07739 & 0.002 & 298.359 \\
\hline 1209 & 5.620 & 0.56535 & 301.681 & 12.7693 & 0.07743 & 0.001 & 298.361 \\
\hline 1211 & 3.341 & 0.16331 & 299.288 & 12.6984 & 0.07667 & 0.008 & 298.329 \\
\hline 1213 & 3.335 & 0.21319 & 299.592 & 12.6827 & 0.07633 & 0.006 & 298.338 \\
\hline 1215 & 3.374 & 0.26970 & 299.925 & 12.6694 & 0.07985 & 0.004 & 298.338 \\
\hline 1217 & 3.390 & 0.33313 & 300.320 & 12.6510 & 0.07673 & 0.003 & 298.346 \\
\hline 1219 & 3.403 & 0.40363 & 300.759 & 12.6300 & 0.07579 & 0.002 & 298.349 \\
\hline 1221 & 3.390 & 0.48084 & 301.208 & 12.6061 & 0.07621 & 0.002 & 298.349 \\
\hline 1223 & 3.355 & 0.56516 & 301.729 & 12.5764 & 0.07586 & 0.001 & 298.354 \\
\hline 1225 & 1.433 & 0.16330 & 299.287 & 12.5234 & 0.07547 & 0.008 & 298.323 \\
\hline 1227 & 1.397 & 0.21322 & 299.611 & 12.5022 & 0.07690 & 0.009 & 298.335 \\
\hline 1229 & 1.386 & 0.26988 & 299.959 & 12.4819 & 0.07555 & 0.004 & 298.336 \\
\hline 1231 & 1.418 & 0.33329 & 300.348 & 12.4636 & 0.07746 & 0.003 & 298.341 \\
\hline 1233 & 1.439 & 0.40350 & 300.771 & 12.4424 & 0.07748 & 0.003 & 298.342 \\
\hline 1235 & 1.447 & 0.48077 & 301.266 & 12.4156 & 0.07387 & 0.002 & 298.347 \\
\hline 1237 & 1.442 & 0.56487 & 301.789 & 12.3856 & 0.07348 & 0.001 & 298.348 \\
\hline 1239 & 9.378 & 0.17058 & 314.510 & 12.4802 & 0.07702 & 0.008 & 313.494 \\
\hline 1241 & 9.381 & 0.22273 & 314.839 & 12.4655 & 0.07506 & 0.005 & 313.501 \\
\hline 1243 & 9.386 & 0.28207 & 315.204 & 12.4493 & 0.07512 & 0.004 & 313.496 \\
\hline 1245 & 9.390 & 0.34858 & 315.619 & 12.4308 & 0.07464 & 0.003 & 313.503 \\
\hline 1247 & 9.392 & 0.42212 & 316.071 & 12.4104 & 0.07430 & 0.002 & 313.502 \\
\hline 1249 & 9.389 & 0.50290 & 316.570 & 12.3873 & 0.07395 & 0.001 & 313.506 \\
\hline 1251 & 9.389 & 0.59091 & 317.121 & 12.3620 & 0.07347 & 0.001 & 313.507 \\
\hline 1253 & 7.545 & 0.17091 & 314.511 & 12.3222 & 0.07369 & 0.007 & 313.470 \\
\hline 1255 & 7.554 & 0.22308 & 314.846 & 12.3067 & 0.07360 & 0.005 & 313.485 \\
\hline 1257 & 7.557 & 0.28219 & 315.221 & 12.2888 & 0.07391 & 0.003 & 313.491 \\
\hline 1259 & 7.558 & 0.34833 & 315.636 & 12.2687 & 0.07329 & 0.003 & 313.493 \\
\hline 1261 & 7.539 & 0.42214 & 316.092 & 12.2446 & 0.07399 & 0.002 & 313.493 \\
\hline 1263 & 7.534 & 0.50324 & 316.602 & 12.2190 & 0.07269 & 0.002 & 313.489 \\
\hline 1265 & 7.531 & 0.59146 & 317.180 & 12.1901 & 0.07205 & 0.001 & 313.497 \\
\hline 1267 & 5.504 & 0.17089 & 314.507 & 12.1215 & 0.07457 & 0.007 & 313.454 \\
\hline 1269 & 5.511 & 0.22293 & 314.850 & 12.1040 & 0.07209 & 0.005 & 313.469 \\
\hline 1271 & 5.515 & 0.28191 & 315.228 & 12.0841 & 0.07188 & 0.003 & 313.468 \\
\hline 1273 & 5.514 & 0.34832 & 315.655 & 12.0610 & 0.07143 & 0.002 & 313.470 \\
\hline 1275 & 5.496 & 0.42226 & 316.138 & 12.0330 & 0.07212 & 0.002 & 313.476 \\
\hline 1277 & 5.491 & 0.50334 & 316.661 & 12.0040 & 0.07135 & 0.001 & 313.482 \\
\hline 1279 & 5.493 & 0.59160 & 317.228 & 11.9731 & 0.07074 & 0.001 & 313.476 \\
\hline 1281 & 3.427 & 0.17091 & 314.513 & 11.8771 & 0.07000 & 0.008 & 313.425 \\
\hline 1283 & 3.441 & 0.22289 & 314.848 & 11.8587 & 0.07263 & 0.005 & 313.436 \\
\hline 1285 & 3.448 & 0.28195 & 315.251 & 11.8352 & 0.06974 & 0.003 & 313.446 \\
\hline
\end{tabular}


Table 11. Thermal conductivity of the ternary $33 \% \mathrm{R} 32$ / $33 \% \mathrm{R} 125$ / $33 \%$ R134a mixture in the compressed liquid phase (continued).

\begin{tabular}{|c|c|c|c|c|c|c|c|}
\hline $\begin{array}{l}\text { Run } \\
\text { point }\end{array}$ & $\begin{array}{l}P_{\text {exp }} \\
\mathrm{MPa}\end{array}$ & $\begin{array}{c}Q \\
W \cdot \mathrm{m}^{-1}\end{array}$ & $\begin{array}{c}T_{\exp } \\
\mathrm{K}\end{array}$ & $\begin{array}{c}\rho_{\text {calc }} \\
\mathrm{mol} \cdot \mathrm{L}^{-1}\end{array}$ & $\begin{array}{c}\lambda_{\exp } \\
\mathrm{W} \cdot \mathrm{m}^{-1} \cdot \mathrm{K}^{-1}\end{array}$ & STAT & $\begin{array}{c}T_{\text {cell }} \\
\mathrm{K}\end{array}$ \\
\hline 1287 & 3.451 & 0.34859 & 315.679 & 11.8093 & 0.06865 & 0.002 & 313.444 \\
\hline 1289 & 3.453 & 0.42239 & 316.171 & 11.7793 & 0.06844 & 0.002 & 313.442 \\
\hline 1291 & 3.455 & 0.50321 & 316.698 & 11.7467 & 0.06797 & 0.002 & 313.444 \\
\hline 1293 & 3.434 & 0.59157 & 317.260 & 11.7085 & 0.06952 & 0.002 & 313.442 \\
\hline 1295 & 2.160 & 0.17088 & 314.495 & 11.6997 & 0.06810 & 0.007 & 313.392 \\
\hline 1297 & 2.156 & 0.22293 & 314.854 & 11.6751 & 0.06737 & 0.004 & 313.416 \\
\hline 1299 & 2.156 & 0.28205 & 315.260 & 11.6475 & 0.06719 & 0.003 & 313.421 \\
\hline 1301 & 2.170 & 0.34865 & 315.680 & 11.6211 & 0.06745 & 0.003 & 313.417 \\
\hline 1303 & 2.174 & 0.42242 & 316.177 & 11.5874 & 0.06674 & 0.002 & 313.414 \\
\hline 1305 & 2.176 & 0.50352 & 316.718 & 11.5501 & 0.06637 & 0.001 & 313.414 \\
\hline 1307 & 2.159 & 0.59185 & 317.311 & 11.5052 & 0.06642 & 0.001 & 313.421 \\
\hline 1309 & 8.511 & 0.17824 & 329.204 & 11.6783 & 0.06974 & 0.007 & 328.059 \\
\hline 1311 & 8.514 & 0.23240 & 329.572 & 11.6592 & 0.06873 & 0.005 & 328.069 \\
\hline 1313 & 8.506 & 0.29428 & 329.992 & 11.6357 & 0.06874 & 0.003 & 328.075 \\
\hline 1315 & 8.489 & 0.36400 & 330.464 & 11.6083 & 0.07032 & 0.006 & 328.076 \\
\hline 1317 & 8.483 & 0.44097 & 330.974 & 11.5800 & 0.06754 & 0.002 & 328.081 \\
\hline 1319 & 8.496 & 0.52521 & 331.500 & 11.5530 & 0.07031 & 0.002 & 328.074 \\
\hline 1321 & 8.504 & 0.61700 & 332.130 & 11.5197 & 0.06832 & 0.001 & 328.081 \\
\hline 1323 & 6.874 & 0.17828 & 329.188 & 11.4687 & 0.06941 & 0.007 & 328.023 \\
\hline 1325 & 6.877 & 0.23253 & 329.575 & 11.4463 & 0.06817 & 0.005 & 328.046 \\
\hline 1327 & 6.878 & 0.29410 & 329.995 & 11.4216 & 0.06866 & 0.003 & 328.053 \\
\hline 1329 & 6.876 & 0.36359 & 330.469 & 11.3932 & 0.06701 & 0.002 & 328.051 \\
\hline 1331 & 6.875 & 0.44056 & 330.997 & 11.3613 & 0.06618 & 0.002 & 328.057 \\
\hline 1333 & 6.874 & 0.52498 & 331.560 & 11.3272 & 0.06746 & 0.002 & 328.059 \\
\hline 1335 & 6.873 & 0.61677 & 332.178 & 11.2894 & 0.06747 & 0.002 & 328.057 \\
\hline 1337 & 5.351 & 0.17826 & 329.201 & 11.2340 & 0.06939 & 0.007 & 328.013 \\
\hline 1339 & 5.353 & 0.23250 & 329.568 & 11.2100 & 0.07115 & 0.005 & 328.027 \\
\hline 1341 & 5.355 & 0.29412 & 330.000 & 11.1813 & 0.06904 & 0.004 & 328.036 \\
\hline 1343 & 5.355 & 0.36352 & 330.475 & 11.1493 & 0.06925 & 0.003 & 328.031 \\
\hline 1349 & 5.354 & 0.61693 & 332.186 & 11.0310 & 0.06996 & 0.003 & 328.035 \\
\hline 1355 & 5.338 & 0.17760 & 329.222 & 11.2303 & 0.07202 & 0.008 & 328.049 \\
\hline 1359 & 5.355 & 0.23234 & 329.587 & 11.2090 & 0.07238 & 0.006 & 328.049 \\
\hline 1361 & 5.359 & 0.26243 & 329.811 & 11.1947 & 0.06921 & 0.004 & 328.055 \\
\hline 1363 & 5.361 & 0.29445 & 330.033 & 11.1802 & 0.06928 & 0.004 & 328.055 \\
\hline 1365 & 4.107 & 0.13083 & 328.879 & 11.0246 & 0.07072 & 0.011 & 328.015 \\
\hline 1369 & 4.109 & 0.17760 & 329.234 & 10.9984 & 0.06846 & 0.007 & 328.042 \\
\hline 1375 & 4.095 & 0.26261 & 329.814 & 10.9509 & 0.06858 & 0.005 & 328.034 \\
\hline 1377 & 4.101 & 0.29456 & 330.031 & 10.9353 & 0.07158 & 0.005 & 328.042 \\
\hline 1381 & 2.968 & 0.15334 & 329.066 & 10.7412 & 0.06776 & 0.009 & 328.032 \\
\hline 1385 & 2.965 & 0.20426 & 329.437 & 10.7066 & 0.06764 & 0.006 & 328.037 \\
\hline 1389 & 2.976 & 0.26261 & 329.829 & 10.6733 & 0.07198 & 0.007 & 328.035 \\
\hline 1393 & 7.590 & 0.13584 & 342.720 & 10.7362 & 0.06170 & 0.015 & 341.791 \\
\hline 1395 & 7.581 & 0.15931 & 342.907 & 10.7218 & 0.06176 & 0.012 & 341.794 \\
\hline
\end{tabular}


Table 11. Thermal conductivity of the ternary $33 \%$ R32 / $33 \%$ R125 / $33 \%$ R134a mixture in the compressed liquid phase (continued).

\begin{tabular}{|ccccccc|c|}
\hline $\begin{array}{c}\text { Run } \\
\text { point }\end{array}$ & $\begin{array}{c}P_{\text {exp }} \\
\mathrm{MPa}\end{array}$ & $\begin{array}{c}Q \\
\mathrm{~W} \cdot \mathrm{m}^{-1}\end{array}$ & $\begin{array}{c}T_{\text {exp }} \\
\mathrm{K}\end{array}$ & $\begin{array}{c}\rho_{\text {calc }} \\
\mathrm{mol} \cdot \mathrm{L}^{-1}\end{array}$ & $\begin{array}{c}\lambda_{\text {exp }} \\
\mathrm{W} \cdot \mathrm{m}^{-1} \cdot \mathrm{K}^{-1}\end{array}$ & STAT & $\begin{array}{c}T_{\text {cell }} \\
\mathrm{K}\end{array}$ \\
\hline 1397 & 7.576 & 0.18474 & 343.097 & 10.7080 & 0.06224 & 0.012 & 341.797 \\
1401 & 7.586 & 0.24128 & 343.512 & 10.6816 & 0.06431 & 0.016 & 341.805 \\
1403 & 7.589 & 0.27259 & 343.741 & 10.6664 & 0.06363 & 0.009 & 341.806 \\
\hline
\end{tabular}


Table 12. Thermal conductivity of the ternary $30 \% \mathrm{R} 32$ / $10 \% \mathrm{R} 125$ / $60 \% \mathrm{R} 134 \mathrm{a}$ mixture in the compressed liquid phase.

\begin{tabular}{|c|c|c|c|c|c|c|c|}
\hline $\begin{array}{l}\text { Run } \\
\text { point }\end{array}$ & $\begin{array}{l}P_{\text {exp }} \\
\mathrm{MPa}\end{array}$ & $\begin{array}{c}Q \\
\mathrm{~W} \cdot \mathrm{m}^{-1}\end{array}$ & $\begin{array}{c}T_{\text {exp }} \\
\mathrm{K}\end{array}$ & $\begin{array}{c}\rho_{\text {calc }} \\
\mathrm{mol} \cdot \mathrm{L}^{-1}\end{array}$ & $\begin{array}{c}\lambda_{\exp } \\
\mathrm{W} \cdot \mathrm{m}^{-1} \cdot \mathrm{K}^{-1}\end{array}$ & STAT & $\begin{array}{c}T_{\text {cell }} \\
\mathrm{K}\end{array}$ \\
\hline 1003 & 0.416 & 0.18275 & 249.003 & 14.9669 & 0.10438 & 0.006 & 248.144 \\
\hline 1005 & 0.416 & 0.23167 & 249.205 & 14.9597 & 0.10373 & 0.004 & 248.126 \\
\hline 1007 & 0.416 & 0.28646 & 249.433 & 14.9515 & 0.10422 & 0.003 & 248.101 \\
\hline 1009 & 0.416 & 0.34707 & 249.698 & 14.9419 & 0.10313 & 0.002 & 248.076 \\
\hline 1011 & 0.415 & 0.41343 & 249.970 & 14.9321 & 0.10401 & 0.002 & 248.047 \\
\hline 1013 & 0.414 & 0.48560 & 250.296 & 14.9203 & 0.10292 & 0.001 & 248.029 \\
\hline 1015 & 0.412 & 0.14003 & 248.631 & 14.9802 & 0.10509 & 0.008 & 247.955 \\
\hline 1017 & 0.412 & 0.18298 & 248.800 & 14.9741 & 0.10438 & 0.005 & 247.936 \\
\hline 1019 & 0.412 & 0.23165 & 248.991 & 14.9672 & 0.10474 & 0.004 & 247.909 \\
\hline 1021 & 0.412 & 0.28612 & 249.230 & 14.9586 & 0.10492 & 0.003 & 247.891 \\
\hline 1023 & 0.412 & 0.34645 & 249.486 & 14.9494 & 0.10328 & 0.002 & 247.868 \\
\hline 1025 & 0.413 & 0.41250 & 249.763 & 14.9395 & 0.10371 & 0.002 & 247.847 \\
\hline 1027 & 0.413 & 0.48437 & 250.085 & 14.9279 & 0.10379 & 0.001 & 247.833 \\
\hline 1029 & 0.425 & 0.15099 & 269.737 & 14.1871 & 0.09683 & 0.007 & 268.963 \\
\hline 1031 & 0.424 & 0.15103 & 269.771 & 14.1856 & 0.09345 & 0.007 & 268.981 \\
\hline 1033 & 0.422 & 0.19732 & 270.007 & 14.1762 & 0.09508 & 0.005 & 268.985 \\
\hline 1035 & 0.423 & 0.19726 & 269.998 & 14.1766 & 0.09438 & 0.005 & 268.985 \\
\hline 1037 & 0.424 & 0.24979 & 270.273 & 14.1657 & 0.09471 & 0.003 & 268.996 \\
\hline 1039 & 0.425 & 0.24975 & 270.262 & 14.1662 & 0.09538 & 0.004 & 268.989 \\
\hline 1041 & 0.425 & 0.30849 & 270.562 & 14.1543 & 0.09470 & 0.003 & 268.996 \\
\hline 1043 & 0.425 & 0.30857 & 270.559 & 14.1544 & 0.09402 & 0.003 & 268.995 \\
\hline 1045 & 0.425 & 0.37374 & 270.918 & 14.1401 & 0.09338 & 0.002 & 269.008 \\
\hline 1047 & 0.424 & 0.37374 & 270.913 & 14.1402 & 0.09427 & 0.002 & 269.003 \\
\hline 1049 & 0.423 & 0.44513 & 271.270 & 14.1259 & 0.09437 & 0.002 & 269.000 \\
\hline 1051 & 0.422 & 0.44510 & 271.256 & 14.1264 & 0.09393 & 0.001 & 269.007 \\
\hline 1053 & 0.422 & 0.52270 & 271.665 & 14.1100 & 0.09473 & 0.001 & 269.014 \\
\hline 1055 & 0.422 & 0.52257 & 271.651 & 14.1106 & 0.09377 & 0.001 & 269.010 \\
\hline 1057 & 1.062 & 0.15866 & 284.488 & 13.6116 & 0.08897 & 0.006 & 283.618 \\
\hline 1059 & 1.053 & 0.15855 & 284.502 & 13.6105 & 0.09142 & 0.006 & 283.620 \\
\hline 1061 & 1.048 & 0.20716 & 284.774 & 13.5984 & 0.08964 & 0.004 & 283.635 \\
\hline 1063 & 1.060 & 0.20709 & 284.769 & 13.5994 & 0.08896 & 0.004 & 283.625 \\
\hline 1065 & 1.070 & 0.26223 & 285.051 & 13.5877 & 0.08857 & 0.003 & 283.627 \\
\hline 1067 & 1.074 & 0.26221 & 285.056 & 13.5878 & 0.08789 & 0.003 & 283.630 \\
\hline 1069 & 1.082 & 0.32388 & 285.381 & 13.5742 & 0.08901 & 0.002 & 283.632 \\
\hline 1071 & 1.086 & 0.32390 & 285.390 & 13.5741 & 0.08889 & 0.002 & 283.637 \\
\hline 1073 & 1.072 & 0.39239 & 285.751 & 13.5575 & 0.08762 & 0.002 & 283.637 \\
\hline 1075 & 1.058 & 0.39245 & 285.742 & 13.5571 & 0.08811 & 0.002 & 283.630 \\
\hline 1077 & 1.050 & 0.46748 & 286.149 & 13.5389 & 0.08758 & 0.001 & 283.634 \\
\hline 1079 & 1.042 & 0.46766 & 286.147 & 13.5385 & 0.08785 & 0.001 & 283.632 \\
\hline 1081 & 1.046 & 0.54933 & 286.590 & 13.5194 & 0.08754 & 0.001 & 283.631 \\
\hline 1083 & 1.061 & 0.54911 & 286.585 & 13.5205 & 0.08788 & 0.001 & 283.638 \\
\hline 1085 & 10.377 & 0.16605 & 299.295 & 13.5307 & 0.09083 & 0.006 & 298.378 \\
\hline 1087 & 10.385 & 0.21663 & 299.559 & 13.5211 & 0.09061 & 0.004 & 298.382 \\
\hline
\end{tabular}


Table 12. Thermal conductivity of the ternary $30 \% \mathrm{R} 32$ / $10 \% \mathrm{R} 125$ / $60 \% \mathrm{R} 134 \mathrm{a}$ mixture in the compressed liquid phase (continued).

\begin{tabular}{|c|c|c|c|c|c|c|c|}
\hline $\begin{array}{l}\text { Run } \\
\text { point }\end{array}$ & $\begin{array}{l}P_{\text {exp }} \\
\mathrm{MPa}\end{array}$ & $\begin{array}{c}Q \\
\mathrm{~W} \cdot \mathrm{m}^{-1}\end{array}$ & $\begin{array}{c}T_{\text {exp }} \\
\mathrm{K}\end{array}$ & $\begin{array}{c}\rho_{\text {calc }} \\
\mathrm{mol} \cdot \mathrm{L}^{-1}\end{array}$ & $\begin{array}{c}\lambda_{\exp } \\
\mathrm{W} \cdot \mathrm{m}^{-1} \cdot \mathrm{K}^{-1}\end{array}$ & STAT & $\begin{array}{c}T_{\text {cell }} \\
\mathrm{K}\end{array}$ \\
\hline 1089 & 10.387 & 0.27454 & 299.873 & 13.5092 & 0.08902 & 0.003 & 298.389 \\
\hline 1091 & 10.381 & 0.33945 & 300.226 & 13.4956 & 0.08886 & 0.002 & 298.391 \\
\hline 1093 & 10.343 & 0.41138 & 300.602 & 13.4792 & 0.08931 & 0.002 & 298.393 \\
\hline 1095 & 10.331 & 0.49002 & 301.018 & 13.4626 & 0.08896 & 0.001 & 298.397 \\
\hline 1097 & 10.320 & 0.57553 & 301.484 & 13.4443 & 0.08863 & 0.001 & 298.395 \\
\hline 1099 & 8.101 & 0.16621 & 299.289 & 13.4065 & 0.08886 & 0.006 & 298.362 \\
\hline 1101 & 8.110 & 0.21688 & 299.565 & 13.3960 & 0.08731 & 0.004 & 298.369 \\
\hline 1103 & 8.122 & 0.27506 & 299.889 & 13.3838 & 0.08649 & 0.003 & 298.379 \\
\hline 1105 & 8.133 & 0.34008 & 300.241 & 13.3704 & 0.08731 & 0.002 & 298.383 \\
\hline 1107 & 8.145 & 0.41187 & 300.629 & 13.3556 & 0.08689 & 0.002 & 298.383 \\
\hline 1109 & 8.154 & 0.49058 & 301.054 & 13.3391 & 0.08746 & 0.001 & 298.377 \\
\hline 1111 & 8.161 & 0.57638 & 301.515 & 13.3210 & 0.08746 & 0.001 & 298.377 \\
\hline 1113 & 6.103 & 0.16610 & 299.281 & 13.2881 & 0.08780 & 0.006 & 298.349 \\
\hline 1115 & 6.109 & 0.21713 & 299.560 & 13.2769 & 0.08681 & 0.004 & 298.351 \\
\hline 1117 & 6.120 & 0.27518 & 299.888 & 13.2638 & 0.08659 & 0.003 & 298.358 \\
\hline 1119 & 6.129 & 0.34000 & 300.235 & 13.2498 & 0.08685 & 0.003 & 298.364 \\
\hline 1121 & 6.132 & 0.41183 & 300.646 & 13.2327 & 0.08589 & 0.002 & 298.365 \\
\hline 1123 & 6.136 & 0.49050 & 301.088 & 13.2143 & 0.08621 & 0.001 & 298.372 \\
\hline 1125 & 6.140 & 0.57607 & 301.550 & 13.1950 & 0.08649 & 0.001 & 298.377 \\
\hline 1127 & 3.985 & 0.16618 & 299.301 & 13.1494 & 0.08495 & 0.006 & 298.340 \\
\hline 1129 & 3.990 & 0.21721 & 299.592 & 13.1369 & 0.08412 & 0.004 & 298.345 \\
\hline 1131 & 3.997 & 0.27525 & 299.907 & 13.1233 & 0.08491 & 0.003 & 298.357 \\
\hline 1133 & 4.005 & 0.34009 & 300.270 & 13.1077 & 0.08438 & 0.002 & 298.363 \\
\hline 1135 & 4.020 & 0.41180 & 300.699 & 13.0895 & 0.08404 & 0.002 & 298.366 \\
\hline 1137 & 4.035 & 0.49040 & 301.124 & 13.0714 & 0.08493 & 0.002 & 298.367 \\
\hline 1139 & 4.040 & 0.57605 & 301.603 & 13.0502 & 0.08454 & 0.001 & 298.372 \\
\hline 1141 & 1.381 & 0.16610 & 299.288 & 12.9582 & 0.08356 & 0.006 & 298.323 \\
\hline 1143 & 1.375 & 0.21718 & 299.614 & 12.9419 & 0.08243 & 0.004 & 298.348 \\
\hline 1145 & 1.376 & 0.27524 & 299.934 & 12.9264 & 0.08279 & 0.003 & 298.349 \\
\hline 1147 & 1.383 & 0.34006 & 300.307 & 12.9086 & 0.08266 & 0.002 & 298.347 \\
\hline 1149 & 1.398 & 0.41181 & 300.706 & 12.8903 & 0.08268 & 0.002 & 298.345 \\
\hline 1151 & 1.410 & 0.49056 & 301.159 & 12.8688 & 0.08269 & 0.002 & 298.348 \\
\hline 1153 & 1.420 & 0.57615 & 301.634 & 12.8461 & 0.08223 & 0.002 & 298.348 \\
\hline 1155 & 10.201 & 0.17372 & 314.405 & 12.9256 & 0.08460 & 0.006 & 313.382 \\
\hline 1157 & 10.217 & 0.22705 & 314.699 & 12.9146 & 0.08336 & 0.004 & 313.388 \\
\hline 1159 & 10.214 & 0.28769 & 315.049 & 12.9000 & 0.08287 & 0.005 & 313.390 \\
\hline 1161 & 10.222 & 0.35547 & 315.434 & 12.8847 & 0.08348 & 0.002 & 313.400 \\
\hline 1163 & 10.224 & 0.43042 & 315.867 & 12.8669 & 0.08260 & 0.002 & 313.408 \\
\hline 1165 & 10.202 & 0.51278 & 316.321 & 12.8466 & 0.08323 & 0.001 & 313.399 \\
\hline 1167 & 10.195 & 0.60249 & 316.836 & 12.8247 & 0.08369 & 0.001 & 313.404 \\
\hline 1173 & 8.009 & 0.20489 & 314.556 & 12.7645 & 0.08216 & 0.004 & 313.344 \\
\hline 1175 & 7.993 & 0.25068 & 314.806 & 12.7523 & 0.08147 & 0.003 & 313.345 \\
\hline 1177 & 7.987 & 0.30095 & 315.084 & 12.7396 & 0.08172 & 0.003 & 313.331 \\
\hline
\end{tabular}


Table 12. Thermal conductivity of the ternary $30 \% \mathrm{R} 32$ / $10 \% \mathrm{R} 125$ / $60 \% \mathrm{R} 134 \mathrm{a}$ mixture in the compressed liquid phase (continued).

\begin{tabular}{|c|c|c|c|c|c|c|c|}
\hline $\begin{array}{l}\text { Run } \\
\text { point }\end{array}$ & $\begin{array}{l}P_{\text {exp }} \\
\text { MPa }\end{array}$ & $\begin{array}{c}Q \\
\mathrm{~W} \cdot \mathrm{m}^{-1}\end{array}$ & $\begin{array}{c}T_{\exp } \\
\mathrm{K}\end{array}$ & $\begin{array}{c}\rho_{\text {calc }} \\
\mathrm{mol} \cdot \mathrm{L}^{-1}\end{array}$ & $\begin{array}{c}\lambda_{\exp } \\
\mathrm{W} \cdot \mathrm{m}^{-1} \cdot \mathrm{K}^{-1}\end{array}$ & STAT & $\begin{array}{c}T_{\text {cell }} \\
\mathrm{K}\end{array}$ \\
\hline 1179 & 7.980 & 0.35589 & 315.409 & 12.7247 & 0.08150 & 0.002 & 313.339 \\
\hline 1181 & 7.975 & 0.41528 & 315.742 & 12.7097 & 0.08143 & 0.002 & 313.334 \\
\hline 1183 & 5.977 & 0.12756 & 314.050 & 12.6275 & 0.08042 & 0.008 & 313.266 \\
\hline 1185 & 5.972 & 0.14980 & 314.195 & 12.6203 & 0.08010 & 0.008 & 313.281 \\
\hline 1189 & 5.966 & 0.19971 & 314.487 & 12.6060 & 0.08078 & 0.006 & 313.288 \\
\hline 1191 & 5.965 & 0.22736 & 314.638 & 12.5988 & 0.08018 & 0.004 & 313.282 \\
\hline 1193 & 5.961 & 0.25679 & 314.814 & 12.5902 & 0.07962 & 0.004 & 313.281 \\
\hline 1195 & 5.964 & 0.28797 & 314.988 & 12.5822 & 0.07962 & 0.003 & 313.280 \\
\hline 1197 & 3.781 & 0.12755 & 314.028 & 12.4320 & 0.07673 & 0.008 & 313.224 \\
\hline 1199 & 3.787 & 0.14973 & 314.161 & 12.4258 & 0.07866 & 0.007 & 313.234 \\
\hline 1201 & 3.791 & 0.17381 & 314.305 & 12.4187 & 0.07811 & 0.005 & 313.232 \\
\hline 1203 & 3.794 & 0.19960 & 314.458 & 12.4112 & 0.07756 & 0.007 & 313.235 \\
\hline 1205 & 3.800 & 0.22720 & 314.629 & 12.4029 & 0.07709 & 0.005 & 313.241 \\
\hline 1207 & 3.804 & 0.25662 & 314.811 & 12.3938 & 0.07771 & 0.003 & 313.249 \\
\hline 1209 & 3.807 & 0.28774 & 314.986 & 12.3851 & 0.07785 & 0.003 & 313.243 \\
\hline 1213 & 1.875 & 0.14979 & 314.160 & 12.2247 & 0.07606 & 0.006 & 313.206 \\
\hline 1215 & 1.878 & 0.17383 & 314.307 & 12.2166 & 0.07736 & 0.005 & 313.219 \\
\hline 1217 & 1.882 & 0.19957 & 314.464 & 12.2081 & 0.07624 & 0.004 & 313.224 \\
\hline 1219 & 1.885 & 0.22715 & 314.634 & 12.1987 & 0.07616 & 0.003 & 313.219 \\
\hline 1221 & 1.889 & 0.25651 & 314.806 & 12.1893 & 0.07583 & 0.004 & 313.215 \\
\hline 1223 & 1.892 & 0.28774 & 315.001 & 12.1783 & 0.07539 & 0.003 & 313.228 \\
\hline 1225 & 9.197 & 0.13315 & 328.900 & 12.2142 & 0.07589 & 0.007 & 328.134 \\
\hline 1227 & 9.206 & 0.15625 & 329.045 & 12.2083 & 0.07759 & 0.008 & 328.134 \\
\hline 1229 & 9.207 & 0.18124 & 329.213 & 12.2006 & 0.07629 & 0.005 & 328.147 \\
\hline 1231 & 9.208 & 0.20814 & 329.379 & 12.1929 & 0.07774 & 0.004 & 328.150 \\
\hline 1233 & 9.209 & 0.23696 & 329.559 & 12.1845 & 0.07696 & 0.003 & 328.152 \\
\hline 1235 & 9.209 & 0.26756 & 329.758 & 12.1752 & 0.07681 & 0.003 & 328.154 \\
\hline 1237 & 9.209 & 0.30009 & 329.953 & 12.1660 & 0.07687 & 0.002 & 328.151 \\
\hline 1241 & 7.386 & 0.15624 & 329.067 & 12.0280 & 0.07616 & 0.008 & 328.130 \\
\hline 1245 & 7.395 & 0.20810 & 329.440 & 12.0100 & 0.07500 & 0.005 & 328.137 \\
\hline 1247 & 7.398 & 0.23690 & 329.636 & 12.0005 & 0.07501 & 0.003 & 328.147 \\
\hline 1249 & 7.403 & 0.26754 & 329.810 & 11.9920 & 0.07565 & 0.003 & 328.134 \\
\hline 1251 & 7.406 & 0.30006 & 330.006 & 11.9824 & 0.07634 & 0.005 & 328.133 \\
\hline 1253 & 5.673 & 0.13314 & 328.978 & 11.8387 & 0.07431 & 0.007 & 328.103 \\
\hline 1255 & 5.673 & 0.15628 & 329.126 & 11.8305 & 0.07443 & 0.007 & 328.113 \\
\hline 1257 & 5.681 & 0.18130 & 329.289 & 11.8224 & 0.07301 & 0.005 & 328.118 \\
\hline 1259 & 5.684 & 0.20822 & 329.447 & 11.8139 & 0.07397 & 0.004 & 328.124 \\
\hline 1261 & 5.689 & 0.23705 & 329.635 & 11.8040 & 0.07351 & 0.003 & 328.126 \\
\hline 1263 & 5.692 & 0.26769 & 329.831 & 11.7935 & 0.07285 & 0.003 & 328.124 \\
\hline 1265 & 5.695 & 0.30027 & 330.045 & 11.7817 & 0.07349 & 0.003 & 328.134 \\
\hline 1267 & 3.718 & 0.13310 & 328.966 & 11.5734 & 0.07086 & 0.007 & 328.080 \\
\hline 1269 & 3.717 & 0.15628 & 329.133 & 11.5626 & 0.07106 & 0.006 & 328.096 \\
\hline 1271 & 3.717 & 0.18131 & 329.307 & 11.5514 & 0.07096 & 0.005 & 328.115 \\
\hline
\end{tabular}


Table 12. Thermal conductivity of the ternary $30 \% \mathrm{R} 32 / 10 \% \mathrm{R} 125 / 60 \% \mathrm{R} 134 \mathrm{a}$ mixture in the compressed liquid phase (continued).

\begin{tabular}{|c|c|c|c|c|c|c|c|}
\hline $\begin{array}{l}\text { Run } \\
\text { point }\end{array}$ & $\begin{array}{l}P_{e x p} \\
\mathrm{MPa}\end{array}$ & $\begin{array}{c}Q \\
\mathrm{~W} \cdot \mathrm{m}^{-1}\end{array}$ & $\begin{array}{c}T_{\text {exp }} \\
\mathrm{K}\end{array}$ & $\begin{array}{c}\rho_{\text {calc }} \\
\mathrm{mol} \cdot \mathrm{L}^{-1}\end{array}$ & $\begin{array}{c}\lambda_{\exp } \\
\mathrm{W} \cdot \mathrm{m}^{-1} \cdot \mathrm{K}^{-1}\end{array}$ & STAT & $\begin{array}{c}T_{\text {cell }} \\
\mathrm{K}\end{array}$ \\
\hline 1273 & 3.721 & 0.20827 & 329.481 & 11.5409 & 0.07131 & 0.004 & 328.109 \\
\hline 1275 & 3.725 & 0.23703 & 329.647 & 11.5306 & 0.07139 & 0.004 & 328.107 \\
\hline 1277 & 3.727 & 0.26772 & 329.850 & 11.5179 & 0.07121 & 0.003 & 328.109 \\
\hline 1279 & 3.731 & 0.30024 & 330.064 & 11.5046 & 0.07178 & 0.002 & 328.108 \\
\hline 1281 & 2.439 & 0.13310 & 328.984 & 11.3575 & 0.07025 & 0.007 & 328.082 \\
\hline 1283 & 2.438 & 0.15629 & 329.133 & 11.3464 & 0.07023 & 0.006 & 328.089 \\
\hline 1285 & 2.437 & 0.18131 & 329.314 & 11.3329 & 0.06910 & 0.005 & 328.104 \\
\hline 1287 & 2.442 & 0.20824 & 329.484 & 11.3213 & 0.06962 & 0.004 & 328.103 \\
\hline 1289 & 2.445 & 0.23703 & 329.677 & 11.3076 & 0.06953 & 0.003 & 328.109 \\
\hline 1291 & 2.448 & 0.26767 & 329.884 & 11.2929 & 0.07004 & 0.005 & 328.113 \\
\hline 1293 & 2.451 & 0.30023 & 330.092 & 11.2779 & 0.07033 & 0.003 & 328.113 \\
\hline 1295 & 8.432 & 0.13885 & 344.143 & 11.3514 & 0.07088 & 0.008 & 343.204 \\
\hline 1297 & 8.434 & 0.16292 & 344.318 & 11.3418 & 0.07042 & 0.007 & 343.220 \\
\hline 1299 & 8.435 & 0.18902 & 344.479 & 11.3329 & 0.07146 & 0.005 & 343.214 \\
\hline 1301 & 8.437 & 0.21716 & 344.660 & 11.3230 & 0.07143 & 0.004 & 343.215 \\
\hline 1303 & 8.439 & 0.24724 & 344.857 & 11.3120 & 0.07105 & 0.004 & 343.219 \\
\hline 1305 & 8.442 & 0.27921 & 345.072 & 11.3004 & 0.07097 & 0.003 & 343.222 \\
\hline 1307 & 8.444 & 0.31314 & 345.283 & 11.2887 & 0.07157 & 0.003 & 343.212 \\
\hline 1309 & 7.472 & 0.13885 & 344.140 & 11.2105 & 0.06976 & 0.008 & 343.190 \\
\hline 1311 & 7.471 & 0.16298 & 344.311 & 11.2001 & 0.06930 & 0.007 & 343.200 \\
\hline 1313 & 7.465 & 0.18914 & 344.482 & 11.1888 & 0.07067 & 0.005 & 343.208 \\
\hline 1315 & 7.472 & 0.21719 & 344.678 & 11.1781 & 0.06978 & 0.004 & 343.213 \\
\hline 1317 & 7.476 & 0.24724 & 344.871 & 11.1670 & 0.07021 & 0.004 & 343.209 \\
\hline 1319 & 7.479 & 0.27921 & 345.080 & 11.1547 & 0.07012 & 0.003 & 343.211 \\
\hline 1321 & 7.480 & 0.31315 & 345.301 & 11.1415 & 0.07024 & 0.003 & 343.206 \\
\hline 1323 & 6.522 & 0.13880 & 344.135 & 11.0540 & 0.06845 & 0.008 & 343.176 \\
\hline 1325 & 6.525 & 0.16297 & 344.312 & 11.0429 & 0.06859 & 0.006 & 343.186 \\
\hline 1327 & 6.526 & 0.18908 & 344.483 & 11.0321 & 0.06868 & 0.005 & 343.189 \\
\hline 1329 & 6.529 & 0.21716 & 344.669 & 11.0203 & 0.06887 & 0.004 & 343.184 \\
\hline 1331 & 6.531 & 0.24723 & 344.879 & 11.0069 & 0.06872 & 0.004 & 343.194 \\
\hline 1333 & 6.532 & 0.27925 & 345.089 & 10.9932 & 0.06914 & 0.003 & 343.192 \\
\hline 1335 & 6.533 & 0.31321 & 345.318 & 10.9783 & 0.06949 & 0.003 & 343.195 \\
\hline 1337 & 5.637 & 0.13882 & 344.151 & 10.8858 & 0.06822 & 0.008 & 343.177 \\
\hline 1339 & 5.639 & 0.16298 & 344.318 & 10.8744 & 0.06743 & 0.006 & 343.180 \\
\hline 1341 & 5.641 & 0.18908 & 344.497 & 10.8620 & 0.06755 & 0.005 & 343.187 \\
\hline 1343 & 5.642 & 0.21721 & 344.691 & 10.8482 & 0.06800 & 0.004 & 343.191 \\
\hline 1345 & 5.645 & 0.24722 & 344.888 & 10.8346 & 0.06802 & 0.004 & 343.193 \\
\hline 1347 & 5.646 & 0.27921 & 345.105 & 10.8191 & 0.06822 & 0.004 & 343.189 \\
\hline 1349 & 5.647 & 0.31313 & 345.334 & 10.8028 & 0.06842 & 0.003 & 343.190 \\
\hline 1351 & 4.429 & 0.13880 & 344.162 & 10.6085 & 0.06595 & 0.009 & 343.170 \\
\hline 1353 & 4.428 & 0.16300 & 344.322 & 10.5948 & 0.06565 & 0.006 & 343.174 \\
\hline 1355 & 4.429 & 0.18902 & 344.510 & 10.5793 & 0.06589 & 0.005 & 343.183 \\
\hline 1357 & 4.432 & 0.21712 & 344.704 & 10.5633 & 0.06625 & 0.004 & 343.184 \\
\hline
\end{tabular}


Table 12. Thermal conductivity of the ternary $30 \% \mathrm{R} 32$ / $10 \% \mathrm{R} 125$ / $60 \% \mathrm{R} 134 \mathrm{a}$ mixture in the compressed liquid phase (continued).

\begin{tabular}{|ccccccc|c|}
\hline $\begin{array}{c}\text { Run } \\
\text { point }\end{array}$ & $\begin{array}{c}P_{\text {exp }} \\
\mathrm{MPa}\end{array}$ & $\begin{array}{c}Q \\
\mathrm{~W} \cdot \mathrm{m}^{-1}\end{array}$ & $\begin{array}{c}T_{\text {exp }} \\
\mathrm{K}\end{array}$ & $\begin{array}{c}\rho_{\text {calc }} \\
\mathrm{mol} \cdot \mathrm{L}^{-1}\end{array}$ & $\begin{array}{c}\lambda_{\text {exp }} \\
\mathrm{W} \cdot \mathrm{m}^{-1} \cdot \mathrm{K}^{-1}\end{array}$ & STAT & $\begin{array}{c}T_{\text {cell }} \\
\mathrm{K}\end{array}$ \\
\hline 1359 & 4.433 & 0.24717 & 344.907 & 10.5463 & 0.06756 & 0.007 & 343.186 \\
1361 & 4.435 & 0.27917 & 345.114 & 10.5290 & 0.06706 & 0.004 & 343.176 \\
1363 & 4.438 & 0.31317 & 345.357 & 10.5085 & 0.06797 & 0.004 & 343.182 \\
1365 & 3.488 & 0.13878 & 344.157 & 10.3270 & 0.06478 & 0.008 & 343.155 \\
1367 & 3.488 & 0.16299 & 344.337 & 10.3087 & 0.06414 & 0.006 & 343.178 \\
1369 & 3.488 & 0.18905 & 344.516 & 10.2902 & 0.06499 & 0.005 & 343.175 \\
1371 & 3.489 & 0.21717 & 344.730 & 10.2682 & 0.06560 & 0.005 & 343.187 \\
1373 & 3.490 & 0.24716 & 344.935 & 10.2469 & 0.06612 & 0.005 & 343.196 \\
1375 & 3.492 & 0.27907 & 345.149 & 10.2246 & 0.06712 & 0.006 & 343.180 \\
1377 & 3.494 & 0.31293 & 345.373 & 10.2009 & 0.06830 & 0.007 & 343.181 \\
\hline
\end{tabular}




\section{Tables of transient results for bare tungsten wires}

Table 13. Thermal conductivity of the binary $30 \% \mathrm{R} 125$ / $70 \% \mathrm{R} 134 \mathrm{a}$ mixture in the vapor phase.

\begin{tabular}{|c|c|c|c|c|c|c|c|}
\hline $\begin{array}{l}\text { Run } \\
\text { point }\end{array}$ & $\begin{array}{l}P_{\text {exp }} \\
\mathrm{MPa}\end{array}$ & $\begin{array}{c}Q \\
\mathrm{~W} \cdot \mathrm{m}^{-1}\end{array}$ & $\begin{array}{c}T_{\text {exp }} \\
\mathrm{K}\end{array}$ & $\begin{array}{c}\rho_{\text {calc }} \\
\mathrm{mol} \cdot \mathrm{L}^{-1}\end{array}$ & $\begin{array}{c}\lambda_{\exp } \\
\mathrm{W} \cdot \mathrm{m}^{-1} \cdot \mathrm{K}^{-1}\end{array}$ & $\overline{\text { STAT }}$ & $\begin{array}{c}T_{\text {cell }} \\
\mathrm{K}\end{array}$ \\
\hline 1001 & 0.217 & 0.02783 & 263.777 & 0.1059 & 0.01116 & 0.011 & 261.132 \\
\hline 1004 & 0.215 & 0.03782 & 264.739 & 0.1044 & 0.01123 & 0.008 & 261.145 \\
\hline 1007 & 0.215 & 0.04931 & 265.843 & 0.1040 & 0.01131 & 0.005 & 261.171 \\
\hline 1010 & 0.217 & 0.06228 & 267.058 & 0.1042 & 0.01145 & 0.004 & 261.179 \\
\hline 1013 & 0.218 & 0.07674 & 268.427 & 0.1041 & 0.01155 & 0.003 & 261.215 \\
\hline 1016 & 0.147 & 0.02780 & 264.062 & 0.0700 & 0.01116 & 0.013 & 261.229 \\
\hline 1019 & 0.147 & 0.03780 & 265.062 & 0.0699 & 0.01121 & 0.009 & 261.249 \\
\hline 1022 & 0.146 & 0.04929 & 266.228 & 0.0691 & 0.01128 & 0.006 & 261.289 \\
\hline 1025 & 0.145 & 0.06225 & 267.486 & 0.0682 & 0.01140 & 0.005 & 261.290 \\
\hline 1028 & 0.146 & 0.07668 & 268.893 & 0.0681 & 0.01151 & 0.004 & 261.305 \\
\hline 1031 & 0.074 & 0.02779 & 264.452 & 0.0344 & 0.01105 & 0.012 & 261.378 \\
\hline 1034 & 0.075 & 0.03776 & 265.514 & 0.0349 & 0.01131 & 0.008 & 261.396 \\
\hline 1037 & 0.077 & 0.04924 & 266.713 & 0.0353 & 0.01139 & 0.006 & 261.408 \\
\hline 1040 & 0.076 & 0.06220 & 268.063 & 0.0347 & 0.01148 & 0.005 & 261.424 \\
\hline 1043 & 0.074 & 0.07660 & 269.544 & 0.0337 & 0.01161 & 0.004 & 261.436 \\
\hline 1046 & 0.297 & 0.02692 & 272.745 & 0.1424 & 0.01199 & 0.013 & 270.456 \\
\hline 1049 & 0.297 & 0.03661 & 273.606 & 0.1420 & 0.01202 & 0.009 & 270.483 \\
\hline 1052 & 0.300 & 0.04775 & 274.545 & 0.1431 & 0.01208 & 0.006 & 270.476 \\
\hline 1055 & 0.301 & 0.06032 & 275.599 & 0.1427 & 0.01216 & 0.005 & 270.467 \\
\hline 1058 & 0.298 & 0.07434 & 276.772 & 0.1402 & 0.01228 & 0.005 & 270.466 \\
\hline 1064 & 0.216 & 0.03660 & 273.699 & 0.1005 & 0.01198 & 0.009 & 270.469 \\
\hline 1067 & 0.219 & 0.04772 & 274.689 & 0.1017 & 0.01206 & 0.007 & 270.469 \\
\hline 1070 & 0.221 & 0.06030 & 275.793 & 0.1021 & 0.01216 & 0.005 & 270.465 \\
\hline 1073 & 0.219 & 0.07433 & 277.013 & 0.1005 & 0.01225 & 0.005 & 270.462 \\
\hline 1076 & 0.151 & 0.02691 & 272.901 & 0.0694 & 0.01188 & 0.014 & 270.444 \\
\hline 1079 & 0.148 & 0.03660 & 273.810 & 0.0676 & 0.01192 & 0.009 & 270.450 \\
\hline 1082 & 0.146 & 0.04771 & 274.840 & 0.0663 & 0.01200 & 0.006 & 270.452 \\
\hline 1085 & 0.149 & 0.06028 & 275.995 & 0.0675 & 0.01212 & 0.005 & 270.457 \\
\hline 1088 & 0.151 & 0.07429 & 277.266 & 0.0682 & 0.01220 & 0.004 & 270.456 \\
\hline 1091 & 0.078 & 0.02690 & 273.028 & 0.0351 & 0.01194 & 0.014 & 270.429 \\
\hline 1094 & 0.077 & 0.03656 & 273.992 & 0.0344 & 0.01205 & 0.009 & 270.434 \\
\hline 1097 & 0.074 & 0.04769 & 275.088 & 0.0329 & 0.01218 & 0.006 & 270.444 \\
\hline 1100 & 0.075 & 0.06026 & 276.291 & 0.0332 & 0.01228 & 0.005 & 270.431 \\
\hline 1103 & 0.078 & 0.07424 & 277.650 & 0.0343 & 0.01237 & 0.004 & 270.443 \\
\hline 1106 & 0.433 & 0.02593 & 283.205 & 0.2058 & 0.01278 & 0.015 & 281.163 \\
\hline 1109 & 0.434 & 0.03527 & 283.950 & 0.2050 & 0.01287 & 0.010 & 281.168 \\
\hline 1112 & 0.431 & 0.04603 & 284.787 & 0.2030 & 0.01300 & 0.007 & 281.174 \\
\hline 1115 & 0.427 & 0.05816 & 285.721 & 0.1997 & 0.01304 & 0.005 & 281.179 \\
\hline 1118 & 0.426 & 0.07169 & 286.744 & 0.1984 & 0.01326 & 0.005 & 281.163 \\
\hline 1121 & 0.346 & 0.02594 & 283.255 & 0.1599 & 0.01282 & 0.015 & 281.143 \\
\hline 1127 & 0.350 & 0.04602 & 284.879 & 0.1610 & 0.01284 & 0.007 & 281.159 \\
\hline 1130 & 0.350 & 0.05815 & 285.822 & 0.1600 & 0.01295 & 0.006 & 281.141 \\
\hline
\end{tabular}


Table 13. Thermal conductivity of the binary $30 \% \mathrm{R} 125 / 70 \% \mathrm{R} 134 \mathrm{a}$ mixture in the vapor phase (continued).

\begin{tabular}{|c|c|c|c|c|c|c|c|}
\hline $\begin{array}{l}\text { Run } \\
\text { point }\end{array}$ & $\begin{array}{l}P_{\text {exp }} \\
\mathrm{MPa} \\
\end{array}$ & $\begin{array}{c}Q \\
\mathrm{~W} \cdot \mathrm{m}^{-1}\end{array}$ & $\begin{array}{c}T_{\exp } \\
\mathrm{K}\end{array}$ & $\begin{array}{c}\rho_{\text {calc }} \\
\mathrm{mol} \cdot \mathrm{L}^{-1}\end{array}$ & $\begin{array}{c}\lambda_{\exp } \\
\mathrm{W} \cdot \mathrm{m}^{-1} \cdot \mathrm{K}^{-1}\end{array}$ & STAT & $\begin{array}{c}T_{\text {cell }} \\
\mathrm{K}\end{array}$ \\
\hline 1133 & 0.347 & 0.07170 & 286.895 & 0.1576 & 0.01309 & 0.004 & 281.152 \\
\hline 1136 & 0.286 & 0.02593 & 283.237 & 0.1302 & 0.01279 & 0.015 & 281.111 \\
\hline 1139 & 0.283 & 0.03527 & 284.014 & 0.1284 & 0.01267 & 0.010 & 281.118 \\
\hline 1142 & 0.285 & 0.04601 & 284.889 & 0.1287 & 0.01290 & 0.007 & 281.112 \\
\hline 1145 & 0.288 & 0.05813 & 285.838 & 0.1294 & 0.01296 & 0.005 & 281.075 \\
\hline 1148 & 0.287 & 0.07166 & 286.956 & 0.1283 & 0.01303 & 0.004 & 281.104 \\
\hline 1151 & 0.216 & 0.02593 & 283.253 & 0.0966 & 0.01274 & 0.014 & 281.082 \\
\hline 1154 & 0.213 & 0.03526 & 284.053 & 0.0947 & 0.01271 & 0.010 & 281.087 \\
\hline 1160 & 0.218 & 0.05812 & 285.973 & 0.0963 & 0.01300 & 0.005 & 281.091 \\
\hline 1163 & 0.216 & 0.07165 & 287.099 & 0.0948 & 0.01305 & 0.004 & 281.096 \\
\hline 1166 & 0.150 & 0.02592 & 283.290 & 0.0659 & 0.01278 & 0.016 & 281.080 \\
\hline 1169 & 0.146 & 0.03525 & 284.113 & 0.0641 & 0.01269 & 0.009 & 281.083 \\
\hline 1172 & 0.146 & 0.04597 & 285.047 & 0.0638 & 0.01289 & 0.007 & 281.081 \\
\hline 1175 & 0.149 & 0.05809 & 286.102 & 0.0645 & 0.01301 & 0.006 & 281.086 \\
\hline 1178 & 0.148 & 0.07158 & 287.244 & 0.0638 & 0.01310 & 0.004 & 281.069 \\
\hline 1196 & 0.548 & 0.02504 & 293.301 & 0.2550 & 0.01347 & 0.017 & 291.489 \\
\hline 1199 & 0.547 & 0.03407 & 293.969 & 0.2533 & 0.01363 & 0.012 & 291.498 \\
\hline 1202 & 0.549 & 0.04445 & 294.697 & 0.2534 & 0.01389 & 0.009 & 291.487 \\
\hline 1205 & 0.550 & 0.05619 & 295.529 & 0.2529 & 0.01385 & 0.006 & 291.491 \\
\hline 1208 & 0.549 & 0.06926 & 296.455 & 0.2511 & 0.01404 & 0.005 & 291.494 \\
\hline 1214 & 0.485 & 0.03406 & 293.906 & 0.2212 & 0.01375 & 0.013 & 291.447 \\
\hline 1217 & 0.488 & 0.04445 & 294.689 & 0.2220 & 0.01381 & 0.009 & 291.473 \\
\hline 1220 & 0.486 & 0.05617 & 295.526 & 0.2200 & 0.01381 & 0.007 & 291.460 \\
\hline 1223 & 0.483 & 0.06925 & 296.462 & 0.2173 & 0.01391 & 0.006 & 291.457 \\
\hline 1226 & 0.424 & 0.02504 & 293.267 & 0.1908 & 0.01360 & 0.018 & 291.456 \\
\hline 1229 & 0.420 & 0.03405 & 293.915 & 0.1885 & 0.01357 & 0.013 & 291.437 \\
\hline 1232 & 0.420 & 0.04443 & 294.686 & 0.1877 & 0.01375 & 0.009 & 291.439 \\
\hline 1235 & 0.423 & 0.05617 & 295.562 & 0.1886 & 0.01383 & 0.006 & 291.454 \\
\hline 1238 & 0.425 & 0.06925 & 296.507 & 0.1886 & 0.01390 & 0.005 & 291.441 \\
\hline 1241 & 0.359 & 0.02503 & 293.178 & 0.1591 & 0.01361 & 0.017 & 291.404 \\
\hline 1244 & 0.356 & 0.03404 & 293.872 & 0.1571 & 0.01368 & 0.011 & 291.409 \\
\hline 1247 & 0.353 & 0.04441 & 294.658 & 0.1553 & 0.01374 & 0.008 & 291.407 \\
\hline 1250 & 0.355 & 0.05614 & 295.557 & 0.1555 & 0.01379 & 0.006 & 291.422 \\
\hline 1253 & 0.358 & 0.06922 & 296.578 & 0.1563 & 0.01404 & 0.056 & 291.406 \\
\hline 1256 & 0.278 & 0.02503 & 293.183 & 0.1210 & 0.01355 & 0.017 & 291.376 \\
\hline 1259 & 0.282 & 0.03404 & 293.899 & 0.1223 & 0.01364 & 0.011 & 291.384 \\
\hline 1262 & 0.284 & 0.04441 & 294.717 & 0.1228 & 0.01372 & 0.008 & 291.388 \\
\hline 1265 & 0.280 & 0.05615 & 295.635 & 0.1208 & 0.01382 & 0.006 & 291.397 \\
\hline 1268 & 0.278 & 0.06922 & 296.624 & 0.1191 & 0.01391 & 0.005 & 291.378 \\
\hline 1271 & 0.218 & 0.02502 & 293.204 & 0.0935 & 0.01358 & 0.018 & 291.364 \\
\hline 1274 & 0.217 & 0.03403 & 293.934 & 0.0927 & 0.01376 & 0.011 & 291.363 \\
\hline 1277 & 0.213 & 0.04441 & 294.772 & 0.0906 & 0.01370 & 0.008 & 291.369 \\
\hline 1280 & 0.214 & 0.05613 & 295.695 & 0.0908 & 0.01380 & 0.006 & 291.358 \\
\hline
\end{tabular}


Table 13. Thermal conductivity of the binary $30 \% \mathrm{R} 125 / 70 \% \mathrm{R} 134 \mathrm{a}$ mixture in the vapor phase (continued).

\begin{tabular}{|c|c|c|c|c|c|c|c|}
\hline $\begin{array}{l}\text { Run } \\
\text { point }\end{array}$ & $\begin{array}{l}P_{\text {exp }} \\
\mathrm{MPa}\end{array}$ & $\begin{array}{c}Q \\
\mathrm{~W} \cdot \mathrm{m}^{-1}\end{array}$ & $\begin{array}{c}T_{\text {exp }} \\
\mathrm{K}\end{array}$ & $\begin{array}{c}\rho_{\text {calc }} \\
\mathrm{mol} \cdot \mathrm{L}^{-1}\end{array}$ & $\begin{array}{c}\lambda_{\exp } \\
\mathrm{W} \cdot \mathrm{m}^{-1} \cdot \mathrm{K}^{-1}\end{array}$ & STAT & $\begin{array}{c}T_{\text {cell }} \\
\mathrm{K}\end{array}$ \\
\hline 1283 & 0.217 & 0.06918 & 296.729 & 0.0917 & 0.01392 & 0.005 & 291.358 \\
\hline 1286 & 0.149 & 0.02502 & 293.243 & 0.0629 & 0.01360 & 0.019 & 291.343 \\
\hline 1289 & 0.149 & 0.03404 & 293.999 & 0.0626 & 0.01378 & 0.011 & 291.341 \\
\hline 1292 & 0.145 & 0.04440 & 294.865 & 0.0607 & 0.01379 & 0.008 & 291.339 \\
\hline 1295 & 0.144 & 0.05610 & 295.840 & 0.0603 & 0.01389 & 0.006 & 291.347 \\
\hline 1298 & 0.148 & 0.06917 & 296.913 & 0.0616 & 0.01398 & 0.005 & 291.338 \\
\hline 1316 & 0.538 & 0.02414 & 303.461 & 0.2371 & 0.01445 & 0.020 & 301.836 \\
\hline 1319 & 0.540 & 0.03283 & 304.069 & 0.2375 & 0.01460 & 0.013 & 301.842 \\
\hline 1322 & 0.538 & 0.04285 & 304.774 & 0.2354 & 0.01466 & 0.010 & 301.854 \\
\hline 1325 & 0.535 & 0.05418 & 305.542 & 0.2333 & 0.01469 & 0.007 & 301.848 \\
\hline 1328 & 0.538 & 0.06681 & 306.374 & 0.2337 & 0.01476 & 0.005 & 301.821 \\
\hline 1331 & 0.487 & 0.02417 & 302.769 & 0.2127 & 0.01440 & 0.021 & 301.369 \\
\hline 1334 & 0.488 & 0.03286 & 303.386 & 0.2126 & 0.01451 & 0.014 & 301.373 \\
\hline 1337 & 0.484 & 0.04288 & 304.100 & 0.2101 & 0.01454 & 0.010 & 301.388 \\
\hline 1340 & 0.482 & 0.05421 & 304.883 & 0.2085 & 0.01465 & 0.007 & 301.381 \\
\hline 1343 & 0.486 & 0.06684 & 305.745 & 0.2093 & 0.01472 & 0.005 & 301.370 \\
\hline 1346 & 0.415 & 0.02416 & 302.738 & 0.1787 & 0.01437 & 0.021 & 301.338 \\
\hline 1349 & 0.417 & 0.03286 & 303.402 & 0.1791 & 0.01449 & 0.014 & 301.381 \\
\hline 1352 & 0.420 & 0.04288 & 304.144 & 0.1798 & 0.01504 & 0.062 & 301.383 \\
\hline 1355 & 0.418 & 0.05419 & 304.879 & 0.1784 & 0.01467 & 0.007 & 301.343 \\
\hline 1358 & 0.415 & 0.06683 & 305.796 & 0.1764 & 0.01470 & 0.005 & 301.373 \\
\hline 1361 & 0.354 & 0.02416 & 302.725 & 0.1503 & 0.01436 & 0.021 & 301.326 \\
\hline 1364 & 0.352 & 0.03285 & 303.382 & 0.1489 & 0.01447 & 0.013 & 301.353 \\
\hline 1367 & 0.353 & 0.04287 & 304.116 & 0.1491 & 0.01457 & 0.009 & 301.359 \\
\hline 1373 & 0.355 & 0.06683 & 305.815 & 0.1490 & 0.01473 & 0.006 & 301.331 \\
\hline 1376 & 0.283 & 0.02416 & 302.720 & 0.1183 & 0.01437 & 0.020 & 301.303 \\
\hline 1379 & 0.285 & 0.03285 & 303.389 & 0.1191 & 0.01453 & 0.013 & 301.320 \\
\hline 1382 & 0.282 & 0.04286 & 304.159 & 0.1173 & 0.01459 & 0.009 & 301.347 \\
\hline 1385 & 0.279 & 0.05418 & 304.989 & 0.1158 & 0.01469 & 0.006 & 301.335 \\
\hline 1388 & 0.281 & 0.06682 & 305.930 & 0.1161 & 0.01477 & 0.005 & 301.348 \\
\hline 1391 & 0.219 & 0.02415 & 302.782 & 0.0903 & 0.01438 & 0.019 & 301.329 \\
\hline 1394 & 0.218 & 0.03285 & 303.452 & 0.0900 & 0.01465 & 0.013 & 301.325 \\
\hline 1397 & 0.215 & 0.04286 & 304.224 & 0.0882 & 0.01460 & 0.009 & 301.327 \\
\hline 1400 & 0.214 & 0.05418 & 305.070 & 0.0877 & 0.01474 & 0.007 & 301.310 \\
\hline 1403 & 0.216 & 0.06680 & 306.027 & 0.0883 & 0.01479 & 0.006 & 301.313 \\
\hline 1406 & 0.142 & 0.02415 & 302.845 & 0.0577 & 0.01465 & 0.020 & 301.317 \\
\hline 1409 & 0.144 & 0.03285 & 303.543 & 0.0585 & 0.01463 & 0.013 & 301.320 \\
\hline 1412 & 0.147 & 0.04286 & 304.309 & 0.0594 & 0.01449 & 0.009 & 301.290 \\
\hline 1415 & 0.144 & 0.05417 & 305.211 & 0.0581 & 0.01480 & 0.007 & 301.300 \\
\hline 1418 & 0.141 & 0.06678 & 306.174 & 0.0569 & 0.01488 & 0.005 & 301.278 \\
\hline 1436 & 0.535 & 0.02322 & 315.652 & 0.2228 & 0.01540 & 0.023 & 314.144 \\
\hline 1439 & 0.538 & 0.03159 & 316.183 & 0.2238 & 0.01554 & 0.015 & 314.103 \\
\hline 1442 & 0.536 & 0.04122 & 316.832 & 0.2224 & 0.01541 & 0.010 & 314.116 \\
\hline
\end{tabular}


Table 13. Thermal conductivity of the binary $30 \% \mathrm{R} 125 / 70 \% \mathrm{R} 134 \mathrm{a}$ mixture in the vapor phase (continued).

\begin{tabular}{|c|c|c|c|c|c|c|c|}
\hline $\begin{array}{l}\text { Run } \\
\text { point }\end{array}$ & $\begin{array}{l}P_{\text {exp }} \\
\mathrm{MPa}\end{array}$ & $\begin{array}{c}Q \\
\mathrm{~W} \cdot \mathrm{m}^{-1}\end{array}$ & $\begin{array}{c}T_{\exp } \\
\mathrm{K}\end{array}$ & $\begin{array}{c}\rho_{\text {calc }} \\
\mathrm{mol} \cdot \mathrm{L}^{-1}\end{array}$ & $\begin{array}{c}\lambda_{\exp } \\
\mathrm{W} \cdot \mathrm{m}^{-1} \cdot \mathrm{K}^{-1}\end{array}$ & STAT & $\begin{array}{c}T_{\text {cell }} \\
\mathrm{K}\end{array}$ \\
\hline 1445 & 0.532 & 0.05211 & 317.550 & 0.2199 & 0.01571 & 0.008 & 314.125 \\
\hline 1448 & 0.534 & 0.06428 & 318.319 & 0.2201 & 0.01578 & 0.006 & 314.109 \\
\hline 1451 & 0.484 & 0.02322 & 315.602 & 0.1996 & 0.01535 & 0.023 & 314.084 \\
\hline 1454 & 0.486 & 0.03159 & 316.182 & 0.2001 & 0.01556 & 0.016 & 314.102 \\
\hline 1457 & 0.489 & 0.04121 & 316.814 & 0.2010 & 0.01535 & 0.011 & 314.089 \\
\hline 1460 & 0.488 & 0.05211 & 317.543 & 0.1997 & 0.01568 & 0.007 & 314.100 \\
\hline 1463 & 0.484 & 0.06429 & 318.317 & 0.1974 & 0.01577 & 0.006 & 314.075 \\
\hline 1466 & 0.421 & 0.02322 & 315.587 & 0.1719 & 0.01553 & 0.023 & 314.061 \\
\hline 1469 & 0.419 & 0.03159 & 316.170 & 0.1707 & 0.01561 & 0.015 & 314.074 \\
\hline 1472 & 0.423 & 0.04122 & 316.803 & 0.1719 & 0.01542 & 0.011 & 314.056 \\
\hline 1475 & 0.425 & 0.05211 & 317.538 & 0.1723 & 0.01567 & 0.008 & 314.060 \\
\hline 1478 & 0.421 & 0.06427 & 318.372 & 0.1700 & 0.01576 & 0.006 & 314.083 \\
\hline 1481 & 0.348 & 0.02322 & 315.555 & 0.1401 & 0.01549 & 0.023 & 314.037 \\
\hline 1484 & 0.352 & 0.03158 & 316.134 & 0.1415 & 0.01570 & 0.016 & 314.039 \\
\hline 1487 & 0.350 & 0.04120 & 316.800 & 0.1404 & 0.01554 & 0.012 & 314.042 \\
\hline 1490 & 0.346 & 0.05210 & 317.521 & 0.1385 & 0.01579 & 0.008 & 314.020 \\
\hline 1493 & 0.349 & 0.06426 & 318.358 & 0.1390 & 0.01581 & 0.006 & 314.027 \\
\hline 1496 & 0.288 & 0.02321 & 315.492 & 0.1148 & 0.01559 & 0.023 & 313.985 \\
\hline 1499 & 0.284 & 0.03158 & 316.111 & 0.1131 & 0.01552 & 0.016 & 314.015 \\
\hline 1502 & 0.282 & 0.04121 & 316.767 & 0.1119 & 0.01565 & 0.011 & 313.991 \\
\hline 1505 & 0.286 & 0.05209 & 317.538 & 0.1130 & 0.01575 & 0.009 & 314.002 \\
\hline 1508 & 0.288 & 0.06424 & 318.374 & 0.1137 & 0.01577 & 0.006 & 313.994 \\
\hline 1511 & 0.219 & 0.02321 & 315.494 & 0.0862 & 0.01562 & 0.025 & 313.967 \\
\hline 1514 & 0.218 & 0.03157 & 316.111 & 0.0857 & 0.01570 & 0.016 & 313.972 \\
\hline 1517 & 0.213 & 0.04120 & 316.798 & 0.0837 & 0.01558 & 0.011 & 313.964 \\
\hline 1520 & 0.214 & 0.05210 & 317.585 & 0.0839 & 0.01586 & 0.008 & 313.968 \\
\hline 1523 & 0.218 & 0.06424 & 318.455 & 0.0849 & 0.01597 & 0.006 & 313.969 \\
\hline 1526 & 0.146 & 0.02321 & 315.526 & 0.0568 & 0.01577 & 0.024 & 313.952 \\
\hline 1529 & 0.148 & 0.03156 & 316.160 & 0.0575 & 0.01578 & 0.016 & 313.956 \\
\hline 1532 & 0.146 & 0.04118 & 316.854 & 0.0568 & 0.01565 & 0.011 & 313.934 \\
\hline 1535 & 0.142 & 0.05208 & 317.669 & 0.0551 & 0.01592 & 0.008 & 313.941 \\
\hline 1538 & 0.144 & 0.06423 & 318.566 & 0.0556 & 0.01599 & 0.006 & 313.944 \\
\hline 1556 & 0.536 & 0.02262 & 323.069 & 0.2163 & 0.01627 & 0.026 & 321.678 \\
\hline 1559 & 0.536 & 0.03076 & 323.594 & 0.2161 & 0.01619 & 0.017 & 321.682 \\
\hline 1562 & 0.537 & 0.04015 & 324.165 & 0.2158 & 0.01625 & 0.012 & 321.656 \\
\hline 1565 & 0.537 & 0.05077 & 324.847 & 0.2154 & 0.01631 & 0.008 & 321.666 \\
\hline 1568 & 0.537 & 0.06263 & 325.577 & 0.2147 & 0.01643 & 0.006 & 321.653 \\
\hline 1571 & 0.488 & 0.02262 & 323.025 & 0.1956 & 0.01614 & 0.026 & 321.650 \\
\hline 1574 & 0.490 & 0.03077 & 323.562 & 0.1960 & 0.01623 & 0.016 & 321.657 \\
\hline 1577 & 0.491 & 0.04016 & 324.164 & 0.1960 & 0.01631 & 0.011 & 321.653 \\
\hline 1580 & 0.492 & 0.05077 & 324.834 & 0.1957 & 0.01638 & 0.008 & 321.641 \\
\hline 1583 & 0.491 & 0.06262 & 325.581 & 0.1949 & 0.01649 & 0.007 & 321.636 \\
\hline 1586 & 0.422 & 0.02261 & 322.989 & 0.1671 & 0.01628 & 0.026 & 321.607 \\
\hline
\end{tabular}


Table 13. Thermal conductivity of the binary $30 \% \mathrm{R} 125$ / $70 \% \mathrm{R} 134 \mathrm{a}$ mixture in the vapor phase (continued).

\begin{tabular}{|c|c|c|c|c|c|c|c|}
\hline $\begin{array}{l}\text { Run } \\
\text { point }\end{array}$ & $\begin{array}{l}P_{\text {exp }} \\
\mathrm{MPa}\end{array}$ & $\begin{array}{c}Q Q \\
W \cdot \mathrm{m}^{-1}\end{array}$ & $\begin{array}{c}T_{e x p} \\
\mathrm{~K}\end{array}$ & $\begin{array}{c}\rho_{\text {calc }} \\
\mathrm{mol} \cdot \mathrm{L}^{-1}\end{array}$ & $\frac{\lambda_{\exp }}{\mathrm{W} \cdot \mathrm{m}^{-1} \cdot \mathrm{K}^{-1}}$ & STAT & $\begin{array}{c}T_{\text {cell }} \\
\mathrm{K}\end{array}$ \\
\hline 1589 & 0.423 & 0.03076 & 323.543 & 0.1675 & 0.01630 & 0.016 & 321.627 \\
\hline 1595 & 0.424 & 0.05078 & 324.860 & 0.1670 & 0.01637 & 0.008 & 321.640 \\
\hline 1598 & 0.423 & 0.06263 & 325.597 & 0.1661 & 0.01642 & 0.006 & 321.609 \\
\hline 1601 & 0.353 & 0.02261 & 322.960 & 0.1384 & 0.01607 & 0.025 & 321.590 \\
\hline 1604 & 0.354 & 0.03076 & 323.502 & 0.1385 & 0.01629 & 0.017 & 321.584 \\
\hline 1607 & 0.355 & 0.04013 & 324.123 & 0.1388 & 0.01641 & 0.011 & 321.584 \\
\hline 1610 & 0.355 & 0.05077 & 324.878 & 0.1382 & 0.01634 & 0.049 & 321.604 \\
\hline 1613 & 0.352 & 0.06262 & 325.627 & 0.1366 & 0.01657 & 0.006 & 321.602 \\
\hline 1616 & 0.279 & 0.02260 & 322.933 & 0.1083 & 0.01639 & 0.027 & 321.565 \\
\hline 1619 & 0.278 & 0.03075 & 323.504 & 0.1076 & 0.01629 & 0.016 & 321.580 \\
\hline 1622 & 0.278 & 0.04014 & 324.134 & 0.1075 & 0.01633 & 0.011 & 321.573 \\
\hline 1625 & 0.280 & 0.05076 & 324.856 & 0.1079 & 0.01651 & 0.009 & 321.568 \\
\hline 1628 & 0.281 & 0.06260 & 325.648 & 0.1081 & 0.01654 & 0.007 & 321.565 \\
\hline 1631 & 0.212 & 0.02260 & 322.925 & 0.0813 & 0.01642 & 0.027 & 321.534 \\
\hline 1634 & 0.210 & 0.03075 & 323.519 & 0.0805 & 0.01629 & 0.016 & 321.555 \\
\hline 1637 & 0.209 & 0.04014 & 324.177 & 0.0799 & 0.01648 & 0.012 & 321.559 \\
\hline 1640 & 0.209 & 0.05076 & 324.908 & 0.0795 & 0.01651 & 0.008 & 321.550 \\
\hline 1643 & 0.210 & 0.06259 & 325.720 & 0.0799 & 0.01661 & 0.007 & 321.546 \\
\hline 1676 & 0.538 & 0.02218 & 332.489 & 0.2095 & 0.01688 & 0.027 & 331.174 \\
\hline 1679 & 0.539 & 0.03017 & 332.982 & 0.2093 & 0.01689 & 0.017 & 331.177 \\
\hline 1682 & 0.540 & 0.03938 & 333.533 & 0.2092 & 0.01717 & 0.012 & 331.163 \\
\hline 1685 & 0.540 & 0.04981 & 334.163 & 0.2088 & 0.01712 & 0.008 & 331.157 \\
\hline 1688 & 0.540 & 0.06145 & 334.874 & 0.2082 & 0.01722 & 0.006 & 331.166 \\
\hline 1691 & 0.488 & 0.02218 & 332.451 & 0.1885 & 0.01710 & 0.026 & 331.143 \\
\hline 1694 & 0.489 & 0.03017 & 332.937 & 0.1885 & 0.01709 & 0.019 & 331.131 \\
\hline 1697 & 0.489 & 0.03938 & 333.514 & 0.1882 & 0.01708 & 0.012 & 331.136 \\
\hline 1700 & 0.489 & 0.04982 & 334.171 & 0.1876 & 0.01724 & 0.010 & 331.148 \\
\hline 1703 & 0.488 & 0.06144 & 334.880 & 0.1869 & 0.01729 & 0.007 & 331.148 \\
\hline 1706 & 0.420 & 0.02218 & 332.408 & 0.1607 & 0.01698 & 0.029 & 331.110 \\
\hline 1709 & 0.420 & 0.03017 & 332.935 & 0.1602 & 0.01716 & 0.019 & 331.122 \\
\hline 1712 & 0.420 & 0.03939 & 333.503 & 0.1599 & 0.01735 & 0.013 & 331.119 \\
\hline 1715 & 0.420 & 0.04979 & 334.161 & 0.1598 & 0.01729 & 0.009 & 331.122 \\
\hline 1718 & 0.421 & 0.06141 & 334.875 & 0.1596 & 0.01731 & 0.007 & 331.113 \\
\hline 1721 & 0.352 & 0.02217 & 332.398 & 0.1334 & 0.01702 & 0.028 & 331.107 \\
\hline 1724 & 0.352 & 0.03018 & 332.891 & 0.1330 & 0.01713 & 0.019 & 331.084 \\
\hline 1727 & 0.352 & 0.03938 & 333.485 & 0.1328 & 0.01712 & 0.013 & 331.091 \\
\hline 1730 & 0.353 & 0.04979 & 334.142 & 0.1329 & 0.01728 & 0.009 & 331.082 \\
\hline 1733 & 0.353 & 0.06141 & 334.871 & 0.1327 & 0.01734 & 0.006 & 331.074 \\
\hline 1736 & 0.282 & 0.02217 & 332.349 & 0.1059 & 0.01717 & 0.029 & 331.060 \\
\hline 1739 & 0.282 & 0.03017 & 332.892 & 0.1055 & 0.01713 & 0.018 & 331.079 \\
\hline 1742 & 0.282 & 0.03938 & 333.499 & 0.1052 & 0.01718 & 0.013 & 331.084 \\
\hline 1745 & 0.282 & 0.04978 & 334.155 & 0.1054 & 0.01732 & 0.009 & 331.061 \\
\hline 1748 & 0.283 & 0.06141 & 334.905 & 0.1054 & 0.01742 & 0.007 & 331.060 \\
\hline
\end{tabular}


Table 13. Thermal conductivity of the binary $30 \% \mathrm{R} 125 / 70 \% \mathrm{R} 134 \mathrm{a}$ mixture in the vapor phase (continued).

\begin{tabular}{|c|c|c|c|c|c|c|c|}
\hline $\begin{array}{l}\text { Run } \\
\text { point }\end{array}$ & $\begin{array}{l}P_{\text {exp }} \\
\mathrm{MPa}\end{array}$ & $\begin{array}{c}Q \\
\mathrm{~W} \cdot \mathrm{m}^{-1}\end{array}$ & $\begin{array}{c}T_{\exp } \\
\mathrm{K}\end{array}$ & $\begin{array}{c}\rho_{\text {calc }} \\
\mathrm{mol} \cdot \mathrm{L}^{-1}\end{array}$ & $\begin{array}{c}\lambda_{\exp } \\
\mathrm{W} \cdot \mathrm{m}^{-1} \cdot \mathrm{K}^{-1}\end{array}$ & STAT & $\begin{array}{c}T_{\text {cell }} \\
\mathrm{K}\end{array}$ \\
\hline 1751 & 0.214 & 0.02217 & 332.359 & 0.0794 & 0.01720 & 0.029 & 331.064 \\
\hline 1754 & 0.212 & 0.03017 & 332.903 & 0.0788 & 0.01719 & 0.019 & 331.068 \\
\hline 1757 & 0.213 & 0.03937 & 333.504 & 0.0787 & 0.01726 & 0.012 & 331.051 \\
\hline 1760 & 0.213 & 0.04977 & 334.192 & 0.0789 & 0.01740 & 0.010 & 331.039 \\
\hline 1763 & 0.214 & 0.06140 & 334.965 & 0.0789 & 0.01755 & 0.008 & 331.044 \\
\hline 1796 & 0.536 & 0.02154 & 343.614 & 0.2001 & 0.01754 & 0.030 & 341.500 \\
\hline 1799 & 0.534 & 0.02931 & 344.088 & 0.1988 & 0.01759 & 0.020 & 341.505 \\
\hline 1802 & 0.534 & 0.03825 & 344.600 & 0.1985 & 0.01798 & 0.013 & 341.488 \\
\hline 1805 & 0.536 & 0.04838 & 345.192 & 0.1987 & 0.01784 & 0.009 & 341.493 \\
\hline 1808 & 0.536 & 0.05968 & 345.876 & 0.1985 & 0.01804 & 0.007 & 341.517 \\
\hline 1811 & 0.491 & 0.02154 & 343.573 & 0.1821 & 0.01782 & 0.030 & 341.477 \\
\hline 1814 & 0.491 & 0.02931 & 344.042 & 0.1819 & 0.01777 & 0.020 & 341.475 \\
\hline 1817 & 0.489 & 0.03825 & 344.580 & 0.1808 & 0.01790 & 0.013 & 341.476 \\
\hline 1820 & 0.489 & 0.04838 & 345.166 & 0.1804 & 0.01809 & 0.010 & 341.463 \\
\hline 1823 & 0.491 & 0.05968 & 345.814 & 0.1806 & 0.01813 & 0.007 & 341.446 \\
\hline 1826 & 0.421 & 0.02155 & 343.351 & 0.1549 & 0.01759 & 0.030 & 341.313 \\
\hline 1829 & 0.421 & 0.02932 & 343.854 & 0.1545 & 0.01788 & 0.021 & 341.339 \\
\hline 1832 & 0.421 & 0.03827 & 344.391 & 0.1542 & 0.01771 & 0.014 & 341.331 \\
\hline 1835 & 0.421 & 0.04840 & 345.001 & 0.1539 & 0.01810 & 0.010 & 341.335 \\
\hline 1838 & 0.421 & 0.05970 & 345.674 & 0.1537 & 0.01812 & 0.007 & 341.330 \\
\hline 1841 & 0.352 & 0.02155 & 343.318 & 0.1283 & 0.01785 & 0.032 & 341.290 \\
\hline 1844 & 0.352 & 0.02932 & 343.816 & 0.1281 & 0.01812 & 0.021 & 341.304 \\
\hline 1847 & 0.351 & 0.03827 & 344.350 & 0.1277 & 0.01800 & 0.014 & 341.283 \\
\hline 1850 & 0.351 & 0.04840 & 344.977 & 0.1273 & 0.01810 & 0.010 & 341.293 \\
\hline 1856 & 0.283 & 0.02155 & 343.285 & 0.1025 & 0.01807 & 0.032 & 341.268 \\
\hline 1859 & 0.283 & 0.02932 & 343.788 & 0.1022 & 0.01822 & 0.021 & 341.274 \\
\hline 1862 & 0.282 & 0.03827 & 344.341 & 0.1018 & 0.01822 & 0.014 & 341.264 \\
\hline 1865 & 0.282 & 0.04839 & 344.977 & 0.1015 & 0.01821 & 0.010 & 341.269 \\
\hline 1868 & 0.282 & 0.05968 & 345.664 & 0.1012 & 0.01831 & 0.008 & 341.255 \\
\hline 1871 & 0.214 & 0.02155 & 343.245 & 0.0770 & 0.01807 & 0.031 & 341.230 \\
\hline 1874 & 0.215 & 0.02931 & 343.766 & 0.0769 & 0.01818 & 0.021 & 341.246 \\
\hline 1877 & 0.214 & 0.03826 & 344.333 & 0.0767 & 0.01832 & 0.014 & 341.236 \\
\hline 1880 & 0.214 & 0.04839 & 344.986 & 0.0764 & 0.01823 & 0.010 & 341.240 \\
\hline 1883 & 0.213 & 0.05968 & 345.697 & 0.0760 & 0.01837 & 0.007 & 341.234 \\
\hline
\end{tabular}


Table 14. Thermal conductivity of the binary $70 \% \mathrm{R} 125$ / $30 \% \mathrm{R} 134 \mathrm{a}$ mixture in the vapor phase.

\begin{tabular}{|c|c|c|c|c|c|c|c|}
\hline $\begin{array}{l}\text { Run } \\
\text { point }\end{array}$ & $\begin{array}{l}P_{\text {exp }} \\
\mathrm{MPa}\end{array}$ & $\begin{array}{c}Q \\
\mathrm{~W} \cdot \mathrm{m}^{-1}\end{array}$ & $\begin{array}{c}T_{\exp } \\
\mathrm{K}\end{array}$ & $\begin{array}{c}\rho_{\text {calc }} \\
\mathrm{mol} \cdot \mathrm{L}^{-1}\end{array}$ & $\begin{array}{c}\lambda_{\exp } \\
\mathrm{W} \cdot \mathrm{m}^{-1} \cdot \mathrm{K}^{-1}\end{array}$ & STAT & $\begin{array}{c}T_{\text {cell }} \\
\mathrm{K}\end{array}$ \\
\hline 1001 & 0.088 & 0.02837 & 259.583 & 0.0418 & 0.01093 & 0.009 & 256.661 \\
\hline 1004 & 0.089 & 0.03856 & 260.646 & 0.0418 & 0.01102 & 0.007 & 256.668 \\
\hline 1007 & 0.090 & 0.05026 & 261.838 & 0.0423 & 0.01117 & 0.006 & 256.665 \\
\hline 1010 & 0.091 & 0.06348 & 263.169 & 0.0424 & 0.01125 & 0.004 & 256.659 \\
\hline 1013 & 0.089 & 0.07821 & 264.660 & 0.0416 & 0.01134 & 0.004 & 256.676 \\
\hline 1016 & 0.178 & 0.02838 & 259.413 & 0.0867 & 0.01102 & 0.012 & 256.682 \\
\hline 1019 & 0.177 & 0.03856 & 260.431 & 0.0859 & 0.01106 & 0.007 & 256.711 \\
\hline 1022 & 0.175 & 0.05028 & 261.527 & 0.0846 & 0.01116 & 0.005 & 256.688 \\
\hline 1025 & 0.176 & 0.06352 & 262.806 & 0.0845 & 0.01122 & 0.003 & 256.710 \\
\hline 1028 & 0.177 & 0.07825 & 264.185 & 0.0847 & 0.01133 & 0.003 & 256.698 \\
\hline 1031 & 0.180 & 0.02796 & 263.712 & 0.0861 & 0.01134 & 0.010 & 261.195 \\
\hline 1034 & 0.180 & 0.03801 & 264.443 & 0.0860 & 0.01145 & 0.008 & 261.051 \\
\hline 1037 & 0.179 & 0.04957 & 265.334 & 0.0852 & 0.01147 & 0.005 & 260.933 \\
\hline 1040 & 0.178 & 0.06266 & 266.346 & 0.0841 & 0.01160 & 0.004 & 260.804 \\
\hline 1043 & 0.180 & 0.07721 & 267.499 & 0.0845 & 0.01167 & 0.003 & 260.683 \\
\hline 1046 & 0.181 & 0.02783 & 264.697 & 0.0861 & 0.01145 & 0.010 & 262.216 \\
\hline 1049 & 0.180 & 0.03785 & 265.352 & 0.0857 & 0.01153 & 0.007 & 262.034 \\
\hline 1052 & 0.180 & 0.04937 & 266.158 & 0.0852 & 0.01161 & 0.005 & 261.859 \\
\hline 1055 & 0.181 & 0.06239 & 267.112 & 0.0852 & 0.01168 & 0.004 & 261.707 \\
\hline 1058 & 0.180 & 0.07692 & 268.203 & 0.0844 & 0.01180 & 0.003 & 261.556 \\
\hline 1061 & 0.092 & 0.02774 & 265.546 & 0.0427 & 0.01148 & 0.010 & 262.831 \\
\hline 1064 & 0.093 & 0.03769 & 266.486 & 0.0431 & 0.01150 & 0.007 & 262.793 \\
\hline 1067 & 0.096 & 0.04914 & 267.590 & 0.0440 & 0.01162 & 0.005 & 262.778 \\
\hline 1070 & 0.096 & 0.06207 & 268.807 & 0.0441 & 0.01174 & 0.004 & 262.749 \\
\hline 1073 & 0.096 & 0.07646 & 270.167 & 0.0439 & 0.01183 & 0.003 & 262.722 \\
\hline 1076 & 0.091 & 0.02774 & 265.609 & 0.0422 & 0.01151 & 0.011 & 262.878 \\
\hline 1079 & 0.091 & 0.03769 & 266.615 & 0.0421 & 0.01157 & 0.007 & 262.890 \\
\hline 1082 & 0.091 & 0.04915 & 267.725 & 0.0420 & 0.01165 & 0.005 & 262.872 \\
\hline 1085 & 0.092 & 0.06207 & 268.983 & 0.0419 & 0.01175 & 0.004 & 262.871 \\
\hline 1088 & 0.092 & 0.07648 & 270.380 & 0.0419 & 0.01185 & 0.003 & 262.873 \\
\hline 1091 & 0.258 & 0.02775 & 265.358 & 0.1252 & 0.01140 & 0.012 & 262.868 \\
\hline 1094 & 0.257 & 0.03772 & 266.248 & 0.1243 & 0.01159 & 0.008 & 262.870 \\
\hline 1097 & 0.257 & 0.04919 & 267.264 & 0.1239 & 0.01165 & 0.005 & 262.871 \\
\hline 1100 & 0.258 & 0.06214 & 268.388 & 0.1236 & 0.01170 & 0.004 & 262.858 \\
\hline 1103 & 0.257 & 0.07657 & 269.644 & 0.1224 & 0.01177 & 0.003 & 262.854 \\
\hline 1106 & 0.264 & 0.02718 & 270.713 & 0.1254 & 0.01192 & 0.012 & 268.379 \\
\hline 1109 & 0.265 & 0.03695 & 271.586 & 0.1253 & 0.01196 & 0.008 & 268.406 \\
\hline 1112 & 0.263 & 0.04820 & 272.534 & 0.1240 & 0.01205 & 0.006 & 268.388 \\
\hline 1115 & 0.263 & 0.06091 & 273.618 & 0.1234 & 0.01215 & 0.004 & 268.383 \\
\hline 1118 & 0.265 & 0.07504 & 274.796 & 0.1233 & 0.01220 & 0.004 & 268.356 \\
\hline 1121 & 0.174 & 0.02718 & 270.744 & 0.0808 & 0.01185 & 0.011 & 268.307 \\
\hline 1124 & 0.175 & 0.03694 & 271.636 & 0.0810 & 0.01192 & 0.008 & 268.313 \\
\hline 1127 & 0.176 & 0.04817 & 272.638 & 0.0811 & 0.01204 & 0.005 & 268.303 \\
\hline 1130 & 0.177 & 0.06087 & 273.763 & 0.0810 & 0.01212 & 0.004 & 268.295 \\
\hline
\end{tabular}


Table 14. Thermal conductivity of the binary $70 \% \mathrm{R} 125 / 30 \% \mathrm{R} 134 \mathrm{a}$ mixture in the vapor phase (continued).

\begin{tabular}{|c|c|c|c|c|c|c|c|}
\hline $\begin{array}{l}\text { Run } \\
\text { point }\end{array}$ & $\begin{array}{l}P_{\text {exp }} \\
\mathrm{MPa}\end{array}$ & $\begin{array}{c}Q \\
\mathrm{~W} \cdot \mathrm{m}^{-1}\end{array}$ & $\begin{array}{c}T_{\exp } \\
\mathrm{K}\end{array}$ & $\begin{array}{c}\rho_{\text {calc }} \\
\mathrm{mol} \cdot \mathrm{L}^{-1}\end{array}$ & $\begin{array}{c}\lambda_{\exp } \\
\mathrm{W} \cdot \mathrm{m}^{-1} \cdot \mathrm{K}^{-1}\end{array}$ & STAT & $\begin{array}{c}T_{\text {cell }} \\
\mathrm{K}\end{array}$ \\
\hline 1133 & 0.175 & 0.07501 & 275.027 & 0.0795 & 0.01220 & 0.003 & 268.304 \\
\hline 1136 & 0.326 & 0.02718 & 270.563 & 0.1578 & 0.01187 & 0.012 & 268.277 \\
\hline 1139 & 0.326 & 0.03695 & 271.401 & 0.1570 & 0.01204 & 0.007 & 268.280 \\
\hline 1142 & 0.326 & 0.04819 & 272.339 & 0.1563 & 0.01210 & 0.005 & 268.278 \\
\hline 1145 & 0.326 & 0.06090 & $273: 409$ & 0.1554 & 0.01215 & 0.004 & 268.287 \\
\hline 1148 & 0.326 & 0.07505 & 274.574 & 0.1546 & 0.01226 & 0.003 & 268.278 \\
\hline 1151 & 0.092 & 0.02625 & 280.251 & 0.0401 & 0.01284 & 0.015 & 277.941 \\
\hline 1154 & 0.092 & 0.03569 & 280.992 & 0.0399 & 0.01277 & 0.010 & 277.870 \\
\hline 1157 & 0.092 & 0.02628 & 280.008 & 0.0403 & 0.01275 & 0.014 & 277.680 \\
\hline 1158 & 0.093 & 0.02618 & 280.660 & 0.0407 & 0.01275 & 0.014 & 278.299 \\
\hline 1161 & 0.093 & 0.03559 & 281.527 & 0.0407 & 0.01290 & 0.009 & 278.307 \\
\hline 1164 & 0.094 & 0.04642 & 282.489 & 0.0409 & 0.01298 & 0.007 & 278.293 \\
\hline 1167 & 0.095 & 0.05865 & 283.592 & 0.0409 & 0.01302 & 0.005 & 278.299 \\
\hline 1170 & 0.094 & 0.07228 & 284.801 & 0.0406 & 0.01314 & 0.004 & 278.298 \\
\hline 1173 & 0.196 & 0.02619 & 280.458 & 0.0876 & 0.01273 & 0.014 & 278.260 \\
\hline 1176 & 0.196 & 0.03561 & 281.261 & 0.0874 & 0.01261 & 0.009 & 278.258 \\
\hline 1179 & 0.196 & 0.04643 & 282.168 & 0.0871 & 0.01276 & 0.007 & 278.246 \\
\hline 1182 & 0.196 & 0.05868 & 283.206 & 0.0865 & 0.01290 & 0.005 & 278.252 \\
\hline 1185 & 0.195 & 0.07235 & 284.359 & 0.0857 & 0.01302 & 0.004 & 278.264 \\
\hline 1188 & 0.325 & 0.02620 & 280.331 & 0.1498 & 0.01276 & 0.014 & 278.230 \\
\hline 1191 & 0.324 & 0.03562 & 281.108 & 0.1489 & 0.01261 & 0.009 & 278.238 \\
\hline 1194 & 0.324 & 0.04645 & 281.976 & 0.1486 & 0.01284 & 0.006 & 278.231 \\
\hline 1197 & 0.325 & 0.05872 & 282.954 & 0.1482 & 0.01291 & 0.005 & 278.228 \\
\hline 1200 & 0.326 & 0.07240 & 284.058 & 0.1477 & 0.01302 & 0.004 & 278.248 \\
\hline 1203 & 0.463 & 0.02620 & 280.209 & 0.2219 & 0.01269 & 0.013 & 278.204 \\
\hline 1206 & 0.463 & 0.03563 & 280.944 & 0.2208 & 0.01285 & 0.008 & 278.218 \\
\hline 1209 & 0.463 & 0.04649 & 281.777 & 0.2196 & 0.01287 & 0.006 & 278.217 \\
\hline 1212 & 0.463 & 0.05874 & 282.705 & 0.2187 & 0.01302 & 0.004 & 278.215 \\
\hline 1215 & 0.464 & 0.07242 & 283.715 & 0.2180 & 0.01312 & 0.003 & 278.198 \\
\hline 1218 & 0.549 & 0.02622 & 280.047 & 0.2694 & 0.01288 & 0.014 & 278.101 \\
\hline 1221 & 0.548 & 0.03564 & 280.754 & 0.2681 & 0.01281 & 0.008 & 278.105 \\
\hline 1224 & 0.548 & 0.04650 & 281.553 & 0.2667 & 0.01298 & 0.007 & 278.089 \\
\hline 1227 & 0.548 & 0.05876 & 282.457 & 0.2652 & 0.01294 & 0.005 & 278.081 \\
\hline 1230 & 0.547 & 0.07244 & 283.470 & 0.2636 & 0.01310 & 0.003 & 278.085 \\
\hline 1233 & 0.570 & 0.02539 & 289.097 & 0.2680 & 0.01344 & 0.014 & 287.316 \\
\hline 1236 & 0.570 & 0.03453 & 289.750 & 0.2668 & 0.01358 & 0.010 & 287.311 \\
\hline 1239 & 0.570 & 0.04505 & 290.501 & 0.2657 & 0.01364 & 0.006 & 287.307 \\
\hline 1242 & 0.571 & 0.05694 & 291.348 & 0.2650 & 0.01374 & 0.004 & 287.308 \\
\hline 1245 & 0.571 & 0.07021 & 292.285 & 0.2642 & 0.01385 & 0.004 & 287.309 \\
\hline 1248 & 0.448 & 0.02539 & 289.167 & 0.2043 & 0.01348 & 0.015 & 287.306 \\
\hline 1251 & 0.448 & 0.03452 & 289.844 & 0.2038 & 0.01354 & 0.010 & 287.299 \\
\hline 1254 & 0.449 & 0.04504 & 290.629 & 0.2033 & 0.01354 & 0.007 & 287.301 \\
\hline 1257 & 0.449 & 0.05693 & 291.497 & 0.2026 & 0.01362 & 0.005 & 287.291 \\
\hline
\end{tabular}


Table 14. Thermal conductivity of the binary $70 \% \mathrm{R} 125 / 30 \% \mathrm{R} 134 \mathrm{a}$ mixture in the vapor phase (continued).

\begin{tabular}{|c|c|c|c|c|c|c|c|}
\hline $\begin{array}{l}\text { Run } \\
\text { point }\end{array}$ & $\begin{array}{l}P_{\text {exp }} \\
\mathrm{MPa}\end{array}$ & $\begin{array}{c}Q \\
\mathrm{~W} \cdot \mathrm{m}^{-1}\end{array}$ & $\begin{array}{c}T_{\exp } \\
\mathrm{K}\end{array}$ & $\begin{array}{c}\rho_{\text {calc }} \\
\mathrm{mol} \cdot \mathrm{L}^{-1}\end{array}$ & $\begin{array}{c}\lambda_{\exp } \\
\mathrm{W} \cdot \mathrm{m}^{-1} \cdot \mathrm{K}^{-1}\end{array}$ & STAT & $\begin{array}{c}T_{\text {cell }} \\
\mathrm{K}\end{array}$ \\
\hline 1260 & 0.449 & 0.07018 & 292.487 & 0.2017 & 0.01368 & 0.004 & 287.312 \\
\hline 1263 & 0.319 & 0.02538 & 289.214 & 0.1413 & 0.01360 & 0.018 & 287.272 \\
\hline 1266 & 0.320 & 0.03451 & 289.936 & 0.1412 & 0.01353 & 0.011 & 287.279 \\
\hline 1269 & 0.320 & 0.04503 & 290.754 & 0.1407 & 0.01350 & 0.008 & 287.283 \\
\hline 1272 & 0.320 & 0.05692 & 291.664 & 0.1401 & 0.01361 & 0.005 & 287.283 \\
\hline 1275 & 0.319 & 0.07017 & 292.690 & 0.1393 & 0.01367 & 0.005 & 287.298 \\
\hline 1278 & 0.188 & 0.02539 & 289.261 & 0.0809 & 0.01346 & 0.016 & 287.222 \\
\hline 1281 & 0.188 & 0.03452 & 290.019 & 0.0807 & 0.01344 & 0.011 & 287.231 \\
\hline 1284 & 0.188 & 0.04504 & 290.868 & 0.0804 & 0.01362 & 0.007 & 287.219 \\
\hline 1287 & 0.188 & 0.05692 & 291.836 & 0.0801 & 0.01366 & 0.005 & 287.225 \\
\hline 1290 & 0.187 & 0.07015 & 292.891 & 0.0795 & 0.01370 & 0.004 & 287.217 \\
\hline 1323 & 0.241 & 0.02423 & 303.095 & 0.0993 & 0.01457 & 0.018 & 301.311 \\
\hline 1326 & 0.241 & 0.03295 & 303.733 & 0.0991 & 0.01458 & 0.012 & 301.293 \\
\hline 1329 & 0.241 & 0.04299 & 304.497 & 0.0990 & 0.01475 & 0.008 & 301.307 \\
\hline 1332 & 0.242 & 0.05436 & 305.331 & 0.0989 & 0.01483 & 0.006 & 301.300 \\
\hline 1335 & 0.242 & 0.06703 & 306.262 & 0.0985 & 0.01489 & 0.005 & 301.298 \\
\hline 1338 & 0.458 & 0.02424 & 302.948 & 0.1965 & 0.01465 & 0.018 & 301.281 \\
\hline 1341 & 0.457 & 0.03297 & 303.563 & 0.1958 & 0.01467 & 0.012 & 301.285 \\
\hline 1344 & 0.457 & 0.04302 & 304.274 & 0.1950 & 0.01472 & 0.009 & 301.295 \\
\hline 1347 & 0.456 & 0.05439 & 305.048 & 0.1940 & 0.01478 & 0.006 & 301.286 \\
\hline 1350 & 0.456 & 0.06707 & 305.916 & 0.1933 & 0.01488 & 0.005 & 301.279 \\
\hline 1353 & 0.600 & 0.02425 & 301.876 & 0.2661 & 0.01448 & 0.018 & 300.272 \\
\hline 1356 & 0.600 & 0.03298 & 302.477 & 0.2656 & 0.01466 & 0.011 & 300.281 \\
\hline 1359 & 0.601 & 0.04303 & 303.153 & 0.2649 & 0.01461 & 0.008 & 300.276 \\
\hline 1362 & 0.602 & 0.05440 & 303.901 & 0.2645 & 0.01468 & 0.007 & 300.266 \\
\hline 1365 & 0.602 & 0.06708 & 304.735 & 0.2635 & 0.01479 & 0.005 & 300.257 \\
\hline 1368 & 0.627 & 0.02328 & 314.617 & 0.2637 & 0.01557 & 0.021 & 313.177 \\
\hline 1371 & 0.627 & 0.03167 & 315.133 & 0.2632 & 0.01576 & 0.013 & 313.159 \\
\hline 1374 & 0.628 & 0.04134 & 315.728 & 0.2628 & 0.01570 & 0.009 & 313.141 \\
\hline 1377 & 0.628 & 0.05227 & 316.428 & 0.2623 & 0.01584 & 0.007 & 313.148 \\
\hline 1380 & 0.628 & 0.06446 & 317.175 & 0.2614 & 0.01585 & 0.005 & 313.129 \\
\hline 1383 & 0.475 & 0.02338 & 313.305 & 0.1957 & 0.01545 & 0.022 & 311.790 \\
\hline 1386 & 0.475 & 0.03179 & 313.876 & 0.1954 & 0.01549 & 0.015 & 311.800 \\
\hline 1389 & 0.476 & 0.04149 & 314.496 & 0.1951 & 0.01538 & 0.010 & 311.782 \\
\hline 1392 & 0.476 & 0.05246 & 315.221 & 0.1946 & 0.01571 & 0.007 & 311.785 \\
\hline 1395 & 0.475 & 0.06470 & 316.005 & 0.1937 & 0.01576 & 0.006 & 311.769 \\
\hline 1398 & 0.290 & 0.02339 & 313.305 & 0.1160 & 0.01565 & 0.022 & 311.705 \\
\hline 1401 & 0.290 & 0.03181 & 313.897 & 0.1158 & 0.01556 & 0.014 & 311.704 \\
\hline 1404 & 0.290 & 0.04151 & 314.557 & 0.1155 & 0.01540 & 0.010 & 311.691 \\
\hline 1407 & 0.290 & 0.05248 & 315.320 & 0.1150 & 0.01565 & 0.007 & 311.692 \\
\hline 1410 & 0.290 & 0.06473 & 316.141 & 0.1149 & 0.01574 & 0.006 & 311.669 \\
\hline 1413 & 0.173 & 0.02339 & 313.280 & 0.0681 & 0.01567 & 0.022 & 311.607 \\
\hline 1416 & 0.173 & 0.03181 & 313.890 & 0.0679 & 0.01574 & 0.015 & 311.599 \\
\hline
\end{tabular}


Table 14. Thermal conductivity of the binary $70 \% \mathrm{R} 125 / 30 \% \mathrm{R} 134 \mathrm{a}$ mixture in the vapor phase (continued).

\begin{tabular}{|c|c|c|c|c|c|c|c|}
\hline $\begin{array}{l}\text { Run } \\
\text { point }\end{array}$ & $\begin{array}{l}P_{\text {exp }} \\
\mathrm{MPa}\end{array}$ & $\begin{array}{c}Q \\
\mathrm{~W} \cdot \mathrm{m}^{-1}\end{array}$ & $\begin{array}{c}T_{\exp } \\
\mathrm{K}\end{array}$ & $\begin{array}{c}\rho_{\text {calc }} \\
\mathrm{mol} \cdot \mathrm{L}^{-1}\end{array}$ & $\frac{\lambda_{\exp }}{\mathrm{W} \cdot \mathrm{m}^{-1} \cdot \mathrm{K}^{-1}}$ & STAT & $\begin{array}{c}T_{\text {cell }} \\
\mathrm{K}\end{array}$ \\
\hline 1419 & 0.173 & 0.04151 & 314.603 & 0.0677 & 0.01574 & 0.010 & 311.600 \\
\hline 1422 & 0.172 & 0.05249 & 315.386 & 0.0673 & 0.01579 & 0.007 & 311.590 \\
\hline 1425 & 0.172 & 0.06473 & 316.265 & 0.0670 & 0.01586 & 0.006 & 311.587 \\
\hline 1458 & 0.183 & 0.02283 & 321.005 & 0.0700 & 0.01647 & 0.024 & 319.444 \\
\hline 1461 & 0.182 & 0.03106 & 321.575 & 0.0696 & 0.01643 & 0.016 & 319.437 \\
\hline 1464 & 0.182 & 0.04052 & 322.209 & 0.0695 & 0.01623 & 0.011 & 319.409 \\
\hline 1467 & 0.182 & 0.05124 & 322.960 & 0.0693 & 0.01644 & 0.008 & 319.416 \\
\hline 1470 & 0.182 & 0.06321 & 323.775 & 0.0691 & 0.01658 & 0.007 & 319.407 \\
\hline 1473 & 0.268 & 0.02278 & 319.602 & 0.1045 & 0.01586 & 0.022 & 318.074 \\
\hline 1476 & 0.268 & 0.03099 & 320.134 & 0.1042 & 0.01620 & 0.014 & 318.048 \\
\hline 1479 & 0.268 & 0.04044 & 320.773 & 0.1041 & 0.01612 & 0.010 & 318.044 \\
\hline 1482 & 0.268 & 0.05113 & 321.505 & 0.1038 & 0.01642 & 0.007 & 318.048 \\
\hline 1485 & 0.268 & 0.06306 & 322.264 & 0.1034 & 0.01642 & 0.005 & 318.005 \\
\hline 1488 & 0.345 & 0.02279 & 319.429 & 0.1364 & 0.01584 & 0.023 & 317.929 \\
\hline 1491 & 0.345 & 0.03100 & 319.960 & 0.1360 & 0.01606 & 0.015 & 317.914 \\
\hline 1494 & 0.345 & 0.04046 & 320.570 & 0.1356 & 0.01620 & 0.011 & 317.897 \\
\hline 1497 & 0.345 & 0.05115 & 321.273 & 0.1351 & 0.01619 & 0.009 & 317.898 \\
\hline 1500 & 0.345 & 0.06309 & 322.015 & 0.1350 & 0.01619 & 0.006 & 317.864 \\
\hline 1503 & 0.485 & 0.02280 & 319.233 & 0.1955 & 0.01604 & 0.023 & 317.798 \\
\hline 1506 & 0.485 & 0.03101 & 319.766 & 0.1951 & 0.01581 & 0.014 & 317.798 \\
\hline 1509 & 0.484 & 0.04048 & 320.355 & 0.1944 & 0.01617 & 0.010 & 317.778 \\
\hline 1512 & 0.484 & 0.05118 & 321.047 & 0.1940 & 0.01614 & 0.007 & 317.784 \\
\hline 1515 & 0.484 & 0.06313 & 321.790 & 0.1933 & 0.01626 & 0.006 & 317.772 \\
\hline 1518 & 0.601 & 0.02280 & 318.957 & 0.2473 & 0.01554 & 0.021 & 317.559 \\
\hline 1521 & 0.602 & 0.03102 & 319.485 & 0.2468 & 0.01616 & 0.014 & 317.574 \\
\hline 1524 & 0.601 & 0.04048 & 320.038 & 0.2461 & 0.01627 & 0.010 & 317.541 \\
\hline 1527 & 0.601 & 0.05118 & 320.694 & 0.2454 & 0.01621 & 0.007 & 317.535 \\
\hline 1530 & 0.601 & 0.06313 & 321.420 & 0.2447 & 0.01628 & 0.006 & 317.525 \\
\hline 1533 & 0.620 & 0.02204 & 329.991 & 0.2444 & 0.01729 & 0.026 & 328.722 \\
\hline 1536 & 0.618 & 0.02998 & 330.465 & 0.2431 & 0.01707 & 0.017 & 328.727 \\
\hline 1539 & 0.617 & 0.03912 & 330.977 & 0.2421 & 0.01710 & 0.013 & 328.699 \\
\hline 1542 & 0.619 & 0.04949 & 331.552 & 0.2424 & 0.01719 & 0.008 & 328.664 \\
\hline 1545 & 0.621 & 0.06105 & 332.203 & 0.2428 & 0.01708 & 0.006 & 328.640 \\
\hline 1548 & 0.464 & 0.02207 & 329.620 & 0.1791 & 0.01709 & 0.027 & 328.295 \\
\hline 1551 & 0.464 & 0.03001 & 330.074 & 0.1790 & 0.01718 & 0.017 & 328.262 \\
\hline 1554 & 0.466 & 0.03917 & 330.630 & 0.1795 & 0.01712 & 0.012 & 328.258 \\
\hline 1557 & 0.468 & 0.04955 & 331.226 & 0.1798 & 0.01720 & 0.008 & 328.221 \\
\hline 1560 & 0.468 & 0.06112 & 331.932 & 0.1795 & 0.01709 & 0.006 & 328.223 \\
\hline 1563 & 0.322 & 0.02207 & 329.450 & 0.1222 & 0.01705 & 0.027 & 328.069 \\
\hline 1566 & 0.325 & 0.03002 & 329.960 & 0.1231 & 0.01713 & 0.017 & 328.074 \\
\hline 1569 & 0.326 & 0.03920 & 330.530 & 0.1233 & 0.01694 & 0.011 & 328.061 \\
\hline 1572 & 0.325 & 0.04957 & 331.175 & 0.1229 & 0.01696 & 0.009 & 328.040 \\
\hline 1575 & 0.324 & 0.06113 & 331.888 & 0.1219 & 0.01716 & 0.007 & 328.023 \\
\hline
\end{tabular}


Table 14. Thermal conductivity of the binary $70 \% \mathrm{R} 125 / 30 \% \mathrm{R} 134 \mathrm{a}$ mixture in the vapor phase (continued).

\begin{tabular}{|c|c|c|c|c|c|c|c|}
\hline $\begin{array}{l}\text { Run } \\
\text { point }\end{array}$ & $\begin{array}{l}P_{\text {exp }} \\
\mathrm{MPa}\end{array}$ & $\begin{array}{c}Q \\
W \cdot m^{-1}\end{array}$ & $\begin{array}{c}T_{\text {exp }} \\
\mathrm{K}\end{array}$ & $\begin{array}{c}\rho_{\text {calc }} \\
\mathrm{mol} \cdot \mathrm{L}^{-1}\end{array}$ & $\begin{array}{c}\lambda_{\text {exp }} \\
\mathrm{W} \cdot \mathrm{m}^{-1} \cdot \mathrm{K}^{-1}\end{array}$ & STAT & $\begin{array}{c}T_{\text {cell }} \\
\mathrm{K}\end{array}$ \\
\hline 1578 & 0.211 & 0.02208 & 329.355 & 0.0789 & 0.01673 & 0.027 & 327.924 \\
\hline 1581 & 0.211 & 0.03004 & 329.868 & 0.0789 & 0.01719 & 0.017 & 327.905 \\
\hline 1584 & 0.211 & 0.03921 & 330.472 & 0.0786 & 0.01737 & 0.011 & 327.902 \\
\hline 1587 & 0.209 & 0.04959 & 331.138 & 0.0780 & 0.01722 & 0.009 & 327.885 \\
\hline 1590 & 0.207 & 0.06116 & 331.883 & 0.0769 & 0.01732 & 0.006 & 327.870 \\
\hline 1648 & 0.204 & 0.02152 & 341.820 & 0.0732 & 0.01848 & 0.031 & 340.528 \\
\hline 1651 & 0.204 & 0.02928 & 342.276 & 0.0732 & 0.01823 & 0.019 & 340.496 \\
\hline 1654 & 0.204 & 0.03822 & 342.787 & 0.0732 & 0.01815 & 0.013 & 340.447 \\
\hline 1657 & 0.205 & 0.04833 & 343.407 & 0.0735 & 0.01852 & 0.009 & 340.436 \\
\hline 1660 & 0.206 & 0.05962 & 344.101 & 0.0736 & 0.01856 & 0.007 & 340.426 \\
\hline 1663 & 0.205 & 0.04832 & 343.345 & 0.0732 & 0.01857 & 0.009 & 340.321 \\
\hline 1666 & 0.204 & 0.05961 & 344.030 & 0.0728 & 0.01861 & 0.007 & 340.307 \\
\hline 1669 & 0.347 & 0.02149 & 341.156 & 0.1269 & 0.01782 & 0.030 & 339.909 \\
\hline 1672 & 0.348 & 0.02924 & 341.597 & 0.1271 & 0.01813 & 0.019 & 339.885 \\
\hline 1675 & 0.349 & 0.03817 & 342.115 & 0.1274 & 0.01817 & 0.013 & 339.869 \\
\hline 1678 & 0.349 & 0.04826 & 342.677 & 0.1272 & 0.01820 & 0.009 & 339.840 \\
\hline 1681 & 0.349 & 0.05954 & 343.318 & 0.1267 & 0.01839 & 0.007 & 339.812 \\
\hline 1687 & 0.348 & 0.05951 & 343.203 & 0.1264 & 0.01837 & 0.007 & 339.634 \\
\hline 1690 & 0.484 & 0.02148 & 341.241 & 0.1797 & 0.01803 & 0.030 & 340.031 \\
\hline 1693 & 0.483 & 0.02922 & 341.664 & 0.1791 & 0.01806 & 0.019 & 340.009 \\
\hline 1696 & 0.482 & 0.03814 & 342.168 & 0.1786 & 0.01812 & 0.012 & 340.001 \\
\hline 1699 & 0.483 & 0.04823 & 342.758 & 0.1785 & 0.01796 & 0.009 & 339.972 \\
\hline 1702 & 0.484 & 0.05950 & 343.363 & 0.1784 & 0.01830 & 0.007 & 339.930 \\
\hline 1705 & 0.599 & 0.02150 & 340.814 & 0.2260 & 0.01781 & 0.030 & 339.629 \\
\hline 1708 & 0.598 & 0.02925 & 341.248 & 0.2253 & 0.01810 & 0.019 & 339.621 \\
\hline 1711 & 0.598 & 0.03818 & 341.745 & 0.2246 & 0.01812 & 0.013 & 339.612 \\
\hline 1714 & 0.597 & 0.04828 & 342.269 & 0.2239 & 0.01789 & 0.009 & 339.565 \\
\hline 1717 & 0.597 & 0.04829 & 342.264 & 0.2240 & 0.01779 & 0.010 & 339.535 \\
\hline 1720 & 0.598 & 0.05958 & 342.875 & 0.2240 & 0.01815 & 0.007 & 339.510 \\
\hline
\end{tabular}


Table 15 . Thermal conductivity of the binary $30 \% \mathrm{R} 32 / 70 \%$ propane mixture in the vapor phase.

\begin{tabular}{|c|c|c|c|c|c|c|c|}
\hline $\begin{array}{c}\text { Run } \\
\text { point }\end{array}$ & $\begin{array}{l}P_{\exp } \\
\mathrm{MPa}\end{array}$ & $\begin{array}{c}Q \\
\mathrm{~W} \cdot \mathrm{m}^{-1}\end{array}$ & $\begin{array}{c}T_{\exp } \\
\mathrm{K} \\
\end{array}$ & $\begin{array}{c}\rho_{\text {calc }} \\
\mathrm{mol} \cdot \mathrm{L}^{-1}\end{array}$ & $\begin{array}{c}\lambda_{\exp } \\
\mathrm{W} \cdot \mathrm{m}^{-1} \cdot \mathrm{K}^{-1}\end{array}$ & STAT & $\begin{array}{c}T_{\text {cell }} \\
\mathrm{K}\end{array}$ \\
\hline 1005 & 0.071 & 0.02137 & 228.983 & 0.0379 & 0.01108 & 0.011 & 226.774 \\
\hline 1009 & 0.069 & 0.03332 & 230.228 & 0.0370 & 0.01121 & 0.006 & 226.762 \\
\hline 1013 & 0.069 & 0.04787 & 231.730 & 0.0364 & 0.01134 & 0.005 & 226.756 \\
\hline 1017 & 0.068 & 0.06497 & 233.481 & 0.0360 & 0.01147 & 0.004 & 226.753 \\
\hline 1025 & 0.029 & 0.02137 & 229.065 & 0.0155 & 0.01190 & 0.012 & 226.712 \\
\hline 1029 & 0.029 & 0.03332 & 230.411 & 0.0151 & 0.01194 & 0.007 & 226.720 \\
\hline 1033 & 0.028 & 0.04785 & 232.005 & 0.0147 & 0.01201 & 0.005 & 226.707 \\
\hline 1037 & 0.028 & 0.06494 & 233.873 & 0.0145 & 0.01210 & 0.005 & 226.712 \\
\hline 1045 & 0.030 & 0.02037 & 241.005 & 0.0150 & 0.01331 & 0.014 & 238.943 \\
\hline 1049 & 0.029 & 0.03178 & 242.170 & 0.0147 & 0.01324 & 0.008 & 238.933 \\
\hline 1053 & 0.030 & 0.04565 & 243.581 & 0.0147 & 0.01338 & 0.006 & 238.931 \\
\hline 1057 & 0.029 & 0.06199 & 245.223 & 0.0145 & 0.01342 & 0.005 & 238.928 \\
\hline 1065 & 0.024 & 0.01993 & 246.872 & 0.0118 & 0.01384 & 0.016 & 244.939 \\
\hline 1069 & 0.024 & 0.03110 & 247.983 & 0.0118 & 0.01386 & 0.009 & 244.936 \\
\hline 1073 & 0.024 & 0.04469 & 249.315 & 0.0117 & 0.01406 & 0.006 & 244.928 \\
\hline 1077 & 0.024 & 0.06069 & 250.866 & 0.0116 & 0.01413 & 0.004 & 244.922 \\
\hline 1085 & 0.058 & 0.01994 & 246.719 & 0.0288 & 0.01314 & 0.015 & 244.872 \\
\hline 1089 & 0.058 & 0.03110 & 247.755 & 0.0285 & 0.01301 & 0.008 & 244.853 \\
\hline 1093 & 0.058 & 0.04470 & 249.028 & 0.0282 & 0.01324 & 0.006 & 244.854 \\
\hline 1097 & 0.057 & 0.06071 & 250.510 & 0.0279 & 0.01333 & 0.005 & 244.855 \\
\hline 1105 & 0.052 & 0.01961 & 251.041 & 0.0250 & 0.01348 & 0.017 & 249.277 \\
\hline 1109 & 0.050 & 0.03059 & 252.043 & 0.0243 & 0.01351 & 0.009 & 249.262 \\
\hline 1113 & 0.051 & 0.04398 & 253.268 & 0.0246 & 0.01368 & 0.006 & 249.257 \\
\hline 1117 & 0.052 & 0.05974 & 254.693 & 0.0247 & 0.01383 & 0.005 & 249.251 \\
\hline 1125 & 0.118 & 0.01959 & 250.192 & 0.0585 & 0.01275 & 0.016 & 248.505 \\
\hline 1129 & 0.118 & 0.03056 & 251.151 & 0.0582 & 0.01274 & 0.008 & 248.493 \\
\hline 1133 & 0.118 & 0.04393 & 252.313 & 0.0577 & 0.01286 & 0.005 & 248.483 \\
\hline 1137 & 0.118 & 0.05967 & 253.697 & 0.0573 & 0.01298 & 0.003 & 248.499 \\
\hline 1145 & 0.123 & 0.01880 & 261.318 & 0.0582 & 0.01369 & 0.018 & 259.826 \\
\hline 1149 & 0.123 & 0.02934 & 262.170 & 0.0578 & 0.01386 & 0.010 & 259.811 \\
\hline 1153 & 0.123 & 0.04219 & 263.218 & 0.0575 & 0.01392 & 0.006 & 259.806 \\
\hline 1157 & 0.123 & 0.05732 & 264.425 & 0.0573 & 0.01408 & 0.004 & 259.790 \\
\hline 1165 & 0.303 & 0.01881 & 261.058 & 0.1491 & 0.01371 & 0.018 & 259.702 \\
\hline 1169 & 0.302 & 0.02936 & 261.827 & 0.1476 & 0.01386 & 0.009 & 259.679 \\
\hline 1173 & 0.300 & 0.04222 & 262.778 & 0.1463 & 0.01396 & 0.005 & 259.670 \\
\hline 1177 & 0.301 & 0.05737 & 263.897 & 0.1456 & 0.01402 & 0.003 & 259.665 \\
\hline 1185 & 0.344 & 0.01755 & 280.892 & 0.1557 & 0.01581 & 0.025 & 279.803 \\
\hline 1189 & 0.343 & 0.02741 & 281.527 & 0.1548 & 0.01599 & 0.013 & 279.793 \\
\hline 1193 & 0.342 & 0.03943 & 282.295 & 0.1541 & 0.01599 & 0.008 & 279.774 \\
\hline 1197 & 0.342 & 0.05360 & 283.219 & 0.1534 & 0.01608 & 0.005 & 279.771 \\
\hline 1205 & 0.622 & 0.01757 & 280.576 & 0.2974 & 0.01618 & 0.025 & 279.566 \\
\hline 1209 & 0.622 & 0.02743 & 281.159 & 0.2965 & 0.01603 & 0.013 & 279.547 \\
\hline 1213 & 0.622 & 0.03946 & 281.891 & 0.2954 & 0.01617 & 0.008 & 279.542 \\
\hline 1217 & 0.622 & 0.05366 & 282.750 & 0.2941 & 0.01627 & 0.005 & 279.542 \\
\hline
\end{tabular}


Table 15. Thermal conductivity of the binary $30 \% \mathrm{R} 32 / 70 \%$ propane mixture in the vapor phase (continued).

\begin{tabular}{|c|c|c|c|c|c|c|c|}
\hline $\begin{array}{l}\text { Run } \\
\text { point }\end{array}$ & $\begin{array}{l}P_{\text {exp }} \\
\mathrm{MPa}\end{array}$ & $\begin{array}{c}Q \\
\mathrm{~W} \cdot \mathrm{m}^{-1}\end{array}$ & $\begin{array}{c}T_{\text {exp }} \\
\mathrm{K}\end{array}$ & $\begin{array}{c}\rho_{\text {calc }} \\
\mathrm{mol} \mathrm{L}^{-1}\end{array}$ & $\frac{\lambda_{\exp }}{\mathrm{W} \cdot \mathrm{m}^{-1} \cdot \mathrm{K}^{-1}}$ & STAT & $\begin{array}{c}T_{\text {cell }} \\
\mathrm{K}\end{array}$ \\
\hline 1225 & 0.655 & 0.01697 & 291.044 & 0.2994 & 0.01716 & 0.034 & 290.152 \\
\hline 1229 & 0.655 & 0.02650 & 291.575 & 0.2987 & 0.01736 & 0.017 & 290.128 \\
\hline 1233 & 0.656 & 0.03813 & 292.227 & 0.2981 & 0.01730 & 0.010 & 290.110 \\
\hline 1237 & 0.656 & 0.05184 & 292.989 & 0.2973 & 0.01728 & 0.006 & 290.087 \\
\hline 1245 & 0.882 & 0.01699 & 290.816 & 0.4210 & 0.01769 & 0.032 & 289.959 \\
\hline 1249 & 0.880 & 0.02654 & 291.335 & 0.4190 & 0.01764 & 0.016 & 289.952 \\
\hline 1253 & 0.879 & 0.03819 & 291.942 & 0.4167 & 0.01753 & 0.009 & 289.920 \\
\hline 1257 & 0.879 & 0.05192 & 292.677 & 0.4152 & 0.01758 & 0.006 & 289.905 \\
\hline 1265 & 0.347 & 0.01696 & 289.183 & 0.1519 & 0.01647 & 0.031 & 288.178 \\
\hline 1269 & 0.346 & 0.02648 & 289.765 & 0.1511 & 0.01657 & 0.018 & 288.156 \\
\hline 1273 & 0.345 & 0.03810 & 290.478 & 0.1504 & 0.01690 & 0.010 & 288.150 \\
\hline 1277 & 0.345 & 0.05180 & 291.336 & 0.1496 & 0.01688 & 0.007 & 288.146 \\
\hline 1285 & 0.082 & 0.01695 & 289.040 & 0.0344 & 0.01776 & 0.032 & 287.916 \\
\hline 1289 & 0.081 & 0.02646 & 289.718 & 0.0340 & 0.01769 & 0.018 & 287.914 \\
\hline 1293 & 0.080 & 0.03806 & 290.518 & 0.0336 & 0.01765 & 0.010 & 287.895 \\
\hline 1297 & 0.079 & 0.05175 & 291.451 & 0.0331 & 0.01765 & 0.007 & 287.856 \\
\hline 1305 & 0.366 & 0.01695 & 288.405 & 0.1610 & 0.01697 & 0.030 & 287.413 \\
\hline 1309 & 0.365 & 0.02646 & 288.986 & 0.1606 & 0.01687 & 0.017 & 287.390 \\
\hline 1313 & 0.364 & 0.03806 & 289.685 & 0.1595 & 0.01675 & 0.020 & 287.391 \\
\hline 1317 & 0.353 & 0.05180 & 290.544 & 0.1541 & 0.01638 & 0.007 & 287.368 \\
\hline 1325 & 0.150 & 0.01677 & 270.067 & 0.0688 & 0.01487 & 0.026 & 268.858 \\
\hline 1329 & 0.150 & 0.02618 & 270.793 & 0.0686 & 0.01455 & 0.014 & 268.866 \\
\hline 1333 & 0.150 & 0.03766 & 271.623 & 0.0684 & 0.01457 & 0.009 & 268.831 \\
\hline 1337 & 0.151 & 0.05120 & 272.632 & 0.0682 & 0.01486 & 0.005 & 268.825 \\
\hline 1345 & 0.373 & 0.01677 & 269.804 & 0.1787 & 0.01483 & 0.025 & 268.693 \\
\hline 1349 & 0.373 & 0.02619 & 270.459 & 0.1780 & 0.01471 & 0.014 & 268.687 \\
\hline 1353 & 0.373 & 0.03768 & 271.264 & 0.1772 & 0.01480 & 0.008 & 268.688 \\
\hline 1357 & 0.373 & 0.05123 & 272.181 & 0.1764 & 0.01479 & 0.005 & 268.665 \\
\hline 1365 & 0.497 & 0.01678 & 269.650 & 0.2448 & 0.01462 & 0.025 & 268.586 \\
\hline 1369 & 0.497 & 0.02620 & 270.294 & 0.2438 & 0.01479 & 0.013 & 268.598 \\
\hline 1373 & 0.496 & 0.03769 & 271.046 & 0.2427 & 0.01494 & 0.008 & 268.585 \\
\hline 1377 & 0.496 & 0.05125 & 271.937 & 0.2416 & 0.01498 & 0.005 & 268.578 \\
\hline 1385 & 0.759 & 0.01523 & 301.662 & 0.3358 & 0.01870 & 0.042 & 300.911 \\
\hline 1389 & 0.759 & 0.02379 & 302.139 & 0.3350 & 0.01847 & 0.024 & 300.918 \\
\hline 1393 & 0.759 & 0.03424 & 302.683 & 0.3341 & 0.01859 & 0.014 & 300.900 \\
\hline 1397 & 0.759 & 0.04656 & 303.348 & 0.3331 & 0.01850 & 0.009 & 300.898 \\
\hline 1405 & 0.319 & 0.01523 & 301.738 & 0.1326 & 0.01780 & 0.042 & 300.901 \\
\hline 1409 & 0.320 & 0.02379 & 302.243 & 0.1324 & 0.01818 & 0.022 & 300.882 \\
\hline 1413 & 0.320 & 0.03424 & 302.863 & 0.1322 & 0.01820 & 0.013 & 300.882 \\
\hline 1417 & 0.320 & 0.04656 & 303.600 & 0.1318 & 0.01796 & 0.008 & 300.887 \\
\hline 1425 & 1.005 & 0.01524 & 301.249 & 0.4640 & 0.01858 & 0.048 & 300.527 \\
\hline 1429 & 1.005 & 0.02381 & 301.710 & 0.4628 & 0.01872 & 0.026 & 300.546 \\
\hline 1433 & 1.005 & 0.03427 & 302.230 & 0.4616 & 0.01882 & 0.016 & 300.520 \\
\hline
\end{tabular}


Table 15. Thermal conductivity of the binary $30 \% \mathrm{R} 32 / 70 \%$ propane mixture in the vapor phase (continued).

\begin{tabular}{|c|c|c|c|c|c|c|c|}
\hline $\begin{array}{l}\text { Run } \\
\text { point }\end{array}$ & $\begin{array}{l}P_{\text {exp }} \\
\mathrm{MPa} \\
\end{array}$ & $\begin{array}{c}Q \\
\mathrm{~W} \cdot \mathrm{m}^{-1}\end{array}$ & $\begin{array}{c}T_{\text {exp }} \\
\mathrm{K}\end{array}$ & $\begin{array}{c}\rho_{\text {calc }} \\
\mathrm{mol} \cdot \mathrm{L}^{-1}\end{array}$ & $\begin{array}{c}\lambda_{\exp } \\
\mathrm{W} \cdot \mathrm{m}^{-1} \cdot \mathrm{K}^{-1}\end{array}$ & STAT & $\begin{array}{c}T_{\text {cell }} \\
\mathrm{K}\end{array}$ \\
\hline 1437 & 1.005 & 0.04661 & 302.853 & 0.4602 & 0.01868 & 0.011 & 300.509 \\
\hline 1445 & 1.041 & 0.01531 & 310.037 & 0.4621 & 0.01953 & 0.048 & 309.363 \\
\hline 1449 & 1.040 & 0.02392 & 310.436 & 0.4610 & 0.01985 & 0.025 & 309.334 \\
\hline 1453 & 1.041 & 0.03443 & 310.943 & 0.4600 & 0.01975 & 0.015 & 309.327 \\
\hline 1457 & 1.041 & 0.04683 & 311.569 & 0.4586 & 0.01976 & 0.009 & 309.346 \\
\hline 1465 & 0.661 & 0.01531 & 309.951 & 0.2778 & 0.01906 & 0.047 & 309.223 \\
\hline 1469 & 0.661 & 0.02392 & 310.405 & 0.2773 & 0.01913 & 0.025 & 309.210 \\
\hline 1473 & 0.661 & 0.03442 & 310.954 & 0.2767 & 0.01888 & 0.016 & 309.209 \\
\hline 1477 & 0.661 & 0.04681 & 311.585 & 0.2760 & 0.01944 & 0.011 & 309.191 \\
\hline 1486 & 0.348 & 0.01532 & 309.926 & 0.1405 & 0.01879 & 0.045 & 309.140 \\
\hline 1491 & 0.348 & 0.02392 & 310.409 & 0.1403 & 0.01901 & 0.027 & 309.129 \\
\hline 1496 & 0.347 & 0.03443 & 310.988 & 0.1398 & 0.01914 & 0.017 & 309.112 \\
\hline 1501 & 0.347 & 0.04683 & 311.685 & 0.1394 & 0.01909 & 0.010 & 309.114 \\
\hline 1510 & 1.061 & 0.01549 & 306.138 & 0.4822 & 0.01926 & 0.045 & 305.434 \\
\hline 1514 & 1.061 & 0.02420 & 306.582 & 0.4810 & 0.01928 & 0.024 & 305.435 \\
\hline 1518 & 1.060 & 0.03482 & 307.110 & 0.4794 & 0.01934 & 0.014 & 305.431 \\
\hline 1522 & 1.060 & 0.04736 & 307.729 & 0.4778 & 0.01940 & 0.009 & 305.427 \\
\hline 1530 & 1.122 & 0.01547 & 320.349 & 0.4794 & 0.02071 & 0.049 & 319.726 \\
\hline 1534 & 1.122 & 0.02416 & 320.747 & 0.4786 & 0.02061 & 0.025 & 319.718 \\
\hline 1538 & 1.121 & 0.03477 & 321.230 & 0.4773 & 0.02099 & 0.015 & 319.713 \\
\hline 1542 & 1.121 & 0.04730 & 321.798 & 0.4758 & 0.02103 & 0.010 & 319.703 \\
\hline 1550 & 0.575 & 0.01547 & 320.270 & 0.2294 & 0.02034 & 0.054 & 319.573 \\
\hline 1554 & 0.574 & 0.02416 & 320.681 & 0.2290 & 0.02038 & 0.023 & 319.551 \\
\hline 1558 & 0.575 & 0.03476 & 321.224 & 0.2288 & 0.02046 & 0.015 & 319.550 \\
\hline 1562 & 0.575 & 0.04727 & 321.828 & 0.2284 & 0.02061 & 0.009 & 319.528 \\
\hline 1570 & 0.220 & 0.01544 & 318.723 & 0.0851 & 0.02031 & 0.050 & 317.937 \\
\hline 1574 & 0.220 & 0.02412 & 319.203 & 0.0850 & 0.02044 & 0.026 & 317.921 \\
\hline 1578 & 0.220 & 0.03471 & 319.799 & 0.0847 & 0.02026 & 0.016 & 317.929 \\
\hline 1582 & 0.220 & 0.04720 & 320.486 & 0.0845 & 0.02027 & 0.010 & 317.918 \\
\hline 1590 & 1.110 & 0.01506 & 330.967 & 0.4515 & 0.02187 & 0.061 & 330.401 \\
\hline 1594 & 1.111 & 0.02353 & 331.319 & 0.4510 & 0.02216 & 0.032 & 330.376 \\
\hline 1598 & 1.111 & 0.03386 & 331.712 & 0.4504 & 0.02216 & 0.019 & 330.315 \\
\hline 1602 & 1.111 & 0.04607 & 332.257 & 0.4492 & 0.02219 & 0.012 & 330.322 \\
\hline 1610 & 0.601 & 0.01507 & 330.758 & 0.2315 & 0.02193 & 0.059 & 330.114 \\
\hline 1614 & 0.601 & 0.02354 & 331.133 & 0.2313 & 0.02159 & 0.029 & 330.073 \\
\hline 1618 & 0.601 & 0.03388 & 331.619 & 0.2308 & 0.02152 & 0.018 & 330.066 \\
\hline 1622 & 0.601 & 0.04608 & 332.181 & 0.2304 & 0.02165 & 0.012 & 330.047 \\
\hline 1634 & 0.117 & 0.02350 & 329.067 & 0.0431 & 0.02244 & 0.033 & 327.834 \\
\hline 1638 & 0.117 & 0.03382 & 329.632 & 0.0431 & 0.02233 & 0.020 & 327.825 \\
\hline 1642 & 0.117 & 0.04600 & 330.284 & 0.0430 & 0.02249 & 0.014 & 327.804 \\
\hline 1650 & 1.087 & 0.01506 & 340.155 & 0.4244 & 0.02287 & 0.063 & 339.642 \\
\hline 1654 & 1.087 & 0.02352 & 340.474 & 0.4237 & 0.02350 & 0.037 & 339.612 \\
\hline 1658 & 1.087 & 0.03387 & 340.853 & 0.4231 & 0.02307 & 0.021 & 339.559 \\
\hline
\end{tabular}


Table 15. Thermal conductivity of the binary $30 \% \mathrm{R} 32 / 70 \%$ propane mixture in the vapor phase (continued).

\begin{tabular}{|c|c|c|c|c|c|c|c|}
\hline $\begin{array}{l}\text { Run } \\
\text { point }\end{array}$ & $\begin{array}{l}P_{\text {exp }} \\
\mathrm{MPa}\end{array}$ & $\begin{array}{c}Q \\
\mathrm{~W} \cdot \mathrm{m}^{-1}\end{array}$ & $\begin{array}{c}T_{\text {exp }} \\
\mathrm{K}\end{array}$ & $\begin{array}{c}\rho_{\text {calc }} \\
\mathrm{mol} \cdot \mathrm{L}^{-1}\end{array}$ & $\begin{array}{c}\lambda_{\exp } \\
\mathrm{W} \cdot \mathrm{m}^{-1} \cdot \mathrm{K}^{-1}\end{array}$ & STAT & $\begin{array}{c}T_{\text {cell }} \\
\mathrm{K}\end{array}$ \\
\hline 1662 & 1.087 & 0.04607 & 341.320 & 0.4223 & 0.02326 & 0.014 & 339.523 \\
\hline 1683 & 0.594 & 0.01505 & 339.460 & 0.2214 & 0.02256 & 0.060 & 338.875 \\
\hline 1687 & 0.593 & 0.02351 & 339.804 & 0.2208 & 0.02255 & 0.033 & 338.829 \\
\hline 1691 & 0.593 & 0.03383 & 340.238 & 0.2207 & 0.02278 & 0.020 & 338.797 \\
\hline 1695 & 0.594 & 0.04602 & 340.752 & 0.2206 & 0.02276 & 0.013 & 338.757 \\
\hline 1703 & 0.117 & 0.01506 & 339.130 & 0.0419 & 0.02403 & 0.071 & 338.444 \\
\hline 1707 & 0.117 & 0.02353 & 339.562 & 0.0418 & 0.02367 & 0.035 & 338.426 \\
\hline 1711 & 0.117 & 0.03386 & 340.074 & 0.0418 & 0.02369 & 0.022 & 338.402 \\
\hline 1715 & 0.117 & 0.04606 & 340.654 & 0.0418 & 0.02334 & 0.015 & 338.351 \\
\hline
\end{tabular}


Table 16. Thermal conductivity of the binary $70 \% \mathrm{R} 32$ / $30 \%$ propane mixture in the vapor phase.

\begin{tabular}{|c|c|c|c|c|c|c|c|}
\hline $\begin{array}{c}\text { Run } \\
\text { point }\end{array}$ & $\begin{array}{l}P_{\text {exp }} \\
\mathrm{MPa}\end{array}$ & $\begin{array}{c}Q \\
\mathrm{~W} \cdot \mathrm{m}^{-1}\end{array}$ & $\begin{array}{c}T_{\exp } \\
\mathrm{K}\end{array}$ & $\begin{array}{c}\rho_{\text {calc }} \\
\mathrm{mol} \cdot \mathrm{L}^{-1}\end{array}$ & $\begin{array}{c}\lambda_{\exp } \\
\mathrm{W} \cdot \mathrm{m}^{-1} \cdot \mathrm{K}^{-1}\end{array}$ & STAT & $\begin{array}{c}T_{\text {cell }} \\
\mathrm{K}\end{array}$ \\
\hline 1001 & 0.502 & 0.02622 & 257.491 & 0.2618 & 0.01226 & 0.013 & 255.468 \\
\hline 1004 & 0.503 & 0.03567 & 258.275 & 0.2610 & 0.01229 & 0.010 & 255.495 \\
\hline 1007 & 0.503 & 0.04655 & 259.140 & 0.2595 & 0.01253 & 0.007 & 255.499 \\
\hline 1010 & 0.501 & 0.05882 & 260.094 & 0.2574 & 0.01260 & 0.005 & 255.489 \\
\hline 1013 & 0.500 & 0.07251 & 261.139 & 0.2552 & 0.01263 & 0.003 & 255.470 \\
\hline 1016 & 0.416 & 0.02622 & 257.567 & 0.2119 & 0.01223 & 0.014 & 255.460 \\
\hline 1022 & 0.414 & 0.04654 & 259.237 & 0.2093 & 0.01230 & 0.007 & 255.469 \\
\hline 1025 & 0.413 & 0.05881 & 260.222 & 0.2073 & 0.01237 & 0.006 & 255.465 \\
\hline 1028 & 0.413 & 0.07249 & 261.340 & 0.2062 & 0.01244 & 0.005 & 255.477 \\
\hline 1031 & 0.312 & 0.02621 & 257.653 & 0.1550 & 0.01202 & 0.014 & 255.476 \\
\hline 1034 & 0.309 & 0.03566 & 258.455 & 0.1531 & 0.01199 & 0.010 & 255.470 \\
\hline 1037 & 0.310 & 0.04652 & 259.386 & 0.1529 & 0.01212 & 0.007 & 255.473 \\
\hline 1040 & 0.312 & 0.05878 & 260.434 & 0.1531 & 0.01224 & 0.005 & 255.486 \\
\hline 1043 & 0.313 & 0.07247 & 261.574 & 0.1527 & 0.01230 & 0.003 & 255.490 \\
\hline 1046 & 0.213 & 0.02620 & 257.723 & 0.1038 & 0.01183 & 0.014 & 255.470 \\
\hline 1049 & 0.214 & 0.03565 & 258.581 & 0.1037 & 0.01195 & 0.009 & 255.473 \\
\hline 1052 & 0.213 & 0.04649 & 259.536 & 0.1029 & 0.01199 & 0.006 & 255.470 \\
\hline 1055 & 0.211 & 0.05875 & 260.621 & 0.1012 & 0.01209 & 0.005 & 255.470 \\
\hline 1058 & 0.212 & 0.07243 & 261.825 & 0.1011 & 0.01216 & 0.003 & 255.475 \\
\hline 1061 & 0.105 & 0.02620 & 257.867 & 0.0498 & 0.01203 & 0.012 & 255.472 \\
\hline 1064 & 0.105 & 0.03564 & 258.778 & 0.0500 & 0.01211 & 0.008 & 255.468 \\
\hline 1067 & 0.108 & 0.04648 & 259.825 & 0.0509 & 0.01221 & 0.006 & 255.478 \\
\hline 1070 & 0.108 & 0.05872 & 260.964 & 0.0510 & 0.01231 & 0.005 & 255.456 \\
\hline 1073 & 0.107 & 0.07238 & 262.255 & 0.0498 & 0.01237 & 0.004 & 255.476 \\
\hline 1076 & 0.752 & 0.02715 & 266.843 & 0.3943 & 0.01361 & 0.013 & 264.930 \\
\hline 1079 & 0.752 & 0.03694 & 267.539 & 0.3928 & 0.01359 & 0.009 & 264.940 \\
\hline 1082 & 0.752 & 0.04819 & 268.329 & 0.3907 & 0.01370 & 0.006 & 264.946 \\
\hline 1085 & 0.753 & 0.06091 & 269.194 & 0.3889 & 0.01371 & 0.004 & 264.926 \\
\hline 1088 & 0.752 & 0.07509 & 270.176 & 0.3861 & 0.01380 & 0.003 & 264.930 \\
\hline 1091 & 0.641 & 0.02715 & 266.886 & 0.3271 & 0.01330 & 0.013 & 264.925 \\
\hline 1094 & 0.642 & 0.03693 & 267.592 & 0.3262 & 0.01326 & 0.009 & 264.919 \\
\hline 1097 & 0.643 & 0.04818 & 268.410 & 0.3253 & 0.01340 & 0.006 & 264.918 \\
\hline 1100 & 0.643 & 0.06089 & 269.325 & 0.3240 & 0.01344 & 0.004 & 264.918 \\
\hline 1103 & 0.642 & 0.07507 & 270.347 & 0.3215 & 0.01352 & 0.003 & 264.924 \\
\hline 1106 & 0.532 & 0.02715 & 266.703 & 0.2653 & 0.01308 & 0.014 & 264.785 \\
\hline 1109 & 0.533 & 0.03692 & 267.419 & 0.2647 & 0.01312 & 0.009 & 264.765 \\
\hline 1112 & 0.533 & 0.04815 & 268.290 & 0.2638 & 0.01320 & 0.007 & 264.783 \\
\hline 1115 & 0.534 & 0.06085 & 269.224 & 0.2626 & 0.01322 & 0.005 & 264.778 \\
\hline 1118 & 0.534 & 0.07501 & 270.266 & 0.2613 & 0.01332 & 0.003 & 264.763 \\
\hline 1121 & 0.421 & 0.02715 & 266.708 & 0.2049 & 0.01292 & 0.012 & 264.747 \\
\hline 1124 & 0.421 & 0.03693 & 267.498 & 0.2042 & 0.01293 & 0.008 & 264.771 \\
\hline 1127 & 0.422 & 0.04817 & 268.373 & 0.2039 & 0.01300 & 0.006 & 264.767 \\
\hline 1130 & 0.423 & 0.06087 & 269.335 & 0.2033 & 0.01306 & 0.004 & 264.743 \\
\hline 1133 & 0.423 & 0.07502 & 270.436 & 0.2025 & 0.01318 & 0.003 & 264.756 \\
\hline
\end{tabular}


Table 16. Thermal conductivity of the binary $70 \% \mathrm{R} 32 / 30 \%$ propane mixture in the vapor phase (continued).

\begin{tabular}{|c|c|c|c|c|c|c|c|}
\hline $\begin{array}{l}\text { Run } \\
\text { point }\end{array}$ & $\begin{array}{l}P_{\text {exp }} \\
\mathrm{MPa}\end{array}$ & $\begin{array}{c}Q \\
W \cdot \mathrm{m}^{-1}\end{array}$ & $\begin{array}{c}T_{\exp } \\
\mathrm{K}\end{array}$ & $\begin{array}{c}\rho_{\text {calc }} \\
\mathrm{mol} \cdot \mathrm{L}^{-1}\end{array}$ & $\begin{array}{c}\lambda_{\exp } \\
\mathrm{W} \cdot \mathrm{m}^{-1} \cdot \mathrm{K}^{-1}\end{array}$ & STAT & $\begin{array}{c}T_{\text {cell }} \\
\mathrm{K}\end{array}$ \\
\hline 1136 & 0.314 & 0.02712 & 266.760 & 0.1497 & 0.01266 & 0.012 & 264.739 \\
\hline 1139 & 0.315 & 0.03691 & 267.559 & 0.1496 & 0.01262 & 0.007 & 264.739 \\
\hline 1142 & 0.316 & 0.04816 & 268.498 & 0.1498 & 0.01287 & 0.005 & 264.763 \\
\hline 1145 & 0.317 & 0.06085 & 269.506 & 0.1495 & 0.01294 & 0.004 & 264.744 \\
\hline 1148 & 0.318 & 0.07499 & 270.639 & 0.1489 & 0.01303 & 0.003 & 264.741 \\
\hline 1151 & 0.213 & 0.02710 & 266.853 & 0.0997 & 0.01256 & 0.011 & 264.717 \\
\hline 1154 & 0.216 & 0.03691 & 267.704 & 0.1006 & 0.01246 & 0.008 & 264.739 \\
\hline 1157 & 0.217 & 0.04815 & 268.650 & 0.1009 & 0.01271 & 0.005 & 264.736 \\
\hline 1160 & 0.217 & 0.06083 & 269.720 & 0.1002 & 0.01279 & 0.004 & 264.738 \\
\hline 1163 & 0.213 & 0.07497 & 270.884 & 0.0981 & 0.01291 & 0.003 & 264.720 \\
\hline 1166 & 0.114 & 0.02709 & 267.007 & 0.0522 & 0.01281 & 0.013 & 264.738 \\
\hline 1169 & 0.112 & 0.03698 & 267.839 & 0.0512 & 0.01305 & 0.009 & 264.716 \\
\hline 1172 & 0.109 & 0.04813 & 268.873 & 0.0498 & 0.01298 & 0.006 & 264.722 \\
\hline 1175 & 0.111 & 0.06081 & 270.017 & 0.0504 & 0.01310 & 0.005 & 264.736 \\
\hline 1178 & 0.113 & 0.07493 & 271.248 & 0.0511 & 0.01322 & 0.004 & 264.716 \\
\hline 1181 & 0.930 & 0.02624 & 278.053 & 0.4735 & 0.01471 & 0.015 & 276.428 \\
\hline 1184 & 0.929 & 0.03567 & 278.691 & 0.4707 & 0.01468 & 0.010 & 276.451 \\
\hline 1187 & 0.928 & 0.04654 & 279.381 & 0.4683 & 0.01469 & 0.007 & 276.440 \\
\hline 1190 & 0.929 & 0.05881 & 280.141 & 0.4664 & 0.01475 & 0.005 & 276.419 \\
\hline 1193 & 0.930 & 0.07253 & 281.025 & 0.4646 & 0.01480 & 0.004 & 276.432 \\
\hline 1196 & 0.833 & 0.02622 & 278.069 & 0.4154 & 0.01438 & 0.015 & 276.414 \\
\hline 1199 & 0.833 & 0.03567 & 278.692 & 0.4134 & 0.01441 & 0.010 & 276.409 \\
\hline 1202 & 0.833 & 0.04653 & 279.421 & 0.4119 & 0.01449 & 0.007 & 276.413 \\
\hline 1205 & 0.834 & 0.05882 & 280.217 & 0.4107 & 0.01459 & 0.005 & 276.406 \\
\hline 1208 & 0.835 & 0.07255 & 281.128 & 0.4090 & 0.01463 & 0.004 & 276.420 \\
\hline 1211 & 0.727 & 0.02622 & 278.090 & 0.3547 & 0.01423 & 0.015 & 276.400 \\
\hline 1214 & 0.727 & 0.03566 & 278.725 & 0.3535 & 0.01425 & 0.010 & 276.392 \\
\hline 1217 & 0.729 & 0.04653 & 279.466 & 0.3533 & 0.01430 & 0.007 & 276.392 \\
\hline 1220 & 0.730 & 0.05883 & 280.307 & 0.3524 & 0.01439 & 0.005 & 276.403 \\
\hline 1223 & 0.731 & 0.07252 & 281.223 & 0.3508 & 0.01444 & 0.004 & 276.400 \\
\hline 1226 & 0.629 & 0.02609 & 278.084 & 0.3012 & 0.01390 & 0.016 & 276.370 \\
\hline 1229 & 0.626 & 0.03566 & 278.721 & 0.2984 & 0.01408 & 0.010 & 276.367 \\
\hline 1232 & 0.628 & 0.04652 & 279.478 & 0.2984 & 0.01417 & 0.007 & 276.361 \\
\hline 1235 & 0.630 & 0.05880 & 280.329 & 0.2981 & 0.01420 & 0.005 & 276.361 \\
\hline 1238 & 0.630 & 0.07250 & 281.276 & 0.2969 & 0.01430 & 0.004 & 276.363 \\
\hline 1241 & 0.529 & 0.02621 & 278.057 & 0.2489 & 0.01392 & 0.014 & 276.339 \\
\hline 1244 & 0.529 & 0.03566 & 278.740 & 0.2479 & 0.01391 & 0.010 & 276.339 \\
\hline 1247 & 0.526 & 0.04652 & 279.519 & 0.2454 & 0.01400 & 0.007 & 276.339 \\
\hline 1250 & 0.527 & 0.05879 & 280.391 & 0.2449 & 0.01411 & 0.006 & 276.334 \\
\hline 1253 & 0.529 & 0.07247 & 281.355 & 0.2450 & 0.01415 & 0.004 & 276.328 \\
\hline 1256 & 0.416 & 0.02621 & 278.080 & 0.1919 & 0.01381 & 0.016 & 276.323 \\
\hline 1259 & 0.418 & 0.03565 & 278.771 & 0.1925 & 0.01375 & 0.010 & 276.306 \\
\hline 1262 & 0.420 & 0.04650 & 279.592 & 0.1924 & 0.01384 & 0.007 & 276.317 \\
\hline
\end{tabular}


Table 16. Thermal conductivity of the binary $70 \% \mathrm{R} 32 / 30 \%$ propane mixture in the vapor phase (continued).

\begin{tabular}{|c|c|c|c|c|c|c|c|}
\hline $\begin{array}{l}\text { Run } \\
\text { point }\end{array}$ & $\begin{array}{l}P_{\text {exp }} \\
\mathrm{MPa}\end{array}$ & $\begin{array}{c}Q \\
\mathrm{~W} \cdot \mathrm{m}^{-1}\end{array}$ & $\begin{array}{c}T_{\exp } \\
\mathrm{K}\end{array}$ & $\begin{array}{c}\rho_{\text {calc }} \\
\mathrm{mol} \cdot \mathrm{L}^{-1}\end{array}$ & $\begin{array}{c}\lambda_{\exp } \\
\mathrm{W} \cdot \mathrm{m}^{-1} \cdot \mathrm{K}^{-1}\end{array}$ & STAT & $\begin{array}{c}T_{\text {cell }} \\
\mathrm{K}\end{array}$ \\
\hline 1265 & 0.418 & 0.05878 & 280.504 & 0.1908 & 0.01391 & 0.005 & 276.324 \\
\hline 1268 & 0.415 & 0.07248 & 281.496 & 0.1887 & 0.01405 & 0.004 & 276.313 \\
\hline 1271 & 0.311 & 0.02621 & 278.139 & 0.1408 & 0.01360 & 0.015 & 276.314 \\
\hline 1274 & 0.310 & 0.03565 & 278.862 & 0.1401 & 0.01373 & 0.011 & 276.308 \\
\hline 1277 & 0.312 & 0.04650 & 279.699 & 0.1407 & 0.01370 & 0.006 & 276.304 \\
\hline 1280 & 0.314 & 0.05876 & 280.628 & 0.1409 & 0.01382 & 0.005 & 276.294 \\
\hline 1283 & 0.313 & 0.07244 & 281.679 & 0.1399 & 0.01395 & 0.004 & 276.302 \\
\hline 1286 & 0.213 & 0.02629 & 275.974 & 0.0959 & 0.01347 & 0.015 & 274.817 \\
\hline 1289 & 0.213 & 0.03575 & 276.756 & 0.0955 & 0.01356 & 0.010 & 274.836 \\
\hline 1292 & 0.212 & 0.04663 & 277.647 & 0.0950 & 0.01366 & 0.007 & 274.852 \\
\hline 1295 & 0.212 & 0.05893 & 278.617 & 0.0945 & 0.01372 & 0.004 & 274.838 \\
\hline 1298 & 0.212 & 0.07264 & 279.700 & 0.0941 & 0.01382 & 0.004 & 274.836 \\
\hline 1301 & 0.109 & 0.02628 & 276.101 & 0.0485 & 0.01387 & 0.016 & 274.833 \\
\hline 1304 & 0.110 & 0.03574 & 276.896 & 0.0485 & 0.01404 & 0.010 & 274.819 \\
\hline 1306 & 0.113 & 0.02616 & 276.996 & 0.0499 & 0.01390 & 0.015 & 274.791 \\
\hline 1309 & 0.113 & 0.03557 & 277.822 & 0.0498 & 0.01385 & 0.010 & 274.807 \\
\hline 1312 & 0.112 & 0.04641 & 278.760 & 0.0491 & 0.01394 & 0.007 & 274.822 \\
\hline 1315 & 0.111 & 0.05865 & 279.765 & 0.0486 & 0.01401 & 0.006 & 274.789 \\
\hline 1318 & 0.111 & 0.07227 & 280.924 & 0.0481 & 0.01411 & 0.005 & 274.804 \\
\hline 1321 & 1.390 & 0.02537 & 285.641 & 0.7456 & 0.01645 & 0.019 & 284.274 \\
\hline 1324 & 1.384 & 0.03451 & 286.156 & 0.7381 & 0.01643 & 0.012 & 284.272 \\
\hline 1327 & 1.376 & 0.04504 & 286.762 & 0.7288 & 0.01634 & 0.008 & 284.285 \\
\hline 1330 & 1.371 & 0.05697 & 287.409 & 0.7220 & 0.01640 & 0.006 & 284.278 \\
\hline 1333 & 1.371 & 0.07027 & 288.145 & 0.7176 & 0.01637 & 0.005 & 284.287 \\
\hline 1336 & 1.244 & 0.02537 & 285.655 & 0.6448 & 0.01590 & 0.019 & 284.256 \\
\hline 1339 & 1.246 & 0.03452 & 286.188 & 0.6438 & 0.01601 & 0.013 & 284.259 \\
\hline 1342 & 1.248 & 0.04505 & 286.809 & 0.6420 & 0.01582 & 0.009 & 284.272 \\
\hline 1345 & 1.246 & 0.05696 & 287.466 & 0.6377 & 0.01600 & 0.007 & 284.241 \\
\hline 1348 & 1.244 & 0.07024 & 288.224 & 0.6330 & 0.01606 & 0.005 & 284.238 \\
\hline 1351 & 1.141 & 0.02537 & 285.631 & 0.5787 & 0.01561 & 0.018 & 284.222 \\
\hline 1354 & 1.142 & 0.03452 & 286.169 & 0.5772 & 0.01559 & 0.011 & 284.217 \\
\hline 1357 & 1.145 & 0.04503 & 286.798 & 0.5764 & 0.01552 & 0.008 & 284.216 \\
\hline 1360 & 1.145 & 0.05694 & 287.489 & 0.5738 & 0.01574 & 0.006 & 284.203 \\
\hline 1363 & 1.142 & 0.07022 & 288.278 & 0.5691 & 0.01579 & 0.005 & 284.212 \\
\hline 1366 & 1.026 & 0.02535 & 285.366 & 0.5090 & 0.01535 & 0.018 & 283.883 \\
\hline 1369 & 1.025 & 0.03449 & 285.949 & 0.5064 & 0.01531 & 0.013 & 283.901 \\
\hline 1372 & 1.025 & 0.04501 & 286.586 & 0.5045 & 0.01527 & 0.009 & 283.893 \\
\hline 1375 & 1.026 & 0.05689 & 287.306 & 0.5032 & 0.01543 & 0.006 & 283.886 \\
\hline 1378 & 1.027 & 0.07016 & 288.110 & 0.5013 & 0.01556 & 0.005 & 283.885 \\
\hline 1384 & 0.928 & 0.03448 & 285.971 & 0.4503 & 0.01519 & 0.012 & 283.904 \\
\hline 1387 & 0.928 & 0.04500 & 286.634 & 0.4487 & 0.01506 & 0.008 & 283.903 \\
\hline 1393 & 0.929 & 0.07015 & 288.190 & 0.4454 & 0.01528 & 0.004 & 283.888 \\
\hline 1396 & 0.835 & 0.02534 & 285.379 & 0.3996 & 0.01487 & 0.017 & 283.869 \\
\hline
\end{tabular}


Table 16. Thermal conductivity of the binary $70 \% \mathrm{R} 32 / 30 \%$ propane mixture in the vapor phase (continued).

\begin{tabular}{|c|c|c|c|c|c|c|c|}
\hline $\begin{array}{l}\text { Run } \\
\text { point }\end{array}$ & $\begin{array}{l}P_{\text {exp }} \\
\mathrm{MPa}\end{array}$ & $\begin{array}{c}Q \\
\mathrm{~W} \cdot \mathrm{m}^{-1}\end{array}$ & $\begin{array}{c}T_{\exp } \\
\mathrm{K}\end{array}$ & $\begin{array}{c}\rho_{\text {calc }} \\
\mathrm{mol} \cdot \mathrm{L}^{-1}\end{array}$ & $\frac{\lambda_{\exp }}{\mathrm{W} \cdot \mathrm{m}^{-1} \cdot \mathrm{K}^{-1}}$ & STAT & $\begin{array}{c}T_{\text {cell }} \\
\mathrm{K}\end{array}$ \\
\hline 1399 & 0.837 & 0.03448 & 285.965 & 0.3991 & 0.01503 & 0.011 & 283.863 \\
\hline 1402 & 0.838 & 0.04498 & 286.637 & 0.3983 & 0.01495 & 0.009 & 283.856 \\
\hline 1405 & 0.838 & 0.05688 & 287.395 & 0.3969 & 0.01509 & 0.005 & 283.853 \\
\hline 1408 & 0.836 & 0.07015 & 288.254 & 0.3943 & 0.01519 & 0.004 & 283.867 \\
\hline 1411 & 0.731 & 0.02534 & 285.380 & 0.3433 & 0.01473 & 0.019 & 283.830 \\
\hline 1414 & 0.728 & 0.03447 & 286.013 & 0.3410 & 0.01482 & 0.011 & 283.854 \\
\hline 1417 & 0.729 & 0.04499 & 286.692 & 0.3400 & 0.01480 & 0.008 & 283.838 \\
\hline 1420 & 0.731 & 0.05688 & 287.485 & 0.3401 & 0.01498 & 0.006 & 283.847 \\
\hline 1423 & 0.732 & 0.07013 & 288.331 & 0.3389 & 0.01500 & 0.004 & 283.827 \\
\hline 1426 & 0.627 & 0.02534 & 285.394 & 0.2897 & 0.01461 & 0.017 & 283.813 \\
\hline 1429 & 0.630 & 0.03448 & 286.010 & 0.2904 & 0.01466 & 0.012 & 283.804 \\
\hline 1432 & 0.630 & 0.04498 & 286.731 & 0.2891 & 0.01471 & 0.008 & 283.808 \\
\hline 1435 & 0.627 & 0.05686 & 287.520 & 0.2866 & 0.01482 & 0.006 & 283.796 \\
\hline 1438 & 0.627 & 0.07012 & 288.412 & 0.2854 & 0.01491 & 0.004 & 283.803 \\
\hline 1441 & 0.526 & 0.02533 & 285.377 & 0.2390 & 0.01450 & 0.017 & 283.768 \\
\hline 1444 & 0.527 & 0.03447 & 286.041 & 0.2388 & 0.01454 & 0.011 & 283.790 \\
\hline 1447 & 0.527 & 0.04498 & 286.774 & 0.2378 & 0.01452 & 0.008 & 283.791 \\
\hline 1450 & 0.524 & 0.05686 & 287.575 & 0.2358 & 0.01469 & 0.006 & 283.771 \\
\hline 1453 & 0.523 & 0.07011 & 288.490 & 0.2341 & 0.01471 & 0.005 & 283.773 \\
\hline 1456 & 0.423 & 0.02533 & 285.405 & 0.1892 & 0.01432 & 0.016 & 283.759 \\
\hline 1459 & 0.423 & 0.03446 & 286.072 & 0.1887 & 0.01439 & 0.011 & 283.769 \\
\hline 1462 & 0.422 & 0.04497 & 286.856 & 0.1876 & 0.01450 & 0.007 & 283.798 \\
\hline 1465 & 0.419 & 0.05685 & 287.685 & 0.1855 & 0.01452 & 0.006 & 283.780 \\
\hline 1468 & 0.420 & 0.07009 & 288.587 & 0.1851 & 0.01462 & 0.004 & 283.747 \\
\hline 1471 & 0.317 & 0.02534 & 285.467 & 0.1393 & 0.01437 & 0.016 & 283.751 \\
\hline 1474 & 0.315 & 0.03446 & 286.154 & 0.1381 & 0.01426 & 0.011 & 283.753 \\
\hline 1477 & 0.314 & 0.04497 & 286.944 & 0.1372 & 0.01439 & 0.008 & 283.757 \\
\hline 1480 & 0.316 & 0.05685 & 287.833 & 0.1378 & 0.01445 & 0.006 & 283.773 \\
\hline 1483 & 0.317 & 0.07009 & 288.780 & 0.1378 & 0.01451 & 0.004 & 283.752 \\
\hline 1486 & 0.213 & 0.02534 & 285.557 & 0.0922 & 0.01418 & 0.017 & 283.748 \\
\hline 1489 & 0.211 & 0.03447 & 286.266 & 0.0911 & 0.01430 & 0.011 & 283.750 \\
\hline 1492 & 0.210 & 0.04496 & 287.052 & 0.0907 & 0.01436 & 0.008 & 283.726 \\
\hline 1495 & 0.212 & 0.05683 & 287.975 & 0.0909 & 0.01443 & 0.006 & 283.742 \\
\hline 1498 & 0.213 & 0.07008 & 288.977 & 0.0912 & 0.01453 & 0.005 & 283.741 \\
\hline 1501 & 0.112 & 0.02532 & 285.471 & 0.0477 & 0.01472 & 0.019 & 283.450 \\
\hline 1504 & 0.111 & 0.03443 & 286.229 & 0.0475 & 0.01478 & 0.012 & 283.461 \\
\hline 1507 & 0.112 & 0.04491 & 287.080 & 0.0474 & 0.01488 & 0.010 & 283.449 \\
\hline 1510 & 0.112 & 0.05677 & 288.034 & 0.0474 & 0.01493 & 0.007 & 283.444 \\
\hline 1513 & 0.112 & 0.06998 & 289.085 & 0.0471 & 0.01503 & 0.006 & 283.436 \\
\hline 1516 & 1.957 & 0.02437 & 298.974 & 1.0741 & 0.01877 & 0.023 & 297.895 \\
\hline 1519 & 1.955 & 0.03316 & 299.394 & 1.0682 & 0.01882 & 0.015 & 297.901 \\
\hline 1522 & 1.951 & 0.04330 & 299.896 & 1.0600 & 0.01868 & 0.010 & 297.927 \\
\hline 1525 & 1.948 & 0.05477 & 300.396 & 1.0533 & 0.01866 & 0.007 & 297.891 \\
\hline
\end{tabular}


Table 16. Thermal conductivity of the binary $70 \% \mathrm{R} 32 / 30 \%$ propane mixture in the vapor phase (continued).

\begin{tabular}{|c|c|c|c|c|c|c|c|}
\hline $\begin{array}{l}\text { Run } \\
\text { point }\end{array}$ & $\begin{array}{l}P_{\text {exp }} \\
\mathrm{MPa}\end{array}$ & $\begin{array}{c}Q \\
\mathrm{~W} \cdot \mathrm{m}^{-1}\end{array}$ & $\begin{array}{c}T_{\exp } \\
\mathrm{K}\end{array}$ & $\begin{array}{c}\rho_{\text {calc }} \\
\mathrm{mol} \cdot \mathrm{L}^{-1}\end{array}$ & $\begin{array}{c}\lambda_{\exp } \\
\mathrm{W} \cdot \mathrm{m}^{-1} \cdot \mathrm{K}^{-1}\end{array}$ & STAT & $\begin{array}{c}T_{\text {cell }} \\
\mathrm{K}\end{array}$ \\
\hline 1528 & 1.949 & 0.06755 & 300.994 & 1.0483 & 0.01878 & 0.005 & 297.892 \\
\hline 1531 & 1.826 & 0.02438 & 298.998 & 0.9712 & 0.01826 & 0.024 & 297.874 \\
\hline 1534 & 1.823 & 0.03317 & 299.405 & 0.9660 & 0.01806 & 0.016 & 297.853 \\
\hline 1537 & 1.823 & 0.04328 & 299.912 & 0.9621 & 0.01825 & 0.011 & 297.868 \\
\hline 1540 & 1.825 & 0.05475 & $300: 478$ & 0.9590 & 0.01829 & 0.008 & 297.875 \\
\hline 1543 & 1.825 & 0.06754 & 301.071 & 0.9543 & 0.01825 & 0.006 & 297.851 \\
\hline 1546 & 1.599 & 0.02437 & 298.964 & 0.8110 & 0.01759 & 0.023 & 297.825 \\
\hline 1549 & 1.600 & 0.03316 & 299.415 & 0.8088 & 0.01750 & 0.016 & 297.819 \\
\hline 1552 & 1.596 & 0.04328 & 299.945 & 0.8036 & 0.01755 & 0.012 & 297.826 \\
\hline 1555 & 1.594 & 0.05474 & 300.536 & 0.7989 & 0.01752 & 0.009 & 297.826 \\
\hline 1558 & 1.596 & 0.06753 & 301.196 & 0.7967 & 0.01762 & 0.007 & 297.830 \\
\hline 1561 & 1.383 & 0.02437 & 298.935 & 0.6740 & 0.01690 & 0.024 & 297.780 \\
\hline 1564 & 1.386 & 0.03316 & 299.439 & 0.6733 & 0.01698 & 0.014 & 297.803 \\
\hline 1567 & 1.384 & 0.04328 & 299.977 & 0.6703 & 0.01684 & 0.010 & 297.791 \\
\hline 1570 & 1.382 & 0.05473 & 300.581 & 0.6665 & 0.01715 & 0.008 & 297.771 \\
\hline 1573 & 1.385 & 0.06749 & 301.263 & 0.6654 & 0.01704 & 0.006 & 297.766 \\
\hline 1576 & 1.179 & 0.02437 & 298.936 & 0.5550 & 0.01659 & 0.027 & 297.743 \\
\hline 1579 & 1.181 & 0.03315 & 299.453 & 0.5542 & 0.01663 & 0.016 & 297.756 \\
\hline 1582 & 1.181 & 0.04327 & 300.020 & 0.5528 & 0.01658 & 0.013 & 297.749 \\
\hline 1585 & 1.181 & 0.05472 & 300.664 & 0.5509 & 0.01662 & 0.010 & 297.744 \\
\hline 1588 & 1.179 & 0.06749 & 301.375 & 0.5474 & 0.01660 & 0.007 & 297.737 \\
\hline 1591 & 0.971 & 0.02436 & 298.917 & 0.4423 & 0.01616 & 0.023 & 297.708 \\
\hline 1594 & 0.972 & 0.03315 & 299.416 & 0.4422 & 0.01617 & 0.015 & 297.680 \\
\hline 1597 & 0.971 & 0.04326 & 300.027 & 0.4402 & 0.01615 & 0.013 & 297.693 \\
\hline 1600 & 0.970 & 0.05470 & 300.723 & 0.4379 & 0.01626 & 0.010 & 297.712 \\
\hline 1603 & 0.972 & 0.06747 & 301.447 & 0.4374 & 0.01633 & 0.007 & 297.686 \\
\hline 1606 & 0.763 & 0.02436 & 298.880 & 0.3374 & 0.01587 & 0.027 & 297.622 \\
\hline 1609 & 0.764 & 0.03315 & 299.428 & 0.3373 & 0.01600 & 0.018 & 297.622 \\
\hline 1615 & 0.761 & 0.05471 & 300.751 & 0.3335 & 0.01603 & 0.009 & 297.607 \\
\hline 1618 & 0.762 & 0.06747 & 301.541 & 0.3329 & 0.01603 & 0.007 & 297.613 \\
\hline 1621 & 0.558 & 0.02436 & 298.917 & 0.2400 & 0.01571 & 0.027 & 297.588 \\
\hline 1624 & 0.559 & 0.03315 & 299.484 & 0.2401 & 0.01574 & 0.018 & 297.579 \\
\hline 1627 & 0.562 & 0.04327 & 300.138 & 0.2407 & 0.01581 & 0.013 & 297.578 \\
\hline 1630 & 0.562 & 0.05471 & 300.876 & 0.2399 & 0.01583 & 0.010 & 297.580 \\
\hline 1633 & 0.559 & 0.06746 & 301.696 & 0.2379 & 0.01594 & 0.008 & 297.580 \\
\hline 1636 & 0.355 & 0.02437 & 298.935 & 0.1489 & 0.01554 & 0.023 & 297.550 \\
\hline 1639 & 0.357 & 0.03315 & 299.549 & 0.1494 & 0.01555 & 0.014 & 297.559 \\
\hline 1642 & 0.355 & 0.04325 & 300.231 & 0.1480 & 0.01563 & 0.010 & 297.552 \\
\hline 1645 & 0.353 & 0.05468 & 300.970 & 0.1468 & 0.01569 & 0.008 & 297.516 \\
\hline 1648 & 0.356 & 0.06742 & 301.835 & 0.1477 & 0.01583 & 0.006 & 297.522 \\
\hline 1651 & 0.218 & 0.02432 & 298.614 & 0.0900 & 0.01551 & 0.021 & 296.906 \\
\hline 1654 & 0.217 & 0.03310 & 299.242 & 0.0895 & 0.01549 & 0.013 & 296.901 \\
\hline 1657 & 0.214 & 0.04319 & 299.970 & 0.0880 & 0.01554 & 0.010 & 296.900 \\
\hline
\end{tabular}


Table 16. Thermal conductivity of the binary $70 \% \mathrm{R} 32 / 30 \%$ propane mixture in the vapor phase (continued).

\begin{tabular}{|c|c|c|c|c|c|c|c|}
\hline $\begin{array}{l}\text { Run } \\
\text { point }\end{array}$ & $\begin{array}{l}P_{\text {exp }} \\
\mathrm{MPa}\end{array}$ & $\begin{array}{c}Q \\
\mathrm{~W} \cdot \mathrm{m}^{-1}\end{array}$ & $\begin{array}{c}T_{\exp } \\
\mathrm{K}\end{array}$ & $\begin{array}{c}\rho_{\text {calc }} \\
\mathrm{mol} \cdot \mathrm{L}^{-1}\end{array}$ & $\begin{array}{c}\lambda_{\exp } \\
\mathrm{W} \cdot \mathrm{m}^{-1} \cdot \mathrm{K}^{-1}\end{array}$ & STAT & $\begin{array}{c}T_{\text {cell }} \\
\mathrm{K}\end{array}$ \\
\hline 1660 & 0.215 & 0.05459 & 300.768 & 0.0882 & 0.01565 & 0.007 & 296.883 \\
\hline 1663 & 0.217 & 0.06730 & 301.647 & 0.0887 & 0.01574 & 0.006 & 296.860 \\
\hline 1666 & 0.116 & 0.02416 & 303.502 & 0.0464 & 0.01604 & 0.023 & 300.552 \\
\hline 1669 & 0.115 & 0.03280 & 305.517 & 0.0459 & 0.01619 & 0.015 & 301.445 \\
\hline 1672 & 0.114 & 0.04272 & 307.600 & 0.0450 & 0.01627 & 0.012 & 302.322 \\
\hline 1675 & 0.116 & 0.05391 & 309.747 & 0.0456 & 0.01641 & 0.010 & 303.174 \\
\hline 1678 & 0.119 & 0.06636 & 311.959 & 0.0464 & 0.01667 & 0.008 & 304.013 \\
\hline 1681 & 2.157 & 0.02362 & 307.464 & 1.1460 & 0.01939 & 0.027 & 306.450 \\
\hline 1684 & 2.187 & 0.03214 & 307.823 & 1.1662 & 0.01949 & 0.018 & 306.459 \\
\hline 1687 & 2.169 & 0.04195 & 308.307 & 1.1470 & 0.02006 & 0.013 & 306.446 \\
\hline 1690 & 2.131 & 0.05305 & 308.772 & 1.1133 & 0.01965 & 0.010 & 306.441 \\
\hline 1693 & 2.148 & 0.06546 & 309.389 & 1.1200 & 0.01991 & 0.008 & 306.455 \\
\hline 1696 & 1.961 & 0.02361 & 307.387 & 1.0019 & 0.01901 & 0.030 & 306.381 \\
\hline 1699 & 1.969 & 0.03213 & 307.823 & 1.0043 & 0.01896 & 0.019 & 306.410 \\
\hline 1702 & 1.991 & 0.04195 & 308.250 & 1.0163 & 0.01907 & 0.013 & 306.392 \\
\hline 1705 & 1.993 & 0.05305 & 308.756 & 1.0131 & 0.01890 & 0.009 & 306.382 \\
\hline 1708 & 1.990 & 0.06544 & 309.327 & 1.0072 & 0.01909 & 0.007 & 306.378 \\
\hline 1711 & 1.655 & 0.02360 & 307.347 & 0.8007 & 0.01802 & 0.029 & 306.325 \\
\hline 1714 & 1.653 & 0.03213 & 307.802 & 0.7969 & 0.01822 & 0.019 & 306.351 \\
\hline 1717 & 1.654 & 0.04194 & 308.288 & 0.7948 & 0.01816 & 0.014 & 306.344 \\
\hline 1720 & 1.657 & 0.05304 & 308.826 & 0.7938 & 0.01802 & 0.009 & 306.322 \\
\hline 1723 & 1.658 & 0.06542 & 309.440 & 0.7913 & 0.01830 & 0.009 & 306.319 \\
\hline 1726 & 1.396 & 0.02355 & 304.939 & 0.6571 & 0.01726 & 0.028 & 303.784 \\
\hline 1729 & 1.396 & 0.03204 & 305.387 & 0.6553 & 0.01738 & 0.021 & 303.778 \\
\hline 1732 & 1.395 & 0.04183 & 305.909 & 0.6529 & 0.01749 & 0.014 & 303.774 \\
\hline 1735 & 1.395 & 0.05291 & 306.515 & 0.6503 & 0.01752 & 0.011 & 303.788 \\
\hline 1738 & 1.395 & 0.06527 & 307.153 & 0.6481 & 0.01744 & 0.008 & 303.773 \\
\hline 1741 & 1.096 & 0.02356 & 304.930 & 0.4935 & 0.01681 & 0.028 & 303.750 \\
\hline 1744 & 1.097 & 0.03205 & 305.417 & 0.4928 & 0.01691 & 0.019 & 303.751 \\
\hline 1747 & 1.097 & 0.04184 & 305.957 & 0.4915 & 0.01692 & 0.013 & 303.732 \\
\hline 1750 & 1.097 & 0.05291 & 306.592 & 0.4898 & 0.01706 & 0.011 & 303.744 \\
\hline 1753 & 1.097 & 0.06526 & 307.264 & 0.4883 & 0.01702 & 0.007 & 303.722 \\
\hline 1756 & 0.830 & 0.02356 & 304.934 & 0.3605 & 0.01665 & 0.025 & 303.707 \\
\hline 1759 & 0.830 & 0.03205 & 305.435 & 0.3597 & 0.01651 & 0.020 & 303.698 \\
\hline 1762 & 0.831 & 0.04184 & 306.034 & 0.3589 & 0.01646 & 0.013 & 303.714 \\
\hline 1765 & 0.831 & 0.05290 & 306.679 & 0.3581 & 0.01665 & 0.010 & 303.700 \\
\hline 1768 & 0.832 & 0.06525 & 307.406 & 0.3571 & 0.01676 & 0.009 & 303.698 \\
\hline 1771 & 0.557 & 0.02356 & 304.981 & 0.2338 & 0.01619 & 0.032 & 303.672 \\
\hline 1774 & 0.558 & 0.03205 & 305.543 & 0.2334 & 0.01615 & 0.019 & 303.696 \\
\hline 1777 & 0.558 & 0.04183 & 306.130 & 0.2330 & 0.01626 & 0.014 & 303.664 \\
\hline 1780 & 0.558 & 0.05289 & 306.849 & 0.2324 & 0.01637 & 0.011 & 303.686 \\
\hline 1783 & 0.559 & 0.06523 & 307.630 & 0.2320 & 0.01645 & 0.008 & 303.691 \\
\hline 1786 & 0.319 & 0.02356 & 305.004 & 0.1303 & 0.01611 & 0.026 & 303.641 \\
\hline
\end{tabular}


Table 16. Thermal conductivity of the binary $70 \% \mathrm{R} 32 / 30 \%$ propane mixture in the vapor phase (continued).

\begin{tabular}{|c|c|c|c|c|c|c|c|}
\hline $\begin{array}{l}\text { Run } \\
\text { point }\end{array}$ & $\begin{array}{l}P_{\text {exp }} \\
\mathrm{MPa}\end{array}$ & $\begin{array}{c}Q \\
\mathrm{~W} \cdot \mathrm{m}^{-1} \\
\end{array}$ & $\begin{array}{c}T_{\exp } \\
\mathrm{K}\end{array}$ & $\begin{array}{c}\rho_{\text {calc }} \\
\mathrm{mol} \cdot \mathrm{L}^{-1}\end{array}$ & $\begin{array}{c}\lambda_{\exp } \\
\mathrm{W} \cdot \mathrm{m}^{-1} \cdot \mathrm{K}^{-1}\end{array}$ & STAT & $\begin{array}{c}T_{\text {cell }} \\
\mathrm{K}\end{array}$ \\
\hline 1789 & 0.320 & 0.03205 & 305.569 & 0.1303 & 0.01609 & 0.017 & 303.631 \\
\hline 1792 & 0.320 & 0.04182 & 306.233 & 0.1302 & 0.01617 & 0.012 & 303.639 \\
\hline 1795 & 0.320 & 0.05289 & 306.973 & 0.1297 & 0.01621 & 0.009 & 303.634 \\
\hline 1798 & 0.320 & 0.06523 & 307.785 & 0.1291 & 0.01631 & 0.007 & 303.637 \\
\hline 1801 & 0.113 & 0.02356 & 305.099 & 0.0450 & 0.01685 & 0.025 & 303.599 \\
\hline 1804 & 0.115 & 0.03205 & 305.713 & 0.0458 & 0.01691 & 0.017 & 303.598 \\
\hline 1807 & 0.116 & 0.04182 & 306.430 & 0.0463 & 0.01686 & 0.013 & 303.601 \\
\hline 1810 & 0.117 & 0.05287 & 307.248 & 0.0463 & 0.01715 & 0.010 & 303.617 \\
\hline 1813 & 0.115 & 0.06520 & 308.108 & 0.0456 & 0.01705 & 0.007 & 303.587 \\
\hline 1831 & 2.617 & 0.02263 & 317.472 & 1.3960 & 0.02155 & 0.033 & 316.689 \\
\hline 1834 & 2.617 & 0.03080 & 317.808 & 1.3915 & 0.02145 & 0.021 & 316.692 \\
\hline 1837 & 2.611 & 0.04021 & 318.174 & 1.3821 & 0.02130 & 0.013 & 316.680 \\
\hline 1840 & 2.607 & 0.05086 & 318.586 & 1.3736 & 0.02145 & 0.010 & 316.659 \\
\hline 1843 & 2.610 & 0.06276 & 319.084 & 1.3691 & 0.02118 & 0.007 & 316.683 \\
\hline 1846 & 2.370 & 0.02263 & 317.453 & 1.2057 & 0.02040 & 0.031 & 316.665 \\
\hline 1849 & 2.375 & 0.03080 & 317.778 & 1.2055 & 0.02055 & 0.020 & 316.641 \\
\hline 1852 & 2.393 & 0.04020 & 318.210 & 1.2145 & 0.02066 & 0.014 & 316.656 \\
\hline 1855 & 2.403 & 0.05085 & 318.605 & 1.2175 & 0.02064 & 0.011 & 316.618 \\
\hline 1858 & 2.408 & 0.06274 & 319.119 & 1.2162 & 0.02058 & 0.009 & 316.640 \\
\hline 1861 & 2.215 & 0.02262 & 317.331 & 1.0971 & 0.02009 & 0.030 & 316.559 \\
\hline 1864 & 2.222 & 0.03079 & 317.721 & 1.0989 & 0.02020 & 0.020 & 316.583 \\
\hline 1867 & 2.175 & 0.04021 & 318.092 & 1.0644 & 0.02029 & 0.015 & 316.573 \\
\hline 1870 & 2.165 & 0.05085 & 318.646 & 1.0533 & 0.02008 & 0.010 & 316.556 \\
\hline 1873 & 2.179 & 0.06274 & 319.145 & 1.0585 & 0.01998 & 0.008 & 316.571 \\
\hline 1876 & 1.950 & 0.02259 & 316.829 & 0.9288 & 0.01916 & 0.031 & 315.829 \\
\hline 1879 & 1.948 & 0.03075 & 317.211 & 0.9250 & 0.01938 & 0.019 & 315.819 \\
\hline 1882 & 1.945 & 0.04014 & 317.629 & 0.9207 & 0.01921 & 0.015 & 315.798 \\
\hline 1885 & 1.946 & 0.05077 & 318.124 & 0.9185 & 0.01933 & 0.010 & 315.795 \\
\hline 1888 & 1.950 & 0.06264 & 318.679 & 0.9173 & 0.01939 & 0.008 & 315.795 \\
\hline 1891 & 1.597 & 0.02259 & 316.759 & 0.7233 & 0.01853 & 0.029 & 315.726 \\
\hline 1894 & 1.594 & 0.03075 & 317.188 & 0.7197 & 0.01871 & 0.019 & 315.741 \\
\hline 1897 & 1.593 & 0.04015 & 317.660 & 0.7173 & 0.01868 & 0.014 & 315.743 \\
\hline 1900 & 1.596 & 0.05078 & 318.178 & 0.7171 & 0.01856 & 0.010 & 315.732 \\
\hline 1903 & 1.598 & 0.06264 & 318.765 & 0.7154 & 0.01875 & 0.008 & 315.730 \\
\hline 1906 & 1.249 & 0.02260 & 316.766 & 0.5405 & 0.01806 & 0.030 & 315.689 \\
\hline 1909 & 1.245 & 0.03074 & 317.216 & 0.5378 & 0.01823 & 0.020 & 315.699 \\
\hline 1912 & 1.246 & 0.04013 & 317.707 & 0.5366 & 0.01805 & 0.014 & 315.697 \\
\hline 1915 & 1.250 & 0.05076 & 318.282 & 0.5370 & 0.01791 & 0.010 & 315.710 \\
\hline 1918 & 1.249 & 0.06262 & 318.895 & 0.5353 & 0.01816 & 0.008 & 315.699 \\
\hline 1921 & 0.971 & 0.02259 & 316.796 & 0.4067 & 0.01765 & 0.029 & 315.674 \\
\hline 1924 & 0.970 & 0.03074 & 317.241 & 0.4054 & 0.01786 & 0.019 & 315.659 \\
\hline 1927 & 0.968 & 0.04013 & 317.755 & 0.4037 & 0.01780 & 0.014 & 315.652 \\
\hline 1930 & 0.968 & 0.05075 & 318.364 & 0.4028 & 0.01773 & 0.011 & 315.674 \\
\hline
\end{tabular}


Table 16. Thermal conductivity of the binary $70 \% \mathrm{R} 32 / 30 \%$ propane mixture in the vapor phase (continued).

\begin{tabular}{|c|c|c|c|c|c|c|c|}
\hline $\begin{array}{l}\text { Run } \\
\text { point }\end{array}$ & $\begin{array}{l}P_{\text {exp }} \\
\mathrm{MPa}\end{array}$ & $\begin{array}{c}Q \\
W \cdot \mathrm{m}^{-1}\end{array}$ & $\begin{array}{c}T_{\text {exp }} \\
\mathrm{K}\end{array}$ & $\begin{array}{c}\rho_{\text {calc }} \\
\mathrm{mol} \cdot \mathrm{L}^{-1}\end{array}$ & $\begin{array}{c}\lambda_{\exp } \\
\mathrm{W} \cdot \mathrm{m}^{-1} \cdot \mathrm{K}^{-1}\end{array}$ & STAT & $\begin{array}{c}T_{\text {cell }} \\
\mathrm{K}\end{array}$ \\
\hline 1933 & 0.971 & 0.06262 & 318.995 & 0.4027 & 0.01784 & 0.008 & 315.654 \\
\hline 1936 & 0.694 & 0.02259 & 316.713 & 0.2823 & 0.01709 & 0.029 & 315.575 \\
\hline 1939 & 0.695 & 0.03074 & 317.168 & 0.2820 & 0.01749 & 0.019 & 315.553 \\
\hline 1942 & 0.697 & 0.04012 & 317.724 & 0.2822 & 0.01755 & 0.011 & 315.559 \\
\hline 1945 & 0.700 & 0.05072 & 318.321 & 0.2828 & 0.01746 & 0.009 & 315.543 \\
\hline 1948 & 0.700 & 0.06258 & 319.020 & 0.2820 & 0.01766 & 0.007 & 315.558 \\
\hline 1951 & 0.390 & 0.02259 & 316.722 & 0.1538 & 0.01714 & 0.027 & 315.532 \\
\hline 1954 & 0.391 & 0.03073 & 317.234 & 0.1538 & 0.01737 & 0.017 & 315.533 \\
\hline 1957 & 0.388 & 0.04012 & 317.813 & 0.1522 & 0.01721 & 0.012 & 315.526 \\
\hline 1960 & 0.387 & 0.05075 & 318.473 & 0.1517 & 0.01741 & 0.009 & 315.529 \\
\hline 1963 & 0.391 & 0.06260 & 319.195 & 0.1526 & 0.01750 & 0.007 & 315.522 \\
\hline 1996 & 3.243 & 0.02236 & 327.783 & 1.7701 & 0.02428 & 0.038 & 327.129 \\
\hline 1999 & 3.243 & 0.03044 & 328.064 & 1.7648 & 0.02374 & 0.023 & 327.121 \\
\hline 2003 & 3.238 & 0.03974 & 328.381 & 1.7544 & 0.02407 & 0.016 & 327.110 \\
\hline 2006 & 3.234 & 0.05029 & 328.769 & 1.7428 & 0.02418 & 0.011 & 327.127 \\
\hline 2009 & 3.233 & 0.06205 & 329.171 & 1.7352 & 0.02406 & 0.008 & 327.119 \\
\hline 2012 & 2.913 & 0.02236 & 327.589 & 1.4959 & 0.02250 & 0.035 & 326.973 \\
\hline 2015 & 2.914 & 0.03044 & 327.887 & 1.4923 & 0.02267 & 0.024 & 326.957 \\
\hline 2018 & 2.911 & 0.03975 & 328.261 & 1.4848 & 0.02240 & 0.018 & 326.973 \\
\hline 2021 & 2.908 & 0.05028 & 328.642 & 1.4775 & 0.02248 & 0.011 & 326.954 \\
\hline 2024 & 2.910 & 0.06204 & 329.093 & 1.4735 & 0.02242 & 0.009 & 326.952 \\
\hline 3001 & 2.533 & 0.02237 & 327.482 & 1.2232 & 0.02141 & 0.034 & 326.869 \\
\hline 3004 & 2.534 & 0.03044 & 327.803 & 1.2214 & 0.02132 & 0.022 & 326.855 \\
\hline 3007 & 2.530 & 0.03974 & 328.180 & 1.2152 & 0.02125 & 0.014 & 326.846 \\
\hline 3010 & 2.528 & 0.05026 & 328.603 & 1.2099 & 0.02133 & 0.011 & 326.831 \\
\hline 3013 & 2.534 & 0.06202 & 329.108 & 1.2097 & 0.02136 & 0.008 & 326.855 \\
\hline 3016 & 2.133 & 0.02237 & 327.396 & 0.9743 & 0.02017 & 0.033 & 326.764 \\
\hline 3019 & 2.175 & 0.03043 & 327.757 & 0.9968 & 0.02052 & 0.020 & 326.766 \\
\hline 3022 & 2.204 & 0.03974 & 328.109 & 1.0120 & 0.02003 & 0.014 & 326.776 \\
\hline 3025 & 2.168 & 0.05027 & 328.603 & 0.9873 & 0.02052 & 0.010 & 326.778 \\
\hline 3028 & 2.175 & 0.06203 & 329.156 & 0.9880 & 0.02079 & 0.008 & 326.765 \\
\hline 3031 & 1.750 & 0.02233 & 326.729 & 0.7647 & 0.01969 & 0.031 & 325.832 \\
\hline 3034 & 1.752 & 0.03038 & 327.097 & 0.7641 & 0.01955 & 0.019 & 325.817 \\
\hline 3037 & 1.750 & 0.03967 & 327.556 & 0.7614 & 0.01970 & 0.013 & 325.834 \\
\hline 3040 & 1.748 & 0.05019 & 328.050 & 0.7585 & 0.01966 & 0.010 & 325.832 \\
\hline 3043 & 1.749 & 0.06192 & 328.596 & 0.7569 & 0.01978 & 0.007 & 325.826 \\
\hline 3046 & 1.332 & 0.02233 & 326.709 & 0.5555 & 0.01887 & 0.031 & 325.772 \\
\hline 3049 & 1.332 & 0.03040 & 327.137 & 0.5543 & 0.01898 & 0.018 & 325.791 \\
\hline 3052 & 1.334 & 0.03968 & 327.591 & 0.5541 & 0.01905 & 0.014 & 325.773 \\
\hline 3055 & 1.335 & 0.05018 & 328.084 & 0.5531 & 0.01917 & 0.010 & 325.739 \\
\hline 3058 & 1.334 & 0.06190 & 328.671 & 0.5514 & 0.01915 & 0.008 & 325.739 \\
\hline 3061 & 0.930 & 0.02234 & 326.690 & 0.3722 & 0.01877 & 0.029 & 325.711 \\
\hline 3064 & 0.929 & 0.03039 & 327.123 & 0.3712 & 0.01875 & 0.020 & 325.704 \\
\hline
\end{tabular}


Table 16. Thermal conductivity of the binary $70 \% \mathrm{R} 32 / 30 \%$ propane mixture in the vapor phase (continued).

\begin{tabular}{|c|c|c|c|c|c|c|c|}
\hline $\begin{array}{l}\text { Run } \\
\text { point }\end{array}$ & $\begin{array}{l}P_{\text {exp }} \\
\mathrm{MPa}\end{array}$ & $\begin{array}{c}Q \\
\mathrm{~W} \cdot \mathrm{m}^{-1}\end{array}$ & $\begin{array}{c}T_{\exp } \\
\mathrm{K}\end{array}$ & $\begin{array}{c}\rho_{\text {calc }} \\
\mathrm{mol} \cdot \mathrm{L}^{-1}\end{array}$ & $\begin{array}{c}\lambda_{\exp } \\
\mathrm{W} \cdot \mathrm{m}^{-1} \cdot \mathrm{K}^{-1}\end{array}$ & STAT & $\begin{array}{c}T_{\text {cell }} \\
\mathrm{K}\end{array}$ \\
\hline 3067 & 0.928 & 0.03967 & 327.629 & 0.3697 & 0.01865 & 0.013 & 325.710 \\
\hline 3070 & 0.928 & 0.05017 & 328.167 & 0.3692 & 0.01872 & 0.010 & 325.694 \\
\hline 3073 & 0.931 & 0.06190 & 328.818 & 0.3692 & 0.01872 & 0.007 & 325.719 \\
\hline 3076 & 0.518 & 0.02233 & 326.631 & 0.1996 & 0.01820 & 0.031 & 325.636 \\
\hline 3079 & 0.520 & 0.03039 & 327.089 & 0.1999 & 0.01845 & 0.020 & 325.615 \\
\hline 3082 & 0.518 & 0.03967 & 327.614 & 0.1988 & 0.01846 & 0.013 & 325.609 \\
\hline 3085 & 0.516 & 0.05016 & 328.195 & 0.1977 & 0.01842 & 0.010 & 325.590 \\
\hline 3088 & 0.518 & 0.06187 & 328.854 & 0.1978 & 0.01853 & 0.009 & 325.578 \\
\hline 3091 & 0.109 & 0.02233 & 326.720 & 0.0404 & 0.01922 & 0.031 & 325.546 \\
\hline 3094 & 0.110 & 0.03038 & 327.276 & 0.0410 & 0.01944 & 0.021 & 325.570 \\
\hline 3097 & 0.112 & 0.03965 & 327.870 & 0.0416 & 0.01972 & 0.015 & 325.552 \\
\hline 3100 & 0.111 & 0.05015 & 328.563 & 0.0411 & 0.01968 & 0.012 & 325.558 \\
\hline 3103 & 0.109 & 0.06186 & 329.324 & 0.0402 & 0.01955 & 0.009 & 325.561 \\
\hline 3136 & 3.142 & 0.02174 & 335.288 & 1.5660 & 0.02393 & 0.041 & 334.544 \\
\hline 3145 & 3.143 & 0.04887 & 336.194 & 1.5544 & 0.02436 & 0.013 & 334.520 \\
\hline 3148 & 3.134 & 0.06030 & 336.655 & 1.5415 & 0.02390 & 0.009 & 334.530 \\
\hline 3151 & 2.722 & 0.02174 & 335.121 & 1.2776 & 0.02224 & 0.035 & 334.442 \\
\hline 3154 & 2.714 & 0.02958 & 335.449 & 1.2700 & 0.02193 & 0.023 & 334.449 \\
\hline 3157 & 2.710 & 0.03862 & 335.784 & 1.2640 & 0.02217 & 0.016 & 334.421 \\
\hline 3160 & 2.711 & 0.04887 & 336.214 & 1.2609 & 0.02222 & 0.012 & 334.448 \\
\hline 3163 & 2.712 & 0.06030 & 336.659 & 1.2577 & 0.02220 & 0.009 & 334.437 \\
\hline 3166 & 2.303 & 0.02173 & 334.314 & 1.0301 & 0.02127 & 0.037 & 333.504 \\
\hline 3169 & 2.327 & 0.02956 & 334.606 & 1.0422 & 0.02136 & 0.023 & 333.469 \\
\hline 3172 & 2.321 & 0.03858 & 334.995 & 1.0363 & 0.02111 & 0.016 & 333.467 \\
\hline 3175 & 2.292 & 0.04882 & 335.475 & 1.0168 & 0.02108 & 0.012 & 333.494 \\
\hline 3178 & 2.295 & 0.06025 & 335.945 & 1.0157 & 0.02105 & 0.008 & 333.478 \\
\hline 3181 & 1.891 & 0.02172 & 334.115 & 0.8075 & 0.02034 & 0.035 & 333.335 \\
\hline 3184 & 1.890 & 0.02956 & 334.488 & 0.8055 & 0.02040 & 0.022 & 333.352 \\
\hline 3187 & 1.887 & 0.03860 & 334.911 & 0.8023 & 0.02049 & 0.016 & 333.361 \\
\hline 3190 & 1.889 & 0.04882 & 335.377 & 0.8012 & 0.02057 & 0.012 & 333.367 \\
\hline 3193 & 1.891 & 0.06023 & 335.858 & 0.8006 & 0.02033 & 0.009 & 333.334 \\
\hline 3196 & 1.474 & 0.02172 & 334.068 & 0.6030 & 0.01985 & 0.033 & 333.274 \\
\hline 3199 & 1.475 & 0.02956 & 334.436 & 0.6022 & 0.01996 & 0.022 & 333.262 \\
\hline 3202 & 1.472 & 0.03860 & 334.850 & 0.6000 & 0.01997 & 0.014 & 333.246 \\
\hline 3205 & 1.470 & 0.04883 & 335.337 & 0.5977 & 0.02004 & 0.011 & 333.245 \\
\hline 3208 & 1.472 & 0.06023 & 335.859 & 0.5972 & 0.01987 & 0.008 & 333.227 \\
\hline 3211 & 1.055 & 0.02172 & 333.891 & 0.4148 & 0.01932 & 0.032 & 333.128 \\
\hline 3214 & 1.055 & 0.02957 & 334.308 & 0.4142 & 0.01934 & 0.021 & 333.141 \\
\hline 3217 & 1.057 & 0.03860 & 334.736 & 0.4144 & 0.01949 & 0.015 & 333.108 \\
\hline 3220 & 1.057 & 0.04881 & 335.228 & 0.4138 & 0.01951 & 0.012 & 333.082 \\
\hline 3223 & 1.056 & 0.06022 & 335.809 & 0.4121 & 0.01949 & 0.008 & 333.088 \\
\hline 3226 & 0.600 & 0.02168 & 334.357 & 0.2264 & 0.01909 & 0.033 & 333.044 \\
\hline 3229 & 0.598 & 0.02950 & 334.787 & 0.2252 & 0.01912 & 0.021 & 333.046 \\
\hline
\end{tabular}


Table 16. Thermal conductivity of the binary $70 \% \mathrm{R} 32 / 30 \%$ propane mixture in the vapor phase (continued).

\begin{tabular}{|ccccccc|c|}
\hline $\begin{array}{c}\text { Run } \\
\text { point }\end{array}$ & $\begin{array}{c}P_{\text {exp }} \\
\mathrm{MPa}\end{array}$ & $\begin{array}{c}Q \\
\mathrm{~W} \cdot \mathrm{m}^{-1}\end{array}$ & $\begin{array}{c}T_{\text {exp }} \\
\mathrm{K}\end{array}$ & $\begin{array}{c}\rho_{\text {calc }} \\
\mathrm{mol} \cdot \mathrm{L}^{-1}\end{array}$ & $\begin{array}{c}\lambda_{\text {exp }} \\
\mathrm{W} \cdot \mathrm{m}^{-1} \cdot \mathrm{K}^{-1}\end{array}$ & STAT & $\begin{array}{c}T_{\text {cell }} \\
\mathrm{K}\end{array}$ \\
\hline 3232 & 0.596 & 0.03851 & 335.267 & 0.2241 & 0.01914 & 0.015 & 333.032 \\
3235 & 0.597 & 0.04871 & 335.817 & 0.2242 & 0.01914 & 0.011 & 333.021 \\
3238 & 0.599 & 0.06008 & 336.433 & 0.2245 & 0.01920 & 0.007 & 333.021 \\
3241 & 0.112 & 0.02167 & 334.426 & 0.0407 & 0.01989 & 0.034 & 332.981 \\
3244 & 0.114 & 0.02948 & 334.918 & 0.0413 & 0.02007 & 0.022 & 332.978 \\
3247 & 0.115 & 0.03848 & 335.477 & 0.0418 & 0.02012 & 0.016 & 332.967 \\
3253 & 0.114 & 0.06006 & 336.831 & 0.0412 & 0.02031 & 0.009 & 332.970 \\
\hline
\end{tabular}


Table 17. Thermal conductivity of the binary $30 \% \mathrm{R} 32 / 70 \% \mathrm{R} 134 \mathrm{a}$ mixture in the vapor phase.

\begin{tabular}{|c|c|c|c|c|c|c|c|}
\hline $\begin{array}{l}\text { Run } \\
\text { point }\end{array}$ & $\begin{array}{l}P_{\text {exp }} \\
\mathrm{MPa}\end{array}$ & $\begin{array}{c}Q \\
\mathrm{~W} \cdot \mathrm{m}^{-1}\end{array}$ & $\begin{array}{c}T_{\exp } \\
\mathrm{K}\end{array}$ & $\begin{array}{c}\rho_{\text {calc }} \\
\mathrm{mol} \cdot \mathrm{L}^{-1}\end{array}$ & $\begin{array}{c}\lambda_{\exp } \\
\mathrm{W} \cdot \mathrm{m}^{-1} \cdot \mathrm{K}^{-1}\end{array}$ & STAT & $\begin{array}{c}T_{\text {cell }} \\
\mathrm{K}\end{array}$ \\
\hline 1001 & 0.097 & 0.02608 & 258.835 & 0.0465 & 0.01055 & 0.011 & 256.047 \\
\hline 1004 & 0.097 & 0.03545 & 259.858 & 0.0464 & 0.01062 & 0.007 & 256.063 \\
\hline 1007 & 0.098 & 0.04623 & 261.003 & 0.0464 & 0.01074 & 0.005 & 256.056 \\
\hline 1010 & 0.098 & 0.05840 & 262.288 & 0.0464 & 0.01083 & 0.004 & 256.056 \\
\hline 1013 & 0.098 & 0.07197 & 263.719 & 0.0461 & 0.01092 & 0.003 & 256.065 \\
\hline 1016 & 0.151 & 0.02609 & 258.699 & 0.0738 & 0.01062 & 0.011 & 256.036 \\
\hline 1019 & 0.151 & 0.03546 & 259.662 & 0.0733 & 0.01062 & 0.007 & 256.031 \\
\hline 1022 & 0.151 & 0.04625 & 260.777 & 0.0727 & 0.01075 & 0.005 & 256.046 \\
\hline 1025 & 0.151 & 0.05844 & 262.012 & 0.0723 & 0.01082 & 0.004 & 256.050 \\
\hline 1028 & 0.151 & 0.07201 & 263.374 & 0.0721 & 0.01088 & 0.003 & 256.047 \\
\hline 1031 & 0.157 & 0.02536 & 266.617 & 0.0738 & 0.01115 & 0.013 & 264.151 \\
\hline 1034 & 0.158 & 0.03447 & 267.534 & 0.0741 & 0.01123 & 0.008 & 264.165 \\
\hline 1037 & 0.158 & 0.04496 & 268.557 & 0.0737 & 0.01130 & 0.006 & 264.163 \\
\hline 1040 & 0.158 & 0.05682 & 269.695 & 0.0735 & 0.01138 & 0.004 & 264.154 \\
\hline 1043 & 0.157 & 0.07004 & 270.976 & 0.0725 & 0.01148 & 0.004 & 264.166 \\
\hline 1046 & 0.095 & 0.02534 & 267.328 & 0.0437 & 0.01124 & 0.013 & 264.746 \\
\hline 1049 & 0.094 & 0.03445 & 268.257 & 0.0433 & 0.01137 & 0.009 & 264.736 \\
\hline 1052 & 0.094 & 0.04494 & 269.337 & 0.0432 & 0.01145 & 0.007 & 264.749 \\
\hline 1055 & 0.095 & 0.05679 & 270.527 & 0.0433 & 0.01150 & 0.005 & 264.741 \\
\hline 1058 & 0.095 & 0.06999 & 271.848 & 0.0433 & 0.01157 & 0.004 & 264.741 \\
\hline 1061 & 0.227 & 0.02535 & 267.105 & 0.1089 & 0.01121 & 0.013 & 264.746 \\
\hline 1064 & 0.227 & 0.03447 & 267.973 & 0.1086 & 0.01141 & 0.009 & 264.766 \\
\hline 1067 & 0.227 & 0.04497 & 268.938 & 0.1082 & 0.01144 & 0.006 & 264.755 \\
\hline 1070 & 0.227 & 0.05683 & 270.058 & 0.1076 & 0.01150 & 0.005 & 264.772 \\
\hline 1073 & 0.227 & 0.07004 & 271.264 & 0.1069 & 0.01157 & 0.004 & 264.771 \\
\hline 1076 & 0.236 & 0.02459 & 276.365 & 0.1088 & 0.01199 & 0.015 & 274.198 \\
\hline 1079 & 0.236 & 0.03343 & 277.156 & 0.1083 & 0.01189 & 0.010 & 274.198 \\
\hline 1082 & 0.235 & 0.04361 & 278.056 & 0.1078 & 0.01208 & 0.007 & 274.192 \\
\hline 1085 & 0.236 & 0.05512 & 279.096 & 0.1077 & 0.01220 & 0.005 & 274.221 \\
\hline 1088 & 0.237 & 0.06796 & 280.198 & 0.1075 & 0.01228 & 0.004 & 274.199 \\
\hline 1091 & 0.154 & 0.02457 & 276.588 & 0.0696 & 0.01202 & 0.015 & 274.323 \\
\hline 1094 & 0.154 & 0.03341 & 277.420 & 0.0694 & 0.01192 & 0.010 & 274.328 \\
\hline 1097 & 0.155 & 0.04358 & 278.359 & 0.0696 & 0.01208 & 0.007 & 274.321 \\
\hline 1100 & 0.156 & 0.05508 & 279.429 & 0.0695 & 0.01216 & 0.005 & 274.334 \\
\hline 1103 & 0.155 & 0.06790 & 280.581 & 0.0690 & 0.01226 & 0.004 & 274.313 \\
\hline 1106 & 0.093 & 0.02457 & 276.613 & 0.0413 & 0.01215 & 0.014 & 274.245 \\
\hline 1109 & 0.093 & 0.03340 & 277.474 & 0.0412 & 0.01220 & 0.009 & 274.242 \\
\hline 1112 & 0.093 & 0.04357 & 278.458 & 0.0408 & 0.01232 & 0.007 & 274.241 \\
\hline 1115 & 0.092 & 0.05507 & 279.563 & 0.0406 & 0.01237 & 0.005 & 274.241 \\
\hline 1118 & 0.092 & 0.06788 & 280.784 & 0.0402 & 0.01246 & 0.004 & 274.243 \\
\hline 1121 & 0.316 & 0.02458 & 276.306 & 0.1493 & 0.01196 & 0.015 & 274.226 \\
\hline 1124 & 0.316 & 0.03343 & 277.100 & 0.1485 & 0.01203 & 0.010 & 274.253 \\
\hline 1127 & 0.316 & 0.04361 & 277.974 & 0.1479 & 0.01217 & 0.007 & 274.257 \\
\hline 1130 & 0.315 & 0.05513 & 278.938 & 0.1471 & 0.01222 & 0.005 & 274.243 \\
\hline
\end{tabular}


Table 17. Thermal conductivity of the binary $30 \% \mathrm{R} 32 / 70 \% \mathrm{R} 134 \mathrm{a}$ mixture in the vapor phase (continued).

\begin{tabular}{|c|c|c|c|c|c|c|c|}
\hline $\begin{array}{l}\text { Run } \\
\text { point }\end{array}$ & $\begin{array}{l}P_{\text {exp }} \\
\mathrm{MPa}\end{array}$ & $\begin{array}{c}Q \\
\mathrm{~W} \cdot \mathrm{m}^{-1}\end{array}$ & $\begin{array}{c}T_{\exp } \\
\mathrm{K}\end{array}$ & $\begin{array}{c}\rho_{\text {calc }} \\
\mathrm{mol} \cdot \mathrm{L}^{-1}\end{array}$ & $\begin{array}{c}\lambda_{\exp } \\
\mathrm{W} \cdot \mathrm{m}^{-1} \cdot \mathrm{K}^{-1}\end{array}$ & STAT & $\begin{array}{c}T_{\text {cell }} \\
\mathrm{K}\end{array}$ \\
\hline 1133 & 0.316 & 0.06797 & 280.011 & 0.1466 & 0.01232 & 0.004 & 274.240 \\
\hline 1136 & 0.330 & 0.02381 & 286.033 & 0.1493 & 0.01278 & 0.016 & 284.121 \\
\hline 1139 & 0.329 & 0.03238 & 286.720 & 0.1485 & 0.01287 & 0.012 & 284.107 \\
\hline 1142 & 0.329 & 0.04225 & 287.538 & 0.1480 & 0.01290 & 0.007 & 284.113 \\
\hline 1145 & 0.328 & 0.05342 & 288.444 & 0.1467 & 0.01296 & 0.006 & 284.120 \\
\hline 1148 & 0.329 & 0.06588 & 289.444 & 0.1464 & 0.01306 & 0.004 & 284.117 \\
\hline 1151 & 0.417 & 0.02381 & 286.022 & 0.1928 & 0.01273 & 0.018 & 284.179 \\
\hline 1154 & 0.416 & 0.03237 & 286.699 & 0.1919 & 0.01288 & 0.011 & 284.174 \\
\hline 1157 & 0.415 & 0.04224 & 287.479 & 0.1908 & 0.01299 & 0.008 & 284.170 \\
\hline 1160 & 0.415 & 0.05342 & 288.352 & 0.1896 & 0.01299 & 0.006 & 284.176 \\
\hline 1163 & 0.415 & 0.06587 & 289.331 & 0.1890 & 0.01310 & 0.004 & 284.187 \\
\hline 1166 & 0.224 & 0.02380 & 286.202 & 0.0987 & 0.01276 & 0.017 & 284.201 \\
\hline 1169 & 0.225 & 0.03237 & 286.908 & 0.0989 & 0.01276 & 0.011 & 284.174 \\
\hline 1172 & 0.225 & 0.04223 & 287.736 & 0.0988 & 0.01288 & 0.008 & 284.161 \\
\hline 1175 & 0.226 & 0.05338 & 288.728 & 0.0985 & 0.01296 & 0.005 & 284.205 \\
\hline 1178 & 0.225 & 0.06582 & 289.768 & 0.0979 & 0.01302 & 0.004 & 284.197 \\
\hline 1181 & 0.159 & 0.02380 & 286.222 & 0.0690 & 0.01275 & 0.019 & 284.163 \\
\hline 1184 & 0.158 & 0.03236 & 286.983 & 0.0683 & 0.01280 & 0.011 & 284.159 \\
\hline 1187 & 0.156 & 0.04222 & 287.860 & 0.0674 & 0.01282 & 0.009 & 284.166 \\
\hline 1190 & 0.157 & 0.05336 & 288.859 & 0.0676 & 0.01293 & 0.006 & 284.187 \\
\hline 1193 & 0.159 & 0.06580 & 289.938 & 0.0682 & 0.01307 & 0.005 & 284.188 \\
\hline 1196 & 0.093 & 0.02379 & 286.325 & 0.0398 & 0.01295 & 0.017 & 284.166 \\
\hline 1199 & 0.095 & 0.03236 & 287.111 & 0.0405 & 0.01290 & 0.011 & 284.152 \\
\hline 1202 & 0.095 & 0.04221 & 288.049 & 0.0403 & 0.01309 & 0.008 & 284.178 \\
\hline 1205 & 0.092 & 0.05335 & 289.052 & 0.0391 & 0.01310 & 0.006 & 284.162 \\
\hline 1208 & 0.092 & 0.06577 & 290.179 & 0.0388 & 0.01323 & 0.005 & 284.159 \\
\hline 1211 & 0.284 & 0.02380 & 286.129 & 0.1271 & 0.01278 & 0.016 & 284.186 \\
\hline 1214 & 0.283 & 0.03237 & 286.817 & 0.1264 & 0.01290 & 0.010 & 284.163 \\
\hline 1217 & 0.285 & 0.04224 & 287.665 & 0.1267 & 0.01292 & 0.007 & 284.190 \\
\hline 1220 & 0.286 & 0.05340 & 288.556 & 0.1267 & 0.01288 & 0.005 & 284.167 \\
\hline 1223 & 0.285 & 0.06585 & 289.568 & 0.1256 & 0.01302 & 0.004 & 284.159 \\
\hline 1226 & 0.453 & 0.02299 & 297.124 & 0.2005 & 0.01392 & 0.019 & 295.449 \\
\hline 1229 & 0.455 & 0.03127 & 297.736 & 0.2010 & 0.01381 & 0.013 & 295.443 \\
\hline 1232 & 0.456 & 0.04081 & 298.449 & 0.2010 & 0.01385 & 0.009 & 295.441 \\
\hline 1235 & 0.454 & 0.05160 & 299.234 & 0.1989 & 0.01390 & 0.007 & 295.430 \\
\hline 1238 & 0.451 & 0.06363 & 300.129 & 0.1971 & 0.01398 & 0.005 & 295.440 \\
\hline 1241 & 0.379 & 0.02298 & 297.120 & 0.1653 & 0.01379 & 0.019 & 295.425 \\
\hline 1244 & 0.381 & 0.03125 & 297.764 & 0.1656 & 0.01378 & 0.012 & 295.438 \\
\hline 1247 & 0.384 & 0.04079 & 298.469 & 0.1664 & 0.01373 & 0.008 & 295.416 \\
\hline 1250 & 0.384 & 0.05158 & 299.308 & 0.1660 & 0.01388 & 0.006 & 295.441 \\
\hline 1253 & 0.382 & 0.06361 & 300.208 & 0.1642 & 0.01397 & 0.005 & 295.435 \\
\hline 1256 & 0.311 & 0.02297 & 297.177 & 0.1337 & 0.01368 & 0.020 & 295.416 \\
\hline 1259 & 0.313 & 0.03125 & 297.848 & 0.1339 & 0.01366 & 0.012 & 295.435 \\
\hline 1262 & 0.309 & 0.04077 & 298.581 & 0.1320 & 0.01380 & 0.009 & 295.419 \\
\hline
\end{tabular}


Table 17. Thermal conductivity of the binary $30 \% \mathrm{R} 32$ / $70 \% \mathrm{R} 134 \mathrm{a}$ mixture in the vapor phase (continued).

\begin{tabular}{|c|c|c|c|c|c|c|c|}
\hline $\begin{array}{l}\text { Run } \\
\text { point }\end{array}$ & $\begin{array}{l}P_{\text {exp }} \\
\mathrm{MPa}\end{array}$ & $\begin{array}{c}Q \\
\mathrm{~W} \cdot \mathrm{m}^{-1}\end{array}$ & $\begin{array}{c}T_{\exp } \\
\mathrm{K}\end{array}$ & $\begin{array}{c}\rho_{\text {calc }} \\
\mathrm{mol} \cdot \mathrm{L}^{-1}\end{array}$ & $\begin{array}{c}\lambda_{\exp } \\
\mathrm{W} \cdot \mathrm{m}^{-1} \cdot \mathrm{K}^{-1}\end{array}$ & STAT & $\begin{array}{c}T_{\text {cell }} \\
\mathrm{K}\end{array}$ \\
\hline 1265 & 0.308 & 0.05154 & 299.428 & 0.1307 & 0.01387 & 0.007 & 295.430 \\
\hline 1268 & 0.310 & 0.06358 & 300.341 & 0.1312 & 0.01396 & 0.005 & 295.415 \\
\hline 1271 & 0.240 & 0.02297 & 297.184 & 0.1014 & 0.01379 & 0.020 & 295.394 \\
\hline 1274 & 0.240 & 0.03124 & 297.843 & 0.1014 & 0.01369 & 0.013 & 295.383 \\
\hline 1277 & 0.237 & 0.04077 & 298.623 & 0.0996 & 0.01381 & 0.009 & 295.393 \\
\hline 1280 & 0.236 & 0.05155 & 299.477 & 0.0990 & 0.01390 & 0.007 & 295.387 \\
\hline 1283 & 0.239 & 0.06357 & 300.431 & 0.0998 & 0.01396 & 0.005 & 295.389 \\
\hline 1286 & 0.171 & 0.02297 & 297.210 & 0.0714 & 0.01361 & 0.019 & 295.360 \\
\hline 1289 & 0.171 & 0.03125 & 297.920 & 0.0711 & 0.01379 & 0.012 & 295.377 \\
\hline 1292 & 0.173 & 0.04077 & 298.723 & 0.0719 & 0.01384 & 0.009 & 295.388 \\
\hline 1295 & 0.174 & 0.05155 & 299.594 & 0.0721 & 0.01388 & 0.006 & 295.371 \\
\hline 1298 & 0.171 & 0.06357 & 300.573 & 0.0704 & 0.01399 & 0.005 & 295.368 \\
\hline 1331 & 0.180 & 0.02231 & 306.479 & 0.0727 & 0.01463 & 0.021 & 304.753 \\
\hline 1334 & 0.182 & 0.03035 & 307.121 & 0.0733 & 0.01431 & 0.014 & 304.758 \\
\hline 1337 & 0.183 & 0.03962 & 307.866 & 0.0734 & 0.01468 & 0.010 & 304.767 \\
\hline 1340 & 0.183 & 0.05008 & 308.673 & 0.0733 & 0.01467 & 0.008 & 304.757 \\
\hline 1343 & 0.180 & 0.06175 & 309.579 & 0.0718 & 0.01475 & 0.006 & 304.757 \\
\hline 1346 & 0.248 & 0.02232 & 306.436 & 0.1014 & 0.01438 & 0.022 & 304.753 \\
\hline 1349 & 0.247 & 0.03037 & 307.061 & 0.1008 & 0.01455 & 0.014 & 304.758 \\
\hline 1352 & 0.247 & 0.03964 & 307.775 & 0.1003 & 0.01462 & 0.011 & 304.756 \\
\hline 1355 & 0.246 & 0.05011 & 308.577 & 0.0996 & 0.01470 & 0.007 & 304.760 \\
\hline 1358 & 0.245 & 0.06180 & 309.456 & 0.0992 & 0.01471 & 0.005 & 304.752 \\
\hline 1361 & 0.321 & 0.02233 & 306.340 & 0.1330 & 0.01447 & 0.023 & 304.720 \\
\hline 1364 & 0.321 & 0.03038 & 306.933 & 0.1328 & 0.01450 & 0.014 & 304.709 \\
\hline 1367 & 0.321 & 0.03966 & 307.635 & 0.1324 & 0.01444 & 0.010 & 304.716 \\
\hline 1370 & 0.319 & 0.05013 & 308.413 & 0.1309 & 0.01466 & 0.007 & 304.716 \\
\hline 1373 & 0.317 & 0.06182 & 309.261 & 0.1298 & 0.01470 & 0.006 & 304.703 \\
\hline 1376 & 0.390 & 0.02235 & 306.239 & 0.1639 & 0.01447 & 0.022 & 304.646 \\
\hline 1379 & 0.389 & 0.03039 & 306.817 & 0.1631 & 0.01449 & 0.014 & 304.632 \\
\hline 1382 & 0.388 & 0.03965 & 307.493 & 0.1619 & 0.01441 & 0.010 & 304.632 \\
\hline 1385 & 0.387 & 0.05016 & 308.263 & 0.1608 & 0.01472 & 0.007 & 304.645 \\
\hline 1388 & 0.388 & 0.06186 & 309.096 & 0.1608 & 0.01472 & 0.006 & 304.633 \\
\hline 1391 & 0.430 & 0.02237 & 305.686 & 0.1826 & 0.01441 & 0.021 & 304.113 \\
\hline 1394 & 0.432 & 0.03042 & 306.275 & 0.1828 & 0.01461 & 0.014 & 304.115 \\
\hline 1397 & 0.433 & 0.03969 & 306.933 & 0.1825 & 0.01439 & 0.009 & 304.104 \\
\hline 1400 & 0.433 & 0.05019 & 307.712 & 0.1819 & 0.01465 & 0.008 & 304.134 \\
\hline 1403 & 0.432 & 0.06191 & 308.521 & 0.1811 & 0.01468 & 0.006 & 304.110 \\
\hline 1406 & 0.446 & 0.02166 & 316.420 & 0.1815 & 0.01516 & 0.026 & 315.005 \\
\hline 1409 & 0.449 & 0.02945 & 316.928 & 0.1822 & 0.01534 & 0.016 & 314.980 \\
\hline 1412 & 0.451 & 0.03844 & 317.536 & 0.1828 & 0.01548 & 0.011 & 314.974 \\
\hline 1415 & 0.451 & 0.04863 & 318.236 & 0.1821 & 0.01554 & 0.008 & 314.983 \\
\hline 1418 & 0.448 & 0.05997 & 318.968 & 0.1802 & 0.01553 & 0.006 & 314.948 \\
\hline 1421 & 0.450 & 0.02944 & 316.890 & 0.1826 & 0.01526 & 0.015 & 314.894 \\
\hline 1424 & 0.448 & 0.03844 & 317.507 & 0.1815 & 0.01550 & 0.011 & 314.897 \\
\hline
\end{tabular}


Table 17. Thermal conductivity of the binary $30 \% \mathrm{R} 32 / 70 \% \mathrm{R} 134 \mathrm{a}$ mixture in the vapor phase (continued).

\begin{tabular}{|c|c|c|c|c|c|c|c|}
\hline $\begin{array}{c}\text { Run } \\
\text { point }\end{array}$ & $\begin{array}{l}P_{\text {exp }} \\
\mathrm{MPa} \\
\end{array}$ & $\begin{array}{c}Q \\
\mathrm{~W} \cdot \mathrm{m}^{-1}\end{array}$ & $\begin{array}{c}T_{\exp } \\
\mathrm{K} \\
\end{array}$ & $\begin{array}{c}\rho_{\text {calc }} \\
\mathrm{mol} \cdot \mathrm{L}^{-1}\end{array}$ & $\begin{array}{c}\lambda_{\exp } \\
\mathrm{W} \cdot \mathrm{m}^{-1} \cdot \mathrm{K}^{-1}\end{array}$ & STAT & $\begin{array}{c}T_{\text {cell }} \\
\mathrm{K}\end{array}$ \\
\hline 1427 & 0.447 & 0.04861 & 318.191 & 0.1806 & 0.01549 & 0.008 & 314.887 \\
\hline 1430 & 0.447 & 0.05995 & 318.944 & 0.1799 & 0.01554 & 0.007 & 314.873 \\
\hline 1433 & 0.371 & 0.02164 & 315.972 & 0.1493 & 0.01537 & 0.026 & 314.492 \\
\hline 1436 & 0.371 & 0.02944 & 316.528 & 0.1490 & 0.01537 & 0.016 & 314.499 \\
\hline 1439 & 0.369 & 0.03843 & 317.134 & 0.1478 & 0.01540 & 0.011 & 314.473 \\
\hline 1442 & 0.367 & 0.04858 & 317.854 & 0.1467 & 0.01543 & 0.008 & 314.486 \\
\hline 1445 & 0.367 & 0.05993 & 318.625 & 0.1461 & 0.01553 & 0.007 & 314.469 \\
\hline 1448 & 0.306 & 0.02165 & 315.809 & 0.1218 & 0.01526 & 0.025 & 314.305 \\
\hline 1451 & 0.306 & 0.02946 & 316.363 & 0.1219 & 0.01544 & 0.016 & 314.300 \\
\hline 1454 & 0.309 & 0.03844 & 317.007 & 0.1225 & 0.01547 & 0.011 & 314.302 \\
\hline 1457 & 0.310 & 0.04860 & 317.764 & 0.1226 & 0.01551 & 0.062 & 314.276 \\
\hline 1460 & 0.309 & 0.05996 & 318.512 & 0.1222 & 0.01556 & 0.006 & 314.281 \\
\hline 1463 & 0.237 & 0.02171 & 314.799 & 0.0938 & 0.01532 & 0.025 & 313.240 \\
\hline 1466 & 0.237 & 0.02953 & 315.383 & 0.0935 & 0.01533 & 0.017 & 313.240 \\
\hline 1469 & 0.237 & 0.03855 & 316.037 & 0.0934 & 0.01522 & 0.011 & 313.232 \\
\hline 1472 & 0.237 & 0.04875 & 316.781 & 0.0930 & 0.01545 & 0.008 & 313.227 \\
\hline 1475 & 0.237 & 0.06014 & 317.592 & 0.0927 & 0.01548 & 0.006 & 313.207 \\
\hline 1478 & 0.169 & 0.02166 & 315.787 & 0.0658 & 0.01548 & 0.025 & 314.190 \\
\hline 1481 & 0.171 & 0.02945 & 316.364 & 0.0668 & 0.01548 & 0.016 & 314.171 \\
\hline 1484 & 0.172 & 0.03844 & 317.021 & 0.0669 & 0.01538 & 0.012 & 314.152 \\
\hline 1487 & 0.170 & 0.04863 & 317.797 & 0.0661 & 0.01562 & 0.009 & 314.161 \\
\hline 1490 & 0.170 & 0.05997 & 318.607 & 0.0657 & 0.01572 & 0.007 & 314.125 \\
\hline 1523 & 0.175 & 0.02104 & 325.826 & 0.0659 & 0.01645 & 0.030 & 324.370 \\
\hline 1526 & 0.173 & 0.02861 & 326.372 & 0.0653 & 0.01638 & 0.018 & 324.364 \\
\hline 1529 & 0.171 & 0.03734 & 326.989 & 0.0643 & 0.01655 & 0.013 & 324.358 \\
\hline 1532 & 0.173 & 0.04724 & 327.688 & 0.0648 & 0.01658 & 0.009 & 324.350 \\
\hline 1535 & 0.174 & 0.05827 & 328.432 & 0.0653 & 0.01669 & 0.007 & 324.313 \\
\hline 1538 & 0.175 & 0.05829 & 328.295 & 0.0654 & 0.01661 & 0.007 & 324.123 \\
\hline 1541 & 0.244 & 0.02106 & 325.451 & 0.0930 & 0.01607 & 0.028 & 324.027 \\
\hline 1544 & 0.240 & 0.02864 & 325.952 & 0.0916 & 0.01626 & 0.017 & 323.992 \\
\hline 1547 & 0.243 & 0.03738 & 326.582 & 0.0924 & 0.01642 & 0.013 & 324.011 \\
\hline 1550 & 0.245 & 0.04728 & 327.260 & 0.0929 & 0.01647 & 0.009 & 324.000 \\
\hline 1553 & 0.244 & 0.05831 & 328.011 & 0.0925 & 0.01653 & 0.006 & 323.990 \\
\hline 1556 & 0.314 & 0.02105 & 325.287 & 0.1209 & 0.01615 & 0.028 & 323.887 \\
\hline 1559 & 0.313 & 0.02866 & 325.793 & 0.1203 & 0.01635 & 0.018 & 323.872 \\
\hline 1562 & 0.310 & 0.03740 & 326.389 & 0.1191 & 0.01638 & 0.013 & 323.872 \\
\hline 1565 & 0.311 & 0.04729 & 327.033 & 0.1189 & 0.01643 & 0.009 & 323.845 \\
\hline 1568 & 0.313 & 0.05834 & 327.775 & 0.1195 & 0.01646 & 0.006 & 323.840 \\
\hline 1571 & 0.379 & 0.02108 & 324.773 & 0.1477 & 0.01616 & 0.028 & 323.412 \\
\hline 1574 & 0.379 & 0.02868 & 325.283 & 0.1473 & 0.01625 & 0.017 & 323.408 \\
\hline 1577 & 0.378 & 0.03744 & 325.840 & 0.1468 & 0.01641 & 0.012 & 323.382 \\
\hline 1580 & 0.377 & 0.04735 & 326.500 & 0.1460 & 0.01639 & 0.009 & 323.381 \\
\hline 1583 & 0.376 & 0.05841 & 327.226 & 0.1451 & 0.01646 & 0.006 & 323.372 \\
\hline 1586 & 0.434 & 0.02102 & 325.848 & 0.1700 & 0.01628 & 0.028 & 324.518 \\
\hline
\end{tabular}


Table 17. Thermal conductivity of the binary $30 \% \mathrm{R} 32 / 70 \% \mathrm{R} 134 \mathrm{a}$ mixture in the vapor phase (continued).

\begin{tabular}{|c|c|c|c|c|c|c|c|}
\hline $\begin{array}{l}\text { Run } \\
\text { point }\end{array}$ & $\begin{array}{l}P_{\text {exp }} \\
\mathrm{MPa}\end{array}$ & $\begin{array}{c}Q \\
\mathrm{~W} \cdot \mathrm{m}^{-1}\end{array}$ & $\begin{array}{c}T_{\exp } \\
\mathrm{K}\end{array}$ & $\begin{array}{c}\rho_{\text {calc }} \\
\mathrm{mol} \cdot \mathrm{L}^{-1}\end{array}$ & $\begin{array}{c}\lambda_{\exp } \\
\mathrm{W} \cdot \mathrm{m}^{-1} \cdot \mathrm{K}^{-1}\end{array}$ & STAT & $\begin{array}{c}T_{\text {cell }} \\
\mathrm{K}\end{array}$ \\
\hline 1589 & 0.433 & 0.02859 & 326.329 & 0.1691 & 0.01606 & 0.018 & 324.497 \\
\hline 1592 & 0.431 & 0.03732 & 326.883 & 0.1679 & 0.01606 & 0.012 & 324.477 \\
\hline 1595 & 0.430 & 0.04720 & 327.533 & 0.1673 & 0.01621 & 0.009 & 324.476 \\
\hline 1598 & 0.430 & 0.05823 & 328.219 & 0.1668 & 0.01650 & 0.006 & 324.446 \\
\hline 1601 & 0.448 & 0.02183 & 334.586 & 0.1699 & 0.01697 & 0.028 & 333.256 \\
\hline 1604 & 0.445 & 0.02970 & 335.090 & 0.1687 & 0.01698 & 0.017 & 333.267 \\
\hline 1607 & 0.444 & 0.03877 & 335.637 & 0.1679 & 0.01694 & 0.012 & 333.247 \\
\hline 1610 & 0.445 & 0.04904 & 336.245 & 0.1680 & 0.01717 & 0.009 & 333.212 \\
\hline 1613 & 0.446 & 0.06049 & 336.955 & 0.1679 & 0.01731 & 0.007 & 333.208 \\
\hline 1616 & 0.440 & 0.04930 & 334.586 & 0.1667 & 0.01688 & 0.008 & 331.483 \\
\hline 1619 & 0.443 & 0.06077 & 335.273 & 0.1676 & 0.01711 & 0.006 & 331.456 \\
\hline 1622 & 0.383 & 0.02187 & 334.205 & 0.1442 & 0.01698 & 0.027 & 332.840 \\
\hline 1625 & 0.384 & 0.02974 & 334.714 & 0.1447 & 0.01676 & 0.017 & 332.845 \\
\hline 1628 & 0.382 & 0.03881 & 335.257 & 0.1435 & 0.01701 & 0.012 & 332.805 \\
\hline 1631 & 0.378 & 0.04908 & 335.900 & 0.1417 & 0.01711 & 0.009 & 332.794 \\
\hline 1634 & 0.378 & 0.06054 & 336.607 & 0.1412 & 0.01719 & 0.007 & 332.776 \\
\hline 1637 & 0.303 & 0.02189 & 333.749 & 0.1134 & 0.01712 & 0.028 & 332.350 \\
\hline 1640 & 0.303 & 0.02977 & 334.263 & 0.1129 & 0.01688 & 0.017 & 332.348 \\
\hline 1643 & 0.307 & 0.03886 & 334.843 & 0.1142 & 0.01705 & 0.012 & 332.333 \\
\hline 1646 & 0.309 & 0.04915 & 335.491 & 0.1150 & 0.01727 & 0.008 & 332.310 \\
\hline 1649 & 0.303 & 0.06062 & 336.223 & 0.1121 & 0.01721 & 0.006 & 332.261 \\
\hline 1652 & 0.235 & 0.02183 & 334.670 & 0.0869 & 0.01682 & 0.027 & 333.252 \\
\hline 1655 & 0.240 & 0.02969 & 335.157 & 0.0884 & 0.01722 & 0.018 & 333.212 \\
\hline 1658 & 0.242 & 0.03876 & 335.747 & 0.0890 & 0.01736 & 0.012 & 333.195 \\
\hline 1661 & 0.240 & 0.04901 & 336.430 & 0.0882 & 0.01730 & 0.009 & 333.194 \\
\hline 1664 & 0.236 & 0.06045 & 337.182 & 0.0867 & 0.01732 & 0.007 & 333.191 \\
\hline 1667 & 0.169 & 0.02182 & 334.366 & 0.0618 & 0.01750 & 0.029 & 332.909 \\
\hline 1670 & 0.167 & 0.02967 & 334.892 & 0.0611 & 0.01705 & 0.018 & 332.885 \\
\hline 1673 & 0.166 & 0.03873 & 335.498 & 0.0608 & 0.01714 & 0.013 & 332.870 \\
\hline 1676 & 0.166 & 0.04898 & 336.164 & 0.0607 & 0.01743 & 0.009 & 332.829 \\
\hline 1679 & 0.167 & 0.06040 & 336.947 & 0.0606 & 0.01746 & 0.007 & 332.804 \\
\hline 1720 & 0.168 & 0.02126 & 342.967 & 0.0601 & 0.01810 & 0.033 & 341.630 \\
\hline 1723 & 0.168 & 0.02892 & 343.433 & 0.0599 & 0.01823 & 0.022 & 341.590 \\
\hline 1726 & 0.167 & 0.02892 & 343.457 & 0.0596 & 0.01818 & 0.022 & 341.588 \\
\hline 1729 & 0.167 & 0.03776 & 343.979 & 0.0594 & 0.01833 & 0.014 & 341.532 \\
\hline 1732 & 0.168 & 0.04777 & 344.638 & 0.0595 & 0.01842 & 0.010 & 341.498 \\
\hline 1735 & 0.168 & 0.05892 & 345.351 & 0.0595 & 0.01848 & 0.008 & 341.485 \\
\hline 1738 & 0.248 & 0.02131 & 342.332 & 0.0896 & 0.01810 & 0.031 & 341.027 \\
\hline 1741 & 0.248 & 0.02897 & 342.857 & 0.0893 & 0.01767 & 0.019 & 341.063 \\
\hline 1744 & 0.248 & 0.03782 & 343.406 & 0.0891 & 0.01797 & 0.014 & 341.022 \\
\hline 1747 & 0.248 & 0.04784 & 344.034 & 0.0892 & 0.01823 & 0.010 & 341.010 \\
\hline 1750 & 0.249 & 0.05901 & 344.679 & 0.0892 & 0.01823 & 0.007 & 340.944 \\
\hline 1751 & 0.250 & 0.05906 & 344.709 & 0.0895 & 0.01816 & 0.007 & 340.930 \\
\hline 1754 & 0.314 & 0.02134 & 342.178 & 0.1142 & 0.01777 & 0.030 & 340.870 \\
\hline
\end{tabular}


Table 17. Thermal conductivity of the binary $30 \% \mathrm{R} 32 / 70 \% \mathrm{R} 134 \mathrm{a}$ mixture in the vapor phase (continued).

\begin{tabular}{|ccccccc|c|}
\hline $\begin{array}{c}\text { Run } \\
\text { point }\end{array}$ & $\begin{array}{c}P_{\text {exp }} \\
\mathrm{MPa}\end{array}$ & $\begin{array}{c}Q \\
\mathrm{~W} \cdot \mathrm{m}^{-1}\end{array}$ & $\begin{array}{c}T_{\text {exp }} \\
\mathrm{K}\end{array}$ & $\begin{array}{c}\rho_{\text {calc }} \\
\mathrm{mol} \cdot \mathrm{L}^{-1}\end{array}$ & $\begin{array}{c}\lambda_{\text {exp }} \\
\mathrm{W} \cdot \mathrm{m}^{-1} \cdot \mathrm{K}^{-1}\end{array}$ & STAT & $\begin{array}{c}T_{\text {cell }} \\
\mathrm{K}\end{array}$ \\
\hline 1757 & 0.314 & 0.02902 & 342.599 & 0.1140 & 0.01794 & 0.019 & 340.810 \\
1760 & 0.313 & 0.03788 & 343.133 & 0.1137 & 0.01796 & 0.013 & 340.791 \\
1763 & 0.314 & 0.04791 & 343.772 & 0.1137 & 0.01797 & 0.010 & 340.778 \\
1766 & 0.315 & 0.05910 & 344.462 & 0.1137 & 0.01809 & 0.007 & 340.774 \\
1769 & 0.373 & 0.02136 & 341.537 & 0.1370 & 0.01761 & 0.030 & 340.251 \\
1772 & 0.375 & 0.02904 & 342.007 & 0.1374 & 0.01776 & 0.020 & 340.248 \\
1775 & 0.377 & 0.03792 & 342.535 & 0.1379 & 0.01771 & 0.013 & 340.224 \\
1778 & 0.377 & 0.04797 & 343.127 & 0.1376 & 0.01812 & 0.017 & 340.194 \\
1781 & 0.375 & 0.05918 & 343.810 & 0.1366 & 0.01790 & 0.007 & 340.190 \\
1784 & 0.430 & 0.02135 & 341.514 & 0.1589 & 0.01762 & 0.032 & 340.272 \\
1787 & 0.430 & 0.02907 & 341.908 & 0.1588 & 0.01773 & 0.019 & 340.201 \\
1790 & 0.433 & 0.02907 & 341.893 & 0.1597 & 0.01743 & 0.019 & 340.144 \\
1793 & 0.432 & 0.03793 & 342.391 & 0.1592 & 0.01764 & 0.013 & 340.102 \\
1796 & 0.430 & 0.04797 & 342.992 & 0.1582 & 0.01780 & 0.010 & 340.064 \\
1799 & 0.430 & 0.05920 & 343.645 & 0.1578 & 0.01804 & 0.007 & 340.040 \\
\hline
\end{tabular}


Table 18. Thermal conductivity of the binary $70 \% \mathrm{R} 32$ / $30 \% \mathrm{R} 134 \mathrm{a}$ mixture in the vapor phase.

\begin{tabular}{|c|c|c|c|c|c|c|c|}
\hline $\begin{array}{l}\text { Run } \\
\text { point }\end{array}$ & $\begin{array}{l}P_{\text {exp }} \\
\mathrm{MPa}\end{array}$ & $\begin{array}{c}Q \\
\mathrm{~W} \cdot \mathrm{m}^{-1}\end{array}$ & $\begin{array}{c}T_{\exp } \\
\mathrm{K}\end{array}$ & $\begin{array}{c}\rho_{\text {calc }} \\
\mathrm{mol} \cdot \mathrm{L}^{-1}\end{array}$ & $\begin{array}{c}\lambda_{\exp } \\
\mathrm{W} \cdot \mathrm{m}^{-1} \cdot \mathrm{K}^{-1}\end{array}$ & STAT & $\begin{array}{c}T_{\text {cell }} \\
\mathrm{K}\end{array}$ \\
\hline 1001 & 0.089 & 0.02810 & 259.029 & 0.0423 & 0.01050 & 0.010 & 255.933 \\
\hline 1004 & 0.089 & 0.03817 & 260.136 & 0.0420 & 0.01068 & 0.007 & 255.919 \\
\hline 1007 & 0.089 & 0.04974 & 261.509 & 0.0418 & 0.01075 & 0.069 & 255.905 \\
\hline 1010 & 0.089 & 0.06283 & 262.843 & 0.0415 & 0.01083 & 0.005 & 255.930 \\
\hline 1013 & 0.089 & 0.07739 & 264.410 & 0.0414 & 0.01097 & 0.004 & 255.928 \\
\hline 1016 & 0.175 & 0.02810 & 258.842 & 0.0850 & 0.01044 & 0.010 & 255.963 \\
\hline 1019 & 0.176 & 0.03816 & 259.890 & 0.0850 & 0.01057 & 0.007 & 255.965 \\
\hline 1022 & 0.176 & 0.04975 & 261.059 & 0.0846 & 0.01063 & 0.005 & 255.944 \\
\hline 1025 & 0.176 & 0.06281 & 262.415 & 0.0841 & 0.01070 & 0.004 & 255.970 \\
\hline 1028 & 0.176 & 0.07740 & 263.899 & 0.0835 & 0.01079 & 0.003 & 255.981 \\
\hline 1031 & 0.214 & 0.02811 & 258.765 & 0.1051 & 0.01054 & 0.009 & 255.964 \\
\hline 1034 & 0.214 & 0.03816 & 259.783 & 0.1046 & 0.01059 & 0.006 & 255.963 \\
\hline 1037 & 0.214 & 0.04977 & 260.955 & 0.1042 & 0.01068 & 0.004 & 255.978 \\
\hline 1040 & 0.215 & 0.06287 & 262.264 & 0.1039 & 0.01075 & 0.003 & 255.986 \\
\hline 1043 & 0.215 & 0.07740 & 263.668 & 0.1036 & 0.01084 & 0.003 & 255.951 \\
\hline 1046 & 0.221 & 0.02719 & 267.670 & 0.1047 & 0.01112 & 0.010 & 265.074 \\
\hline 1049 & 0.222 & 0.03693 & 268.601 & 0.1045 & 0.01122 & 0.007 & 265.058 \\
\hline 1052 & 0.222 & 0.04819 & 269.697 & 0.1042 & 0.01128 & 0.005 & 265.081 \\
\hline 1055 & 0.222 & 0.06089 & 270.897 & 0.1035 & 0.01136 & 0.004 & 265.074 \\
\hline 1058 & 0.222 & 0.07500 & 272.205 & 0.1027 & 0.01142 & 0.003 & 265.047 \\
\hline 1061 & 0.110 & 0.02718 & 267.813 & 0.0505 & 0.01118 & 0.011 & 265.040 \\
\hline 1062 & 0.110 & 0.02717 & 267.871 & 0.0506 & 0.01123 & 0.011 & 265.061 \\
\hline 1065 & 0.110 & 0.03693 & 268.887 & 0.0505 & 0.01123 & 0.007 & 265.057 \\
\hline 1068 & 0.110 & 0.04814 & 270.036 & 0.0500 & 0.01132 & 0.006 & 265.051 \\
\hline 1071 & 0.109 & 0.06080 & 271.350 & 0.0496 & 0.01141 & 0.004 & 265.071 \\
\hline 1074 & 0.110 & 0.07494 & 272.775 & 0.0494 & 0.01152 & 0.004 & 265.061 \\
\hline 1077 & 0.343 & 0.02720 & 267.481 & 0.1675 & 0.01132 & 0.020 & 265.049 \\
\hline 1080 & 0.342 & 0.03695 & 268.378 & 0.1664 & 0.01135 & 0.006 & 265.060 \\
\hline 1083 & 0.342 & 0.04820 & 269.410 & 0.1652 & 0.01141 & 0.004 & 265.079 \\
\hline 1086 & 0.342 & 0.06088 & 270.529 & 0.1643 & 0.01145 & 0.003 & 265.057 \\
\hline 1089 & 0.342 & 0.07502 & 271.792 & 0.1636 & 0.01155 & 0.003 & 265.059 \\
\hline 1092 & 0.106 & 0.02623 & 277.797 & 0.0468 & 0.01205 & 0.013 & 275.227 \\
\hline 1095 & 0.105 & 0.03561 & 278.740 & 0.0462 & 0.01210 & 0.008 & 275.232 \\
\hline 1098 & 0.105 & 0.04645 & 279.822 & 0.0459 & 0.01213 & 0.006 & 275.240 \\
\hline 1101 & 0.105 & 0.05868 & 281.014 & 0.0456 & 0.01221 & 0.005 & 275.240 \\
\hline 1104 & 0.106 & 0.07232 & 282.335 & 0.0459 & 0.01230 & 0.004 & 275.235 \\
\hline 1107 & 0.211 & 0.02624 & 277.616 & 0.0953 & 0.01182 & 0.013 & 275.211 \\
\hline 1110 & 0.212 & 0.03565 & 278.512 & 0.0953 & 0.01190 & 0.008 & 275.229 \\
\hline 1113 & 0.211 & 0.04649 & 279.510 & 0.0946 & 0.01198 & 0.005 & 275.227 \\
\hline 1116 & 0.210 & 0.05872 & 280.622 & 0.0937 & 0.01209 & 0.004 & 275.216 \\
\hline 1119 & 0.210 & 0.07237 & 281.878 & 0.0931 & 0.01214 & 0.004 & 275.231 \\
\hline 1122 & 0.317 & 0.02624 & 277.483 & 0.1464 & 0.01199 & 0.013 & 275.208 \\
\hline 1125 & 0.318 & 0.03565 & 278.307 & 0.1464 & 0.01199 & 0.008 & 275.197 \\
\hline 1128 & 0.317 & 0.04650 & 279.279 & 0.1456 & 0.01204 & 0.005 & 275.216 \\
\hline
\end{tabular}


Table 18. Thermal conductivity of the binary $70 \% \mathrm{R} 32 / 30 \% \mathrm{R} 134 \mathrm{a}$ mixture in the vapor phase (continued).

\begin{tabular}{|c|c|c|c|c|c|c|c|}
\hline $\begin{array}{l}\text { Run } \\
\text { point }\end{array}$ & $\begin{array}{l}P_{e x p} \\
\mathrm{MPa}\end{array}$ & $\begin{array}{c}Q \\
W \cdot \mathrm{m}^{-1}\end{array}$ & $\begin{array}{c}T_{\exp } \\
\mathrm{K}\end{array}$ & $\begin{array}{c}\rho_{\text {calc }} \\
\mathrm{mol}^{-\mathrm{L}^{-1}}\end{array}$ & $\begin{array}{c}\lambda_{\exp } \\
\mathrm{W} \cdot \mathrm{m}^{-1} \cdot \mathrm{K}^{-1}\end{array}$ & STAT & $\begin{array}{c}T_{\text {cell }} \\
\mathrm{K}\end{array}$ \\
\hline 1131 & 0.317 & 0.05878 & 280.350 & 0.1444 & 0.01213 & 0.004 & 275.218 \\
\hline 1134 & 0.315 & 0.07245 & 281.530 & 0.1431 & 0.01223 & 0.003 & 275.215 \\
\hline 1137 & 0.426 & 0.02625 & 277.228 & 0.2024 & 0.01211 & 0.012 & 275.042 \\
\hline 1140 & 0.425 & 0.03566 & 278.026 & 0.2011 & 0.01199 & 0.008 & 275.040 \\
\hline 1143 & 0.424 & 0.04654 & 278.968 & 0.1994 & 0.01217 & 0.005 & 275.065 \\
\hline 1146 & 0.423 & 0.05882 & 279.979 & 0.1981 & 0.01224 & 0.004 & 275.052 \\
\hline 1149 & 0.423 & 0.07248 & 281.102 & 0.1970 & 0.01230 & 0.003 & 275.040 \\
\hline 1152 & 0.484 & 0.02626 & 277.151 & 0.2334 & 0.01214 & 0.012 & 275.026 \\
\hline 1155 & 0.486 & 0.03569 & 277.936 & 0.2333 & 0.01204 & 0.008 & 275.033 \\
\hline 1158 & 0.487 & 0.04656 & 278.821 & 0.2329 & 0.01221 & 0.005 & 275.023 \\
\hline 1161 & 0.488 & 0.05882 & 279.810 & 0.2317 & 0.01230 & 0.004 & 275.015 \\
\hline 1164 & 0.486 & 0.07247 & 280.933 & 0.2296 & 0.01239 & 0.003 & 275.030 \\
\hline 1167 & 0.503 & 0.02524 & 288.735 & 0.2295 & 0.01290 & 0.015 & 286.801 \\
\hline 1170 & 0.504 & 0.03431 & 289.442 & 0.2290 & 0.01297 & 0.009 & 286.793 \\
\hline 1173 & 0.505 & 0.04473 & 290.267 & 0.2288 & 0.01301 & 0.006 & 286.798 \\
\hline 1176 & 0.505 & 0.05652 & 291.232 & 0.2277 & 0.01318 & 0.056 & 286.789 \\
\hline 1179 & 0.504 & 0.06970 & 292.193 & 0.2263 & 0.01317 & 0.004 & 286.791 \\
\hline 1182 & 0.554 & 0.02524 & 288.641 & 0.2557 & 0.01295 & 0.015 & 286.736 \\
\hline 1185 & 0.555 & 0.03430 & 289.342 & 0.2551 & 0.01302 & 0.009 & 286.733 \\
\hline 1188 & 0.555 & 0.04473 & 290.146 & 0.2539 & 0.01304 & 0.006 & 286.732 \\
\hline 1191 & 0.554 & 0.05655 & 291.060 & 0.2524 & 0.01312 & 0.005 & 286.739 \\
\hline 1194 & 0.553 & 0.06974 & 292.067 & 0.2506 & 0.01318 & 0.004 & 286.742 \\
\hline 1197 & 0.388 & 0.02525 & 288.682 & 0.1731 & 0.01279 & 0.014 & 286.664 \\
\hline 1200 & 0.387 & 0.03429 & 289.436 & 0.1722 & 0.01295 & 0.009 & 286.675 \\
\hline 1203 & 0.387 & 0.04475 & 290.306 & 0.1711 & 0.01297 & 0.006 & 286.694 \\
\hline 1206 & 0.388 & 0.05656 & 291.249 & 0.1709 & 0.01297 & 0.005 & 286.675 \\
\hline 1209 & 0.388 & 0.06969 & 292.310 & 0.1705 & 0.01306 & 0.017 & 286.677 \\
\hline 1212 & 0.291 & 0.02526 & 288.621 & 0.1276 & 0.01262 & 0.014 & 286.521 \\
\hline 1215 & 0.290 & 0.03433 & 289.368 & 0.1268 & 0.01268 & 0.009 & 286.491 \\
\hline 1218 & 0.291 & 0.04475 & 290.264 & 0.1265 & 0.01287 & 0.006 & 286.501 \\
\hline 1221 & 0.292 & 0.05656 & 291.252 & 0.1265 & 0.01290 & 0.004 & 286.501 \\
\hline 1224 & 0.293 & 0.06975 & 292.340 & 0.1263 & 0.01301 & 0.003 & 286.492 \\
\hline 1227 & 0.190 & 0.02526 & 288.662 & 0.0816 & 0.01265 & 0.015 & 286.458 \\
\hline 1230 & 0.191 & 0.03433 & 289.463 & 0.0819 & 0.01258 & 0.010 & 286.448 \\
\hline 1233 & 0.191 & 0.04475 & 290.371 & 0.0817 & 0.01281 & 0.007 & 286.435 \\
\hline 1236 & 0.190 & 0.05657 & 291.432 & 0.0810 & 0.01290 & 0.005 & 286.462 \\
\hline 1239 & 0.190 & 0.06974 & 292.583 & 0.0804 & 0.01299 & 0.004 & 286.464 \\
\hline 1272 & 0.174 & 0.02452 & 297.238 & 0.0724 & 0.01344 & 0.017 & 295.185 \\
\hline 1275 & 0.175 & 0.03333 & 298.009 & 0.0727 & 0.01354 & 0.010 & 295.197 \\
\hline 1278 & 0.174 & 0.04348 & 298.862 & 0.0718 & 0.01358 & 0.007 & 295.177 \\
\hline 1281 & 0.172 & 0.05495 & 299.825 & 0.0707 & 0.01367 & 0.005 & 295.163 \\
\hline 1284 & 0.172 & 0.06775 & 300.928 & 0.0705 & 0.01374 & 0.004 & 295.190 \\
\hline 1287 & 0.277 & 0.02454 & 297.130 & 0.1171 & 0.01338 & 0.016 & 295.179 \\
\hline 1290 & 0.276 & 0.03334 & 297.854 & 0.1163 & 0.01342 & 0.010 & 295.173 \\
\hline
\end{tabular}


Table 18. Thermal conductivity of the binary $70 \% \mathrm{R} 32 / 30 \% \mathrm{R} 134 \mathrm{a}$ mixture in the vapor phase (continued).

\begin{tabular}{|c|c|c|c|c|c|c|c|}
\hline $\begin{array}{l}\text { Run } \\
\text { point }\end{array}$ & $\begin{array}{l}P_{\exp } \\
\mathrm{MPa}\end{array}$ & $\begin{array}{c}Q \\
\mathrm{~W} \cdot \mathrm{m}^{-1}\end{array}$ & $\begin{array}{c}T_{\exp } \\
\mathrm{K}\end{array}$ & $\begin{array}{c}\rho_{\text {calc }} \\
\mathrm{mol} \cdot \mathrm{L}^{-1}\end{array}$ & $\begin{array}{c}\lambda_{\exp } \\
\mathrm{W} \cdot \mathrm{m}^{-1} \cdot \mathrm{K}^{-1}\end{array}$ & STAT & $\begin{array}{c}T_{\text {cell }} \\
\mathrm{K}\end{array}$ \\
\hline 1293 & 0.274 & 0.04349 & 298.683 & 0.1151 & 0.01355 & 0.007 & 295.166 \\
\hline 1296 & 0.275 & 0.05496 & 299.612 & 0.1148 & 0.01363 & 0.005 & 295.162 \\
\hline 1299 & 0.276 & 0.06777 & 300.638 & 0.1151 & 0.01364 & 0.004 & 295.159 \\
\hline 1302 & 0.384 & 0.02454 & 297.024 & 0.1650 & 0.01332 & 0.016 & 295.152 \\
\hline 1305 & 0.384 & 0.03335 & 297.707 & 0.1645 & 0.01348 & 0.010 & 295.131 \\
\hline 1308 & 0.381 & 0.04351 & 298.524 & 0.1627 & 0.01357 & 0.007 & 295.146 \\
\hline 1311 & 0.381 & 0.05500 & 299.419 & 0.1621 & 0.01339 & 0.005 & 295.137 \\
\hline 1314 & 0.383 & 0.06781 & 300.421 & 0.1623 & 0.01362 & 0.004 & 295.142 \\
\hline 1317 & 0.479 & 0.02456 & 296.931 & 0.2094 & 0.01344 & 0.016 & 295.114 \\
\hline 1320 & 0.478 & 0.03336 & 297.626 & 0.2082 & 0.01354 & 0.010 & 295.121 \\
\hline 1323 & 0.479 & 0.04352 & 298.406 & 0.2082 & 0.01364 & 0.006 & 295.124 \\
\hline 1326 & 0.480 & 0.05502 & 299.285 & 0.2077 & 0.01370 & 0.005 & 295.131 \\
\hline 1329 & 0.478 & 0.06783 & 300.308 & 0.2060 & 0.01356 & 0.049 & 295.125 \\
\hline 1332 & 0.544 & 0.02455 & 296.887 & 0.2407 & 0.01363 & 0.016 & 295.102 \\
\hline 1335 & 0.545 & 0.03335 & 297.571 & 0.2403 & 0.01369 & 0.010 & 295.123 \\
\hline 1338 & 0.544 & 0.04350 & 298.319 & 0.2390 & 0.01374 & 0.007 & 295.120 \\
\hline 1341 & 0.543 & 0.05503 & 299.178 & 0.2375 & 0.01378 & 0.005 & 295.119 \\
\hline 1344 & 0.543 & 0.06783 & 300.126 & 0.2366 & 0.01379 & 0.004 & 295.117 \\
\hline 1347 & 0.564 & 0.02373 & 307.235 & 0.2392 & 0.01434 & 0.019 & 305.602 \\
\hline 1350 & 0.567 & 0.03225 & 307.814 & 0.2396 & 0.01442 & 0.012 & 305.573 \\
\hline 1353 & 0.567 & 0.04207 & 308.525 & 0.2390 & 0.01436 & 0.008 & 305.573 \\
\hline 1356 & 0.565 & 0.05321 & 309.321 & 0.2374 & 0.01447 & 0.006 & 305.577 \\
\hline 1359 & 0.564 & 0.06563 & 310.204 & 0.2358 & 0.01463 & 0.005 & 305.584 \\
\hline 1362 & 0.479 & 0.02374 & 307.143 & 0.2005 & 0.01413 & 0.018 & 305.474 \\
\hline 1365 & 0.476 & 0.03227 & 307.769 & 0.1985 & 0.01431 & 0.012 & 305.462 \\
\hline 1368 & 0.476 & 0.04209 & 308.468 & 0.1980 & 0.01423 & 0.008 & 305.439 \\
\hline 1371 & 0.478 & 0.05320 & 309.280 & 0.1985 & 0.01450 & 0.006 & 305.442 \\
\hline 1374 & 0.478 & 0.06562 & 310.260 & 0.1975 & 0.01426 & 0.046 & 305.452 \\
\hline 1377 & 0.378 & 0.02374 & 307.124 & 0.1558 & 0.01426 & 0.019 & 305.396 \\
\hline 1380 & 0.381 & 0.03227 & 307.763 & 0.1566 & 0.01439 & 0.012 & 305.383 \\
\hline 1383 & 0.381 & 0.04209 & 308.496 & 0.1565 & 0.01442 & 0.009 & 305.370 \\
\hline 1386 & 0.380 & 0.05321 & 309.335 & 0.1553 & 0.01442 & 0.006 & 305.380 \\
\hline 1389 & 0.377 & 0.06564 & 310.253 & 0.1538 & 0.01454 & 0.005 & 305.372 \\
\hline 1392 & 0.292 & 0.02380 & 306.401 & 0.1193 & 0.01398 & 0.017 & 304.610 \\
\hline 1395 & 0.292 & 0.03234 & 307.061 & 0.1190 & 0.01423 & 0.013 & 304.592 \\
\hline 1398 & 0.292 & 0.04219 & 307.838 & 0.1188 & 0.01427 & 0.009 & 304.603 \\
\hline 1401 & 0.293 & 0.05336 & 308.718 & 0.1187 & 0.01442 & 0.006 & 304.618 \\
\hline 1404 & 0.294 & 0.06578 & 309.660 & 0.1188 & 0.01450 & 0.005 & 304.606 \\
\hline 1407 & 0.188 & 0.02381 & 306.370 & 0.0758 & 0.01409 & 0.018 & 304.499 \\
\hline 1410 & 0.187 & 0.03236 & 307.084 & 0.0751 & 0.01424 & 0.012 & 304.513 \\
\hline 1413 & 0.187 & 0.04220 & 307.879 & 0.0747 & 0.01429 & 0.009 & 304.502 \\
\hline 1416 & 0.187 & 0.05335 & 308.800 & 0.0746 & 0.01445 & 0.006 & 304.515 \\
\hline 1419 & 0.188 & 0.06579 & 309.784 & 0.0749 & 0.01455 & 0.005 & 304.501 \\
\hline 1452 & 0.178 & 0.02300 & 316.929 & 0.0690 & 0.01514 & 0.022 & 315.210 \\
\hline
\end{tabular}


Table 18. Thermal conductivity of the binary $70 \% \mathrm{R} 32 / 30 \% \mathrm{R} 134 \mathrm{a}$ mixture in the vapor phase (continued).

\begin{tabular}{|c|c|c|c|c|c|c|c|}
\hline $\begin{array}{l}\text { Run } \\
\text { point }\end{array}$ & $\begin{array}{l}P_{\text {exp }} \\
\mathrm{MPa}\end{array}$ & $\begin{array}{c}Q \\
\mathrm{~W} \cdot \mathrm{m}^{-1}\end{array}$ & $\begin{array}{c}T_{\exp } \\
\mathrm{K}\end{array}$ & $\begin{array}{c}\rho_{\text {calc }} \\
\mathrm{mol} \cdot \mathrm{L}^{-1}\end{array}$ & $\begin{array}{c}\lambda_{\exp } \\
\mathrm{W} \cdot \mathrm{m}^{-1} \cdot \mathrm{K}^{-1}\end{array}$ & STAT & $\begin{array}{c}T_{\text {cell }} \\
\mathrm{K}\end{array}$ \\
\hline 1455 & 0.179 & 0.03128 & 317.542 & 0.0691 & 0.01544 & 0.014 & 315.181 \\
\hline 1458 & 0.177 & 0.04081 & 318.291 & 0.0684 & 0.01545 & 0.010 & 315.186 \\
\hline 1461 & 0.176 & 0.05160 & 319.104 & 0.0678 & 0.01539 & 0.007 & 315.170 \\
\hline 1464 & 0.176 & 0.06363 & 320.029 & 0.0675 & 0.01547 & 0.006 & 315.176 \\
\hline 1467 & 0.274 & 0.02302 & 316.664 & 0.1076 & 0.01498 & 0.021 & 315.023 \\
\hline 1470 & 0.275 & 0.03130 & 317.287 & 0.1075 & 0.01508 & 0.014 & 315.018 \\
\hline 1473 & 0.277 & 0.04085 & 317.978 & 0.1081 & 0.01515 & 0.009 & 314.995 \\
\hline 1476 & 0.278 & 0.05165 & 318.773 & 0.1082 & 0.01522 & 0.007 & 314.993 \\
\hline 1479 & 0.276 & 0.06368 & 319.684 & 0.1072 & 0.01531 & 0.005 & 315.005 \\
\hline 1482 & 0.373 & 0.02303 & 316.543 & 0.1485 & 0.01502 & 0.021 & 314.925 \\
\hline 1485 & 0.373 & 0.03132 & 317.133 & 0.1480 & 0.01525 & 0.014 & 314.912 \\
\hline 1488 & 0.373 & 0.04088 & 317.814 & 0.1477 & 0.01499 & 0.010 & 314.901 \\
\hline 1491 & 0.375 & 0.05170 & 318.574 & 0.1481 & 0.01528 & 0.007 & 314.887 \\
\hline 1494 & 0.377 & 0.06375 & 319.429 & 0.1485 & 0.01529 & 0.005 & 314.882 \\
\hline 1497 & 0.473 & 0.02305 & 316.319 & 0.1908 & 0.01510 & 0.021 & 314.744 \\
\hline 1500 & 0.474 & 0.03135 & 316.879 & 0.1908 & 0.01512 & 0.013 & 314.717 \\
\hline 1503 & 0.472 & 0.04091 & 317.549 & 0.1893 & 0.01499 & 0.010 & 314.706 \\
\hline 1506 & 0.470 & 0.05174 & 318.308 & 0.1881 & 0.01529 & 0.007 & 314.707 \\
\hline 1509 & 0.470 & 0.06382 & 319.125 & 0.1872 & 0.01536 & 0.005 & 314.690 \\
\hline 1512 & 0.541 & 0.02306 & 316.161 & 0.2200 & 0.01502 & 0.020 & 314.611 \\
\hline 1515 & 0.542 & 0.03136 & 316.787 & 0.2200 & 0.01520 & 0.052 & 314.637 \\
\hline 1518 & 0.544 & 0.04093 & 317.417 & 0.2205 & 0.01507 & 0.009 & 314.617 \\
\hline 1521 & 0.545 & 0.05176 & 318.146 & 0.2203 & 0.01525 & 0.007 & 314.609 \\
\hline 1524 & 0.543 & 0.06384 & 318.961 & 0.2185 & 0.01538 & 0.005 & 314.596 \\
\hline 1527 & 0.562 & 0.02227 & 327.334 & 0.2197 & 0.01601 & 0.024 & 325.920 \\
\hline 1530 & 0.565 & 0.03030 & 327.844 & 0.2204 & 0.01611 & 0.016 & 325.901 \\
\hline 1533 & 0.565 & 0.03955 & 328.449 & 0.2198 & 0.01624 & 0.011 & 325.898 \\
\hline 1536 & 0.563 & 0.05001 & 329.126 & 0.2184 & 0.01620 & 0.008 & 325.885 \\
\hline 1539 & 0.560 & 0.06169 & 329.860 & 0.2168 & 0.01628 & 0.006 & 325.857 \\
\hline 1542 & 0.466 & 0.02231 & 326.870 & 0.1803 & 0.01593 & 0.025 & 325.424 \\
\hline 1545 & 0.468 & 0.03034 & 327.433 & 0.1808 & 0.01598 & 0.015 & 325.430 \\
\hline 1548 & 0.470 & 0.03960 & 328.057 & 0.1811 & 0.01611 & 0.011 & 325.430 \\
\hline 1551 & 0.470 & 0.05008 & 328.734 & 0.1806 & 0.01611 & 0.008 & 325.406 \\
\hline 1554 & 0.467 & 0.06177 & 329.492 & 0.1789 & 0.01625 & 0.006 & 325.390 \\
\hline 1557 & 0.385 & 0.02233 & 326.667 & 0.1479 & 0.01588 & 0.025 & 325.177 \\
\hline 1560 & 0.383 & 0.03036 & 327.191 & 0.1467 & 0.01594 & 0.015 & 325.148 \\
\hline 1563 & 0.381 & 0.03961 & 327.824 & 0.1455 & 0.01607 & 0.010 & 325.132 \\
\hline 1566 & 0.385 & 0.05009 & 328.531 & 0.1467 & 0.01620 & 0.007 & 325.120 \\
\hline 1569 & 0.385 & 0.06179 & 329.319 & 0.1464 & 0.01629 & 0.006 & 325.117 \\
\hline 1572 & 0.272 & 0.02233 & 326.577 & 0.1033 & 0.01586 & 0.023 & 325.030 \\
\hline 1575 & 0.270 & 0.03037 & 327.129 & 0.1022 & 0.01606 & 0.016 & 325.010 \\
\hline 1578 & 0.268 & 0.03964 & 327.770 & 0.1013 & 0.01620 & 0.011 & 324.996 \\
\hline 1581 & 0.272 & 0.05013 & 328.490 & 0.1023 & 0.01597 & 0.008 & 324.975 \\
\hline 1584 & 0.273 & 0.06183 & 329.294 & 0.1024 & 0.01625 & 0.006 & 324.958 \\
\hline
\end{tabular}


Table 18. Thermal conductivity of the binary $70 \% \mathrm{R} 32 / 30 \% \mathrm{R} 134 \mathrm{a}$ mixture in the vapor phase (continued).

\begin{tabular}{|c|c|c|c|c|c|c|c|}
\hline $\begin{array}{l}\text { Run } \\
\text { point }\end{array}$ & $\begin{array}{l}P_{\text {exp }} \\
\mathrm{MPa}\end{array}$ & $\begin{array}{c}Q \\
\mathrm{~W} \cdot \mathrm{m}^{-1}\end{array}$ & $\begin{array}{c}T_{e x p} \\
\mathrm{~K}\end{array}$ & $\begin{array}{c}\rho_{\text {calc }} \\
\mathrm{mol} \cdot \mathrm{L}^{-1}\end{array}$ & $\begin{array}{c}\lambda_{\exp } \\
\mathrm{W} \cdot \mathrm{m}^{-1} \cdot \mathrm{K}^{-1}\end{array}$ & STAT & $\begin{array}{c}T_{\text {cell }} \\
\mathrm{K}\end{array}$ \\
\hline 1587 & 0.184 & 0.02235 & 326.801 & 0.0689 & 0.01598 & 0.025 & 325.213 \\
\hline 1590 & 0.186 & 0.03038 & 327.374 & 0.0699 & 0.01632 & 0.016 & 325.180 \\
\hline 1593 & 0.189 & 0.03964 & 328.030 & 0.0706 & 0.01613 & 0.011 & 325.156 \\
\hline 1596 & 0.191 & 0.05010 & 328.773 & 0.0712 & 0.01649 & 0.008 & 325.136 \\
\hline 1599 & 0.192 & 0.06177 & 329.612 & 0.0714 & 0.01646 & 0.006 & 325.127 \\
\hline 1636 & 0.179 & 0.02170 & 336.999 & 0.0651 & 0.01738 & 0.028 & 335.514 \\
\hline 1639 & 0.180 & 0.02951 & 337.539 & 0.0651 & 0.01735 & 0.019 & 335.504 \\
\hline 1642 & 0.178 & 0.03852 & 338.157 & 0.0643 & 0.01719 & 0.030 & 335.482 \\
\hline 1645 & 0.176 & 0.04872 & 338.823 & 0.0635 & 0.01748 & 0.009 & 335.447 \\
\hline 1648 & 0.175 & 0.06009 & 339.592 & 0.0629 & 0.01745 & 0.007 & 335.429 \\
\hline 1651 & 0.281 & 0.02168 & 337.242 & 0.1030 & 0.01684 & 0.028 & 335.852 \\
\hline 1654 & 0.283 & 0.02949 & 337.755 & 0.1036 & 0.01717 & 0.019 & 335.830 \\
\hline 1657 & 0.282 & 0.03848 & 338.337 & 0.1030 & 0.01717 & 0.013 & 335.795 \\
\hline 1660 & 0.281 & 0.04867 & 339.027 & 0.1022 & 0.01722 & 0.009 & 335.788 \\
\hline 1663 & 0.279 & 0.06003 & 339.759 & 0.1014 & 0.01732 & 0.007 & 335.766 \\
\hline 1666 & 0.387 & 0.02170 & 337.030 & 0.1435 & 0.01711 & 0.028 & 335.663 \\
\hline 1669 & 0.388 & 0.02951 & 337.520 & 0.1435 & 0.01687 & 0.018 & 335.641 \\
\hline 1672 & 0.390 & 0.03850 & 338.104 & 0.1441 & 0.01677 & 0.012 & 335.633 \\
\hline 1675 & 0.389 & 0.04870 & 338.754 & 0.1434 & 0.01699 & 0.008 & 335.608 \\
\hline 1678 & 0.388 & 0.06007 & 339.483 & 0.1424 & 0.01722 & 0.007 & 335.604 \\
\hline 1681 & 0.537 & 0.02173 & 336.796 & 0.2019 & 0.01649 & 0.027 & 335.480 \\
\hline 1684 & 0.539 & 0.02954 & 337.289 & 0.2026 & 0.01686 & 0.018 & 335.468 \\
\hline 1687 & 0.542 & 0.03855 & 337.842 & 0.2032 & 0.01708 & 0.012 & 335.447 \\
\hline 1690 & 0.542 & 0.04875 & 338.468 & 0.2029 & 0.01710 & 0.009 & 335.426 \\
\hline 1693 & 0.540 & 0.06014 & 339.156 & 0.2017 & 0.01712 & 0.006 & 335.400 \\
\hline 1696 & 0.465 & 0.02175 & 336.349 & 0.1738 & 0.01701 & 0.027 & 335.006 \\
\hline 1699 & 0.463 & 0.02958 & 336.842 & 0.1728 & 0.01706 & 0.019 & 334.974 \\
\hline 1702 & 0.463 & 0.03860 & 337.423 & 0.1726 & 0.01703 & 0.012 & 334.959 \\
\hline 1705 & 0.467 & 0.04882 & 338.060 & 0.1735 & 0.01696 & 0.009 & 334.944 \\
\hline 1708 & 0.467 & 0.06022 & 338.758 & 0.1734 & 0.01713 & 0.006 & 334.922 \\
\hline 1711 & 0.559 & 0.02114 & 345.841 & 0.2044 & 0.01788 & 0.032 & 344.616 \\
\hline 1714 & 0.562 & 0.02876 & 346.272 & 0.2050 & 0.01794 & 0.052 & 344.566 \\
\hline 1717 & 0.564 & 0.03754 & 346.774 & 0.2054 & 0.01783 & 0.014 & 344.553 \\
\hline 1720 & 0.564 & 0.04746 & 347.342 & 0.2051 & 0.01790 & 0.010 & 344.526 \\
\hline 1723 & 0.562 & 0.05855 & 347.988 & 0.2037 & 0.01800 & 0.007 & 344.474 \\
\hline 1726 & 0.468 & 0.02116 & 345.533 & 0.1697 & 0.01799 & 0.032 & 344.292 \\
\hline 1729 & 0.467 & 0.02878 & 345.975 & 0.1690 & 0.01790 & 0.020 & 344.254 \\
\hline 1732 & 0.467 & 0.03757 & 346.490 & 0.1688 & 0.01790 & 0.014 & 344.220 \\
\hline 1735 & 0.468 & 0.04750 & 347.129 & 0.1690 & 0.01789 & 0.010 & 344.218 \\
\hline 1738 & 0.470 & 0.05861 & 347.769 & 0.1692 & 0.01792 & 0.007 & 344.186 \\
\hline 1741 & 0.377 & 0.02119 & 345.098 & 0.1358 & 0.01793 & 0.032 & 343.828 \\
\hline 1744 & 0.377 & 0.02882 & 345.563 & 0.1356 & 0.01787 & 0.020 & 343.807 \\
\hline 1747 & 0.377 & 0.03762 & 346.109 & 0.1353 & 0.01770 & 0.014 & 343.788 \\
\hline 1750 & 0.377 & 0.04757 & 346.735 & 0.1352 & 0.01785 & 0.010 & 343.765 \\
\hline
\end{tabular}


Table 18. Thermal conductivity of the binary $70 \% \mathrm{R} 32 / 30 \% \mathrm{R} 134 \mathrm{a}$ mixture in the vapor phase (continued).

\begin{tabular}{|ccccccc|c|}
\hline $\begin{array}{c}\text { Run } \\
\text { point }\end{array}$ & $\begin{array}{c}P_{\text {exp }} \\
\mathrm{MPa}\end{array}$ & $\begin{array}{c}Q \\
\mathrm{~W} \cdot \mathrm{m}^{-1}\end{array}$ & $\begin{array}{c}T_{\text {exp }} \\
\mathrm{K}\end{array}$ & $\begin{array}{c}\rho_{\text {calc }} \\
\mathrm{mol} \cdot \mathrm{L}^{-1}\end{array}$ & $\begin{array}{c}\lambda_{\text {exp }} \\
\mathrm{W} \cdot \mathrm{m}^{-1} \cdot \mathrm{K}^{-1}\end{array}$ & STAT & $\begin{array}{c}T_{\text {cell }} \\
\mathrm{K}\end{array}$ \\
\hline 1753 & 0.379 & 0.05868 & 347.407 & 0.1354 & 0.01798 & 0.007 & 343.735 \\
1756 & 0.283 & 0.02121 & 344.876 & 0.1013 & 0.01786 & 0.033 & 343.570 \\
1759 & 0.284 & 0.02884 & 345.353 & 0.1012 & 0.01766 & 0.020 & 343.552 \\
1762 & 0.285 & 0.03763 & 345.920 & 0.1015 & 0.01764 & 0.014 & 343.528 \\
1765 & 0.286 & 0.04759 & 346.547 & 0.1016 & 0.01802 & 0.010 & 343.477 \\
1768 & 0.286 & 0.05870 & 347.274 & 0.1014 & 0.01799 & 0.007 & 343.489 \\
1771 & 0.186 & 0.02116 & 345.473 & 0.0659 & 0.01846 & 0.033 & 344.106 \\
1774 & 0.182 & 0.02878 & 345.926 & 0.0642 & 0.01827 & 0.021 & 344.051 \\
1777 & 0.184 & 0.03755 & 346.503 & 0.0650 & 0.01816 & 0.015 & 344.039 \\
1780 & 0.188 & 0.04749 & 347.174 & 0.0662 & 0.01846 & 0.010 & 344.038 \\
1783 & 0.187 & 0.05859 & 347.841 & 0.0658 & 0.01839 & 0.008 & 343.970 \\
\hline
\end{tabular}


Table 19. Thermal conductivity of the binary $30 \% \mathrm{R} 134 \mathrm{a} / 70 \%$ propane mixture in the vapor phase.

\begin{tabular}{|c|c|c|c|c|c|c|c|}
\hline $\begin{array}{c}\text { Run } \\
\text { point }\end{array}$ & $\begin{array}{l}P_{\exp } \\
\mathrm{MPa}\end{array}$ & $\begin{array}{c}Q \\
\mathrm{~W} \cdot \mathrm{m}^{-1} \\
\end{array}$ & $\begin{array}{c}T_{\exp } \\
\mathrm{K}\end{array}$ & $\begin{array}{c}\rho_{\text {calc }} \\
\mathrm{mol} \cdot \mathrm{L}^{-1}\end{array}$ & $\begin{array}{c}\lambda_{\exp } \\
\mathrm{W} \cdot \mathrm{m}^{-1} \cdot \mathrm{K}^{-1} \\
\end{array}$ & STAT & $\begin{array}{c}T_{\text {cell }} \\
\mathrm{K}\end{array}$ \\
\hline 1106 & 0.131 & 0.02756 & 243.296 & 0.0670 & 0.01172 & 0.088 & 240.803 \\
\hline 1111 & 0.131 & 0.03964 & 244.419 & 0.0668 & 0.01195 & 0.053 & 240.812 \\
\hline 1116 & 0.131 & 0.05386 & 245.706 & 0.0665 & 0.01200 & 0.034 & 240.809 \\
\hline 1126 & 0.136 & 0.02757 & 243.229 & 0.0695 & 0.01183 & 0.088 & 240.737 \\
\hline 1131 & 0.136 & 0.03964 & 244.338 & 0.0690 & 0.01194 & 0.052 & 240.737 \\
\hline 1136 & 0.135 & 0.05388 & 245.622 & 0.0686 & 0.01195 & 0.034 & 240.733 \\
\hline 1146 & 0.124 & 0.02761 & 242.614 & 0.0637 & 0.01175 & 0.089 & 240.132 \\
\hline 1151 & 0.125 & 0.03971 & 243.693 & 0.0635 & 0.01194 & 0.053 & 240.105 \\
\hline 1156 & 0.125 & 0.05397 & 244.963 & 0.0631 & 0.01208 & 0.034 & 240.091 \\
\hline 1171 & 0.136 & 0.03744 & 261.578 & 0.0641 & 0.01356 & 0.071 & 258.588 \\
\hline 1176 & 0.135 & 0.05090 & 262.632 & 0.0637 & 0.01370 & 0.046 & 258.563 \\
\hline 1191 & 0.281 & 0.03755 & 260.591 & 0.1376 & 0.01352 & 0.071 & 257.775 \\
\hline 1196 & 0.280 & 0.05104 & 261.590 & 0.1368 & 0.01383 & 0.046 & 257.753 \\
\hline 1211 & 0.294 & 0.03620 & 272.127 & 0.1374 & 0.01465 & 0.085 & 269.625 \\
\hline 1216 & 0.294 & 0.04923 & 273.037 & 0.1366 & 0.01479 & 0.054 & 269.620 \\
\hline 1251 & 0.389 & 0.03620 & 271.788 & 0.1854 & 0.01470 & 0.084 & 269.354 \\
\hline 1256 & 0.389 & 0.04922 & 272.692 & 0.1846 & 0.01485 & 0.054 & 269.361 \\
\hline 1271 & 0.524 & 0.03511 & 282.126 & 0.2453 & 0.01596 & 0.099 & 279.985 \\
\hline 1276 & 0.524 & 0.04774 & 282.918 & 0.2443 & 0.01604 & 0.063 & 279.989 \\
\hline 1291 & 0.668 & 0.03513 & 281.775 & 0.3229 & 0.01599 & 0.100 & 279.729 \\
\hline 1296 & 0.668 & 0.04778 & 282.529 & 0.3217 & 0.01609 & 0.063 & 279.723 \\
\hline 1311 & 0.161 & 0.03511 & 281.847 & 0.0703 & 0.01556 & 0.096 & 279.414 \\
\hline 1316 & 0.158 & 0.04773 & 282.731 & 0.0691 & 0.01591 & 0.063 & 279.399 \\
\hline 1331 & 0.304 & 0.03531 & 280.200 & 0.1372 & 0.01562 & 0.095 & 277.867 \\
\hline 1336 & 0.304 & 0.04802 & 281.036 & 0.1368 & 0.01553 & 0.060 & 277.849 \\
\hline 1351 & 0.692 & 0.03411 & 291.711 & 0.3196 & 0.01731 & 0.117 & 289.832 \\
\hline 1356 & 0.692 & 0.04640 & 292.377 & 0.3186 & 0.01711 & 0.074 & 289.800 \\
\hline 1371 & 0.166 & 0.03410 & 291.883 & 0.0701 & 0.01701 & 0.115 & 289.633 \\
\hline 1376 & 0.166 & 0.04636 & 292.670 & 0.0701 & 0.01713 & 0.073 & 289.603 \\
\hline 1391 & 0.280 & 0.03414 & 291.541 & 0.1202 & 0.01661 & 0.113 & 289.422 \\
\hline 1396 & 0.277 & 0.04643 & 292.314 & 0.1189 & 0.01658 & 0.071 & 289.404 \\
\hline 1401 & 0.521 & 0.01524 & 290.189 & 0.2348 & 0.01664 & 0.044 & 289.309 \\
\hline 1406 & 0.521 & 0.02379 & 290.672 & 0.2342 & 0.01683 & 0.023 & 289.291 \\
\hline 1411 & 0.520 & 0.03423 & 291.284 & 0.2332 & 0.01686 & 0.013 & 289.273 \\
\hline 1416 & 0.520 & 0.04657 & 291.992 & 0.2323 & 0.01669 & 0.009 & 289.245 \\
\hline 1421 & 0.745 & 0.01535 & 299.478 & 0.3349 & 0.01809 & 0.049 & 298.729 \\
\hline 1426 & 0.745 & 0.02396 & 299.938 & 0.3342 & 0.01794 & 0.025 & 298.711 \\
\hline 1431 & 0.745 & 0.03448 & 300.450 & 0.3333 & 0.01800 & 0.014 & 298.657 \\
\hline 1436 & 0.745 & 0.04689 & 301.087 & 0.3322 & 0.01812 & 0.009 & 298.626 \\
\hline 1441 & 0.137 & 0.01534 & 299.230 & 0.0559 & 0.01757 & 0.046 & 298.320 \\
\hline 1446 & 0.137 & 0.02395 & 299.744 & 0.0562 & 0.01774 & 0.025 & 298.276 \\
\hline 1451 & 0.139 & 0.03446 & 300.416 & 0.0568 & 0.01788 & 0.015 & 298.269 \\
\hline 1456 & 0.141 & 0.04687 & 301.242 & 0.0573 & 0.01772 & 0.009 & 298.247 \\
\hline 1461 & 0.227 & 0.01531 & 301.478 & 0.0934 & 0.01741 & 0.045 & 300.590 \\
\hline
\end{tabular}


Table 19. Thermal conductivity of the binary $30 \% \mathrm{R} 134 \mathrm{a} / 70 \%$ propane mixture in the vapor phase (continued).

\begin{tabular}{|ccccccc|c|}
\hline $\begin{array}{c}\text { Run } \\
\text { point }\end{array}$ & $\begin{array}{c}P_{\text {exp }} \\
\mathrm{MPa}\end{array}$ & $\begin{array}{c}\mathrm{Q} \cdot \mathrm{m}^{-1} \\
1466\end{array}$ & $\begin{array}{c}T_{\text {exp }} \\
\mathrm{K}\end{array}$ & $\begin{array}{c}\rho_{\text {calc }} \\
\mathrm{mol} \cdot \mathrm{L}^{-1}\end{array}$ & $\begin{array}{c}\lambda_{\text {exp }} \\
\mathrm{W} \cdot \mathrm{m}^{-1} \cdot \mathrm{K}^{-1}\end{array}$ & $\mathrm{STAT}$ & $\begin{array}{c}T_{\text {cell }} \\
\mathrm{K}\end{array}$ \\
\hline 1471 & 0.227 & 0.02391 & 302.008 & 0.0931 & 0.01768 & 0.024 & 300.576 \\
1476 & 0.227 & 0.03441 & 302.600 & 0.0928 & 0.01770 & 0.014 & 300.513 \\
1481 & 0.226 & 0.04679 & 303.347 & 0.0925 & 0.01771 & 0.009 & 300.487 \\
1486 & 0.523 & 0.01533 & 301.188 & 0.2248 & 0.01755 & 0.047 & 300.404 \\
1491 & 0.521 & 0.02393 & 301.648 & 0.2237 & 0.01792 & 0.024 & 300.381 \\
1496 & 0.521 & 0.03443 & 302.213 & 0.2227 & 0.01799 & 0.014 & 300.348 \\
\hline
\end{tabular}


Table 20 . Thermal conductivity of the binary $70 \% \mathrm{R} 134 \mathrm{a} / 30 \%$ propane mixture in the vapor phase.

\begin{tabular}{|c|c|c|c|c|c|c|c|}
\hline $\begin{array}{c}\text { Run } \\
\text { point }\end{array}$ & $\begin{array}{l}P_{\text {exp }} \\
\mathrm{MPa}\end{array}$ & $\begin{array}{c}Q \\
\mathrm{~W} \cdot \mathrm{m}^{-1}\end{array}$ & $\begin{array}{c}T_{\exp } \\
\mathrm{K} \\
\end{array}$ & $\begin{array}{c}\rho_{\text {calc }} \\
\mathrm{mol} \cdot \mathrm{L}^{-1}\end{array}$ & $\begin{array}{c}\lambda_{\exp } \\
\mathrm{W} \cdot \mathrm{m}^{-1} \cdot \mathrm{K}^{-1}\end{array}$ & STAT & $\begin{array}{c}T_{\text {cell }} \\
\mathrm{K}\end{array}$ \\
\hline 1016 & 0.087 & 0.02392 & 258.299 & 0.0415 & 0.01187 & 0.015 & 256.020 \\
\hline 1019 & 0.088 & 0.03252 & 259.156 & 0.0415 & 0.01174 & 0.009 & 256.040 \\
\hline 1022 & 0.088 & 0.04244 & 260.096 & 0.0415 & 0.01195 & 0.007 & 256.030 \\
\hline 1025 & 0.087 & 0.05365 & 261.184 & 0.0409 & 0.01213 & 0.005 & 256.047 \\
\hline 1028 & 0.087 & 0.06615 & 262.343 & 0.0408 & 0.01220 & 0.004 & 256.033 \\
\hline 1031 & 0.163 & 0.02394 & 258.188 & 0.0788 & 0.01176 & 0.016 & 256.025 \\
\hline 1034 & 0.162 & 0.03255 & 258.986 & 0.0781 & 0.01173 & 0.010 & 256.033 \\
\hline 1037 & 0.162 & 0.04246 & 259.897 & 0.0779 & 0.01198 & 0.007 & 256.046 \\
\hline 1040 & 0.163 & 0.05367 & 260.889 & 0.0779 & 0.01206 & 0.005 & 256.028 \\
\hline 1043 & 0.163 & 0.06617 & 262.005 & 0.0777 & 0.01212 & 0.004 & 256.027 \\
\hline 1046 & 0.094 & 0.02325 & 267.417 & 0.0433 & 0.01269 & 0.018 & 265.332 \\
\hline 1049 & 0.093 & 0.03161 & 268.207 & 0.0427 & 0.01270 & 0.011 & 265.360 \\
\hline 1052 & 0.092 & 0.04123 & 269.063 & 0.0419 & 0.01288 & 0.008 & 265.340 \\
\hline 1055 & 0.093 & 0.05211 & 270.023 & 0.0420 & 0.01289 & 0.006 & 265.324 \\
\hline 1058 & 0.093 & 0.06425 & 271.120 & 0.0422 & 0.01300 & 0.005 & 265.342 \\
\hline 1061 & 0.192 & 0.02325 & 267.282 & 0.0903 & 0.01246 & 0.016 & 265.341 \\
\hline 1064 & 0.193 & 0.03161 & 267.988 & 0.0903 & 0.01258 & 0.010 & 265.334 \\
\hline 1067 & 0.193 & 0.04125 & 268.815 & 0.0902 & 0.01273 & 0.007 & 265.342 \\
\hline 1070 & 0.193 & 0.05215 & 269.714 & 0.0895 & 0.01281 & 0.005 & 265.326 \\
\hline 1073 & 0.192 & 0.06431 & 270.742 & 0.0889 & 0.01288 & 0.004 & 265.344 \\
\hline 1076 & 0.286 & 0.02326 & 267.149 & 0.1376 & 0.01254 & 0.016 & 265.301 \\
\hline 1079 & 0.285 & 0.03162 & 267.825 & 0.1367 & 0.01268 & 0.011 & 265.298 \\
\hline 1082 & 0.285 & 0.04125 & 268.620 & 0.1363 & 0.01276 & 0.008 & 265.308 \\
\hline 1085 & 0.286 & 0.05216 & 269.499 & 0.1362 & 0.01312 & 0.015 & 265.310 \\
\hline 1088 & 0.286 & 0.06432 & 270.454 & 0.1354 & 0.01294 & 0.004 & 265.290 \\
\hline 1091 & 0.083 & 0.02254 & 277.227 & 0.0367 & 0.01355 & 0.020 & 275.314 \\
\hline 1094 & 0.084 & 0.03065 & 277.932 & 0.0370 & 0.01376 & 0.014 & 275.314 \\
\hline 1097 & 0.085 & 0.04000 & 278.760 & 0.0375 & 0.01385 & 0.010 & 275.329 \\
\hline 1100 & 0.086 & 0.05058 & 279.660 & 0.0376 & 0.01391 & 0.007 & 275.318 \\
\hline 1103 & 0.086 & 0.06238 & 280.660 & 0.0375 & 0.01402 & 0.006 & 275.316 \\
\hline 1106 & 0.178 & 0.02257 & 277.106 & 0.0800 & 0.01342 & 0.020 & 275.311 \\
\hline 1109 & 0.179 & 0.03068 & 277.778 & 0.0801 & 0.01359 & 0.012 & 275.320 \\
\hline 1112 & 0.180 & 0.04002 & 278.532 & 0.0805 & 0.01364 & 0.008 & 275.312 \\
\hline 1115 & 0.181 & 0.05060 & 279.378 & 0.0805 & 0.01371 & 0.006 & 275.309 \\
\hline 1118 & 0.180 & 0.06239 & 280.327 & 0.0799 & 0.01381 & 0.005 & 275.314 \\
\hline 1121 & 0.262 & 0.02257 & 277.008 & 0.1199 & 0.01334 & 0.019 & 275.298 \\
\hline 1124 & 0.262 & 0.03068 & 277.634 & 0.1198 & 0.01349 & 0.012 & 275.290 \\
\hline 1127 & 0.261 & 0.04003 & 278.371 & 0.1186 & 0.01357 & 0.009 & 275.293 \\
\hline 1130 & 0.260 & 0.05062 & 279.199 & 0.1180 & 0.01366 & 0.006 & 275.290 \\
\hline 1133 & 0.261 & 0.06244 & 280.114 & 0.1180 & 0.01380 & 0.005 & 275.299 \\
\hline 1136 & 0.341 & 0.02257 & 276.946 & 0.1588 & 0.01349 & 0.020 & 275.287 \\
\hline 1139 & 0.342 & 0.03070 & 277.563 & 0.1587 & 0.01369 & 0.013 & 275.288 \\
\hline 1142 & 0.341 & 0.04005 & 278.287 & 0.1577 & 0.01368 & 0.008 & 275.299 \\
\hline 1145 & 0.339 & 0.05064 & 279.077 & 0.1564 & 0.01372 & 0.006 & 275.297 \\
\hline
\end{tabular}


Table 20. Thermal conductivity of the binary $70 \% \mathrm{R} 134 \mathrm{a} / 30 \%$ propane mixture in the vapor phase (continued).

\begin{tabular}{|c|c|c|c|c|c|c|c|}
\hline $\begin{array}{l}\text { Run } \\
\text { point }\end{array}$ & $\begin{array}{l}P_{\text {exp }} \\
\mathrm{MPa}\end{array}$ & $\begin{array}{c}Q \\
W \cdot m^{-1}\end{array}$ & $\begin{array}{c}T_{\text {exp }} \\
\mathrm{K}\end{array}$ & $\begin{array}{c}\rho_{\text {calc }} \\
\mathrm{mol} \cdot \mathrm{L}^{-1}\end{array}$ & $\begin{array}{c}\lambda_{\exp } \\
\mathrm{W} \cdot \mathrm{m}^{-1} \cdot \mathrm{K}^{-1}\end{array}$ & STAT & $\begin{array}{c}T_{\text {cell }} \\
\mathrm{K}\end{array}$ \\
\hline 1148 & 0.340 & 0.06244 & 279.950 & 0.1563 & 0.01379 & 0.005 & 275.290 \\
\hline 1151 & 0.411 & 0.02258 & 276.892 & 0.1949 & 0.01353 & 0.019 & 275.274 \\
\hline 1154 & 0.410 & 0.03070 & 277.516 & 0.1938 & 0.01359 & 0.012 & 275.300 \\
\hline 1157 & 0.410 & 0.04005 & 278.184 & 0.1930 & 0.01359 & 0.008 & 275.278 \\
\hline 1163 & 0.411 & 0.06246 & 279.835 & 0.1922 & 0.01384 & 0.005 & 275.287 \\
\hline 1166 & 0.426 & 0.02270 & 286.779 & 0.1932 & 0.01441 & 0.021 & 285.250 \\
\hline 1169 & 0.427 & 0.03085 & 287.353 & 0.1935 & 0.01456 & 0.013 & 285.253 \\
\hline 1172 & 0.431 & 0.04025 & 288.005 & 0.1947 & 0.01439 & 0.009 & 285.249 \\
\hline 1175 & 0.431 & 0.05089 & 288.761 & 0.1943 & 0.01468 & 0.007 & 285.264 \\
\hline 1178 & 0.429 & 0.06276 & 289.585 & 0.1924 & 0.01478 & 0.005 & 285.259 \\
\hline 1181 & 0.555 & 0.02270 & 286.737 & 0.2591 & 0.01457 & 0.021 & 285.246 \\
\hline 1184 & 0.559 & 0.03085 & 287.248 & 0.2606 & 0.01474 & 0.014 & 285.249 \\
\hline 1187 & 0.561 & 0.04026 & 287.867 & 0.2605 & 0.01464 & 0.009 & 285.237 \\
\hline 1190 & 0.556 & 0.05090 & 288.594 & 0.2575 & 0.01483 & 0.007 & 285.248 \\
\hline 1193 & 0.554 & 0.06278 & 289.388 & 0.2553 & 0.01487 & 0.005 & 285.259 \\
\hline 1196 & 0.555 & 0.02271 & 286.685 & 0.2594 & 0.01463 & 0.022 & 285.195 \\
\hline 1199 & 0.559 & 0.03086 & 287.264 & 0.2606 & 0.01460 & 0.013 & 285.224 \\
\hline 1202 & 0.559 & 0.04026 & 287.872 & 0.2599 & 0.01453 & 0.009 & 285.200 \\
\hline 1205 & 0.555 & 0.05091 & 288.590 & 0.2569 & 0.01482 & 0.006 & 285.209 \\
\hline 1208 & 0.555 & 0.06280 & 289.375 & 0.2558 & 0.01487 & 0.005 & 285.208 \\
\hline 1211 & 0.351 & 0.02268 & 286.767 & 0.1568 & 0.01455 & 0.021 & 285.188 \\
\hline 1214 & 0.354 & 0.03084 & 287.371 & 0.1578 & 0.01465 & 0.013 & 285.199 \\
\hline 1217 & 0.353 & 0.04024 & 288.041 & 0.1569 & 0.01446 & 0.009 & 285.190 \\
\hline 1223 & 0.350 & 0.06276 & 289.646 & 0.1543 & 0.01466 & 0.005 & 285.203 \\
\hline 1226 & 0.269 & 0.02271 & 286.447 & 0.1186 & 0.01439 & 0.021 & 284.820 \\
\hline 1229 & 0.269 & 0.03088 & 287.067 & 0.1180 & 0.01439 & 0.013 & 284.823 \\
\hline 1232 & 0.268 & 0.04029 & 287.773 & 0.1176 & 0.01455 & 0.009 & 284.827 \\
\hline 1235 & 0.269 & 0.05094 & 288.554 & 0.1174 & 0.01468 & 0.007 & 284.829 \\
\hline 1238 & 0.270 & 0.06283 & 289.413 & 0.1176 & 0.01475 & 0.005 & 284.827 \\
\hline 1241 & 0.552 & 0.03103 & 286.298 & 0.2582 & 0.01464 & 0.014 & 284.234 \\
\hline 1244 & 0.553 & 0.04048 & 286.947 & 0.2579 & 0.01446 & 0.009 & 284.247 \\
\hline 1247 & 0.552 & 0.05118 & 287.659 & 0.2565 & 0.01462 & 0.007 & 284.240 \\
\hline 1250 & 0.551 & 0.06312 & 288.463 & 0.2550 & 0.01477 & 0.005 & 284.244 \\
\hline 1253 & 0.552 & 0.07630 & 289.336 & 0.2543 & 0.01481 & 0.004 & 284.239 \\
\hline 1256 & 0.446 & 0.03103 & 286.370 & 0.2034 & 0.01446 & 0.013 & 284.237 \\
\hline 1259 & 0.445 & 0.04048 & 287.020 & 0.2027 & 0.01446 & 0.009 & 284.231 \\
\hline 1262 & 0.444 & 0.05117 & 287.763 & 0.2015 & 0.01446 & 0.006 & 284.233 \\
\hline 1265 & 0.443 & 0.06311 & 288.565 & 0.2003 & 0.01469 & 0.005 & 284.216 \\
\hline 1268 & 0.444 & 0.07627 & 289.490 & 0.1999 & 0.01474 & 0.004 & 284.242 \\
\hline 1271 & 0.347 & 0.03103 & 286.386 & 0.1550 & 0.01455 & 0.013 & 284.222 \\
\hline 1274 & 0.346 & 0.04047 & 287.064 & 0.1542 & 0.01441 & 0.009 & 284.225 \\
\hline 1277 & 0.345 & 0.05116 & 287.832 & 0.1532 & 0.01463 & 0.006 & 284.227 \\
\hline 1280 & 0.344 & 0.06309 & 288.659 & 0.1524 & 0.01466 & 0.005 & 284.214 \\
\hline
\end{tabular}


Table 20. Thermal conductivity of the binary $70 \% \mathrm{R} 134 \mathrm{a} / 30 \%$ propane mixture in the vapor phase (continued).

\begin{tabular}{|c|c|c|c|c|c|c|c|}
\hline $\begin{array}{l}\text { Run } \\
\text { point }\end{array}$ & $\begin{array}{l}P_{\text {exp }} \\
\mathrm{MPa}\end{array}$ & $\begin{array}{c}Q \\
\mathrm{~W} \cdot \mathrm{m}^{-1}\end{array}$ & $\begin{array}{c}T_{e x p} \\
\mathrm{~K}\end{array}$ & $\begin{array}{c}\rho_{\text {calc }} \\
\mathrm{mol} \cdot \mathrm{L}^{-1}\end{array}$ & $\begin{array}{c}\lambda_{\exp } \\
\mathrm{W} \cdot \mathrm{m}^{-1} \cdot \mathrm{K}^{-1}\end{array}$ & STAT & $\begin{array}{c}T_{\text {cell }} \\
\mathrm{K}\end{array}$ \\
\hline 1283 & 0.345 & 0.07627 & 289.610 & 0.1521 & 0.01476 & 0.004 & 284.238 \\
\hline 1286 & 0.218 & 0.03102 & 286.482 & 0.0951 & 0.01445 & 0.014 & 284.220 \\
\hline 1289 & 0.218 & 0.04047 & 287.210 & 0.0947 & 0.01420 & 0.009 & 284.221 \\
\hline 1292 & 0.218 & 0.05116 & 288.027 & 0.0944 & 0.01432 & 0.007 & 284.234 \\
\hline 1295 & 0.218 & 0.06309 & 288.909 & 0.0944 & 0.01461 & 0.006 & 284.218 \\
\hline 1298 & 0.219 & 0.07625 & 289.904 & 0.0944 & 0.01472 & 0.004 & 284.234 \\
\hline 1301 & 0.101 & 0.09063 & 291.387 & 0.0426 & 0.01491 & 0.004 & 284.217 \\
\hline 1304 & 0.102 & 0.10618 & 292.597 & 0.0424 & 0.01503 & 0.003 & 284.220 \\
\hline 1307 & 0.103 & 0.12295 & 293.895 & 0.0428 & 0.01522 & 0.003 & 284.218 \\
\hline 1310 & 0.104 & 0.14088 & 295.271 & 0.0431 & 0.01539 & 0.003 & 284.218 \\
\hline 1313 & 0.104 & 0.16000 & 296.730 & 0.0427 & 0.01552 & 0.003 & 284.220 \\
\hline 1316 & 0.558 & 0.03054 & 296.832 & 0.2483 & 0.01548 & 0.015 & 294.905 \\
\hline 1319 & 0.557 & 0.03986 & 297.431 & 0.2473 & 0.01552 & 0.010 & 294.907 \\
\hline 1322 & 0.557 & 0.05040 & 298.116 & 0.2466 & 0.01562 & 0.007 & 294.913 \\
\hline 1325 & 0.558 & 0.06215 & 298.857 & 0.2463 & 0.01565 & 0.005 & 294.904 \\
\hline 1328 & 0.560 & 0.07512 & 299.678 & 0.2463 & 0.01578 & 0.004 & 294.904 \\
\hline 1331 & 0.439 & 0.03054 & 296.858 & 0.1913 & 0.01553 & 0.016 & 294.887 \\
\hline 1334 & 0.439 & 0.03985 & 297.479 & 0.1905 & 0.01560 & 0.010 & 294.900 \\
\hline 1337 & 0.438 & 0.05040 & 298.164 & 0.1897 & 0.01555 & 0.007 & 294.885 \\
\hline 1340 & 0.438 & 0.06216 & 298.951 & 0.1890 & 0.01562 & 0.006 & 294.897 \\
\hline 1343 & 0.439 & 0.07511 & 299.790 & 0.1887 & 0.01574 & 0.005 & 294.895 \\
\hline 1346 & 0.315 & 0.03055 & 296.912 & 0.1343 & 0.01524 & 0.014 & 294.862 \\
\hline 1349 & 0.314 & 0.03985 & 297.591 & 0.1333 & 0.01529 & 0.010 & 294.893 \\
\hline 1352 & 0.315 & 0.05037 & 298.307 & 0.1333 & 0.01549 & 0.007 & 294.883 \\
\hline 1355 & 0.316 & 0.06212 & 299.103 & 0.1336 & 0.01560 & 0.005 & 294.873 \\
\hline 1358 & 0.316 & 0.07511 & 299.998 & 0.1331 & 0.01564 & 0.004 & 294.888 \\
\hline 1361 & 0.212 & 0.03053 & 296.990 & 0.0887 & 0.01553 & 0.014 & 294.848 \\
\hline 1364 & 0.213 & 0.03983 & 297.673 & 0.0889 & 0.01530 & 0.010 & 294.858 \\
\hline 1367 & 0.213 & 0.05037 & 298.448 & 0.0885 & 0.01552 & 0.008 & 294.879 \\
\hline 1370 & 0.211 & 0.06212 & 299.284 & 0.0876 & 0.01559 & 0.005 & 294.877 \\
\hline 1373 & 0.210 & 0.07509 & 300.201 & 0.0869 & 0.01573 & 0.004 & 294.869 \\
\hline 1376 & 0.103 & 0.03053 & 297.118 & 0.0425 & 0.01564 & 0.015 & 294.835 \\
\hline 1379 & 0.103 & 0.03982 & 297.855 & 0.0422 & 0.01577 & 0.011 & 294.856 \\
\hline 1382 & 0.104 & 0.05035 & 298.651 & 0.0426 & 0.01584 & 0.008 & 294.853 \\
\hline 1385 & 0.105 & 0.06210 & 299.535 & 0.0430 & 0.01591 & 0.006 & 294.854 \\
\hline 1388 & 0.106 & 0.07506 & 300.521 & 0.0431 & 0.01598 & 0.005 & 294.867 \\
\hline 1391 & 0.555 & 0.02952 & 306.708 & 0.2365 & 0.01639 & 0.018 & 304.932 \\
\hline 1394 & 0.553 & 0.03852 & 307.260 & 0.2348 & 0.01648 & 0.012 & 304.928 \\
\hline 1397 & 0.551 & 0.04872 & 307.900 & 0.2335 & 0.01651 & 0.008 & 304.944 \\
\hline 1400 & 0.553 & 0.06010 & 308.589 & 0.2337 & 0.01667 & 0.006 & 304.939 \\
\hline 1403 & 0.555 & 0.07268 & 309.341 & 0.2339 & 0.01677 & 0.005 & 304.929 \\
\hline 1406 & 0.445 & 0.02953 & 306.706 & 0.1864 & 0.01639 & 0.018 & 304.910 \\
\hline 1409 & 0.445 & 0.03853 & 307.273 & 0.1856 & 0.01654 & 0.012 & 304.912 \\
\hline
\end{tabular}


Table 20. Thermal conductivity of the binary $70 \% \mathrm{R} 134 \mathrm{a} / 30 \%$ propane mixture in the vapor phase (continued).

\begin{tabular}{|c|c|c|c|c|c|c|c|}
\hline $\begin{array}{l}\text { Run } \\
\text { point }\end{array}$ & $\begin{array}{l}P_{\text {exp }} \\
\mathrm{MPa}\end{array}$ & $\begin{array}{c}Q \\
\mathrm{~W} \cdot \mathrm{m}^{-1}\end{array}$ & $\begin{array}{c}T_{\exp } \\
\mathrm{K}\end{array}$ & $\begin{array}{c}\rho_{\text {calc }} \\
\mathrm{mol} \cdot \mathrm{L}^{-1}\end{array}$ & $\begin{array}{c}\lambda_{\exp } \\
\mathrm{W} \cdot \mathrm{m}^{-1} \cdot \mathrm{K}^{-1}\end{array}$ & STAT & $\begin{array}{c}T_{\text {cell }} \\
\mathrm{K}\end{array}$ \\
\hline 1412 & 0.446 & 0.04871 & 307.932 & 0.1859 & 0.01655 & 0.008 & 304.927 \\
\hline 1415 & 0.448 & 0.06009 & 308.635 & 0.1859 & 0.01635 & 0.006 & 304.900 \\
\hline 1418 & 0.447 & 0.07265 & 309.440 & 0.1848 & 0.01659 & 0.005 & 304.924 \\
\hline 1421 & 0.313 & 0.02953 & 306.755 & 0.1282 & 0.01627 & 0.018 & 304.908 \\
\hline 1424 & 0.312 & 0.03852 & 307.327 & 0.1275 & 0.01634 & 0.012 & 304.894 \\
\hline 1427 & 0.310 & 0.04872 & 308.010 & 0.1264 & 0.01656 & 0.009 & 304.902 \\
\hline 1430 & 0.309 & 0.06010 & 308.793 & 0.1254 & 0.01658 & 0.006 & 304.904 \\
\hline 1433 & 0.310 & 0.07265 & 309.595 & 0.1255 & 0.01670 & 0.005 & 304.891 \\
\hline 1436 & 0.216 & 0.02953 & 306.820 & 0.0872 & 0.01633 & 0.018 & 304.876 \\
\hline 1439 & 0.215 & 0.03853 & 307.440 & 0.0866 & 0.01651 & 0.011 & 304.887 \\
\hline 1442 & 0.218 & 0.04872 & 308.146 & 0.0876 & 0.01646 & 0.009 & 304.892 \\
\hline 1445 & 0.220 & 0.06008 & 308.897 & 0.0883 & 0.01667 & 0.006 & 304.876 \\
\hline 1448 & 0.218 & 0.07261 & 309.730 & 0.0871 & 0.01671 & 0.005 & 304.866 \\
\hline 1451 & 0.109 & 0.02945 & 306.386 & 0.0434 & 0.01674 & 0.018 & 304.287 \\
\hline 1454 & 0.105 & 0.03843 & 307.044 & 0.0419 & 0.01684 & 0.013 & 304.294 \\
\hline 1457 & 0.103 & 0.04860 & 307.782 & 0.0410 & 0.01678 & 0.009 & 304.293 \\
\hline 1460 & 0.104 & 0.05993 & 308.593 & 0.0410 & 0.01686 & 0.007 & 304.294 \\
\hline 1463 & 0.107 & 0.07242 & 309.485 & 0.0422 & 0.01700 & 0.006 & 304.298 \\
\hline 1466 & 0.552 & 0.03050 & 316.729 & 0.2256 & 0.01763 & 0.017 & 314.981 \\
\hline 1469 & 0.551 & 0.03979 & 317.317 & 0.2247 & 0.01764 & 0.012 & 315.019 \\
\hline 1472 & 0.551 & 0.05030 & 317.913 & 0.2238 & 0.01762 & 0.009 & 314.991 \\
\hline 1475 & 0.551 & 0.06204 & 318.594 & 0.2235 & 0.01751 & 0.006 & 314.990 \\
\hline 1478 & 0.554 & 0.07499 & 319.347 & 0.2239 & 0.01763 & 0.005 & 314.993 \\
\hline 1481 & 0.427 & 0.03049 & 316.756 & 0.1715 & 0.01727 & 0.017 & 314.975 \\
\hline 1484 & 0.427 & 0.03978 & 317.295 & 0.1708 & 0.01750 & 0.012 & 314.952 \\
\hline 1487 & 0.426 & 0.05030 & 317.959 & 0.1703 & 0.01758 & 0.008 & 314.981 \\
\hline 1490 & 0.428 & 0.06203 & 318.638 & 0.1705 & 0.01764 & 0.006 & 314.956 \\
\hline 1493 & 0.429 & 0.07500 & 319.413 & 0.1707 & 0.01769 & 0.005 & 314.957 \\
\hline 1496 & 0.313 & 0.03047 & 316.735 & 0.1238 & 0.01747 & 0.017 & 314.879 \\
\hline 1499 & 0.316 & 0.03975 & 317.316 & 0.1248 & 0.01753 & 0.012 & 314.879 \\
\hline 1502 & 0.319 & 0.05028 & 317.966 & 0.1255 & 0.01753 & 0.009 & 314.873 \\
\hline 1505 & 0.319 & 0.06201 & 318.692 & 0.1253 & 0.01767 & 0.007 & 314.873 \\
\hline 1508 & 0.316 & 0.07496 & 319.494 & 0.1238 & 0.01774 & 0.005 & 314.874 \\
\hline 1511 & 0.204 & 0.03047 & 316.787 & 0.0796 & 0.01752 & 0.018 & 314.861 \\
\hline 1514 & 0.208 & 0.03975 & 317.369 & 0.0809 & 0.01758 & 0.012 & 314.833 \\
\hline 1517 & 0.209 & 0.05024 & 318.072 & 0.0812 & 0.01757 & 0.009 & 314.854 \\
\hline 1520 & 0.206 & 0.06197 & 318.836 & 0.0797 & 0.01763 & 0.007 & 314.861 \\
\hline 1523 & 0.204 & 0.07491 & 319.648 & 0.0787 & 0.01769 & 0.005 & 314.844 \\
\hline 1526 & 0.102 & 0.03046 & 316.842 & 0.0392 & 0.01773 & 0.018 & 314.807 \\
\hline 1529 & 0.105 & 0.03974 & 317.467 & 0.0405 & 0.01803 & 0.013 & 314.792 \\
\hline 1532 & 0.109 & 0.05024 & 318.215 & 0.0417 & 0.01801 & 0.009 & 314.820 \\
\hline 1535 & 0.106 & 0.06196 & 318.996 & 0.0405 & 0.01816 & 0.008 & 314.802 \\
\hline 1538 & 0.104 & 0.07489 & 319.867 & 0.0396 & 0.01816 & 0.006 & 314.799 \\
\hline
\end{tabular}


Table 20. Thermal conductivity of the binary $70 \% \mathrm{R} 134 \mathrm{a} / 30 \%$ propane mixture in the vapor phase (continued).

\begin{tabular}{|c|c|c|c|c|c|c|c|}
\hline $\begin{array}{l}\text { Run } \\
\text { point }\end{array}$ & $\begin{array}{l}P_{\text {exp }} \\
\mathrm{MPa}\end{array}$ & $\begin{array}{c}Q \\
\mathrm{~W} \cdot \mathrm{m}^{-1}\end{array}$ & $\begin{array}{c}T_{e x p} \\
\mathrm{~K}\end{array}$ & $\begin{array}{c}\rho_{\text {calc }} \\
\mathrm{mol} \cdot \mathrm{L}^{-1}\end{array}$ & $\begin{array}{c}\lambda_{\exp } \\
\mathrm{W} \cdot \mathrm{m}^{-1} \cdot \mathrm{K}^{-1}\end{array}$ & STAT & $\begin{array}{c}T_{\text {cell }} \\
\mathrm{K}\end{array}$ \\
\hline 1541 & 0.556 & 0.03042 & 326.371 & 0.2188 & 0.01862 & 0.018 & 324.721 \\
\hline 1544 & 0.558 & 0.03968 & 326.907 & 0.2193 & 0.01852 & 0.013 & 324.728 \\
\hline 1547 & 0.557 & 0.05017 & 327.480 & 0.2183 & 0.01852 & 0.009 & 324.709 \\
\hline 1550 & 0.553 & 0.06189 & 328.143 & 0.2162 & 0.01864 & 0.007 & 324.717 \\
\hline 1553 & 0.555 & 0.07484 & 328.850 & 0.2163 & 0.01850 & 0.005 & 324.712 \\
\hline 1556 & 0.428 & 0.03042 & 326.365 & 0.1656 & 0.01855 & 0.019 & 324.694 \\
\hline 1559 & 0.429 & 0.03968 & 326.894 & 0.1657 & 0.01861 & 0.013 & 324.681 \\
\hline 1562 & 0.425 & 0.05017 & 327.519 & 0.1640 & 0.01866 & 0.009 & 324.698 \\
\hline 1565 & 0.425 & 0.06189 & 328.192 & 0.1636 & 0.01872 & 0.007 & 324.697 \\
\hline 1568 & 0.427 & 0.07481 & 328.939 & 0.1637 & 0.01875 & 0.005 & 324.706 \\
\hline 1571 & 0.303 & 0.03039 & 326.454 & 0.1155 & 0.01839 & 0.018 & 324.675 \\
\hline 1574 & 0.305 & 0.03966 & 327.001 & 0.1160 & 0.01850 & 0.013 & 324.666 \\
\hline 1577 & 0.306 & 0.05015 & 327.654 & 0.1162 & 0.01873 & 0.009 & 324.687 \\
\hline 1580 & 0.305 & 0.06188 & 328.349 & 0.1157 & 0.01871 & 0.007 & 324.692 \\
\hline 1583 & 0.303 & 0.07481 & 329.126 & 0.1145 & 0.01877 & 0.005 & 324.704 \\
\hline 1586 & 0.206 & 0.03041 & 326.451 & 0.0778 & 0.01858 & 0.018 & 324.632 \\
\hline 1589 & 0.207 & 0.03966 & 327.051 & 0.0781 & 0.01857 & 0.013 & 324.654 \\
\hline 1592 & 0.209 & 0.05015 & 327.715 & 0.0784 & 0.01870 & 0.009 & 324.666 \\
\hline 1595 & 0.208 & 0.06185 & 328.414 & 0.0778 & 0.01878 & 0.007 & 324.647 \\
\hline 1598 & 0.206 & 0.07475 & 329.197 & 0.0770 & 0.01897 & 0.005 & 324.638 \\
\hline 1616 & 0.566 & 0.02945 & 336.404 & 0.2150 & 0.01931 & 0.021 & 334.879 \\
\hline 1619 & 0.565 & 0.03840 & 336.880 & 0.2142 & 0.01936 & 0.014 & 334.867 \\
\hline 1622 & 0.564 & 0.04856 & 337.427 & 0.2135 & 0.01928 & 0.011 & 334.866 \\
\hline 1625 & 0.565 & 0.05991 & 338.037 & 0.2133 & 0.01966 & 0.008 & 334.873 \\
\hline 1628 & 0.565 & 0.07243 & 338.688 & 0.2128 & 0.01974 & 0.006 & 334.857 \\
\hline 1631 & 0.439 & 0.02944 & 336.364 & 0.1646 & 0.01947 & 0.021 & 334.825 \\
\hline 1634 & 0.439 & 0.03839 & 336.864 & 0.1641 & 0.01959 & 0.015 & 334.827 \\
\hline 1637 & 0.440 & 0.04855 & 337.439 & 0.1642 & 0.01953 & 0.010 & 334.842 \\
\hline 1640 & 0.440 & 0.05989 & 338.052 & 0.1637 & 0.01957 & 0.008 & 334.831 \\
\hline 1643 & 0.439 & 0.07241 & 338.746 & 0.1631 & 0.01966 & 0.006 & 334.840 \\
\hline 1646 & 0.312 & 0.02943 & 336.399 & 0.1151 & 0.01934 & 0.021 & 334.809 \\
\hline 1649 & 0.311 & 0.03839 & 336.908 & 0.1146 & 0.01994 & 0.015 & 334.810 \\
\hline 1652 & 0.313 & 0.04854 & 337.480 & 0.1153 & 0.01947 & 0.011 & 334.802 \\
\hline 1655 & 0.315 & 0.05988 & 338.121 & 0.1155 & 0.01978 & 0.008 & 334.799 \\
\hline 1658 & 0.314 & 0.07239 & 338.832 & 0.1150 & 0.01986 & 0.006 & 334.807 \\
\hline 1661 & 0.213 & 0.02943 & 336.386 & 0.0780 & 0.01985 & 0.022 & 334.747 \\
\hline 1664 & 0.212 & 0.03839 & 336.943 & 0.0774 & 0.01971 & 0.016 & 334.772 \\
\hline 1667 & 0.213 & 0.04854 & 337.551 & 0.0776 & 0.01962 & 0.011 & 334.782 \\
\hline 1670 & 0.214 & 0.05988 & 338.217 & 0.0777 & 0.01974 & 0.008 & 334.783 \\
\hline 1673 & 0.214 & 0.07240 & 338.927 & 0.0774 & 0.02007 & 0.006 & 334.763 \\
\hline 1691 & 0.554 & 0.03719 & 346.114 & 0.2032 & 0.02044 & 0.016 & 344.260 \\
\hline 1694 & 0.554 & 0.04702 & 346.601 & 0.2028 & 0.02072 & 0.012 & 344.239 \\
\hline 1697 & 0.554 & 0.05800 & 347.166 & 0.2023 & 0.02080 & 0.009 & 344.241 \\
\hline
\end{tabular}


Table 20. Thermal conductivity of the binary $70 \% \mathrm{R} 134 \mathrm{a} / 30 \%$ propane mixture in the vapor phase (continued).

\begin{tabular}{|ccccccc|c|}
\hline $\begin{array}{c}\text { Run } \\
\text { point }\end{array}$ & $\begin{array}{c}P_{\text {exp }} \\
\mathrm{MPa}\end{array}$ & $\begin{array}{c}Q \\
\mathrm{~W} \cdot \mathrm{m}^{-1}\end{array}$ & $\begin{array}{c}T_{\text {exp }} \\
\mathrm{K}\end{array}$ & $\begin{array}{c}\rho_{\text {calc }} \\
\mathrm{mol} \cdot \mathrm{L}^{-1}\end{array}$ & $\begin{array}{c}\lambda_{\text {exp }} \\
\mathrm{W} \cdot \mathrm{m}^{-1} \cdot \mathrm{K}^{-1}\end{array}$ & STAT & $\begin{array}{c}T_{\text {cell }} \\
\mathrm{K}\end{array}$ \\
\hline 1700 & 0.554 & 0.07014 & 347.763 & 0.2020 & 0.02071 & 0.007 & 344.224 \\
1703 & 0.554 & 0.08340 & 348.424 & 0.2016 & 0.02094 & 0.005 & 344.219 \\
1706 & 0.447 & 0.03719 & 346.045 & 0.1622 & 0.02073 & 0.017 & 344.155 \\
1709 & 0.447 & 0.04702 & 346.572 & 0.1620 & 0.02075 & 0.012 & 344.174 \\
1712 & 0.447 & 0.05799 & 347.132 & 0.1616 & 0.02084 & 0.009 & 344.156 \\
1715 & 0.448 & 0.07012 & 347.755 & 0.1614 & 0.02081 & 0.007 & 344.147 \\
1718 & 0.448 & 0.08338 & 348.439 & 0.1611 & 0.02093 & 0.005 & 344.145 \\
1721 & 0.313 & 0.03718 & 345.978 & 0.1119 & 0.02067 & 0.017 & 344.044 \\
1724 & 0.313 & 0.04700 & 346.480 & 0.1117 & 0.02073 & 0.012 & 344.014 \\
1727 & 0.312 & 0.05798 & 347.098 & 0.1113 & 0.02093 & 0.009 & 344.034 \\
1730 & 0.312 & 0.07011 & 347.721 & 0.1112 & 0.02105 & 0.007 & 344.004 \\
1733 & 0.312 & 0.08338 & 348.439 & 0.1108 & 0.02100 & 0.006 & 344.015 \\
1736 & 0.210 & 0.03717 & 345.952 & 0.0744 & 0.02092 & 0.018 & 343.930 \\
1739 & 0.210 & 0.04699 & 346.495 & 0.0741 & 0.02102 & 0.012 & 343.923 \\
1742 & 0.209 & 0.05795 & 347.108 & 0.0739 & 0.02091 & 0.009 & 343.923 \\
1745 & 0.209 & 0.07007 & 347.770 & 0.0738 & 0.02109 & 0.007 & 343.909 \\
1748 & 0.209 & 0.08333 & 348.504 & 0.0736 & 0.02118 & 0.006 & 343.904 \\
\hline
\end{tabular}


Table 21. Thermal conductivity of the ternary $33 \% \mathrm{R} 32$ / $33 \% \mathrm{R} 125$ / $33 \% \mathrm{R} 134 \mathrm{a}$ mixture in the vapor phase.

\begin{tabular}{|c|c|c|c|c|c|c|c|}
\hline $\begin{array}{l}\text { Run } \\
\text { point }\end{array}$ & $\begin{array}{l}P_{\text {exp }} \\
\mathrm{MPa}\end{array}$ & $\begin{array}{c}Q \\
\mathrm{~W} \cdot \mathrm{m}^{-1}\end{array}$ & $\begin{array}{c}T_{\exp } \\
\mathrm{K}\end{array}$ & $\begin{array}{c}\rho_{\text {calc }} \\
\mathrm{mol} \cdot \mathrm{L}^{-1}\end{array}$ & $\begin{array}{c}\lambda_{\exp } \\
\mathrm{W} \cdot \mathrm{m}^{-1} \cdot \mathrm{K}^{-1}\end{array}$ & STAT & $\begin{array}{c}T_{\text {cell }} \\
\mathrm{K}\end{array}$ \\
\hline 1001 & 0.221 & 0.03460 & 263.817 & 0.1066 & 0.01139 & 0.009 & 260.587 \\
\hline 1004 & 0.219 & 0.04240 & 264.593 & 0.1051 & 0.01150 & 0.007 & 260.622 \\
\hline 1007 & 0.221 & 0.05095 & 265.459 & 0.1061 & 0.01154 & 0.006 & 260.673 \\
\hline 1010 & 0.222 & 0.06024 & 266.382 & 0.1059 & 0.01162 & 0.005 & 260.717 \\
\hline 1013 & 0.220 & 0.07034 & 267.373 & 0.1043 & 0.01168 & 0.004 & 260.766 \\
\hline 1016 & 0.214 & 0.03512 & 259.684 & 0.1053 & 0.01104 & 0.010 & 256.348 \\
\hline 1019 & 0.214 & 0.04300 & 260.408 & 0.1049 & 0.01112 & 0.008 & 256.319 \\
\hline 1022 & 0.214 & 0.05165 & 261.245 & 0.1046 & 0.01120 & 0.006 & 256.337 \\
\hline 1025 & 0.214 & 0.06110 & 262.121 & 0.1040 & 0.01127 & 0.005 & 256.336 \\
\hline 1031 & 0.092 & 0.03518 & 259.505 & 0.0438 & 0.01102 & 0.008 & 255.837 \\
\hline 1034 & 0.092 & 0.04308 & 260.324 & 0.0438 & 0.01113 & 0.007 & 255.833 \\
\hline 1037 & 0.092 & 0.05177 & 261.210 & 0.0434 & 0.01123 & 0.006 & 255.823 \\
\hline 1040 & 0.092 & 0.06123 & 262.198 & 0.0432 & 0.01123 & 0.005 & 255.835 \\
\hline 1043 & 0.092 & 0.07145 & 263.241 & 0.0429 & 0.01133 & 0.004 & 255.834 \\
\hline 1046 & 0.096 & 0.03383 & 270.908 & 0.0434 & 0.01202 & 0.010 & 267.626 \\
\hline 1049 & 0.096 & 0.04143 & 271.646 & 0.0435 & 0.01207 & 0.008 & 267.628 \\
\hline 1052 & 0.096 & 0.03383 & 270.868 & 0.0435 & 0.01198 & 0.010 & 267.604 \\
\hline 1055 & 0.096 & 0.04143 & 271.602 & 0.0435 & 0.01201 & 0.008 & 267.602 \\
\hline 1058 & 0.096 & 0.04979 & 272.397 & 0.0435 & 0.01215 & 0.007 & 267.587 \\
\hline 1061 & 0.096 & 0.05890 & 273.288 & 0.0432 & 0.01221 & 0.006 & 267.603 \\
\hline 1064 & 0.095 & 0.06875 & 274.222 & 0.0423 & 0.01230 & 0.005 & 267.593 \\
\hline 1067 & 0.279 & 0.03388 & 270.392 & 0.1328 & 0.01177 & 0.012 & 267.439 \\
\hline 1070 & 0.279 & 0.04149 & 271.067 & 0.1322 & 0.01204 & 0.009 & 267.445 \\
\hline 1073 & 0.279 & 0.04986 & 271.798 & 0.1317 & 0.01204 & 0.007 & 267.447 \\
\hline 1076 & 0.279 & 0.05898 & 272.578 & 0.1313 & 0.01208 & 0.006 & 267.437 \\
\hline 1079 & 0.279 & 0.06885 & 273.449 & 0.1307 & 0.01216 & 0.004 & 267.454 \\
\hline 1082 & 0.285 & 0.03369 & 276.748 & 0.1319 & 0.01239 & 0.012 & 273.916 \\
\hline 1085 & 0.286 & 0.04125 & 277.388 & 0.1318 & 0.01248 & 0.009 & 273.913 \\
\hline 1088 & 0.286 & 0.04957 & 278.091 & 0.1316 & 0.01251 & 0.007 & 273.914 \\
\hline 1091 & 0.286 & 0.05865 & 278.854 & 0.1313 & 0.01260 & 0.006 & 273.916 \\
\hline 1094 & 0.287 & 0.06850 & 279.675 & 0.1313 & 0.01268 & 0.005 & 273.919 \\
\hline 1097 & 0.098 & 0.03367 & 277.009 & 0.0434 & 0.01244 & 0.017 & 273.861 \\
\hline 1100 & 0.097 & 0.04123 & 277.731 & 0.0431 & 0.01253 & 0.013 & 273.870 \\
\hline 1103 & 0.098 & 0.04953 & 278.508 & 0.0433 & 0.01263 & 0.012 & 273.869 \\
\hline 1106 & 0.099 & 0.05862 & 279.341 & 0.0436 & 0.01270 & 0.010 & 273.863 \\
\hline 1109 & 0.100 & 0.06844 & 280.259 & 0.0438 & 0.01282 & 0.008 & 273.876 \\
\hline 1112 & 0.103 & 0.03276 & 287.560 & 0.0440 & 0.01350 & 0.016 & 284.684 \\
\hline 1115 & 0.104 & 0.04013 & 288.198 & 0.0441 & 0.01342 & 0.011 & 284.669 \\
\hline 1118 & 0.104 & 0.04824 & 288.902 & 0.0441 & 0.01354 & 0.009 & 284.660 \\
\hline 1121 & 0.104 & 0.05706 & 289.674 & 0.0439 & 0.01370 & 0.007 & 284.658 \\
\hline 1124 & 0.103 & 0.06662 & 290.470 & 0.0436 & 0.01374 & 0.006 & 284.622 \\
\hline 1127 & 0.280 & 0.03277 & 287.193 & 0.1234 & 0.01321 & 0.014 & 284.584 \\
\hline 1130 & 0.281 & 0.04015 & 287.802 & 0.1236 & 0.01331 & 0.010 & 284.596 \\
\hline
\end{tabular}


Table 21. Thermal conductivity of the ternary $33 \% \mathrm{R} 32$ / $33 \% \mathrm{R} 125$ / $33 \% \mathrm{R} 134$ a mixture in the vapor phase (continued).

\begin{tabular}{|c|c|c|c|c|c|c|c|}
\hline $\begin{array}{l}\text { Run } \\
\text { point }\end{array}$ & $\begin{array}{l}P_{\text {exp }} \\
\mathrm{MPa}\end{array}$ & $\begin{array}{c}Q \\
\mathrm{~W} \cdot \mathrm{m}^{-1}\end{array}$ & $\begin{array}{c}T_{\exp } \\
\mathrm{K} \\
\end{array}$ & $\begin{array}{c}\rho_{\text {calc }} \\
\mathrm{mol} \cdot \mathrm{L}^{-1}\end{array}$ & $\begin{array}{c}\lambda_{\exp } \\
\mathrm{W} \cdot \mathrm{m}^{-1} \cdot \mathrm{K}^{-1} \\
\end{array}$ & STAT & $\begin{array}{c}T_{\text {cell }} \\
\mathrm{K}\end{array}$ \\
\hline 1133 & 0.281 & 0.04827 & 288.448 & 0.1235 & 0.01344 & 0.008 & 284.589 \\
\hline 1136 & 0.281 & 0.05710 & 289.156 & 0.1230 & 0.01346 & 0.007 & 284.581 \\
\hline 1139 & 0.280 & 0.06666 & 289.913 & 0.1224 & 0.01350 & 0.006 & 284.579 \\
\hline 1142 & 0.292 & 0.03157 & 298.653 & 0.1233 & 0.01414 & 0.014 & 296.279 \\
\hline 1145 & 0.292 & 0.03868 & 299.188 & 0.1232 & 0.01414 & 0.012 & 296.274 \\
\hline 1148 & 0.293 & 0.04652 & 299.801 & 0.1231 & 0.01439 & 0.009 & 296.293 \\
\hline 1151 & 0.293 & 0.05504 & 300.429 & 0.1230 & 0.01444 & 0.007 & 296.274 \\
\hline 1154 & 0.293 & 0.06425 & 301.112 & 0.1225 & 0.01449 & 0.006 & 296.268 \\
\hline 1172 & 0.103 & 0.03103 & 304.445 & 0.0413 & 0.01505 & 0.016 & 301.959 \\
\hline 1175 & 0.103 & 0.03802 & 304.977 & 0.0411 & 0.01507 & 0.014 & 301.922 \\
\hline 1178 & 0.102 & 0.04570 & 305.582 & 0.0408 & 0.01524 & 0.009 & 301.907 \\
\hline 1181 & 0.102 & 0.05406 & 306.239 & 0.0407 & 0.01532 & 0.009 & 301.889 \\
\hline 1184 & 0.103 & 0.06315 & 306.963 & 0.0409 & 0.01535 & 0.007 & 301.891 \\
\hline 1187 & 0.284 & 0.03110 & 303.583 & 0.1176 & 0.01463 & 0.015 & 301.290 \\
\hline 1190 & 0.284 & 0.03810 & 304.115 & 0.1174 & 0.01450 & 0.012 & 301.295 \\
\hline 1193 & 0.283 & 0.04582 & 304.704 & 0.1167 & 0.01485 & 0.009 & 301.308 \\
\hline 1196 & 0.282 & 0.05422 & 305.301 & 0.1161 & 0.01485 & 0.007 & 301.280 \\
\hline 1199 & 0.282 & 0.06330 & 305.985 & 0.1157 & 0.01493 & 0.007 & 301.294 \\
\hline 1202 & 0.293 & 0.03022 & 312.920 & 0.1172 & 0.01550 & 0.015 & 310.820 \\
\hline 1205 & 0.293 & 0.03702 & 313.399 & 0.1170 & 0.01569 & 0.011 & 310.819 \\
\hline 1208 & 0.293 & 0.04450 & 313.920 & 0.1168 & 0.01570 & 0.009 & 310.816 \\
\hline 1211 & 0.293 & 0.05267 & 314.474 & 0.1166 & 0.01579 & 0.007 & 310.792 \\
\hline 1214 & 0.293 & 0.06151 & 315.081 & 0.1163 & 0.01582 & 0.006 & 310.782 \\
\hline 1247 & 0.297 & 0.02942 & 322.086 & 0.1151 & 0.01659 & 0.019 & 320.146 \\
\hline 1250 & 0.298 & 0.03605 & 322.520 & 0.1150 & 0.01652 & 0.013 & 320.132 \\
\hline 1253 & 0.297 & 0.04335 & 322.993 & 0.1148 & 0.01644 & 0.010 & 320.118 \\
\hline 1256 & 0.297 & 0.05130 & 323.510 & 0.1143 & 0.01670 & 0.008 & 320.106 \\
\hline 1259 & 0.296 & 0.05992 & 324.081 & 0.1139 & 0.01680 & 0.006 & 320.103 \\
\hline 1262 & 0.305 & 0.02961 & 330.817 & 0.1146 & 0.01730 & 0.019 & 328.951 \\
\hline 1265 & 0.305 & 0.03628 & 331.231 & 0.1147 & 0.01736 & 0.013 & 328.939 \\
\hline 1268 & 0.305 & 0.04363 & 331.653 & 0.1145 & 0.01713 & 0.010 & 328.891 \\
\hline 1271 & 0.306 & 0.05163 & 332.169 & 0.1144 & 0.01740 & 0.008 & 328.900 \\
\hline 1274 & 0.305 & 0.06031 & 332.696 & 0.1139 & 0.01747 & 0.007 & 328.878 \\
\hline 1307 & 0.284 & 0.02972 & 339.169 & 0.1035 & 0.01787 & 0.019 & 337.355 \\
\hline 1310 & 0.283 & 0.03642 & 339.561 & 0.1033 & 0.01816 & 0.014 & 337.330 \\
\hline 1313 & 0.283 & 0.04378 & 339.987 & 0.1031 & 0.01797 & 0.011 & 337.294 \\
\hline 1316 & 0.284 & 0.05183 & 340.453 & 0.1032 & 0.01801 & 0.009 & 337.266 \\
\hline 1319 & 0.284 & 0.06054 & 340.962 & 0.1031 & 0.01806 & 0.007 & 337.238 \\
\hline
\end{tabular}


Table 22. Thermal conductivity of the ternary $30 \% \mathrm{R} 32$ / $10 \% \mathrm{R} 125$ / $60 \% \mathrm{R} 134 \mathrm{a}$ mixture in the vapor phase.

\begin{tabular}{|c|c|c|c|c|c|c|c|}
\hline $\begin{array}{c}\text { Run } \\
\text { point }\end{array}$ & $\begin{array}{l}P_{e x p} \\
\mathrm{MPa}\end{array}$ & $\begin{array}{c}Q \\
W \cdot \mathrm{m}^{-1}\end{array}$ & $\begin{array}{c}T_{\exp } \\
\mathrm{K}\end{array}$ & $\begin{array}{c}\rho_{\text {calc }} \\
\mathrm{mol} \cdot \mathrm{L}^{-1}\end{array}$ & $\begin{array}{c}\lambda_{\exp } \\
\mathrm{W} \cdot \mathrm{m}^{-1} \cdot \mathrm{K}^{-1}\end{array}$ & STAT & $\begin{array}{c}T_{\text {cell }} \\
\mathrm{K}\end{array}$ \\
\hline 1001 & 0.086 & 0.02304 & 257.680 & 0.0413 & 0.01071 & 0.015 & 255.203 \\
\hline 1004 & 0.087 & 0.03132 & 258.576 & 0.0417 & 0.01082 & 0.010 & 255.201 \\
\hline 1007 & 0.087 & 0.04086 & 259.591 & 0.0414 & 0.01087 & 0.007 & 255.192 \\
\hline 1010 & 0.086 & 0.05165 & 260.740 & 0.0407 & 0.01092 & 0.006 & 255.192 \\
\hline 1013 & 0.086 & 0.06368 & 262.021 & 0.0406 & 0.01102 & 0.004 & 255.202 \\
\hline 1016 & 0.138 & 0.02304 & 257.528 & 0.0673 & 0.01065 & 0.015 & 255.187 \\
\hline 1019 & 0.138 & 0.03133 & 258.401 & 0.0668 & 0.01066 & 0.010 & 255.191 \\
\hline 1022 & 0.139 & 0.04087 & 259.383 & 0.0672 & 0.01081 & 0.007 & 255.194 \\
\hline 1025 & 0.140 & 0.05168 & 260.478 & 0.0672 & 0.01085 & 0.005 & 255.188 \\
\hline 1028 & 0.139 & 0.06372 & 261.702 & 0.0666 & 0.01095 & 0.004 & 255.195 \\
\hline 1031 & 0.143 & 0.02235 & 267.399 & 0.0670 & 0.01147 & 0.018 & 265.257 \\
\hline 1034 & 0.143 & 0.03040 & 268.170 & 0.0666 & 0.01147 & 0.011 & 265.251 \\
\hline 1037 & 0.144 & 0.03968 & 269.065 & 0.0668 & 0.01156 & 0.007 & 265.247 \\
\hline 1040 & 0.145 & 0.05016 & 270.071 & 0.0669 & 0.01162 & 0.006 & 265.245 \\
\hline 1043 & 0.145 & 0.06186 & 271.195 & 0.0665 & 0.01170 & 0.004 & 265.250 \\
\hline 1046 & 0.088 & 0.02228 & 269.933 & 0.0402 & 0.01172 & 0.017 & 267.732 \\
\hline 1049 & 0.088 & 0.03030 & 270.737 & 0.0398 & 0.01166 & 0.011 & 267.715 \\
\hline 1052 & 0.087 & 0.03953 & 271.669 & 0.0391 & 0.01190 & 0.008 & 267.722 \\
\hline 1055 & 0.087 & 0.04998 & 272.696 & 0.0394 & 0.01196 & 0.006 & 267.712 \\
\hline 1058 & 0.088 & 0.06163 & 273.863 & 0.0395 & 0.01206 & 0.005 & 267.723 \\
\hline 1061 & 0.212 & 0.02229 & 269.735 & 0.1000 & 0.01160 & 0.017 & 267.709 \\
\hline 1064 & 0.211 & 0.03031 & 270.481 & 0.0991 & 0.01172 & 0.011 & 267.716 \\
\hline 1067 & 0.210 & 0.03955 & 271.326 & 0.0982 & 0.01177 & 0.008 & 267.707 \\
\hline 1070 & 0.211 & 0.05001 & 272.291 & 0.0982 & 0.01186 & 0.006 & 267.716 \\
\hline 1073 & 0.212 & 0.06168 & 273.360 & 0.0981 & 0.01193 & 0.004 & 267.728 \\
\hline 1076 & 0.274 & 0.02229 & 269.645 & 0.1315 & 0.01164 & 0.017 & 267.695 \\
\hline 1079 & 0.273 & 0.03032 & 270.363 & 0.1305 & 0.01170 & 0.011 & 267.695 \\
\hline 1082 & 0.271 & 0.03956 & 271.191 & 0.1293 & 0.01184 & 0.007 & 267.693 \\
\hline 1085 & 0.271 & 0.05002 & 272.121 & 0.1287 & 0.01188 & 0.005 & 267.700 \\
\hline 1088 & 0.273 & 0.06168 & 273.135 & 0.1289 & 0.01195 & 0.004 & 267.686 \\
\hline 1091 & 0.283 & 0.02170 & 278.844 & 0.1303 & 0.01232 & 0.019 & 277.043 \\
\hline 1094 & 0.284 & 0.02951 & 279.520 & 0.1305 & 0.01243 & 0.013 & 277.039 \\
\hline 1097 & 0.285 & 0.03851 & 280.302 & 0.1306 & 0.01255 & 0.009 & 277.055 \\
\hline 1100 & 0.285 & 0.04870 & 281.138 & 0.1301 & 0.01258 & 0.006 & 277.035 \\
\hline 1103 & 0.284 & 0.06006 & 282.098 & 0.1292 & 0.01265 & 0.005 & 277.041 \\
\hline 1106 & 0.087 & 0.02169 & 279.043 & 0.0383 & 0.01263 & 0.021 & 276.998 \\
\hline 1109 & 0.087 & 0.02949 & 279.780 & 0.0379 & 0.01258 & 0.013 & 276.991 \\
\hline 1112 & 0.085 & 0.03848 & 280.648 & 0.0372 & 0.01270 & 0.010 & 276.998 \\
\hline 1115 & 0.086 & 0.04866 & 281.610 & 0.0372 & 0.01278 & 0.007 & 276.997 \\
\hline 1118 & 0.086 & 0.06002 & 282.696 & 0.0374 & 0.01285 & 0.006 & 277.001 \\
\hline 1121 & 0.184 & 0.02170 & 278.880 & 0.0829 & 0.01211 & 0.020 & 276.963 \\
\hline 1124 & 0.184 & 0.02951 & 279.585 & 0.0824 & 0.01235 & 0.013 & 276.964 \\
\hline 1127 & 0.183 & 0.03850 & 280.403 & 0.0817 & 0.01243 & 0.008 & 276.976 \\
\hline
\end{tabular}


Table 22. Thermal conductivity of the ternary $30 \% \mathrm{R} 32$ / $10 \% \mathrm{R} 125$ / $60 \% \mathrm{R} 134 \mathrm{a}$ mixture in the vapor phase (continued).

\begin{tabular}{|c|c|c|c|c|c|c|c|}
\hline $\begin{array}{l}\text { Run } \\
\text { point }\end{array}$ & $\begin{array}{l}P_{\text {exp }} \\
\mathrm{MPa}\end{array}$ & $\begin{array}{c}Q \\
\mathrm{~W} \cdot \mathrm{m}^{-1}\end{array}$ & $\begin{array}{c}T_{\text {exp }} \\
\mathrm{K}\end{array}$ & $\begin{array}{c}\rho_{\text {calc }} \\
\mathrm{mol} \cdot \mathrm{L}^{-1}\end{array}$ & $\begin{array}{c}\lambda_{\exp } \\
\mathrm{W} \cdot \mathrm{m}^{-1} \cdot \mathrm{K}^{-1}\end{array}$ & STAT & $\begin{array}{c}T_{\text {cell }} \\
\mathrm{K}\end{array}$ \\
\hline 1130 & 0.184 & 0.04868 & 281.317 & 0.0817 & 0.01251 & 0.006 & 276.987 \\
\hline 1133 & 0.185 & 0.06004 & 282.290 & 0.0819 & 0.01261 & 0.005 & 276.956 \\
\hline 1136 & 0.350 & 0.02169 & 277.984 & 0.1651 & 0.01233 & 0.019 & 276.217 \\
\hline 1139 & 0.351 & 0.02950 & 278.643 & 0.1651 & 0.01235 & 0.013 & 276.218 \\
\hline 1142 & 0.350 & 0.03851 & 279.400 & 0.1639 & 0.01248 & 0.009 & 276.220 \\
\hline 1145 & 0.349 & 0.04870 & 280.245 & 0.1626 & 0.01257 & 0.006 & 276.220 \\
\hline 1148 & 0.349 & 0.06007 & 281.164 & 0.1617 & 0.01260 & 0.005 & 276.202 \\
\hline 1151 & 0.407 & 0.02171 & 277.902 & 0.1949 & 0.01246 & 0.020 & 276.173 \\
\hline 1154 & 0.408 & 0.02951 & 278.521 & 0.1952 & 0.01254 & 0.013 & 276.161 \\
\hline 1157 & 0.409 & 0.03851 & 279.265 & 0.1950 & 0.01254 & 0.009 & 276.172 \\
\hline 1160 & 0.408 & 0.04872 & 280.106 & 0.1933 & 0.01261 & 0.006 & 276.192 \\
\hline 1163 & 0.406 & 0.06009 & 281.007 & 0.1917 & 0.01262 & 0.005 & 276.182 \\
\hline 1166 & 0.426 & 0.02183 & 287.579 & 0.1953 & 0.01302 & 0.021 & 285.933 \\
\hline 1169 & 0.425 & 0.02968 & 288.212 & 0.1942 & 0.01322 & 0.014 & 285.943 \\
\hline 1172 & 0.425 & 0.03874 & 288.895 & 0.1936 & 0.01315 & 0.009 & 285.926 \\
\hline 1175 & 0.427 & 0.04899 & 289.709 & 0.1938 & 0.01335 & 0.006 & 285.954 \\
\hline 1178 & 0.428 & 0.06043 & 290.570 & 0.1935 & 0.01334 & 0.005 & 285.938 \\
\hline 1181 & 0.331 & 0.02183 & 287.633 & 0.1483 & 0.01296 & 0.021 & 285.914 \\
\hline 1184 & 0.332 & 0.02968 & 288.271 & 0.1487 & 0.01316 & 0.014 & 285.916 \\
\hline 1187 & 0.333 & 0.03874 & 288.991 & 0.1485 & 0.01323 & 0.009 & 285.906 \\
\hline 1190 & 0.332 & 0.04898 & 289.803 & 0.1477 & 0.01328 & 0.006 & 285.914 \\
\hline 1193 & 0.331 & 0.06043 & 290.702 & 0.1465 & 0.01338 & 0.005 & 285.908 \\
\hline 1196 & 0.249 & 0.02183 & 287.635 & 0.1097 & 0.01306 & 0.021 & 285.869 \\
\hline 1199 & 0.250 & 0.02968 & 288.287 & 0.1096 & 0.01317 & 0.014 & 285.858 \\
\hline 1202 & 0.249 & 0.03873 & 289.050 & 0.1091 & 0.01312 & 0.009 & 285.867 \\
\hline 1205 & 0.248 & 0.04898 & 289.905 & 0.1081 & 0.01321 & 0.006 & 285.879 \\
\hline 1208 & 0.247 & 0.06041 & 290.819 & 0.1070 & 0.01337 & 0.005 & 285.856 \\
\hline 1211 & 0.183 & 0.02184 & 287.462 & 0.0794 & 0.01268 & 0.020 & 285.637 \\
\hline 1214 & 0.183 & 0.02969 & 288.148 & 0.0792 & 0.01296 & 0.013 & 285.631 \\
\hline 1217 & 0.183 & 0.03874 & 288.917 & 0.0789 & 0.01316 & 0.009 & 285.624 \\
\hline 1220 & 0.183 & 0.04899 & 289.799 & 0.0787 & 0.01328 & 0.006 & 285.634 \\
\hline 1223 & 0.183 & 0.06042 & 290.768 & 0.0786 & 0.01336 & 0.005 & 285.640 \\
\hline 1241 & 0.490 & 0.02185 & 287.225 & 0.2290 & 0.01319 & 0.021 & 285.609 \\
\hline 1244 & 0.491 & 0.02972 & 287.820 & 0.2290 & 0.01328 & 0.014 & 285.608 \\
\hline 1247 & 0.492 & 0.03878 & 288.525 & 0.2286 & 0.01320 & 0.009 & 285.625 \\
\hline 1250 & 0.492 & 0.04903 & 289.289 & 0.2276 & 0.01337 & 0.007 & 285.616 \\
\hline 1253 & 0.491 & 0.06048 & 290.145 & 0.2258 & 0.01344 & 0.005 & 285.615 \\
\hline 1256 & 0.524 & 0.02118 & 297.729 & 0.2344 & 0.01404 & 0.024 & 296.252 \\
\hline 1259 & 0.524 & 0.02881 & 298.301 & 0.2336 & 0.01405 & 0.016 & 296.258 \\
\hline 1262 & 0.524 & 0.03761 & 298.944 & 0.2327 & 0.01415 & 0.011 & 296.261 \\
\hline 1265 & 0.525 & 0.04756 & 299.635 & 0.2326 & 0.01410 & 0.008 & 296.241 \\
\hline 1268 & 0.527 & 0.05868 & 300.441 & 0.2328 & 0.01420 & 0.006 & 296.253 \\
\hline 1271 & 0.440 & 0.02124 & 297.046 & 0.1938 & 0.01395 & 0.025 & 295.519 \\
\hline
\end{tabular}


Table 22. Thermal conductivity of the ternary $30 \% \mathrm{R} 32$ / $10 \% \mathrm{R} 125$ / $60 \% \mathrm{R} 134 \mathrm{a}$ mixture in the vapor phase (continued).

\begin{tabular}{|c|c|c|c|c|c|c|c|}
\hline $\begin{array}{l}\text { Run } \\
\text { point }\end{array}$ & $\begin{array}{l}P_{\text {exp }} \\
\mathrm{MPa}\end{array}$ & $\begin{array}{c}Q \\
\mathrm{~W} \cdot \mathrm{m}^{-1}\end{array}$ & $\begin{array}{c}T_{\exp } \\
\mathrm{K}\end{array}$ & $\begin{array}{c}\rho_{\text {calc }} \\
\mathrm{mol} \cdot \mathrm{L}^{-1}\end{array}$ & $\begin{array}{c}\lambda_{\exp } \\
\mathrm{W} \cdot \mathrm{m}^{-1} \cdot \mathrm{K}^{-1}\end{array}$ & STAT & $\begin{array}{c}T_{\text {cell }} \\
\mathrm{K}\end{array}$ \\
\hline 1274 & 0.439 & 0.02888 & 297.620 & 0.1927 & 0.01391 & 0.016 & 295.522 \\
\hline 1277 & 0.438 & 0.03769 & 298.272 & 0.1916 & 0.01395 & 0.011 & 295.523 \\
\hline 1280 & 0.440 & 0.04767 & 299.013 & 0.1920 & 0.01406 & 0.008 & 295.522 \\
\hline 1283 & 0.441 & 0.05881 & 299.813 & 0.1917 & 0.01416 & 0.006 & 295.507 \\
\hline 1286 & 0.348 & 0.02124 & 297.081 & 0.1505 & 0.01381 & 0.024 & 295.497 \\
\hline 1289 & 0.346 & 0.02889 & 297.667 & 0.1492 & 0.01382 & 0.014 & 295.501 \\
\hline 1292 & 0.347 & 0.03770 & 298.339 & 0.1488 & 0.01375 & 0.010 & 295.504 \\
\hline 1295 & 0.347 & 0.04767 & 299.086 & 0.1488 & 0.01398 & 0.007 & 295.495 \\
\hline 1298 & 0.349 & 0.05880 & 299.919 & 0.1489 & 0.01403 & 0.005 & 295.485 \\
\hline 1301 & 0.257 & 0.02124 & 297.112 & 0.1089 & 0.01362 & 0.025 & 295.481 \\
\hline 1304 & 0.258 & 0.02888 & 297.727 & 0.1094 & 0.01396 & 0.017 & 295.489 \\
\hline 1307 & 0.258 & 0.03769 & 298.412 & 0.1090 & 0.01388 & 0.011 & 295.478 \\
\hline 1310 & 0.257 & 0.04766 & 299.185 & 0.1082 & 0.01395 & 0.008 & 295.464 \\
\hline 1313 & 0.256 & 0.05879 & 300.085 & 0.1073 & 0.01411 & 0.006 & 295.496 \\
\hline 1316 & 0.178 & 0.02124 & 297.097 & 0.0744 & 0.01372 & 0.023 & 295.410 \\
\hline 1319 & 0.177 & 0.02887 & 297.727 & 0.0738 & 0.01376 & 0.015 & 295.411 \\
\hline 1322 & 0.179 & 0.03768 & 298.446 & 0.0744 & 0.01400 & 0.011 & 295.407 \\
\hline 1325 & 0.180 & 0.04765 & 299.284 & 0.0746 & 0.01401 & 0.007 & 295.434 \\
\hline 1328 & 0.178 & 0.05878 & 300.154 & 0.0736 & 0.01416 & 0.006 & 295.402 \\
\hline 1361 & 0.183 & 0.02132 & 307.476 & 0.0737 & 0.01486 & 0.025 & 305.849 \\
\hline 1364 & 0.181 & 0.02899 & 308.073 & 0.0728 & 0.01489 & 0.016 & 305.847 \\
\hline 1367 & 0.181 & 0.03783 & 308.770 & 0.0725 & 0.01476 & 0.011 & 305.853 \\
\hline 1370 & 0.184 & 0.04783 & 309.535 & 0.0734 & 0.01507 & 0.008 & 305.848 \\
\hline 1373 & 0.183 & 0.05900 & 310.396 & 0.0731 & 0.01508 & 0.006 & 305.854 \\
\hline 1376 & 0.269 & 0.02133 & 307.273 & 0.1100 & 0.01487 & 0.026 & 305.701 \\
\hline 1379 & 0.267 & 0.02900 & 307.865 & 0.1088 & 0.01488 & 0.016 & 305.720 \\
\hline 1382 & 0.268 & 0.03785 & 308.525 & 0.1089 & 0.01480 & 0.011 & 305.723 \\
\hline 1385 & 0.270 & 0.04787 & 309.241 & 0.1095 & 0.01501 & 0.008 & 305.692 \\
\hline 1388 & 0.270 & 0.05906 & 310.074 & 0.1092 & 0.01509 & 0.006 & 305.695 \\
\hline 1391 & 0.348 & 0.02134 & 307.063 & 0.1442 & 0.01468 & 0.025 & 305.554 \\
\hline 1394 & 0.347 & 0.02902 & 307.632 & 0.1434 & 0.01483 & 0.017 & 305.539 \\
\hline 1397 & 0.350 & 0.03788 & 308.280 & 0.1442 & 0.01474 & 0.011 & 305.546 \\
\hline 1400 & 0.351 & 0.04789 & 309.002 & 0.1443 & 0.01482 & 0.008 & 305.537 \\
\hline 1403 & 0.349 & 0.05907 & 309.783 & 0.1432 & 0.01494 & 0.006 & 305.514 \\
\hline 1406 & 0.438 & 0.02133 & 306.616 & 0.1846 & 0.01461 & 0.026 & 305.122 \\
\hline 1409 & 0.436 & 0.02901 & 307.163 & 0.1834 & 0.01481 & 0.016 & 305.124 \\
\hline 1412 & 0.435 & 0.03785 & 307.793 & 0.1823 & 0.01483 & 0.011 & 305.129 \\
\hline 1415 & 0.437 & 0.04787 & 308.478 & 0.1828 & 0.01483 & 0.008 & 305.107 \\
\hline 1418 & 0.437 & 0.05906 & 309.263 & 0.1825 & 0.01491 & 0.006 & 305.111 \\
\hline 1421 & 0.492 & 0.02134 & 306.540 & 0.2097 & 0.01470 & 0.026 & 305.084 \\
\hline 1424 & 0.494 & 0.02901 & 307.053 & 0.2102 & 0.01466 & 0.016 & 305.062 \\
\hline 1427 & 0.497 & 0.03786 & 307.694 & 0.2110 & 0.01490 & 0.011 & 305.083 \\
\hline 1430 & 0.495 & 0.04789 & 308.393 & 0.2094 & 0.01485 & 0.008 & 305.081 \\
\hline
\end{tabular}


Table 22. Thermal conductivity of the ternary $30 \% \mathrm{R} 32$ / $10 \% \mathrm{R} 125$ / $60 \% \mathrm{R} 134 \mathrm{a}$ mixture in the vapor phase (continued).

\begin{tabular}{|c|c|c|c|c|c|c|c|}
\hline $\begin{array}{l}\text { Run } \\
\text { point }\end{array}$ & $\begin{array}{l}P_{e x p} \\
\mathrm{MPa}\end{array}$ & $\begin{array}{c}Q \\
\mathrm{~W} \cdot \mathrm{m}^{-1}\end{array}$ & $\begin{array}{c}T_{e x p} \\
\mathrm{~K}\end{array}$ & $\begin{array}{c}\rho_{\text {calc }} \\
\mathrm{mol} \cdot \mathrm{L}^{-1}\end{array}$ & $\begin{array}{c}\lambda_{\exp } \\
\mathrm{W} \cdot \mathrm{m}^{-1} \cdot \mathrm{K}^{-1}\end{array}$ & STAT & $\begin{array}{c}T_{\text {cell }} \\
\mathrm{K}\end{array}$ \\
\hline 1433 & 0.493 & 0.05908 & 309.165 & 0.2078 & 0.01490 & 0.006 & 305.079 \\
\hline 1436 & 0.514 & 0.02135 & 318.554 & 0.2090 & 0.01578 & 0.027 & 317.176 \\
\hline 1439 & 0.517 & 0.02904 & 319.067 & 0.2099 & 0.01594 & 0.018 & 317.182 \\
\hline 1442 & 0.518 & 0.03790 & 319.630 & 0.2098 & 0.01580 & 0.012 & 317.163 \\
\hline 1445 & 0.516 & 0.04793 & 320.282 & 0.2083 & 0.01591 & 0.008 & 317.154 \\
\hline 1448 & 0.514 & 0.05913 & 321.012 & 0.2070 & 0.01588 & 0.006 & 317.151 \\
\hline 1451 & 0.436 & 0.02136 & 318.428 & 0.1752 & 0.01589 & 0.028 & 317.026 \\
\hline 1454 & 0.436 & 0.02905 & 318.923 & 0.1751 & 0.01593 & 0.018 & 317.004 \\
\hline 1457 & 0.435 & 0.03790 & 319.529 & 0.1739 & 0.01592 & 0.011 & 317.019 \\
\hline 1460 & 0.433 & 0.04794 & 320.188 & 0.1728 & 0.01601 & 0.008 & 317.005 \\
\hline 1463 & 0.435 & 0.05914 & 320.906 & 0.1732 & 0.01592 & 0.006 & 316.981 \\
\hline 1466 & 0.344 & 0.02137 & 318.271 & 0.1366 & 0.01605 & 0.028 & 316.831 \\
\hline 1469 & 0.346 & 0.02906 & 318.813 & 0.1372 & 0.01567 & 0.017 & 316.828 \\
\hline 1472 & 0.345 & 0.03792 & 319.440 & 0.1361 & 0.01571 & 0.011 & 316.845 \\
\hline 1475 & 0.342 & 0.04796 & 320.100 & 0.1348 & 0.01585 & 0.008 & 316.820 \\
\hline 1478 & 0.344 & 0.05915 & 320.855 & 0.1353 & 0.01596 & 0.006 & 316.810 \\
\hline 1481 & 0.262 & 0.02144 & 317.186 & 0.1031 & 0.01571 & 0.028 & 315.700 \\
\hline 1484 & 0.260 & 0.02915 & 317.756 & 0.1021 & 0.01583 & 0.017 & 315.708 \\
\hline 1487 & 0.258 & 0.03804 & 318.366 & 0.1008 & 0.01583 & 0.012 & 315.686 \\
\hline 1490 & 0.258 & 0.04809 & 319.093 & 0.1008 & 0.01589 & 0.008 & 315.701 \\
\hline 1493 & 0.262 & 0.05932 & 319.865 & 0.1021 & 0.01593 & 0.006 & 315.673 \\
\hline 1496 & 0.177 & 0.02143 & 317.185 & 0.0689 & 0.01574 & 0.030 & 315.641 \\
\hline 1499 & 0.177 & 0.02915 & 317.741 & 0.0688 & 0.01579 & 0.019 & 315.627 \\
\hline 1502 & 0.180 & 0.03805 & 318.391 & 0.0695 & 0.01598 & 0.012 & 315.623 \\
\hline 1505 & 0.180 & 0.04813 & 319.117 & 0.0697 & 0.01596 & 0.008 & 315.608 \\
\hline 1508 & 0.179 & 0.05937 & 319.930 & 0.0690 & 0.01591 & 0.007 & 315.600 \\
\hline 1541 & 0.179 & 0.02141 & 328.401 & 0.0669 & 0.01676 & 0.030 & 326.932 \\
\hline 1544 & 0.177 & 0.02912 & 328.916 & 0.0661 & 0.01676 & 0.018 & 326.899 \\
\hline 1547 & 0.178 & 0.03801 & 329.535 & 0.0662 & 0.01693 & 0.013 & 326.896 \\
\hline 1550 & 0.179 & 0.04807 & 330.223 & 0.0667 & 0.01708 & 0.009 & 326.883 \\
\hline 1553 & 0.182 & 0.05929 & 330.960 & 0.0677 & 0.01715 & 0.007 & 326.844 \\
\hline 1556 & 0.265 & 0.02140 & 326.331 & 0.1008 & 0.01660 & 0.029 & 324.922 \\
\hline 1559 & 0.261 & 0.02910 & 326.850 & 0.0994 & 0.01659 & 0.018 & 324.912 \\
\hline 1562 & 0.260 & 0.03798 & 327.464 & 0.0988 & 0.01659 & 0.012 & 324.894 \\
\hline 1565 & 0.264 & 0.04803 & 328.126 & 0.0998 & 0.01670 & 0.009 & 324.876 \\
\hline 1568 & 0.265 & 0.05925 & 328.904 & 0.0999 & 0.01674 & 0.007 & 324.876 \\
\hline 1571 & 0.357 & 0.02131 & 326.148 & 0.1376 & 0.01627 & 0.029 & 324.760 \\
\hline 1574 & 0.354 & 0.02898 & 326.650 & 0.1362 & 0.01637 & 0.019 & 324.743 \\
\hline 1577 & 0.351 & 0.03782 & 327.218 & 0.1347 & 0.01658 & 0.012 & 324.727 \\
\hline 1580 & 0.353 & 0.04783 & 327.870 & 0.1353 & 0.01661 & 0.009 & 324.721 \\
\hline 1583 & 0.357 & 0.05900 & 328.594 & 0.1365 & 0.01671 & 0.007 & 324.711 \\
\hline 1586 & 0.431 & 0.02132 & 325.771 & 0.1683 & 0.01614 & 0.028 & 324.431 \\
\hline 1589 & 0.429 & 0.02900 & 326.279 & 0.1671 & 0.01629 & 0.018 & 324.419 \\
\hline
\end{tabular}


Table 22. Thermal conductivity of the ternary $30 \% \mathrm{R} 32$ / $10 \% \mathrm{R} 125$ / $60 \% \mathrm{R} 134 \mathrm{a}$ mixture in the vapor phase (continued).

\begin{tabular}{|c|c|c|c|c|c|c|c|}
\hline $\begin{array}{l}\text { Run } \\
\text { point }\end{array}$ & $\begin{array}{l}P_{\text {exp }} \\
\mathrm{MPa}\end{array}$ & $\begin{array}{c}Q \\
\mathrm{~W} \cdot \mathrm{m}^{-1}\end{array}$ & $\begin{array}{c}T_{\exp } \\
\mathrm{K}\end{array}$ & $\begin{array}{c}\rho_{\text {calc }} \\
\mathrm{mol} \cdot \mathrm{L}^{-1}\end{array}$ & $\begin{array}{c}\lambda_{\exp } \\
\mathrm{W} \cdot \mathrm{m}^{-1} \cdot \mathrm{K}^{-1}\end{array}$ & STAT & $\begin{array}{c}T_{\text {cell }} \\
\mathrm{K}\end{array}$ \\
\hline 1592 & 0.432 & 0.03785 & 326.857 & 0.1682 & 0.01660 & 0.012 & 324.422 \\
\hline 1595 & 0.434 & 0.04787 & 327.479 & 0.1684 & 0.01656 & 0.009 & 324.394 \\
\hline 1598 & 0.431 & 0.05905 & 328.169 & 0.1670 & 0.01671 & 0.007 & 324.364 \\
\hline 1601 & 0.507 & 0.02133 & 325.602 & 0.2004 & 0.01647 & 0.029 & 324.270 \\
\hline 1604 & 0.509 & 0.02901 & 326.113 & 0.2008 & 0.01710 & 0.064 & 324.275 \\
\hline 1607 & 0.514 & 0.03786 & 326.652 & 0.2023 & 0.01657 & 0.013 & 324.263 \\
\hline 1610 & 0.515 & 0.04788 & 327.271 & 0.2025 & 0.01667 & 0.009 & 324.247 \\
\hline 1613 & 0.513 & 0.05906 & 327.953 & 0.2012 & 0.01668 & 0.007 & 324.228 \\
\hline 1616 & 0.532 & 0.02125 & 337.764 & 0.2012 & 0.01762 & 0.032 & 336.521 \\
\hline 1619 & 0.532 & 0.02889 & 338.208 & 0.2011 & 0.01776 & 0.020 & 336.510 \\
\hline 1622 & 0.533 & 0.03771 & 338.719 & 0.2013 & 0.01764 & 0.015 & 336.479 \\
\hline 1625 & 0.534 & 0.04770 & 339.325 & 0.2012 & 0.01818 & 0.063 & 336.466 \\
\hline 1628 & 0.534 & 0.05885 & 339.962 & 0.2007 & 0.01761 & 0.007 & 336.438 \\
\hline 1631 & 0.442 & 0.02126 & 337.610 & 0.1657 & 0.01782 & 0.032 & 336.337 \\
\hline 1634 & 0.441 & 0.02891 & 338.049 & 0.1649 & 0.01757 & 0.020 & 336.309 \\
\hline 1637 & 0.441 & 0.03773 & 338.581 & 0.1647 & 0.01750 & 0.014 & 336.287 \\
\hline 1640 & 0.443 & 0.04772 & 339.185 & 0.1649 & 0.01740 & 0.010 & 336.267 \\
\hline 1643 & 0.443 & 0.05887 & 339.809 & 0.1648 & 0.01773 & 0.007 & 336.213 \\
\hline 1646 & 0.353 & 0.02128 & 337.084 & 0.1312 & 0.01729 & 0.034 & 335.767 \\
\hline 1649 & 0.354 & 0.02894 & 337.516 & 0.1311 & 0.01754 & 0.020 & 335.725 \\
\hline 1652 & 0.355 & 0.03778 & 338.104 & 0.1315 & 0.01730 & 0.013 & 335.727 \\
\hline 1655 & 0.355 & 0.04778 & 338.712 & 0.1312 & 0.01781 & 0.020 & 335.705 \\
\hline 1658 & 0.354 & 0.05895 & 339.393 & 0.1303 & 0.01771 & 0.007 & 335.688 \\
\hline 1661 & 0.255 & 0.02130 & 336.920 & 0.0937 & 0.01780 & 0.033 & 335.558 \\
\hline 1664 & 0.254 & 0.02897 & 337.389 & 0.0932 & 0.01738 & 0.020 & 335.528 \\
\hline 1667 & 0.253 & 0.03781 & 337.942 & 0.0925 & 0.01765 & 0.014 & 335.506 \\
\hline 1670 & 0.253 & 0.04781 & 338.560 & 0.0926 & 0.01781 & 0.010 & 335.474 \\
\hline 1673 & 0.255 & 0.05898 & 339.291 & 0.0929 & 0.01767 & 0.007 & 335.485 \\
\hline 1676 & 0.176 & 0.02132 & 335.567 & 0.0645 & 0.01768 & 0.031 & 334.165 \\
\hline 1679 & 0.176 & 0.02899 & 336.103 & 0.0643 & 0.01765 & 0.019 & 334.167 \\
\hline 1682 & 0.178 & 0.03783 & 336.670 & 0.0649 & 0.01770 & 0.014 & 334.141 \\
\hline 1685 & 0.179 & 0.04785 & 337.320 & 0.0650 & 0.01789 & 0.010 & 334.116 \\
\hline 1688 & 0.178 & 0.05903 & 338.076 & 0.0644 & 0.01786 & 0.008 & 334.106 \\
\hline 1721 & 0.185 & 0.02109 & 344.646 & 0.0659 & 0.01863 & 0.034 & 343.325 \\
\hline 1724 & 0.187 & 0.02868 & 345.101 & 0.0664 & 0.01854 & 0.022 & 343.303 \\
\hline 1727 & 0.188 & 0.03743 & 345.639 & 0.0667 & 0.01861 & 0.015 & 343.261 \\
\hline 1730 & 0.187 & 0.04734 & 346.266 & 0.0662 & 0.01871 & 0.011 & 343.246 \\
\hline 1733 & 0.186 & 0.05841 & 346.955 & 0.0656 & 0.01884 & 0.008 & 343.205 \\
\hline 1736 & 0.268 & 0.02112 & 344.258 & 0.0962 & 0.01842 & 0.033 & 342.971 \\
\hline 1739 & 0.268 & 0.02871 & 344.719 & 0.0963 & 0.01833 & 0.021 & 342.958 \\
\hline 1742 & 0.270 & 0.03747 & 345.244 & 0.0966 & 0.01839 & 0.015 & 342.944 \\
\hline 1745 & 0.269 & 0.04740 & 345.875 & 0.0962 & 0.01840 & 0.011 & 342.906 \\
\hline 1748 & 0.268 & 0.05848 & 346.542 & 0.0955 & 0.01849 & 0.008 & 342.886 \\
\hline
\end{tabular}


Table 22. Thermal conductivity of the ternary $30 \% \mathrm{R} 32$ / $10 \% \mathrm{R} 125$ / $60 \% \mathrm{R} 134 \mathrm{a}$ mixture in the vapor phase (continued).

\begin{tabular}{|c|c|c|c|c|c|c|c|}
\hline $\begin{array}{l}\text { Run } \\
\text { point }\end{array}$ & $\begin{array}{l}P_{\text {exp }} \\
\mathrm{MPa}\end{array}$ & $\begin{array}{c}Q \\
\mathrm{~W} \cdot \mathrm{m}^{-1}\end{array}$ & $\begin{array}{c}T_{\text {exp }} \\
\mathrm{K}\end{array}$ & $\begin{array}{c}\rho_{\text {calc }} \\
\mathrm{mol} \cdot \mathrm{L}^{-1}\end{array}$ & $\frac{\lambda_{\exp }}{\mathrm{W} \cdot \mathrm{m}^{-1} \cdot \mathrm{K}^{-1}}$ & STAT & $\begin{array}{c}T_{\text {cell }} \\
\mathrm{K}\end{array}$ \\
\hline 1751 & 0.353 & 0.02116 & 343.582 & 0.1282 & 0.01821 & 0.033 & 342.329 \\
\hline 1754 & 0.353 & 0.02877 & 344.064 & 0.1281 & 0.01806 & 0.021 & 342.323 \\
\hline 1757 & 0.352 & 0.03755 & 344.562 & 0.1274 & 0.01826 & 0.014 & 342.290 \\
\hline 1760 & 0.351 & 0.04748 & 345.167 & 0.1269 & 0.01825 & 0.010 & 342.270 \\
\hline 1763 & 0.353 & 0.05858 & 345.804 & 0.1273 & 0.01843 & 0.008 & 342.218 \\
\hline 1766 & 0.435 & 0.02117 & 343.257 & 0.1595 & 0.01844 & 0.032 & 342.021 \\
\hline 1769 & 0.433 & 0.02880 & 343.708 & 0.1586 & 0.01799 & 0.021 & 342.011 \\
\hline 1772 & 0.432 & 0.03759 & 344.212 & 0.1579 & 0.01820 & 0.049 & 341.973 \\
\hline 1775 & 0.433 & 0.04754 & 344.799 & 0.1582 & 0.01802 & 0.010 & 341.971 \\
\hline 1778 & 0.434 & 0.05866 & 345.422 & 0.1582 & 0.01820 & 0.007 & 341.909 \\
\hline 1781 & 0.521 & 0.02119 & 342.956 & 0.1934 & 0.01798 & 0.033 & 341.736 \\
\hline 1784 & 0.522 & 0.02882 & 343.380 & 0.1933 & 0.01799 & 0.020 & 341.712 \\
\hline 1787 & 0.520 & 0.03762 & 343.902 & 0.1924 & 0.01803 & 0.014 & 341.697 \\
\hline 1790 & 0.519 & 0.04758 & 344.475 & 0.1916 & 0.01791 & 0.010 & 341.672 \\
\hline 1793 & 0.520 & 0.05870 & 345.089 & 0.1915 & 0.01808 & 0.007 & 341.641 \\
\hline
\end{tabular}




\section{Tables of steady-state results for bare tungsten wires}

Table 23. Thermal conductivity of the binary $30 \% \mathrm{R} 125 / 70 \% \mathrm{R} 134 \mathrm{a}$ mixture in the dilute gas measured with the steady-state technique.

\begin{tabular}{|c|c|c|c|c|c|c|c|c|c|c|}
\hline $\begin{array}{l}\text { Run } \\
\text { point }\end{array}$ & $\begin{array}{l}P_{\text {exp }} \\
\mathrm{MPa}\end{array}$ & $\begin{array}{c}Q \\
\mathrm{~W} \cdot \mathrm{m}^{-1}\end{array}$ & $\begin{array}{c}T_{\exp } \\
\mathrm{K}\end{array}$ & $\begin{array}{c}\rho_{\text {calc }} \\
\mathrm{mol} \cdot \mathrm{L}^{-1}\end{array}$ & $\begin{array}{c}\lambda_{\exp } \\
\mathrm{W} \cdot \mathrm{m}^{-1} \cdot \mathrm{K}^{-1}\end{array}$ & $\begin{array}{c}\text { TBAND } \\
\%\end{array}$ & $\begin{array}{c}\Delta T_{a v g} \\
\mathrm{~K}\end{array}$ & $\begin{array}{c}t_{\text {start }} \\
\mathrm{s}\end{array}$ & $\begin{array}{c}t_{\text {end }} \\
\mathrm{s}\end{array}$ & $N_{R a}$ \\
\hline 1003 & 0.216 & 0.02804 & 262.687 & 0.1058 & 0.01081 & 0.900 & 3.118 & 4.96 & 40.00 & 12817. \\
\hline 1006 & 0.215 & 0.03810 & 263.234 & 0.1051 & 0.01088 & 0.590 & 4.178 & 4.96 & 40.00 & 16740. \\
\hline 1009 & 0.216 & 0.04966 & 263.822 & 0.1055 & 0.01098 & 0.850 & 5.353 & 3.36 & 40.00 & 21401. \\
\hline 1012 & 0.218 & 0.06273 & 264.483 & 0.1059 & 0.01109 & 0.840 & 6.637 & 3.36 & 40.00 & 26445. \\
\hline 1015 & 0.218 & 0.07729 & 265.208 & 0.1055 & 0.01116 & 1.480 & 8.053 & 1.76 & 40.00 & 31408. \\
\hline 1018 & 0.147 & 0.02802 & 262.866 & 0.0706 & 0.01043 & 0.960 & 3.274 & 4.96 & 40.00 & 5274. \\
\hline 1021 & 0.147 & 0.03808 & 263.438 & 0.0702 & 0.01055 & 0.590 & 4.386 & 4.96 & 40.00 & 6916. \\
\hline 1024 & 0.146 & 0.04964 & 264.100 & 0.0693 & 0.01065 & 0.420 & 5.644 & 4.96 & 40.00 & 8579. \\
\hline 1027 & 0.146 & 0.06268 & 264.802 & 0.0691 & 0.01074 & 0.710 & 7.044 & 3.36 & 40.00 & 10505. \\
\hline 1030 & 0.147 & 0.07720 & 265.581 & 0.0695 & 0.01083 & 0.510 & 8.569 & 3.36 & 40.00 & 12781. \\
\hline 1036 & 0.076 & 0.03805 & 263.662 & 0.0355 & 0.01021 & 0.980 & 4.568 & 1.76 & 40.00 & 1649. \\
\hline 1039 & 0.076 & 0.04961 & 264.328 & 0.0356 & 0.01039 & 0.800 & 5.853 & 1.76 & 40.00 & 2097. \\
\hline 1042 & 0.074 & 0.06265 & 265.065 & 0.0345 & 0.01052 & 0.700 & 7.294 & 1.76 & 40.00 & 2428. \\
\hline 1045 & 0.074 & 0.07713 & 265.871 & 0.0342 & 0.01063 & 0.750 & 8.879 & 1.76 & 40.00 & 2869. \\
\hline 1048 & 0.296 & 0.02712 & 271.823 & 0.1428 & 0.01205 & 1.250 & 2.674 & 4.96 & 40.00 & 19162. \\
\hline 1051 & 0.299 & 0.03686 & 272.270 & 0.1441 & 0.01210 & 1.310 & 3.577 & 3.36 & 40.00 & 26013. \\
\hline 1054 & 0.301 & 0.04806 & 272.789 & 0.1447 & 0.01207 & 1.180 & 4.612 & 3.36 & 40.00 & 33546 . \\
\hline 1057 & 0.299 & 0.06072 & 273.342 & 0.1433 & 0.01214 & 1.780 & 5.728 & 1.76 & 40.00 & 40259. \\
\hline 1060 & 0.296 & 0.07485 & 273.961 & 0.1412 & 0.01217 & 1.340 & 6.964 & 1.76 & 40.00 & 46689. \\
\hline 1063 & 0.216 & 0.02712 & 271.845 & 0.1014 & 0.01193 & 0.990 & 2.750 & 6.56 & 40.00 & 8733. \\
\hline 1066 & 0.218 & 0.03685 & 272.333 & 0.1021 & 0.01188 & 0.820 & 3.733 & 4.96 & 40.00 & 11959. \\
\hline 1069 & 0.220 & 0.04804 & 272.889 & 0.1032 & 0.01188 & 0.540 & 4.832 & 4.96 & 40.00 & 15731. \\
\hline 1072 & 0.220 & 0.06071 & 273.480 & 0.1028 & 0.01196 & 0.650 & 6.031 & 3.36 & 40.00 & 19287. \\
\hline 1075 & 0.217 & 0.07481 & 274.132 & 0.1008 & 0.01207 & 1.070 & 7.328 & 3.36 & 40.00 & 22132 . \\
\hline 1078 & 0.149 & 0.02711 & 271.857 & 0.0689 & 0.01188 & 1.100 & 2.788 & 4.96 & 40.00 & 3714. \\
\hline 1081 & 0.146 & 0.03685 & 272.368 & 0.0671 & 0.01182 & 0.670 & 3.800 & 4.96 & 40.00 & 4751. \\
\hline 1084 & 0.148 & 0.04803 & 272.944 & 0.0677 & 0.01181 & 0.500 & 4.945 & 4.96 & 40.00 & 6252. \\
\hline 1087 & 0.151 & 0.06068 & 273.576 & 0.0690 & 0.01182 & 0.780 & 6.224 & 3.36 & 40.00 & 8111. \\
\hline 1090 & 0.151 & 0.07478 & 274.283 & 0.0687 & 0.01184 & 0.620 & 7.631 & 3.36 & 40.00 & 9761. \\
\hline 1093 & 0.078 & 0.02710 & 271.867 & 0.0353 & 0.01179 & 1.020 & 2.823 & 1.76 & 40.00 & 901. \\
\hline 1096 & 0.075 & 0.03683 & 272.381 & 0.0337 & 0.01175 & 0.720 & 3.848 & 1.76 & 40.00 & 1103. \\
\hline 1099 & 0.074 & 0.04805 & 272.954 & 0.0331 & 0.01175 & 0.710 & 5.015 & 1.76 & 40.00 & 1376. \\
\hline 1102 & 0.077 & 0.06068 & 273.611 & 0.0345 & 0.01176 & 0.810 & 6.327 & 1.76 & 40.00 & 1873. \\
\hline 1105 & 0.078 & 0.07474 & 274.339 & 0.0350 & 0.01178 & 0.700 & 7.772 & 1.76 & 40.00 & 2353. \\
\hline 1108 & 0.434 & 0.02611 & 282.379 & 0.2068 & 0.01242 & 1.480 & 2.424 & 4.96 & 40.00 & 36212. \\
\hline 1111 & 0.433 & 0.03552 & 282.761 & 0.2061 & 0.01255 & 1.370 & 3.202 & 3.36 & 40.00 & 47035. \\
\hline 1114 & 0.429 & 0.04633 & 283.201 & 0.2031 & 0.01270 & 1.930 & 4.062 & 1.76 & 40.00 & 57017. \\
\hline 1123 & 0.346 & 0.02612 & 282.400 & 0.1607 & 0.01241 & 1.400 & 2.498 & 4.96 & 40.00 & 19811. \\
\hline 1126 & 0.350 & 0.03552 & 282.816 & 0.1622 & 0.01253 & 1.300 & 3.324 & 3.36 & 40.00 & 26787. \\
\hline 1129 & 0.351 & 0.04631 & 283.277 & 0.1624 & 0.01260 & 1.150 & 4.252 & 3.36 & 40.00 & 34109. \\
\hline 1135 & 0.346 & 0.07217 & 284.338 & 0.1591 & 0.01276 & 1.500 & 6.393 & 1.76 & 40.00 & 47938. \\
\hline
\end{tabular}


Table 23. Thermal conductivity of the binary $30 \% \mathrm{R} 125$ / $70 \% \mathrm{R} 134 \mathrm{a}$ mixture in the dilute gas measured with the steady-state technique (continued).

\begin{tabular}{|c|c|c|c|c|c|c|c|c|c|c|}
\hline $\begin{array}{l}\text { Run } \\
\text { point }\end{array}$ & $\begin{array}{l}P_{e x p} \\
\mathrm{MPa}\end{array}$ & $\begin{array}{c}Q \\
W \cdot m^{-1}\end{array}$ & $\begin{array}{c}T_{\text {exp }} \\
\mathrm{K}\end{array}$ & $\begin{array}{c}\rho_{\text {calc }} \\
\mathrm{mol} \cdot \mathrm{L}^{-1}\end{array}$ & $\begin{array}{c}\lambda_{\exp } \\
\mathrm{W} \cdot \mathrm{m}^{-1} \cdot \mathrm{K}^{-1}\end{array}$ & $\begin{array}{c}\text { TBAND } \\
\%\end{array}$ & $\begin{array}{c}\Delta T_{\text {avg }} \\
\mathrm{K}\end{array}$ & $\begin{array}{c}t_{\text {start }} \\
\mathrm{s}\end{array}$ & $\begin{array}{c}t_{\text {end }} \\
\mathrm{s}\end{array}$ & $N_{R a}$ \\
\hline 1138 & 0.284 & 0.02612 & 282.362 & 0.1298 & 0.01251 & 1.040 & 2.512 & 6.56 & 40.00 & 11988. \\
\hline 1141 & 0.283 & 0.03551 & 282.802 & 0.1292 & 0.01257 & 0.760 & 3.377 & 4.96 & 40.00 & 15820. \\
\hline 1144 & 0.287 & 0.04631 & 283.279 & 0.1305 & 0.01265 & 1.100 & 4.337 & 3.36 & 40.00 & 20676 . \\
\hline 1147 & 0.288 & 0.05851 & 283.805 & 0.1306 & 0.01273 & 0.960 & 5.400 & 3.36 & 40.00 & 25563. \\
\hline 1150 & 0.284 & 0.07213 & 284.398 & 0.1286 & 0.01281 & 0.900 & 6.569 & 3.36 & 40.00 & 29730. \\
\hline 1153 & 0.214 & 0.02612 & 282.345 & 0.0959 & 0.01263 & 1.080 & 2.516 & 6.56 & 40.00 & 6017. \\
\hline 1156 & 0.213 & 0.03551 & 282.800 & 0.0953 & 0.01261 & 0.820 & 3.412 & 4.96 & 40.00 & 7981. \\
\hline 1159 & 0.217 & 0.04630 & 283.301 & 0.0969 & 0.01261 & 0.630 & 4.428 & 4.96 & 40.00 & 10678. \\
\hline 1162 & 0.217 & 0.05852 & 283.865 & 0.0968 & 0.01265 & 0.430 & 5.554 & 4.96 & 40.00 & 13257. \\
\hline 1165 & 0.214 & 0.07212 & 284.482 & 0.0951 & 0.01272 & 0.670 & 6.781 & 3.36 & 40.00 & 15420 . \\
\hline 1168 & 0.148 & 0.02612 & 282.324 & 0.0652 & 0.01288 & 1.160 & 2.483 & 3.36 & 40.00 & 2539. \\
\hline 1171 & 0.145 & 0.03550 & 282.772 & 0.0639 & 0.01281 & 0.970 & 3.389 & 3.36 & 40.00 & 3305. \\
\hline 1174 & 0.148 & 0.04630 & 283.283 & 0.0651 & 0.01276 & 0.760 & 4.428 & 3.36 & 40.00 & 4462 \\
\hline 1177 & 0.148 & 0.05849 & 283.870 & 0.0648 & 0.01275 & 0.710 & 5.588 & 3.36 & 40.00 & 5526. \\
\hline 1180 & 0.148 & 0.07209 & 284.501 & 0.0646 & 0.01275 & 0.600 & 6.871 & 3.36 & 40.00 & 6702. \\
\hline 1183 & 0.078 & 0.02611 & 282.279 & 0.0339 & 0.01303 & 1.390 & 2.462 & 1.76 & 40.00 & 632. \\
\hline 1186 & 0.076 & 0.03551 & 282.748 & 0.0330 & 0.01286 & 1.010 & 3.391 & 1.76 & 40.00 & 816. \\
\hline 1189 & 0.078 & 0.04631 & 283.285 & 0.0339 & 0.01278 & 0.770 & 4.449 & 1.76 & 40.00 & 1125. \\
\hline 1192 & 0.080 & 0.05850 & 283.879 & 0.0346 & 0.01272 & 0.650 & 5.642 & 1.76 & 40.00 & 1479. \\
\hline 1195 & 0.078 & 0.07208 & 284.539 & 0.0335 & 0.01271 & 0.620 & 6.955 & 1.76 & 40.00 & 1693. \\
\hline 1198 & 0.547 & 0.02522 & 292.570 & 0.2553 & 0.01334 & 1.660 & 2.145 & 3.36 & 40.00 & 45652. \\
\hline 1201 & 0.548 & 0.03430 & 292.903 & 0.2553 & 0.01354 & 2.850 & 2.808 & 1.76 & 40.00 & 59424. \\
\hline 1213 & 0.484 & 0.02521 & 292.542 & 0.2219 & 0.01380 & 1.850 & 2.124 & 4.96 & 40.00 & 31532. \\
\hline 1216 & 0.488 & 0.03429 & 292.909 & 0.2235 & 0.01374 & 1.320 & 2.846 & 3.36 & 40.00 & 42734. \\
\hline 1219 & 0.488 & 0.04473 & 293.308 & 0.2232 & 0.01372 & 1.580 & 3.646 & 3.36 & 40.00 & 54144. \\
\hline 1228 & 0.422 & 0.02521 & 292.530 & 0.1903 & 0.01400 & 1.770 & 2.131 & 4.96 & 40.00 & 21587. \\
\hline 1231 & 0.420 & 0.03428 & 292.895 & 0.1891 & 0.01397 & 1.400 & 2.868 & 4.96 & 40.00 & 28426. \\
\hline 1234 & 0.422 & 0.04473 & 293.304 & 0.1899 & 0.01395 & 1.590 & 3.694 & 3.36 & 40.00 & 36762. \\
\hline 1240 & 0.424 & 0.06971 & 294.241 & 0.1901 & 0.01394 & 1.930 & 5.588 & 1.76 & 40.00 & 54932. \\
\hline 1249 & 0.354 & 0.04472 & 293.265 & 0.1565 & 0.01425 & 1.320 & 3.703 & 3.36 & 40.00 & 23196. \\
\hline 1252 & 0.357 & 0.05653 & 293.743 & 0.1578 & 0.01416 & 1.120 & 4.658 & 3.36 & 40.00 & 29552. \\
\hline 1255 & 0.359 & 0.06971 & 294.257 & 0.1582 & 0.01410 & 1.230 & 5.703 & 3.36 & 40.00 & 36135 \\
\hline 1264 & 0.282 & 0.04473 & 293.297 & 0.1227 & 0.01410 & 0.670 & 3.806 & 4.96 & 40.00 & 13590. \\
\hline 1267 & 0.278 & 0.05654 & 293.797 & 0.1207 & 0.01405 & 1.080 & 4.804 & 3.36 & 40.00 & 16430. \\
\hline 1270 & 0.279 & 0.06969 & 294.342 & 0.1207 & 0.01402 & 0.810 & 5.896 & 3.36 & 40.00 & 20002. \\
\hline 1282 & 0.216 & 0.05651 & 293.817 & 0.0923 & 0.01397 & 0.560 & 4.892 & 4.96 & 40.00 & 9185. \\
\hline 1285 & 0.218 & 0.06967 & 294.390 & 0.0932 & 0.01389 & 0.350 & 6.043 & 4.96 & 40.00 & 11513. \\
\hline 1297 & 0.146 & 0.05651 & 293.811 & 0.0617 & 0.01394 & 0.660 & 4.953 & 3.36 & 40.00 & 3888. \\
\hline 1300 & 0.149 & 0.06966 & 294.416 & 0.0627 & 0.01382 & 0.550 & 6.143 & 3.36 & 40.00 & 4958 \\
\hline 1303 & 0.073 & 0.02519 & 292.103 & 0.0307 & 0.01328 & 1.300 & 2.331 & 1.76 & 40.00 & 432. \\
\hline 1306 & 0.075 & 0.03424 & 292.546 & 0.0311 & 0.01323 & 0.850 & 3.180 & 1.76 & 40.00 & 603. \\
\hline 1309 & 0.076 & 0.04467 & 293.023 & 0.0315 & 0.01323 & 0.740 & 4.146 & 1.76 & 40.00 & 803. \\
\hline 1312 & 0.076 & 0.05646 & 293.561 & 0.0315 & 0.01325 & 0.680 & 5.233 & 1.76 & 40.00 & 1005. \\
\hline
\end{tabular}


Table 23. Thermal conductivity of the binary $30 \% \mathrm{R} 125 / 70 \% \mathrm{R} 134 \mathrm{a}$ mixture in the dilute gas measured with the steady-state technique (continued).

\begin{tabular}{|c|c|c|c|c|c|c|c|c|c|c|}
\hline $\begin{array}{l}\text { Run } \\
\text { point }\end{array}$ & $\begin{array}{l}P_{\text {exp }} \\
\mathrm{MPa}\end{array}$ & $\begin{array}{c}Q \\
\mathrm{~W} \cdot \mathrm{m}^{-1} \\
\end{array}$ & $\begin{array}{c}T_{\exp } \\
\mathrm{K}\end{array}$ & $\begin{array}{c}\rho_{\text {calc }} \\
\mathrm{mol} \cdot \mathrm{L}^{-1}\end{array}$ & $\begin{array}{c}\lambda_{\exp } \\
\mathrm{W} \cdot \mathrm{m}^{-1} \cdot \mathrm{K}^{-1}\end{array}$ & $\begin{array}{c}\text { TBAND } \\
\% \\
\end{array}$ & $\begin{array}{c}\Delta T_{\text {avg }} \\
\mathrm{K} \\
\end{array}$ & $\begin{array}{c}t_{\text {start }} \\
\mathrm{s}\end{array}$ & $\begin{array}{c}t_{\text {end }} \\
\mathrm{s}\end{array}$ & $N_{R a}$ \\
\hline 1315 & 0.074 & 0.06958 & 294.161 & 0.0307 & 0.01327 & 0.460 & 6.436 & 1.76 & 40.00 & 1167. \\
\hline 1438 & 0.537 & 0.02337 & 315.035 & 0.2245 & 0.01510 & 1.750 & 1.837 & 6.56 & 40.00 & 19779. \\
\hline 1441 & 0.538 & 0.03179 & 315.330 & 0.2246 & 0.01523 & 1.380 & 2.449 & 4.96 & 40.00 & 26297. \\
\hline 1444 & 0.534 & 0.04147 & 315.685 & 0.2223 & 0.01528 & 1.430 & 3.148 & 3.36 & 40.00 & 32819. \\
\hline 1447 & 0.533 & 0.05244 & 316.060 & 0.2215 & 0.01533 & 1.350 & 3.918 & 3.36 & 40.00 & 40308. \\
\hline 1450 & 0.537 & 0.06468 & 316.482 & 0.2229 & 0.01531 & 1.180 & 4.764 & 3.36 & 40.00 & 49463. \\
\hline 1453 & 0.484 & 0.02338 & 315.008 & 0.2004 & 0.01526 & 1.690 & 1.833 & 8.16 & 40.00 & 15090 . \\
\hline 1456 & 0.488 & 0.03179 & 315.319 & 0.2017 & 0.01531 & 1.440 & 2.460 & 4.96 & 40.00 & 20494. \\
\hline 1459 & 0.489 & 0.04147 & 315.665 & 0.2020 & 0.01534 & 1.100 & 3.172 & 4.96 & 40.00 & 26369. \\
\hline 1462 & 0.485 & 0.05245 & 316.053 & 0.1998 & 0.01541 & 1.140 & 3.953 & 3.36 & 40.00 & 31894. \\
\hline 1465 & 0.484 & 0.06469 & 316.475 & 0.1991 & 0.01544 & 1.280 & 4.813 & 3.36 & 40.00 & 38275 . \\
\hline 1468 & 0.420 & 0.02338 & 314.969 & 0.1716 & 0.01549 & 1.900 & 1.821 & 6.56 & 40.00 & 10477. \\
\hline 1471 & 0.421 & 0.03179 & 315.293 & 0.1722 & 0.01542 & 0.990 & 2.471 & 6.56 & 40.00 & 14268. \\
\hline 1474 & 0.425 & 0.04147 & 315.657 & 0.1735 & 0.01541 & 0.900 & 3.199 & 4.96 & 40.00 & 18703. \\
\hline 1477 & 0.423 & 0.05244 & 316.052 & 0.1723 & 0.01547 & 1.200 & 4.000 & 3.36 & 40.00 & 22907. \\
\hline 1480 & 0.419 & 0.06468 & 316.489 & 0.1703 & 0.01552 & 0.990 & 4.883 & 3.36 & 40.00 & 27066 . \\
\hline 1489 & 0.347 & 0.04147 & 315.617 & 0.1400 & 0.01566 & 0.800 & 3.191 & 6.56 & 40.00 & 11469. \\
\hline 1492 & 0.347 & 0.05245 & 316.024 & 0.1396 & 0.01562 & 0.810 & 4.025 & 4.96 & 40.00 & 14307. \\
\hline 1495 & 0.351 & 0.06467 & 316.481 & 0.1409 & 0.01560 & 0.600 & 4.936 & 4.96 & 40.00 & 17832. \\
\hline 1504 & 0.284 & 0.04148 & 315.567 & 0.1132 & 0.01592 & 0.980 & 3.165 & 4.96 & 40.00 & 7118. \\
\hline 1507 & 0.288 & 0.05244 & 316.007 & 0.1146 & 0.01576 & 0.780 & 4.024 & 4.96 & 40.00 & 9243. \\
\hline 1510 & 0.286 & 0.06466 & 316.466 & 0.1138 & 0.01568 & 0.590 & 4.971 & 4.96 & 40.00 & 11185. \\
\hline 1519 & 0.213 & 0.04149 & 315.548 & 0.0839 & 0.01597 & 0.850 & 3.173 & 4.96 & 40.00 & 3733. \\
\hline 1522 & 0.217 & 0.05245 & 315.987 & 0.0853 & 0.01580 & 0.760 & 4.047 & 4.96 & 40.00 & 4905. \\
\hline 1525 & 0.219 & 0.06465 & 316.467 & 0.0860 & 0.01567 & 0.470 & 5.018 & 4.96 & 40.00 & 6154. \\
\hline 1534 & 0.144 & 0.04147 & 315.531 & 0.0560 & 0.01601 & 0.750 & 3.178 & 3.36 & 40.00 & 1587. \\
\hline 1537 & 0.143 & 0.05245 & 315.969 & 0.0555 & 0.01584 & 0.660 & 4.059 & 3.36 & 40.00 & 1985. \\
\hline 1540 & 0.147 & 0.06466 & 316.455 & 0.0571 & 0.01571 & 0.450 & 5.039 & 3.36 & 40.00 & 2597. \\
\hline 1549 & 0.075 & 0.04148 & 315.532 & 0.0291 & 0.01576 & 0.760 & 3.234 & 1.76 & 40.00 & 417. \\
\hline 1552 & 0.072 & 0.05243 & 315.982 & 0.0276 & 0.01562 & 0.590 & 4.126 & 1.76 & 40.00 & 475. \\
\hline 1555 & 0.070 & 0.06465 & 316.473 & 0.0271 & 0.01554 & 0.440 & 5.114 & 1.76 & 40.00 & 564. \\
\hline 1558 & 0.536 & 0.02276 & 322.503 & 0.2169 & 0.01633 & 1.990 & 1.669 & 6.56 & 40.00 & 14989. \\
\hline 1561 & 0.536 & 0.03096 & 322.787 & 0.2169 & 0.01627 & 1.500 & 2.256 & 4.96 & 40.00 & 20185. \\
\hline 1564 & 0.537 & 0.04040 & 323.127 & 0.2169 & 0.01622 & 1.350 & 2.924 & 4.96 & 40.00 & 26041. \\
\hline 1567 & 0.537 & 0.05108 & 323.486 & 0.2166 & 0.01623 & 1.240 & 3.655 & 3.36 & 40.00 & 32296 . \\
\hline 1570 & 0.537 & 0.06301 & 323.884 & 0.2161 & 0.01626 & 1.400 & 4.447 & 3.36 & 40.00 & 38873. \\
\hline 1573 & 0.489 & 0.02277 & 322.465 & 0.1966 & 0.01665 & 1.940 & 1.646 & 8.16 & 40.00 & 11763. \\
\hline 1576 & 0.491 & 0.03096 & 322.762 & 0.1969 & 0.01645 & 1.270 & 2.249 & 6.56 & 40.00 & 16071. \\
\hline 1579 & 0.491 & 0.04040 & 323.103 & 0.1970 & 0.01636 & 1.100 & 2.924 & 4.96 & 40.00 & 20824 . \\
\hline 1582 & 0.492 & 0.05108 & 323.464 & 0.1968 & 0.01633 & 1.020 & 3.671 & 4.96 & 40.00 & 25963. \\
\hline 1585 & 0.490 & 0.06300 & 323.870 & 0.1958 & 0.01632 & 1.020 & 4.489 & 3.36 & 40.00 & 31227. \\
\hline 1594 & 0.424 & 0.04041 & 323.080 & 0.1683 & 0.01647 & 0.830 & 2.938 & 6.56 & 40.00 & 14601. \\
\hline 1597 & 0.424 & 0.05110 & 323.460 & 0.1677 & 0.01640 & 0.680 & 3.708 & 4.96 & 40.00 & 18206. \\
\hline
\end{tabular}


Table 23. Thermal conductivity of the binary $30 \% \mathrm{R} 125 / 70 \% \mathrm{R} 134 \mathrm{a}$ mixture in the dilute gas measured with the steady-state technique (continued).

\begin{tabular}{|c|c|c|c|c|c|c|c|c|c|c|}
\hline $\begin{array}{l}\text { Run } \\
\text { point }\end{array}$ & $\begin{array}{l}P_{\text {exp }} \\
\mathrm{MPa}\end{array}$ & $\begin{array}{c}Q \\
\mathrm{~W} \cdot \mathrm{m}^{-1}\end{array}$ & $\begin{array}{c}T_{\exp } \\
\mathrm{K}\end{array}$ & $\begin{array}{c}\rho_{\text {calc }} \\
\mathrm{mol} \cdot \mathrm{L}^{-1}\end{array}$ & $\begin{array}{c}\lambda_{\exp } \\
\mathrm{W} \cdot \mathrm{m}^{-1} \cdot \mathrm{K}^{-1}\end{array}$ & $\begin{array}{c}\text { TBAND } \\
\%\end{array}$ & $\begin{array}{c}\Delta T_{\text {avg }} \\
\mathrm{K}\end{array}$ & $\begin{array}{c}t_{\text {start }} \\
\mathrm{s}\end{array}$ & $\begin{array}{c}t_{\text {end }} \\
\mathrm{s}\end{array}$ & $N_{R a}$ \\
\hline 1600 & 0.422 & 0.06300 & 323.881 & 0.1669 & 0.01638 & 1.130 & 4.546 & 3.36 & 40.00 & 21961. \\
\hline 1609 & 0.355 & 0.04040 & 323.037 & 0.1393 & 0.01673 & 0.950 & 2.920 & 6.56 & 40.00 & 9506. \\
\hline 1612 & 0.353 & 0.05110 & 323.455 & 0.1384 & 0.01656 & 0.810 & 3.715 & 4.96 & 40.00 & 11860. \\
\hline 1615 & 0.350 & 0.06300 & 323.887 & 0.1367 & 0.01646 & 0.670 & 4.587 & 4.96 & 40.00 & 14179. \\
\hline 1627 & 0.281 & 0.05109 & 323.421 & 0.1088 & 0.01674 & 0.900 & 3.706 & 4.96 & 40.00 & 6990. \\
\hline 1630 & 0.282 & 0.06300 & 323.854 & 0.1089 & 0.01657 & 0.600 & 4.604 & 4.96 & 40.00 & 8665. \\
\hline 1642 & 0.210 & 0.05109 & 323.399 & 0.0803 & 0.01678 & 0.680 & 3.720 & 4.96 & 40.00 & 3656 . \\
\hline 1645 & 0.211 & 0.06299 & 323.854 & 0.0807 & 0.01660 & 0.960 & 4.629 & 3.36 & 40.00 & 4578. \\
\hline 1657 & 0.144 & 0.05109 & 323.392 & 0.0545 & 0.01675 & 0.800 & 3.740 & 3.36 & 40.00 & 1628. \\
\hline 1660 & 0.143 & 0.06301 & 323.834 & 0.0540 & 0.01657 & 0.530 & 4.659 & 3.36 & 40.00 & 1982. \\
\hline 1672 & 0.078 & 0.05109 & 323.375 & 0.0293 & 0.01671 & 0.690 & 3.757 & 1.76 & 40.00 & 455. \\
\hline 1675 & 0.079 & 0.06299 & 323.830 & 0.0298 & 0.01651 & 0.500 & 4.688 & 1.76 & 40.00 & 585. \\
\hline 1678 & 0.539 & 0.02232 & 331.974 & 0.2100 & 0.01692 & 2.070 & 1.588 & 6.56 & 40.00 & 11746. \\
\hline 1681 & 0.539 & 0.03036 & 332.257 & 0.2101 & 0.01686 & 1.380 & 2.152 & 6.56 & 40.00 & 15876. \\
\hline 1684 & 0.540 & 0.03962 & 332.559 & 0.2100 & 0.01688 & 1.210 & 2.782 & 4.96 & 40.00 & 20444 . \\
\hline 1687 & 0.540 & 0.05012 & 332.909 & 0.2098 & 0.01692 & 1.010 & 3.481 & 4.96 & 40.00 & 25414. \\
\hline 1690 & 0.540 & 0.06181 & 333.289 & 0.2094 & 0.01694 & 1.090 & 4.249 & 3.36 & 40.00 & 30736 . \\
\hline 1696 & 0.489 & 0.03036 & 332.209 & 0.1891 & 0.01706 & 1.420 & 2.140 & 6.56 & 40.00 & 12429. \\
\hline 1699 & 0.489 & 0.03963 & 332.529 & 0.1889 & 0.01701 & 1.040 & 2.784 & 6.56 & 40.00 & 16064. \\
\hline 1702 & 0.488 & 0.05012 & 332.891 & 0.1884 & 0.01699 & 0.960 & 3.499 & 4.96 & $40 . \dot{00}$ & 19977. \\
\hline 1705 & 0.488 & 0.06180 & 333.273 & 0.1880 & 0.01700 & 1.340 & 4.280 & 3.36 & 40.00 & 24235. \\
\hline 1714 & 0.420 & 0.03963 & 332.487 & 0.1606 & 0.01721 & 1.070 & 2.776 & 6.56 & 40.00 & 11132. \\
\hline 1717 & 0.421 & 0.05010 & 332.849 & 0.1607 & 0.01710 & 1.030 & 3.513 & 4.96 & 40.00 & 14049. \\
\hline 1720 & 0.421 & 0.06180 & 333.269 & 0.1605 & 0.01707 & 0.740 & 4.317 & 4.96 & 40.00 & 17145. \\
\hline 1768 & 0.146 & 0.02235 & 331.601 & 0.0539 & 0.01669 & 1.930 & 1.645 & 1.76 & 40.00 & 644. \\
\hline 1771 & 0.146 & 0.03039 & 331.924 & 0.0538 & 0.01658 & 1.170 & 2.250 & 3.36 & 40.00 & 876. \\
\hline 1774 & 0.146 & 0.03966 & 332.252 & 0.0540 & 0.01656 & 0.850 & 2.940 & 3.36 & 40.00 & 1147. \\
\hline 1777 & 0.146 & 0.05016 & 332.628 & 0.0539 & 0.01655 & 0.650 & 3.717 & 3.36 & 40.00 & 1442. \\
\hline 1780 & 0.146 & 0.06186 & 333.063 & 0.0538 & 0.01655 & 0.480 & 4.583 & 3.36 & 40.00 & 1763. \\
\hline 1786 & 0.076 & 0.03040 & 331.884 & 0.0279 & 0.01664 & 1.390 & 2.245 & 1.76 & 40.00 & 227. \\
\hline 1789 & 0.077 & 0.03967 & 332.232 & 0.0281 & 0.01654 & 0.910 & 2.949 & 1.76 & 40.00 & 301. \\
\hline 1792 & 0.077 & 0.05015 & 332.615 & 0.0279 & 0.01649 & 0.630 & 3.737 & 1.76 & 40.00 & 376. \\
\hline 1795 & 0.074 & 0.06186 & 333.064 & 0.0271 & 0.01650 & 0.470 & 4.609 & 1.76 & 40.00 & 435. \\
\hline
\end{tabular}


Table 24. Thermal conductivity of the binary $70 \% \mathrm{R} 125$ / $30 \% \mathrm{R} 134 \mathrm{a}$ mixture in the dilute gas measured with the steady-state technique.

\begin{tabular}{|c|c|c|c|c|c|c|c|c|c|c|}
\hline $\begin{array}{l}\text { Run } \\
\text { point }\end{array}$ & $\begin{array}{l}P_{\text {exp }} \\
\mathrm{MPa}\end{array}$ & $\begin{array}{c}Q \\
\mathrm{~W} \cdot \mathrm{m}^{-1}\end{array}$ & $\begin{array}{c}T_{\text {exp }} \\
\mathrm{K}\end{array}$ & $\begin{array}{c}\rho_{\text {calc }} \\
\mathrm{mol} \cdot \mathrm{L}^{-1}\end{array}$ & $\begin{array}{c}\lambda_{\exp } \\
\mathrm{W} \cdot \mathrm{m}^{-1} \cdot \mathrm{K}^{-1}\end{array}$ & $\begin{array}{c}\text { TBAND } \\
\%\end{array}$ & $\begin{array}{c}\Delta T_{\text {avg }} \\
\mathrm{K}\end{array}$ & $\begin{array}{c}t_{\text {start }} \\
\mathrm{s}\end{array}$ & $\begin{array}{c}t_{\text {end }} \\
\mathrm{s}\end{array}$ & $\overline{N_{R a}}$ \\
\hline 1003 & 0.088 & 0.02860 & 258.216 & 0.0420 & 0.01080 & 0.770 & 3.246 & 3.36 & 40.00 & 1817. \\
\hline 1006 & 0.089 & 0.03886 & 258.801 & 0.0425 & 0.01082 & 0.530 & 4.398 & 3.36 & 40.00 & 2509. \\
\hline 1009 & 0.091 & 0.05064 & 259.450 & 0.0430 & 0.01084 & 0.370 & 5.712 & 3.36 & 40.00 & 3309. \\
\hline 1012 & 0.090 & 0.06396 & 260.196 & 0.0428 & 0.01089 & 0.350 & 7.170 & 3.36 & 40.00 & 4070. \\
\hline 1015 & 0.088 & 0.07877 & 261.000 & 0.0415 & 0.01094 & 0.300 & 8.781 & 3.36 & 40.00 & 4616. \\
\hline 1018 & 0.178 & 0.02859 & 258.226 & 0.0872 & 0.01094 & 0.960 & 3.163 & 4.96 & 40.00 & 8701. \\
\hline 1021 & 0.176 & 0.03885 & 258.770 & 0.0860 & 0.01095 & 0.560 & 4.275 & 4.96 & 40.00 & 11317. \\
\hline 1024 & 0.175 & 0.05065 & 259.403 & 0.0855 & 0.01099 & 0.390 & 5.522 & 4.96 & 40.00 & 14268. \\
\hline 1027 & 0.177 & 0.06397 & 260.080 & 0.0860 & 0.01108 & 0.600 & 6.875 & 3.36 & 40.00 & 17815. \\
\hline 1030 & 0.178 & 0.07879 & 260.808 & 0.0861 & 0.01118 & 1.710 & 8.338 & 1.76 & 40.00 & 21417 \\
\hline 1063 & 0.093 & 0.02794 & 264.295 & 0.0433 & 0.01146 & 0.940 & 2.989 & 3.36 & 40.00 & 1639. \\
\hline 1066 & 0.095 & 0.03797 & 264.810 & 0.0440 & 0.01148 & 0.550 & 4.050 & 3.36 & 40.00 & 2285 \\
\hline 1069 & 0.096 & 0.04949 & 265.390 & 0.0447 & 0.01150 & 0.400 & 5.264 & 3.36 & 40.00 & 3055 . \\
\hline 1072 & 0.097 & 0.06251 & 266.047 & 0.0447 & 0.01152 & 0.370 & 6.628 & 3.36 & 40.00 & 3808 . \\
\hline 1075 & 0.096 & 0.07701 & 266.777 & 0.0442 & 0.01155 & 0.250 & 8.130 & 3.36 & 40.00 & 4506 \\
\hline 1078 & 0.091 & 0.02794 & 264.352 & 0.0425 & 0.01134 & 0.700 & 3.023 & 3.36 & 40.00 & 1593. \\
\hline 1081 & 0.091 & 0.03797 & 264.905 & 0.0424 & 0.01132 & 0.470 & 4.108 & 3.36 & 40.00 & 2142 . \\
\hline 1084 & 0.092 & 0.04950 & 265.516 & 0.0425 & 0.01134 & 0.350 & 5.340 & 3.36 & 40.00 & 2774 . \\
\hline 1087 & 0.092 & 0.06251 & 266.205 & 0.0426 & 0.01137 & 0.280 & 6.717 & 3.36 & 40.00 & 3481. \\
\hline 1090 & 0.091 & 0.07702 & 266.967 & 0.0419 & 0.01142 & 0.270 & 8.234 & 3.36 & 40.00 & 4084 \\
\hline 1093 & 0.257 & 0.02795 & 264.291 & 0.1255 & 0.01146 & 0.910 & 2.910 & 4.96 & 40.00 & 16787. \\
\hline 1096 & 0.257 & 0.03798 & 264.778 & 0.1253 & 0.01156 & 1.080 & 3.883 & 3.36 & 40.00 & 22122 . \\
\hline 1099 & 0.258 & 0.04953 & 265.311 & 0.1254 & 0.01166 & 1.050 & 4.970 & 3.36 & 40.00 & 28104. \\
\hline 1102 & 0.257 & 0.06257 & 265.920 & 0.1247 & 0.01172 & 1.760 & 6.180 & 1.76 & 40.00 & 34173. \\
\hline 1105 & 0.257 & 0.07709 & 266.570 & 0.1242 & 0.01178 & 1.410 & 7.487 & 1.76 & 40.00 & 40580. \\
\hline 1108 & 0.265 & 0.02737 & 269.739 & 0.1263 & 0.01201 & 1.160 & 2.731 & 4.96 & 40.00 & 14602 . \\
\hline 1111 & 0.264 & 0.03722 & 270.198 & 0.1258 & 0.01205 & 0.850 & 3.668 & 4.96 & 40.00 & 19299. \\
\hline 1114 & 0.263 & 0.04854 & 270.718 & 0.1249 & 0.01213 & 1.060 & 4.714 & 3.36 & 40.00 & 24205 . \\
\hline 1117 & 0.264 & 0.06132 & 271.297 & 0.1251 & 0.01215 & 0.940 & 5.883 & 3.36 & 40.00 & 30057. \\
\hline 1120 & 0.265 & 0.07555 & 271.920 & 0.1252 & 0.01220 & 1.640 & 7.141 & 1.76 & 40.00 & 36191. \\
\hline 1123 & 0.175 & 0.02737 & 269.710 & 0.0814 & 0.01190 & 1.130 & 2.800 & 4.96 & 40.00 & 5557. \\
\hline 1126 & 0.176 & 0.03720 & 270.198 & 0.0818 & 0.01188 & 0.660 & 3.799 & 4.96 & 40.00 & 7583. \\
\hline 1129 & 0.177 & 0.04850 & 270.760 & 0.0821 & 0.01188 & 0.520 & 4.935 & 4.96 & 40.00 & 9825. \\
\hline 1132 & 0.176 & 0.06127 & 271.384 & 0.0813 & 0.01189 & 0.350 & 6.200 & 4.96 & 40.00 & 12001. \\
\hline 1135 & 0.174 & 0.07550 & 272.061 & 0.0802 & 0.01196 & 0.570 & 7.567 & 3.36 & 40.00 & 14057. \\
\hline 1138 & 0.326 & 0.02737 & 269.591 & 0.1584 & 0.01203 & 1.330 & 2.677 & 4.96 & 40.00 & 24564. \\
\hline 1141 & 0.326 & 0.03721 & 270.039 & 0.1581 & 0.01208 & 1.240 & 3.575 & 3.36 & 40.00 & 32380 . \\
\hline 1144 & 0.326 & 0.04853 & 270.541 & 0.1576 & 0.01211 & 2.090 & 4.585 & 1.76 & 40.00 & 40873. \\
\hline 1147 & 0.326 & 0.06131 & 271.101 & 0.1572 & 0.01212 & 1.640 & 5.697 & 1.76 & 40.00 & 50018. \\
\hline 1160 & 0.093 & 0.02638 & 279.602 & 0.0409 & 0.01246 & 0.960 & 2.599 & 3.36 & 40.00 & 1042. \\
\hline 1163 & 0.094 & 0.03586 & 280.063 & 0.0411 & 0.01247 & 0.620 & 3.529 & 3.36 & 40.00 & 1424. \\
\hline 1166 & 0.094 & 0.04676 & 280.586 & 0.0413 & 0.01249 & 0.370 & 4.589 & 3.36 & 40.00 & 1855. \\
\hline 1169 & 0.095 & 0.05907 & 281.185 & 0.0412 & 0.01252 & 0.340 & 5.778 & 3.36 & 40.00 & 2312. \\
\hline
\end{tabular}


Table 24. Thermal conductivity of the binary $70 \% \mathrm{R} 125 / 30 \% \mathrm{R} 134 \mathrm{a}$ mixture in the dilute gas measured with the steady-state technique (continued).

\begin{tabular}{|c|c|c|c|c|c|c|c|c|c|c|}
\hline $\begin{array}{l}\text { Run } \\
\text { point }\end{array}$ & $\begin{array}{l}P_{\text {exp }} \\
\mathrm{MPa}\end{array}$ & $\begin{array}{c}Q \\
\mathrm{~W} \cdot \mathrm{m}^{-1}\end{array}$ & $\begin{array}{c}T_{\exp } \\
\mathrm{K}\end{array}$ & $\begin{array}{c}\rho_{\text {calc }} \\
\mathrm{mol} \cdot \mathrm{L}^{-1}\end{array}$ & $\begin{array}{c}\lambda_{\exp } \\
\mathrm{W} \cdot \mathrm{m}^{-1} \cdot \mathrm{K}^{-1}\end{array}$ & $\begin{array}{c}\text { TBAND } \\
\%\end{array}$ & $\begin{array}{c}\Delta T_{a v g} \\
\mathrm{~K}\end{array}$ & $\begin{array}{c}t_{\text {start }} \\
\mathrm{s}\end{array}$ & $\begin{array}{c}t_{\text {end }} \\
\mathrm{s}\end{array}$ & $N_{R a}$ \\
\hline 1172 & 0.094 & 0.07279 & 281.835 & 0.0409 & 0.01256 & 0.270 & 7.091 & 3.36 & 40.00 & 2774 . \\
\hline 1175 & 0.196 & 0.02638 & 279.525 & 0.0880 & 0.01271 & 1.130 & 2.529 & 4.96 & 40.00 & 5209 . \\
\hline 1178 & 0.196 & 0.03585 & 279.969 & 0.0879 & 0.01265 & 0.730 & 3.442 & 4.96 & 40.00 & 7017. \\
\hline 1181 & 0.196 & 0.04676 & 280.493 & 0.0876 & 0.01263 & 0.690 & 4.478 & 4.96 & 40.00 & 9001. \\
\hline 1184 & 0.195 & 0.05910 & 281.067 & 0.0870 & 0.01265 & 0.390 & 5.632 & 4.96 & 40.00 & 11068. \\
\hline 1187 & 0.195 & 0.07283 & 281.693 & 0.0866 & 0.01269 & 0.650 & 6.889 & 3.36 & 40.00 & 13298. \\
\hline 1190 & 0.324 & 0.02639 & 279.485 & 0.1501 & 0.01266 & 1.020 & 2.485 & 6.56 & 40.00 & 17071. \\
\hline 1193 & 0.324 & 0.03586 & 279.899 & 0.1500 & 0.01270 & 1.050 & 3.334 & 4.96 & 40.00 & 22706 . \\
\hline 1196 & 0.325 & 0.04677 & 280.371 & 0.1499 & 0.01275 & 1.010 & 4.283 & 3.36 & 40.00 & 28925 . \\
\hline 1199 & 0.325 & 0.05912 & 280.901 & 0.1498 & 0.01279 & 1.170 & 5.335 & 3.36 & 40.00 & 35689 . \\
\hline 1202 & 0.326 & 0.07287 & 281.466 & 0.1495 & 0.01283 & 1.620 & 6.477 & 1.76 & 40.00 & 42770 . \\
\hline 1250 & 0.448 & 0.02558 & 288.393 & 0.2051 & 0.01361 & 1.540 & 2.199 & 4.96 & 40.00 & 27611 . \\
\hline 1253 & 0.449 & 0.03477 & 288.780 & 0.2050 & 0.01358 & 1.480 & 2.949 & 3.36 & 40.00 & 36736 . \\
\hline 1256 & 0.449 & 0.04536 & 289.197 & 0.2047 & 0.01354 & 1.420 & 3.794 & 3.36 & 40.00 & 46800 . \\
\hline 1265 & 0.319 & 0.02557 & 288.438 & 0.1420 & 0.01339 & 1.250 & 2.297 & 6.56 & 40.00 & 12165. \\
\hline 1268 & 0.320 & 0.03476 & 288.843 & 0.1419 & 0.01337 & 1.050 & 3.104 & 4.96 & 40.00 & 16338. \\
\hline 1271 & 0.319 & 0.04535 & 289.288 & 0.1415 & 0.01344 & 1.110 & 3.997 & 3.36 & 40.00 & 20753 . \\
\hline 1274 & 0.320 & 0.05732 & 289.785 & 0.1412 & 0.01351 & 0.970 & 4.984 & 3.36 & 40.00 & 25600 . \\
\hline 1277 & 0.319 & 0.07064 & 290.326 & 0.1406 & 0.01354 & 0.940 & 6.076 & 3.36 & 40.00 & 30671 . \\
\hline 1280 & 0.188 & 0.02557 & 288.388 & 0.0812 & 0.01339 & 1.020 & 2.333 & 4.96 & 40.00 & 3592 . \\
\hline 1283 & 0.188 & 0.03477 & 288.811 & 0.0810 & 0.01333 & 0.730 & 3.180 & 4.96 & 40.00 & 4850. \\
\hline 1286 & 0.188 & 0.04535 & 289.282 & 0.0809 & 0.01331 & 0.560 & 4.143 & 4.96 & 40.00 & 6256. \\
\hline 1289 & 0.187 & 0.05730 & 289.834 & 0.0805 & 0.01331 & 0.490 & 5.222 & 4.96 & 40.00 & 7757. \\
\hline 1292 & 0.187 & 0.07062 & 290.421 & 0.0802 & 0.01332 & 0.380 & 6.410 & 4.96 & 40.00 & 9361. \\
\hline 1295 & 0.093 & 0.02557 & 288.394 & 0.0395 & 0.01331 & 1.460 & 2.359 & 1.76 & 40.00 & 792. \\
\hline 1298 & 0.093 & 0.03476 & 288.817 & 0.0396 & 0.01326 & 0.990 & 3.218 & 1.76 & 40.00 & 1080. \\
\hline 1301 & 0.094 & 0.04534 & 289.310 & 0.0397 & 0.01325 & 0.470 & 4.198 & 3.36 & 40.00 & 1408. \\
\hline 1304 & 0.094 & 0.05729 & 289.863 & 0.0397 & 0.01326 & 0.790 & 5.299 & 1.76 & 40.00 & 1770. \\
\hline 1307 & 0.095 & 0.07060 & 290.473 & 0.0398 & 0.01327 & 0.890 & 6.516 & 1.76 & 40.00 & 2175 . \\
\hline 1310 & 0.099 & 0.02440 & 302.377 & 0.0402 & 0.01428 & 1.350 & 2.100 & 1.76 & 40.00 & 625. \\
\hline 1313 & 0.099 & 0.03317 & 302.758 & 0.0401 & 0.01427 & 1.160 & 2.855 & 1.76 & 40.00 & 845. \\
\hline 1316 & 0.099 & 0.04328 & 303.185 & 0.0399 & 0.01428 & 0.910 & 3.721 & 1.76 & 40.00 & 1083. \\
\hline 1319 & 0.098 & 0.05471 & 303.658 & 0.0396 & 0.01431 & 0.880 & 4.692 & 1.76 & 40.00 & 1336. \\
\hline 1322 & 0.099 & 0.06745 & 304.205 & 0.0398 & 0.01434 & 0.700 & 5.770 & 1.76 & 40.00 & 1649. \\
\hline 1325 & 0.241 & 0.02440 & 302.344 & 0.0997 & 0.01441 & 1.510 & 2.067 & 4.96 & 40.00 & 4176. \\
\hline 1328 & 0.241 & 0.03318 & 302.697 & 0.0995 & 0.01438 & 1.040 & 2.810 & 4.96 & 40.00 & 5640 . \\
\hline 1331 & 0.242 & 0.04329 & 303.127 & 0.0997 & 0.01436 & 0.710 & 3.658 & 4.96 & 40.00 & 7323. \\
\hline 1334 & 0.242 & 0.05472 & 303.601 & 0.0995 & 0.01436 & 0.550 & 4.608 & 4.96 & 40.00 & 9149. \\
\hline 1337 & 0.241 & 0.06746 & 304.115 & 0.0992 & 0.01439 & 0.450 & 5.653 & 4.96 & 40.00 & 11067. \\
\hline 1340 & 0.457 & 0.02440 & 302.275 & 0.1969 & 0.01462 & 1.470 & 1.986 & 6.56 & 40.00 & 18431. \\
\hline 1343 & 0.457 & 0.03318 & 302.619 & 0.1964 & 0.01466 & 1.220 & 2.665 & 4.96 & 40.00 & 24467. \\
\hline 1346 & 0.456 & 0.04330 & 302.992 & 0.1958 & 0.01468 & 1.270 & 3.432 & 3.36 & 40.00 & 31122 . \\
\hline 1349 & 0.456 & 0.05474 & 303.421 & 0.1953 & 0.01468 & 1.340 & 4.282 & 3.36 & 40.00 & 38381. \\
\hline
\end{tabular}


Table 24. Thermal conductivity of the binary $70 \% \mathrm{R} 125 / 30 \% \mathrm{R} 134 \mathrm{a}$ mixture in the dilute gas measured with the steady-state technique (continued).

\begin{tabular}{|c|c|c|c|c|c|c|c|c|c|c|}
\hline $\begin{array}{l}\text { Run } \\
\text { point }\end{array}$ & $\begin{array}{l}P_{\text {exp }} \\
\mathrm{MPa}\end{array}$ & $\begin{array}{c}Q \\
\mathrm{~W} \cdot \mathrm{m}^{-1}\end{array}$ & $\begin{array}{c}T_{\exp } \\
\mathrm{K}\end{array}$ & $\begin{array}{c}\rho_{\text {calc }} \\
\mathrm{mol} \cdot \mathrm{L}^{-1}\end{array}$ & $\begin{array}{c}\lambda_{\exp } \\
\mathrm{W} \cdot \mathrm{m}^{-1} \cdot \mathrm{K}^{-1}\end{array}$ & $\begin{array}{c}\text { TBAND } \\
\%\end{array}$ & $\begin{array}{c}\Delta T_{a v g} \\
\mathrm{~K}\end{array}$ & $\begin{array}{c}t_{\text {start }} \\
\mathrm{s}\end{array}$ & $\begin{array}{c}t_{\text {end }} \\
\mathrm{s}\end{array}$ & $N_{R a}$ \\
\hline 1352 & 0.456 & 0.06749 & 303.872 & 0.1950 & 0.01470 & 2.060 & 5.205 & 1.76 & 40.00 & 46197. \\
\hline 1355 & 0.600 & 0.02441 & 301.240 & 0.2671 & 0.01472 & 1.640 & 1.908 & 4.96 & 40.00 & 37294. \\
\hline 1358 & 0.600 & 0.03320 & 301.562 & 0.2669 & 0.01464 & 1.490 & 2.555 & 3.36 & 40.00 & 49596. \\
\hline 1370 & 0.627 & 0.02344 & 314.016 & 0.2644 & 0.01594 & 1.740 & 1.723 & 4.96 & 40.00 & 27217. \\
\hline 1373 & 0.628 & 0.03189 & 314.302 & 0.2644 & 0.01585 & 1.380 & 2.319 & 4.96 & 40.00 & 36498. \\
\hline 1376 & 0.628 & 0.04161 & 314.635 & 0.2643 & 0.01576 & 1.280 & 2.990 & 3.36 & 40.00 & 46796. \\
\hline 1385 & 0.475 & 0.02354 & 312.711 & 0.1963 & 0.01554 & 1.620 & 1.814 & 6.56 & 40.00 & 14544. \\
\hline 1388 & 0.475 & 0.03201 & 313.016 & 0.1961 & 0.01555 & 1.590 & 2.445 & 4.96 & 40.00 & 19478. \\
\hline 1391 & 0.476 & 0.04177 & 313.376 & 0.1961 & 0.01556 & 1.060 & 3.155 & 4.96 & 40.00 & 25021. \\
\hline 1394 & 0.475 & 0.05281 & 313.767 & 0.1955 & 0.01560 & 1.220 & 3.939 & 3.36 & 40.00 & 30855 . \\
\hline 1397 & 0.475 & 0.06512 & 314.179 & 0.1952 & 0.01562 & 1.170 & 4.798 & 3.36 & 40.00 & 37247. \\
\hline 1400 & 0.290 & 0.02354 & 312.623 & 0.1163 & 0.01536 & 1.440 & 1.870 & 6.56 & 40.00 & 4681. \\
\hline 1403 & 0.290 & 0.03202 & 312.948 & 0.1162 & 0.01533 & 1.100 & 2.539 & 4.96 & 40.00 & 6320. \\
\hline 1406 & 0.290 & 0.04179 & 313.331 & 0.1159 & 0.01531 & 0.830 & 3.308 & 4.96 & 40.00 & 8152. \\
\hline 1409 & 0.290 & 0.05283 & 313.756 & 0.1158 & 0.01530 & 0.790 & 4.168 & 4.96 & 40.00 & 10201. \\
\hline 1412 & 0.290 & 0.06514 & 314.213 & 0.1157 & 0.01533 & 0.530 & 5.111 & 4.96 & 40.00 & 12426. \\
\hline 1415 & 0.173 & 0.02355 & 312.535 & 0.0682 & 0.01531 & 1.410 & 1.887 & 3.36 & 40.00 & 1516. \\
\hline 1418 & 0.173 & 0.03203 & 312.870 & 0.0681 & 0.01529 & 1.060 & 2.568 & 3.36 & 40.00 & 2051. \\
\hline 1421 & 0.173 & 0.04180 & 313.252 & 0.0679 & 0.01527 & 0.940 & 3.351 & 3.36 & 40.00 & 2644. \\
\hline 1424 & 0.172 & 0.05284 & 313.685 & 0.0676 & 0.01528 & 0.750 & 4.229 & 3.36 & 40.00 & 3297. \\
\hline 1427 & 0.172 & 0.06516 & 314.175 & 0.0675 & 0.01529 & 0.580 & 5.204 & 3.36 & 40.00 & 4022. \\
\hline 1430 & 0.088 & 0.02356 & 312.429 & 0.0344 & 0.01518 & 1.500 & 1.909 & 1.76 & 40.00 & 372. \\
\hline 1433 & 0.089 & 0.03204 & 312.769 & 0.0347 & 0.01515 & 0.980 & 2.599 & 1.76 & 40.00 & 513. \\
\hline 1436 & 0.089 & 0.04181 & 313.156 & 0.0347 & 0.01515 & 0.670 & 3.391 & 1.76 & 40.00 & 668. \\
\hline 1439 & 0.089 & 0.05286 & 313.589 & 0.0347 & 0.01516 & 0.850 & 4.283 & 1.76 & 40.00 & 839. \\
\hline 1442 & 0.089 & 0.06517 & 314.086 & 0.0346 & 0.01518 & 0.510 & 5.272 & 1.76 & 40.00 & 1023. \\
\hline 1445 & 0.091 & 0.02298 & 320.464 & 0.0347 & 0.01589 & 1.620 & 1.778 & 1.76 & 40.00 & 326. \\
\hline 1448 & 0.091 & 0.03125 & 320.777 & 0.0346 & 0.01585 & 1.330 & 2.424 & 1.76 & 40.00 & 440. \\
\hline 1451 & 0.091 & 0.04078 & 321.142 & 0.0346 & 0.01584 & 0.930 & 3.164 & 1.76 & 40.00 & 570. \\
\hline 1454 & 0.092 & 0.05155 & 321.550 & 0.0349 & 0.01586 & 0.730 & 3.993 & 1.76 & 40.00 & 730. \\
\hline 1457 & 0.093 & 0.06359 & 321.991 & 0.0352 & 0.01589 & 0.520 & 4.915 & 1.76 & 40.00 & 912. \\
\hline 1460 & 0.182 & 0.02299 & 320.299 & 0.0700 & 0.01611 & 1.820 & 1.751 & 3.36 & 40.00 & 1368. \\
\hline 1463 & 0.182 & 0.03126 & 320.618 & 0.0698 & 0.01603 & 1.270 & 2.390 & 3.36 & 40.00 & 1854. \\
\hline 1466 & 0.182 & 0.04079 & 320.966 & 0.0699 & 0.01600 & 1.000 & 3.121 & 3.36 & 40.00 & 2416. \\
\hline 1469 & 0.182 & 0.05158 & 321.373 & 0.0696 & 0.01601 & 0.800 & 3.942 & 3.36 & 40.00 & 3014. \\
\hline 1472 & 0.182 & 0.06362 & 321.826 & 0.0697 & 0.01601 & 0.590 & 4.854 & 3.36 & 40.00 & 3698. \\
\hline 1475 & 0.268 & 0.02293 & 318.935 & 0.1047 & 0.01584 & 1.390 & 1.770 & 4.96 & 40.00 & 3288. \\
\hline 1478 & 0.268 & 0.03118 & 319.241 & 0.1047 & 0.01583 & 1.040 & 2.403 & 4.96 & 40.00 & 4449. \\
\hline 1481 & 0.268 & 0.04069 & 319.591 & 0.1045 & 0.01580 & 0.820 & 3.134 & 4.96 & 40.00 & 5765. \\
\hline 1484 & 0.268 & 0.05144 & 319.996 & 0.1043 & 0.01582 & 0.590 & 3.948 & 4.96 & 40.00 & 7196. \\
\hline 1487 & 0.267 & 0.06344 & 320.432 & 0.1039 & 0.01583 & 0.540 & 4.851 & 4.96 & 40.00 & 8730. \\
\hline 1490 & 0.345 & 0.02293 & 318.814 & 0.1366 & 0.01582 & 1.720 & 1.764 & 6.56 & 40.00 & 5837. \\
\hline 1493 & 0.345 & 0.03119 & 319.114 & 0.1364 & 0.01583 & 1.170 & 2.389 & 6.56 & 40.00 & 7853. \\
\hline
\end{tabular}


Table 24 . Thermal conductivity of the binary $70 \% \mathrm{R} 125 / 30 \% \mathrm{R} 134 \mathrm{a}$ mixture in the dilute gas measured with the steady-state technique (continued).

\begin{tabular}{|c|c|c|c|c|c|c|c|c|c|c|}
\hline $\begin{array}{l}\text { Run } \\
\text { point }\end{array}$ & $\begin{array}{l}P_{\text {exp }} \\
\mathrm{MPa}\end{array}$ & $\begin{array}{c}Q \\
\mathrm{~W} \cdot \mathrm{m}^{-1}\end{array}$ & $\begin{array}{c}T_{e x p} \\
\mathrm{~K}\end{array}$ & $\begin{array}{c}\rho_{\text {calc }} \\
\mathrm{mol} \cdot \mathrm{L}^{-1}\end{array}$ & $\begin{array}{c}\lambda_{\exp } \\
\mathrm{W} \cdot \mathrm{m}^{-1} \cdot \mathrm{K}^{-1}\end{array}$ & $\begin{array}{c}\text { TBAND } \\
\%\end{array}$ & $\begin{array}{c}\Delta T_{\text {avg }} \\
\mathrm{K}\end{array}$ & $\begin{array}{c}t_{\text {start }} \\
\mathrm{s}\end{array}$ & $\begin{array}{c}t_{\text {end }} \\
\mathrm{s}\end{array}$ & $N_{R a}$ \\
\hline 1496 & 0.345 & 0.04070 & 319.449 & 0.1360 & 0.01585 & 0.900 & 3.102 & 4.96 & 40.00 & 10102. \\
\hline 1499 & 0.345 & 0.05146 & 319.841 & 0.1359 & 0.01589 & 0.730 & 3.894 & 4.96 & 40.00 & 12597. \\
\hline 1502 & 0.346 & 0.06346 & 320.259 & 0.1360 & 0.01596 & 0.690 & 4.756 & 4.96 & 40.00 & 15340 . \\
\hline 1505 & 0.485 & 0.02294 & 318.644 & 0.1960 & 0.01599 & 1.510 & 1.724 & 6.56 & 40.00 & 12778. \\
\hline 1508 & 0.484 & 0.03120 & 318.953 & 0.1955 & 0.01595 & 1.080 & 2.333 & 6.56 & 40.00 & 17130. \\
\hline 1511 & 0.485 & 0.04073 & 319.281 & 0.1954 & 0.01601 & 1.090 & 3.008 & 4.96 & 40.00 & 21954. \\
\hline 1514 & 0.484 & 0.05150 & 319.636 & 0.1949 & 0.01607 & 1.220 & 3.754 & 3.36 & 40.00 & 27126 . \\
\hline 1517 & 0.484 & 0.06351 & 320.046 & 0.1946 & 0.01611 & 1.040 & 4.572 & 3.36 & 40.00 & 32766 . \\
\hline 1520 & 0.601 & 0.02294 & 318.385 & 0.2478 & 0.01616 & 1.900 & 1.680 & 6.56 & 40.00 & 21461. \\
\hline 1523 & 0.602 & 0.03121 & 318.672 & 0.2476 & 0.01618 & 1.400 & 2.253 & 4.96 & 40.00 & 28612 . \\
\hline 1526 & 0.601 & 0.04073 & 318.984 & 0.2471 & 0.01618 & 1.180 & 2.901 & 4.96 & 40.00 & 36509 . \\
\hline 1529 & 0.601 & 0.05149 & 319.348 & 0.2468 & 0.01614 & 0.960 & 3.622 & 3.36 & 40.00 & 45229 . \\
\hline 1532 & 0.601 & 0.06352 & 319.707 & 0.2463 & 0.01622 & 1.740 & 4.380 & 3.36 & 40.00 & 54193. \\
\hline 1535 & 0.619 & 0.02218 & 329.485 & 0.2443 & 0.01724 & 1.990 & 1.536 & 6.56 & 40.00 & 16486. \\
\hline 1538 & 0.617 & 0.03017 & 329.732 & 0.2434 & 0.01727 & 1.630 & 2.066 & 6.56 & 40.00 & 21913. \\
\hline 1541 & 0.618 & 0.03937 & 330.008 & 0.2434 & 0.01731 & 1.270 & 2.661 & 4.96 & 40.00 & 28130 . \\
\hline 1544 & 0.620 & 0.04980 & 330.338 & 0.2442 & 0.01726 & 0.970 & 3.332 & 4.96 & 40.00 & 35353. \\
\hline 1547 & 0.623 & 0.06143 & 330.697 & 0.2448 & 0.01719 & 0.930 & 4.072 & 3.36 & 40.00 & 43229. \\
\hline 1550 & 0.464 & 0.02221 & 329.077 & 0.1795 & 0.01693 & 1.730 & 1.588 & 8.16 & 40.00 & 8533. \\
\hline 1553 & 0.465 & 0.03020 & 329.333 & 0.1800 & 0.01693 & 1.010 & 2.148 & 8.16 & 40.00 & 11583. \\
\hline 1556 & 0.467 & 0.03942 & 329.637 & 0.1807 & 0.01696 & 0.850 & 2.781 & 6.56 & 40.00 & 15068. \\
\hline 1559 & 0.468 & 0.04986 & 329.986 & 0.1808 & 0.01699 & 0.880 & 3.489 & 4.96 & 40.00 & 18858. \\
\hline 1562 & 0.468 & 0.06150 & 330.360 & 0.1803 & 0.01704 & 0.770 & 4.261 & 4.96 & 40.00 & 22810 . \\
\hline 1565 & 0.324 & 0.02222 & 328.879 & 0.1231 & 0.01683 & 1.780 & 1.613 & 6.56 & 40.00 & 3813. \\
\hline 1568 & 0.326 & 0.03023 & 329.161 & 0.1238 & 0.01680 & 1.390 & 2.192 & 4.96 & 40.00 & 5231. \\
\hline 1571 & 0.326 & 0.03946 & 329.474 & 0.1237 & 0.01679 & 1.100 & 2.854 & 4.96 & 40.00 & 6778. \\
\hline 1574 & 0.324 & 0.04988 & 329.837 & 0.1230 & 0.01678 & 0.880 & 3.601 & 4.96 & 40.00 & 8419. \\
\hline 1577 & 0.322 & 0.06151 & 330.231 & 0.1221 & 0.01679 & 0.690 & 4.424 & 4.96 & 40.00 & 10130. \\
\hline 1580 & 0.211 & 0.02223 & 328.734 & 0.0792 & 0.01674 & 1.710 & 1.628 & 3.36 & 40.00 & 1511. \\
\hline 1583 & 0.211 & 0.03024 & 329.008 & 0.0790 & 0.01675 & 1.170 & 2.212 & 3.36 & 40.00 & 2038. \\
\hline 1586 & 0.210 & 0.03947 & 329.343 & 0.0787 & 0.01675 & 0.990 & 2.885 & 3.36 & 40.00 & 2628. \\
\hline 1589 & 0.208 & 0.04991 & 329.712 & 0.0778 & 0.01675 & 0.920 & 3.644 & 3.36 & 40.00 & 3224 . \\
\hline 1592 & 0.207 & 0.06156 & 330.113 & 0.0774 & 0.01676 & 0.590 & 4.486 & 3.36 & 40.00 & 3918. \\
\hline 1595 & 0.096 & 0.02221 & 326.679 & 0.0358 & 0.01634 & 1.510 & 1.671 & 1.76 & 40.00 & 307. \\
\hline 1598 & 0.096 & 0.03020 & 326.967 & 0.0356 & 0.01636 & 0.990 & 2.269 & 1.76 & 40.00 & 410. \\
\hline 1601 & 0.095 & 0.03941 & 327.293 & 0.0353 & 0.01638 & 0.810 & 2.957 & 1.76 & 40.00 & 525. \\
\hline 1604 & 0.094 & 0.04984 & 327.668 & 0.0350 & 0.01640 & 0.640 & 3.735 & 1.76 & 40.00 & 649. \\
\hline 1607 & 0.094 & 0.06147 & 328.089 & 0.0349 & 0.01642 & 0.560 & 4.597 & 1.76 & 40.00 & 791. \\
\hline 1611 & 0.097 & 0.02206 & 327.815 & 0.0361 & 0.01643 & 1.630 & 1.650 & 1.76 & 40.00 & 304. \\
\hline 1614 & 0.097 & 0.03000 & 328.094 & 0.0359 & 0.01644 & 1.100 & 2.243 & 1.76 & 40.00 & 409. \\
\hline 1617 & 0.097 & 0.03916 & 328.421 & 0.0358 & 0.01646 & 0.740 & 2.924 & 1.76 & 40.00 & 529. \\
\hline 1620 & 0.096 & 0.04952 & 328.800 & 0.0356 & 0.01647 & 0.680 & 3.693 & 1.76 & 40.00 & 657. \\
\hline 1623 & 0.096 & 0.06107 & 329.213 & 0.0355 & 0.01650 & 0.620 & 4.545 & 1.76 & 40.00 & 800. \\
\hline
\end{tabular}


Table 24. Thermal conductivity of the binary $70 \% \mathrm{R} 125 / 30 \% \mathrm{R} 134 \mathrm{a}$ mixture in the dilute gas measured with the steady-state technique (continued).

\begin{tabular}{|c|c|c|c|c|c|c|c|c|c|c|}
\hline $\begin{array}{l}\text { Run } \\
\text { point }\end{array}$ & $\begin{array}{l}P_{\text {exp }} \\
\mathrm{MPa}\end{array}$ & $\begin{array}{c}Q \\
\mathrm{~W} \cdot \mathrm{m}^{-1}\end{array}$ & $\begin{array}{c}T_{e x p} \\
\mathrm{~K}\end{array}$ & $\begin{array}{c}\rho_{\text {calc }} \\
\mathrm{mol} \cdot \mathrm{L}^{-1}\end{array}$ & $\begin{array}{c}\lambda_{\exp } \\
\mathrm{W} \cdot \mathrm{m}^{-1} \cdot \mathrm{K}^{-1}\end{array}$ & $\begin{array}{c}\text { TBAND } \\
\%\end{array}$ & $\begin{array}{c}\Delta T_{\text {avg }} \\
\mathrm{K}\end{array}$ & $\begin{array}{c}t_{\text {start }} \\
\mathrm{s}\end{array}$ & $\begin{array}{c}t_{\text {end }} \\
\mathrm{s}\end{array}$ & $N_{R a}$ \\
\hline 1626 & 0.104 & 0.02163 & 342.043 & 0.0368 & 0.01803 & 1.990 & 1.475 & 1.76 & 40.00 & 249. \\
\hline 1629 & 0.103 & 0.02942 & 342.27 .9 & 0.0367 & 0.01804 & 1.110 & 2.005 & 1.76 & 40.00 & 335. \\
\hline 1632 & 0.104 & 0.03839 & 342.559 & 0.0367 & 0.01801 & 0.870 & 2.620 & 1.76 & 40.00 & 438. \\
\hline 1635 & 0.104 & 0.04855 & 342.890 & 0.0367 & 0.01801 & 0.730 & 3.313 & 1.76 & 40.00 & 550. \\
\hline 1638 & 0.104 & 0.05989 & 343.242 & 0.0367 & 0.01802 & 0.700 & 4.082 & 1.76 & 40.00 & 675. \\
\hline 1641 & 0.101 & 0.03845 & 342.092 & 0.0358 & 0.01763 & 0.970 & 2.680 & 1.76 & 40.00 & 427. \\
\hline 1644 & 0.098 & 0.04863 & 342.424 & 0.0349 & 0.01768 & 0.850 & 3.380 & 1.76 & 40.00 & 508. \\
\hline 1647 & 0.100 & 0.05998 & 342.796 & 0.0353 & 0.01773 & 0.580 & 4.156 & 1.76 & 40.00 & 640. \\
\hline 1650 & 0.204 & 0.02166 & 341.233 & 0.0734 & 0.01815 & 2.220 & 1.465 & 3.36 & 40.00 & 1029. \\
\hline 1653 & 0.204 & 0.02947 & 341.467 & 0.0734 & 0.01811 & 1.330 & 1.996 & 3.36 & 40.00 & 1398. \\
\hline 1656 & 0.205 & 0.03846 & 341.750 & 0.0737 & 0.01810 & 1.000 & 2.605 & 3.36 & 40.00 & 1834. \\
\hline 1659 & 0.206 & 0.04864 & 342.069 & 0.0740 & 0.01807 & 0.920 & 3.296 & 3.36 & 40.00 & 2336 . \\
\hline 1662 & 0.207 & 0.06000 & 342.444 & 0.0742 & 0.01805 & 0.620 & 4.067 & 3.36 & 40.00 & 2883. \\
\hline 1665 & 0.204 & 0.04862 & 342.015 & 0.0734 & 0.01766 & 0.750 & 3.372 & 3.36 & 40.00 & 2351. \\
\hline 1668 & 0.204 & 0.05998 & 342.358 & 0.0732 & 0.01774 & 0.660 & 4.138 & 3.36 & 40.00 & 2852 . \\
\hline 1671 & 0.347 & 0.02162 & 340.628 & 0.1273 & 0.01811 & 2.060 & 1.460 & 4.96 & 40.00 & 3284. \\
\hline 1674 & 0.349 & 0.02942 & 340.875 & 0.1278 & 0.01805 & 1.330 & 1.988 & 6.56 & 40.00 & 4500 . \\
\hline 1677 & 0.349 & 0.03839 & 341.134 & 0.1279 & 0.01815 & 1.230 & 2.574 & 4.96 & 40.00 & 5816. \\
\hline 1680 & 0.349 & 0.04855 & 341.462 & 0.1276 & 0.01812 & 0.900 & 3.251 & 4.96 & 40.00 & 7289. \\
\hline 1683 & 0.348 & 0.05989 & 341.796 & 0.1272 & 0.01809 & 0.790 & 4.005 & 4.96 & 40.00 & 8885. \\
\hline 1686 & 0.348 & 0.04852 & 341.299 & 0.1273 & 0.01765 & 0.730 & 3.336 & 4.96 & 40.00 & 7457. \\
\hline 1689 & 0.348 & 0.05986 & 341.649 & 0.1270 & 0.01770 & 0.680 & 4.091 & 4.96 & 40.00 & 9062. \\
\hline 1692 & 0.483 & 0.02161 & 340.756 & 0.1799 & 0.01810 & 1.950 & 1.450 & 8.16 & 40.00 & 6884. \\
\hline 1695 & 0.482 & 0.02940 & 340.980 & 0.1794 & 0.01815 & 1.570 & 1.959 & 6.56 & 40.00 & 9223. \\
\hline 1698 & 0.482 & 0.03837 & 341.251 & 0.1791 & 0.01815 & 0.970 & 2.544 & 6.56 & 40.00 & 11903. \\
\hline 1701 & 0.484 & 0.04852 & 341.560 & 0.1795 & 0.01788 & 0.950 & 3.247 & 4.96 & 40.00 & 15217. \\
\hline 1704 & 0.483 & 0.05985 & 341.907 & 0.1791 & 0.01796 & 0.690 & 3.964 & 4.96 & 40.00 & 18427. \\
\hline 1707 & 0.599 & 0.02163 & 340.360 & 0.2262 & 0.01807 & 1.960 & 1.442 & 8.16 & 40.00 & 11434. \\
\hline 1710 & 0.598 & 0.02942 & 340.577 & 0.2257 & 0.01810 & 1.510 & 1.945 & 6.56 & 40.00 & 15308. \\
\hline 1713 & 0.597 & 0.03840 & 340.846 & 0.2252 & 0.01815 & 1.390 & 2.511 & 4.96 & 40.00 & 19620. \\
\hline 1716 & 0.596 & 0.04857 & 341.122 & 0.2246 & 0.01825 & 1.060 & 3.133 & 4.96 & 40.00 & 24259 . \\
\hline 1719 & 0.598 & 0.04858 & 341.087 & 0.2252 & 0.01798 & 0.970 & 3.178 & 4.96 & 40.00 & 24760 . \\
\hline 1722 & 0.598 & 0.05993 & 341.413 & 0.2252 & 0.01805 & 1.150 & 3.871 & 3.36 & 40.00 & 30045 . \\
\hline
\end{tabular}


Table 25 . Thermal conductivity of the binary $30 \% \mathrm{R} 32 / 70 \%$ propane mixture in the dilute gas measured with the steady-state technique.

\begin{tabular}{|c|c|c|c|c|c|c|c|c|c|c|}
\hline $\begin{array}{l}\text { Run } \\
\text { point }\end{array}$ & $\begin{array}{l}P_{\text {exp }} \\
\mathrm{MPa}\end{array}$ & $\begin{array}{c}Q \\
\mathrm{~W} \cdot \mathrm{m}^{-1}\end{array}$ & $\begin{array}{c}T_{\exp } \\
\mathrm{K}\end{array}$ & $\begin{array}{c}\rho_{\text {calc }} \\
\mathrm{mol} \cdot \mathrm{L}^{-1}\end{array}$ & $\begin{array}{c}\lambda_{\exp } \\
\mathrm{W} \cdot \mathrm{m}^{-1} \cdot \mathrm{K}^{-1}\end{array}$ & $\begin{array}{c}\text { TBAND } \\
\%\end{array}$ & $\begin{array}{c}\Delta T_{\text {avg }} \\
\mathrm{K}\end{array}$ & $\begin{array}{c}t_{\text {start }} \\
\mathrm{s}\end{array}$ & $\begin{array}{c}t_{\text {end }} \\
\mathrm{s}\end{array}$ & $N_{R a}$ \\
\hline 1008 & 0.069 & 0.02155 & 227.921 & 0.0375 & 0.01120 & 1.110 & 2.365 & 1.76 & 40.00 & 521. \\
\hline 1011 & 0.069 & 0.03361 & 228.581 & 0.0371 & 0.01118 & 0.620 & 3.692 & 1.78 & 4.00 & 787. \\
\hline 1012 & 0.069 & 0.03360 & 228.586 & 0.0370 & 0.01117 & 0.590 & 3.695 & 1.76 & 40.00 & 784. \\
\hline 1015 & 0.069 & 0.04827 & 229.376 & 0.0368 & 0.01120 & 0.470 & 5.288 & 1.78 & 4.00 & 1092. \\
\hline 1016 & 0.069 & 0.04826 & 229.386 & 0.0367 & 0.01119 & 0.520 & 5.294 & 1.76 & 40.00 & 1088. \\
\hline 1019 & 0.068 & 0.06550 & 230.298 & 0.0364 & 0.01126 & 0.480 & 7.139 & 1.62 & 4.00 & 1422. \\
\hline 1020 & 0.068 & 0.06549 & 230.311 & 0.0363 & 0.01124 & 0.390 & 7.150 & 1.76 & 40.00 & 1417. \\
\hline 1027 & 0.029 & 0.02155 & 227.873 & 0.0154 & 0.01111 & 1.100 & 2.385 & 0.66 & 4.00 & 83. \\
\hline 1028 & 0.029 & 0.02155 & 227.872 & 0.0153 & 0.01112 & 1.120 & 2.384 & 1.76 & 40.00 & 81. \\
\hline 1031 & 0.029 & 0.03360 & 228.544 & 0.0152 & 0.01109 & 0.780 & 3.727 & 0.66 & 4.00 & 124. \\
\hline 1032 & 0.028 & 0.03361 & 228.535 & 0.0151 & 0.01108 & 0.530 & 3.729 & 1.76 & 40.00 & 123. \\
\hline 1035 & 0.028 & 0.04827 & 229.337 & 0.0149 & 0.01111 & 0.630 & 5.340 & 0.66 & 4.00 & 169. \\
\hline 1036 & 0.028 & 0.04827 & 229.346 & 0.0149 & 0.01111 & 0.300 & 5.342 & 1.76 & 40.00 & 169. \\
\hline 1039 & 0.028 & 0.06550 & 230.281 & 0.0147 & 0.01117 & 0.440 & 7.212 & 0.66 & 4.00 & 221. \\
\hline 1040 & 0.028 & 0.06550 & 230.278 & 0.0148 & 0.01116 & 0.210 & 7.215 & 1.76 & 40.00 & 223. \\
\hline 1047 & 0.030 & 0.02054 & 240.001 & 0.0150 & 0.01215 & 0.850 & 2.078 & 0.66 & 4.00 & 58. \\
\hline 1048 & 0.030 & 0.02054 & 240.002 & 0.0149 & 0.01216 & 0.810 & 2.078 & 1.76 & 40.00 & 57. \\
\hline 1051 & 0.029 & 0.03203 & 240.582 & 0.0148 & 0.01212 & 0.560 & 3.252 & 0.66 & 4.00 & 88. \\
\hline 1052 & 0.029 & 0.03204 & 240.574 & 0.0148 & 0.01211 & 0.490 & 3.253 & 1.76 & 40.00 & 88. \\
\hline 1055 & 0.030 & 0.04603 & 241.274 & 0.0148 & 0.01213 & 0.480 & 4.668 & 0.66 & 4.00 & 125. \\
\hline 1056 & 0.029 & 0.04603 & 241.283 & 0.0148 & 0.01212 & 0.240 & 4.670 & 1.76 & 40.00 & 124. \\
\hline 1059 & 0.029 & 0.06249 & 242.096 & 0.0146 & 0.01217 & 0.410 & 6.314 & 0.66 & 4.00 & 162. \\
\hline 1060 & 0.029 & 0.06250 & 242.093 & 0.0146 & 0.01217 & 0.150 & 6.316 & 1.76 & 40.00 & 163. \\
\hline 1067 & 0.024 & 0.02010 & 245.933 & 0.0119 & 0.01284 & 1.500 & 1.924 & 0.66 & 4.00 & 31. \\
\hline 1068 & 0.024 & 0.02010 & 245.936 & 0.0119 & 0.01284 & 1.500 & 1.924 & 1.76 & 40.00 & 31 . \\
\hline 1071 & 0.024 & 0.03135 & 246.482 & 0.0119 & 0.01275 & 0.750 & 3.024 & 0.66 & 4.00 & 49. \\
\hline 1072 & 0.024 & 0.03135 & 246.483 & 0.0119 & 0.01276 & 0.760 & 3.022 & 1.76 & 40.00 & 48. \\
\hline 1075 & 0.024 & 0.04505 & 247.133 & 0.0118 & 0.01275 & 0.650 & 4.346 & 0.50 & 4.00 & 68. \\
\hline 1076 & 0.024 & 0.04505 & 247.138 & 0.0118 & 0.01275 & 0.490 & 4.347 & 1.76 & 40.00 & 68. \\
\hline 1079 & 0.024 & 0.06117 & 247.907 & 0.0118 & 0.01278 & 0.440 & 5.889 & 0.50 & 4.00 & 91. \\
\hline 1080 & 0.024 & 0.06117 & 247.895 & 0.0117 & 0.01277 & 0.290 & 5.890 & 1.76 & 40.00 & 90. \\
\hline 1087 & 0.058 & 0.02009 & 245.876 & 0.0288 & 0.01274 & 1.320 & 1.940 & 1.14 & 4.00 & 190. \\
\hline 1088 & 0.058 & 0.02009 & 245.872 & 0.0288 & 0.01273 & 1.540 & 1.941 & 1.76 & 40.00 & 190. \\
\hline 1091 & 0.058 & 0.03134 & 246.424 & 0.0286 & 0.01270 & 0.910 & 3.034 & 1.30 & 4.00 & 292. \\
\hline 1092 & 0.058 & 0.03134 & 246.412 & 0.0286 & 0.01269 & 1.010 & 3.036 & 1.76 & 40.00 & 291. \\
\hline 1095 & 0.058 & 0.04504 & 247.079 & 0.0284 & 0.01271 & 0.510 & 4.355 & 1.30 & 4.00 & 407. \\
\hline 1096 & 0.057 & 0.04505 & 247.064 & 0.0283 & 0.01271 & 0.420 & 4.356 & 1.76 & 40.00 & 405. \\
\hline 1099 & 0.057 & 0.06117 & 247.829 & 0.0282 & 0.01276 & 0.480 & 5.889 & 1.14 & 4.00 & 536. \\
\hline 1100 & 0.057 & 0.06117 & 247.839 & 0.0282 & 0.01275 & 0.280 & 5.895 & 1.76 & 40.00 & 537. \\
\hline 1107 & 0.050 & 0.01976 & 250.202 & 0.0243 & 0.01327 & 1.490 & 1.832 & 0.98 & 4.00 & 120. \\
\hline 1108 & 0.050 & 0.01976 & 250.206 & 0.0243 & 0.01327 & 1.520 & 1.831 & 1.76 & 40.00 & 120. \\
\hline 1111 & 0.051 & 0.03083 & 250.712 & 0.0247 & 0.01318 & 0.770 & 2.877 & 0.98 & 4.00 & 193. \\
\hline 1112 & 0.051 & 0.03083 & 250.722 & 0.0248 & 0.01317 & 0.710 & 2.879 & 1.76 & 40.00 & 194. \\
\hline
\end{tabular}


Table 25 . Thermal conductivity of the binary $30 \% \mathrm{R} 32 / 70 \%$ propane mixture in the dilute gas measured with the steady-state technique (continued).

\begin{tabular}{|c|c|c|c|c|c|c|c|c|c|c|}
\hline $\begin{array}{l}\text { Run } \\
\text { point }\end{array}$ & $\begin{array}{l}P_{\text {exp }} \\
\mathrm{MPa}\end{array}$ & $\begin{array}{c}Q \\
\mathrm{~W} \cdot \mathrm{m}^{-1}\end{array}$ & $\begin{array}{c}T_{\text {exp }} \\
\mathrm{K}\end{array}$ & $\begin{array}{c}\rho_{\text {calc }} \\
\mathrm{mol} \cdot \mathrm{L}^{-1}\end{array}$ & $\begin{array}{c}\lambda_{\exp } \\
\mathrm{W} \cdot \mathrm{m}^{-1} \cdot \mathrm{K}^{-1}\end{array}$ & $\begin{array}{c}\text { TBAND } \\
\%\end{array}$ & $\begin{array}{c}\Delta T_{\text {avg }} \\
\mathrm{K}\end{array}$ & $\begin{array}{c}t_{\text {start }} \\
\mathrm{s}\end{array}$ & $\begin{array}{c}t_{\text {end }} \\
\mathrm{s}\end{array}$ & $N_{R a}$ \\
\hline 1115 & 0.052 & 0.04432 & 251.351 & 0.0249 & 0.01316 & 0.530 & 4.141 & 1.14 & 4.00 & 281. \\
\hline 1116 & 0.052 & 0.04432 & 251.356 & 0.0250 & 0.01315 & 0.420 & 4.143 & 1.76 & 40.00 & 282. \\
\hline 1119 & 0.052 & 0.06020 & 252.082 & 0.0250 & 0.01318 & 0.490 & 5.613 & 1.14 & 4.00 & 379. \\
\hline 1120 & 0.052 & 0.06020 & 252.075 & 0.0250 & 0.01318 & 0.300 & 5.615 & 1.76 & 40.00 & 380 . \\
\hline 1127 & 0.118 & 0.01974 & 249.446 & 0.0586 & 0.01320 & 1.730 & 1.837 & 2.10 & 4.00 & 760. \\
\hline 1128 & 0.118 & 0.01974 & 249.446 & 0.0586 & 0.01318 & 1.620 & 1.840 & 1.76 & 40.00 & 761. \\
\hline 1131 & 0.118 & 0.03079 & 249.963 & 0.0584 & 0.01310 & 0.920 & 2.885 & 2.26 & 4.00 & 1178. \\
\hline 1132 & 0.118 & 0.03079 & 249.962 & 0.0584 & 0.01307 & 1.110 & 2.891 & 1.76 & 40.00 & 1180. \\
\hline 1135 & 0.118 & 0.04426 & 250.584 & 0.0581 & 0.01307 & 0.640 & 4.153 & 2.26 & 4.00 & 1660. \\
\hline 1136 & 0.118 & 0.04426 & 250.589 & 0.0581 & 0.01304 & 1.170 & 4.162 & 1.76 & 40.00 & 1663. \\
\hline 1139 & 0.118 & 0.06010 & 251.325 & 0.0579 & 0.01308 & 0.510 & 5.629 & 2.26 & 4.00 & 2212 . \\
\hline 1140 & 0.118 & 0.06010 & 251.334 & 0.0579 & 0.01304 & 0.300 & 5.644 & 3.36 & 40.00 & 2217. \\
\hline 1151 & 0.123 & 0.02955 & 261.112 & 0.0580 & 0.01424 & 1.060 & 2.549 & 2.10 & 4.00 & 882. \\
\hline 1152 & 0.123 & 0.02955 & 261.128 & 0.0580 & 0.01420 & 1.520 & 2.555 & 1.76 & 40.00 & 884. \\
\hline 1155 & 0.123 & 0.04249 & 261.676 & 0.0579 & 0.01416 & 0.600 & 3.681 & 2.26 & 4.00 & 1259. \\
\hline 1156 & 0.123 & 0.04249 & 261.660 & 0.0579 & 0.01414 & 0.920 & 3.687 & 1.76 & 40.00 & 1263. \\
\hline 1159 & 0.123 & 0.05772 & 262.327 & 0.0577 & 0.01416 & 0.610 & 4.998 & 2.10 & 4.00 & 1687. \\
\hline 1160 & 0.123 & 0.05772 & 262.329 & 0.0578 & 0.01413 & 0.820 & 5.010 & 1.76 & 40.00 & 1693. \\
\hline 1168 & 0.302 & 0.01895 & 260.505 & 0.1489 & 0.01453 & 2.060 & 1.591 & 6.56 & 40.00 & 4352. \\
\hline 1171 & 0.301 & 0.02957 & 260.940 & 0.1479 & 0.01466 & 0.890 & 2.452 & 3.22 & 4.00 & 6554. \\
\hline 1172 & 0.301 & 0.02957 & 260.949 & 0.1477 & 0.01437 & 1.060 & 2.500 & 4.96 & 40.00 & 6663. \\
\hline 1175 & 0.301 & 0.04252 & 261.460 & 0.1473 & 0.01458 & 0.840 & 3.528 & 3.06 & 4.00 & 9274. \\
\hline 1176 & 0.301 & 0.04251 & 261.500 & 0.1473 & 0.01428 & 0.800 & 3.598 & 4.96 & 40.00 & 9448. \\
\hline 1179 & 0.301 & 0.05777 & 262.087 & 0.1469 & 0.01449 & 0.580 & 4.793 & 3.22 & 4.00 & 12392. \\
\hline 1180 & 0.301 & 0.05776 & 262.120 & 0.1468 & 0.01425 & 0.510 & 4.873 & 4.96 & 40.00 & 12581. \\
\hline 1191 & 0.343 & 0.02761 & 280.762 & 0.1552 & 0.01710 & 1.500 & 1.971 & 3.06 & 4.00 & 4376. \\
\hline 1192 & 0.342 & 0.02761 & 280.784 & 0.1552 & 0.01676 & 1.590 & 2.010 & 4.96 & 40.00 & 4457. \\
\hline 1195 & 0.342 & 0.03972 & 281.195 & 0.1548 & 0.01693 & 0.880 & 2.852 & 3.06 & 4.00 & 6256. \\
\hline 1196 & 0.342 & 0.03971 & 281.242 & 0.1547 & 0.01661 & 1.160 & 2.907 & 4.96 & 40.00 & 6367. \\
\hline 1199 & 0.342 & 0.05398 & 281.694 & 0.1543 & 0.01683 & 0.940 & 3.885 & 3.06 & 4.00 & 8401. \\
\hline 1200 & 0.342 & 0.05397 & 281.737 & 0.1543 & 0.01652 & 0.790 & 3.957 & 4.96 & 40.00 & 8553. \\
\hline 1211 & 0.622 & 0.02762 & 280.492 & 0.2976 & 0.01764 & 1.400 & 1.861 & 3.22 & 4.00 & 18755. \\
\hline 1212 & 0.622 & 0.02762 & 280.516 & 0.2975 & 0.01687 & 2.160 & 1.944 & 6.56 & 40.00 & 19567. \\
\hline 1215 & 0.622 & 0.03973 & 280.879 & 0.2969 & 0.01726 & 1.040 & 2.698 & 3.22 & 4.00 & 26870. \\
\hline 1216 & 0.622 & 0.03973 & 280.921 & 0.2968 & 0.01687 & 1.330 & 2.758 & 4.96 & 40.00 & 27414. \\
\hline 1219 & 0.622 & 0.05402 & 281.364 & 0.2962 & 0.01699 & 0.970 & 3.668 & 3.06 & 4.00 & 36018. \\
\hline 1220 & 0.622 & 0.05401 & 281.372 & 0.2962 & 0.01689 & 1.680 & 3.687 & 3.36 & 40.00 & 36204. \\
\hline 1231 & 0.656 & 0.02669 & 290.940 & 0.2998 & 0.01927 & 1.880 & 1.660 & 3.22 & 4.00 & 14451. \\
\hline 1232 & 0.656 & 0.02669 & 290.991 & 0.2998 & 0.01849 & 2.080 & 1.727 & 6.56 & 40.00 & 15024. \\
\hline 1235 & 0.656 & 0.03840 & 291.294 & 0.2996 & 0.01883 & 1.470 & 2.415 & 3.06 & 4.00 & 20882. \\
\hline 1236 & 0.656 & 0.03839 & 291.319 & 0.2997 & 0.01835 & 1.680 & 2.476 & 4.96 & 40.00 & 21407. \\
\hline 1239 & 0.656 & 0.05220 & 291.732 & 0.2992 & 0.01845 & 0.930 & 3.308 & 3.22 & 4.00 & 28310. \\
\hline 1240 & 0.657 & 0.05220 & 291.729 & 0.2993 & 0.01834 & 1.710 & 3.325 & 3.36 & 40.00 & 28486. \\
\hline
\end{tabular}


Table 25. Thermal conductivity of the binary $30 \%$ R32 / $70 \%$ propane mixture in the dilute gas measured with the steady-state technique (continued).

\begin{tabular}{|c|c|c|c|c|c|c|c|c|c|c|}
\hline $\begin{array}{l}\text { Run } \\
\text { point }\end{array}$ & $\begin{array}{l}P_{\text {exp }} \\
\text { MPa }\end{array}$ & $\begin{array}{c}Q \\
\mathrm{~W} \cdot \mathrm{m}^{-1}\end{array}$ & $\begin{array}{c}T_{\exp } \\
\mathrm{K}\end{array}$ & $\begin{array}{c}\rho_{\text {calc }} \\
\mathrm{mol}^{\prime} \mathrm{L}^{-1}\end{array}$ & $\begin{array}{c}\lambda_{\exp } \\
\mathrm{W} \cdot \mathrm{m}^{-1} \cdot \mathrm{K}^{-1}\end{array}$ & $\begin{array}{c}\text { TBAND } \\
\%\end{array}$ & $\begin{array}{c}\Delta T_{a v g} \\
\mathrm{~K}\end{array}$ & $\begin{array}{c}t_{\text {start }} \\
\mathrm{s}\end{array}$ & $\begin{array}{c}t_{\text {end }} \\
\mathrm{s}\end{array}$ & $N_{R o}$ \\
\hline 1248 & 0.881 & 0.01711 & 290.445 & 0.4215 & 0.01898 & 2.670 & 1.066 & 9.76 & 40.00 & 21747 . \\
\hline 1251 & 0.880 & 0.02672 & 290.701 & 0.4201 & 0.01936 & 1.620 & 1.602 & 3.22 & 4.00 & 32243. \\
\hline 1252 & 0.879 & 0.02672 & 290.733 & 0.4197 & 0.01859 & 2.450 & 1.666 & 4.96 & 40.00 & 33419 . \\
\hline 1255 & 0.879 & 0.03844 & 291.048 & 0.4189 & 0.01871 & 1.060 & 2.329 & 3.06 & 4.00 & 46234 . \\
\hline 1256 & 0.879 & 0.03844 & 291.066 & 0.4188 & 0.01840 & 2.060 & 2.366 & 3.36 & 40.00 & 46941. \\
\hline 1259 & 0.879 & 0.05227 & 291.469 & 0.4179 & 0.01812 & 0.570 & 3.182 & 3.22 & 4.00 & 62346. \\
\hline 1260 & 0.879 & 0.05227 & 291.441 & 0.4180 & 0.01819 & 2.110 & 3.170 & 3.36 & 40.00 & 62206. \\
\hline 1271 & 0.346 & 0.02667 & 289.048 & 0.1514 & 0.01798 & 1.930 & 1.813 & 2.90 & 4.00 & 3423. \\
\hline 1272 & 0.346 & 0.02667 & 289.069 & 0.1513 & 0.01766 & 1.650 & 1.846 & 6.56 & 40.00 & 3480 . \\
\hline 1275 & 0.345 & 0.03837 & 289.435 & 0.1510 & 0.01784 & 1.210 & 2.622 & 2.90 & 4.00 & 4893. \\
\hline 1276 & 0.345 & 0.03837 & 289.469 & 0.1508 & 0.01749 & 1.060 & 2.673 & 4.96 & 40.00 & 4977. \\
\hline 1279 & 0.345 & 0.05215 & 289.916 & 0.1503 & 0.01765 & 0.820 & 3.591 & 3.06 & 4.00 & 6601. \\
\hline 1280 & 0.345 & 0.05215 & 289.943 & 0.1503 & 0.01736 & 0.780 & 3.650 & 4.96 & 40.00 & 6705. \\
\hline 1291 & 0.081 & 0.02666 & 288.834 & 0.0340 & 0.01759 & 1.800 & 1.864 & 1.14 & 4.00 & 154. \\
\hline 1292 & 0.080 & 0.02666 & 288.823 & 0.0339 & 0.01757 & 1.760 & 1.866 & 1.76 & 40.00 & 153. \\
\hline 1295 & 0.080 & 0.03835 & 289.248 & 0.0336 & 0.01734 & 0.890 & 2.720 & 1.30 & 4.00 & 217. \\
\hline 1296 & 0.079 & 0.03836 & 289.233 & 0.0334 & 0.01735 & 0.900 & 2.719 & 1.76 & 40.00 & 216 . \\
\hline 1299 & 0.079 & 0.05214 & 289.733 & 0.0332 & 0.01724 & 0.890 & 3.717 & 1.14 & 4.00 & 288 . \\
\hline 1300 & 0.079 & 0.05214 & 289.735 & 0.0331 & 0.01723 & 0.690 & 3.721 & 1.76 & 40.00 & 287. \\
\hline 1308 & 0.365 & 0.01706 & 287.970 & 0.1612 & 0.01791 & 3.090 & 1.166 & 4.96 & 40.00 & 2563. \\
\hline 1311 & 0.365 & 0.02663 & 288.289 & 0.1611 & 0.01793 & 2.010 & 1.814 & 3.06 & 4.00 & 3959 . \\
\hline 1312 & 0.365 & 0.02663 & 288.300 & 0.1606 & 0.01748 & 1.930 & 1.861 & 8.16 & 40.00 & 4035 . \\
\hline 1315 & 0.356 & 0.03836 & 288.657 & 0.1564 & 0.01788 & 1.130 & 2.613 & 3.06 & 4.00 & 5320 . \\
\hline 1316 & 0.353 & 0.03836 & 288.688 & 0.1550 & 0.01755 & 1.940 & 2.662 & 3.36 & 40.00 & 5312 . \\
\hline 1319 & 0.349 & 0.05215 & 289.136 & 0.1528 & 0.01770 & 0.860 & 3.578 & 3.06 & 4.00 & 6878. \\
\hline 1320 & 0.349 & 0.05215 & 289.169 & 0.1527 & 0.01738 & 0.690 & 3.643 & 4.96 & 40.00 & 6999. \\
\hline 1327 & 0.150 & 0.01689 & 269.531 & 0.0689 & 0.01560 & 2.620 & 1.330 & 1.94 & 4.00 & 595. \\
\hline 1328 & 0.150 & 0.01689 & 269.542 & 0.0689 & 0.01555 & 3.080 & 1.334 & 3.36 & 40.00 & 596. \\
\hline 1331 & 0.150 & 0.02636 & 269.903 & 0.0688 & 0.01541 & 1.410 & 2.101 & 2.10 & 4.00 & 931. \\
\hline 1332 & 0.150 & 0.02636 & 269.926 & 0.0688 & 0.01535 & 1.510 & 2.109 & 3.36 & 40.00 & 935. \\
\hline 1335 & 0.150 & 0.03791 & 270.377 & 0.0688 & 0.01530 & 0.980 & 3.041 & 2.26 & 4.00 & 1338. \\
\hline 1336 & 0.151 & 0.03791 & 270.372 & 0.0688 & 0.01525 & 0.900 & 3.051 & 3.36 & 40.00 & 1346. \\
\hline 1339 & 0.151 & 0.05154 & 270.915 & 0.0687 & 0.01525 & 0.670 & 4.142 & 2.42 & 4.00 & 1808. \\
\hline 1340 & 0.151 & 0.05154 & 270.918 & 0.0688 & 0.01520 & 0.550 & 4.156 & 3.36 & 40.00 & 1818. \\
\hline 1348 & 0.373 & 0.01688 & 269.364 & 0.1790 & 0.01556 & 3.120 & 1.323 & 6.56 & 40.00 & 4813. \\
\hline 1351 & 0.373 & 0.02636 & 269.719 & 0.1787 & 0.01573 & 1.160 & 2.034 & 3.22 & 4.00 & 7326. \\
\hline 1352 & 0.373 & 0.02636 & 269.740 & 0.1785 & 0.01530 & 1.540 & 2.090 & 6.56 & 40.00 & 7506. \\
\hline 1355 & 0.373 & 0.03792 & 270.164 & 0.1781 & 0.01556 & 0.920 & 2.940 & 3.22 & 4.00 & 10443. \\
\hline 1356 & 0.373 & 0.03791 & 270.208 & 0.1781 & 0.01517 & 0.980 & 3.016 & 6.56 & 40.00 & 10700. \\
\hline 1359 & 0.373 & 0.05155 & 270.695 & 0.1776 & 0.01543 & 0.850 & 4.005 & 3.22 & 4.00 & 14025 . \\
\hline 1360 & 0.373 & 0.05154 & 270.724 & 0.1776 & 0.01512 & 0.810 & 4.086 & 4.96 & 40.00 & 14292. \\
\hline 1368 & 0.497 & 0.01689 & 269.241 & 0.2451 & 0.01574 & 2.710 & 1.296 & 8.16 & 40.00 & 9984. \\
\hline 1371 & 0.497 & 0.02637 & 269.562 & 0.2446 & 0.01614 & 1.030 & 1.956 & 3.22 & 4.00 & 14912. \\
\hline
\end{tabular}


Table 25 . Thermal conductivity of the binary $30 \%$ R $32 / 70 \%$ propane mixture in the dilute gas measured with the steady-state technique (continued).

\begin{tabular}{|c|c|c|c|c|c|c|c|c|c|c|}
\hline $\begin{array}{l}\text { Run } \\
\text { point }\end{array}$ & $\begin{array}{l}P_{\text {exp }} \\
\mathrm{MPa}\end{array}$ & $\begin{array}{c}Q \\
\mathrm{~W} \cdot \mathrm{m}^{-1}\end{array}$ & $\begin{array}{c}T_{\text {exp }} \\
\mathrm{K}\end{array}$ & $\begin{array}{c}\rho_{\text {calc }} \\
\mathrm{mol} \cdot \mathrm{L}^{-1}\end{array}$ & $\begin{array}{c}\lambda_{\exp } \\
\mathrm{W} \cdot \mathrm{m}^{-1} \cdot \mathrm{K}^{-1}\end{array}$ & $\begin{array}{c}\text { TBAND } \\
\%\end{array}$ & $\begin{array}{c}\Delta T_{\text {avg }} \\
\mathrm{K}\end{array}$ & $\begin{array}{c}t_{\text {start }} \\
\mathrm{s}\end{array}$ & $\begin{array}{c}t_{\text {end }} \\
\mathrm{s}\end{array}$ & $N_{R a}$ \\
\hline 1372 & 0.497 & 0.02636 & 269.607 & 0.2446 & 0.01555 & 1.400 & 2.028 & 6.56 & 40.00 & 15440 . \\
\hline 1375 & 0.496 & 0.03793 & 270.011 & 0.2439 & 0.01588 & 0.830 & 2.827 & 3.22 & 4.00 & 21237. \\
\hline 1376 & 0.496 & 0.03793 & 270.030 & 0.2439 & 0.01555 & 1.250 & 2.884 & 4.96 & 40.00 & 21659 . \\
\hline 1379 & 0.496 & 0.05157 & 270.502 & 0.2432 & 0.01570 & 0.810 & 3.839 & 3.06 & 4.00 & 28392. \\
\hline 1380 & 0.496 & 0.05157 & 270.507 & 0.2433 & 0.01558 & 1.300 & 3.867 & 3.36 & 40.00 & 28619 . \\
\hline 1391 & 0.759 & 0.02395 & 301.608 & 0.3358 & 0.02029 & 2.430 & 1.415 & 3.22 & 4.00 & 13823. \\
\hline 1392 & 0.759 & 0.02394 & 301.643 & 0.3357 & 0.01937 & 2.450 & 1.481 & 9.76 & 40.00 & 14448. \\
\hline 1395 & 0.759 & 0.03446 & 301.940 & 0.3353 & 0.01987 & 1.420 & 2.057 & 3.22 & 4.00 & 19931. \\
\hline 1396 & 0.759 & 0.03445 & 301.960 & 0.3352 & 0.01916 & 1.610 & 2.130 & 6.56 & 40.00 & 20625 . \\
\hline 1399 & 0.759 & 0.04687 & 302.299 & 0.3347 & 0.01951 & 1.060 & 2.814 & 3.22 & 4.00 & 27021 . \\
\hline 1400 & 0.759 & 0.04686 & 302.329 & 0.3348 & 0.01912 & 1.480 & 2.869 & 4.96 & 40.00 & 27534 . \\
\hline 1411 & 0.320 & 0.02395 & 301.626 & 0.1327 & 0.01916 & 1.650 & 1.532 & 2.74 & 4.00 & 1870. \\
\hline 1412 & 0.320 & 0.02395 & 301.626 & 0.1327 & 0.01894 & 2.460 & 1.550 & 3.36 & 40.00 & 1891. \\
\hline 1415 & 0.320 & 0.03446 & 301.976 & 0.1326 & 0.01890 & 1.400 & 2.232 & 3.06 & 4.00 & 2708 . \\
\hline 1416 & 0.320 & 0.03446 & 301.996 & 0.1326 & 0.01873 & 1.050 & 2.252 & 4.96 & 40.00 & 2730 . \\
\hline 1419 & 0.320 & 0.04686 & 302.386 & 0.1323 & 0.01883 & 0.920 & 3.041 & 2.90 & 4.00 & 3655 . \\
\hline 1420 & 0.320 & 0.04686 & 302.398 & 0.1323 & 0.01860 & 0.880 & 3.077 & 4.96 & 40.00 & 3699. \\
\hline 1432 & 1.005 & 0.02396 & 301.187 & 0.4642 & 0.01974 & 3.300 & 1.413 & 4.96 & 40.00 & 30774 . \\
\hline 1435 & 1.005 & 0.03448 & 301.476 & 0.4635 & 0.01983 & 1.940 & 1.982 & 3.06 & 4.00 & 42809 . \\
\hline 1436 & 1.005 & 0.03448 & 301.505 & 0.4634 & 0.01949 & 2.550 & 2.015 & 4.96 & 40.00 & 43476. \\
\hline 1439 & 1.005 & 0.04689 & 301.857 & 0.4625 & 0.01918 & 1.320 & 2.716 & 3.22 & 4.00 & 58005. \\
\hline 1452 & 1.040 & 0.02407 & 309.986 & 0.4621 & 0.02132 & 3.670 & 1.328 & 4.96 & 40.00 & 24938. \\
\hline 1455 & 1.041 & 0.03464 & 310.257 & 0.4616 & 0.02148 & 1.820 & 1.864 & 3.06 & 4.00 & 34782 . \\
\hline 1456 & 1.041 & 0.03464 & 310.255 & 0.4616 & 0.02101 & 2.700 & 1.904 & 4.96 & 40.00 & 35522 . \\
\hline 1459 & 1.041 & 0.04712 & 310.577 & 0.4609 & 0.02086 & 1.420 & 2.556 & 3.06 & 4.00 & 47280 . \\
\hline 1460 & 1.041 & 0.04712 & 310.585 & 0.4609 & 0.02081 & 2.250 & 2.562 & 3.36 & 40.00 & 47384 \\
\hline 1468 & 0.661 & 0.01542 & 309.659 & 0.2782 & 0.02103 & 5.550 & 0.894 & 9.76 & 40.00 & 5060. \\
\hline 1471 & 0.661 & 0.02408 & 309.895 & 0.2779 & 0.02129 & 2.450 & 1.372 & 3.06 & 4.00 & 7726 \\
\hline 1472 & 0.661 & 0.02407 & 309.917 & 0.2779 & 0.02046 & 3.140 & 1.427 & 8.16 & 40.00 & 8033. \\
\hline 1475 & 0.661 & 0.03465 & 310.202 & 0.2776 & 0.02089 & 1.530 & 1.999 & 3.22 & 4.00 & 11183. \\
\hline 1476 & 0.661 & 0.03465 & 310.233 & 0.2775 & 0.02015 & 2.080 & 2.071 & 6.56 & 40.00 & 11576. \\
\hline 1479 & 0.661 & 0.04712 & 310.563 & 0.2771 & 0.02063 & 1.070 & 2.734 & 3.22 & 4.00 & 15165. \\
\hline 1480 & 0.661 & 0.04712 & 310.586 & 0.2770 & 0.02001 & 1.110 & 2.815 & 6.56 & 40.00 & 15605. \\
\hline 1493 & 0.348 & 0.02409 & 309.832 & 0.1405 & 0.02052 & 2.470 & 1.439 & 2.74 & 4.00 & 1808. \\
\hline 1494 & 0.348 & 0.02409 & 309.840 & 0.1405 & 0.02026 & 2.480 & 1.457 & 4.96 & 40.00 & 1831. \\
\hline 1495 & 0.347 & 0.02409 & 309.816 & 0.1405 & 0.02070 & 1.900 & 1.427 & 2.41 & 3.00 & 1792. \\
\hline 1498 & 0.347 & 0.03466 & 310.159 & 0.1403 & 0.02021 & 1.550 & 2.100 & 2.74 & 4.00 & 2620 . \\
\hline 1499 & 0.347 & 0.03467 & 310.154 & 0.1403 & 0.01996 & 1.370 & 2.126 & 4.96 & 40.00 & 2652 . \\
\hline 1500 & 0.347 & 0.03466 & 310.153 & 0.1402 & 0.02040 & 1.400 & 2.080 & 2.41 & 3.00 & 2595. \\
\hline 1503 & 0.347 & 0.04714 & 310.536 & 0.1401 & 0.02002 & 1.200 & 2.877 & 2.74 & 4.00 & 3564. \\
\hline 1504 & 0.348 & 0.04714 & 310.548 & 0.1402 & 0.01978 & 1.050 & 2.913 & 4.96 & 40.00 & 3612 . \\
\hline 1505 & 0.348 & 0.04714 & 310.526 & 0.1402 & 0.02025 & 1.060 & 2.845 & 2.29 & 3.00 & 3532 . \\
\hline 1516 & 1.061 & 0.02435 & 306.077 & 0.4822 & 0.02125 & 2.680 & 1.336 & 3.06 & 4.00 & 29646 . \\
\hline
\end{tabular}


Table 25. Thermal conductivity of the binary $30 \% \mathrm{R} 32 / 70 \%$ propane mixture in the dilute gas measured with the steady-state technique (continued).

\begin{tabular}{|c|c|c|c|c|c|c|c|c|c|c|}
\hline $\begin{array}{l}\text { Run } \\
\text { point }\end{array}$ & $\begin{array}{l}P_{\text {exp }} \\
\mathrm{MPa}\end{array}$ & $\begin{array}{c}Q \\
W \cdot \mathrm{m}^{-1}\end{array}$ & $\begin{array}{c}T_{\text {exp }} \\
\mathrm{K}\end{array}$ & $\begin{array}{c}\rho_{\text {calc }} \\
\mathrm{mol} \cdot \mathrm{L}^{-1}\end{array}$ & $\begin{array}{c}\lambda_{\exp } \\
\mathrm{W} \cdot \mathrm{m}^{-1} \cdot \mathrm{K}^{-1}\end{array}$ & $\begin{array}{c}\text { TBAND } \\
\%\end{array}$ & $\begin{array}{c}\Delta T_{\text {avg }} \\
\mathrm{K}\end{array}$ & $\begin{array}{c}t_{\text {start }} \\
\mathrm{s}\end{array}$ & $\begin{array}{c}t_{\text {end }} \\
\mathrm{s}\end{array}$ & $N_{R a}$ \\
\hline 1517 & 1.060 & 0.02435 & 306.108 & 0.4820 & 0.02049 & 3.340 & 1.384 & 4.96 & 40.00 & 30657. \\
\hline 1520 & 1.060 & 0.03504 & 306.380 & 0.4811 & 0.02055 & 1.720 & 1.945 & 3.22 & 4.00 & 42708. \\
\hline 1521 & 1.060 & 0.03503 & 306.398 & 0.4811 & 0.02025 & 2.630 & 1.971 & 3.36 & 40.00 & 43267. \\
\hline 1524 & 1.060 & 0.04764 & 306.747 & 0.4802 & 0.01995 & 1.400 & 2.655 & 3.06 & 4.00 & 57677. \\
\hline 1525 & 1.060 & 0.04765 & 306.729 & 0.4802 & 0.02018 & 3.510 & 2.628 & 1.76 & 40.00 & 57131. \\
\hline 1537 & 1.122 & 0.02433 & 320.329 & 0.4795 & 0.02311 & 3.780 & 1.244 & 6.56 & 40.00 & 22067 . \\
\hline 1540 & 1.121 & 0.03501 & 320.555 & 0.4787 & 0.02339 & 2.040 & 1.742 & 3.06 & 4.00 & 30678. \\
\hline 1541 & 1.121 & 0.03501 & 320.595 & 0.4786 & 0.02274 & 2.790 & 1.790 & 4.96 & 40.00 & 31486. \\
\hline 1544 & 1.121 & 0.04762 & 320.897 & 0.4780 & 0.02267 & 1.110 & 2.399 & 3.22 & 4.00 & 41895 \\
\hline 1545 & 1.121 & 0.04762 & 320.899 & 0.4780 & 0.02249 & 2.600 & 2.417 & 3.36 & 40.00 & 42205 \\
\hline 1556 & 0.575 & 0.02432 & 320.178 & 0.2296 & 0.02303 & 2.840 & 1.289 & 2.74 & 4.00 & 4186 \\
\hline 1557 & 0.575 & 0.02432 & 320.208 & 0.2297 & 0.02233 & 2.570 & 1.329 & 6.56 & 40.00 & 4318. \\
\hline 1560 & 0.575 & 0.03499 & 320.488 & 0.2295 & 0.02245 & 1.440 & 1.896 & 3.22 & 4.00 & 6132. \\
\hline 1561 & 0.575 & 0.03499 & 320.508 & 0.2295 & 0.02182 & 1.230 & 1.950 & 6.56 & 40.00 & 6306. \\
\hline 1564 & 0.576 & 0.04759 & 320.809 & 0.2294 & 0.02211 & 0.930 & 2.607 & 3.22 & 4.00 & 8396. \\
\hline 1565 & 0.576 & 0.04759 & 320.835 & 0.2295 & 0.02150 & 0.750 & 2.680 & 6.56 & 40.00 & 8635. \\
\hline 1576 & 0.220 & 0.02429 & 318.615 & 0.0851 & 0.02135 & 2.550 & 1.398 & 1.94 & 4.00 & 557. \\
\hline 1577 & 0.220 & 0.02429 & 318.612 & 0.0850 & 0.02131 & 2.290 & 1.401 & 3.36 & 40.00 & 558. \\
\hline 1580 & 0.220 & 0.03495 & 318.905 & 0.0849 & 0.02109 & 1.630 & 2.036 & 2.10 & 4.00 & 806. \\
\hline 1581 & 0.220 & 0.03495 & 318.931 & 0.0849 & 0.02102 & 1.530 & 2.042 & 3.36 & 40.00 & 807. \\
\hline 1584 & 0.220 & 0.04753 & 319.279 & 0.0848 & 0.02090 & 1.060 & 2.791 & 2.26 & 4.00 & 1098. \\
\hline 1585 & 0.220 & 0.04753 & 319.298 & 0.0848 & 0.02084 & 0.970 & 2.799 & 3.36 & 40.00 & 1101. \\
\hline 1597 & 1.111 & 0.02368 & 330.935 & 0.4519 & 0.02469 & 3.590 & 1.147 & 9.76 & 40.00 & 15345. \\
\hline 1600 & 1.111 & 0.03408 & 331.153 & 0.4514 & 0.02519 & 2.620 & 1.602 & 3.06 & 4.00 & 21312. \\
\hline 1601 & 1.111 & 0.03408 & 331.180 & 0.4514 & 0.02423 & 3.190 & 1.663 & 4.96 & 40.00 & 22111. \\
\hline 1604 & 1.111 & 0.04636 & 331.427 & 0.4509 & 0.02452 & 1.720 & 2.207 & 3.06 & 4.00 & 29188. \\
\hline 1605 & 1.111 & 0.04636 & 331.461 & 0.4508 & 0.02396 & 2.200 & 2.256 & 4.96 & 40.00 & 29814. \\
\hline 1616 & 0.601 & 0.02369 & 330.682 & 0.2316 & 0.02398 & 2.480 & 1.207 & 2.90 & 4.00 & 3560 . \\
\hline 1617 & 0.601 & 0.02369 & 330.687 & 0.2316 & 0.02336 & 2.240 & 1.240 & 6.56 & 40.00 & 3656. \\
\hline 1620 & 0.601 & 0.03410 & 330.938 & 0.2314 & 0.02359 & 1.670 & 1.762 & 3.06 & 4.00 & 5172. \\
\hline 1621 & 0.601 & 0.03410 & 330.952 & 0.2314 & 0.02300 & 1.310 & 1.806 & 6.56 & 40.00 & 5299. \\
\hline 1624 & 0.601 & 0.04638 & 331.248 & 0.2311 & 0.02332 & 1.050 & 2.414 & 3.22 & 4.00 & 7045 . \\
\hline 1625 & 0.601 & 0.04637 & 331.281 & 0.2311 & 0.02274 & 0.890 & 2.476 & 6.56 & 40.00 & 7221. \\
\hline 1636 & 0.117 & 0.02366 & 328.466 & 0.0432 & 0.02271 & 3.420 & 1.281 & 1.14 & 4.00 & 116. \\
\hline 1637 & 0.117 & 0.02366 & 328.447 & 0.0432 & 0.02282 & 3.310 & 1.275 & 1.76 & 40.00 & 115. \\
\hline 1640 & 0.117 & 0.03405 & 328.743 & 0.0432 & 0.02233 & 2.060 & 1.875 & 1.30 & 4.00 & 169. \\
\hline 1641 & 0.117 & 0.03405 & 328.736 & 0.0432 & 0.02239 & 1.960 & 1.870 & 1.76 & 40.00 & 168. \\
\hline 1644 & 0.117 & 0.04631 & 329.080 & 0.0431 & 0.02213 & 1.050 & 2.573 & 1.30 & 4.00 & 231. \\
\hline 1645 & 0.117 & 0.04631 & 329.093 & 0.0431 & 0.02216 & 1.460 & 2.570 & 1.76 & 40.00 & 230. \\
\hline 1660 & 1.087 & 0.03408 & 340.293 & 0.4240 & 0.02812 & 2.430 & 1.451 & 3.22 & 4.00 & 14940. \\
\hline 1661 & 1.087 & 0.03408 & 340.321 & 0.4240 & 0.02684 & 2.780 & 1.518 & 6.56 & 40.00 & 15619. \\
\hline 1664 & 1.086 & 0.04636 & 340.561 & 0.4235 & 0.02712 & 1.380 & 2.025 & 3.22 & 4.00 & 20725 . \\
\hline 1665 & 1.086 & 0.04636 & 340.544 & 0.4235 & 0.02632 & 2.190 & 2.085 & 4.96 & 40.00 & 21342. \\
\hline
\end{tabular}


Table 25 . Thermal conductivity of the binary $30 \% \mathrm{R} 32$ / $70 \%$ propane mixture in the dilute gas measured with the steady-state technique (continued).

\begin{tabular}{|c|c|c|c|c|c|c|c|c|c|c|}
\hline $\begin{array}{l}\text { Run } \\
\text { point }\end{array}$ & $\begin{array}{l}P_{\text {exp }} \\
\mathrm{MPa}\end{array}$ & $\begin{array}{c}Q \\
\mathrm{~W} \cdot \mathrm{m}^{-1}\end{array}$ & $\begin{array}{c}T_{\exp } \\
\mathrm{K}\end{array}$ & $\begin{array}{c}\rho_{\text {calc }} \\
\mathrm{mol}^{-1} \mathrm{~L}^{-1}\end{array}$ & $\begin{array}{c}\lambda_{\exp } \\
\mathrm{W} \cdot \mathrm{m}^{-1} \cdot \mathrm{K}^{-1}\end{array}$ & $\begin{array}{c}\text { TBAND } \\
\%\end{array}$ & $\begin{array}{c}\Delta T_{\text {avg }} \\
\mathrm{K}\end{array}$ & $\begin{array}{c}t_{\text {start }} \\
\mathrm{s}\end{array}$ & $\begin{array}{c}t_{\text {end }} \\
\mathrm{s}\end{array}$ & $N_{R a}$ \\
\hline 1693 & 0.593 & 0.03406 & 339.617 & 0.2213 & 0.02573 & 2.140 & 1.617 & 3.06 & 4.00 & 3933. \\
\hline 1694 & 0.594 & 0.03405 & 339.649 & 0.2213 & 0.02518 & 2.820 & 1.651 & 4.96 & 40.00 & 4018. \\
\hline 1697 & 0.594 & 0.04632 & 339.906 & 0.2212 & 0.02527 & 1.440 & 2.232 & 3.06 & 4.00 & 5410. \\
\hline 1698 & 0.594 & 0.04632 & 339.917 & 0.2212 & 0.02478 & 1.730 & 2.276 & 4.96 & 40.00 & 5516. \\
\hline 1709 & 0.117 & 0.02369 & 338.997 & 0.0420 & 0.02512 & 2.850 & 1.160 & 1.14 & 4.00 & 89. \\
\hline 1710 & 0.117 & 0.02369 & 338.981 & 0.0420 & 0.02529 & 3.060 & 1.152 & 1.76 & 40.00 & 89. \\
\hline 1713 & 0.117 & 0.03410 & 339.249 & 0.0420 & 0.02454 & 1.810 & 1.709 & 0.98 & 4.00 & 131. \\
\hline 1714 & 0.117 & 0.03410 & 339.223 & 0.0420 & 0.02460 & 1.760 & 1.705 & 1.76 & 40.00 & 131. \\
\hline 1717 & 0.117 & 0.04638 & 339.554 & 0.0420 & 0.02411 & 1.110 & 2.365 & 1.14 & 4.00 & 182. \\
\hline 1718 & 0.117 & 0.04638 & 339.544 & 0.0420 & 0.02417 & 1.140 & 2.360 & 1.76 & 40.00 & 181. \\
\hline
\end{tabular}


Table 26. Thermal conductivity of the binary $70 \% \mathrm{R} 32$ / $30 \%$ propane mixture in the dilute gas measured with the steady-state technique.

\begin{tabular}{|c|c|c|c|c|c|c|c|c|c|c|}
\hline $\begin{array}{l}\text { Run } \\
\text { point }\end{array}$ & $\begin{array}{l}P_{\text {exp }} \\
\mathrm{MPa}\end{array}$ & $\begin{array}{c}Q \\
\mathrm{~W} \cdot \mathrm{m}^{-1}\end{array}$ & $\begin{array}{c}T_{\exp } \\
\mathrm{K}\end{array}$ & $\begin{array}{c}\rho_{\text {calc }} \\
\mathrm{mol} \cdot \mathrm{L}^{-1}\end{array}$ & $\begin{array}{c}\lambda_{\exp } \\
\mathrm{W} \cdot \mathrm{m}^{-1} \cdot \mathrm{K}^{-1}\end{array}$ & $\begin{array}{c}\text { TBAND } \\
\%\end{array}$ & $\begin{array}{c}\Delta T_{\text {avg }} \\
\mathrm{K}\end{array}$ & $\begin{array}{c}t_{\text {start }} \\
\mathrm{s}\end{array}$ & $\begin{array}{c}t_{\text {end }} \\
\mathrm{s}\end{array}$ & $N_{R o}$ \\
\hline 1003 & 0.503 & 0.02641 & 256.675 & 0.2635 & 0.01286 & 1.490 & 2.415 & 4.96 & 40.00 & 24787 . \\
\hline 1006 & 0.503 & 0.03592 & 257.089 & 0.2627 & 0.01288 & 1.720 & 3.236 & 3.36 & 40.00 & 32614 . \\
\hline 1009 & 0.502 & 0.04685 & 257.543 & 0.2615 & 0.01288 & 1.380 & 4.162 & 3.36 & 40.00 & 41011 . \\
\hline 1012 & 0.501 & 0.05920 & 258.051 & 0.2600 & 0.01292 & 1.970 & 5.165 & 1.76 & 40.00 & 49488. \\
\hline 1015 & 0.500 & 0.07299 & 258.608 & 0.2588 & 0.01289 & 1.640 & 6.283 & 1.76 & 40.00 & 58738. \\
\hline 1018 & 0.416 & 0.02641 & 256.723 & 0.2129 & 0.01266 & 0.980 & 2.499 & 6.56 & 40.00 & 14746 . \\
\hline 1021 & 0.415 & 0.03591 & 257.156 & 0.2121 & 0.01263 & 0.820 & 3.375 & 4.96 & 40.00 & 19553. \\
\hline 1024 & 0.413 & 0.04684 & 257.637 & 0.2105 & 0.01272 & 1.130 & 4.336 & 3.36 & 40.00 & 24419 . \\
\hline 1027 & 0.412 & 0.05919 & 258.160 & 0.2093 & 0.01280 & 1.340 & 5.394 & 3.36 & 40.00 & 29631. \\
\hline 1030 & 0.413 & 0.07298 & 258.751 & 0.2093 & 0.01284 & 2.100 & 6.563 & 1.76 & 40.00 & 35608 . \\
\hline 1033 & 0.310 & 0.02641 & 256.732 & 0.1549 & 0.01263 & 1.070 & 2.540 & 6.56 & 40.00 & 6930. \\
\hline 1036 & 0.309 & 0.03591 & 257.203 & 0.1541 & 0.01254 & 0.980 & 3.462 & 4.96 & 40.00 & 9265. \\
\hline 1039 & 0.311 & 0.04682 & 257.725 & 0.1548 & 0.01251 & 0.650 & 4.505 & 4.96 & 40.00 & 12063. \\
\hline 1042 & 0.312 & 0.05917 & 258.292 & 0.1550 & 0.01251 & 0.430 & 5.661 & 4.96 & 40.00 & 15054 . \\
\hline 1045 & 0.313 & 0.07294 & 258.933 & 0.1546 & 0.01255 & 0.710 & 6.916 & 3.36 & 40.00 & 18098. \\
\hline 1051 & 0.214 & 0.03590 & 257.208 & 0.1044 & 0.01258 & 0.660 & 3.487 & 4.96 & 40.00 & 3837. \\
\hline 1054 & 0.212 & 0.04681 & 257.740 & 0.1030 & 0.01251 & 0.710 & 4.563 & 3.36 & 40.00 & 4833. \\
\hline 1057 & 0.211 & 0.05917 & 258.354 & 0.1023 & 0.01248 & 0.590 & 5.767 & 3.36 & 40.00 & 5966. \\
\hline 1060 & 0.213 & 0.07293 & 259.014 & 0.1030 & 0.01246 & 0.610 & 7.101 & 3.36 & 40.00 & 7379 . \\
\hline 1066 & 0.107 & 0.03590 & 257.232 & 0.0511 & 0.01243 & 0.850 & 3.546 & 1.76 & 40.00 & 836. \\
\hline 1069 & 0.108 & 0.04681 & 257.784 & 0.0516 & 0.01237 & 0.720 & 4.645 & 1.76 & 40.00 & 1110. \\
\hline 1072 & 0.108 & 0.05916 & 258.393 & 0.0512 & 0.01235 & 0.530 & 5.875 & 1.76 & 40.00 & 1367. \\
\hline 1075 & 0.105 & 0.07292 & 259.085 & 0.0496 & 0.01236 & 0.450 & 7.237 & 1.76 & 40.00 & 1562. \\
\hline 1078 & 0.753 & 0.02736 & 266.076 & 0.3967 & 0.01346 & 1.740 & 2.264 & 3.36 & 40.00 & 56603. \\
\hline 1081 & 0.752 & 0.03720 & 266.431 & 0.3955 & 0.01360 & 2.460 & 2.967 & 1.76 & 40.00 & 72804 . \\
\hline 1093 & 0.641 & 0.02735 & 266.103 & 0.3288 & 0.01357 & 1.810 & 2.330 & 3.36 & 40.00 & 34539 . \\
\hline 1096 & 0.642 & 0.03719 & 266.483 & 0.3287 & 0.01360 & 1.580 & 3.104 & 3.36 & 40.00 & 45565 . \\
\hline 1099 & 0.644 & 0.04852 & 266.913 & 0.3286 & 0.01359 & 2.350 & 3.969 & 1.76 & 40.00 & 57628. \\
\hline 1102 & 0.643 & 0.06133 & 267.376 & 0.3272 & 0.01366 & 2.840 & 4.894 & 1.76 & 40.00 & 69525 . \\
\hline 1108 & 0.532 & 0.02736 & 265.895 & 0.2667 & 0.01434 & 1.310 & 2.266 & 6.56 & 40.00 & 19537. \\
\hline 1111 & 0.533 & 0.03719 & 266.303 & 0.2665 & 0.01420 & 1.740 & 3.072 & 3.36 & 40.00 & 26232 . \\
\hline 1114 & 0.534 & 0.04850 & 266.758 & 0.2661 & 0.01410 & 1.730 & 3.983 & 3.36 & 40.00 & 33581 . \\
\hline 1117 & 0.534 & 0.06129 & 267.257 & 0.2654 & 0.01402 & 1.480 & 4.996 & 3.36 & 40.00 & 41429. \\
\hline 1120 & 0.533 & 0.07556 & 267.810 & 0.2645 & 0.01399 & 2.060 & 6.090 & 1.76 & 40.00 & 49514 . \\
\hline 1126 & 0.421 & 0.03721 & 266.349 & 0.2057 & 0.01404 & 0.790 & 3.176 & 6.56 & 40.00 & 14340. \\
\hline 1129 & 0.422 & 0.04852 & 266.825 & 0.2057 & 0.01392 & 0.680 & 4.147 & 4.96 & 40.00 & 18567. \\
\hline 1132 & 0.423 & 0.06131 & 267.356 & 0.2054 & 0.01389 & 1.160 & 5.208 & 3.36 & 40.00 & 23038 . \\
\hline 1135 & 0.423 & 0.07556 & 267.928 & 0.2051 & 0.01391 & 2.410 & 6.355 & 1.76 & 40.00 & 27725 . \\
\hline 1141 & 0.316 & 0.03720 & 266.351 & 0.1509 & 0.01400 & 0.790 & 3.225 & 4.96 & 40.00 & 7075 . \\
\hline 1144 & 0.317 & 0.04852 & 266.872 & 0.1512 & 0.01378 & 0.570 & 4.259 & 4.96 & 40.00 & 9308. \\
\hline 1147 & 0.318 & 0.06129 & 267.448 & 0.1510 & 0.01363 & 0.490 & 5.416 & 4.96 & 40.00 & 11700. \\
\hline 1150 & 0.317 & 0.07552 & 268.079 & 0.1504 & 0.01356 & 0.250 & 6.677 & 4.96 & 40.00 & 14149. \\
\hline 1156 & 0.217 & 0.03720 & 266.379 & 0.1016 & 0.01387 & 0.950 & 3.282 & 3.36 & 40.00 & 2977. \\
\hline
\end{tabular}


Table 26. Thermal conductivity of the binary $70 \% \mathrm{R} 32 / 30 \%$ propane mixture in the dilute gas measured with the steady-state technique (continued).

\begin{tabular}{|c|c|c|c|c|c|c|c|c|c|c|}
\hline $\begin{array}{l}\text { Run } \\
\text { point }\end{array}$ & $\begin{array}{l}P_{\text {exp }} \\
\mathrm{MPa} \\
\end{array}$ & $\begin{array}{c}Q \\
W \cdot m^{-1}\end{array}$ & $\begin{array}{c}T_{\text {exp }} \\
\mathrm{K}\end{array}$ & $\begin{array}{c}\rho_{\text {calc }} \\
\mathrm{mol} \cdot \mathrm{L}^{-1}\end{array}$ & $\begin{array}{c}\lambda_{\exp } \\
\mathrm{W} \cdot \mathrm{m}^{-1} \cdot \mathrm{K}^{-1}\end{array}$ & $\begin{array}{c}\text { TBAND } \\
\%\end{array}$ & $\begin{array}{c}\Delta T_{a v g} \\
\mathrm{~K} \\
\end{array}$ & $\begin{array}{c}t_{\text {start }} \\
\mathrm{s}\end{array}$ & $\begin{array}{c}t_{\text {end }} \\
\mathrm{s}\end{array}$ & $N_{R a}$ \\
\hline 1159 & 0.218 & 0.04851 & 266.910 & 0.1018 & 0.01364 & 0.670 & 4.343 & 3.36 & 40.00 & 3928. \\
\hline 1162 & 0.215 & 0.06128 & 267.496 & 0.1001 & 0.01351 & 0.530 & 5.531 & 3.36 & 40.00 & 4787. \\
\hline 1165 & 0.213 & 0.07552 & 268.151 & 0.0990 & 0.01346 & 0.510 & 6.832 & 3.36 & 40.00 & 5720. \\
\hline 1171 & 0.110 & 0.03720 & 266.377 & 0.0505 & 0.01379 & 0.740 & 3.313 & 1.76 & 40.00 & 677. \\
\hline 1174 & 0.110 & 0.04851 & 266.926 & 0.0506 & 0.01358 & 0.550 & 4.387 & 1.76 & 40.00 & 893. \\
\hline 1177 & 0.112 & 0.06129 & 267.519 & 0.0515 & 0.01346 & 0.470 & 5.591 & 1.76 & 40.00 & 1171. \\
\hline 1180 & 0.114 & 0.07550 & 268.186 & 0.0520 & 0.01336 & 0.450 & 6.934 & 1.76 & 40.00 & 1471. \\
\hline 1183 & 0.930 & 0.02641 & 277.407 & 0.4749 & 0.01510 & 2.720 & 1.936 & 3.36 & 40.00 & 60285. \\
\hline 1186 & 0.928 & 0.03592 & 277.733 & 0.4731 & 0.01506 & 3.290 & 2.563 & 1.76 & 40.00 & 78342 . \\
\hline 1198 & 0.833 & 0.02642 & 277.416 & 0.4167 & 0.01529 & 2.020 & 1.970 & 4.96 & 40.00 & 42753. \\
\hline 1201 & 0.833 & 0.03591 & 277.746 & 0.4157 & 0.01508 & 1.600 & 2.653 & 3.36 & 40.00 & 56815. \\
\hline 1204 & 0.833 & 0.04685 & 278.108 & 0.4153 & 0.01508 & 2.940 & 3.377 & 1.76 & 40.00 & 71571. \\
\hline 1213 & 0.726 & 0.02641 & 277.413 & 0.3555 & 0.01534 & 1.960 & 2.011 & 4.96 & 40.00 & 28725 . \\
\hline 1216 & 0.728 & 0.03591 & 277.757 & 0.3562 & 0.01519 & 1.740 & 2.715 & 3.36 & 40.00 & 38712 . \\
\hline 1219 & 0.730 & 0.04686 & 278.155 & 0.3563 & 0.01507 & 2.790 & 3.505 & 1.76 & 40.00 & 49611. \\
\hline 1222 & 0.731 & 0.05923 & 278.586 & 0.3559 & 0.01492 & 2.040 & 4.388 & 1.76 & 40.00 & 61442. \\
\hline 1225 & 0.729 & 0.07302 & 279.044 & 0.3537 & 0.01495 & 3.760 & 5.300 & 1.76 & 40.00 & 72415 . \\
\hline 1231 & 0.626 & 0.03592 & 277.733 & 0.3004 & 0.01537 & 1.520 & 2.745 & 4.96 & 40.00 & 25483. \\
\hline 1234 & 0.629 & 0.04684 & 278.147 & 0.3012 & 0.01521 & 1.660 & 3.570 & 3.36 & 40.00 & 33089. \\
\hline 1237 & 0.630 & 0.05922 & 278.612 & 0.3007 & 0.01509 & 1.470 & 4.488 & 3.36 & 40.00 & 41116. \\
\hline 1240 & 0.629 & 0.07302 & 279.106 & 0.2995 & 0.01503 & 2.050 & 5.478 & 1.76 & 40.00 & 49253. \\
\hline 1246 & 0.527 & 0.03592 & 277.758 & 0.2480 & 0.01529 & 0.840 & 2.805 & 6.56 & 40.00 & 16336. \\
\hline 1249 & 0.525 & 0.04685 & 278.184 & 0.2469 & 0.01513 & 1.040 & 3.668 & 4.96 & 40.00 & 20976 . \\
\hline 1252 & 0.528 & 0.05921 & 278.644 & 0.2476 & 0.01506 & 1.380 & 4.610 & 3.36 & 40.00 & 26365. \\
\hline 1255 & 0.530 & 0.07299 & 279.165 & 0.2480 & 0.01501 & 1.320 & 5.646 & 3.36 & 40.00 & 32128. \\
\hline 1261 & 0.419 & 0.03591 & 277.758 & 0.1939 & 0.01522 & 0.960 & 2.853 & 6.56 & 40.00 & 9333. \\
\hline 1264 & 0.419 & 0.04684 & 278.215 & 0.1934 & 0.01493 & 0.860 & 3.774 & 4.96 & 40.00 & 12193. \\
\hline 1267 & 0.416 & 0.05920 & 278.723 & 0.1913 & 0.01481 & 0.490 & 4.787 & 4.96 & 40.00 & 14962. \\
\hline 1270 & 0.416 & 0.07298 & 279.278 & 0.1909 & 0.01475 & 0.900 & 5.887 & 3.36 & 40.00 & 18164. \\
\hline 1276 & 0.311 & 0.03591 & 277.766 & 0.1414 & 0.01513 & 1.000 & 2.896 & 4.96 & 40.00 & 4648 \\
\hline 1279 & 0.313 & 0.04683 & 278.238 & 0.1421 & 0.01481 & 0.630 & 3.845 & 4.96 & 40.00 & 6199. \\
\hline 1282 & 0.314 & 0.05918 & 278.764 & 0.1421 & 0.01464 & 0.460 & 4.903 & 4.96 & 40.00 & 7841. \\
\hline 1285 & 0.310 & 0.07295 & 279.334 & 0.1400 & 0.01453 & 0.370 & 6.072 & 4.96 & 40.00 & 9329. \\
\hline 1308 & 0.113 & 0.02636 & 275.991 & 0.0502 & 0.01367 & 1.090 & 2.371 & 1.76 & 40.00 & 426. \\
\hline 1311 & 0.113 & 0.03585 & 276.426 & 0.0498 & 0.01366 & 0.660 & 3.226 & 1.76 & 40.00 & 567. \\
\hline 1314 & 0.111 & 0.04676 & 276.914 & 0.0492 & 0.01367 & 0.450 & 4.203 & 1.76 & 40.00 & 717. \\
\hline 1317 & 0.111 & 0.05908 & 277.457 & 0.0488 & 0.01369 & 0.530 & 5.300 & 1.76 & 40.00 & 883. \\
\hline 1320 & 0.110 & 0.07281 & 278.066 & 0.0485 & 0.01373 & 0.400 & 6.512 & 1.76 & 40.00 & 1063. \\
\hline 1383 & 0.929 & 0.02554 & 284.764 & 0.4536 & 0.01650 & 2.670 & 1.770 & 4.96 & 40.00 & 41276. \\
\hline 1386 & 0.928 & 0.03473 & 285.083 & 0.4526 & 0.01614 & 1.810 & 2.402 & 3.36 & 40.00 & 55327. \\
\hline 1398 & 0.836 & 0.02554 & 284.764 & 0.4014 & 0.01655 & 2.390 & 1.798 & 4.96 & 40.00 & 30459. \\
\hline 1401 & 0.837 & 0.03472 & 285.081 & 0.4014 & 0.01620 & 1.840 & 2.450 & 3.36 & 40.00 & 41274. \\
\hline 1404 & 0.838 & 0.04532 & 285.438 & 0.4011 & 0.01598 & 2.310 & 3.177 & 3.36 & 40.00 & 53048. \\
\hline
\end{tabular}


Table 26. Thermal conductivity of the binary $70 \%$ R32/30\% propane mixture in the dilute gas measured with the steady-state technique (continued).

\begin{tabular}{|c|c|c|c|c|c|c|c|c|c|c|}
\hline $\begin{array}{l}\text { Run } \\
\text { point }\end{array}$ & $\begin{array}{l}P_{e x p} \\
\mathrm{MPa}\end{array}$ & $\begin{array}{c}Q \\
\mathrm{~W} \cdot \mathrm{m}^{-1}\end{array}$ & $\begin{array}{c}T_{\text {exp }} \\
\mathrm{K}\end{array}$ & $\begin{array}{c}\rho_{\text {calc }} \\
\mathrm{mol} \cdot^{\cdot} \mathrm{L}^{-1}\end{array}$ & $\begin{array}{c}\lambda_{\exp } \\
\mathrm{W} \cdot \mathrm{m}^{-1} \cdot \mathrm{K}^{-1}\end{array}$ & $\begin{array}{c}\text { TBAND } \\
\%\end{array}$ & $\begin{array}{c}\Delta T_{\text {avg }} \\
\mathrm{K}\end{array}$ & $\begin{array}{c}t_{\text {start }} \\
\mathrm{s}\end{array}$ & $\begin{array}{c}t_{\text {end }} \\
\mathrm{s}\end{array}$ & $N_{R a}$ \\
\hline 1407 & 0.837 & 0.05731 & 285.823 & 0.3999 & 0.01596 & 2.900 & 3.945 & 1.76 & 40.00 & 64887. \\
\hline 1413 & 0.729 & 0.02553 & 284.761 & 0.3437 & 0.01625 & 1.670 & 1.860 & 6.56 & 40.00 & 21291. \\
\hline 1416 & 0.728 & 0.03473 & 285.103 & 0.3423 & 0.01604 & 1.720 & 2.530 & 4.96 & 40.00 & 28519 . \\
\hline 1419 & 0.730 & 0.04532 & 285.481 & 0.3430 & 0.01588 & 1.540 & 3.286 & 3.36 & 40.00 & 36969 . \\
\hline 1422 & 0.732 & 0.05728 & 285.892 & 0.3431 & 0.01576 & 2.780 & 4.121 & 1.76 & 40.00 & 46080. \\
\hline 1425 & 0.731 & 0.07062 & 286.351 & 0.3420 & 0.01563 & 2.020 & 5.041 & 1.76 & 40.00 & 55473. \\
\hline 1428 & 0.629 & 0.02554 & 284.759 & 0.2916 & 0.01613 & 1.370 & 1.897 & 8.16 & 40.00 & 14537. \\
\hline 1431 & 0.631 & 0.03473 & 285.104 & 0.2918 & 0.01585 & 1.190 & 2.600 & 6.56 & 40.00 & 19847. \\
\hline 1434 & 0.628 & 0.04530 & 285.500 & 0.2899 & 0.01576 & 1.520 & 3.379 & 3.36 & 40.00 & 25223. \\
\hline 1437 & 0.627 & 0.05727 & 285.921 & 0.2885 & 0.01570 & 1.430 & 4.243 & 3.36 & 40.00 & 31108 . \\
\hline 1440 & 0.629 & 0.07062 & 286.402 & 0.2890 & 0.01560 & 1.280 & 5.204 & 3.36 & 40.00 & 37995. \\
\hline 1446 & 0.527 & 0.03472 & 285.098 & 0.2397 & 0.01581 & 0.880 & 2.640 & 6.56 & 40.00 & 12649. \\
\hline 1449 & 0.525 & 0.04530 & 285.517 & 0.2385 & 0.01559 & 1.010 & 3.469 & 4.96 & 40.00 & 16334. \\
\hline 1452 & 0.523 & 0.05727 & 285.979 & 0.2369 & 0.01552 & 0.840 & 4.377 & 4.96 & 40.00 & 20133. \\
\hline 1455 & 0.524 & 0.07060 & 286.466 & 0.2367 & 0.01551 & 1.090 & 5.357 & 3.36 & 40.00 & 24430 . \\
\hline 1461 & 0.423 & 0.03472 & 285.098 & 0.1896 & 0.01584 & 1.010 & 2.660 & 6.56 & 40.00 & 7438. \\
\hline 1464 & 0.420 & 0.04530 & 285.532 & 0.1877 & 0.01555 & 0.890 & 3.522 & 4.96 & 40.00 & 9578. \\
\hline 1467 & 0.419 & 0.05725 & 286.017 & 0.1867 & 0.01537 & 0.630 & 4.482 & 4.96 & 40.00 & 11955. \\
\hline 1470 & 0.421 & 0.07057 & 286.535 & 0.1874 & 0.01528 & 0.380 & 5.530 & 4.96 & 40.00 & 14775. \\
\hline 1476 & 0.314 & 0.03471 & 285.115 & 0.1381 & 0.01557 & 0.990 & 2.723 & 4.96 & 40.00 & 3765. \\
\hline 1479 & 0.315 & 0.04530 & 285.550 & 0.1386 & 0.01533 & 0.760 & 3.602 & 4.96 & 40.00 & 4993. \\
\hline 1482 & 0.317 & 0.05725 & 286.048 & 0.1393 & 0.01518 & 0.480 & 4.586 & 4.96 & 40.00 & 6385. \\
\hline 1485 & 0.317 & 0.07056 & 286.587 & 0.1390 & 0.01508 & 0.390 & 5.674 & 4.96 & 40.00 & 7812. \\
\hline 1491 & 0.210 & 0.03472 & 285.118 & 0.0913 & 0.01541 & 0.980 & 2.764 & 3.36 & 40.00 & 1566. \\
\hline 1494 & 0.211 & 0.04528 & 285.561 & 0.0915 & 0.01520 & 0.640 & 3.652 & 3.36 & 40.00 & 2066 . \\
\hline 1497 & 0.213 & 0.05724 & 286.057 & 0.0921 & 0.01508 & 0.470 & 4.646 & 3.36 & 40.00 & 2647. \\
\hline 1500 & 0.213 & 0.07057 & 286.605 & 0.0921 & 0.01501 & 0.360 & 5.747 & 3.36 & 40.00 & 3259 . \\
\hline 1503 & 0.111 & 0.02552 & 284.524 & 0.0478 & 0.01452 & 1.310 & 2.160 & 1.76 & 40.00 & 318. \\
\hline 1506 & 0.112 & 0.03469 & 284.928 & 0.0478 & 0.01444 & 0.840 & 2.952 & 1.76 & 40.00 & 432. \\
\hline 1509 & 0.112 & 0.04525 & 285.368 & 0.0478 & 0.01443 & 0.620 & 3.854 & 1.76 & 40.00 & 562. \\
\hline 1512 & 0.112 & 0.05719 & 285.862 & 0.0478 & 0.01444 & 0.450 & 4.866 & 1.76 & 40.00 & 704. \\
\hline 1515 & 0.111 & 0.07050 & 286.434 & 0.0474 & 0.01447 & 0.460 & 5.982 & 1.76 & 40.00 & 849. \\
\hline 1596 & 0.972 & 0.03339 & 298.692 & 0.4437 & 0.01922 & 2.760 & 2.010 & 4.96 & 40.00 & 34350. \\
\hline 1599 & 0.970 & 0.04358 & 299.018 & 0.4419 & 0.01860 & 2.420 & 2.662 & 3.36 & 40.00 & 44787. \\
\hline 1602 & 0.971 & 0.05510 & 299.369 & 0.4415 & 0.01819 & 2.070 & 3.376 & 3.36 & 40.00 & 56338. \\
\hline 1611 & 0.763 & 0.03339 & 298.699 & 0.3380 & 0.01881 & 2.080 & 2.111 & 4.96 & 40.00 & 18572. \\
\hline 1614 & 0.761 & 0.04358 & 299.032 & 0.3363 & 0.01827 & 1.960 & 2.808 & 4.96 & 40.00 & 24296 . \\
\hline 1617 & 0.761 & 0.05510 & 299.416 & 0.3358 & 0.01794 & 1.780 & 3.576 & 3.36 & 40.00 & 30659. \\
\hline 1620 & 0.763 & 0.06795 & 299.833 & 0.3361 & 0.01764 & 1.570 & 4.429 & 3.36 & 40.00 & 37816 . \\
\hline 1626 & 0.561 & 0.03339 & 298.708 & 0.2418 & 0.01829 & 2.090 & 2.209 & 6.56 & 40.00 & 8916 . \\
\hline 1629 & 0.563 & 0.04358 & 299.072 & 0.2420 & 0.01771 & 1.200 & 2.961 & 6.56 & 40.00 & 11923. \\
\hline 1632 & 0.560 & 0.05509 & 299.489 & 0.2403 & 0.01737 & 1.120 & 3.797 & 4.96 & 40.00 & 14964. \\
\hline 1635 & 0.560 & 0.06792 & 299.942 & 0.2401 & 0.01718 & 0.870 & 4.703 & 4.96 & 40.00 & 18383. \\
\hline
\end{tabular}


Table 26. Thermal conductivity of the binary $70 \% \mathrm{R} 32 / 30 \%$ propane mixture in the dilute gas measured with the steady-state technique (continued).

\begin{tabular}{|c|c|c|c|c|c|c|c|c|c|c|}
\hline $\begin{array}{l}\text { Run } \\
\text { point }\end{array}$ & $\begin{array}{l}P_{\text {exp }} \\
\mathrm{MPa}\end{array}$ & $\begin{array}{c}Q \\
W \cdot \mathrm{m}^{-1}\end{array}$ & $\begin{array}{c}T_{\text {exp }} \\
\mathrm{K}\end{array}$ & $\begin{array}{c}\rho_{\text {calc }} \\
{\mathrm{mol} \cdot \mathrm{L}^{-1}}^{-1}\end{array}$ & $\begin{array}{c}\lambda_{\exp } \\
\mathrm{W} \cdot \mathrm{m}^{-1} \cdot \mathrm{K}^{-1}\end{array}$ & $\begin{array}{c}\text { TBAND } \\
\% \\
\end{array}$ & $\begin{array}{c}\Delta T_{a v g} \\
\mathrm{~K}\end{array}$ & $\begin{array}{c}t_{\text {start }} \\
\mathrm{s}\end{array}$ & $\begin{array}{c}t_{\text {end }} \\
\mathrm{s}\end{array}$ & $\overline{N_{R a}}$ \\
\hline 1644 & 0.353 & 0.04356 & 299.043 & 0.1481 & 0.01778 & 1.210 & 2.992 & 4.96 & 40.00 & 4052. \\
\hline 1647 & 0.355 & 0.05507 & 299.477 & 0.1484 & 0.01737 & 0.870 & 3.863 & 4.96 & 40.00 & 5228. \\
\hline 1650 & 0.358 & 0.06790 & 299.961 & 0.1496 & 0.01709 & 0.540 & 4.827 & 4.96 & 40.00 & 6614. \\
\hline 1653 & 0.218 & 0.02451 & 297.848 & 0.0903 & 0.01584 & 1.460 & 1.900 & 3.36 & 40.00 & 907. \\
\hline 1656 & 0.215 & 0.03334 & 298.194 & 0.0889 & 0.01579 & 0.950 & 2.592 & 3.36 & 40.00 & 1194. \\
\hline 1659 & 0.214 & 0.04350 & 298.584 & 0.0885 & 0.01576 & 0.720 & 3.385 & 3.36 & 40.00 & 1537. \\
\hline 1662 & 0.216 & 0.05498 & 299.014 & 0.0892 & 0.01577 & 0.480 & 4.274 & 3.36 & 40.00 & 1965. \\
\hline 1665 & 0.218 & 0.06777 & 299.510 & 0.0899 & 0.01578 & 0.400 & 5.258 & 3.36 & 40.00 & 2446. \\
\hline 1743 & 1.097 & 0.02373 & 304.466 & 0.4950 & 0.01957 & 3.500 & 1.416 & 4.96 & 40.00 & 28948. \\
\hline 1746 & 1.098 & 0.03228 & 304.741 & 0.4946 & 0.01892 & 2.250 & 1.956 & 4.96 & 40.00 & 39720 . \\
\hline 1749 & 1.097 & 0.04213 & 305.037 & 0.4936 & 0.01847 & 2.040 & 2.562 & 3.36 & 40.00 & 51535. \\
\hline 1752 & 1.097 & 0.05328 & 305.347 & 0.4929 & 0.01840 & 3.330 & 3.188 & 1.76 & 40.00 & 63584. \\
\hline 1758 & 0.830 & 0.02373 & 304.453 & 0.3612 & 0.01928 & 2.820 & 1.476 & 8.16 & 40.00 & 13987. \\
\hline 1761 & 0.830 & 0.03228 & 304.748 & 0.3609 & 0.01865 & 2.030 & 2.056 & 6.56 & 40.00 & 19370. \\
\hline 1764 & 0.831 & 0.04213 & 305.052 & 0.3606 & 0.01832 & 1.840 & 2.702 & 4.96 & 40.00 & 25300 . \\
\hline 1767 & 0.831 & 0.05327 & 305.420 & 0.3602 & 0.01810 & 1.950 & 3.420 & 3.36 & 40.00 & 31759 . \\
\hline 1770 & 0.832 & 0.06569 & 305.816 & 0.3598 & 0.01792 & 1.580 & 4.209 & 3.36 & 40.00 & 38774 . \\
\hline 1773 & 0.557 & 0.02373 & 304.442 & 0.2344 & 0.01892 & 2.900 & 1.528 & 8.16 & 40.00 & 5339. \\
\hline 1776 & 0.558 & 0.03228 & 304.767 & 0.2343 & 0.01819 & 1.610 & 2.153 & 6.56 & 40.00 & 7485. \\
\hline 1779 & 0.558 & 0.04212 & 305.108 & 0.2340 & 0.01775 & 1.170 & 2.867 & 6.56 & 40.00 & 9894. \\
\hline 1782 & 0.558 & 0.05325 & 305.511 & 0.2339 & 0.01748 & 1.040 & 3.661 & 4.96 & 40.00 & 12560. \\
\hline 1785 & 0.559 & 0.06566 & 305.941 & 0.2338 & 0.01733 & 0.750 & 4.530 & 4.96 & 40.00 & 15444. \\
\hline 1791 & 0.320 & 0.03228 & 304.699 & 0.1309 & 0.01841 & 1.660 & 2.149 & 3.36 & 40.00 & 2091. \\
\hline 1794 & 0.320 & 0.04212 & 305.075 & 0.1306 & 0.01787 & 1.520 & 2.884 & 3.36 & 40.00 & 2782. \\
\hline 1797 & 0.320 & 0.05326 & 305.474 & 0.1303 & 0.01759 & 1.080 & 3.700 & 3.36 & 40.00 & 3534 . \\
\hline 1800 & 0.319 & 0.06567 & 305.932 & 0.1299 & 0.01738 & 0.760 & 4.612 & 3.36 & 40.00 & 4356. \\
\hline 1806 & 0.116 & 0.03228 & 304.676 & 0.0464 & 0.01832 & 1.550 & 2.166 & 1.76 & 40.00 & 242. \\
\hline 1809 & 0.117 & 0.04213 & 305.045 & 0.0466 & 0.01780 & 1.010 & 2.911 & 1.76 & 40.00 & 327. \\
\hline 1812 & 0.116 & 0.05326 & 305.466 & 0.0462 & 0.01745 & 0.690 & 3.752 & 1.76 & 40.00 & 412. \\
\hline 1908 & 1.246 & 0.02275 & 316.366 & 0.5405 & 0.02025 & 3.260 & 1.315 & 4.96 & 40.00 & 27792 . \\
\hline 1911 & 1.245 & 0.03096 & 316.610 & 0.5391 & 0.01979 & 2.510 & 1.799 & 4.96 & 40.00 & 37649. \\
\hline 1914 & 1.248 & 0.04041 & 316.878 & 0.5400 & 0.01941 & 1.820 & 2.349 & 4.96 & 40.00 & 49148. \\
\hline 1917 & 1.250 & 0.05111 & 317.167 & 0.5402 & 0.01919 & 2.230 & 2.943 & 3.36 & 40.00 & 61388. \\
\hline 1923 & 0.971 & 0.02275 & 316.353 & 0.4074 & 0.01983 & 2.940 & 1.374 & 8.16 & 40.00 & 14687. \\
\hline 1926 & 0.969 & 0.03095 & 316.610 & 0.4059 & 0.01937 & 2.030 & 1.896 & 6.56 & 40.00 & 20017. \\
\hline 1929 & 0.968 & 0.04040 & 316.888 & 0.4051 & 0.01916 & 1.670 & 2.475 & 4.96 & 40.00 & 25903. \\
\hline 1932 & 0.970 & 0.05110 & 317.218 & 0.4054 & 0.01900 & 1.850 & 3.121 & 3.36 & 40.00 & 32575 . \\
\hline 1935 & 0.971 & 0.06303 & 317.563 & 0.4056 & 0.01886 & 1.470 & 3.829 & 3.36 & 40.00 & 39845 . \\
\hline 1941 & 0.696 & 0.03095 & 316.528 & 0.2831 & 0.01967 & 1.680 & 1.904 & 8.16 & 40.00 & 8770. \\
\hline 1944 & 0.699 & 0.04038 & 316.831 & 0.2840 & 0.01917 & 1.070 & 2.536 & 6.56 & 40.00 & 11720 . \\
\hline 1947 & 0.700 & 0.05107 & 317.178 & 0.2844 & 0.01888 & 0.810 & 3.237 & 6.56 & 40.00 & 14940. \\
\hline 1950 & 0.697 & 0.06301 & 317.562 & 0.2826 & 0.01874 & 0.790 & 4.003 & 4.96 & 40.00 & 18123. \\
\hline 1956 & 0.389 & 0.03095 & 316.492 & 0.1534 & 0.01986 & 1.730 & 1.909 & 4.96 & 40.00 & 2292. \\
\hline
\end{tabular}


Table 26. Thermal conductivity of the binary $70 \% \mathrm{R} 32 / 30 \%$ propane mixture in the dilute gas measured with the steady-state technique (continued).

\begin{tabular}{|ccccccc|cccc|}
\hline $\begin{array}{c}\text { Run } \\
\text { point }\end{array}$ & $\begin{array}{c}P_{\text {exp }} \\
\mathrm{MPa}\end{array}$ & $\mathrm{W} \cdot \mathrm{m}^{-1}$ & $\begin{array}{c}\text { exp } \\
\mathrm{K}\end{array}$ & $\begin{array}{c}\rho_{\text {calc }} \\
\mathrm{mol} \cdot \mathrm{L}^{-1}\end{array}$ & $\begin{array}{c}\mathrm{W}_{\text {exp }} \mathrm{m}^{-1} \cdot \mathrm{K}^{-1} \\
\text { TBAND }\end{array}$ & $\begin{array}{c}\Delta T_{\text {avg }} \\
t_{\text {start }}\end{array}$ & $t_{\text {end }}$ & $N_{R a}$ \\
\hline 1959 & 0.387 & 0.04041 & 316.815 & 0.1522 & 0.01931 & 1.210 & 2.559 & 4.96 & 40.00 & 3010. \\
1962 & 0.389 & 0.05110 & 317.176 & 0.1532 & 0.01893 & 0.850 & 3.297 & 4.96 & 40.00 & 3915. \\
1965 & 0.392 & 0.06301 & 317.602 & 0.1539 & 0.01854 & 0.670 & 4.143 & 4.96 & 40.00 & 4948. \\
1971 & 0.111 & 0.03096 & 316.455 & 0.0427 & 0.01981 & 1.910 & 1.922 & 1.76 & 40.00 & 161. \\
1974 & 0.113 & 0.04041 & 316.780 & 0.0435 & 0.01918 & 1.250 & 2.590 & 1.76 & 40.00 & 225. \\
1977 & 0.111 & 0.05110 & 317.160 & 0.0426 & 0.01876 & 0.790 & 3.348 & 1.76 & 40.00 & 278. \\
1980 & 0.109 & 0.06301 & 317.579 & 0.0419 & 0.01851 & 0.600 & 4.184 & 1.76 & 40.00 & 333. \\
3051 & 1.334 & 0.03061 & 326.558 & 0.5564 & 0.02235 & 3.280 & 1.593 & 4.96 & 40.00 & 31229. \\
3054 & 1.335 & 0.03995 & 326.811 & 0.5563 & 0.02161 & 2.170 & 2.114 & 4.96 & 40.00 & 41273. \\
3057 & 1.335 & 0.05052 & 327.097 & 0.5555 & 0.02106 & 1.680 & 2.692 & 3.36 & 40.00 & 52188. \\
3060 & 1.333 & 0.06232 & 327.391 & 0.5541 & 0.02098 & 3.460 & 3.275 & 1.76 & 40.00 & 62834. \\
3066 & 0.929 & 0.03060 & 326.562 & 0.3717 & 0.02185 & 1.990 & 1.683 & 8.16 & 40.00 & 12750. \\
3069 & 0.927 & 0.03994 & 326.834 & 0.3708 & 0.02115 & 1.330 & 2.252 & 6.56 & 40.00 & 16911. \\
3072 & 0.930 & 0.05052 & 327.141 & 0.3715 & 0.02077 & 1.260 & 2.878 & 4.96 & 40.00 & 21626. \\
3075 & 0.930 & 0.06232 & 327.488 & 0.3711 & 0.02054 & 1.610 & 3.558 & 3.36 & 40.00 & 26566. \\
3084 & 0.517 & 0.03994 & 326.735 & 0.1989 & 0.02156 & 1.510 & 2.261 & 6.56 & 40.00 & 4255. \\
3087 & 0.517 & 0.05051 & 327.075 & 0.1986 & 0.02089 & 1.100 & 2.944 & 4.96 & 40.00 & 5503. \\
3090 & 0.520 & 0.06230 & 327.445 & 0.1995 & 0.02044 & 0.950 & 3.701 & 4.96 & 40.00 & 6956. \\
3099 & 0.112 & 0.03994 & 326.722 & 0.0417 & 0.02110 & 1.500 & 2.328 & 1.76 & 40.00 & 168. \\
3102 & 0.110 & 0.05052 & 327.068 & 0.0408 & 0.02047 & 1.040 & 3.034 & 1.76 & 40.00 & 210. \\
3105 & 0.109 & 0.06231 & 327.458 & 0.0404 & 0.02007 & 0.580 & 3.818 & 1.76 & 40.00 & 258. \\
\hline
\end{tabular}


Table 27. Thermal conductivity of the binary $30 \% \mathrm{R} 32 / 70 \% \mathrm{R} 134 \mathrm{a}$ mixture in the dilute gas measured with the steady-state technique with bare tungsten hot wires.

\begin{tabular}{|c|c|c|c|c|c|c|c|c|c|c|}
\hline $\begin{array}{l}\text { Run } \\
\text { point }\end{array}$ & $\begin{array}{l}P_{\text {exp }} \\
\mathrm{MPa} \\
\end{array}$ & $\begin{array}{c}Q \\
W \cdot m^{-1}\end{array}$ & $\begin{array}{c}T_{\exp } \\
\mathrm{K}\end{array}$ & $\begin{array}{c}\rho_{\text {calc }} \\
\mathrm{mol} \cdot \mathrm{L}^{-1}\end{array}$ & $\begin{array}{c}\lambda_{\exp } \\
\mathrm{W} \cdot \mathrm{m}^{-1} \cdot \mathrm{K}^{-1}\end{array}$ & $\begin{array}{c}\text { TBAND } \\
\%\end{array}$ & $\begin{array}{c}\Delta T_{a v g} \\
\mathrm{~K}\end{array}$ & $\begin{array}{c}t_{\text {start }} \\
\mathrm{s}\end{array}$ & $\begin{array}{c}t_{\text {end }} \\
\mathrm{s}\end{array}$ & $N_{R a}$ \\
\hline 1003 & 0.097 & 0.02627 & 257.573 & 0.0469 & 0.01045 & 0.810 & 3.082 & 3.36 & 40.00 & 1577. \\
\hline 1006 & 0.097 & 0.03570 & 258.132 & 0.0468 & 0.01046 & 0.500 & 4.184 & 3.36 & 40.00 & 2114. \\
\hline 1009 & 0.098 & 0.04655 & 258.754 & 0.0469 & 0.01048 & 0.380 & 5.436 & 3.36 & 40.00 & 2739. \\
\hline 1012 & 0.098 & 0.05881 & 259.449 & 0.0470 & 0.01051 & 0.360 & 6.837 & 3.36 & 40.00 & 3418. \\
\hline 1015 & 0.098 & 0.07247 & 260.227 & 0.0467 & 0.01055 & 0.260 & 8.385 & 3.36 & 40.00 & 4080. \\
\hline 1018 & 0.151 & 0.02627 & 257.549 & 0.0741 & 0.01055 & 0.760 & 3.038 & 4.96 & 40.00 & 4320. \\
\hline 1021 & 0.151 & 0.03571 & 258.090 & 0.0736 & 0.01055 & 0.520 & 4.120 & 4.96 & 40.00 & 5718. \\
\hline 1024 & 0.151 & 0.04656 & 258.704 & 0.0734 & 0.01057 & 0.680 & 5.347 & 3.36 & 40.00 & 7284. \\
\hline 1027 & 0.151 & 0.05882 & 259.395 & 0.0733 & 0.01059 & 0.580 & 6.722 & 3.36 & 40.00 & 9012. \\
\hline 1030 & 0.151 & 0.07247 & 260.147 & 0.0732 & 0.01062 & 0.530 & 8.226 & 3.36 & 40.00 & 10858. \\
\hline 1033 & 0.157 & 0.02553 & 265.565 & 0.0746 & 0.01111 & 1.100 & 2.808 & 3.36 & 40.00 & 3536. \\
\hline 1036 & 0.158 & 0.03470 & 266.060 & 0.0746 & 0.01110 & 0.950 & 3.812 & 3.36 & 40.00 & 4759. \\
\hline 1039 & 0.158 & 0.04526 & 266.633 & 0.0744 & 0.01111 & 0.380 & 4.956 & 4.96 & 40.00 & 6101. \\
\hline 1042 & 0.157 & 0.05719 & 267.271 & 0.0740 & 0.01113 & 0.600 & 6.233 & 3.36 & 40.00 & 7493. \\
\hline 1045 & 0.157 & 0.07047 & 267.983 & 0.0734 & 0.01115 & 0.620 & 7.645 & 3.36 & 40.00 & 8943. \\
\hline 1048 & 0.094 & 0.02552 & 266.168 & 0.0437 & 0.01105 & 1.300 & 2.835 & 1.76 & 40.00 & 1098. \\
\hline 1051 & 0.094 & 0.03469 & 266.680 & 0.0436 & 0.01105 & 0.670 & 3.851 & 3.36 & 40.00 & 1472. \\
\hline 1054 & 0.095 & 0.04524 & 267.255 & 0.0438 & 0.01108 & 0.890 & 5.007 & 1.76 & 40.00 & 1916. \\
\hline 1057 & 0.095 & 0.05716 & 267.899 & 0.0438 & 0.01110 & 0.280 & 6.304 & 3.36 & 40.00 & 2396. \\
\hline 1060 & 0.095 & 0.07043 & 268.618 & 0.0439 & 0.01114 & 0.220 & 7.734 & 3.36 & 40.00 & 2914. \\
\hline 1063 & 0.227 & 0.02552 & 266.123 & 0.1095 & 0.01129 & 1.100 & 2.738 & 4.96 & 40.00 & 8317. \\
\hline 1066 & 0.227 & 0.03470 & 266.602 & 0.1094 & 0.01127 & 1.010 & 3.712 & 4.96 & 40.00 & 11146. \\
\hline 1069 & 0.227 & 0.04525 & 267.165 & 0.1091 & 0.01129 & 0.530 & 4.805 & 4.96 & 40.00 & 14198. \\
\hline 1072 & 0.227 & 0.05718 & 267.761 & 0.1088 & 0.01134 & 0.810 & 6.009 & 3.36 & 40.00 & 17417. \\
\hline 1075 & 0.226 & 0.07047 & 268.400 & 0.1082 & 0.01145 & 0.840 & 7.295 & 3.36 & 40.00 & 20639. \\
\hline 1078 & 0.236 & 0.02474 & 275.465 & 0.1091 & 0.01192 & 1.090 & 2.524 & 4.96 & 40.00 & 6459. \\
\hline 1081 & 0.235 & 0.03364 & 275.906 & 0.1089 & 0.01190 & 0.840 & 3.422 & 4.96 & 40.00 & 8643. \\
\hline 1084 & 0.236 & 0.04389 & 276.416 & 0.1088 & 0.01191 & 0.630 & 4.441 & 4.96 & 40.00 & 11104. \\
\hline 1087 & 0.236 & 0.05547 & 276.980 & 0.1089 & 0.01195 & 0.380 & 5.567 & 4.96 & 40.00 & 13823. \\
\hline 1090 & 0.237 & 0.06838 & 277.589 & 0.1087 & 0.01201 & 0.570 & 6.793 & 3.36 & 40.00 & 16657. \\
\hline 1093 & 0.154 & 0.02474 & 275.596 & 0.0699 & 0.01184 & 1.110 & 2.559 & 3.36 & 40.00 & 2392 . \\
\hline 1096 & 0.155 & 0.03363 & 276.052 & 0.0701 & 0.01183 & 0.870 & 3.477 & 3.36 & 40.00 & 3256 . \\
\hline 1099 & 0.156 & 0.04387 & 276.584 & 0.0702 & 0.01184 & 0.690 & 4.524 & 3.36 & 40.00 & 4218. \\
\hline 1102 & 0.156 & 0.05544 & 277.166 & 0.0701 & 0.01186 & 0.480 & 5.696 & 3.36 & 40.00 & 5244. \\
\hline 1105 & 0.155 & 0.06833 & 277.826 & 0.0696 & 0.01189 & 0.430 & 6.990 & 3.36 & 40.00 & 6274. \\
\hline 1108 & 0.093 & 0.02474 & 275.566 & 0.0416 & 0.01174 & 0.900 & 2.588 & 3.36 & 40.00 & 793. \\
\hline 1111 & 0.093 & 0.03364 & 276.029 & 0.0413 & 0.01175 & 0.990 & 3.514 & 1.76 & 40.00 & 1056. \\
\hline 1114 & 0.092 & 0.04387 & 276.556 & 0.0410 & 0.01177 & 0.690 & 4.572 & 1.76 & 40.00 & 1345. \\
\hline 1117 & 0.092 & 0.05545 & 277.151 & 0.0409 & 0.01181 & 0.600 & 5.759 & 1.76 & 40.00 & 1667. \\
\hline 1120 & 0.092 & 0.06834 & 277.808 & 0.0406 & 0.01185 & 0.600 & 7.067 & 1.76 & 40.00 & 2002 . \\
\hline 1123 & 0.316 & 0.02474 & 275.490 & 0.1497 & 0.01203 & 1.090 & 2.468 & 6.56 & 40.00 & 13455. \\
\hline 1126 & 0.316 & 0.03364 & 275.916 & 0.1493 & 0.01206 & 1.020 & 3.322 & 4.96 & 40.00 & 17848. \\
\hline 1129 & 0.316 & 0.04389 & 276.378 & 0.1490 & 0.01214 & 0.960 & 4.268 & 3.36 & 40.00 & 22591 . \\
\hline
\end{tabular}


Table 27. Thermal conductivity of the binary $30 \% \mathrm{R} 32 / 70 \% \mathrm{R} 134 \mathrm{a}$ mixture in the dilute gas measured with the steady-state technique with bare tungsten hot wires (continued).

\begin{tabular}{|c|c|c|c|c|c|c|c|c|c|c|}
\hline $\begin{array}{l}\text { Run } \\
\text { point }\end{array}$ & $\begin{array}{l}P_{\text {exp }} \\
\mathrm{MPa}\end{array}$ & $\begin{array}{c}Q \\
W \cdot \mathrm{m}^{-1}\end{array}$ & $\begin{array}{c}\overline{T_{e x p}} \\
\mathrm{~K}\end{array}$ & $\begin{array}{c}\rho_{\text {calc }} \\
\mathrm{mol}^{-1} \mathrm{~L}^{-1}\end{array}$ & $\begin{array}{c}\lambda_{\exp } \\
\mathrm{W} \cdot \mathrm{m}^{-1} \cdot \mathrm{K}^{-1}\end{array}$ & $\begin{array}{c}\text { TBAND } \\
\%\end{array}$ & $\begin{array}{c}\Delta T_{\text {avg }} \\
\mathrm{K}\end{array}$ & $\begin{array}{c}t_{\text {start }} \\
\mathrm{s}\end{array}$ & $\begin{array}{c}t_{\text {end }} \\
\mathrm{s}\end{array}$ & $\overline{N_{R a}}$ \\
\hline 1132 & 0.316 & 0.05547 & 276.906 & 0.1486 & 0.01222 & 1.220 & 5.313 & 3.36 & 40.00 & 27670 . \\
\hline 1135 & 0.316 & 0.06838 & 277.487 & 0.1486 & 0.01225 & 1.000 & 6.468 & 3.36 & 40.00 & 33327. \\
\hline 1138 & 0.329 & 0.02396 & 285.233 & 0.1496 & 0.01276 & 1.440 & 2.266 & 6.56 & 40.00 & 10386 . \\
\hline 1141 & 0.329 & 0.03259 & 285.638 & 0.1493 & 0.01274 & 0.910 & 3.067 & 6.56 & 40.00 & 13880 . \\
\hline 1144 & 0.328 & 0.04252 & 286.092 & 0.1486 & 0.01278 & 0.810 & 3.964 & 4.96 & 40.00 & 17614. \\
\hline 1147 & 0.328 & 0.05375 & 286.571 & 0.1481 & 0.01286 & 0.950 & 4.944 & 3.36 & 40.00 & 21626 . \\
\hline 1150 & 0.329 & 0.06628 & 287.101 & 0.1482 & 0.01293 & 1.040 & 6.015 & 3.36 & 40.00 & 26135. \\
\hline 1153 & 0.416 & 0.02396 & 285.280 & 0.1934 & 0.01293 & 1.330 & 2.202 & 6.56 & 40.00 & 18906. \\
\hline 1156 & 0.416 & 0.03257 & 285.659 & 0.1928 & 0.01296 & 1.270 & 2.954 & 4.96 & 40.00 & 24998. \\
\hline 1159 & 0.415 & 0.04251 & 286.081 & 0.1917 & 0.01301 & 1.330 & 3.798 & 3.36 & 40.00 & 31455 . \\
\hline 1162 & 0.415 & 0.05375 & 286.552 & 0.1913 & 0.01305 & 1.440 & 4.729 & 3.36 & 40.00 & 38619 . \\
\hline 1165 & 0.416 & 0.06628 & 287.052 & 0.1914 & 0.01307 & 1.800 & 5.743 & 1.76 & 40.00 & 46525. \\
\hline 1168 & 0.225 & 0.02396 & 285.329 & 0.0994 & 0.01270 & 1.280 & 2.304 & 4.96 & 40.00 & 4098. \\
\hline 1171 & 0.225 & 0.03258 & 285.752 & 0.0996 & 0.01266 & 0.910 & 3.133 & 4.96 & 40.00 & 5559 . \\
\hline 1174 & 0.226 & 0.04251 & 286.208 & 0.0995 & 0.01264 & 0.550 & 4.082 & 4.96 & 40.00 & 7191. \\
\hline 1177 & 0.225 & 0.05373 & 286.750 & 0.0992 & 0.01264 & 0.450 & 5.145 & 4.96 & 40.00 & 8922 . \\
\hline 1180 & 0.225 & 0.06624 & 287.332 & 0.0987 & 0.01266 & 0.320 & 6.313 & 4.96 & 40.00 & 10738. \\
\hline 1183 & 0.158 & 0.02396 & 285.333 & 0.0690 & 0.01269 & 1.290 & 2.315 & 3.36 & 40.00 & 1842. \\
\hline 1186 & 0.157 & 0.03258 & 285.755 & 0.0683 & 0.01265 & 0.950 & 3.154 & 3.36 & 40.00 & 2439 . \\
\hline 1189 & 0.157 & 0.04250 & 286.233 & 0.0680 & 0.01263 & 0.820 & 4.115 & 3.36 & 40.00 & 3133. \\
\hline 1192 & 0.158 & 0.05372 & 286.766 & 0.0687 & 0.01263 & 0.620 & 5.193 & 3.36 & 40.00 & 4014. \\
\hline 1195 & 0.160 & 0.06623 & 287.371 & 0.0691 & 0.01264 & 0.470 & 6.388 & 3.36 & 40.00 & 4959 . \\
\hline 1198 & 0.094 & 0.02396 & 285.350 & 0.0405 & 0.01255 & 1.330 & 2.346 & 1.76 & 40.00 & 600. \\
\hline 1201 & 0.095 & 0.03258 & 285.772 & 0.0410 & 0.01250 & 1.000 & 3.201 & 1.76 & 40.00 & 835. \\
\hline 1204 & 0.093 & 0.04250 & 286.261 & 0.0400 & 0.01250 & 0.810 & 4.175 & 1.76 & 40.00 & 1028. \\
\hline 1207 & 0.091 & 0.05371 & 286.800 & 0.0390 & 0.01251 & 0.630 & 5.268 & 1.76 & 40.00 & 1227. \\
\hline 1210 & 0.094 & 0.06621 & 287.405 & 0.0400 & 0.01254 & 0.570 & 6.474 & 1.76 & 40.00 & 1573. \\
\hline 1213 & 0.283 & 0.02396 & 285.320 & 0.1271 & 0.01274 & 1.080 & 2.284 & 6.56 & 40.00 & 7120 . \\
\hline 1216 & 0.284 & 0.03258 & 285.726 & 0.1275 & 0.01270 & 0.720 & 3.100 & 6.56 & 40.00 & 9662 . \\
\hline 1219 & 0.286 & 0.04250 & 286.189 & 0.1278 & 0.01269 & 0.710 & 4.027 & 4.96 & 40.00 & 12545. \\
\hline 1222 & 0.286 & 0.05373 & 286.703 & 0.1275 & 0.01271 & 0.490 & 5.053 & 4.96 & 40.00 & 15522 . \\
\hline 1225 & 0.284 & 0.06625 & 287.263 & 0.1262 & 0.01277 & 0.720 & 6.172 & 3.36 & 40.00 & 18381. \\
\hline 1228 & 0.454 & 0.02313 & 296.450 & 0.2018 & 0.01377 & 1.640 & 2.009 & 6.56 & 40.00 & 15773. \\
\hline 1231 & 0.456 & 0.03146 & 296.798 & 0.2024 & 0.01378 & 1.290 & 2.702 & 4.96 & 40.00 & 21249. \\
\hline 1234 & 0.455 & 0.04106 & 297.181 & 0.2015 & 0.01382 & 1.410 & 3.482 & 3.36 & 40.00 & 26929 . \\
\hline 1237 & 0.451 & 0.05191 & 297.616 & 0.1993 & 0.01386 & 1.120 & 4.348 & 3.36 & 40.00 & 32509 . \\
\hline 1240 & 0.453 & 0.06401 & 298.092 & 0.1998 & 0.01387 & 1.330 & 5.292 & 3.36 & 40.00 & 39509 . \\
\hline 1243 & 0.380 & 0.02313 & 296.435 & 0.1659 & 0.01387 & 1.460 & 2.014 & 8.16 & 40.00 & 9888. \\
\hline 1246 & 0.383 & 0.03145 & 296.795 & 0.1673 & 0.01380 & 1.040 & 2.735 & 6.56 & 40.00 & 13615. \\
\hline 1249 & 0.385 & 0.04105 & 297.205 & 0.1676 & 0.01379 & 0.810 & 3.547 & 4.96 & 40.00 & 17626. \\
\hline 1252 & 0.383 & 0.05190 & 297.649 & 0.1665 & 0.01384 & 1.200 & 4.435 & 3.36 & 40.00 & 21557. \\
\hline 1255 & 0.380 & 0.06400 & 298.144 & 0.1650 & 0.01390 & 1.010 & 5.411 & 3.36 & 40.00 & 25559 . \\
\hline 1258 & 0.313 & 0.02313 & 296.461 & 0.1346 & 0.01358 & 1.310 & 2.070 & 6.56 & 40.00 & 6252 . \\
\hline
\end{tabular}


Table 27. Thermal conductivity of the binary $30 \% \mathrm{R} 32$ / $70 \% \mathrm{R} 134 \mathrm{a}$ mixture in the dilute gas measured with the steady-state technique with bare tungsten hot wires (continued).

\begin{tabular}{|c|c|c|c|c|c|c|c|c|c|c|}
\hline $\begin{array}{l}\text { Run } \\
\text { point }\end{array}$ & $\begin{array}{l}P_{\text {exp }} \\
\mathrm{MPa}\end{array}$ & $\begin{array}{c}Q \\
W \cdot \mathrm{m}^{-1}\end{array}$ & $\begin{array}{c}T_{\exp } \\
\mathrm{K}\end{array}$ & $\begin{array}{c}\rho_{\text {calc }} \\
\mathrm{mol} \cdot \mathrm{L}^{-1}\end{array}$ & $\frac{\lambda_{\exp }}{\mathrm{W} \cdot \mathrm{m}^{-1} \cdot \mathrm{K}^{-1}}$ & $\begin{array}{c}\text { TBAND } \\
\%\end{array}$ & $\begin{array}{c}\Delta T_{a v g} \\
\mathrm{~K}\end{array}$ & $\begin{array}{c}t_{\text {start }} \\
\mathrm{s}\end{array}$ & $\begin{array}{c}t_{\text {end }} \\
\mathrm{s}\end{array}$ & $N_{R a}$ \\
\hline 1261 & 0.311 & 0.03145 & 296.839 & 0.1336 & 0.01354 & 0.970 & 2.815 & 6.56 & 40.00 & 8304. \\
\hline 1264 & 0.308 & 0.04103 & 297.255 & 0.1319 & 0.01352 & 0.780 & 3.663 & 4.96 & 40.00 & 10443. \\
\hline 1267 & 0.309 & 0.05188 & 297.730 & 0.1321 & 0.01354 & 0.670 & 4.602 & 4.96 & 40.00 & 13074. \\
\hline 1270 & 0.311 & 0.06398 & 298.244 & 0.1330 & 0.01358 & 0.410 & 5.628 & 4.96 & 40.00 & 16116. \\
\hline 1273 & 0.240 & 0.02313 & 296.415 & 0.1019 & 0.01374 & 1.470 & 2.058 & 4.96 & 40.00 & 3324 . \\
\hline 1276 & 0.238 & 0.03145 & 296.794 & 0.1010 & 0.01365 & 1.040 & 2.811 & 4.96 & 40.00 & 4431 \\
\hline 1279 & 0.236 & 0.04104 & 297.228 & 0.0997 & 0.01359 & 0.640 & 3.675 & 4.96 & 40.00 & 5597. \\
\hline 1282 & 0.238 & 0.05188 & 297.699 & 0.1005 & 0.01357 & 0.620 & 4.640 & 4.96 & 40.00 & 7142 . \\
\hline 1285 & 0.240 & 0.06397 & 298.241 & 0.1011 & 0.01357 & 0.400 & 5.707 & 4.96 & 40.00 & 8840. \\
\hline 1288 & 0.170 & 0.02313 & 296.422 & 0.0712 & 0.01364 & 1.620 & 2.080 & 3.36 & 40.00 & 1538. \\
\hline 1291 & 0.172 & 0.03145 & 296.793 & 0.0719 & 0.01357 & 0.900 & 2.840 & 3.36 & 40.00 & 2137. \\
\hline 1294 & 0.174 & 0.04104 & 297.238 & 0.0728 & 0.01353 & 0.770 & 3.713 & 3.36 & 40.00 & 2853. \\
\hline 1297 & 0.172 & 0.05188 & 297.726 & 0.0717 & 0.01352 & 0.530 & 4.692 & 3.36 & 40.00 & 3465 . \\
\hline 1300 & 0.169 & 0.06398 & 298.262 & 0.0705 & 0.01352 & 0.510 & 5.776 & 3.36 & 40.00 & 4085 \\
\hline 1303 & 0.099 & 0.02313 & 296.445 & 0.0408 & 0.01329 & 1.550 & 2.139 & 1.76 & 40.00 & 489. \\
\hline 1306 & 0.099 & 0.03145 & 296.826 & 0.0406 & 0.01328 & 1.010 & 2.909 & 1.76 & 40.00 & 655. \\
\hline 1309 & 0.094 & 0.04104 & 297.276 & 0.0388 & 0.01329 & 0.680 & 3.793 & 1.76 & 40.00 & 772. \\
\hline 1312 & 0.095 & 0.05188 & 297.764 & 0.0 & 0.01331 & 0.640 & 4.786 & 1.76 & 40.00 & 971. \\
\hline 1315 & 0.098 & 0.06396 & 298.324 & 0.0402 & 0.01334 & 0.520 & 5.885 & 1.76 & 40.00 & 1273. \\
\hline 1318 & 0.103 & 0.02234 & 307.809 & 0.0 & 0.01427 & 1.750 & 1.925 & 1.76 & 40.00 & 388. \\
\hline 1321 & 0.100 & 0.03039 & 308.155 & 0.0397 & 0.01424 & 1.140 & 2.622 & 1.76 & 40.00 & 497. \\
\hline 1324 & 0.099 & 0.03964 & 308.545 & 0.0 & 0.01423 & 0.880 & 3.422 & 1.76 & 40.00 & 634. \\
\hline 1327 & 0.101 & 0.05012 & 308.985 & 0.0399 & 0.01425 & 0.670 & 4.319 & 1.76 & 40.00 & 817. \\
\hline 1330 & 0.102 & 0.06182 & 309.486 & 0.0404 & 0.01428 & 0.470 & 5.316 & 1.76 & 40.00 & 1028. \\
\hline 1333 & 0.181 & 0.02245 & 305.735 & 0.0733 & 0.01414 & 1.370 & 1.948 & 3.36 & 40.00 & 1372. \\
\hline 1336 & 0.182 & 0.03055 & 306.090 & 0.0738 & 0.01416 & 1.220 & 2.645 & 3.36 & 40.00 & 1885. \\
\hline 1339 & 0.183 & 0.03987 & 306.485 & 0.0740 & 0.01415 & 0.810 & 3.450 & 3.36 & 40.00 & 2458 \\
\hline 1342 & 0.181 & 0.05039 & 306.930 & 0.0731 & 0.01416 & 0.630 & 4.352 & 3.36 & 40.00 & 3010. \\
\hline 1345 & 0.179 & 0.06215 & 307.415 & 0.0720 & 0.01420 & 0.590 & 5.349 & 3.36 & 40.00 & 3557. \\
\hline 1348 & 0.247 & 0.02247 & 305.721 & 0.1015 & 0.01419 & 1.540 & 1.938 & 4.96 & 40.00 & 2756. \\
\hline 1351 & 0.247 & 0.03057 & 306.062 & 0.1011 & 0.01417 & 1.080 & 2.635 & 4.96 & 40.00 & 3705. \\
\hline 1354 & 0.246 & 0.03988 & 306.468 & 0.1005 & 0.01416 & 0.780 & 3.435 & 4.96 & 40.00 & 4745 \\
\hline 1357 & 0.246 & 0.05042 & 306.914 & 0.1002 & 0.01416 & 0.550 & 4.332 & 4.96 & 40.00 & 5907. \\
\hline 1360 & 0.245 & 0.06218 & 307.408 & 0.0998 & 0.01419 & 0.450 & 5.319 & 4.96 & 40.00 & 7149. \\
\hline 1363 & 0.321 & 0.02247 & 305.662 & 0.1334 & 0.01441 & 1.520 & 1.900 & 6.56 & 40.00 & 4961. \\
\hline 1366 & 0.321 & 0.03057 & 306.012 & 0.1333 & 0.01434 & 1.320 & 2.589 & 4.96 & 40.00 & 6718. \\
\hline 1369 & 0.320 & 0.03989 & 306.401 & 0.1324 & 0.01431 & 0.880 & 3.376 & 4.96 & 40.00 & 8589. \\
\hline 1372 & 0.317 & 0.05042 & 306.830 & 0.1312 & 0.01430 & 0.660 & 4.253 & 4.96 & 40.00 & 10546. \\
\hline 1375 & 0.317 & 0.06219 & 307.304 & 0.1307 & 0.01434 & 0.540 & 5.213 & 4.96 & 40.00 & 12734 . \\
\hline 1378 & 0.390 & 0.02248 & 305.588 & 0.1642 & 0.01437 & 1.750 & 1.896 & 6.56 & 40.00 & 7959. \\
\hline 1381 & 0.389 & 0.03056 & 305.923 & 0.1634 & 0.01433 & 0.990 & 2.573 & 6.56 & 40.00 & 10626. \\
\hline 1384 & 0.387 & 0.03990 & 306.298 & 0.1623 & 0.01435 & 1.130 & 3.337 & 4.96 & 40.00 & 13509. \\
\hline 1387 & 0.387 & 0.05046 & 306.721 & 0.1621 & 0.01439 & 0.710 & 4.183 & 4.96 & 40.00 & 16792. \\
\hline
\end{tabular}


Table 27. Thermal conductivity of the binary $30 \% \mathrm{R} 32 / 70 \% \mathrm{R} 134 \mathrm{a}$ mixture in the dilute gas measured with the steady-state technique with bare tungsten hot wires (continued).

\begin{tabular}{|c|c|c|c|c|c|c|c|c|c|c|}
\hline $\begin{array}{l}\text { Run } \\
\text { point }\end{array}$ & $\begin{array}{l}P_{\text {exp }} \\
\mathrm{MPa}\end{array}$ & $\begin{array}{c}Q \\
\mathrm{~W} \cdot \mathrm{m}^{-1}\end{array}$ & $\begin{array}{c}T_{\exp } \\
\mathrm{K}\end{array}$ & $\begin{array}{c}\rho_{\text {calc }} \\
\mathrm{mol}^{-1} \mathrm{~L}^{-1}\end{array}$ & $\begin{array}{c}\lambda_{\exp } \\
\mathrm{W} \cdot \mathrm{m}^{-1} \cdot \mathrm{K}^{-1}\end{array}$ & $\begin{array}{c}\text { TBAND } \\
\%\end{array}$ & $\begin{array}{c}\Delta T_{a v g} \\
\mathrm{~K}\end{array}$ & $\begin{array}{c}t_{\text {start }} \\
\mathrm{s}\end{array}$ & $\begin{array}{c}t_{\text {end }} \\
\mathrm{s}\end{array}$ & $N_{R a}$ \\
\hline 1390 & 0.389 & 0.06221 & 307.175 & 0.1628 & 0.01446 & 1.060 & 5.100 & 3.36 & 40.00 & 20537. \\
\hline 1393 & 0.431 & 0.02250 & 305.054 & 0.1835 & 0.01443 & 1.690 & 1.882 & 6.56 & 40.00 & 10321. \\
\hline 1396 & 0.432 & 0.03059 & 305.380 & 0.1837 & 0.01439 & 1.180 & 2.550 & 6.56 & 40.00 & 13950. \\
\hline 1399 & 0.433 & 0.03993 & 305.746 & 0.1835 & 0.01442 & 0.980 & 3.297 & 4.96 & 40.00 & 17886. \\
\hline 1402 & 0.432 & 0.05050 & 306.157 & 0.1830 & 0.01449 & 1.290 & 4.119 & 3.36 & 40.00 & 22075 . \\
\hline 1405 & 0.431 & 0.06227 & 306.604 & 0.1822 & 0.01455 & 1.110 & 5.019 & 3.36 & 40.00 & 26460 . \\
\hline 1408 & 0.448 & 0.02179 & 315.842 & 0.1825 & 0.01575 & 2.000 & 1.678 & 8.16 & 40.00 & 7842. \\
\hline 1411 & 0.450 & 0.02963 & 316.138 & 0.1835 & 0.01559 & 1.390 & 2.292 & 6.56 & 40.00 & 10799. \\
\hline 1414 & 0.451 & 0.03868 & 316.468 & 0.1837 & 0.01552 & 0.850 & 2.989 & 6.56 & 40.00 & 14061. \\
\hline 1417 & 0.448 & 0.04892 & 316.848 & 0.1821 & 0.01551 & 0.830 & 3.761 & 4.96 & 40.00 & 17255. \\
\hline 1420 & 0.447 & 0.06032 & 317.251 & 0.1813 & 0.01554 & 1.140 & 4.599 & 3.36 & 40.00 & 20777. \\
\hline 1423 & 0.449 & 0.02962 & 316.082 & 0.1829 & 0.01514 & 1.230 & 2.358 & 6.56 & 40.00 & 11034. \\
\hline 1426 & 0.448 & 0.03867 & 316.436 & 0.1821 & 0.01514 & 0.790 & 3.061 & 6.56 & 40.00 & 14116. \\
\hline 1429 & 0.447 & 0.04889 & 316.813 & 0.1814 & 0.01521 & 0.850 & 3.832 & 4.96 & 40.00 & 17442. \\
\hline 1432 & 0.448 & 0.06030 & 317.215 & 0.1817 & 0.01528 & 1.200 & 4.671 & 3.36 & 40.00 & 21224 . \\
\hline 1435 & 0.371 & 0.02178 & 315.353 & 0.1497 & 0.01524 & 1.820 & 1.741 & 4.96 & 40.00 & 5211. \\
\hline 1438 & 0.370 & 0.02963 & 315.663 & 0.1490 & 0.01518 & 1.120 & 2.371 & 6.56 & 40.00 & 6999. \\
\hline 1441 & 0.368 & 0.03865 & 316.028 & 0.1480 & 0.01514 & 0.880 & 3.090 & 6.56 & 40.00 & 8940. \\
\hline 1444 & 0.367 & 0.04888 & 316.406 & 0.1473 & 0.01514 & 0.810 & 3.890 & 4.96 & 40.00 & 11098. \\
\hline 1447 & 0.368 & 0.06030 & 316.845 & 0.1475 & 0.01516 & 0.560 & 4.772 & 4.96 & 40.00 & 13567. \\
\hline 1450 & 0.306 & 0.02179 & 315.182 & 0.1222 & 0.01529 & 1.990 & 1.742 & 4.96 & 40.00 & 3327. \\
\hline 1453 & 0.308 & 0.02964 & 315.477 & 0.1228 & 0.01522 & 1.290 & 2.375 & 4.96 & 40.00 & 4572. \\
\hline 1456 & 0.309 & 0.03867 & 315.830 & 0.1234 & 0.01517 & 0.860 & 3.101 & 4.96 & 40.00 & 6005. \\
\hline 1459 & 0.310 & 0.04891 & 316.240 & 0.1234 & 0.01516 & 0.680 & 3.915 & 4.96 & 40.00 & 7539. \\
\hline 1462 & 0.308 & 0.06033 & 316.687 & 0.1223 & 0.01515 & 0.580 & 4.818 & 4.96 & 40.00 & 9062 . \\
\hline 1465 & 0.237 & 0.02185 & 314.117 & 0.0940 & 0.01507 & 2.110 & 1.778 & 3.36 & 40.00 & 1940. \\
\hline 1468 & 0.237 & 0.02973 & 314.432 & 0.0939 & 0.01504 & 1.440 & 2.420 & 3.36 & 40.00 & 2623. \\
\hline 1471 & 0.237 & 0.03880 & 314.796 & 0.0938 & 0.01501 & 1.020 & 3.159 & 3.36 & 40.00 & 3403. \\
\hline 1474 & 0.237 & 0.04907 & 315.201 & 0.0935 & 0.01500 & 0.820 & 3.992 & 3.36 & 40.00 & 4258. \\
\hline 1477 & 0.236 & 0.06051 & 315.655 & 0.0933 & 0.01501 & 0.760 & 4.911 & 3.36 & 40.00 & 5183. \\
\hline 1480 & 0.171 & 0.02180 & 315.064 & 0.0667 & 0.01506 & 1.970 & 1.777 & 3.36 & 40.00 & 924. \\
\hline 1483 & 0.172 & 0.02965 & 315.368 & 0.0673 & 0.01502 & 1.140 & 2.423 & 3.36 & 40.00 & 1277. \\
\hline 1486 & 0.171 & 0.03870 & 315.736 & 0.0667 & 0.01500 & 0.790 & 3.164 & 3.36 & 40.00 & 1631. \\
\hline 1489 & 0.170 & 0.04893 & 316.145 & 0.0664 & 0.01500 & 0.570 & 3.998 & 3.36 & 40.00 & 2035 . \\
\hline 1492 & 0.169 & 0.06033 & 316.595 & 0.0657 & 0.01501 & 0.460 & 4.921 & 3.36 & 40.00 & 2435. \\
\hline 1495 & 0.083 & 0.02180 & 314.932 & 0.0322 & 0.01477 & 1.660 & 1.815 & 1.76 & 40.00 & 207. \\
\hline 1498 & 0.085 & 0.02966 & 315.252 & 0.0329 & 0.01476 & 1.110 & 2.470 & 1.76 & 40.00 & 295. \\
\hline 1501 & 0.087 & 0.03870 & 315.627 & 0.0335 & 0.01476 & 0.870 & 3.224 & 1.76 & 40.00 & 398. \\
\hline 1504 & 0.087 & 0.04893 & 316.034 & 0.0335 & 0.01479 & 0.530 & 4.067 & 1.76 & 40.00 & 498. \\
\hline 1507 & 0.086 & 0.06037 & 316.491 & 0.0331 & 0.01482 & 0.430 & 5.004 & 1.76 & 40.00 & 597. \\
\hline 1510 & 0.088 & 0.02111 & 326.167 & 0.0327 & 0.01575 & 1.760 & 1.648 & 1.76 & 40.00 & 174. \\
\hline 1513 & 0.088 & 0.02872 & 326.451 & 0.0327 & 0.01575 & 1.240 & 2.243 & 1.76 & 40.00 & 236. \\
\hline 1516 & 0.089 & 0.03748 & 326.782 & 0.0331 & 0.01576 & 0.740 & 2.924 & 1.76 & 40.00 & 315. \\
\hline
\end{tabular}


Table 27. Thermal conductivity of the binary $30 \%$ R32 / $70 \%$ R 134a mixture in the dilute gas measured with the steady-state technique with bare tungsten hot wires (continued).

\begin{tabular}{|c|c|c|c|c|c|c|c|c|c|c|}
\hline $\begin{array}{l}\text { Run } \\
\text { point }\end{array}$ & $\begin{array}{l}P_{\text {exp }} \\
\mathrm{MPa}\end{array}$ & $\begin{array}{c}Q \\
\mathrm{~W} \cdot \mathrm{m}^{-1}\end{array}$ & $\begin{array}{c}T_{\exp } \\
\mathrm{K} \\
\end{array}$ & $\begin{array}{c}\rho_{\text {calc }} \\
\mathrm{mol} \cdot \mathrm{L}^{-1}\end{array}$ & $\begin{array}{c}\lambda_{\exp } \\
\mathrm{W} \cdot \mathrm{m}^{-1} \cdot \mathrm{K}^{-1} \\
\end{array}$ & $\begin{array}{c}\text { TBAND } \\
\%\end{array}$ & $\begin{array}{c}\Delta T_{\text {avg }} \\
\mathrm{K} \\
\end{array}$ & $\begin{array}{c}t_{\text {start }} \\
\mathrm{s}\end{array}$ & $\begin{array}{c}t_{\text {end }} \\
\mathrm{s}\end{array}$ & $N_{R a}$ \\
\hline 1519 & 0.091 & 0.04739 & 327.158 & 0.0337 & 0.01577 & 0.570 & 3.695 & 1.76 & 40.00 & 411. \\
\hline 1522 & 0.091 & 0.05847 & 327.580 & 0.0338 & 0.01580 & 0.470 & 4.547 & 1.76 & 40.00 & 506. \\
\hline 1525 & 0.174 & 0.02117 & 325.175 & 0.0658 & 0.01605 & 2.000 & 1.620 & 3.36 & 40.00 & 736. \\
\hline 1528 & 0.172 & 0.02879 & 325.456 & 0.0648 & 0.01599 & 1.290 & 2.211 & 3.36 & 40.00 & 969. \\
\hline 1531 & 0.172 & 0.03758 & 325.778 & 0.0649 & 0.01597 & 0.940 & 2.887 & 3.36 & 40.00 & 1265. \\
\hline 1534 & 0.174 & 0.04753 & 326.152 & 0.0656 & 0.01595 & 0.600 & 3.654 & 3.36 & 40.00 & 1631. \\
\hline 1537 & 0.174 & 0.05861 & 326.576 & 0.0654 & 0.01594 & 0.540 & 4.505 & 3.36 & 40.00 & 1990. \\
\hline 1540 & 0.173 & 0.05862 & 326.428 & 0.0652 & 0.01572 & 0.440 & 4.570 & 3.36 & 40.00 & 2008. \\
\hline 1543 & 0.242 & 0.02118 & 324.838 & 0.0923 & 0.01598 & 2.300 & 1.626 & 3.36 & 40.00 & 1517. \\
\hline 1546 & 0.242 & 0.02881 & 325.104 & 0.0924 & 0.01596 & 1.520 & 2.212 & 3.36 & 40.00 & 2061. \\
\hline 1549 & 0.244 & 0.03761 & 325.431 & 0.0933 & 0.01595 & 1.060 & 2.886 & 3.36 & 40.00 & 2736. \\
\hline 1552 & 0.245 & 0.04756 & 325.812 & 0.0934 & 0.01593 & 0.850 & 3.648 & 3.36 & 40.00 & 3455 . \\
\hline 1555 & 0.243 & 0.05865 & 326.213 & 0.0923 & 0.01595 & 0.770 & 4.489 & 3.36 & 40.00 & 4129. \\
\hline 1558 & 0.313 & 0.02119 & 324.689 & 0.1210 & 0.01597 & 1.950 & 1.623 & 4.96 & 40.00 & 2721. \\
\hline 1561 & 0.311 & 0.02882 & 324.963 & 0.1200 & 0.01594 & 1.150 & 2.209 & 4.96 & 40.00 & 3626. \\
\hline 1564 & 0.310 & 0.03761 & 325.289 & 0.1194 & 0.01592 & 0.920 & 2.881 & 4.96 & 40.00 & 4661. \\
\hline 1567 & 0.312 & 0.04756 & 325.658 & 0.1201 & 0.01592 & 0.700 & 3.635 & 4.96 & 40.00 & 5930. \\
\hline 1570 & 0.313 & 0.05868 & 326.065 & 0.1203 & 0.01592 & 0.660 & 4.473 & 4.96 & 40.00 & 7291. \\
\hline 1573 & 0.379 & 0.02121 & 324.217 & 0.1480 & 0.01613 & 1.930 & 1.605 & 4.96 & 40.00 & 4212. \\
\hline 1576 & 0.378 & 0.02885 & 324.492 & 0.1476 & 0.01609 & 1.520 & 2.183 & 4.96 & 40.00 & 5675. \\
\hline 1579 & 0.377 & 0.03766 & 324.811 & 0.1470 & 0.01607 & 0.990 & 2.845 & 4.96 & 40.00 & 7309. \\
\hline 1582 & 0.376 & 0.04763 & 325.166 & 0.1464 & 0.01601 & 0.810 & 3.598 & 4.96 & 40.00 & 9119. \\
\hline 1585 & 0.376 & 0.05875 & 325.572 & 0.1460 & 0.01601 & 0.660 & 4.423 & 4.96 & 40.00 & 11084 . \\
\hline 1588 & 0.434 & 0.02114 & 325.273 & 0.1701 & 0.01624 & 2.020 & 1.585 & 6.56 & 40.00 & 5612. \\
\hline 1591 & 0.431 & 0.02876 & 325.538 & 0.1690 & 0.01618 & 1.350 & 2.157 & 6.56 & 40.00 & 7498. \\
\hline 1594 & 0.431 & 0.03754 & 325.862 & 0.1685 & 0.01613 & 1.040 & 2.813 & 6.56 & 40.00 & 9682. \\
\hline 1597 & 0.430 & 0.04747 & 326.218 & 0.1679 & 0.01612 & 0.870 & 3.543 & 4.96 & 40.00 & 12042 . \\
\hline 1600 & 0.431 & 0.05856 & 326.598 & 0.1680 & 0.01613 & 0.820 & 4.347 & 4.96 & 40.00 & 14729. \\
\hline 1603 & 0.447 & 0.02197 & 334.026 & 0.1699 & 0.01710 & 1.860 & 1.566 & 4.96 & 40.00 & 4994. \\
\hline 1606 & 0.444 & 0.02988 & 334.312 & 0.1687 & 0.01703 & 1.170 & 2.132 & 6.56 & 40.00 & 6672. \\
\hline 1609 & 0.445 & 0.03901 & 334.611 & 0.1688 & 0.01700 & 0.820 & 2.778 & 6.56 & 40.00 & 8673. \\
\hline 1612 & 0.446 & 0.04933 & 334.954 & 0.1690 & 0.01696 & 0.610 & 3.507 & 6.56 & 40.00 & 10951 \\
\hline 1615 & 0.446 & 0.06084 & 335.334 & 0.1687 & 0.01694 & 0.700 & 4.311 & 4.96 & 40.00 & 13337. \\
\hline 1618 & 0.441 & 0.04956 & 333.277 & 0.1681 & 0.01666 & 0.740 & 3.585 & 4.96 & 40.00 & 11266. \\
\hline 1621 & 0.445 & 0.06112 & 333.670 & 0.1693 & 0.01667 & 0.630 & 4.398 & 4.96 & 40.00 & 13968. \\
\hline 1624 & 0.384 & 0.02200 & 333.632 & 0.1451 & 0.01688 & 2.140 & 1.593 & 4.96 & 40.00 & 3596. \\
\hline 1627 & 0.384 & 0.02993 & 333.901 & 0.1448 & 0.01685 & 1.150 & 2.165 & 4.96 & 40.00 & 4857. \\
\hline 1630 & 0.379 & 0.03905 & 334.215 & 0.1430 & 0.01682 & 1.070 & 2.824 & 4.96 & 40.00 & 6138. \\
\hline 1633 & 0.377 & 0.04938 & 334.566 & 0.1420 & 0.01681 & 0.860 & 3.562 & 4.96 & 40.00 & 7598. \\
\hline 1636 & 0.381 & 0.06091 & 334.950 & 0.1432 & 0.01682 & 0.620 & 4.379 & 4.96 & 40.00 & 9469. \\
\hline 1639 & 0.302 & 0.02203 & 333.160 & 0.1132 & 0.01680 & 1.830 & 1.606 & 4.96 & 40.00 & 2124. \\
\hline 1642 & 0.305 & 0.02996 & 333.435 & 0.1142 & 0.01678 & 1.160 & 2.185 & 4.96 & 40.00 & 2935. \\
\hline 1645 & 0.308 & 0.03911 & 333.750 & 0.1154 & 0.01677 & 0.790 & 2.849 & 4.96 & 40.00 & 3903. \\
\hline
\end{tabular}


Table 27. Thermal conductivity of the binary $30 \% \mathrm{R} 32$ / $70 \% \mathrm{R} 134 \mathrm{a}$ mixture in the dilute gas measured with the steady-state technique with bare tungsten hot wires (continued).

\begin{tabular}{|c|c|c|c|c|c|c|c|c|c|c|}
\hline $\begin{array}{l}\text { Run } \\
\text { point }\end{array}$ & $\begin{array}{l}P_{\text {exp }} \\
\mathrm{MPa}\end{array}$ & $\begin{array}{c}Q \\
\mathrm{~W} \cdot \mathrm{m}^{-1}\end{array}$ & $\begin{array}{c}T_{\exp } \\
\mathrm{K}\end{array}$ & $\begin{array}{c}\rho_{\text {calc }} \\
\mathrm{mol} \cdot \mathrm{L}^{-1}\end{array}$ & $\begin{array}{c}\lambda_{\exp } \\
\mathrm{W} \cdot \mathrm{m}^{-1} \cdot \mathrm{K}^{-1}\end{array}$ & $\begin{array}{c}\text { TBAND } \\
\%\end{array}$ & $\begin{array}{c}\Delta T_{a v g} \\
\mathrm{~K}\end{array}$ & $\begin{array}{c}t_{\text {start }} \\
\mathrm{s}\end{array}$ & $\begin{array}{c}t_{\text {end }} \\
\mathrm{s}\end{array}$ & $N_{R a}$ \\
\hline 1648 & 0.309 & 0.04946 & 334.108 & 0.1153 & 0.01675 & 0.620 & 3.599 & 4.96 & 40.00 & 4902. \\
\hline 1651 & 0.304 & 0.06099 & 334.505 & 0.1132 & 0.01656 & 0.440 & 4.481 & 4.96 & 40.00 & 5844. \\
\hline 1654 & 0.238 & 0.02197 & 334.031 & 0.0881 & 0.01678 & 2.010 & 1.607 & 3.36 & 40.00 & 1234. \\
\hline 1657 & 0.241 & 0.02989 & 334.307 & 0.0893 & 0.01679 & 1.340 & 2.183 & 3.36 & 40.00 & 1718. \\
\hline 1660 & 0.241 & 0.03901 & 334.620 & 0.0892 & 0.01678 & 0.930 & 2.848 & 3.36 & 40.00 & 2232 . \\
\hline 1663 & 0.238 & 0.04933 & 334.983 & 0.0877 & 0.01676 & 0.830 & 3.601 & 3.36 & 40.00 & 2711. \\
\hline 1666 & 0.235 & 0.06084 & 335.377 & 0.0866 & 0.01679 & 0.660 & 4.431 & 3.36 & 40.00 & 3232. \\
\hline 1669 & 0.168 & 0.02196 & 333.680 & 0.0616 & 0.01673 & 1.790 & 1.613 & 3.36 & 40.00 & 586. \\
\hline 1672 & 0.167 & 0.02987 & 333.947 & 0.0612 & 0.01670 & 1.420 & 2.198 & 1.76 & 40.00 & 784. \\
\hline 1675 & 0.167 & 0.03899 & 334.263 & 0.0611 & 0.01669 & 0.870 & 2.869 & 3.36 & 40.00 & 1019. \\
\hline 1678 & 0.166 & 0.04930 & 334.627 & 0.0609 & 0.01668 & 0.640 & 3.626 & 3.36 & 40.00 & 1272. \\
\hline 1681 & 0.167 & 0.06079 & 335.044 & 0.0611 & 0.01653 & 0.950 & 4.510 & 1.76 & 40.00 & 1588. \\
\hline 1684 & 0.090 & 0.02181 & 335.975 & 0.0325 & 0.01674 & 2.080 & 1.602 & 1.76 & 40.00 & 152. \\
\hline 1687 & 0.091 & 0.02967 & 336.245 & 0.0327 & 0.01678 & 1.250 & 2.174 & 1.76 & 40.00 & 209. \\
\hline 1690 & 0.090 & 0.03872 & 336.553 & 0.0326 & 0.01676 & 0.840 & 2.841 & 1.76 & 40.00 & 270. \\
\hline 1693 & 0.088 & 0.04897 & 336.930 & 0.0318 & 0.01676 & 0.660 & 3.593 & 1.76 & 40.00 & 325. \\
\hline 1698 & 0.089 & 0.06040 & 337.334 & 0.0320 & 0.01651 & 0.440 & 4.495 & 1.76 & 40.00 & 410. \\
\hline 1701 & 0.091 & 0.02142 & 342.070 & 0.0323 & 0.01737 & 2.240 & 1.516 & 1.76 & 40.00 & 134. \\
\hline 1704 & 0.091 & 0.02914 & 342.309 & 0.0324 & 0.01732 & 1.230 & 2.068 & 1.76 & 40.00 & 185. \\
\hline 1707 & 0.090 & 0.03803 & 342.601 & 0.0318 & 0.01734 & 0.790 & 2.696 & 1.76 & 40.00 & 231. \\
\hline 1710 & 0.089 & 0.04811 & 342.924 & 0.0314 & 0.01736 & 0.590 & 3.408 & 1.76 & 40.00 & 284. \\
\hline 1713 & 0.089 & 0.05933 & 343.310 & 0.0315 & 0.01735 & 0.440 & 4.203 & 1.76 & 40.00 & 351. \\
\hline 1716 & 0.090 & 0.04808 & 342.997 & 0.0320 & 0.01720 & 0.660 & 3.436 & 1.76 & 40.00 & 297. \\
\hline 1719 & 0.090 & 0.05931 & 343.408 & 0.0319 & 0.01702 & 0.480 & 4.283 & 1.76 & 40.00 & 365. \\
\hline 1722 & 0.168 & 0.02140 & 342.363 & 0.0601 & 0.01797 & 2.400 & 1.464 & 1.76 & 40.00 & 466. \\
\hline 1725 & 0.168 & 0.02910 & 342.604 & 0.0601 & 0.01791 & 1.740 & 1.997 & 1.76 & 40.00 & 632. \\
\hline 1728 & 0.167 & 0.02912 & 342.566 & 0.0597 & 0.01755 & 1.720 & 2.038 & 1.76 & 40.00 & 637. \\
\hline 1731 & 0.167 & 0.03800 & 342.875 & 0.0597 & 0.01754 & 1.000 & 2.661 & 3.36 & 40.00 & 830. \\
\hline 1734 & 0.168 & 0.04807 & 343.193 & 0.0599 & 0.01734 & 1.210 & 3.403 & 1.76 & 40.00 & 1064. \\
\hline 1737 & 0.168 & 0.05928 & 343.564 & 0.0599 & 0.01739 & 0.840 & 4.184 & 1.76 & 40.00 & 1305. \\
\hline 1740 & 0.248 & 0.02144 & 341.776 & 0.0896 & 0.01795 & 2.440 & 1.466 & 3.36 & 40.00 & 1080. \\
\hline 1743 & 0.248 & 0.02915 & 342.037 & 0.0896 & 0.01781 & 1.650 & 2.008 & 3.36 & 40.00 & 1475. \\
\hline 1746 & 0.248 & 0.03806 & 342.327 & 0.0895 & 0.01755 & 1.200 & 2.658 & 3.36 & 40.00 & 1944. \\
\hline 1749 & 0.249 & 0.04813 & 342.656 & 0.0898 & 0.01754 & 0.980 & 3.359 & 3.36 & 40.00 & 2463 \\
\hline 1753 & 0.250 & 0.05942 & 343.029 & 0.0900 & 0.01730 & 0.670 & 4.201 & 3.36 & 40.00 & 3086. \\
\hline 1756 & 0.314 & 0.02146 & 341.592 & 0.1144 & 0.01751 & 2.310 & 1.503 & 4.96 & 40.00 & 1867. \\
\hline 1759 & 0.313 & 0.02920 & 341.834 & 0.1141 & 0.01755 & 1.420 & 2.037 & 4.96 & 40.00 & 2511. \\
\hline 1762 & 0.314 & 0.03811 & 342.128 & 0.1141 & 0.01736 & 0.900 & 2.684 & 4.96 & 40.00 & 3298. \\
\hline 1765 & 0.315 & 0.04820 & 342.462 & 0.1145 & 0.01740 & 0.630 & 3.382 & 4.96 & 40.00 & 4166. \\
\hline 1768 & 0.315 & 0.05945 & 342.819 & 0.1143 & 0.01743 & 0.520 & 4.156 & 4.96 & 40.00 & 5092. \\
\hline 1771 & 0.374 & 0.02148 & 341.005 & 0.1374 & 0.01754 & 1.920 & 1.498 & 4.96 & 40.00 & 2780 . \\
\hline 1774 & 0.376 & 0.02922 & 341.256 & 0.1382 & 0.01754 & 1.410 & 2.035 & 4.96 & 40.00 & 3815. \\
\hline 1777 & 0.377 & 0.03815 & 341.549 & 0.1384 & 0.01754 & 1.050 & 2.651 & 4.96 & 40.00 & 4965. \\
\hline
\end{tabular}


Table 27. Thermal conductivity of the binary $30 \% \mathrm{R} 32 / 70 \% \mathrm{R} 134 \mathrm{a}$ mixture in the dilute gas measured with the steady-state technique with bare tungsten hot wires (continued).

\begin{tabular}{|ccccccc|cccc|}
\hline $\begin{array}{c}\text { Run } \\
\text { point }\end{array}$ & $\begin{array}{c}P_{\text {exp }} \\
\mathrm{MPa}\end{array}$ & $\begin{array}{c}Q \\
\mathrm{~W} \cdot \mathrm{m}^{-1}\end{array}$ & $\begin{array}{c}T_{\text {exp }} \\
\mathrm{K}\end{array}$ & $\begin{array}{c}\rho_{\text {calc }} \\
\mathrm{mol} \cdot \mathrm{L}^{-1}\end{array}$ & $\begin{array}{c}\lambda_{\text {exp }} \cdot \mathrm{m}^{-1} \cdot \mathrm{K}^{-1} \\
\%\end{array}$ & $\begin{array}{c}\text { TBAND } \\
\%\end{array}$ & $\begin{array}{c}\Delta T_{\text {avg }} \\
t_{\text {start }}\end{array}$ & $t_{\text {end }}$ & $N_{\text {Ra }}$ \\
\hline 1780 & 0.376 & 0.04825 & 341.875 & 0.1378 & 0.01753 & 0.730 & 3.349 & 4.96 & 40.00 & 6195. \\
1783 & 0.374 & 0.05952 & 342.247 & 0.1368 & 0.01753 & 0.660 & 4.119 & 4.96 & 40.00 & 7471. \\
1786 & 0.430 & 0.02149 & 340.968 & 0.1593 & 0.01808 & 2.430 & 1.452 & 6.56 & 40.00 & 3721. \\
1789 & 0.430 & 0.02924 & 341.196 & 0.1592 & 0.01800 & 1.910 & 1.979 & 4.96 & 40.00 & 5054. \\
1792 & 0.432 & 0.02924 & 341.135 & 0.1600 & 0.01744 & 1.590 & 2.043 & 4.96 & 40.00 & 5280. \\
1795 & 0.431 & 0.03815 & 341.416 & 0.1595 & 0.01749 & 0.920 & 2.650 & 6.56 & 40.00 & 6784. \\
1798 & 0.430 & 0.04826 & 341.735 & 0.1586 & 0.01731 & 0.860 & 3.377 & 4.96 & 40.00 & 8508. \\
1801 & 0.431 & 0.05954 & 342.093 & 0.1590 & 0.01735 & 0.830 & 4.142 & 4.96 & 40.00 & 10457. \\
\hline
\end{tabular}


Table 28. Thermal conductivity of the binary $70 \% \mathrm{R} 32$ / $30 \% \mathrm{R} 134 \mathrm{a}$ mixture in the dilute gas measured with the steady-state technique.

\begin{tabular}{|c|c|c|c|c|c|c|c|c|c|c|}
\hline $\begin{array}{l}\text { Run } \\
\text { point }\end{array}$ & $\begin{array}{l}P_{\exp } \\
\mathrm{MPa}\end{array}$ & $\begin{array}{c}Q \\
\mathrm{~W} \cdot \mathrm{m}^{-1}\end{array}$ & $\begin{array}{c}T_{e x p} \\
\mathrm{~K}\end{array}$ & $\begin{array}{c}\rho_{\text {calc }} \\
\mathrm{mol} \cdot \mathrm{L}^{-1}\end{array}$ & $\begin{array}{c}\lambda_{\exp } \\
\mathrm{W} \cdot \mathrm{m}^{-1} \cdot \mathrm{K}^{-1}\end{array}$ & $\begin{array}{c}\text { TBAND } \\
\%\end{array}$ & $\begin{array}{c}\Delta T_{a v g} \\
\mathrm{~K}\end{array}$ & $\begin{array}{c}t_{\text {start }} \\
\mathrm{s}\end{array}$ & $\begin{array}{c}t_{\text {end }} \\
\mathrm{s}\end{array}$ & $N_{R a}$ \\
\hline 1003 & 0.089 & 0.02831 & 257.587 & 0.0424 & 0.01039 & 0.950 & 3.349 & 1.76 & 40.00 & 778. \\
\hline 1006 & 0.089 & 0.03845 & 258.178 & 0.0424 & 0.01039 & 0.520 & 4.543 & 1.76 & 40.00 & 1044. \\
\hline 1009 & 0.089 & 0.05011 & 258.865 & 0.0423 & 0.01042 & 0.610 & 5.903 & 1.76 & 40.00 & 1335. \\
\hline 1012 & 0.089 & 0.06330 & 259.624 & 0.0421 & 0.01046 & 0.560 & 7.424 & 1.76 & 40.00 & 1645. \\
\hline 1015 & 0.089 & 0.07793 & 260.457 & 0.0422 & 0.01050 & 0.440 & 9.099 & 1.76 & 40.00 & 2005. \\
\hline 1018 & 0.175 & 0.02829 & 257.582 & 0.0858 & 0.01060 & 1.070 & 3.262 & 3.36 & 40.00 & 3575 . \\
\hline 1021 & 0.176 & 0.03842 & 258.163 & 0.0857 & 0.01059 & 0.740 & 4.424 & 3.36 & 40.00 & 4794. \\
\hline 1024 & 0.176 & 0.05008 & 258.829 & 0.0855 & 0.01060 & 0.640 & 5.748 & 3.36 & 40.00 & 6123. \\
\hline 1027 & 0.176 & 0.06324 & 259.562 & 0.0853 & 0.01062 & 0.510 & 7.223 & 3.36 & 40.00 & 7548. \\
\hline 1030 & 0.175 & 0.07791 & 260.382 & 0.0846 & 0.01065 & 0.500 & 8.848 & 3.36 & 40.00 & 8970. \\
\hline 1033 & 0.214 & 0.02829 & 257.556 & 0.1057 & 0.01072 & 0.790 & 3.213 & 4.96 & 40.00 & 5723. \\
\hline 1036 & 0.214 & 0.03843 & 258.131 & 0.1056 & 0.01069 & 0.520 & 4.360 & 4.96 & 40.00 & 7678 . \\
\hline 1039 & 0.215 & 0.05012 & 258.784 & 0.1055 & 0.01069 & 0.400 & 5.662 & 4.96 & 40.00 & 9834. \\
\hline 1042 & 0.215 & 0.06326 & 259.512 & 0.1055 & 0.01071 & 0.700 & 7.106 & 3.36 & 40.00 & 12156. \\
\hline 1045 & 0.215 & 0.07788 & 260.299 & 0.1052 & 0.01075 & 0.430 & 8.677 & 3.36 & 40.00 & 14552. \\
\hline 1048 & 0.222 & 0.02737 & 266.556 & 0.1054 & 0.01122 & 0.780 & 2.975 & 4.96 & 40.00 & 4498. \\
\hline 1051 & 0.222 & 0.03721 & 267.089 & 0.1053 & 0.01121 & 0.570 & 4.039 & 4.96 & 40.00 & 6046 . \\
\hline 1054 & 0.222 & 0.04852 & 267.693 & 0.1051 & 0.01122 & 0.380 & 5.245 & 4.96 & 40.00 & 7743. \\
\hline 1057 & 0.222 & 0.06127 & 268.362 & 0.1045 & 0.01123 & 0.650 & 6.596 & 3.36 & 40.00 & 9501. \\
\hline 1060 & 0.222 & 0.07545 & 269.106 & 0.1041 & 0.01127 & 0.620 & 8.069 & 3.36 & 40.00 & 11381. \\
\hline 1064 & 0.110 & 0.02738 & 266.599 & 0.0510 & 0.01096 & 0.810 & 3.067 & 3.36 & 40.00 & 931. \\
\hline 1067 & 0.110 & 0.03719 & 267.141 & 0.0506 & 0.01097 & 0.860 & 4.163 & 1.76 & 40.00 & 1236 . \\
\hline 1070 & 0.109 & 0.04847 & 267.762 & 0.0503 & 0.01099 & 0.690 & 5.409 & 1.76 & 40.00 & 1569. \\
\hline 1073 & 0.109 & 0.06125 & 268.463 & 0.0502 & 0.01104 & 0.760 & 6.801 & 1.76 & 40.00 & 1948. \\
\hline 1076 & 0.110 & 0.07545 & 269.228 & 0.0503 & 0.01108 & 0.680 & 8.343 & 1.76 & 40.00 & 2375 . \\
\hline 1079 & 0.343 & 0.02737 & 266.493 & 0.1683 & 0.01149 & 0.770 & 2.859 & 6.56 & 40.00 & 13342. \\
\hline 1082 & 0.342 & 0.03721 & $266: 984$ & 0.1674 & 0.01152 & 0.700 & 3.850 & 4.96 & 40.00 & 17532 . \\
\hline 1085 & 0.342 & 0.04851 & 267.538 & 0.1667 & 0.01158 & 0.880 & 4.951 & 3.36 & 40.00 & 22074 . \\
\hline 1088 & 0.342 & 0.06129 & 268.135 & 0.1665 & 0.01166 & 2.220 & 6.157 & 1.76 & 40.00 & 27018 . \\
\hline 1091 & 0.343 & 0.07554 & 268.804 & 0.1662 & 0.01170 & 1.670 & 7.497 & 1.76 & 40.00 & 32331. \\
\hline 1094 & 0.105 & 0.02640 & 276.632 & 0.0468 & 0.01164 & 1.050 & 2.786 & 1.76 & 40.00 & 618. \\
\hline 1097 & 0.105 & 0.03587 & 277.130 & 0.0464 & 0.01162 & 0.920 & 3.791 & 1.76 & 40.00 & 822. \\
\hline 1100 & 0.105 & 0.04678 & 277.700 & 0.0462 & 0.01164 & 0.610 & 4.933 & 1.76 & 40.00 & 1052. \\
\hline 1103 & 0.105 & 0.05910 & 278.347 & 0.0463 & 0.01168 & 0.550 & 6.210 & 1.76 & 40.00 & 1321. \\
\hline 1106 & 0.106 & 0.07285 & 279.048 & 0.0467 & 0.01172 & 0.590 & 7.621 & 1.76 & 40.00 & 1635. \\
\hline 1109 & 0.212 & 0.02642 & 276.593 & 0.0960 & 0.01183 & 1.100 & 2.733 & 3.36 & 40.00 & 2865 . \\
\hline 1112 & 0.211 & 0.03590 & 277.074 & 0.0957 & 0.01181 & 0.910 & 3.712 & 3.36 & 40.00 & 3841. \\
\hline 1115 & 0.211 & 0.04680 & 277.633 & 0.0951 & 0.01183 & 0.720 & 4.824 & 3.36 & 40.00 & 4882. \\
\hline 1118 & 0.210 & 0.05912 & 278.263 & 0.0944 & 0.01184 & 0.580 & 6.076 & 3.36 & 40.00 & 6004. \\
\hline 1121 & 0.210 & 0.07285 & 278.949 & 0.0942 & 0.01187 & 0.540 & 7.449 & 3.36 & 40.00 & 7244. \\
\hline 1124 & 0.317 & 0.02640 & 276.552 & 0.1473 & 0.01205 & 0.980 & 2.659 & 6.56 & 40.00 & 7447. \\
\hline 1127 & 0.318 & 0.03590 & 277.027 & 0.1473 & 0.01201 & 0.550 & 3.610 & 6.56 & 40.00 & 10022 . \\
\hline 1130 & 0.317 & 0.04682 & 277.558 & 0.1464 & 0.01201 & 0.540 & 4.686 & 4.96 & 40.00 & 12718. \\
\hline
\end{tabular}


Table 28. Thermal conductivity of the binary $70 \% \mathrm{R} 32$ / $30 \% \mathrm{R} 134 \mathrm{a}$ mixture in the dilute gas measured with the steady-state technique (continued).

\begin{tabular}{|c|c|c|c|c|c|c|c|c|c|c|}
\hline $\begin{array}{l}\text { Run } \\
\text { point }\end{array}$ & $\begin{array}{l}P_{\text {exp }} \\
\mathrm{MPa}\end{array}$ & $\begin{array}{c}Q \\
\mathrm{~W} \cdot \mathrm{m}^{-1}\end{array}$ & $\begin{array}{c}T_{\exp } \\
\mathrm{K}\end{array}$ & $\begin{array}{c}\rho_{\text {calc }} \\
\mathrm{mol} \cdot \mathrm{L}^{-1}\end{array}$ & $\begin{array}{c}\lambda_{\exp } \\
\mathrm{W} \cdot \mathrm{m}^{-1} \cdot \mathrm{K}^{-1}\end{array}$ & $\begin{array}{c}\text { TBAND } \\
\%\end{array}$ & $\begin{array}{c}\Delta T_{a v g} \\
\mathrm{~K}\end{array}$ & $\begin{array}{c}t_{\text {start }} \\
\mathrm{s}\end{array}$ & $\begin{array}{c}t_{\text {end }} \\
\mathrm{s}\end{array}$ & $N_{R a}$ \\
\hline 1133 & 0.316 & 0.05917 & 278.156 & 0.1455 & 0.01205 & 0.430 & 5.871 & 4.96 & 40.00 & 15559. \\
\hline 1136 & 0.316 & 0.07291 & 278.794 & 0.1451 & 0.01213 & 0.590 & 7.149 & 3.36 & 40.00 & 18615. \\
\hline 1139 & 0.426 & 0.02641 & 276.350 & 0.2031 & 0.01214 & 0.870 & 2.600 & 6.56 & 40.00 & 15995. \\
\hline 1142 & 0.425 & 0.03591 & 276.786 & 0.2019 & 0.01220 & 0.880 & 3.486 & 4.96 & 40.00 & 20943. \\
\hline 1145 & 0.424 & 0.04686 & 277.287 & 0.2008 & 0.01229 & 1.180 & 4.473 & 3.36 & 40.00 & 26264. \\
\hline 1148 & 0.423 & 0.05919 & 277.834 & 0.2001 & 0.01234 & 1.190 & 5.571 & 3.36 & 40.00 & 32066 . \\
\hline 1151 & 0.424 & 0.07293 & 278.423 & 0.2000 & 0.01242 & 1.590 & 6.748 & 1.76 & 40.00 & 38355. \\
\hline 1154 & 0.486 & 0.02643 & 276.284 & 0.2350 & 0.01241 & 1.240 & 2.515 & 4.96 & 40.00 & 22559 . \\
\hline 1157 & 0.487 & 0.03593 & 276.713 & 0.2354 & 0.01246 & 1.520 & 3.362 & 3.36 & 40.00 & 29999. \\
\hline 1160 & 0.488 & 0.04686 & 277.197 & 0.2350 & 0.01246 & 1.270 & 4.324 & 3.36 & 40.00 & 38042 . \\
\hline 1163 & 0.487 & 0.05919 & 277.712 & 0.2339 & 0.01251 & 1.830 & 5.365 & 1.76 & 40.00 & 46087. \\
\hline 1166 & 0.485 & 0.07296 & 278.298 & 0.2323 & 0.01248 & 1.490 & 6.536 & 1.76 & 40.00 & 54528. \\
\hline 1169 & 0.503 & 0.02541 & 287.960 & 0.2304 & 0.01314 & 1.110 & 2.311 & 6.56 & 40.00 & 15716. \\
\hline 1172 & 0.505 & 0.03453 & 288.358 & 0.2307 & 0.01314 & 0.930 & 3.111 & 4.96 & 40.00 & 21099. \\
\hline 1175 & 0.505 & 0.04502 & 288.804 & 0.2305 & 0.01321 & 1.260 & 3.995 & 3.36 & 40.00 & 26820. \\
\hline 1178 & 0.505 & 0.05691 & 289.289 & 0.2296 & 0.01324 & 1.100 & 4.984 & 3.36 & 40.00 & 32867. \\
\hline 1181 & 0.504 & 0.07017 & 289.828 & 0.2285 & 0.01329 & 1.980 & 6.057 & 1.76 & 40.00 & 39105. \\
\hline 1184 & 0.555 & 0.02540 & 287.880 & 0.2569 & 0.01321 & 1.230 . & 2.280 & 6.56 & 40.00 & 20411. \\
\hline 1187 & 0.555 & 0.03452 & 288.262 & 0.2564 & 0.01326 & 1.520 & 3.051 & 3.36 & 40.00 & 26991. \\
\hline 1190 & 0.554 & 0.04503 & 288.701 & 0.2556 & 0.01329 & 1.320 & 3.921 & 3.36 & 40.00 & 34147. \\
\hline 1193 & 0.553 & 0.05694 & 289.185 & 0.2543 & 0.01332 & 2.310 & 4.884 & 1.76 & 40.00 & 41625. \\
\hline 1196 & 0.552 & 0.07020 & 289.703 & 0.2532 & 0.01332 & 1.700 & 5.940 & 1.76 & 40.00 & 49579 . \\
\hline 1199 & 0.388 & 0.02540 & 287.873 & 0.1736 & 0.01292 & 0.930 & 2.383 & 6.56 & 40.00 & 8192. \\
\hline 1202 & 0.387 & 0.03453 & 288.294 & 0.1726 & 0.01288 & 0.730 & 3.233 & 6.56 & 40.00 & 10905. \\
\hline 1205 & 0.387 & 0.04505 & 288.775 & 0.1725 & 0.01288 & 0.670 & 4.194 & 4.96 & 40.00 & 14020 . \\
\hline 1208 & 0.388 & 0.05691 & 289.305 & 0.1726 & 0.01291 & 0.470 & 5.253 & 4.96 & 40.00 & 17440 . \\
\hline 1211 & 0.388 & 0.07013 & 289.873 & 0.1721 & 0.01300 & 0.800 & 6.387 & 3.36 & 40.00 & 20866 . \\
\hline 1214 & 0.291 & 0.02543 & 287.728 & 0.1278 & 0.01276 & 0.980 & 2.433 & 4.96 & 40.00 & 4144. \\
\hline 1217 & 0.291 & 0.03455 & 288.167 & 0.1275 & 0.01271 & 0.790 & 3.310 & 4.96 & 40.00 & 5573. \\
\hline 1220 & 0.291 & 0.04505 & 288.655 & 0.1276 & 0.01272 & 0.560 & 4.301 & 4.96 & 40.00 & 7205. \\
\hline 1223 & 0.293 & 0.05696 & 289.220 & 0.1279 & 0.01273 & 0.370 & 5.415 & 4.96 & 40.00 & 9038. \\
\hline 1226 & 0.293 & 0.07019 & 289.819 & 0.1276 & 0.01274 & 0.370 & 6.641 & 4.96 & 40.00 & 10938. \\
\hline 1229 & 0.191 & 0.02544 & 287.697 & 0.0823 & 0.01264 & 1.120 & 2.467 & 3.36 & 40.00 & 1588. \\
\hline 1232 & 0.191 & 0.03455 & 288.138 & 0.0825 & 0.01262 & 0.670 & 3.355 & 3.36 & 40.00 & 2159. \\
\hline 1235 & 0.191 & 0.04507 & 288.647 & 0.0821 & 0.01262 & 0.540 & 4.370 & 3.36 & 40.00 & 2766. \\
\hline 1238 & 0.190 & 0.05697 & 289.219 & 0.0816 & 0.01265 & 0.470 & 5.507 & 3.36 & 40.00 & 3416. \\
\hline 1241 & 0.190 & 0.07019 & 289.845 & 0.0812 & 0.01266 & 0.350 & 6.768 & 3.36 & 40.00 & 4121. \\
\hline 1244 & 0.085 & 0.02543 & 287.719 & 0.0362 & 0.01243 & 0.980 & 2.514 & 1.76 & 40.00 & 285. \\
\hline 1247 & 0.084 & 0.03455 & 288.148 & 0.0357 & 0.01240 & 0.680 & 3.423 & 1.76 & 40.00 & 377. \\
\hline 1250 & 0.084 & 0.04506 & 288.667 & 0.0356 & 0.01242 & 0.420 & 4.460 & 1.76 & 40.00 & 484. \\
\hline 1253 & 0.085 & 0.05695 & 289.259 & 0.0358 & 0.01243 & 0.350 & 5.628 & 1.76 & 40.00 & 613. \\
\hline 1256 & 0.086 & 0.07017 & 289.901 & 0.0361 & 0.01246 & 0.310 & 6.916 & 1.76 & 40.00 & 760. \\
\hline 1259 & 0.088 & 0.02477 & 295.236 & 0.0365 & 0.01299 & 1.160 & 2.345 & 1.76 & 40.00 & 249. \\
\hline
\end{tabular}


Table 28. Thermal conductivity of the binary $70 \% \mathrm{R} 32$ / $30 \% \mathrm{R} 134 \mathrm{a}$ mixture in the dilute gas measured with the steady-state technique (continued).

\begin{tabular}{|c|c|c|c|c|c|c|c|c|c|c|}
\hline $\begin{array}{l}\text { Run } \\
\text { point }\end{array}$ & $\begin{array}{l}P_{\text {exp }} \\
\mathrm{MPa}\end{array}$ & $\begin{array}{c}Q \\
\mathrm{~W} \cdot \mathrm{m}^{-1}\end{array}$ & $\begin{array}{c}T_{e x p} \\
\mathrm{~K}\end{array}$ & $\begin{array}{c}\rho_{\text {calc }} \\
\mathrm{mol}^{\circ} \mathrm{L}^{-1}\end{array}$ & $\begin{array}{c}\lambda_{\exp } \\
\mathrm{W} \cdot \mathrm{m}^{-1} \cdot \mathrm{K}^{-1}\end{array}$ & $\begin{array}{c}\text { TBAND } \\
\%\end{array}$ & $\begin{array}{c}\Delta T_{\text {avg }} \\
\mathrm{K}\end{array}$ & $\begin{array}{c}t_{\text {start }} \\
\mathrm{s}\end{array}$ & $\begin{array}{c}t_{\text {end }} \\
\mathrm{s}\end{array}$ & $N_{R a}$ \\
\hline 1262 & 0.089 & 0.03368 & 295.669 & 0.0367 & 0.01297 & 0.740 & 3.192 & 1.76 & 40.00 & 341. \\
\hline 1265 & 0.088 & 0.04394 & 296.149 & 0.0362 & 0.01298 & 0.520 & 4.162 & 1.76 & 40.00 & 430. \\
\hline 1268 & 0.087 & 0.05553 & 296.694 & 0.0356 & 0.01300 & 0.330 & 5.250 & 1.76 & 40.00 & 522. \\
\hline 1271 & 0.087 & 0.06843 & 297.286 & 0.0357 & 0.01303 & 0.380 & 6.453 & 1.76 & 40.00 & 642 . \\
\hline 1274 & 0.175 & 0.02469 & 296.327 & 0.0730 & 0.01325 & 1.280 & 2.287 & 3.36 & 40.00 & 1026. \\
\hline 1277 & 0.175 & 0.03356 & 296.738 & 0.0728 & 0.01322 & 0.740 & 3.115 & 3.36 & 40.00 & 1380. \\
\hline 1280 & 0.173 & 0.04378 & 297.213 & 0.0717 & 0.01322 & 0.510 & 4.061 & 3.36 & 40.00 & 1732. \\
\hline 1283 & 0.172 & 0.05533 & 297.740 & 0.0711 & 0.01324 & 0.380 & 5.121 & 3.36 & 40.00 & 2135. \\
\hline 1286 & 0.173 & 0.06821 & 298.329 & 0.0717 & 0.01327 & 0.310 & 6.294 & 3.36 & 40.00 & 2654 . \\
\hline 1289 & 0.277 & 0.02469 & 296.303 & 0.1172 & 0.01343 & 1.180 & 2.251 & 4.96 & 40.00 & 2817. \\
\hline 1292 & 0.275 & 0.03356 & 296.704 & 0.1163 & 0.01336 & 0.750 & 3.068 & 4.96 & 40.00 & 3750 . \\
\hline 1295 & 0.274 & 0.04377 & 297.170 & 0.1157 & 0.01335 & 0.520 & 3.999 & 4.96 & 40.00 & 4804. \\
\hline 1298 & 0.276 & 0.05532 & 297.690 & 0.1161 & 0.01335 & 0.450 & 5.041 & 4.96 & 40.00 & 6068. \\
\hline 1301 & 0.277 & 0.06821 & 298.263 & 0.1164 & 0.01335 & 0.300 & 6.198 & 4.96 & 40.00 & 7447. \\
\hline 1304 & 0.384 & 0.02469 & 296.249 & 0.1657 & 0.01356 & 1.210 & 2.216 & 6.56 & 40.00 & 6043. \\
\hline 1307 & 0.382 & 0.03356 & 296.648 & 0.1645 & 0.01348 & 1.010 & 3.017 & 6.56 & 40.00 & 8049. \\
\hline 1310 & 0.381 & 0.04378 & 297.095 & 0.1636 & 0.01346 & 0.790 & 3.928 & 4.96 & 40.00 & 10283. \\
\hline 1313 & 0.382 & 0.05534 & 297.601 & 0.1639 & 0.01346 & 0.610 & 4.940 & 4.96 & 40.00 & 12898. \\
\hline 1316 & 0.384 & 0.06822 & 298.164 & 0.1643 & 0.01348 & 0.400 & 6.049 & 4.96 & 40.00 & 15768. \\
\hline 1319 & 0.478 & 0.02470 & 296.202 & 0.2096 & 0.01365 & 1.220 & 2.184 & 6.56 & 40.00 & 10322. \\
\hline 1322 & 0.479 & 0.03356 & 296.589 & 0.2095 & 0.01360 & 0.710 & 2.960 & 6.56 & 40.00 & 13898. \\
\hline 1325 & 0.480 & 0.04379 & 297.038 & 0.2098 & 0.01361 & 0.680 & 3.831 & 4.96 & 40.00 & 17930 . \\
\hline 1328 & 0.479 & 0.05536 & 297.510 & 0.2090 & 0.01369 & 0.990 & 4.781 & 3.36 & 40.00 & 22000 . \\
\hline 1331 & 0.477 & 0.06822 & 298.017 & 0.2077 & 0.01376 & 1.120 & 5.817 & 3.36 & 40.00 & 26184 . \\
\hline 1334 & 0.545 & 0.02469 & 296.179 & 0.2418 & 0.01377 & 1.180 & 2.149 & 6.56 & 40.00 & 14335. \\
\hline 1337 & 0.544 & 0.03356 & 296.536 & 0.2412 & 0.01388 & 1.200 & 2.874 & 4.96 & 40.00 & 18954. \\
\hline 1340 & 0.543 & 0.04381 & 296.955 & 0.2402 & 0.01391 & 1.550 & 3.709 & 3.36 & 40.00 & 24049 . \\
\hline 1343 & 0.542 & 0.05537 & 297.428 & 0.2392 & 0.01394 & 1.130 & 4.635 & 3.36 & 40.00 & 29534. \\
\hline 1346 & 0.544 & 0.06824 & 297.935 & 0.2396 & 0.01392 & 1.100 & 5.655 & 3.36 & 40.00 & 35873 . \\
\hline 1349 & 0.566 & 0.02388 & 306.577 & 0.2406 & 0.01465 & 1.610 & 1.965 & 8.16 & 40.00 & 11056. \\
\hline 1352 & 0.567 & 0.03246 & 306.921 & 0.2409 & 0.01457 & 1.060 & 2.667 & 6.56 & 40.00 & 14966. \\
\hline 1355 & 0.566 & 0.04236 & 307.305 & 0.2398 & 0.01458 & 0.940 & 3.453 & 4.96 & 40.00 & 19071. \\
\hline 1358 & 0.564 & 0.05356 & 307.728 & 0.2386 & 0.01464 & 1.390 & 4.315 & 3.36 & 40.00 & 23410 . \\
\hline 1361 & 0.564 & 0.06602 & 308.195 & 0.2381 & 0.01469 & 1.110 & 5.257 & 3.36 & 40.00 & 28188 . \\
\hline 1364 & 0.477 & 0.02389 & 306.465 & 0.2002 & 0.01454 & 1.440 & 1.995 & 8.16 & 40.00 & 7312 . \\
\hline 1367 & 0.475 & 0.03247 & 306.832 & 0.1990 & 0.01442 & 0.900 & 2.721 & 6.56 & 40.00 & 9784. \\
\hline 1370 & 0.478 & 0.04236 & 307.225 & 0.1997 & 0.01438 & 0.770 & 3.540 & 6.56 & 40.00 & 12767. \\
\hline 1373 & 0.479 & 0.05355 & 307.665 & 0.2000 & 0.01440 & 0.650 & 4.445 & 4.96 & 40.00 & 15978. \\
\hline 1376 & 0.477 & 0.06603 & 308.168 & 0.1986 & 0.01445 & 0.990 & 5.429 & 3.36 & 40.00 & 19082. \\
\hline 1379 & 0.379 & 0.02389 & 306.415 & 0.1569 & 0.01437 & 1.380 & 2.029 & 6.56 & 40.00 & 4268. \\
\hline 1382 & 0.382 & 0.03248 & 306.772 & 0.1577 & 0.01429 & 1.040 & 2.765 & 4.96 & 40.00 & 5856 . \\
\hline 1385 & 0.381 & 0.04237 & 307.182 & 0.1570 & 0.01426 & 0.820 & 3.604 & 4.96 & 40.00 & 7525 . \\
\hline 1388 & 0.378 & 0.05358 & 307.641 & 0.1557 & 0.01426 & 0.660 & 4.543 & 4.96 & 40.00 & 9248. \\
\hline
\end{tabular}


Table 28. Thermal conductivity of the binary $70 \% \mathrm{R} 32 / 30 \% \mathrm{R} 134 \mathrm{a}$ mixture in the dilute gas measured with the steady-state technique (continued).

\begin{tabular}{|c|c|c|c|c|c|c|c|c|c|c|}
\hline $\begin{array}{l}\text { Run } \\
\text { point }\end{array}$ & $\begin{array}{l}P_{\text {exp }} \\
\mathrm{MPa}\end{array}$ & $\begin{array}{c}Q \\
\mathrm{~W} \cdot \mathrm{m}^{-1}\end{array}$ & $\begin{array}{c}T_{\exp } \\
\mathrm{K}\end{array}$ & $\begin{array}{c}\rho_{\text {calc }} \\
\mathrm{mol} \cdot \mathrm{L}^{-1}\end{array}$ & $\begin{array}{c}\lambda_{\exp } \\
\mathrm{W} \cdot \mathrm{m}^{-1} \cdot \mathrm{K}^{-1}\end{array}$ & $\begin{array}{c}\text { TBAND } \\
\%\end{array}$ & $\begin{array}{c}\Delta T_{\text {avg }} \\
\mathrm{K}\end{array}$ & $\begin{array}{c}t_{\text {start }} \\
\mathrm{s}\end{array}$ & $\begin{array}{c}t_{\text {end }} \\
\mathrm{s}\end{array}$ & $N_{R a}$ \\
\hline 1391 & 0.378 & 0.06605 & 308.161 & 0.1552 & 0.01426 & 0.490 & 5.580 & 4.96 & 40.00 & 11210. \\
\hline 1394 & 0.292 & 0.02395 & 305.643 & 0.1196 & 0.01420 & 1.500 & 2.065 & 4.96 & 40.00 & 2406 . \\
\hline 1397 & 0.292 & 0.03255 & 306.010 & 0.1195 & 0.01415 & 0.910 & 2.813 & 4.96 & 40.00 & 3256. \\
\hline 1400 & 0.293 & 0.04249 & 306.437 & 0.1196 & 0.01412 & 0.650 & 3.673 & 4.96 & 40.00 & 4235. \\
\hline 1403 & 0.294 & 0.05371 & 306.923 & 0.1198 & 0.01411 & 0.500 & 4.636 & 4.96 & 40.00 & 5339. \\
\hline 1406 & 0.294 & 0.06618 & 307.452 & 0.1197 & 0.01411 & 0.420 & 5.702 & 4.96 & 40.00 & 6515. \\
\hline 1409 & 0.187 & 0.02396 & 305.563 & 0.0756 & 0.01410 & 1.720 & 2.087 & 3.36 & 40.00 & 906. \\
\hline 1412 & 0.187 & 0.03257 & 305.938 & 0.0753 & 0.01404 & 0.920 & 2.848 & 3.36 & 40.00 & 1221. \\
\hline 1415 & 0.187 & 0.04249 & 306.378 & 0.0752 & 0.01396 & 0.580 & 3.732 & 3.36 & 40.00 & 1588. \\
\hline 1418 & 0.187 & 0.05372 & 306.870 & 0.0753 & 0.01398 & 0.510 & 4.710 & 3.36 & 40.00 & 2000 . \\
\hline 1421 & 0.190 & 0.06621 & 307.396 & 0.0762 & 0.01399 & 0.400 & 5.793 & 3.36 & 40.00 & 2505. \\
\hline 1424 & 0.087 & 0.02396 & 305.497 & 0.0346 & 0.01386 & 1.380 & 2.127 & 1.76 & 40.00 & 181. \\
\hline 1427 & 0.088 & 0.03258 & 305.877 & 0.0351 & 0.01382 & 0.790 & 2.899 & 1.76 & 40.00 & 253. \\
\hline 1430 & 0.088 & 0.04251 & 306.313 & 0.0348 & 0.01378 & 0.620 & 3.791 & 1.76 & 40.00 & 324. \\
\hline 1433 & 0.086 & 0.05372 & 306.808 & 0.0342 & 0.01379 & 0.410 & 4.787 & 1.76 & 40.00 & 394. \\
\hline 1436 & 0.086 & 0.06620 & 307.361 & 0.0340 & 0.01382 & 0.320 & 5.888 & 1.76 & 40.00 & 474. \\
\hline 1439 & 0.090 & 0.02308 & 317.374 & 0.0344 & 0.01504 & 1.590 & 1.887 & 1.76 & 40.00 & 141. \\
\hline 1442 & 0.090 & 0.03137 & 317.698 & 0.0345 & 0.01480 & 1.120 & 2.607 & 1.76 & 40.00 & 195. \\
\hline 1445 & 0.089 & 0.04093 & 318.098 & 0.0342 & 0.01474 & 0.690 & 3.415 & 1.76 & 40.00 & 250. \\
\hline 1448 & 0.088 & 0.05177 & 318.535 & 0.0335 & 0.01474 & 0.510 & 4.319 & 1.76 & 40.00 & 303. \\
\hline 1451 & 0.090 & 0.06382 & 319.033 & 0.0341 & 0.01476 & 0.360 & 5.313 & 1.76 & 40.00 & 384. \\
\hline 1454 & 0.179 & 0.02316 & 316.149 & 0.0694 & 0.01498 & 1.840 & 1.900 & 1.76 & 40.00 & 614. \\
\hline 1457 & 0.178 & 0.03149 & 316.486 & 0.0691 & 0.01491 & 0.980 & 2.595 & 3.36 & 40.00 & 830. \\
\hline 1460 & 0.177 & 0.04109 & 316.883 & 0.0684 & 0.01488 & 0.710 & 3.390 & 3.36 & 40.00 & 1057. \\
\hline 1463 & 0.176 & 0.05194 & 317.323 & 0.0681 & 0.01487 & 0.550 & 4.287 & 3.36 & 40.00 & 1317. \\
\hline 1466 & 0.176 & 0.06403 & 317.802 & 0.0681 & 0.01488 & 0.360 & 5.276 & 3.36 & 40.00 & 1611. \\
\hline 1469 & 0.274 & 0.02317 & 315.935 & 0.1078 & 0.01523 & 1.800 & 1.866 & 3.36 & 40.00 & 1541. \\
\hline 1472 & 0.276 & 0.03151 & 316.281 & 0.1085 & 0.01511 & 1.070 & 2.556 & 3.36 & 40.00 & 2130. \\
\hline 1475 & 0.278 & 0.04112 & 316.660 & 0.1090 & 0.01505 & 0.900 & 3.344 & 3.36 & 40.00 & 2803. \\
\hline 1478 & 0.277 & 0.05198 & 317.083 & 0.1086 & 0.01502 & 0.890 & 4.229 & 3.36 & 40.00 & 3500 . \\
\hline 1481 & 0.275 & 0.06411 & 317.581 & 0.1075 & 0.01497 & 0.590 & 5.226 & 3.36 & 40.00 & 4209. \\
\hline 1484 & 0.373 & 0.02318 & 315.845 & 0.1487 & 0.01502 & 1.650 & 1.888 & 4.96 & 40.00 & 3142. \\
\hline 1487 & 0.373 & 0.03152 & 316.175 & 0.1484 & 0.01497 & 1.060 & 2.569 & 4.96 & 40.00 & 4243. \\
\hline 1490 & 0.374 & 0.04114 & 316.561 & 0.1489 & 0.01496 & 0.820 & 3.349 & 4.96 & 40.00 & 5542. \\
\hline 1493 & 0.377 & 0.05202 & 316.997 & 0.1495 & 0.01496 & 0.700 & 4.221 & 4.96 & 40.00 & 7019. \\
\hline 1496 & 0.377 & 0.06414 & 317.473 & 0.1494 & 0.01496 & 0.560 & 5.190 & 4.96 & 40.00 & 8561. \\
\hline 1499 & 0.474 & 0.02320 & 315.641 & 0.1918 & 0.01515 & 1.530 & 1.865 & 6.56 & 40.00 & 5496. \\
\hline 1502 & 0.473 & 0.03155 & 315.969 & 0.1910 & 0.01509 & 1.040 & 2.537 & 6.56 & 40.00 & 7377. \\
\hline 1505 & 0.471 & 0.04117 & 316.355 & 0.1898 & 0.01502 & 0.770 & 3.315 & 6.56 & 40.00 & 9452. \\
\hline 1508 & 0.470 & 0.05206 & 316.784 & 0.1890 & 0.01504 & 0.780 & 4.168 & 4.96 & 40.00 & 11715 . \\
\hline 1511 & 0.470 & 0.06420 & 317.246 & 0.1885 & 0.01506 & 0.550 & 5.111 & 4.96 & 40.00 & 14207. \\
\hline 1514 & 0.541 & 0.02321 & 315.530 & 0.2208 & 0.01519 & 1.630 & 1.854 & 6.56 & 40.00 & 7546 . \\
\hline 1517 & 0.543 & 0.03157 & 315.867 & 0.2215 & 0.01504 & 1.210 & 2.534 & 6.56 & 40.00 & 10349. \\
\hline
\end{tabular}


Table 28. Thermal conductivity of the binary $70 \% \mathrm{R} 32 / 30 \% \mathrm{R} 134 \mathrm{a}$ mixture in the dilute gas measured with the steady-state technique (continued).

\begin{tabular}{|c|c|c|c|c|c|c|c|c|c|c|}
\hline $\begin{array}{l}\text { Run } \\
\text { point }\end{array}$ & $\begin{array}{l}P_{\text {exp }} \\
\mathrm{MPa}\end{array}$ & $\begin{array}{c}Q \\
W \cdot m^{-1}\end{array}$ & $\begin{array}{c}T_{\exp } \\
\mathrm{K}\end{array}$ & $\begin{array}{c}\rho_{\text {calc }} \\
\mathrm{mol}: \mathrm{L}^{-1}\end{array}$ & $\begin{array}{c}\lambda_{\exp } \\
\mathrm{W} \cdot \mathrm{m}^{-1} \cdot \mathrm{K}^{-1}\end{array}$ & $\begin{array}{c}\text { TBAND } \\
\%\end{array}$ & $\begin{array}{c}\Delta T_{a v g} \\
\mathrm{~K}\end{array}$ & $\begin{array}{c}t_{\text {start }} \\
\mathrm{s}\end{array}$ & $\begin{array}{c}t_{\text {end }} \\
\mathrm{s}\end{array}$ & $N_{R a}$ \\
\hline 1520 & 0.545 & 0.04119 & 316.241 & 0.2220 & 0.01504 & 0.760 & 3.286 & 6.56 & 40.00 & 13421. \\
\hline 1523 & 0.544 & 0.05208 & 316.635 & 0.2211 & 0.01509 & 0.730 & 4.118 & 4.96 & 40.00 & 16583. \\
\hline 1526 & 0.541 & 0.06422 & 317.088 & 0.2195 & 0.01517 & 1.180 & 5.023 & 3.36 & 40.00 & 19771. \\
\hline 1529 & 0.564 & 0.02242 & 326.735 & 0.2211 & 0.01618 & 1.720 & 1.686 & 6.56 & 40.00 & 5989. \\
\hline 1532 & 0.566 & 0.03049 & 327.033 & 0.2213 & 0.01609 & 1.150 & 2.296 & 6.56 & 40.00 & 8143. \\
\hline 1535 & 0.564 & 0.03979 & 327.370 & 0.2202 & 0.01602 & 0.810 & 2.997 & 6.56 & 40.00 & 10471. \\
\hline 1538 & 0.561 & 0.05032 & 327.745 & 0.2189 & 0.01600 & 0.610 & 3.779 & 6.56 & 40.00 & 12963. \\
\hline 1541 & 0.560 & 0.06206 & 328.156 & 0.2179 & 0.01605 & 0.640 & 4.622 & 4.96 & 40.00 & 15618. \\
\hline 1544 & 0.467 & 0.02245 & 326.288 & 0.1812 & 0.01579 & 7.910 & 1.736 & 1.76 & 40.00 & 3967. \\
\hline 1547 & 0.469 & 0.03053 & 326.587 & 0.1818 & 0.01587 & 1.040 & 2.343 & 6.56 & 40.00 & 5374. \\
\hline 1550 & 0.470 & 0.03985 & 326.929 & 0.1821 & 0.01588 & 1.000 & 3.047 & 4.96 & 40.00 & 6985. \\
\hline 1553 & 0.468 & 0.05038 & 327.305 & 0.1808 & 0.01589 & 0.550 & 3.840 & 6.56 & 40.00 & 8629. \\
\hline 1556 & 0.466 & 0.06214 & 327.721 & 0.1796 & 0.01591 & 0.620 & 4.715 & 4.96 & 40.00 & 10394. \\
\hline 1559 & 0.385 & 0.02246 & 326.016 & 0.1479 & 0.01589 & 1.710 & 1.731 & 6.56 & 40.00 & 2537. \\
\hline 1562 & 0.381 & 0.03054 & 326.322 & 0.1463 & 0.01576 & 1.340 & 2.369 & 4.96 & 40.00 & 3381. \\
\hline 1565 & 0.383 & 0.03985 & 326.672 & 0.1468 & 0.01578 & 0.990 & 3.081 & 4.96 & 40.00 & 4414. \\
\hline 1568 & 0.386 & 0.05039 & 327.065 & 0.1479 & 0.01581 & 0.630 & 3.881 & 4.96 & 40.00 & 5626. \\
\hline 1571 & 0.383 & 0.06215 & 327.494 & 0.1463 & 0.01584 & 0.450 & 4.766 & 4.96 & 40.00 & 6713. \\
\hline 1574 & 0.271 & 0.02247 & 325.880 & 0.1031 & 0.01584 & 1.870 & 1.742 & 3.36 & 40.00 & 1175 . \\
\hline 1577 & 0.268 & 0.03057 & 326.186 & 0.1018 & 0.01583 & 1.320 & 2.369 & 3.36 & 40.00 & 1549. \\
\hline 1580 & 0.270 & 0.03990 & 326.533 & 0.1024 & 0.01581 & 0.760 & 3.093 & 3.36 & 40.00 & 2043. \\
\hline 1583 & 0.273 & 0.05046 & 326.924 & 0.1034 & 0.01582 & 0.820 & 3.905 & 3.36 & 40.00 & 2622 . \\
\hline 1586 & 0.270 & 0.06222 & 327.367 & 0.1022 & 0.01582 & 0.680 & 4.810 & 3.36 & 40.00 & 3132. \\
\hline 1589 & 0.185 & 0.02249 & 326.070 & 0.0697 & 0.01570 & 1.840 & 1.760 & 1.76 & 40.00 & 520. \\
\hline 1592 & 0.188 & 0.03058 & 326.376 & 0.0706 & 0.01564 & 1.540 & 2.402 & 1.76 & 40.00 & 726. \\
\hline 1595 & 0.190 & 0.03989 & 326.724 & 0.0714 & 0.01565 & 1.040 & 3.129 & 1.76 & 40.00 & 964. \\
\hline 1598 & 0.192 & 0.05042 & 327.110 & 0.0719 & 0.01566 & 0.850 & 3.951 & 1.76 & 40.00 & 1230. \\
\hline 1601 & 0.191 & 0.06216 & 327.554 & 0.0715 & 0.01570 & 0.930 & 4.856 & 1.76 & 40.00 & 1487. \\
\hline 1604 & 0.095 & 0.022 .49 & 325.740 & 0.0354 & 0.01552 & 1.560 & 1.782 & 1.76 & 40.00 & 130. \\
\hline 1607 & 0.093 & 0.03059 & 326.037 & 0.0348 & 0.01549 & 1.060 & 2.428 & 1.76 & 40.00 & 171. \\
\hline 1610 & 0.091 & 0.03991 & 326.405 & 0.0339 & 0.01550 & 0.680 & 3.166 & 1.76 & 40.00 & 211. \\
\hline 1613 & 0.090 & 0.05047 & 326.811 & 0.0333 & 0.01552 & 0.490 & 3.999 & 1.76 & 40.00 & 256. \\
\hline 1616 & 0.092 & 0.06224 & 327.254 & 0.0342 & 0.01554 & 0.320 & 4.924 & 1.76 & 40.00 & 331. \\
\hline 1619 & 0.099 & 0.02175 & 336.927 & 0.0357 & 0.01660 & 2.400 & 1.611 & 1.76 & 40.00 & 108. \\
\hline 1622 & 0.099 & 0.02958 & 337.190 & 0.0355 & 0.01662 & 1.580 & 2.189 & 1.76 & 40.00 & 144. \\
\hline 1625 & 0.096 & 0.03861 & 337.484 & 0.0347 & 0.01664 & 1.020 & 2.853 & 1.76 & 40.00 & 179. \\
\hline 1628 & 0.095 & 0.04881 & 337.848 & 0.0340 & 0.01664 & 0.620 & 3.607 & 1.76 & 40.00 & 217. \\
\hline 1631 & 0.094 & 0.06019 & 338.240 & 0.0336 & 0.01664 & 0.450 & 4.448 & 1.76 & 40.00 & 261. \\
\hline 1632 & 0.099 & 0.04906 & 337.502 & 0.0354 & 0.01641 & 0.650 & 3.676 & 1.76 & 40.00 & 241. \\
\hline 1635 & 0.098 & 0.06047 & 337.891 & 0.0353 & 0.01646 & 0.460 & 4.515 & 1.76 & 40.00 & 294. \\
\hline 1638 & 0.180 & 0.02183 & 336.310 & 0.0654 & 0.01644 & 1.820 & 1.633 & 1.76 & 40.00 & 382. \\
\hline 1641 & 0.179 & 0.02970 & 336.586 & 0.0649 & 0.01650 & 1.250 & 2.212 & 1.76 & 40.00 & 508. \\
\hline 1644 & 0.177 & 0.03877 & 336.900 & 0.0641 & 0.01654 & 1.320 & 2.880 & 1.76 & 40.00 & 643. \\
\hline
\end{tabular}


Table 28. Thermal conductivity of the binary $70 \% \mathrm{R} 32$ / $30 \% \mathrm{R} 134 \mathrm{a}$ mixture in the dilute gas measured with the steady-state technique (continued).

\begin{tabular}{|c|c|c|c|c|c|c|c|c|c|c|}
\hline $\begin{array}{l}\text { Run } \\
\text { point }\end{array}$ & $\begin{array}{l}P_{\text {exp }} \\
\mathrm{MPa}\end{array}$ & $\begin{array}{c}Q \\
\mathrm{~W} \cdot \mathrm{m}^{-1}\end{array}$ & $\begin{array}{c}T_{\exp } \\
\mathrm{K}\end{array}$ & $\begin{array}{c}\rho_{\text {calc }} \\
\mathrm{mol} \cdot \mathrm{L}^{-1}\end{array}$ & $\frac{\lambda_{\exp }}{\mathrm{W} \cdot \mathrm{m}^{-1} \cdot \mathrm{K}^{-1}}$ & $\begin{array}{c}\text { TBAND } \\
\%\end{array}$ & $\begin{array}{c}\Delta T_{a v g} \\
\mathrm{~K}\end{array}$ & $\begin{array}{c}t_{\text {start }} \\
\mathrm{s}\end{array}$ & $\begin{array}{c}t_{\text {end }} \\
\mathrm{s}\end{array}$ & $N_{R a}$ \\
\hline 1647 & 0.175 & 0.04903 & 337.250 & 0.0635 & 0.01657 & 0.880 & 3.636 & 1.76 & 40.00 & 793. \\
\hline 1650 & 0.175 & 0.06047 & 337.649 & 0.0633 & 0.01661 & 0.760 & 4.470 & 1.76 & 40.00 & 966. \\
\hline 1653 & 0.283 & 0.02182 & 336.638 & 0.1038 & 0.01700 & 2.080 & 1.576 & 3.36 & 40.00 & 968. \\
\hline 1656 & 0.283 & 0.02967 & 336.875 & 0.1037 & 0.01693 & 1.340 & 2.150 & 3.36 & 40.00 & 1314. \\
\hline 1659 & 0.282 & 0.03872 & 337.217 & 0.1032 & 0.01667 & 0.920 & 2.848 & 3.36 & 40.00 & 1716. \\
\hline 1662 & 0.280 & 0.04896 & 337.584 & 0.1024 & 0.01669 & 0.710 & 3.595 & 3.36 & 40.00 & 2121. \\
\hline 1665 & 0.280 & 0.06038 & 337.969 & 0.1021 & 0.01669 & 0.580 & 4.428 & 3.36 & 40.00 & 2592. \\
\hline 1668 & 0.387 & 0.02183 & 336.444 & 0.1436 & 0.01696 & 2.400 & 1.578 & 4.96 & 40.00 & 1941. \\
\hline 1671 & 0.390 & 0.02968 & 336.727 & 0.1445 & 0.01686 & 1.420 & 2.156 & 4.96 & 40.00 & 2679 . \\
\hline 1674 & 0.390 & 0.03874 & 337.019 & 0.1446 & 0.01675 & 0.900 & 2.826 & 4.96 & 40.00 & 3508 . \\
\hline 1677 & 0.389 & 0.04899 & 337.378 & 0.1437 & 0.01677 & 0.690 & 3.564 & 4.96 & 40.00 & 4349. \\
\hline 1680 & 0.386 & 0.06042 & 337.764 & 0.1427 & 0.01676 & 0.480 & 4.392 & 4.96 & 40.00 & 5258. \\
\hline 1683 & 0.538 & 0.02185 & 336.223 & 0.2029 & 0.01725 & 2.310 & 1.547 & 6.56 & 40.00 & 4065. \\
\hline 1686 & 0.541 & 0.02971 & 336.515 & 0.2038 & 0.01696 & 1.560 & 2.132 & 6.56 & 40.00 & 5642 . \\
\hline 1689 & 0.542 & 0.03877 & 336.827 & 0.2042 & 0.01693 & 1.090 & 2.778 & 6.56 & 40.00 & 7355. \\
\hline 1692 & 0.541 & 0.04903 & 337.163 & 0.2033 & 0.01681 & 0.690 & 3.528 & 6.56 & 40.00 & 9216. \\
\hline 1695 & 0.539 & 0.06047 & 337.571 & 0.2024 & 0.01684 & 0.840 & 4.328 & 4.96 & 40.00 & 11147. \\
\hline 1698 & 0.464 & 0.02188 & 335.768 & 0.1737 & 0.01690 & 1.930 & 1.583 & 6.56 & 40.00 & 2968. \\
\hline 1701 & 0.462 & 0.02975 & 336.053 & 0.1731 & 0.01661 & 1.470 & 2.186 & 4.96 & 40.00 & 4055. \\
\hline 1704 & 0.465 & 0.03883 & 336.363 & 0.1741 & 0.01666 & 0.930 & 2.839 & 4.96 & 40.00 & 5311. \\
\hline 1707 & 0.468 & 0.04910 & 336.706 & 0.1748 & 0.01670 & 0.800 & 3.572 & 4.96 & 40.00 & 6719. \\
\hline 1710 & 0.465 & 0.06056 & 337.098 & 0.1737 & 0.01673 & 0.590 & 4.387 & 4.96 & 40.00 & 8103. \\
\hline 1713 & 0.561 & 0.02127 & 345.326 & 0.2053 & 0.01784 & 2.550 & 1.457 & 4.96 & 40.00 & 3563. \\
\hline 1716 & 0.563 & 0.02893 & 345.550 & 0.2060 & 0.01780 & 1.470 & 1.982 & 6.56 & 40.00 & 4871. \\
\hline 1719 & 0.564 & 0.03775 & 345.835 & 0.2062 & 0.01779 & 1.010 & 2.581 & 6.56 & 40.00 & 6343. \\
\hline 1722 & 0.563 & 0.04774 & 346.148 & 0.2054 & 0.01759 & 0.720 & 3.291 & 6.56 & 40.00 & 7988. \\
\hline 1725 & 0.561 & 0.05889 & 346.511 & 0.2044 & 0.01760 & 0.550 & 4.044 & 6.56 & 40.00 & 9672. \\
\hline 1728 & 0.467 & 0.02129 & 345.006 & 0.1697 & 0.01797 & 2.470 & 1.451 & 6.56 & 40.00 & 2347. \\
\hline 1731 & 0.467 & 0.02895 & 345.247 & 0.1694 & 0.01794 & 1.620 & 1.974 & 4.96 & 40.00 & 3173. \\
\hline 1734 & 0.468 & 0.03778 & 345.549 & 0.1696 & 0.01745 & 1.120 & 2.643 & 4.96 & 40.00 & 4247. \\
\hline 1737 & 0.469 & 0.04778 & 345.873 & 0.1701 & 0.01748 & 0.900 & 3.330 & 4.96 & 40.00 & 5367. \\
\hline 1740 & 0.470 & 0.05894 & 346.233 & 0.1703 & 0.01753 & 0.660 & 4.086 & 4.96 & 40.00 & 6576. \\
\hline 1743 & 0.377 & 0.02132 & 344.565 & 0.1360 & 0.01792 & 2.510 & 1.460 & 3.36 & 40.00 & 1473. \\
\hline 1746 & 0.377 & 0.02899 & 344.815 & 0.1359 & 0.01762 & 1.730 & 2.016 & 3.36 & 40.00 & 2025 . \\
\hline 1749 & 0.377 & 0.03784 & 345.098 & 0.1357 & 0.01761 & 1.310 & 2.630 & 3.36 & 40.00 & 2625. \\
\hline 1752 & 0.378 & 0.04784 & 345.441 & 0.1360 & 0.01740 & 1.030 & 3.361 & 3.36 & 40.00 & 3363. \\
\hline 1755 & 0.379 & 0.05902 & 345.782 & 0.1363 & 0.01746 & 0.820 & 4.126 & 3.36 & 40.00 & 4129. \\
\hline 1758 & 0.283 & 0.02134 & 344.302 & 0.1014 & 0.01792 & 2.640 & 1.463 & 3.36 & 40.00 & 794. \\
\hline 1761 & 0.284 & 0.02901 & 344.562 & 0.1017 & 0.01757 & 1.860 & 2.027 & 3.36 & 40.00 & 1103. \\
\hline 1764 & 0.285 & 0.03786 & 344.868 & 0.1020 & 0.01728 & 1.090 & 2.688 & 3.36 & 40.00 & 1469. \\
\hline 1767 & 0.286 & 0.04788 & 345.194 & 0.1021 & 0.01725 & 0.800 & 3.402 & 3.36 & 40.00 & 1856. \\
\hline 1770 & 0.286 & 0.05905 & 345.561 & 0.1020 & 0.01732 & 0.590 & 4.176 & 3.36 & 40.00 & 2266 . \\
\hline 1773 & 0.183 & 0.02129 & 344.836 & 0.0650 & 0.01757 & 2.450 & 1.490 & 1.76 & 40.00 & 318. \\
\hline
\end{tabular}


Table 28. Thermal conductivity of the binary $70 \% \mathrm{R} 32$ / $30 \% \mathrm{R} 134 \mathrm{a}$ mixture in the dilute gas measured with the steady-state technique (continued).

\begin{tabular}{|ccccccc|cccc|}
\hline $\begin{array}{c}\text { Run } \\
\text { point }\end{array}$ & $\begin{array}{c}P_{\text {exp }} \\
\mathrm{MPa}\end{array}$ & $\begin{array}{c}Q \\
\mathrm{~W} \cdot \mathrm{m}^{-1}\end{array}$ & $\begin{array}{c}T_{\exp } \\
\mathrm{K}\end{array}$ & $\begin{array}{c}\rho_{\text {calc }} \\
\mathrm{mol} \cdot \mathrm{L}^{-1}\end{array}$ & $\begin{array}{c}\lambda_{\exp } \cdot \mathrm{m}^{-1} \cdot \mathrm{K}^{-1} \\
\text { TBAND }\end{array}$ & $\begin{array}{c}\Delta T_{\text {avg }} \\
t_{\text {start }}\end{array}$ & $t_{\text {end }}$ & $N_{\text {Ra }}$ \\
\hline 1776 & 0.182 & 0.02895 & 345.082 & 0.0645 & 0.01747 & 1.570 & 2.037 & 1.76 & 40.00 & 427. \\
1779 & 0.187 & 0.03779 & 345.365 & 0.0662 & 0.01748 & 1.100 & 2.656 & 1.76 & 40.00 & 586. \\
1782 & 0.188 & 0.04779 & 345.676 & 0.0665 & 0.01744 & 0.830 & 3.366 & 1.76 & 40.00 & 749. \\
1785 & 0.184 & 0.05896 & 346.083 & 0.0651 & 0.01724 & 0.900 & 4.199 & 1.76 & 40.00 & 889. \\
1788 & 0.095 & 0.02132 & 344.452 & 0.0333 & 0.01733 & 2.140 & 1.513 & 1.76 & 40.00 & 82. \\
1791 & 0.096 & 0.02900 & 344.713 & 0.0337 & 0.01708 & 1.350 & 2.089 & 1.76 & 40.00 & 116. \\
1794 & 0.095 & 0.03785 & 345.002 & 0.0335 & 0.01705 & 0.870 & 2.730 & 1.76 & 40.00 & 149. \\
1797 & 0.094 & 0.04786 & 345.346 & 0.0331 & 0.01695 & 0.570 & 3.473 & 1.76 & 40.00 & 185. \\
1800 & 0.093 & 0.05903 & 345.711 & 0.0325 & 0.01703 & 0.440 & 4.262 & 1.76 & 40.00 & 218. \\
\hline
\end{tabular}


Table 29. Thermal conductivity of the binary $30 \% \mathrm{R} 134 \mathrm{a} / 70 \%$ propane mixture in the dilute gas measured with the steady-state technique.

\begin{tabular}{|c|c|c|c|c|c|c|c|c|c|c|}
\hline $\begin{array}{l}\text { Run } \\
\text { point }\end{array}$ & $\begin{array}{l}P_{\exp } \\
\mathrm{MPa}\end{array}$ & $\begin{array}{c}Q \\
W \cdot \mathrm{m}^{-1}\end{array}$ & $\begin{array}{c}T_{e x p} \\
\mathrm{~K}\end{array}$ & $\begin{array}{c}\rho_{\text {calc }} \\
\mathrm{mol} \cdot \mathrm{L}^{-1}\end{array}$ & $\begin{array}{c}\lambda_{\exp } \\
\mathrm{W} \cdot \mathrm{m}^{-1} \cdot \mathrm{K}^{-1}\end{array}$ & $\begin{array}{c}\text { TBAND } \\
\%\end{array}$ & $\begin{array}{c}\Delta \bar{T}_{\text {avg }} \\
\mathrm{K}\end{array}$ & $\begin{array}{c}t_{\text {start }} \\
\mathrm{s}\end{array}$ & $\begin{array}{c}t_{\text {end }} \\
\mathrm{s}\end{array}$ & $N_{R a}$ \\
\hline 1118 & 0.132 & 0.05420 & 243.502 & 0.0672 & 0.01227 & 2.480 & 5.388 & 2.29 & 3.00 & 4516 . \\
\hline 1119 & 0.132 & 0.05420 & 243.511 & 0.0673 & 0.01222 & 2.670 & 5.411 & 2.42 & 4.00 & 4540. \\
\hline 1120 & 0.132 & 0.05419 & 243.537 & 0.0673 & 0.01210 & 2.460 & 5.464 & 3.36 & 40.00 & 4587. \\
\hline 1135 & 0.136 & 0.03989 & 242.761 & 0.0695 & 0.01208 & 3.120 & 4.034 & 3.36 & 40.00 & 3682 . \\
\hline 1138 & 0.135 & 0.05421 & 243.433 & 0.0688 & 0.01229 & 2.660 & 5.378 & 2.05 & 3.00 & 4742. \\
\hline 1139 & 0.134 & 0.05421 & 243.452 & 0.0683 & 0.01220 & 2.980 & 5.417 & 2.26 & 4.00 & 4703. \\
\hline 1140 & 0.133 & 0.05421 & 243.468 & 0.0681 & 0.01208 & 2.660 & 5.470 & 3.36 & 40.00 & 4719. \\
\hline 1158 & 0.124 & 0.05433 & 242.777 & 0.0636 & 0.01242 & 2.510 & 5.340 & 2.05 & 3.00 & 4014. \\
\hline 1159 & 0.124 & 0.05433 & 242.792 & 0.0636 & 0.01237 & 3.040 & 5.362 & 1.94 & 4.00 & 4029. \\
\hline 1160 & 0.124 & 0.05432 & 242.823 & 0.0636 & 0.01226 & 2.920 & 5.410 & 3.36 & 40.00 & 4067. \\
\hline 1178 & 0.135 & 0.05121 & 260.800 & 0.0642 & 0.01413 & 3.450 & 4.437 & 2.05 & 3.00 & 2632. \\
\hline 1179 & 0.136 & 0.05121 & 260.806 & 0.0643 & 0.01408 & 3.980 & 4.451 & 1.94 & 4.00 & 2642 . \\
\hline 1180 & 0.135 & 0.05121 & 260.828 & 0.0642 & 0.01398 & 3.370 & 4.485 & 3.36 & 40.00 & 2658. \\
\hline 1198 & 0.280 & 0.05135 & 259.874 & 0.1379 & 0.01440 & 3.300 & 4.279 & 2.29 & 3.00 & 13619. \\
\hline 1199 & 0.280 & 0.05134 & 259.916 & 0.1379 & 0.01411 & 3.640 & 4.365 & 3.06 & 4.00 & 13875 . \\
\hline 1200 & 0.280 & 0.05134 & 259.947 & 0.1378 & 0.01387 & 3.490 & 4.438 & 4.96 & 40.00 & 14078 . \\
\hline 1218 & 0.294 & 0.04953 & 271.513 & 0.1375 & 0.01575 & 4.240 & 3.796 & 2.29 & 3.00 & 10114. \\
\hline 1219 & 0.294 & 0.04952 & 271.530 & 0.1375 & 0.01559 & 4.300 & 3.836 & 2.42 & 4.00 & 10209. \\
\hline 1220 & 0.294 & 0.04951 & 271.582 & 0.1374 & 0.01522 & 4.180 & 3.925 & 4.96 & 40.00 & 10433. \\
\hline 1238 & 0.111 & 0.04955 & 271.442 & 0.0500 & 0.01510 & 3.390 & 4.027 & 1.93 & 3.00 & 1242. \\
\hline 1239 & 0.111 & 0.04955 & 271.427 & 0.0500 & 0.01509 & 3.340 & 4.030 & 1.78 & 4.00 & 1244. \\
\hline 1240 & 0.111 & 0.04955 & 271.427 & 0.0501 & 0.01504 & 3.010 & 4.044 & 1.76 & 40.00 & 1251. \\
\hline 1258 & 0.389 & 0.04952 & 271.205 & 0.1859 & 0.01578 & 4.200 & 3.724 & 2.41 & 3.00 & 19906. \\
\hline 1259 & 0.389 & 0.04951 & 271.231 & 0.1860 & 0.01556 & 3.690 & 3.773 & 2.90 & 4.00 & 20174 . \\
\hline 1260 & 0.389 & 0.04951 & 271.245 & 0.1860 & 0.01546 & 4.210 & 3.798 & 3.36 & 40.00 & 20307. \\
\hline 1318 & 0.160 & 0.04806 & 281.237 & 0.0704 & 0.01615 & 3.520 & 3.647 & 2.29 & 3.00 & 2034. \\
\hline 1319 & 0.161 & 0.04809 & 281.213 & 0.0705 & 0.01616 & 4.080 & 3.647 & 1.94 & 4.00 & 2041. \\
\hline 1320 & 0.160 & 0.04809 & 281.237 & 0.0701 & 0.01607 & 3.680 & 3.668 & 3.36 & 40.00 & 2029 . \\
\hline 1338 & 0.304 & 0.04833 & 279.583 & 0.1376 & 0.01656 & 4.170 & 3.535 & 2.17 & 3.00 & 8430 . \\
\hline 1339 & 0.304 & 0.04833 & 279.608 & 0.1375 & 0.01634 & 4.210 & 3.582 & 2.58 & 4.00 & 8534. \\
\hline 1340 & 0.304 & 0.04833 & 279.649 & 0.1376 & 0.01600 & 4.190 & 3.657 & 4.96 & 40.00 & 8707. \\
\hline 1378 & 0.167 & 0.04666 & 291.258 & 0.0705 & 0.01715 & 4.100 & 3.337 & 2.17 & 3.00 & 1662. \\
\hline 1379 & 0.167 & 0.04666 & 291.251 & 0.0705 & 0.01714 & 4.780 & 3.339 & 1.78 & 4.00 & 1663. \\
\hline 1380 & 0.167 & 0.04666 & 291.262 & 0.0705 & 0.01703 & 4.250 & 3.359 & 3.36 & 40.00 & 1673. \\
\hline 1398 & 0.277 & 0.04673 & 290.999 & 0.1192 & 0.01773 & 4.370 & 3.213 & 2.41 & 3.00 & 4858. \\
\hline 1399 & 0.277 & 0.04673 & 291.015 & 0.1191 & 0.01770 & 5.300 & 3.218 & 1.78 & 4.00 & 4859. \\
\hline 1400 & 0.276 & 0.04673 & 291.026 & 0.1190 & 0.01731 & 4.240 & 3.291 & 4.96 & 40.00 & 4957. \\
\hline 1448 & 0.138 & 0.02411 & 299.091 & 0.0565 & 0.01873 & 2.360 & 1.582 & 1.93 & 3.00 & 457. \\
\hline 1449 & 0.138 & 0.02411 & 299.068 & 0.0567 & 0.01873 & 2.450 & 1.582 & 1.94 & 4.00 & 460. \\
\hline 1450 & 0.139 & 0.02411 & 299.065 & 0.0568 & 0.01874 & 2.410 & 1.581 & 3.36 & 40.00 & 462. \\
\hline 1453 & 0.140 & 0.03469 & 299.419 & 0.0573 & 0.01850 & 1.430 & 2.303 & 1.93 & 3.00 & 681. \\
\hline 1454 & 0.140 & 0.03469 & 299.415 & 0.0574 & 0.01847 & 1.420 & 2.308 & 2.26 & 4.00 & 686. \\
\hline 1455 & 0.140 & 0.03469 & 299.413 & 0.0574 & 0.01845 & 1.900 & 2.310 & 1.76 & 40.00 & 688. \\
\hline
\end{tabular}


Table 29. Thermal conductivity of the binary $30 \% \mathrm{R} 134 \mathrm{a} / 70 \%$ propane mixture in the dilute gas measured with the steady-state technique (continued).

\begin{tabular}{|c|c|c|c|c|c|c|c|c|c|c|}
\hline $\begin{array}{l}\text { Run } \\
\text { point }\end{array}$ & $\begin{array}{l}P_{\text {exp }} \\
\mathrm{MPa}\end{array}$ & $\begin{array}{c}Q \\
\mathrm{~W} \cdot \mathrm{m}^{-1}\end{array}$ & $\begin{array}{c}T_{e x p} \\
\mathrm{~K}\end{array}$ & $\begin{array}{c}\rho_{\text {calc }} \\
\mathrm{mol} \cdot \mathrm{L}^{-1}\end{array}$ & $\begin{array}{c}\lambda_{\exp } \\
\mathrm{W} \cdot \mathrm{m}^{-1} \cdot \mathrm{K}^{-1}\end{array}$ & $\begin{array}{c}\text { TBAND } \\
\%\end{array}$ & $\begin{array}{c}\Delta T_{\text {avg }} \\
\mathrm{K}\end{array}$ & $\begin{array}{c}t_{\text {start }} \\
\mathrm{s}\end{array}$ & $\begin{array}{c}t_{\text {end }} \\
\mathrm{s}\end{array}$ & $N_{R a}$ \\
\hline 1458 & 0.140 & 0.04717 & 299.832 & 0.0571 & 0.01808 & 1.000 & 3.204 & 1.93 & 3.00 & 937. \\
\hline 1459 & 0.139 & 0.04717 & 299.825 & 0.0568 & 0.01805 & 1.070 & 3.209 & 1.94 & 4.00 & 929. \\
\hline 1460 & 0.139 & 0.04717 & 299.831 & 0.0569 & 0.01802 & 1.240 & 3.214 & 1.76 & 40.00 & 935. \\
\hline 1468 & 0.227 & 0.02407 & 301.351 & 0.0933 & 0.01868 & 2.500 & 1.581 & 2.29 & 3.00 & 1262. \\
\hline 1469 & 0.227 & 0.02406 & 301.353 & 0.0933 & 0.01859 & 2.460 & 1.588 & 2.58 & 4.00 & 1268. \\
\hline 1470 & 0.227 & 0.02406 & 301.342 & 0.0933 & 0.01845 & 2.300 & 1.600 & 3.36 & 40.00 & 1278. \\
\hline 1473 & 0.227 & 0.03462 & 301.680 & 0.0931 & 0.01851 & 1.400 & 2.294 & 2.29 & 3.00 & 1819. \\
\hline 1474 & 0.227 & 0.03463 & 301.682 & 0.0931 & 0.01840 & 1.360 & 2.307 & 2.74 & 4.00 & 1829. \\
\hline 1475 & 0.227 & 0.03463 & 301.678 & 0.0931 & 0.01824 & 1.560 & 2.327 & 3.36 & 40.00 & 1844. \\
\hline 1478 & 0.227 & 0.04709 & 302.061 & 0.0929 & 0.01841 & 1.180 & 3.133 & 2.29 & 3.00 & 2463. \\
\hline 1479 & 0.226 & 0.04709 & 302.065 & 0.0929 & 0.01829 & 0.920 & 3.153 & 2.74 & 4.00 & 2476 . \\
\hline 1480 & 0.226 & 0.04709 & 302.066 & 0.0929 & 0.01816 & 1.050 & 3.175 & 3.36 & 40.00 & 2493. \\
\hline
\end{tabular}


Table 30. Thermal conductivity of the binary $70 \% \mathrm{R} 134 \mathrm{a} / 30 \%$ propane mixture in the dilute gas measured using the steady-state technique.

\begin{tabular}{|c|c|c|c|c|c|c|c|c|c|c|}
\hline $\begin{array}{l}\text { Run } \\
\text { point }\end{array}$ & $\begin{array}{l}P_{\text {exp }} \\
\mathrm{MPa}\end{array}$ & $\begin{array}{c}Q \\
\mathrm{~W} \cdot \mathrm{m}^{-1}\end{array}$ & $\begin{array}{c}T_{\text {exp }} \\
\mathrm{K}\end{array}$ & $\begin{array}{c}\rho_{\text {calc }} \\
\mathrm{mol} \cdot \mathrm{L}^{-1}\end{array}$ & $\begin{array}{c}\lambda_{\exp } \\
\mathrm{W} \cdot \mathrm{m}^{-1} \cdot \mathrm{K}^{-1}\end{array}$ & $\begin{array}{c}\text { TBAND } \\
\%\end{array}$ & $\begin{array}{c}\Delta T_{\text {avg }} \\
\mathrm{K}\end{array}$ & $\begin{array}{c}t_{\text {start }} \\
\mathrm{s}\end{array}$ & $\begin{array}{c}t_{\text {end }} \\
\mathrm{s}\end{array}$ & $N_{R a}$ \\
\hline 1018 & 0.087 & 0.02407 & 257.272 & 0.0417 & 0.01176 & 0.950 & 2.513 & 3.36 & 40.00 & 974. \\
\hline 1021 & 0.088 & 0.03274 & 257.728 & 0.0420 & 0.01176 & 0.560 & 3.415 & 3.36 & 40.00 & 1336. \\
\hline 1024 & 0.087 & 0.04271 & 258.246 & 0.0416 & 0.01178 & 0.380 & 4.445 & 3.36 & 40.00 & 1690. \\
\hline 1027 & 0.087 & 0.05399 & 258.825 & 0.0414 & 0.01182 & 0.290 & 5.598 & 3.36 & 40.00 & 2090 . \\
\hline 1030 & 0.088 & 0.06655 & 259.455 & 0.0414 & 0.01186 & 0.210 & 6.872 & 3.36 & 40.00 & 2555 . \\
\hline 1033 & 0.162 & 0.02409 & 257.243 & 0.0789 & 0.01190 & 1.100 & 2.473 & 4.96 & 40.00 & 3788. \\
\hline 1036 & 0.161 & 0.03275 & 257.690 & 0.0784 & 0.01187 & 0.720 & 3.361 & 4.96 & 40.00 & 5052. \\
\hline 1039 & 0.162 & 0.04271 & 258.208 & 0.0787 & 0.01187 & 0.520 & 4.372 & 4.96 & 40.00 & 6557. \\
\hline 1042 & 0.163 & 0.05399 & 258.764 & 0.0789 & 0.01189 & 0.430 & 5.504 & 4.96 & 40.00 & 8231. \\
\hline 1045 & 0.162 & 0.06655 & 259.391 & 0.0783 & 0.01192 & 0.660 & 6.744 & 3.36 & 40.00 & 9824. \\
\hline 1048 & 0.094 & 0.02339 & 266.473 & 0.0433 & 0.01257 & 1.450 & 2.285 & 1.76 & 40.00 & 844. \\
\hline 1051 & 0.092 & 0.03179 & 266.894 & 0.0425 & 0.01255 & 1.400 & 3.111 & 1.76 & 40.00 & 1100. \\
\hline 1054 & 0.092 & 0.04148 & 267.360 & 0.0423 & 0.01256 & 0.910 & 4.052 & 1.76 & 40.00 & 1413. \\
\hline 1057 & 0.093 & 0.05242 & 267.890 & 0.0426 & 0.01258 & 0.940 & 5.110 & 1.76 & 40.00 & 1796. \\
\hline 1060 & 0.093 & 0.06464 & 268.473 & 0.0427 & 0.01262 & 0.810 & 6.277 & 1.76 & 40.00 & 2193. \\
\hline 1063 & 0.193 & 0.02339 & 266.450 & 0.0908 & 0.01276 & 1.070 & 2.239 & 4.96 & 40.00 & 4043 \\
\hline 1066 & 0.193 & 0.03180 & 266.853 & 0.0910 & 0.01273 & 0.770 & 3.041 & 4.96 & 40.00 & 5477. \\
\hline 1069 & 0.193 & 0.04148 & 267.329 & 0.0906 & 0.01272 & 0.610 & 3.960 & 4.96 & 40.00 & 7025 . \\
\hline 1072 & 0.192 & 0.05245 & 267.833 & 0.0900 & 0.01272 & 0.440 & 4.990 & 4.96 & 40.00 & 8642 . \\
\hline 1075 & 0.193 & 0.06467 & 268.393 & 0.0900 & 0.01276 & 0.700 & 6.115 & 3.36 & 40.00 & 10513. \\
\hline 1078 & 0.285 & 0.02339 & 266.398 & 0.1378 & 0.01292 & 1.210 & 2.186 & 6.56 & 40.00 & 10271. \\
\hline 1081 & 0.285 & 0.03180 & 266.776 & 0.1374 & 0.01290 & 0.870 & 2.958 & 4.96 & 40.00 & 13705. \\
\hline 1084 & 0.286 & 0.04149 & 267.200 & 0.1375 & 0.01297 & 1.190 & 3.812 & 3.36 & 40.00 & 17564. \\
\hline 1087 & 0.286 & 0.05246 & 267.687 & 0.1373 & 0.01297 & 1.050 & 4.783 & 3.36 & 40.00 & 21735. \\
\hline 1090 & 0.285 & 0.06468 & 268.203 & 0.1365 & 0.01306 & 0.970 & 5.814 & 3.36 & 40.00 & 25826 . \\
\hline 1093 & 0.084 & 0.02268 & 276.358 & 0.0370 & 0.01344 & 1.440 & 2.074 & 1.76 & 40.00 & 487. \\
\hline 1096 & 0.085 & 0.03085 & 276.725 & 0.0376 & 0.01341 & 1.040 & 2.826 & 1.76 & 40.00 & 682. \\
\hline 1099 & 0.086 & 0.04025 & 277.172 & 0.0379 & 0.01337 & 0.860 & 3.697 & 1.76 & 40.00 & 906. \\
\hline 1102 & 0.086 & 0.05090 & 277.649 & 0.0380 & 0.01339 & 0.660 & 4.666 & 1.76 & 40.00 & 1144. \\
\hline 1105 & 0.085 & 0.06276 & 278.188 & 0.0375 & 0.01342 & 0.540 & 5.739 & 1.76 & 40.00 & 1357. \\
\hline 1108 & 0.178 & 0.02270 & 276.348 & 0.0803 & 0.01358 & 1.540 & 2.047 & 3.36 & 40.00 & 2454 \\
\hline 1111 & 0.179 & 0.03086 & 276.709 & 0.0808 & 0.01353 & 1.180 & 2.789 & 3.36 & 40.00 & 3368. \\
\hline 1114 & 0.181 & 0.04026 & 277.129 & 0.0811 & 0.01349 & 0.600 & 3.642 & 4.96 & 40.00 & 4412. \\
\hline 1117 & 0.180 & 0.05089 & 277.611 & 0.0809 & 0.01350 & 0.800 & 4.590 & 3.36 & 40.00 & 5493. \\
\hline 1120 & 0.180 & 0.06275 & 278.134 & 0.0804 & 0.01351 & 0.650 & 5.643 & 3.36 & 40.00 & 6609. \\
\hline 1123 & 0.263 & 0.02269 & 276.288 & 0.1205 & 0.01378 & 1.530 & 2.003 & 4.96 & 40.00 & 5852. \\
\hline 1126 & 0.262 & 0.03085 & 276.657 & 0.1198 & 0.01372 & 0.960 & 2.727 & 4.96 & 40.00 & 7820 . \\
\hline 1129 & 0.260 & 0.04026 & 277.082 & 0.1190 & 0.01360 & 0.800 & 3.576 & 4.96 & 40.00 & 10036. \\
\hline 1132 & 0.261 & 0.05091 & 277.535 & 0.1191 & 0.01364 & 0.490 & 4.488 & 4.96 & 40.00 & 12524. \\
\hline 1135 & 0.261 & 0.06278 & 278.038 & 0.1191 & 0.01370 & 0.770 & 5.483 & 3.36 & 40.00 & 15184. \\
\hline 1138 & 0.341 & 0.02270 & 276.282 & 0.1595 & 0.01386 & 1.350 & 1.975 & 6.56 & 40.00 & 10993. \\
\hline 1141 & 0.341 & 0.03087 & 276.624 & 0.1592 & 0.01382 & 1.110 & 2.674 & 4.96 & 40.00 & 14730. \\
\hline 1144 & 0.339 & 0.04028 & 277.008 & 0.1580 & 0.01388 & 1.390 & 3.451 & 3.36 & 40.00 & 18552. \\
\hline
\end{tabular}


Table 30. Thermal conductivity of the binary $70 \% \mathrm{R} 134 \mathrm{a} / 30 \%$ propane mixture in the dilute gas measured using the steady-state technique (continued).

\begin{tabular}{|c|c|c|c|c|c|c|c|c|c|c|}
\hline $\begin{array}{l}\text { Run } \\
\text { point }\end{array}$ & $\begin{array}{l}P_{e x p} \\
\mathrm{MPa}\end{array}$ & $\begin{array}{c}Q \\
W \cdot \mathrm{m}^{-1}\end{array}$ & $\begin{array}{c}T_{\exp } \\
\mathrm{K}\end{array}$ & $\begin{array}{c}\rho_{\text {calc }} \\
\mathrm{mol} \cdot \mathrm{L}^{-1}\end{array}$ & $\begin{array}{c}\lambda_{\exp } \\
\mathrm{W} \cdot \mathrm{m}^{-1} \cdot \mathrm{K}^{-1}\end{array}$ & $\begin{array}{c}\text { TBAND } \\
\%\end{array}$ & $\begin{array}{c}\Delta T_{\text {avg }} \\
\mathrm{K}\end{array}$ & $\begin{array}{c}t_{\text {start }} \\
\mathrm{s}\end{array}$ & $\begin{array}{c}t_{\text {end }} \\
\mathrm{s}\end{array}$ & $N_{R a}$ \\
\hline 1147 & 0.340 & 0.05092 & 277.446 & 0.1579 & 0.01392 & 1.080 & 4.316 & 3.36 & 40.00 & 23001. \\
\hline 1150 & 0.341 & 0.06280 & 277.921 & 0.1582 & 0.01393 & 0.990 & 5.271 & 3.36 & 40.00 & 27977 \\
\hline 1153 & 0.411 & 0.02270 & 276.244 & 0.1953 & 0.01404 & 1.650 & 1.927 & 4.96 & 40.00 & 17514 \\
\hline 1156 & 0.410 & 0.03087 & 276.583 & 0.1945 & 0.01403 & 1.320 & 2.595 & 4.96 & 40.00 & 23195 \\
\hline 1159 & 0.410 & 0.04027 & 276.971 & 0.1942 & 0.01400 & 1.220 & 3.354 & 3.36 & 40.00 & 29661. \\
\hline 1162 & 0.411 & 0.05092 & 277.381 & 0.1943 & 0.01401 & 2.340 & 4.189 & 1.76 & 40.00 & 36768 . \\
\hline 1165 & 0.412 & 0.06281 & 277.836 & 0.1941 & 0.01402 & 1.700 & 5.096 & 1.76 & 40.00 & 44269 \\
\hline 1168 & 0.426 & 0.02282 & 286.171 & 0.1938 & 0.01498 & 1.890 & 1.827 & 6.56 & 40.00 & 13677. \\
\hline 1171 & 0.430 & 0.03103 & 286.487 & 0.1955 & 0.01493 & 1.200 & 2.471 & 4.96 & 40.00 & 18795 \\
\hline 1174 & 0.432 & 0.04049 & 286.849 & 0.1961 & 0.01493 & 1.390 & 3.193 & 3.36 & 40.00 & 24334 \\
\hline 1177 & 0.430 & 0.05119 & 287.275 & 0.1948 & 0.01487 & 1.060 & 4.013 & 3.36 & 40.00 & 29901. \\
\hline 1180 & 0.428 & 0.06314 & 287.701 & 0.1933 & 0.01490 & 1.220 & 4.893 & 3.36 & 40.00 & 35527 \\
\hline 1213 & 0.353 & 0.02282 & 286.133 & 0.1582 & 0.01466 & 1.450 & 1.884 & 8.16 & 40.00 & 8801. \\
\hline 1216 & 0.354 & 0.03103 & 286.480 & 0.1586 & 0.01457 & 0.950 & 2.563 & 6.56 & 40.00 & 11979. \\
\hline 1219 & 0.350 & 0.04050 & 286.851 & 0.1565 & 0.01461 & 0.750 & 3.319 & 4.96 & 40.00 & 14968. \\
\hline 1222 & 0.348 & 0.05120 & 287.278 & 0.1552 & 0.01469 & 1.170 & 4.148 & 3.36 & 40.00 & 18232. \\
\hline 1225 & 0.352 & 0.06315 & 287.724 & 0.1567 & 0.01474 & 0.930 & 5.059 & 3.36 & 40.00 & 22605 \\
\hline 1228 & 0.269 & 0.02284 & 285.804 & 0.1187 & 0.01452 & 1.520 & 1.919 & 6.56 & 40.00 & 4728 \\
\hline 1231 & 0.268 & 0.03106 & 286.140 & 0.1183 & 0.01447 & 0.860 & 2.610 & 6.56 & 40.00 & 6361. \\
\hline 1234 & 0.269 & 0.04053 & 286.533 & 0.1182 & 0.01445 & 0.870 & 3.399 & 4.96 & 40.00 & 8223. \\
\hline 1237 & 0.270 & 0.05125 & 286.974 & 0.1185 & 0.01446 & 0.530 & 4.279 & 4.96 & 40.00 & 10337. \\
\hline 1240 & 0.271 & 0.06321 & 287.456 & 0.1189 & 0.01449 & 0.450 & 5.243 & 4.96 & 40.00 & 12694. \\
\hline 1243 & 0.553 & 0.03121 & 285.440 & 0.2597 & 0.01491 & 1.710 & 2.407 & 4.96 & 40.00 & 37559 \\
\hline 1246 & 0.553 & 0.04071 & 285.781 & 0.2592 & 0.01493 & 1.660 & 3.084 & 3.36 & 40.00 & 47579 \\
\hline 1258 & 0.446 & 0.03121 & 285.478 & 0.2044 & 0.01472 & 1.460 & 2.509 & 4.96 & 40.00 & 21571 \\
\hline 1261 & 0.445 & 0.04071 & 285.839 & 0.2035 & 0.01478 & 1.290 & 3.226 & 3.36 & 40.00 & 27296 \\
\hline 1264 & 0.444 & 0.05146 & 286.253 & 0.2025 & 0.01482 & 1.450 & 4.022 & 3.36 & 40.00 & 33400 . \\
\hline 1267 & 0.444 & 0.06346 & 286.681 & 0.2023 & 0.01485 & 2.230 & 4.895 & 1.76 & 40.00 & 40271 \\
\hline 1270 & 0.445 & 0.07672 & 287.159 & 0.2024 & 0.01489 & 1.790 & 5.826 & 1.76 & 40.00 & 47622 \\
\hline 1273 & 0.346 & 0.03121 & 285.499 & 0.1554 & 0.01486 & 1.190 & 2.530 & 6.56 & 40.00 & 11446 . \\
\hline 1276 & 0.345 & 0.04071 & 285.876 & 0.1546 & 0.01483 & 0.850 & 3.289 & 4.96 & 40.00 & 14642 . \\
\hline 1279 & 0.344 & 0.05147 & 286.299 & 0.1540 & 0.01485 & 1.100 & 4.125 & 3.36 & 40.00 & 18077 \\
\hline 1282 & 0.344 & 0.06348 & 286.739 & 0.1538 & 0.01490 & 0.960 & 5.039 & 3.36 & 40.00 & 21869 \\
\hline 1285 & 0.345 & 0.07673 & 287.255 & 0.1539 & 0.01491 & 0.950 & 6.041 & 3.36 & 40.00 & 26082 . \\
\hline 1288 & 0.218 & 0.03121 & 285.504 & 0.0954 & 0.01480 & 1.160 & 2.576 & 4.96 & 40.00 & 3957. \\
\hline 1291 & 0.218 & 0.04072 & 285.917 & 0.0951 & 0.01464 & 0.830 & 3.390 & 4.96 & 40.00 & 5150. \\
\hline 1294 & 0.218 & 0.05147 & 286.374 & 0.0952 & 0.01455 & 0.490 & 4.300 & 4.96 & 40.00 & 6511. \\
\hline 1297 & 0.219 & 0.06347 & 286.876 & 0.0954 & 0.01452 & 0.440 & 5.300 & 4.96 & 40.00 & 8007. \\
\hline 1300 & 0.220 & 0.07669 & 287.421 & 0.0955 & 0.01451 & 0.740 & 6.390 & 3.36 & 40.00 & 9595. \\
\hline 1303 & 0.101 & 0.09117 & 288.075 & 0.0430 & 0.01449 & 0.650 & 7.712 & 1.76 & 40.00 & 2153. \\
\hline 1318 & 0.557 & 0.03072 & 296.048 & 0.2489 & 0.01588 & 1.580 & 2.269 & 4.96 & 40.00 & 26335. \\
\hline 1321 & 0.557 & 0.04009 & 296.371 & 0.2485 & 0.01587 & 1.480 & 2.927 & 3.36 & 40.00 & 33662 . \\
\hline 1324 & 0.558 & 0.05068 & 296.745 & 0.2484 & 0.01582 & 1.160 & 3.658 & 3.36 & 40.00 & 41794 \\
\hline
\end{tabular}


Table 30. Thermal conductivity of the binary $70 \% \mathrm{R} 134 \mathrm{a} / 30 \%$ propane mixture in the dilute gas measured using the steady-state technique (continued).

\begin{tabular}{|c|c|c|c|c|c|c|c|c|c|c|}
\hline $\begin{array}{l}\text { Run } \\
\text { point }\end{array}$ & $\begin{array}{l}P_{\text {exp }} \\
\mathrm{MPa}\end{array}$ & $\begin{array}{c}Q \\
\mathrm{~W} \cdot \mathrm{m}^{-1} \\
\end{array}$ & $\begin{array}{c}T_{\exp } \\
\mathrm{K}\end{array}$ & $\begin{array}{c}\rho_{\text {calc }} \\
\mathrm{mol} \cdot \mathrm{L}^{-1}\end{array}$ & $\begin{array}{c}\lambda_{\exp } \\
\mathrm{W} \cdot \mathrm{m}^{-1} \cdot \mathrm{K}^{-1}\end{array}$ & $\begin{array}{c}\text { TBAND } \\
\% \\
\end{array}$ & $\begin{array}{c}\Delta T_{\text {avg }} \\
\mathrm{K} \\
\end{array}$ & $\begin{array}{c}t_{\text {start }} \\
\mathrm{s}\end{array}$ & $\begin{array}{c}t_{\text {end }} \\
\mathrm{s}\end{array}$ & $N_{R a}$ \\
\hline 1333 & 0.439 & 0.03072 & 296.062 & 0.1917 & 0.01584 & 1.170 & 2.322 & 6.56 & 40.00 & 14565. \\
\hline 1336 & 0.439 & 0.04009 & 296.399 & 0.1913 & 0.01586 & 1.110 & 3.005 & 4.96 & 40.00 & 18658. \\
\hline 1339 & 0.438 & 0.05070 & 296.780 & 0.1907 & 0.01590 & 1.130 & 3.762 & 3.36 & 40.00 & 23071 . \\
\hline 1342 & 0.439 & 0.06251 & 297.185 & 0.1907 & 0.01592 & 1.010 & 4.593 & 3.36 & 40.00 & 27995 . \\
\hline 1345 & 0.439 & 0.07556 & 297.626 & 0.1905 & 0.01594 & 1.190 & 5.495 & 3.36 & 40.00 & 33236 . \\
\hline 1348 & 0.314 & 0.03073 & 296.079 & 0.1343 & 0.01561 & 1.030 & 2.392 & 6.56 & 40.00 & 6749. \\
\hline 1351 & 0.314 & 0.04008 & 296.451 & 0.1340 & 0.01551 & 0.850 & 3.129 & 4.96 & 40.00 & 8734. \\
\hline 1354 & 0.316 & 0.05067 & 296.852 & 0.1345 & 0.01548 & 0.680 & 3.946 & 4.96 & 40.00 & 11047. \\
\hline 1357 & 0.317 & 0.06250 & 297.299 & 0.1347 & 0.01550 & 0.460 & 4.839 & 4.96 & 40.00 & 13520. \\
\hline 1360 & 0.315 & 0.07556 & 297.786 & 0.1338 & 0.01556 & 0.770 & 5.804 & 3.36 & 40.00 & 15878. \\
\hline 1363 & 0.213 & 0.03072 & 296.074 & 0.0893 & 0.01550 & 1.280 & 2.424 & 3.36 & 40.00 & 2833. \\
\hline 1366 & 0.213 & 0.04009 & 296.447 & 0.0893 & 0.01542 & 0.730 & 3.176 & 4.96 & 40.00 & 3698. \\
\hline 1369 & 0.212 & 0.05068 & 296.868 & 87 & 0.01540 & 0.820 & 4.015 & 3.36 & 40.00 & 4583. \\
\hline 1372 & 0.211 & 0.06250 & 297.350 & & 0.01534 & 0.670 & 4.962 & 3.36 & 40.00 & 5534. \\
\hline 1375 & 0.210 & 0.07554 & 297.854 & 0.0876 & 0.01536 & 0.640 & $5.979^{\circ}$ & 3.36 & 40.00 & 6560. \\
\hline 1378 & 0.103 & 0.03072 & 296.098 & & 0.01527 & 1.150 & 2.471 & 1.76 & 40.00 & 612. \\
\hline 1381 & 0.104 & 0.04007 & 296.466 & 0.0427 & 0.01520 & 0.770 & 3.238 & 1.76 & 40.00 & 807. \\
\hline 1384 & 0.105 & 0.05067 & 296.894 & & 0.01520 & 0.670 & 4.092 & 1.76 & 40.00 & 1042 . \\
\hline 1387 & 0.106 & 0.06249 & 297.364 & 0.0436 & 0.01521 & 0.850 & 5.042 & 1.76 & 40.00 & 1297. \\
\hline 1390 & 0.106 & 0.07553 & 297.893 & & 0.01523 & 0.540 & 6.084 & 1.76 & 40.00 & 1550. \\
\hline 1393 & 0.554 & 0.02968 & 305.977 & 0.2366 & 0.01675 & 1.550 & 2.108 & 4.96 & 40.00 & 18702. \\
\hline 1396 & 0.552 & 0.03874 & 306.282 & 0.2352 & 0.01676 & 1.210 & 2.724 & 4.96 & 40.00 & 23756 . \\
\hline 1399 & 0.552 & 0.04900 & 306.626 & 0.2351 & 0.01679 & 1.220 & 3.404 & 3.36 & 40.00 & 29519. \\
\hline 1402 & 0.555 & 0.06044 & 307.021 & 0.2358 & 0.01679 & 1.290 & 4.153 & 3.36 & 40.00 & 36063. \\
\hline 1405 & 0.555 & 0.07308 & 307.411 & 0.2358 & 0.01679 & 1.940 & 4.964 & 1.76 & 40.00 & 42846. \\
\hline 1408 & 0.445 & 0.02970 & 305.965 & 0.1866 & 0.01690 & 1.300 & 2.119 & 8.16 & 40.00 & 10935. \\
\hline 1411 & 0.446 & 0.03875 & 306.294 & 0.1867 & 0.01682 & 1.080 & 2.762 & 6.56 & 40.00 & 14215. \\
\hline 1414 & 0.447 & 0.04900 & 306.646 & & 0.01667 & 0.880 & 3.499 & 4.96 & 40.00 & 18020. \\
\hline 1417 & 0.447 & 0.06044 & 307.047 & 0.1869 & 0.01670 & 1.020 & 4.279 & 3.36 & 40.00 & 21843. \\
\hline 1420 & 0.446 & 0.07307 & 307.472 & 0.1859 & 0.01674 & 0.950 & 5.127 & 3.36 & 40.00 & 25724 . \\
\hline 1423 & 0.312 & 0.02970 & 305.963 & 0.1284 & 0.01706 & 1.200 & 2.122 & 4.96 & 40.00 & 4805. \\
\hline 1426 & 0.311 & 0.03875 & 306.297 & 0.1276 & 0.01686 & 1.070 & 2.796 & 4.96 & 40.00 & 6217. \\
\hline 1429 & 0.309 & 0.04901 & 306.672 & 0.1265 & 0.01673 & 0.830 & 3.553 & 4.96 & 40.00 & 7729. \\
\hline 1432 & 0.309 & 0.06046 & 307.114 & 0.1263 & 0.01641 & 0.590 & 4.452 & 4.96 & 40.00 & 9600. \\
\hline 1435 & 0.311 & 0.07308 & 307.571 & 0.1269 & 0.01640 & 0.410 & 5.365 & 4.96 & 40.00 & 11621. \\
\hline 1438 & 0.215 & 0.02971 & 305.978 & 0.0872 & 0.01662 & 1.320 & 2.190 & 3.36 & 40.00 & 2171. \\
\hline 1441 & 0.216 & 0.03877 & 306.318 & 0.0875 & 0.01648 & 1.280 & 2.878 & 3.36 & 40.00 & 2864. \\
\hline 1444 & 0.220 & 0.04901 & 306.716 & 0.0888 & 0.01640 & 0.710 & 3.650 & 3.36 & 40.00 & 3729. \\
\hline 1447 & 0.219 & 0.06044 & 307.133 & 0.0884 & 0.01637 & 0.770 & 4.504 & 3.36 & 40.00 & 4537. \\
\hline 1450 & 0.216 & 0.07304 & 307.606 & 0.0870 & 0.01635 & 0.600 & 5.441 & 3.36 & 40.00 & 5264. \\
\hline 1453 & 0.107 & 0.02963 & 305.431 & 0.0426 & 0.01603 & 1.210 & 2.272 & 1.76 & 40.00 & 511. \\
\hline 1456 & 0.104 & 0.03867 & 305.761 & 0.0415 & 0.01600 & 0.960 & 2.969 & 1.76 & 40.00 & 633. \\
\hline 1459 & 0.103 & 0.04889 & 306.158 & 0.0412 & 0.01602 & 0.770 & 3.750 & 1.76 & 40.00 & 782. \\
\hline
\end{tabular}


Table 30. Thermal conductivity of the binary $70 \% \mathrm{R} 134 \mathrm{a} / 30 \%$ propane mixture in the dilute gas measured using the steady-state technique (continued).

\begin{tabular}{|c|c|c|c|c|c|c|c|c|c|c|}
\hline $\begin{array}{l}\text { Run } \\
\text { point }\end{array}$ & $\begin{array}{l}P_{\text {exp }} \\
\mathrm{MPa}\end{array}$ & $\begin{array}{c}Q \\
\mathrm{~W} \cdot \mathrm{m}^{-1}\end{array}$ & $\begin{array}{c}T_{\exp } \\
\mathrm{K}\end{array}$ & $\begin{array}{c}\rho_{\text {calc }} \\
\mathrm{mol} \cdot \mathrm{L}^{-1}\end{array}$ & $\begin{array}{c}\lambda_{\exp } \\
\mathrm{W} \cdot \mathrm{m}^{-1} \cdot \mathrm{K}^{-1}\end{array}$ & $\begin{array}{c}\text { TBAND } \\
\%\end{array}$ & $\begin{array}{c}\Delta T_{a v g} \\
\mathrm{~K}\end{array}$ & $\begin{array}{c}t_{\text {start }} \\
\mathrm{s}\end{array}$ & $\begin{array}{c}t_{\text {end }} \\
\mathrm{s}\end{array}$ & $N_{R a}$ \\
\hline 1462 & 0.106 & 0.06028 & 306.595 & 0.0420 & 0.01604 & 0.740 & 4.615 & 1.76 & 40.00 & 998. \\
\hline 1465 & 0.109 & 0.07286 & 307.061 & 0.0431 & 0.01606 & 0.540 & 5.568 & 1.76 & 40.00 & 1265. \\
\hline 1468 & 0.552 & 0.03068 & 316.018 & 0.2259 & 0.01766 & 1.250 & 2.081 & 6.56 & 40.00 & 14570 . \\
\hline 1471 & 0.551 & 0.04002 & 316.338 & 0.2253 & 0.01763 & 1.100 & 2.699 & 4.96 & 40.00 & 18711. \\
\hline 1474 & 0.551 & 0.05060 & 316.680 & 0.2250 & 0.01765 & 1.010 & 3.381 & 4.96 & 40.00 & 23265 . \\
\hline 1477 & 0.553 & 0.06240 & 317.042 & 0.2255 & 0.01768 & 1.180 & 4.125 & 3.36 & 40.00 & 28388. \\
\hline 1480 & 0.555 & 0.07543 & 317.452 & 0.2261 & 0.01769 & 1.120 & 4.935 & 3.36 & 40.00 & 34025 . \\
\hline 1483 & 0.427 & 0.03067 & 316.017 & 0.1717 & 0.01784 & 1.800 & 2.084 & 6.56 & 40.00 & 7914. \\
\hline 1486 & 0.426 & 0.04002 & 316.342 & 0.1712 & 0.01768 & 1.000 & 2.732 & 6.56 & 40.00 & 10268. \\
\hline 1489 & 0.427 & 0.05060 & 316.685 & 0.1715 & 0.01764 & 1.080 & 3.446 & 4.96 & 40.00 & 12933. \\
\hline 1492 & 0.429 & 0.06241 & 317.083 & 0.1719 & 0.01764 & 0.650 & 4.229 & 4.96 & 40.00 & 15890. \\
\hline 1495 & 0.430 & 0.07545 & 317.500 & 0.1722 & 0.01768 & 1.070 & 5.072 & 3.36 & 40.00 & 19031. \\
\hline 1498 & 0.315 & 0.03066 & 315.927 & 0.1249 & 0.01756 & 1.240 & 2.132 & 4.96 & 40.00 & 4057. \\
\hline 1501 & 0.318 & 0.04001 & 316.268 & 0.1260 & 0.01746 & 1.050 & 2.791 & 4.96 & 40.00 & 5392. \\
\hline 1504 & 0.319 & 0.05058 & 316.653 & 0.1264 & 0.01739 & 0.660 & 3.533 & 4.96 & 40.00 & 6846. \\
\hline 1507 & 0.317 & 0.06239 & 317.046 & 0.1254 & 0.01735 & 0.550 & 4.358 & 4.96 & 40.00 & 8260. \\
\hline 1510 & 0.315 & 0.07541 & 317.494 & 0.1243 & 0.01734 & 0.410 & 5.255 & 4.96 & 40.00 & 9740. \\
\hline 1513 & 0.207 & 0.03066 & 315.934 & 0.0809 & 0.01740 & 1.460 & 2.161 & 3.36 & 40.00 & 1641. \\
\hline 1516 & 0.210 & 0.04000 & 316.260 & 0.0818 & 0.01731 & 0.930 & 2.831 & 3.36 & 40.00 & 2194. \\
\hline 1519 & 0.208 & 0.05057 & 316.639 & 0.0810 & 0.01726 & 0.740 & 3.587 & 3.36 & 40.00 & 2713. \\
\hline 1522 & 0.205 & 0.06236 & 317.070 & 0.0796 & 0.01724 & 0.620 & 4.423 & 3.36 & 40.00 & 3212. \\
\hline 1525 & 0.204 & 0.07539 & 317.510 & 0.0792 & 0.01724 & 0.530 & 5.341 & 3.36 & 40.00 & 3816 . \\
\hline 1528 & 0.103 & 0.03065 & 315.907 & 0.0399 & 0.01724 & 1.130 & 2.186 & 1.76 & 40.00 & 385. \\
\hline 1531 & 0.108 & 0.03999 & 316.232 & 0.0417 & 0.01717 & 0.970 & 2.862 & 1.76 & 40.00 & 551. \\
\hline 1534 & 0.108 & 0.05057 & 316.623 & 0.0415 & 0.01713 & 0.720 & 3.626 & 1.76 & 40.00 & 688. \\
\hline 1537 & 0.105 & 0.06236 & 317.035 & 0.0403 & 0.01712 & 0.570 & 4.475 & 1.76 & 40.00 & 798. \\
\hline 1540 & 0.104 & 0.07538 & 317.489 & 0.0399 & 0.01713 & 0.460 & 5.404 & 1.76 & 40.00 & 940. \\
\hline 1543 & 0.557 & 0.03059 & 325.689 & 0.2200 & 0.01863 & 1.240 & 1.977 & 9.76 & 40.00 & 11622 . \\
\hline 1546 & 0.558 & 0.03991 & 325.993 & 0.2200 & 0.01859 & 0.990 & 2.570 & 6.56 & 40.00 & 15058. \\
\hline 1549 & 0.554 & 0.05047 & 326.301 & 0.2183 & 0.01858 & 0.800 & 3.231 & 4.96 & 40.00 & 18538. \\
\hline 1552 & 0.554 & 0.06226 & 326.670 & 0.2176 & 0.01864 & 1.350 & 3.947 & 3.36 & 40.00 & 22398 . \\
\hline 1555 & 0.556 & 0.07527 & 327.063 & 0.2184 & 0.01872 & 0.920 & 4.714 & 3.36 & 40.00 & 26845. \\
\hline 1558 & 0.429 & 0.03060 & 325.666 & 0.1664 & 0.01908 & 1.290 & 1.951 & 6.56 & 40.00 & 6205. \\
\hline 1561 & 0.427 & 0.03992 & 325.977 & 0.1655 & 0.01883 & 0.980 & 2.569 & 6.56 & 40.00 & 8042 . \\
\hline 1564 & 0.425 & 0.05048 & 326.328 & 0.1646 & 0.01869 & 0.670 & 3.262 & 6.56 & 40.00 & 10053. \\
\hline 1567 & 0.426 & 0.06227 & 326.692 & 0.1648 & 0.01863 & 0.710 & 4.019 & 4.96 & 40.00 & 12366 . \\
\hline 1570 & 0.427 & 0.07526 & 327.116 & 0.1651 & 0.01861 & 0.520 & 4.842 & 4.96 & 40.00 & 14905. \\
\hline 1573 & 0.304 & 0.03059 & 325.703 & 0.1163 & 0.01836 & 1.230 & 2.038 & 4.96 & 40.00 & 3001. \\
\hline 1576 & 0.305 & 0.03992 & 326.020 & 0.1167 & 0.01830 & 0.880 & 2.665 & 4.96 & 40.00 & 3940 . \\
\hline 1579 & 0.306 & 0.05049 & 326.367 & 0.1167 & 0.01825 & 0.750 & 3.371 & 4.96 & 40.00 & 4971. \\
\hline 1582 & 0.304 & 0.06228 & 326.750 & 0.1157 & 0.01824 & 0.480 & 4.154 & 4.96 & 40.00 & 5988. \\
\hline 1585 & 0.302 & 0.07528 & 327.187 & 0.1148 & 0.01824 & 0.420 & 5.011 & 4.96 & 40.00 & 7072. \\
\hline 1588 & 0.207 & 0.03059 & 325.646 & 0.0782 & 0.01854 & 1.300 & 2.025 & 3.36 & 40.00 & 1296. \\
\hline
\end{tabular}


Table 30. Thermal conductivity of the binary $70 \% \mathrm{R} 134 \mathrm{a} / 30 \%$ propane mixture in the dilute gas measured using the steady-state technique (continued).

\begin{tabular}{|c|c|c|c|c|c|c|c|c|c|c|}
\hline $\begin{array}{l}\text { Run } \\
\text { point }\end{array}$ & $\begin{array}{l}P_{e x p} \\
\mathrm{MPa}\end{array}$ & $\begin{array}{c}Q \\
W \cdot \mathrm{m}^{-1}\end{array}$ & $\begin{array}{c}T_{\exp } \\
\mathrm{K}\end{array}$ & $\begin{array}{c}\rho_{\text {calc }} \\
\mathrm{mol} \cdot \mathrm{L}^{-1}\end{array}$ & $\begin{array}{c}\lambda_{\exp } \\
\mathrm{W} \cdot \mathrm{m}^{-1} \cdot \mathrm{K}^{-1}\end{array}$ & $\begin{array}{c}\text { TBAND } \\
\%\end{array}$ & $\begin{array}{c}\Delta T_{a v g} \\
\mathrm{~K}\end{array}$ & $\begin{array}{c}t_{\text {start }} \\
\mathrm{s}\end{array}$ & $\begin{array}{c}t_{\text {end }} \\
\mathrm{s}\end{array}$ & $N_{R a}$ \\
\hline 1591 & 0.209 & 0.03992 & 325.961 & 0.0788 & 0.01841 & 0.900 & 2.659 & 3.36 & 40.00 & 1726. \\
\hline 1594 & 0.208 & 0.05048 & 326.325 & 0.0786 & 0.01832 & 0.680 & 3.376 & 3.36 & 40.00 & 2174. \\
\hline 1597 & 0.207 & 0.06224 & 326.734 & 0.0778 & 0.01828 & 0.680 & 4.169 & 3.36 & 40.00 & 2615. \\
\hline 1600 & 0.206 & 0.07522 & 327.165 & 0.0776 & 0.01826 & 0.470 & 5.039 & 3.36 & 40.00 & 3128. \\
\hline 1603 & 0.108 & 0.03059 & 325.641 & 0.0403 & 0.01834 & 1.150 & 2.050 & 1.76 & 40.00 & 335. \\
\hline 1606 & 0.107 & 0.03991 & 325.971 & 0.0401 & 0.01822 & 0.900 & 2.692 & 1.76 & 40.00 & 436. \\
\hline 1609 & 0.106 & 0.05047 & 326.335 & 0.0394 & 0.01814 & 0.640 & 3.418 & 1.76 & 40.00 & 532. \\
\hline 1612 & 0.107 & 0.06226 & 326.717 & 0.0397 & 0.01812 & 0.530 & 4.221 & 1.76 & 40.00 & 663. \\
\hline 1615 & 0.108 & 0.07525 & 327.167 & 0.0401 & 0.01811 & 0.460 & 5.104 & 1.76 & 40.00 & 817. \\
\hline 1618 & 0.566 & 0.02960 & 335.785 & 0.2152 & 0.01972 & 1.480 & 1.816 & 8.16 & 40.00 & 9095. \\
\hline 1621 & 0.565 & 0.03862 & 336.049 & 0.2147 & 0.01960 & 1.230 & 2.372 & 6.56 & 40.00 & 11784. \\
\hline 1624 & 0.565 & 0.04885 & 336.364 & 0.2145 & 0.01956 & 1.020 & 2.991 & 4.96 & 40.00 & 14776. \\
\hline 1627 & 0.565 & 0.06025 & 336.695 & 0.2145 & 0.01958 & 0.800 & 3.664 & 4.96 & 40.00 & 18033. \\
\hline 1630 & 0.565 & 0.07284 & 337.059 & 0.2140 & 0.01961 & 1.030 & 4.395 & 3.36 & 40.00 & 21443. \\
\hline 1633 & 0.439 & 0.02959 & 335.743 & 0.1647 & 0.02010 & 1.530 & 1.794 & 6.56 & 40.00 & 5019. \\
\hline 1636 & 0.440 & 0.03862 & 336.019 & 0.1648 & 0.01986 & 1.090 & 2.363 & 6.56 & 40.00 & 6600. \\
\hline 1639 & 0.440 & 0.04884 & 336.339 & 0.1648 & 0.01970 & 0.830 & 3.002 & 6.56 & 40.00 & 8356. \\
\hline 1642 & 0.439 & 0.06024 & 336.692 & 0.1643 & 0.01961 & 0.760 & 3.709 & 4.96 & 40.00 & 10211. \\
\hline 1645 & 0.439 & 0.07283 & 337.072 & 0.1639 & 0.01957 & 0.630 & 4.477 & 4.96 & 40.00 & 12220 . \\
\hline 1648 & 0.311 & 0.02960 & 335.720 & 0.11 & 0.02011 & 1.470 & 1.803 & 4.96 & 40.00 & 2343 . \\
\hline 1651 & 0.312 & 0.03862 & 335.996 & 0.1155 & 0.01986 & 1.090 & 2.379 & 4.96 & 40.00 & 3110. \\
\hline 1654 & 0.314 & 0.04884 & 336.324 & 0.1161 & 0.01969 & 0.820 & 3.028 & 4.96 & 40.00 & 3997. \\
\hline 1657 & 0.314 & 0.06025 & 336.674 & 0.1160 & 0.01961 & 0.590 & 3.746 & 4.96 & 40.00 & 4915. \\
\hline 1660 & 0.313 & 0.07283 & 337.070 & 0.1154 & 0.01953 & 0.420 & 4.537 & 4.96 & 40.00 & 5869. \\
\hline 1663 & 0.213 & 0.02960 & 335.671 & 0.0779 & 0.02008 & 1.580 & 1.810 & 3.36 & 40.00 & 1042. \\
\hline 1666 & 0.213 & 0.03862 & 335.967 & 0.0778 & 0.01982 & 1.190 & 2.391 & 3.36 & 40.00 & 1370 . \\
\hline 1669 & 0.214 & 0.04886 & 336.309 & 0.0781 & 0.01965 & 0.860 & 3.049 & 3.36 & 40.00 & 1757. \\
\hline 1672 & 0.214 & 0.06027 & 336.645 & 0.0781 & 0.01956 & 0.620 & 3.776 & 3.36 & 40.00 & 2167. \\
\hline 1675 & 0.213 & 0.07285 & 337.054 & 0.0776 & 0.01949 & 0.490 & 4.575 & 3.36 & 40.00 & 2582. \\
\hline 1678 & 0.106 & 0.02961 & 335.690 & 0.0385 & 0.01931 & 1.350 & 1.885 & 1.76 & 40.00 & 256. \\
\hline 1681 & 0.107 & 0.03864 & 335.977 & 0.0387 & 0.01921 & 1.060 & 2.473 & 1.76 & 40.00 & 338. \\
\hline 1684 & 0.106 & 0.04885 & 336.309 & 0.0382 & 0.01911 & 0.770 & 3.141 & 1.76 & 40.00 & 416. \\
\hline 1687 & 0.105 & 0.06025 & 336.687 & 0.0380 & 0.01909 & 0.530 & 3.879 & 1.76 & 40.00 & 509. \\
\hline 1690 & 0.107 & 0.07286 & 337.071 & 0.0385 & 0.01908 & 0.480 & 4.692 & 1.76 & 40.00 & 628. \\
\hline 1693 & 0.554 & 0.03739 & 345.343 & 0.2037 & 0.02078 & 1.250 & 2.178 & 6.56 & 40.00 & 8755. \\
\hline 1696 & 0.554 & 0.04728 & 345.615 & 0.2035 & 0.02065 & 0.870 & 2.759 & 6.56 & 40.00 & 11034. \\
\hline 1699 & 0.554 & 0.05832 & 345.938 & 0.2032 & 0.02065 & 0.890 & 3.391 & 4.96 & 40.00 & 13482. \\
\hline 1702 & 0.554 & 0.07052 & 346.252 & 0.2031 & 0.02070 & 0.670 & 4.070 & 4.96 & 40.00 & 16105. \\
\hline 1705 & 0.554 & 0.08385 & 346.607 & 0.2029 & 0.02077 & 1.080 & 4.798 & 3.36 & 40.00 & 18884. \\
\hline 1708 & 0.447 & 0.03740 & 345.267 & 0.1627 & 0.02088 & 1.200 & 2.181 & 6.56 & 40.00 & 5399. \\
\hline 1711 & 0.447 & 0.04728 & 345.546 & 0.1625 & 0.02075 & 1.140 & 2.768 & 4.96 & 40.00 & 6813. \\
\hline 1714 & 0.447 & 0.05832 & 345.882 & 0.1623 & 0.02065 & 1.040 & 3.421 & 4.96 & 40.00 & 8378. \\
\hline 1717 & 0.448 & 0.07051 & 346.204 & 0.1623 & 0.02060 & 0.730 & 4.134 & 4.96 & 40.00 & 10083. \\
\hline
\end{tabular}


Table 30. Thermal conductivity of the binary $70 \% \mathrm{R} 134 \mathrm{a} / 30 \%$ propane mixture in the dilute gas measured using the steady-state technique (continued).

\begin{tabular}{|ccccccc|cccc|}
\hline $\begin{array}{c}\text { Run } \\
\text { point }\end{array}$ & $\begin{array}{c}P_{\text {exp }} \\
\mathrm{MPa}\end{array}$ & $\mathrm{W} \cdot \mathrm{m}^{-1}$ & $\begin{array}{c}T_{\text {exp }} \\
\mathrm{K}\end{array}$ & $\begin{array}{c}\rho_{\text {calc }} \\
\mathrm{mol} \cdot \mathrm{L}^{-1}\end{array}$ & $\begin{array}{c}\lambda_{\text {exp }} \cdot \mathrm{m}^{-1} \cdot \mathrm{K}^{-1} \\
\text { TBAND }\end{array}$ & $\begin{array}{c}\Delta T_{\text {avg }} \\
t_{\text {start }}\end{array}$ & $t_{\text {end }}$ & $N_{R a}$ \\
\hline 1720 & 0.448 & 0.08383 & 346.599 & 0.1621 & 0.02057 & 0.600 & 4.905 & 4.96 & 40.00 & 11897. \\
1723 & 0.313 & 0.03738 & 345.133 & 0.1122 & 0.02104 & 1.140 & 2.176 & 4.96 & 40.00 & 2454. \\
1726 & 0.312 & 0.04727 & 345.425 & 0.1119 & 0.02087 & 0.820 & 2.770 & 4.96 & 40.00 & 3100. \\
1729 & 0.312 & 0.05832 & 345.741 & 0.1119 & 0.02074 & 0.620 & 3.434 & 4.96 & 40.00 & 3830. \\
1732 & 0.312 & 0.07052 & 346.102 & 0.1117 & 0.02067 & 0.510 & 4.161 & 4.96 & 40.00 & 4605. \\
1735 & 0.312 & 0.08386 & 346.483 & 0.1113 & 0.02062 & 0.350 & 4.954 & 4.96 & 40.00 & 5429. \\
1738 & 0.210 & 0.03738 & 345.047 & 0.0745 & 0.02070 & 1.130 & 2.217 & 3.36 & 40.00 & 1067. \\
1741 & 0.209 & 0.04726 & 345.339 & 0.0743 & 0.02058 & 0.850 & 2.818 & 3.36 & 40.00 & 1344. \\
1744 & 0.209 & 0.05830 & 345.660 & 0.0742 & 0.02050 & 0.570 & 3.488 & 3.36 & 40.00 & 1658. \\
1747 & 0.209 & 0.07050 & 346.019 & 0.0742 & 0.02044 & 0.490 & 4.226 & 3.36 & 40.00 & 1998. \\
1750 & 0.209 & 0.08382 & 346.424 & 0.0741 & 0.02041 & 0.430 & 5.029 & 3.36 & 40.00 & 2362. \\
1753 & 0.111 & 0.03735 & 345.035 & 0.0392 & 0.01996 & 1.130 & 2.300 & 1.76 & 40.00 & 298. \\
1756 & 0.110 & 0.04725 & 345.334 & 0.0387 & 0.01995 & 0.680 & 2.911 & 1.76 & 40.00 & 366. \\
1759 & 0.108 & 0.05829 & 345.669 & 0.0379 & 0.01996 & 0.520 & 3.589 & 1.76 & 40.00 & 430. \\
1762 & 0.109 & 0.07047 & 346.014 & 0.0384 & 0.01999 & 0.430 & 4.333 & 1.76 & 40.00 & 533. \\
1765 & 0.111 & 0.08379 & 346.433 & 0.0388 & 0.02001 & 0.420 & 5.144 & 1.76 & 40.00 & 644. \\
\hline
\end{tabular}


Table 31. Thermal conductivity of the ternary $33 \% \mathrm{R} 32$ / $33 \% \mathrm{R} 125$ / $33 \% \mathrm{R} 134 \mathrm{a}$ mixture in the dilute gas measured using the steady-state technique.

\begin{tabular}{|c|c|c|c|c|c|c|c|c|c|c|}
\hline $\begin{array}{l}\text { Run } \\
\text { point }\end{array}$ & $\begin{array}{l}P_{\text {exp }} \\
\mathrm{MPa} \\
\end{array}$ & $\begin{array}{c}Q \\
\mathrm{~W} \cdot \mathrm{m}^{-1}\end{array}$ & $\begin{array}{c}T_{\exp } \\
\mathrm{K} \\
\end{array}$ & $\begin{array}{c}\rho_{\text {calc }} \\
\mathrm{mol} \cdot \mathrm{L}^{-1}\end{array}$ & $\begin{array}{c}\lambda_{\exp } \\
\mathrm{W} \cdot \mathrm{m}^{-1} \cdot \mathrm{K}^{-1}\end{array}$ & $\begin{array}{c}\text { TBAND } \\
\%\end{array}$ & $\begin{array}{c}\Delta T_{\text {avg }} \\
\mathrm{K} \\
\end{array}$ & $\begin{array}{c}t_{\text {start }} \\
\mathrm{s} \\
\end{array}$ & $\begin{array}{c}t_{\text {end }} \\
\mathrm{s}\end{array}$ & $N_{R a}$ \\
\hline 1003 & 0.219 & 0.03483 & 262.493 & 0.1064 & 0.01117 & 0.980 & 3.763 & 4.96 & 40.00 & 10600 . \\
\hline 1006 & 0.220 & 0.04267 & 262.954 & 0.1066 & 0.01112 & 0.610 & 4.608 & 4.96 & 40.00 & 12932. \\
\hline 1009 & 0.222 & 0.05124 & 263.458 & 0.1075 & 0.01111 & 0.450 & 5.516 & 4.96 & 40.00 & 15634. \\
\hline 1012 & 0.220 & 0.06061 & 263.968 & 0.1064 & 0.01114 & 0.640 & 6.481 & 3.36 & 40.00 & 17783. \\
\hline 1015 & 0.219 & 0.07077 & 264.514 & 0.1055 & 0.01120 & 0.600 & 7.494 & 3.36 & 40.00 & 20011. \\
\hline 1018 & 0.214 & 0.03534 & 258.277 & 0.1062 & 0.01100 & 0.860 & 3.869 & 4.96 & 40.00 & 11652. \\
\hline 1021 & 0.214 & 0.04326 & 258.694 & 0.1058 & 0.01102 & 0.600 & 4.708 & 4.96 & 40.00 & 13962. \\
\hline 1024 & 0.214 & 0.05197 & 259.131 & 0.1056 & 0.01110 & 0.680 & 5.591 & 3.36 & 40.00 & 16397. \\
\hline 1027 & 0.214 & 0.06149 & 259.605 & 0.1052 & 0.01117 & 0.540 & 6.545 & 3.36 & 40.00 & 18843. \\
\hline 1030 & 0.213 & 0.07179 & 260.118 & 0.1047 & 0.01124 & 0.940 & 7.557 & 3.36 & 40.00 & 21320 . \\
\hline 1033 & 0.093 & 0.03543 & 257.835 & 0.0443 & 0.01080 & 0.630 & 4.022 & 3.36 & 40.00 & 1748. \\
\hline 1036 & 0.092 & 0.04338 & 258.275 & 0.0440 & 0.01082 & 0.490 & 4.913 & 3.36 & 40.00 & 2093. \\
\hline 1039 & 0.092 & 0.05211 & 258.770 & 0.0439 & 0.01084 & 0.410 & 5.886 & 3.36 & 40.00 & 2475. \\
\hline 1042 & 0.092 & 0.06163 & 259.295 & & 0.01086 & 0.320 & 6.943 & 3.36 & 40.00 & 2880 . \\
\hline 1045 & 0.091 & 0.07192 & 259.858 & 0.0434 & 0.01089 & 0.260 & 8.073 & 3.36 & 40.00 & 3262 . \\
\hline 1048 & 0.096 & 0.03407 & 269.424 & & 0.01168 & 0.980 & 3.580 & 1.76 & 40 & 1296. \\
\hline 1051 & 0.096 & 0.04172 & 269.814 & 0.0439 & 0.01169 & 0.890 & 4.376 & 1.76 & 40.00 & 1586. \\
\hline 1054 & 0.096 & 0.03407 & 269.375 & & 0.01177 & 1.200 & 3.552 & 1.76 & 40.00 & 1296. \\
\hline 1057 & 0.096 & 0.04173 & 269.773 & 0.0439 & 0.01177 & 0.980 & 4.349 & 1.76 & 40.00 & 1582. \\
\hline 1060 & 0.096 & 0.05014 & 270.214 & & 0.01177 & 0.800 & 5.222 & 1.76 & 40.00 & 1885. \\
\hline 1063 & 0.095 & 0.05930 & 270.683 & 0.0431 & 0.01178 & 0.790 & 6.167 & 1.76 & 40.00 & 2127. \\
\hline 1066 & 0.095 & 0.06921 & 271.194 & 0.0429 & 0.01180 & 0.760 & 7.183 & 1.76 & 40.00 & 2435 . \\
\hline 1069 & 0.279 & 0.03410 & 269.163 & 0.1334 & 0.01185 & 0.830 & 3.445 & 4.96 & 40.00 & 14686. \\
\hline 1072 & 0.279 & 0.04175 & 269.537 & 0.1331 & 0.01188 & 0.670 & 4.186 & 4.96 & 40.00 & 17636. \\
\hline 1075 & 0.279 & 0.05017 & 269.933 & 0.1330 & 0.01194 & 0.850 & 4.979 & 3.36 & 40.00 & 20811. \\
\hline 1078 & 0.279 & 0.05935 & 270.354 & 0.1325 & 0.01201 & 1.000 & 5.823 & 3.36 & 40.00 & 23979 . \\
\hline 1081 & 0.279 & 0.06928 & 270.806 & 0.1325 & 0.01206 & 2.000 & 6.723 & 1.76 & 40.00 & 27441. \\
\hline 1084 & 0.286 & 0.03390 & 275.589 & 0.1329 & 0.01231 & 0.880 & 3.310 & 4.96 & 40.00 & 12589. \\
\hline 1087 & 0.286 & 0.04151 & 275.943 & 0.1327 & 0.01234 & 0.690 & 4.026 & 4.96 & 40.00 & 15199. \\
\hline 1090 & 0.286 & 0.04988 & 276.328 & 0.1327 & 0.01238 & 0.620 & 4.798 & 4.96 & 40.00 & 18007. \\
\hline 1093 & 0.287 & 0.05904 & 276.721 & 0.1 & 0.01244 & 0.970 & 5.617 & 3.36 & 40.00 & 21039. \\
\hline 1096 & 0.287 & 0.06893 & 277.172 & $0.1328^{\circ}$ & 0.01251 & 1.030 & 6.490 & 3.36 & 40.00 & 24041. \\
\hline 1099 & 0.098 & 0.03391 & 275.602 & 0.0435 & 0.01211 & 1.060 & 3.437 & 3.36 & 40.00 & 1135. \\
\hline 1102 & 0.098 & 0.04151 & 275.971 & 0.0434 & 0.01213 & 1.180 & 4.200 & 1.76 & 40.00 & 1376. \\
\hline 1105 & 0.099 & 0.04990 & 276.387 & 0.0440 & 0.01215 & 0.910 & 5.035 & 1.76 & 40.00 & 1688. \\
\hline 1108 & 0.100 & 0.05904 & 276.838 & 0.0442 & 0.01218 & 0.780 & 5.940 & 1.76 & 40.00 & 2003. \\
\hline 1111 & 0.100 & 0.06891 & 277.324 & 0.0444 & 0.01220 & 1.140 & 6.916 & 1.76 & 40.00 & 2337. \\
\hline 1114 & 0.103 & 0.03300 & 286.232 & 0.0443 & 0.01294 & 1.340 & 3.132 & 1.76 & 40.00 & 947. \\
\hline 1117 & 0.104 & 0.04042 & 286.577 & 0.0445 & 0.01295 & 1.040 & 3.831 & 1.76 & 40.00 & 1164. \\
\hline 1120 & 0.104 & 0.04857 & 286.956 & 0.0444 & 0.01297 & 0.810 & 4.596 & 1.76 & 40.00 & 1382. \\
\hline 1123 & 0.103 & 0.05746 & 287.359 & 0.0441 & 0.01299 & 0.780 & 5.426 & 1.76 & 40.00 & 1603. \\
\hline 1126 & 0.103 & 0.06707 & 287.803 & 0.0440 & 0.01302 & 0.740 & 6.317 & 1.76 & 40.00 & 1847. \\
\hline 1129 & 0.280 & 0.03299 & 286.125 & 0.1242 & 0.01319 & 0.980 & 3.030 & 6.56 & 40.00 & 8459. \\
\hline
\end{tabular}


Table 31. Thermal conductivity of the ternary $33 \% \mathrm{R} 32$ / $33 \% \mathrm{R} 125$ / $33 \% \mathrm{R} 134 \mathrm{a}$ mixture in the dilute gas measured using the steady-state technique (continued).

\begin{tabular}{|c|c|c|c|c|c|c|c|c|c|c|}
\hline $\begin{array}{l}\text { Run } \\
\text { point }\end{array}$ & $\begin{array}{l}P_{\text {exp }} \\
\mathrm{MPa}\end{array}$ & $\begin{array}{c}Q \\
W \cdot \mathrm{m}^{-1}\end{array}$ & $\begin{array}{c}T_{e x p} \\
\mathrm{~K}\end{array}$ & $\begin{array}{c}\rho_{\text {calc }} \\
\mathrm{mol} \cdot \mathrm{L}^{-1}\end{array}$ & $\frac{\lambda_{e x p}}{\mathrm{~W} \cdot \mathrm{m}^{-1} \cdot \mathrm{K}^{-1}}$ & $\begin{array}{c}\text { TBAND } \\
\%\end{array}$ & $\begin{array}{c}\Delta T_{\text {avg }} \\
\mathrm{K}\end{array}$ & $\begin{array}{c}t_{\text {start }} \\
\mathrm{s}\end{array}$ & $\begin{array}{c}t_{\text {end }} \\
\mathrm{s}\end{array}$ & $N_{R a}$ \\
\hline 1132 & 0.281 & 0.04043 & 286.453 & 0.1245 & 0.01317 & 0.860 & 3.706 & 4.96 & 40.00 & 10351. \\
\hline 1135 & 0.281 & 0.04858 & 286.824 & 0.1242 & 0.01315 & 0.710 & 4.442 & 4.96 & 40.00 & 12285. \\
\hline 1138 & 0.281 & 0.05746 & 287.209 & 0.1239 & 0.01316 & 0.590 & 5.231 & 4.96 & 40.00 & 14311. \\
\hline 1141 & 0.280 & 0.06708 & 287.620 & 0.1232 & 0.01321 & 0.930 & 6.066 & 3.36 & 40.00 & 16274. \\
\hline 1144 & 0.292 & 0.03178 & 297.658 & 0.1239 & 0.01404 & 1.200 & 2.752 & 6.56 & 40.00 & 6541. \\
\hline 1147 & 0.292 & 0.03894 & 297.955 & 0.1239 & 0.01403 & 1.000 & 3.365 & 4.96 & 40.00 & 7969. \\
\hline 1150 & 0.293 & 0.04681 & 298.294 & 0.1239 & 0.01402 & 0.700 & 4.035 & 4.96 & 40.00 & 9522. \\
\hline 1153 & 0.293 & 0.05537 & 298.650 & 0.1237 & 0.01403 & 0.600 & 4.758 & 4.96 & 40.00 & 11131. \\
\hline 1156 & 0.293 & 0.06464 & 299.033 & 0.1235 & 0.01404 & 0.380 & 5.532 & 4.96 & 40.00 & 12829. \\
\hline 1159 & 0.101 & 0.03179 & 297.657 & 0.0414 & 0.01379 & 1.150 & 2.831 & 1.76 & 40.00 & 651. \\
\hline 1162 & 0.102 & 0.03895 & 297.960 & 0.0417 & 0.01382 & 0.900 & 3.462 & 1.76 & 40.00 & 806. \\
\hline 1165 & 0.102 & 0.04682 & 298.321 & 0.0420 & 0.01384 & 1.000 & 4.154 & 1.76 & 40.00 & 978. \\
\hline 1168 & 0.102 & 0.05538 & 298.682 & 0.0419 & 0.01386 & 0.590 & 4.904 & 1.76 & 40.00 & 1147. \\
\hline 1171 & 0.102 & 0.06466 & 299.079 & 0.0415 & 0.01389 & 0.630 & 5.713 & 1.76 & 40.00 & 1302. \\
\hline 1174 & 0.103 & 0.03126 & 303.279 & 0.0414 & 0.01427 & 1.050 & 2.691 & 1.76 & 40.00 & 582. \\
\hline 1177 & 0.102 & 0.03828 & 303.569 & 0.0411 & 0.01428 & 0.850 & 3.294 & 1.76 & 40.00 & 701. \\
\hline 1180 & 0.102 & 0.04600 & 303.891 & 0.0410 & 0.01430 & 0.720 & 3.951 & 1.76 & 40.00 & 832. \\
\hline 1183 & 0.103 & 0.05444 & 304.255 & 0.0411 & 0.01433 & 0.750 & 4.665 & 1.76 & 40.00 & 987. \\
\hline 1186 & 0.104 & 0.06359 & 304.622 & 0.0416 & 0.01436 & 0.690 & 5.434 & 1.76 & 40.00 & 1174. \\
\hline 1189 & 0.284 & 0.03131 & 302.629 & 0.1181 & 0.01436 & 1.080 & 2.655 & 4.96 & 40.00 & 5338. \\
\hline 1192 & 0.284 & 0.03836 & 302.922 & 0.1176 & 0.01436 & 0.820 & 3.248 & 4.96 & 40.00 & 6453. \\
\hline 1195 & 0.283 & 0.04612 & 303.239 & 0.1171 & 0.01436 & 0.730 & 3.896 & 4.96 & 40.00 & 7631. \\
\hline 1198 & 0.282 & 0.05455 & 303.579 & 0.1167 & 0.01436 & 0.640 & 4.598 & 4.96 & 40.00 & 8904. \\
\hline 1201 & 0.283 & 0.06369 & 303.956 & 0.1168 & 0.01437 & 0.510 & 5.350 & 4.96 & 40.00 & 10339. \\
\hline 1204 & 0.293 & 0.03041 & 312.023 & 0.1177 & 0.01540 & 1.080 & 2.410 & 4.96 & 40.00 & 4304 \\
\hline 1207 & 0.293 & 0.03725 & 312.285 & 0.1175 & 0.01538 & 0.970 & 2.952 & 4.96 & 40.00 & 5236. \\
\hline 1210 & 0.293 & 0.04478 & 312.569 & 0.1175 & 0.01536 & 0.690 & 3.545 & 4.96 & 40.00 & 6260. \\
\hline 1213 & 0.293 & 0.05300 & 312.879 & 0.1172 & 0.01537 & 0.550 & 4.185 & 4.96 & 40.00 & 7336. \\
\hline 1216 & 0.293 & 0.06190 & 313.212 & 0.1171 & 0.01537 & 0.540 & 4.877 & 4.96 & 40.00 & 8488. \\
\hline 1219 & 0.101 & 0.03040 & 311.869 & 0.0395 & 0.01521 & 1.110 & 2.456 & 1.76 & 40.00 & 440. \\
\hline 1222 & 0.101 & 0.03724 & 312.151 & 0.0394 & 0.01522 & 1.010 & 3.007 & 1.76 & 40.00 & 536. \\
\hline 1225 & 0.101 & 0.04477 & 312.433 & 0.0394 & 0.01522 & 0.680 & 3.613 & 1.76 & 40.00 & 642. \\
\hline 1228 & 0.101 & 0.05298 & 312.758 & 0.0393 & 0.01524 & 0.620 & 4.271 & 1.76 & 40.00 & 753. \\
\hline 1231 & 0.101 & 0.06187 & 313.104 & 0.0392 & 0.01524 & 0.480 & 4.986 & 1.76 & 40.00 & 870. \\
\hline 1234 & 0.103 & 0.02961 & 321.478 & 0.0392 & 0.01601 & 1.240 & 2.273 & 1.76 & 40.00 & 365. \\
\hline 1237 & 0.104 & 0.03628 & 321.717 & 0.0394 & 0.01604 & 0.940 & 2.781 & 1.76 & 40.00 & 450 \\
\hline 1240 & 0.104 & 0.04362 & 321.990 & 0.0395 & 0.01606 & 0.830 & 3.338 & 1.76 & 40.00 & 542. \\
\hline 1243 & 0.105 & 0.05162 & 322.271 & 0.0395 & 0.01608 & 0.580 & 3.945 & 1.76 & 40.00 & 640. \\
\hline 1246 & 0.104 & 0.06028 & 322.588 & 0.0394 & 0.01608 & 0.570 & 4.604 & 1.76 & 40.00 & 739. \\
\hline 1249 & 0.298 & 0.02961 & 321.231 & 0.1156 & 0.01624 & 1.280 & 2.229 & 4.96 & 40.00 & 3453. \\
\hline 1252 & 0.297 & 0.03628 & 321.465 & 0.1154 & 0.01625 & 0.970 & 2.725 & 4.96 & 40.00 & 4192. \\
\hline 1255 & 0.297 & 0.04362 & 321.724 & 0.1151 & 0.01625 & 0.870 & 3.271 & 4.96 & 40.00 & 4993. \\
\hline 1258 & 0.296 & 0.05162 & 322.002 & 0.1147 & 0.01625 & 0.790 & 3.865 & 4.96 & 40.00 & 5839. \\
\hline
\end{tabular}


Table 31. Thermal conductivity of the ternary $33 \%$ R32 / $33 \%$ R125 / $33 \%$ R134a mixture in the dilute gas measured using the steady-state technique (continued).

\begin{tabular}{|c|c|c|c|c|c|c|c|c|c|c|}
\hline $\begin{array}{l}\text { Run } \\
\text { point }\end{array}$ & $\begin{array}{l}P_{\text {exp }} \\
\mathrm{MPa}\end{array}$ & $\begin{array}{c}Q \\
W \cdot \mathrm{m}^{-1}\end{array}$ & $\begin{array}{c}T_{\text {exp }} \\
\mathrm{K}\end{array}$ & $\begin{array}{c}\rho_{\text {calc }} \\
\mathrm{mol} \cdot \mathrm{L}^{-1}\end{array}$ & $\begin{array}{c}\lambda_{\text {exp }} \\
\mathrm{W} \cdot \mathrm{m}^{-1} \cdot \mathrm{K}^{-1}\end{array}$ & $\begin{array}{c}\text { TBAND } \\
\% \\
\end{array}$ & $\begin{array}{c}\Delta T_{\text {avg }} \\
\mathrm{K}\end{array}$ & $\begin{array}{c}t_{\text {start }} \\
\mathrm{s} \\
\end{array}$ & $\begin{array}{c}t_{\text {end }} \\
\mathrm{s} \\
\end{array}$ & $N_{R a}$ \\
\hline 1261 & 0.296 & 0.06029 & 322.321 & 0.1145 & 0.01626 & 0.570 & 4.506 & 4.96 & 40.00 & 6761. \\
\hline 1264 & 0.305 & 0.02981 & 329.992 & 0.1151 & 0.01711 & 1.340 & 2.131 & 4.96 & 40.00 & 2980. \\
\hline 1267 & 0.305 & 0.03652 & 330.220 & 0.1149 & 0.01713 & 0.950 & 2.605 & 4.96 & 40.00 & 3620 . \\
\hline 1270 & 0.306 & 0.04390 & 330.469 & 0.1150 & 0.01713 & 0.930 & 3.127 & 4.96 & 40.00 & 4344. \\
\hline 1273 & 0.305 & 0.05196 & 330.728 & 0.1148 & 0.01713 & 0.770 & 3.695 & 4.96 & 40.00 & 5095. \\
\hline 1276 & 0.304 & 0.06068 & 331.030 & 0.1143 & 0.01713 & 0.500 & 4.310 & 4.96 & 40.00 & 5867. \\
\hline 1279 & 0.092 & 0.02986 & 329.418 & 0.0341 & 0.01679 & 1.170 & 2.186 & 1.76 & 40.00 & 245. \\
\hline 1282 & 0.091 & 0.03658 & 329.632 & 0.0336 & 0.01682 & 0.870 & 2.673 & 1.76 & 40.00 & 289 . \\
\hline 1285 & 0.091 & 0.04398 & 329.904 & 0.0334 & 0.01683 & 0.680 & 3.212 & 1.76 & 40.00 & 342 . \\
\hline 1288 & 0.091 & 0.05204 & 330.164 & 0.0335 & 0.01685 & 0.510 & 3.797 & 1.76 & 40.00 & 408. \\
\hline 1291 & 0.091 & 0.06077 & 330.463 & 0.0336 & 0.01686 & 0.460 & 4.431 & 1.76 & 40.00 & 476. \\
\hline 1294 & 0.094 & 0.02983 & 338.811 & 0.0338 & 0.01757 & 1.650 & 2.087 & 1.76 & 40.00 & 211. \\
\hline 1297 & 0.095 & 0.03654 & 339.029 & 0.0339 & 0.01762 & 1.050 & 2.550 & 1.76 & 40.00 & 258. \\
\hline 1300 & 0.095 & 0.04393 & 339.243 & 0.0339 & 0.01765 & 0.860 & 3.060 & 1.76 & 40.00 & 309. \\
\hline 1303 & 0.095 & 0.05199 & 339.513 & 0.0339 & 0.01768 & 0.670 & 3.614 & 1.76 & 40.00 & 365 . \\
\hline 1306 & 0.095 & 0.06073 & 339.770 & 0.0338 & 0.01771 & 0.590 & 4.215 & 1.76 & 40.00 & 420. \\
\hline 1309 & 0.284 & 0.02991 & 338.359 & 0.1037 & 0.01791 & 1.430 & 2.046 & 4.96 & 40.00 & 2107. \\
\hline 1312 & 0.283 & 0.03665 & 338.570 & 0.1036 & 0.01787 & 1.260 & 2.511 & 3.36 & 40.00 & 2571. \\
\hline 1315 & 0.284 & 0.04406 & 338.788 & 0.1037 & 0.01786 & 1.040 & 3.017 & 3.36 & 40.00 & 3092 . \\
\hline 1318 & 0.284 & 0.05215 & 339.036 & 0.1037 & 0.01790 & 0.940 & 3.560 & 3.36 & 40.00 & 3641. \\
\hline 1321 & 0.284 & 0.06092 & 339.301 & 0.1036 & 0.01791 & 0.750 & 4.153 & 3.36 & 40.00 & 4228. \\
\hline
\end{tabular}


Table 32. Thermal conductivity of the ternary $30 \% \mathrm{R} 32$ / $10 \% \mathrm{R} 125$ / $60 \% \mathrm{R} 134 \mathrm{a}$ mixture in the dilute gas measured using the steady-state technique.

\begin{tabular}{|c|c|c|c|c|c|c|c|c|c|c|}
\hline $\begin{array}{l}\text { Run } \\
\text { point }\end{array}$ & $\begin{array}{l}P_{\text {exp }} \\
\mathrm{MPa}\end{array}$ & $\begin{array}{c}Q \\
\mathrm{~W} \cdot \mathrm{m}^{-1}\end{array}$ & $\begin{array}{c}T_{\text {exp }} \\
\mathrm{K}\end{array}$ & $\begin{array}{c}\rho_{\text {calc }} \\
\mathrm{mol} \cdot \mathrm{L}^{-1}\end{array}$ & $\begin{array}{c}\lambda_{\exp } \\
\mathrm{W} \cdot \mathrm{m}^{-1} \cdot \mathrm{K}^{-1}\end{array}$ & $\begin{array}{c}\text { TBAND } \\
\%\end{array}$ & $\begin{array}{c}\Delta T_{\text {avg }} \\
\mathrm{K}\end{array}$ & $\begin{array}{c}t_{\text {start }} \\
\mathrm{s}\end{array}$ & $\begin{array}{l}t_{\text {end }} \\
\mathrm{s}\end{array}$ & $N_{R a}$ \\
\hline 1003 & 0.087 & 0.02318 & 256.536 & 0.0419 & 0.01047 & 1.490 & 2.719 & 3.36 & 40.00 & 1108. \\
\hline 1006 & 0.087 & 0.03151 & 257.022 & 0.0420 & 0.01047 & 0.660 & 3.692 & 3.36 & 40.00 & 1500. \\
\hline 1009 & 0.086 & 0.04111 & 257.576 & 0.0413 & 0.01050 & 0.680 & 4.800 & 3.36 & 40.00 & 1872. \\
\hline 1012 & 0.086 & 0.05196 & 258.194 & 0.0411 & 0.01053 & 0.340 & 6.043 & 3.36 & 40.00 & 2305 . \\
\hline 1015 & 0.087 & 0.06407 & 258.878 & 0.0415 & 0.01057 & 0.310 & 7.419 & 3.36 & 40.00 & 2864. \\
\hline 1018 & 0.138 & 0.02318 & 256.516 & 0.0673 & 0.01065 & 1.620 & 2.662 & 4.96 & 40.00 & 3077. \\
\hline 1021 & 0.138 & 0.03152 & 256.979 & 0.0676 & 0.01063 & 1.010 & 3.618 & 3.36 & 40.00 & 4179. \\
\hline 1024 & 0.139 & 0.04112 & 257.525 & 0.0680 & 0.01064 & 0.700 & 4.708 & 3.36 & 40.00 & 5472. \\
\hline 1027 & 0.140 & 0.05199 & 258.135 & 0.0679 & 0.01065 & 0.770 & 5.931 & 3.36 & 40.00 & 6797. \\
\hline 1030 & 0.139 & 0.06409 & 258.810 & 0.0671 & 0.01067 & 0.470 & 7.278 & 3.36 & 40.00 & 8040 . \\
\hline 1033 & 0.143 & 0.02248 & 266.478 & 0.0669 & 0.01143 & 1.250 & 2.408 & 3.36 & 40.00 & 2341. \\
\hline 1036 & 0.144 & 0.03058 & 266.891 & 0.0672 & 0.01142 & 1.180 & 3.276 & 3.36 & 40.00 & 3197. \\
\hline 1039 & 0.145 & 0.03991 & 267.369 & 0.0676 & 0.01141 & 0.680 & 4.268 & 3.36 & 40.00 & 4190. \\
\hline 1042 & 0.145 & 0.05045 & 267.927 & 0.0676 & 0.01141 & 0.600 & 5.384 & 3.36 & 40.00 & 5232 . \\
\hline 1045 & 0.144 & 0.06220 & 268.537 & 0.0670 & 0.01143 & 0.610 & 6.619 & 3.36 & 40.00 & 6247. \\
\hline 1048 & 0.088 & 0.02241 & 268.925 & 0.0403 & 0.01142 & 1.560 & 2.410 & 1.76 & 40.00 & 758. \\
\hline 1051 & 0.087 & 0.03047 & 269.370 & 0.0398 & 0.01139 & 0.870 & 3.285 & 1.76 & 40.00 & 1003. \\
\hline 1054 & 0.087 & 0.03977 & 269.851 & 0.0397 & 0.01141 & 1.080 & 4.278 & 1.76 & 40.00 & 1286. \\
\hline 1057 & 0.088 & 0.05027 & 270.421 & 0.0399 & 0.01143 & 0.810 & 5.396 & 1.76 & 40.00 & 1630. \\
\hline 1060 & 0.088 & 0.06198 & 271.041 & 0.0398 & 0.01146 & 0.750 & 6.631 & 1.76 & 40.00 & 1979. \\
\hline 1063 & 0.212 & 0.02242 & 268.897 & 0.1002 & 0.01164 & 1.410 & 2.346 & 4.96 & 40.00 & 5458. \\
\hline 1066 & 0.210 & 0.03048 & 269.316 & 0.0991 & 0.01161 & 0.810 & 3.187 & 4.96 & 40.00 & 7167. \\
\hline 1069 & 0.210 & 0.03978 & 269.792 & 0.0992 & 0.01161 & 0.770 & 4.145 & 4.96 & 40.00 & 9271. \\
\hline 1072 & 0.212 & 0.05029 & 270.330 & 0.0995 & 0.01161 & 0.560 & 5.218 & 4.96 & 40.00 & 11649. \\
\hline 1075 & 0.211 & 0.06202 & 270.907 & 0.0989 & 0.01165 & 0.770 & 6.385 & 3.36 & 40.00 & 13949. \\
\hline 1081 & 0.272 & 0.03049 & 269.248 & 0.1308 & 0.01174 & 1.690 & 3.116 & 4.96 & 40.00 & 13534. \\
\hline 1084 & 0.271 & 0.03979 & 269.712 & 0.1302 & 0.01176 & 0.690 & 4.035 & 4.96 & 40.00 & 17163. \\
\hline 1087 & 0.272 & 0.05030 & 270.205 & 0.1304 & 0.01182 & 0.950 & 5.036 & 3.36 & 40.00 & 21318. \\
\hline 1090 & 0.273 & 0.06203 & 270.755 & 0.1305 & 0.01188 & 1.070 & 6.131 & 3.36 & 40.00 & 25762 . \\
\hline 1093 & 0.283 & 0.02182 & 278.123 & 0.1311 & 0.01241 & 1.290 & 2.132 & 6.56 & 40.00 & 7963. \\
\hline 1096 & 0.285 & 0.02968 & 278.489 & 0.1315 & 0.01238 & 1.180 & 2.890 & 4.96 & 40.00 & 10814. \\
\hline 1099 & 0.285 & 0.03872 & 278.937 & 0.1315 & 0.01237 & 0.850 & 3.754 & 4.96 & 40.00 & 13933. \\
\hline 1102 & 0.284 & 0.04897 & 279.406 & 0.1309 & 0.01240 & 0.630 & 4.707 & 4.96 & 40.00 & 17147. \\
\hline 1105 & 0.284 & 0.06039 & 279.928 & 0.1302 & 0.01247 & 0.750 & 5.738 & 3.36 & 40.00 & 20496 . \\
\hline 1108 & 0.087 & 0.02182 & 278.115 & 0.0383 & 0.01213 & 1.500 & 2.211 & 1.76 & 40.00 & 556. \\
\hline 1111 & 0.086 & 0.02967 & 278.510 & 0.0378 & 0.01212 & 1.040 & 3.007 & 1.76 & 40.00 & 728. \\
\hline 1114 & 0.085 & 0.03872 & 278.960 & 0.0375 & 0.01212 & 0.720 & 3.922 & 1.76 & 40.00 & 931. \\
\hline 1117 & 0.086 & 0.04896 & 279.471 & 0.0378 & 0.01214 & 0.760 & 4.949 & 1.76 & 40.00 & 1187. \\
\hline 1120 & 0.086 & 0.06038 & 280.058 & 0.0377 & 0.01212 & 0.690 & 6.113 & 1.76 & 40.00 & 1449. \\
\hline 1123 & 0.184 & 0.02182 & 278.078 & 0.0831 & 0.01217 & 1.280 & 2.194 & 4.96 & 40.00 & 2906. \\
\hline 1126 & 0.183 & 0.02968 & 278.465 & 0.0826 & 0.01217 & 1.040 & 2.979 & 3.36 & 40.00 & 3863. \\
\hline 1129 & 0.183 & 0.03873 & 278.925 & 0.0822 & 0.01217 & 0.610 & 3.879 & 4.96 & 40.00 & 4955. \\
\hline 1132 & 0.184 & 0.04897 & 279.418 & 0.0827 & 0.01218 & 0.430 & 4.888 & 4.96 & 40.00 & 6286. \\
\hline
\end{tabular}


Table 32. Thermal conductivity of the ternary $30 \% \mathrm{R} 32$ / $10 \% \mathrm{R} 125$ / $60 \% \mathrm{R} 134 \mathrm{a}$ mixture in the dilute gas measured using the steady-state technique (continued).

\begin{tabular}{|c|c|c|c|c|c|c|c|c|c|c|}
\hline $\begin{array}{l}\text { Run } \\
\text { point }\end{array}$ & $\begin{array}{l}P_{\text {exp }} \\
\mathrm{MPa} \\
\end{array}$ & $\begin{array}{c}Q \\
\mathrm{~W} \cdot \mathrm{m}^{-1}\end{array}$ & $\begin{array}{c}T_{\text {exp }} \\
\mathrm{K}\end{array}$ & $\begin{array}{c}\rho_{\text {calc }} \\
\mathrm{mol} \cdot \mathrm{L}^{-1}\end{array}$ & $\begin{array}{c}\lambda_{\exp } \\
\mathrm{W} \cdot \mathrm{m}^{-1} \cdot \mathrm{K}^{-1} \\
\end{array}$ & $\begin{array}{c}\text { TBAND } \\
\%\end{array}$ & $\begin{array}{c}\Delta T_{\text {avg }} \\
\mathrm{K} \\
\end{array}$ & $\begin{array}{c}t_{\text {start }} \\
\mathrm{s}\end{array}$ & $\begin{array}{c}t_{\text {end }} \\
\mathrm{s}\end{array}$ & $N_{R a}$ \\
\hline 1135 & 0.185 & 0.06038 & 279.977 & 0.0827 & 0.01221 & 0.760 & 5.999 & 3.36 & 40.00 & 7655 . \\
\hline 1138 & 0.351 & 0.02182 & 277.285 & 0.1662 & 0.01240 & 1.320 & 2.109 & 6.56 & 40.00 & 14179. \\
\hline 1141 & 0.350 & 0.02968 & 277.649 & 0.1655 & 0.01238 & 1.250 & 2.850 & 4.96 & 40.00 & 18839. \\
\hline 1144 & 0.349 & 0.03873 & 278.075 & 0.1645 & 0.01242 & 1.260 & 3.674 & 3.36 & 40.00 & 23741. \\
\hline 1147 & 0.349 & 0.04898 & 278.520 & $0: 1638$ & 0.01246 & 1.150 & 4.589 & 3.36 & 40.00 & 29095. \\
\hline 1150 & 0.349 & 0.06039 & 279.032 & 0.1636 & 0.01247 & 1.030 & 5.596 & 3.36 & 40.00 & 35028. \\
\hline 1153 & 0.408 & 0.02182 & 277.218 & 0.1962 & 0.01254 & 1.940 & 2.061 & 4.96 & 40.00 & 21123. \\
\hline 1156 & 0.409 & 0.02967 & 277.549 & 0.1966 & 0.01256 & 1.300 & 2.761 & 4.96 & 40.00 & 28276 . \\
\hline 1159 & 0.409 & 0.03873 & 277.955 & 0.1959 & 0.01258 & 1.350 & 3.554 & 3.36 & 40.00 & 35744 . \\
\hline 1162 & 0.407 & 0.04899 & 278.397 & 0.1945 & 0.01259 & 2.170 & 4.432 & 1.76 & 40.00 & 43359. \\
\hline 1165 & 0.406 & 0.06042 & 278.874 & 0.1938 & 0.01257 & 1.640 & 5.398 & 1.76 & 40.00 & 51822. \\
\hline 1168 & $0: 425$ & 0.02195 & 286.936 & 0.1956 & 0.01324 & 1.650 & 1.979 & -6.56 & 40.00 & 16720. \\
\hline 1171 & 0.425 & 0.02986 & 287.281 & 0.1950 & 0.01326 & 1.190 & 2.662 & 4.96 & 40.00 & 22193. \\
\hline 1174 & 0.426 & 0.03897 & 287.660 & 0.1952 & 0.01329 & 1.340 & 3.428 & 3.36 & 40.00 & 28478 . \\
\hline 1177 & 0.427 & 0.04928 & 288.077 & 0.1956 & 0.01329 & 1.210 & 4.281 & 3.36 & 40.00 & 35455 . \\
\hline 1180 & 0.427 & 0.06079 & 288.541 & 0.1952 & 0.01331 & 2.080 & 5.210 & 1.76 & 40.00 & 42606. \\
\hline 1183 & 0.332 & 0.02196 & 286.938 & 0.1492 & 0.01303 & 1.540 & 2.040 & 6.56 & 40.00 & 8972 . \\
\hline 1186 & 0.333 & 0.02986 & 287.301 & 0.1497 & 0.01298 & 0.980 & 2.767 & 6.56 & 40.00 & 12194. \\
\hline 1189 & 0.333 & 0.03897 & 287.706 & 0.1493 & 0.01306 & 0.840 & 3.567 & 4.96 & 40.00 & 15515. \\
\hline 1192 & 0.332 & 0.04928 & 288.150 & 0.1484 & 0.01310 & 0.720 & 4.470 & 4.96 & 40.00 & 19049. \\
\hline 1195 & 0.331 & 0.06079 & 288.631 & 0.1477 & 0.01318 & 0.920 & 5.445 & 3.36 & 40.00 & 22798 . \\
\hline 1198 & 0.250 & 0.02196 & 286.894 & 0.1103 & 0.01305 & 1.520 & 2.053 & 4.96 & 40.00 & 4508. \\
\hline 1201 & 0.250 & 0.02986 & 287.283 & 0.1100 & 0.01297 & 1.040 & 2.801 & 4.96 & 40.00 & 6081. \\
\hline 1204 & 0.249 & 0.03896 & 287.712 & 0.1095 & 0.01294 & 0.650 & 3.652 & 4.96 & 40.00 & 7802 . \\
\hline 1207 & 0.247 & 0.04927 & 288.175 & 0.1085 & 0.01294 & 0.600 & 4.602 & 4.96 & 40.00 & 9561. \\
\hline 1210 & 0.246 & 0.06077 & 288.687 & 0.1076 & 0.01296 & 0.450 & 5.649 & 4.96 & 40.00 & 11452. \\
\hline 1213 & 0.183 & 0.02197 & 286.688 & 0.0798 & 0.01290 & 1.820 & 2.087 & 3.36 & 40.00 & 2248 \\
\hline 1216 & 0.183 & 0.02987 & 287.052 & 0.0795 & 0.01286 & 1.080 & 2.841 & 3.36 & 40.00 & 3018. \\
\hline 1219 & 0.183 & 0.03898 & 287.492 & 0.0795 & 0.01285 & 0.900 & 3.704 & 3.36 & 40.00 & 3914. \\
\hline 1222 & 0.183 & 0.04929 & 287.973 & 0.0793 & 0.01286 & 0.800 & 4.671 & 3.36 & 40.00 & 4880. \\
\hline 1225 & 0.184 & 0.06079 & 288.512 & 0.0795 & 0.01287 & 0.680 & 5.745 & 3.36 & 40.00 & 5982. \\
\hline 1228 & 0.090 & 0.02197 & 286.680 & 0.0383 & 0.01280 & 1.270 & 2.109 & 1.76 & 40.00 & 475. \\
\hline 1231 & 0.090 & 0.02988 & 287.065 & 0.0383 & 0.01276 & 0.960 & 2.876 & 1.76 & 40.00 & 647. \\
\hline 1234 & 0.089 & 0.03899 & 287.504 & 0.0378 & 0.01272 & 0.830 & 3.764 & 1.76 & 40.00 & 819. \\
\hline 1237 & 0.087 & 0.04930 & 287.989 & 0.0370 & 0.01274 & 0.570 & 4.751 & 1.76 & 40.00 & 984. \\
\hline 1240 & 0.087 & 0.06080 & 288.546 & 0.0370 & 0.01277 & 0.520 & 5.844 & 1.76 & 40.00 & 1199. \\
\hline 1243 & 0.491 & 0.02197 & 286.575 & 0.2301 & 0.01349 & 1.990 & 1.917 & 4.96 & 40.00 & 24628. \\
\hline 1246 & 0.492 & 0.02989 & 286.909 & 0.2304 & 0.01345 & 1.630 & 2.577 & 3.36 & 40.00 & 32999. \\
\hline 1249 & 0.492 & 0.03900 & 287.280 & 0.2302 & 0.01337 & 1.600 & 3.328 & 3.36 & 40.00 & 42197. \\
\hline 1252 & 0.491 & 0.04932 & 287.688 & 0.2289 & 0.01336 & 2.080 & 4.149 & 1.76 & 40.00 & 51461. \\
\hline 1258 & 0.524 & 0.02130 & 297.132 & 0.2351 & 0.01414 & 1.740 & 1.787 & 6.56 & 40.00 & 20019 . \\
\hline 1261 & 0.524 & 0.02898 & 297.458 & 0.2345 & 0.01405 & 1.380 & 2.418 & 4.96 & 40.00 & 26754 . \\
\hline 1264 & 0.524 & 0.03783 & 297.806 & 0.2342 & 0.01407 & 1.390 & 3.112 & 3.36 & 40.00 & 34134 . \\
\hline
\end{tabular}


Table 32. Thermal conductivity of the ternary $30 \% \mathrm{R} 32$ / $10 \% \mathrm{R} 125$ / $60 \% \mathrm{R} 134 \mathrm{a}$ mixture in the dilute gas measured using the steady-state technique (continued).

\begin{tabular}{|c|c|c|c|c|c|c|c|c|c|c|}
\hline $\begin{array}{l}\text { Run } \\
\text { point }\end{array}$ & $\begin{array}{l}P_{\text {exp }} \\
\mathrm{MPa}\end{array}$ & $\begin{array}{c}Q \\
\mathrm{~W} \cdot \mathrm{m}^{-1}\end{array}$ & $\begin{array}{c}T_{\exp } \\
\mathrm{K}\end{array}$ & $\begin{array}{c}\rho_{\text {calc }} \\
\mathrm{mol} \cdot \mathrm{L}^{-1}\end{array}$ & $\begin{array}{c}\lambda_{\exp } \\
\mathrm{W} \cdot \mathrm{m}^{-1} \cdot \mathrm{K}^{-1}\end{array}$ & $\begin{array}{c}\text { TBAND } \\
\%\end{array}$ & $\begin{array}{c}\Delta T_{\text {avg }} \\
\mathrm{K}\end{array}$ & $\begin{array}{c}t_{\text {start }} \\
\mathrm{s}\end{array}$ & $\begin{array}{c}t_{\text {end }} \\
\mathrm{s}\end{array}$ & $N_{R a}$ \\
\hline 1267 & 0.527 & 0.04784 & 298.182 & 0.2350 & 0.01406 & 1.420 & 3.882 & 3.36 & 40.00 & 42677. \\
\hline 1270 & 0.526 & 0.05902 & 298.609 & 0.2343 & 0.01405 & 2.160 & 4.723 & 1.76 & 40.00 & 51187. \\
\hline 1273 & 0.439 & 0.02136 & 296.439 & 0.1940 & 0.01404 & 1.930 & 1.828 & 6.56 & 40.00 & 12932. \\
\hline 1276 & 0.438 & 0.02905 & 296.759 & 0.1930 & 0.01400 & 1.270 & 2.475 & 6.56 & 40.00 & 17211. \\
\hline 1279 & 0.439 & 0.03792 & 297.124 & 0.1932 & 0.01397 & 1.120 & 3.206 & 4.96 & 40.00 & 22239 . \\
\hline 1282 & 0.441 & 0.04796 & 297.515 & 0.1937 & 0.01402 & 1.260 & 4.003 & 3.36 & 40.00 & 27752 . \\
\hline 1285 & 0.440 & 0.05915 & 297.963 & 0.1929 & 0.01404 & 1.070 & 4.884 & 3.36 & 40.00 & 33317. \\
\hline 1288 & 0.347 & 0.02137 & 296.428 & 0.1502 & 0.01386 & 1.540 & 1.871 & 6.56 & 40.00 & 7258. \\
\hline 1291 & 0.346 & 0.02906 & 296.771 & 0.1497 & 0.01383 & 1.060 & 2.539 & 6.56 & 40.00 & 9723. \\
\hline 1294 & 0.347 & 0.03792 & 297.149 & 0.1497 & 0.01380 & 0.730 & 3.304 & 6.56 & 40.00 & 12586. \\
\hline 1297 & 0.348 & 0.04795 & 297.580 & 0.1501 & 0.01379 & 0.850 & 4.155 & 4.96 & 40.00 & 15817. \\
\hline 1300 & 0.350 & 0.05914 & 298.038 & 0.1506 & 0.01385 & 0.970 & 5.071 & 3.36 & 40.00 & 19348. \\
\hline 1303 & 0.258 & 0.02136 & 296.418 & 0.1096 & 0.01380 & 1.680 & 1.891 & 4.96 & 40.00 & 3599. \\
\hline 1306 & 0.259 & 0.02906 & 296.768 & 0.1099 & 0.01375 & 1.090 & 2.576 & 4.96 & 40.00 & 4909. \\
\hline 1309 & 0.258 & 0.03792 & 297.174 & 0.1093 & 0.01372 & 0.840 & 3.360 & 4.96 & 40.00 & 6293. \\
\hline 1312 & 0.256 & 0.04795 & 297.596 & 0.1085 & 0.01370 & 0.560 & 4.243 & 4.96 & 40.00 & 7767. \\
\hline 1315 & 0.256 & 0.05913 & 298.086 & 0.1082 & 0.01370 & 0.490 & 5.220 & 4.96 & 40.00 & 9447. \\
\hline 1318 & 0.177 & 0.02136 & 296.369 & 0.0743 & 0.01376 & 1.670 & 1.904 & 3.36 & 40.00 & 1554. \\
\hline 1321 & 0.178 & 0.02905 & 296.725 & 0.0745 & 0.01370 & 1.030 & 2.599 & 3.36 & 40.00 & 2121. \\
\hline 1324 & 0.180 & 0.03791 & 297.109 & 0.0752 & 0.01367 & 0.830 & 3.394 & 3.36 & 40.00 & 2815. \\
\hline 1327 & 0.179 & 0.04794 & 297.564 & 0.0747 & 0.01364 & 0.860 & 4.296 & 3.36 & 40.00 & 3495 . \\
\hline 1330 & 0.178 & 0.05914 & 298.065 & 0.0739 & 0.01365 & 0.570 & 5.289 & 3.36 & 40.00 & 4176. \\
\hline 1333 & 0.092 & 0.02137 & 296.367 & & 0.01349 & 1.740 & 1.948 & 1.76 & 40.00 & 384. \\
\hline 1336 & 0.093 & 0.02907 & 296.726 & 0.0384 & 0.01346 & 1.100 & 2.655 & 1.76 & 40.00 & 536. \\
\hline 1339 & 0.094 & 0.03793 & 297.142 & 0.0385 & 0.01344 & 0.790 & 3.468 & 1.76 & 40.00 & 703. \\
\hline 1342 & 0.092 & 0.04795 & 297.582 & 0.0376 & 0.01346 & 0.710 & 4.376 & 1.76 & 40.00 & 840. \\
\hline 1345 & 0.092 & 0.05914 & 298.093 & 0.0376 & 0.01348 & 0.640 & 5.388 & 1.76 & 40.00 & 1025. \\
\hline 1348 & 0.097 & 0.02144 & 306.844 & 0.0385 & 0.01429 & 1.790 & 1.844 & 1.76 & 40.00 & 335. \\
\hline 1351 & 0.097 & 0.02916 & 307.168 & 0.0385 & 0.01428 & 1.250 & 2.510 & 1.76 & 40.00 & 455. \\
\hline 1354 & 0.096 & 0.03806 & 307.545 & 0.0383 & 0.01430 & 0.780 & 3.270 & 1.76 & 40.00 & 582. \\
\hline 1357 & 0.096 & 0.04814 & 307.954 & 0.0379 & 0.01432 & 0.660 & 4.129 & 1.76 & 40.00 & 719. \\
\hline 1360 & 0.095 & 0.05937 & 308.432 & 0.0374 & 0.01435 & 0.450 & 5.082 & 1.76 & 40.00 & 855. \\
\hline 1363 & 0.182 & 0.02145 & 306.774 & 0.0733 & 0.01437 & 1.620 & 1.831 & 3.36 & 40.00 & 1284. \\
\hline 1366 & 0.181 & 0.02916 & 307.087 & 0.0727 & 0.01436 & 1.320 & 2.491 & 3.36 & 40.00 & 1710. \\
\hline 1369 & 0.183 & 0.03806 & 307.472 & 0.0736 & 0.01435 & 0.810 & 3.248 & 3.36 & 40.00 & 2275 . \\
\hline 1372 & 0.184 & 0.04812 & 307.888 & 0.0738 & 0.01437 & 0.660 & 4.097 & 3.36 & 40.00 & 2878. \\
\hline 1375 & 0.182 & 0.05936 & 308.349 & 0.0732 & 0.01440 & 0.450 & 5.039 & 3.36 & 40.00 & 3454. \\
\hline 1378 & 0.268 & 0.02145 & 306.616 & 0.1097 & 0.01450 & 1.880 & 1.809 & 4.96 & 40.00 & 3031. \\
\hline 1381 & 0.267 & 0.02917 & 306.934 & 0.1091 & 0.01448 & 1.180 & 2.459 & 4.96 & 40.00 & 4060. \\
\hline 1384 & 0.269 & 0.03807 & 307.304 & 0.1100 & 0.01446 & 0.810 & 3.206 & 4.96 & 40.00 & 5356. \\
\hline 1387 & 0.270 & 0.04815 & 307.716 & 0.1103 & 0.01446 & 0.750 & 4.044 & 4.96 & 40.00 & 6771. \\
\hline 1390 & 0.269 & 0.05939 & 308.172 & 0.1097 & 0.01445 & 0.490 & 4.980 & 4.96 & 40.00 & 8184. \\
\hline 1393 & 0.347 & 0.02146 & 306.434 & 0.1440 & 0.01453 & 1.720 & 1.798 & 6.56 & 40.00 & 5529. \\
\hline
\end{tabular}


Table 32. Thermal conductivity of the ternary $30 \% \mathrm{R} 32 / 10 \% \mathrm{R} 125$ / $60 \% \mathrm{R} 134 \mathrm{a}$ mixture in the dilute gas measured using the steady-state technique (continued).

\begin{tabular}{|c|c|c|c|c|c|c|c|c|c|c|}
\hline $\begin{array}{l}\text { Run } \\
\text { point }\end{array}$ & $\begin{array}{l}P_{\text {exp }} \\
\text { MPa }\end{array}$ & $\begin{array}{c}Q \\
W \cdot m^{-1}\end{array}$ & $\begin{array}{c}T_{\text {exp }} \\
\mathrm{K}\end{array}$ & $\begin{array}{c}\rho_{\text {calc }} \\
\mathrm{mol} \cdot \mathrm{L}^{-1}\end{array}$ & $\begin{array}{c}\lambda_{\exp } \\
\mathrm{W} \cdot \mathrm{m}^{-1} \cdot \mathrm{K}^{-1}\end{array}$ & $\begin{array}{c}\text { TBAND } \\
\%\end{array}$ & $\begin{array}{c}\Delta T_{\text {avg }} \\
\mathrm{K}\end{array}$ & $\begin{array}{c}t_{\text {start }} \\
\mathrm{s}\end{array}$ & $\begin{array}{c}t_{\text {end }} \\
\mathrm{s}\end{array}$ & $N_{R a}$ \\
\hline 1396 & 0.349 & 0.02919 & 306.741 & 0.1448 & 0.01450 & 1.120 & 2.442 & 6.56 & 40.00 & 7571. \\
\hline 1399 & 0.351 & 0.03809 & 307.108 & 0.1454 & 0.01448 & 1.180 & 3.178 & 4.96 & 40.00 & 9902. \\
\hline 1402 & 0.350 & 0.04816 & 307.521 & 0.1447 & 0.01449 & 0.710 & 3.999 & 4.96 & 40.00 & 12268. \\
\hline 1405 & 0.348 & 0.05940 & 307.976 & 0.1438 & 0.01453 & 0.540 & 4.896 & 4.96 & 40.00 & 14711. \\
\hline 1408 & 0.437 & 0.02145 & 306.010 & 0.1846 & 0.01460 & 1.760 & 1.775 & 6.56 & 40.00 & 9705. \\
\hline 1411 & 0.435 & 0.02917 & 306.311 & 0.1837 & 0.01458 & 1.100 & 2.404 & 6.56 & 40.00 & 12928. \\
\hline 1414 & 0.436 & 0.03807 & 306.667 & 0.1837 & 0.01461 & 0.970 & 3.111 & 4.96 & 40.00 & 16661. \\
\hline 1417 & 0.437 & 0.04815 & 307.050 & 0.1842 & 0.01468 & 0.920 & 3.886 & 4.96 & 40.00 & 20831. \\
\hline 1420 & 0.436 & 0.05939 & 307.463 & 0.1834 & 0.01476 & 1.010 & 4.730 & 3.36 & 40.00 & 24943. \\
\hline 1423 & 0.493 & 0.02145 & 305.950 & 0.2107 & 0.01478 & 1.980 & 1.744 & 8.16 & 40.00 & 13022. \\
\hline 1426 & 0.496 & 0.02918 & 306.243 & 0.2119 & 0.01474 & 1.260 & 2.358 & 6.56 & 40.00 & 17775. \\
\hline 1429 & 0.496 & 0.03808 & 306.605 & 0.2116 & 0.01474 & 1.340 & 3.049 & 4.96 & 40.00 & 22789 . \\
\hline 1432 & 0.494 & 0.04816 & 306.973 & 0.2101 & 0.01480 & 1.280 & 3.807 & 3.36 & 40.00 & 27837. \\
\hline 1435 & 0.492 & 0.05942 & 307.377 & 0.2088 & 0.01483 & 1.040 & 4.644 & 3.36 & 40.00 & 33278. \\
\hline 1438 & 0.515 & 0.02148 & 317.980 & 0.2102 & 0.01565 & 1.710 & 1.656 & 8.16 & 40.00 & 10422. \\
\hline 1441 & 0.518 & 0.02921 & 318.273 & 0.2111 & 0.01562 & 1.390 & 2.242 & 6.56 & 40.00 & 14184. \\
\hline 1444 & 0.517 & 0.03813 & 318.602 & 0.2102 & 0.01564 & 0.910 & 2.902 & 6.56 & 40.00 & 18101. \\
\hline 1447 & 0.514 & 0.04822 & 318.943 & 0.2089 & 0.01570 & 0.870 & 3.628 & 4.96 & 40.00 & 22212 . \\
\hline 1450 & 0.513 & 0.05947 & 319.329 & 0.2081 & 0.01576 & 1.210 & 4.425 & 3.36 & 40.00 & 26720 . \\
\hline 1453 & 0.437 & 0.02149 & 317.843 & 0.1760 & 0.01571 & 2.110 & 1.661 & 6.56 & 40.00 & 6957. \\
\hline 1456 & 0.435 & 0.02922 & 318.142 & 0.1751 & 0.01566 & 1.310 & 2.256 & 6.56 & 40.00 & 9313. \\
\hline 1459 & 0.433 & 0.03813 & 318.460 & 0.1741 & 0.01565 & 0.910 & 2.933 & 6.56 & 40.00 & 11906. \\
\hline 1462 & 0.434 & 0.04822 & 318.833 & 0.1741 & 0.01565 & 0.850 & 3.690 & 4.96 & 40.00 & 14900 . \\
\hline 1465 & 0.436 & 0.05949 & 319.224 & 0.1749 & 0.01568 & 0.550 & 4.515 & 4.96 & 40.00 & 18340. \\
\hline 1468 & 0.346 & 0.02150 & 317.684 & 0.1376 & 0.01544 & 1.710 & 1.700 & 8.16 & 40.00 & 4112. \\
\hline 1471 & 0.346 & 0.02924 & 317.977 & 0.1373 & 0.01545 & 1.120 & 2.304 & 4.96 & 40.00 & 5529. \\
\hline 1474 & 0.343 & 0.03815 & 318.322 & 0.1360 & 0.01547 & 0.920 & 2.994 & 4.96 & 40.00 & 7000 \\
\hline 1477 & 0.343 & 0.04824 & 318.700 & 0.1359 & 0.01548 & 0.750 & 3.773 & 4.96 & 40.00 & 8772. \\
\hline 1480 & 0.346 & 0.05950 & 319.112 & 0.1369 & 0.01548 & 0.630 & 4.636 & 4.96 & 40.00 & 10905. \\
\hline 1483 & 0.261 & 0.02157 & 316.556 & 0.1029 & 0.01557 & 1.740 & 1.697 & 4.96 & 40.00 & 2201 . \\
\hline 1486 & 0.259 & 0.02933 & 316.855 & 0.1019 & 0.01543 & 1.130 & 2.325 & 4.96 & 40.00 & 2943. \\
\hline 1489 & 0.257 & 0.03827 & 317.209 & 0.1010 & 0.01541 & 0.770 & 3.033 & 4.96 & 40.00 & 3753. \\
\hline 1492 & 0.261 & 0.04839 & 317.596 & 0.1023 & 0.01540 & 0.550 & 3.830 & 4.96 & 40.00 & 4854 \\
\hline 1495 & 0.262 & 0.05968 & 318.024 & 0.1030 & 0.01535 & 0.560 & 4.729 & 4.96 & 40.00 & 6045 \\
\hline 1498 & 0.177 & 0.02157 & 316.482 & 0.0691 & 0.01542 & 1.910 & 1.718 & 3.36 & 40.00 & 954. \\
\hline 1501 & 0.179 & 0.02934 & 316.791 & 0.0695 & 0.01537 & 1.260 & 2.342 & 3.36 & 40.00 & 1314. \\
\hline 1504 & 0.180 & 0.03829 & 317.143 & 0.0701 & 0.01534 & 0.950 & 3.061 & 3.36 & 40.00 & 1741. \\
\hline 1507 & 0.180 & 0.04843 & 317.519 & 0.0697 & 0.01548 & 0.720 & 3.832 & 3.36 & 40.00 & 2147. \\
\hline 1510 & 0.178 & 0.05973 & 317.991 & 0.0689 & 0.01529 & 0.460 & 4.783 & 3.36 & 40.00 & 2595. \\
\hline 1513 & 0.090 & 0.02159 & 316.401 & 0.0347 & 0.01525 & 2.050 & 1.740 & 1.76 & 40.00 & 231. \\
\hline 1516 & 0.087 & 0.02936 & 316.758 & 0.0335 & 0.01504 & 1.320 & 2.399 & 1.76 & 40.00 & 296. \\
\hline 1519 & 0.090 & 0.03832 & 317.108 & 0.0344 & 0.01505 & 0.870 & 3.129 & 1.76 & 40.00 & 405. \\
\hline 1522 & 0.092 & 0.04845 & 317.519 & 0.0352 & 0.01507 & 0.630 & 3.949 & 1.76 & 40.00 & 534. \\
\hline
\end{tabular}


Table 32. Thermal conductivity of the ternary $30 \% \mathrm{R} 32$ / $10 \% \mathrm{R} 125$ / $60 \% \mathrm{R} 134 \mathrm{a}$ mixture in the dilute gas measured using the steady-state technique (continued).

\begin{tabular}{|c|c|c|c|c|c|c|c|c|c|c|}
\hline $\begin{array}{l}\text { Run } \\
\text { point }\end{array}$ & $\begin{array}{l}P_{\exp } \\
\mathrm{MPa}\end{array}$ & $\begin{array}{c}Q \\
\mathrm{w} \cdot \mathrm{m}^{-1}\end{array}$ & $\begin{array}{c}T_{\exp } \\
\mathrm{K}\end{array}$ & $\begin{array}{c}\rho_{\text {calc }} \\
\mathrm{mol} \cdot \mathrm{L}^{-1}\end{array}$ & $\begin{array}{c}\lambda_{\exp } \\
\mathrm{W} \cdot \mathrm{m}^{-1} \cdot \mathrm{K}^{-1}\end{array}$ & $\begin{array}{c}\text { TBAND } \\
\%\end{array}$ & $\begin{array}{c}\Delta T_{\text {avg }} \\
\mathrm{K}\end{array}$ & $\begin{array}{c}t_{\text {start }} \\
\mathrm{s}\end{array}$ & $\begin{array}{c}t_{\text {end }} \\
\mathrm{s}\end{array}$ & $N_{R a}$ \\
\hline 1525 & 0.089 & 0.05975 & 317.955 & 0.0341 & 0.01510 & 0.450 & 4.861 & 1.76 & 40.00 & 612. \\
\hline 1528 & 0.095 & 0.02154 & 327.975 & 0.0352 & 0.01589 & 2.040 & 1.667 & 1.76 & 40.00 & 204. \\
\hline 1531 & 0.093 & 0.02929 & 328.275 & 0.0344 & 0.01594 & 1.200 & 2.259 & 1.76 & 40.00 & 262. \\
\hline 1534 & 0.090 & 0.03824 & 328.584 & 0.0333 & 0.01600 & 0.800 & 2.938 & 1.76 & 40.00 & 319. \\
\hline 1537 & 0.093 & 0.04835 & 328.958 & $0: 0344$ & 0.01604 & 0.580 & 3.704 & 1.76 & 40.00 & 428. \\
\hline 1540 & 0.095 & 0.05964 & 329.381 & 0.0349 & 0.01608 & 0.430 & 4.556 & 1.76 & 40.00 & 539. \\
\hline 1543 & 0.178 & 0.02155 & 327.732 & 0.0666 & 0.01613 & 1.630 & 1.641 & 3.36 & 40.00 & 750 . \\
\hline 1546 & 0.177 & 0.02931 & 328.007 & 0.0663 & 0.01616 & 1.060 & 2.227 & 3.36 & 40.00 & 1007. \\
\hline 1549 & 0.178 & 0.03826 & 328.317 & 0.0668 & 0.01619 & 0.780 & 2.900 & 3.36 & 40.00 & 1326. \\
\hline 1552 & 0.181 & 0.04837 & 328.702 & 0.0679 & 0.01620 & 0.580 & 3.660 & 3.36 & 40.00 & 1725. \\
\hline 1555 & 0.183 & 0.05965 & 329.098 & 0.0683 & 0.01624 & 0.410 & 4.501 & 3.36 & 40.00 & 2139 . \\
\hline 1558 & 0.262 & 0.02153 & 325.711 & 0.1002 & 0.01649 & 2.220 & 1.601 & 3.36 & 40.00 & 1775 . \\
\hline 1561 & 0.260 & 0.02928 & 326.001 & 0.0992 & 0.01615 & 1.260 & 2.221 & 4.96 & 40.00 & 2403. \\
\hline 1564 & 0.262 & 0.03822 & 326.327 & 0.1000 & 0.01616 & 1.120 & 2.892 & 3.36 & 40.00 & 3169. \\
\hline 1567 & 0.265 & 0.04832 & 326.694 & 0.1009 & 0.01616 & 0.590 & 3.651 & 4.96 & 40.00 & 4061. \\
\hline 1570 & 0.262 & 0.05960 & 327.120 & 0.0994 & 0.01606 & 0.460 & 4.525 & 4.96 & 40.00 & 4853. \\
\hline 1573 & 0.355 & 0.02143 & 325.553 & 0.1373 & 0.01611 & 1.920 & 1.626 & 4.96 & 40.00 & 3575 . \\
\hline 1576 & 0.351 & 0.02915 & 325.828 & 0.1357 & 0.01613 & 1.310 & 2.203 & 4.96 & 40.00 & 4703. \\
\hline 1579 & 0.351 & 0.03804 & 326.156 & 0.1355 & 0.01615 & 1.080 & 2.866 & 4.96 & 40.00 & 6078. \\
\hline 1582 & 0.355 & 0.04811 & 326.517 & 0.1370 & 0.01616 & 0.770 & 3.610 & 4.96 & 40.00 & 7812 . \\
\hline 1585 & 0.358 & 0.05934 & 326.926 & 0.1378 & 0.01616 & 0.600 & 4.437 & 4.96 & 40.00 & 9677. \\
\hline 1588 & 0.429 & 0.02145 & 325.196 & 0.1677 & 0.01624 & 2.080 & 1.608 & 6.56 & 40.00 & 5530 . \\
\hline 1591 & 0.431 & 0.02917 & 325.492 & 0.1685 & 0.01622 & 1.470 & 2.182 & 6.56 & 40.00 & 7550 . \\
\hline 1594 & 0.434 & 0.03807 & 325.802 & 0.1694 & 0.01620 & 0.990 & 2.838 & 6.56 & 40.00 & 9913. \\
\hline 1597 & 0.433 & 0.04814 & 326.162 & 0.1689 & 0.01621 & 0.780 & 3.571 & 6.56 & 40.00 & 12328. \\
\hline 1600 & 0.429 & 0.05938 & 326.553 & 0.1671 & 0.01626 & 0.750 & 4.374 & 4.96 & 40.00 & 14688. \\
\hline 1603 & 0.507 & 0.02145 & 325.060 & 0.2009 & 0.01636 & 1.700 & 1.589 & 8.16 & 40.00 & 8230 . \\
\hline 1606 & 0.512 & 0.02918 & 325.345 & 0.2027 & 0.01622 & 1.930 & 2.167 & 6.56 & 40.00 & 11421. \\
\hline 1609 & 0.515 & 0.03807 & 325.654 & 0.2037 & 0.01627 & 0.820 & 2.801 & 6.56 & 40.00 & 14866. \\
\hline 1612 & 0.515 & 0.04815 & 325.994 & 0.2032 & 0.01634 & 0.900 & 3.506 & 4.96 & 40.00 & 18437. \\
\hline 1615 & 0.511 & 0.05939 & 326.372 & 0.2016 & 0.01641 & 1.420 & 4.278 & 3.36 & 40.00 & 21978 . \\
\hline 1618 & 0.532 & 0.02137 & 337.264 & 0.2017 & 0.01760 & 2.150 & 1.475 & 6.56 & 40.00 & 6680. \\
\hline 1621 & 0.533 & 0.02907 & 337.498 & 0.2020 & 0.01744 & 1.410 & 2.017 & 8.16 & 40.00 & 9136. \\
\hline 1624 & 0.534 & 0.03794 & 337.796 & 0.2022 & 0.01742 & 0.980 & 2.622 & 6.56 & 40.00 & 11873. \\
\hline 1627 & 0.535 & 0.04799 & 338.093 & 0.2022 & 0.01742 & 0.670 & 3.299 & 6.56 & 40.00 & 14884. \\
\hline 1630 & 0.534 & 0.05920 & 338.466 & 0.2016 & 0.01731 & 0.780 & 4.070 & 4.96 & 40.00 & 18157. \\
\hline 1633 & 0.441 & 0.02139 & 337.043 & 0.1656 & 0.01756 & 2.050 & 1.486 & 6.56 & 40.00 & 4349. \\
\hline 1636 & 0.441 & 0.02909 & 337.299 & 0.1653 & 0.01750 & 1.520 & 2.023 & 6.56 & 40.00 & 5877. \\
\hline 1639 & 0.442 & 0.03796 & 337.597 & 0.1656 & 0.01720 & 1.020 & 2.676 & 6.56 & 40.00 & 7781. \\
\hline 1642 & 0.443 & 0.04801 & 337.915 & 0.1658 & 0.01722 & 0.770 & 3.368 & 6.56 & 40.00 & 9787. \\
\hline 1645 & 0.443 & 0.05921 & 338.301 & 0.1655 & 0.01723 & 0.560 & 4.136 & 6.56 & 40.00 & 11912. \\
\hline 1648 & 0.353 & 0.02141 & 336.516 & 0.1315 & 0.01737 & 2.090 & 1.509 & 4.96 & 40.00 & 2678. \\
\hline 1651 & 0.355 & 0.02912 & 336.785 & 0.1319 & 0.01718 & 1.280 & 2.071 & 4.96 & 40.00 & 3695. \\
\hline
\end{tabular}


Table 32. Thermal conductivity of the ternary $30 \% \mathrm{R} 32$ / $10 \% \mathrm{R} 125$ / $60 \% \mathrm{R} 134 \mathrm{a}$ mixture in the dilute gas measured using the steady-state technique (continued).

\begin{tabular}{|c|c|c|c|c|c|c|c|c|c|c|}
\hline $\begin{array}{l}\text { Run } \\
\text { point }\end{array}$ & $\begin{array}{l}P_{\text {exp }} \\
\mathrm{MPa}\end{array}$ & $\begin{array}{c}Q \\
W \cdot \mathrm{m}^{-1}\end{array}$ & $\begin{array}{c}T_{\exp } \\
\mathrm{K}\end{array}$ & $\begin{array}{c}\rho_{\text {calc }} \\
\mathrm{mol} \cdot \mathrm{L}^{-1}\end{array}$ & $\begin{array}{c}\lambda_{\exp } \\
\mathrm{W} \cdot \mathrm{m}^{-1} \cdot \mathrm{K}^{-1}\end{array}$ & $\begin{array}{c}\text { TBAND } \\
\%\end{array}$ & $\begin{array}{c}\Delta T_{\text {avg }} \\
\mathrm{K}\end{array}$ & $\begin{array}{c}t_{\text {start }} \\
\mathrm{s}\end{array}$ & $\begin{array}{c}t_{\text {end }} \\
\mathrm{s}\end{array}$ & $N_{R a}$ \\
\hline 1654 & 0.355 & 0.03801 & 337.090 & 0.1320 & 0.01700 & 0.930 & 2.727 & 4.96 & 40.00 & 4858. \\
\hline 1657 & 0.354 & 0.04808 & 337.412 & 0.1314 & 0.01705 & 0.820 & 3.430 & 4.96 & 40.00 & 6029 \\
\hline 1660 & 0.353 & 0.05931 & 337.784 & 0.1308 & 0.01707 & 0.550 & 4.216 & 4.96 & 40.00 & 7300 \\
\hline 1663 & 0.255 & 0.02143 & 336.309 & 0.0937 & 0.01703 & 1.700 & 1.544 & 3.36 & 40.00 & 1331. \\
\hline 1666 & 0.253 & 0.02915 & 336.577 & 0.0931 & 0.01708 & 1.210 & 2.093 & 3.36 & 40.00 & 1774 . \\
\hline 1669 & 0.253 & 0.03805 & 336.858 & 0.0929 & 0.01711 & 0.960 & 2.724 & 3.36 & 40.00 & 2290 . \\
\hline 1672 & 0.254 & 0.04811 & 337.190 & 0.0933 & 0.01712 & 0.860 & 3.438 & 3.36 & 40.00 & 2909. \\
\hline 1675 & 0.255 & 0.05934 & 337.561 & 0.0933 & 0.01715 & 0.730 & 4.229 & 3.36 & 40.00 & 3569 . \\
\hline 1678 & 0.176 & 0.02145 & 334.949 & 0.0645 & 0.01681 & 1.840 & 1.568 & 3.36 & 40.00 & 624. \\
\hline 1681 & 0.177 & 0.02917 & 335.209 & 0.0649 & 0.01685 & 1.170 & 2.126 & 3.36 & 40.00 & 856. \\
\hline 1684 & 0.179 & 0.03808 & 335.514 & 0.0654 & 0.01688 & 0.920 & 2.769 & 3.36 & 40.00 & 1130. \\
\hline 1687 & 0.178 & 0.04816 & 335.870 & 0.0651 & 0.01677 & 0.700 & 3.524 & 3.36 & 40.00 & 1417. \\
\hline 1690 & 0.177 & 0.05940 & 336.267 & 0.0644 & 0.01682 & 0.410 & 4.330 & 3.36 & 40.00 & 1699. \\
\hline 1693 & 0.089 & 0.02147 & 334.745 & 0.0324 & 0.01659 & 2.140 & 1.591 & 1.76 & 40.00 & 154. \\
\hline 1696 & 0.090 & 0.02919 & 335.004 & 0.0327 & 0.01652 & 1.140 & 2.173 & 1.76 & 40.00 & 213. \\
\hline 1699 & 0.092 & 0.03810 & 335.316 & 0.0332 & 0.01658 & 0.870 & 2.826 & 1.76 & 40.00 & 285. \\
\hline 1702 & 0.091 & 0.04819 & 335.658 & 0.0328 & 0.01662 & 0.570 & 3.563 & 1.76 & 40.00 & 350. \\
\hline 1705 & 0.090 & 0.05943 & 336.056 & 0.0324 & 0.01668 & 0.440 & 4.380 & 1.76 & 40.00 & 419. \\
\hline 1708 & 0.093 & 0.02119 & 344.361 & 0.0327 & 0.01756 & 2.020 & 1.484 & 1.76 & 40.00 & 134. \\
\hline 1711 & 0.092 & 0.02884 & 344.603 & 0.0323 & 0.01762 & 1.340 & 2.012 & 1.76 & 40.00 & 177. \\
\hline 1714 & 0.093 & 0.03764 & 344.892 & 0.0327 & 0.01745 & 0.890 & 2.653 & 1.76 & 40.00 & 238. \\
\hline 1717 & 0.094 & 0.04761 & 345.219 & 0.0331 & 0.01739 & 0.580 & 3.365 & 1.76 & 40.00 & 308. \\
\hline 1720 & 0.093 & 0.05873 & 345.578 & 0.0327 & 0.01747 & 0.460 & 4.131 & 1.76 & 40.00 & 368. \\
\hline 1723 & 0.186 & 0.02122 & 344.036 & 0.0663 & 0.01805 & 2.470 & 1.445 & 1.76 & 40.00 & 559. \\
\hline 1726 & 0.188 & 0.02886 & 344.281 & 0.0669 & 0.01777 & 1.340 & 1.995 & 3.36 & 40.00 & 786. \\
\hline 1729 & 0.188 & 0.03767 & 344.556 & 0.0667 & 0.01775 & 0.880 & 2.606 & 3.36 & 40.00 & 1017. \\
\hline 1732 & 0.186 & 0.04764 & 344.879 & 0.0662 & 0.01779 & 0.660 & 3.286 & 3.36 & 40.00 & 1257. \\
\hline 1735 & 0.186 & 0.05877 & 345.249 & 0.0660 & 0.01765 & 0.460 & 4.084 & 3.36 & 40.00 & 1546 . \\
\hline 1738 & 0.268 & 0.02124 & 343.702 & 0.0964 & 0.01794 & 2.370 & 1.453 & 3.36 & 40.00 & 1237. \\
\hline 1741 & 0.269 & 0.02889 & 343.927 & 0.0969 & 0.01798 & 1.520 & 1.971 & 3.36 & 40.00 & 1691. \\
\hline 1744 & 0.269 & 0.03771 & 344.220 & 0.0968 & 0.01779 & 1.060 & 2.597 & 3.36 & 40.00 & 2216 . \\
\hline 1747 & 0.268 & 0.04769 & 344.562 & 0.0962 & 0.01759 & 0.710 & 3.318 & 3.36 & 40.00 & 2789. \\
\hline 1750 & 0.268 & 0.05883 & 344.915 & 0.0961 & 0.01767 & 0.820 & 4.070 & 3.36 & 40.00 & 3399. \\
\hline 1753 & 0.353 & 0.02128 & 343.082 & 0.1286 & 0.01775 & 2.190 & 1.469 & 4.96 & 40.00 & 2326 . \\
\hline 1756 & 0.353 & 0.02894 & 343.299 & 0.1282 & 0.01781 & 1.370 & 1.987 & 4.96 & 40.00 & 3116. \\
\hline 1759 & 0.351 & 0.03776 & 343.587 & 0.1276 & 0.01779 & 0.990 & 2.592 & 4.96 & 40.00 & 4012 . \\
\hline 1762 & 0.352 & 0.04776 & 343.901 & 0.1277 & 0.01767 & 0.800 & 3.294 & 4.96 & 40.00 & 5096. \\
\hline 1765 & 0.353 & 0.05892 & 344.279 & 0.1281 & 0.01761 & 0.660 & 4.069 & 4.96 & 40.00 & 6313. \\
\hline 1768 & 0.434 & 0.02129 & 342.741 & 0.1595 & 0.01789 & 2.130 & 1.454 & 6.56 & 40.00 & 3684. \\
\hline 1771 & 0.432 & 0.02897 & 342.956 & 0.1588 & 0.01795 & 1.630 & 1.967 & 4.96 & 40.00 & 4923. \\
\hline 1774 & 0.433 & 0.03781 & 343.244 & 0.1587 & 0.01782 & 1.050 & 2.579 & 6.56 & 40.00 & 6432. \\
\hline 1777 & 0.434 & 0.04782 & 343.559 & 0.1592 & 0.01766 & 0.990 & 3.282 & 4.96 & 40.00 & 8217. \\
\hline 1780 & 0.433 & 0.05899 & 343.928 & 0.1587 & 0.01767 & 0.770 & 4.032 & 4.96 & 40.00 & 9986. \\
\hline
\end{tabular}


Table 32. Thermal conductivity of the ternary $30 \% \mathrm{R} 32$ / $10 \% \mathrm{R} 125$ / $60 \% \mathrm{R} 134$ a mixture in the dilute gas measured using the steady-state technique (continued).

\begin{tabular}{|ccccccc|cccc|}
\hline $\begin{array}{c}\text { Run } \\
\text { point }\end{array}$ & $\begin{array}{c}P_{\text {exp }} \\
\mathrm{MPa}\end{array}$ & $\begin{array}{c}Q \\
\mathrm{~W} \cdot \mathrm{m}^{-1}\end{array}$ & $\begin{array}{c}T_{\text {exp }} \\
\mathrm{K}\end{array}$ & $\begin{array}{c}\rho_{\text {calc }} \\
\mathrm{mol} \cdot \mathrm{L}^{-1}\end{array}$ & $\begin{array}{c}\lambda_{\text {exp }} \\
\mathrm{W} \cdot \mathrm{m}^{-1} \cdot \mathrm{K}^{-1}\end{array}$ & $\begin{array}{c}\text { TBAND } \\
\%\end{array}$ & \multicolumn{1}{c}{$\Delta T_{\text {avg }}$} & $t_{\text {start }}$ & $t_{\text {end }}$ & $N_{\text {Ra }}$ \\
\hline 1783 & 0.522 & 0.02132 & 342.452 & 0.1940 & 0.01796 & 1.930 & 1.445 & 8.16 & 40.00 & 5661. \\
1786 & 0.521 & 0.02899 & 342.671 & 0.1935 & 0.01795 & 1.490 & 1.959 & 6.56 & 40.00 & 7610. \\
1789 & 0.519 & 0.03784 & 342.977 & 0.1927 & 0.01756 & 1.070 & 2.603 & 6.56 & 40.00 & 9989. \\
1792 & 0.519 & 0.04786 & 343.280 & 0.1925 & 0.01766 & 0.940 & 3.259 & 4.96 & 40.00 & 12437. \\
1795 & 0.521 & 0.05904 & 343.610 & 0.1930 & 0.01775 & 0.760 & 3.980 & 4.96 & 40.00 & 15220. \\
\hline
\end{tabular}


